\title{
Site Environmental Report for 2001
}

\author{
Volume II
}

August 2002

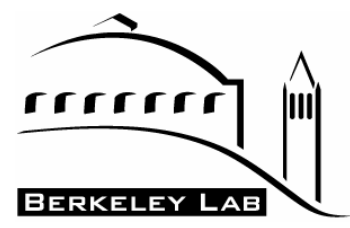

Ernest Orlando Lawrence Berkeley National Laboratory

Prepared for the U.S. Department of Energy under Contract Number DE-AC03-76SF00098 


\section{Contents}

\section{Volume I}

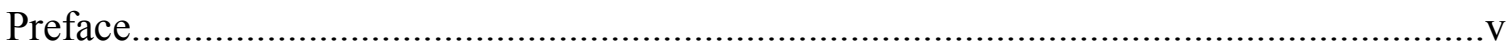

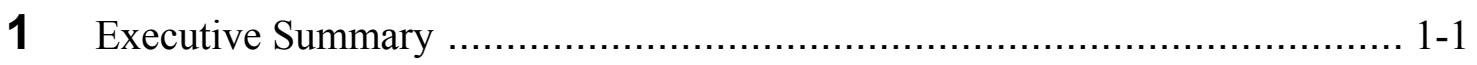

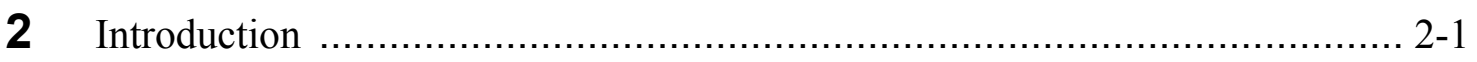

3 Environmental Program Summary f........................................................... 3-1

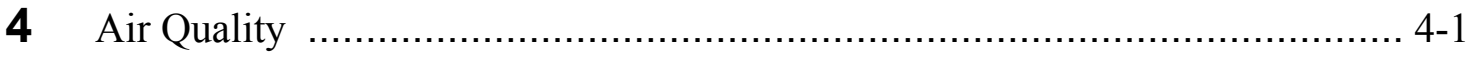

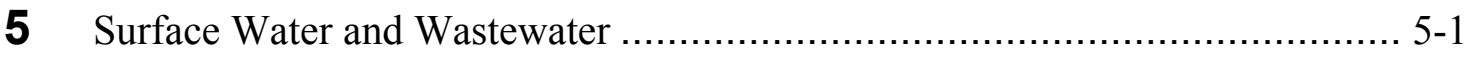

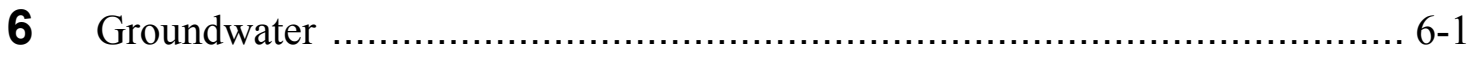

$7 \quad$ Soil and Sediment ................................................................................ $7-1$

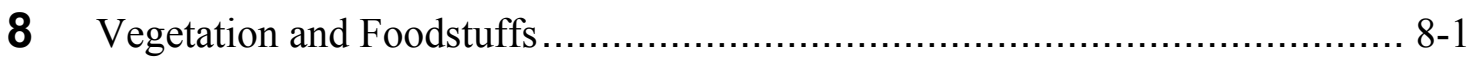

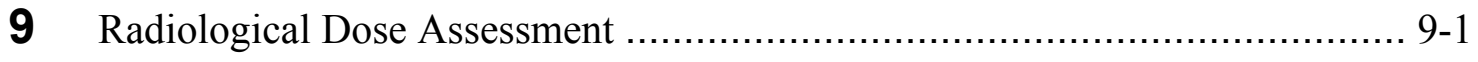

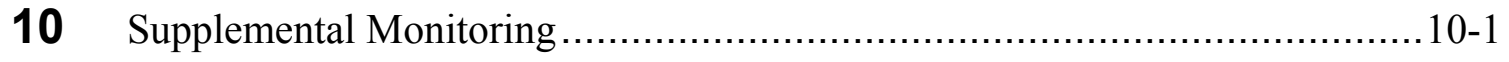

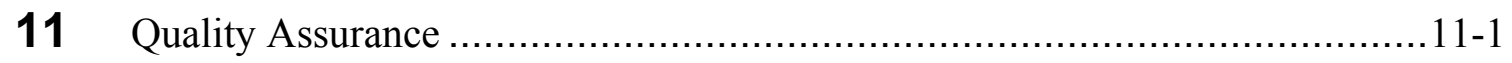

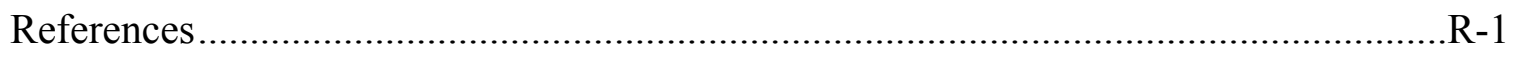

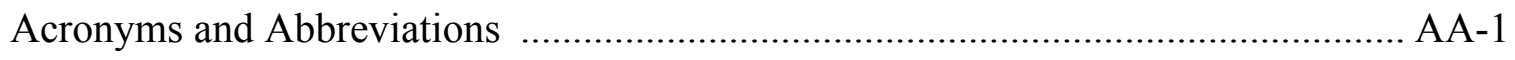

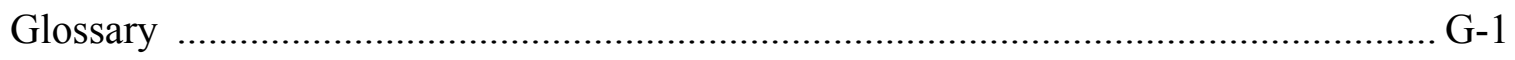

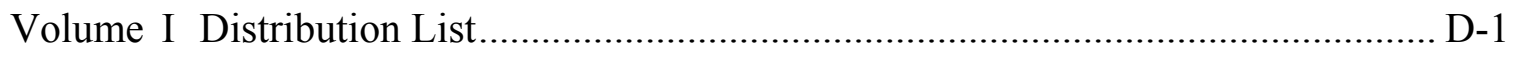

\section{Volume II}

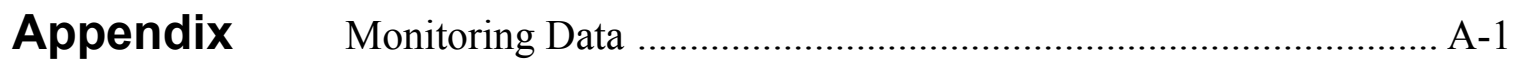

Stack Air .............................................................. A-9 
Ambient Air A-67

Rainwater A-77

Creeks A-81

Lakes A-101

Stormwater A-103

Sewer A-115

Fixed Treatment Units A-143

Soil A-159

Sediment A-177

Vegetation A-179 


\section{Appendix Monitoring Data}

Volume II of the Site Environmental Report for 2001 is provided by Ernest Orlando Lawrence Berkeley National Laboratory as a supplemental appendix to the report printed in Volume I. Volume II contains the environmental monitoring and sampling data used to generate summary results in the main report for routine and nonroutine activities at the Laboratory (except for groundwater sampling data, which may be found in the reports referred to in Chapter 6). For completeness, results from sample collections beginning or ending in CY 2001 are included in this volume but samples representing CY 2000 data are not used in summary results reported in Volume 1 (for example, Ambient Air samples collected on January 9, 2001, represent December 2000 data and are not included in Tables 4-5 and 4-6). Data presented in the tables are given in International System of Units (SI) units of measure.

The list below categorizes the Volume II data sections with corresponding summary result tables in Volume I:

\begin{tabular}{|c|c|c|}
\hline Volume II section & Vo & ume I summary tables \\
\hline Stack Air & $4-4$ & Summary of Radiological Air Emissions \\
\hline \multirow[t]{3}{*}{ Ambient Air } & $4-5$ & Summary of Ambient Tritium Sampling \\
\hline & $4-6$ & $\begin{array}{l}\text { Summary of Gross Alpha and Gross Beta Ambient Air } \\
\text { Particulate Sampling Network Results }\end{array}$ \\
\hline & $10-1$ & Summary of Supplemental Ambient Tritium Sampling \\
\hline Rainwater & & No summary table; results discussed in Section 5.2.1 \\
\hline Creeks & & No summary table; results discussed in Section 5.2 .2 \\
\hline Lakes & & No summary table; results discussed in Section 5.2.3 \\
\hline Stormwater & & No summary table; results discussed in Section 5.2.4 \\
\hline Sewer & & No summary table; results discussed in Section 5.4.1 \\
\hline Fixed Treatment Units & & $\begin{array}{l}\text { No summary table; results discussed in Sections } \\
5.4 .2-5.4 .3\end{array}$ \\
\hline Soil & $7-1$ & $\begin{array}{l}\text { Metals and Oil/Grease Results in Soil and Sediment } \\
\text { Sampling }\end{array}$ \\
\hline Sediment & $7-1$ & $\begin{array}{l}\text { Metals and Oil/Grease Results in Soil and Sediment } \\
\text { Sampling }\end{array}$ \\
\hline Vegetation & & No summary table; results discussed in Section 8.2 \\
\hline
\end{tabular}


The results listed in Volume II reference sampling locations with a station identifier code. The following list cross-references these codes with a more meaningful and descriptive label:

\begin{tabular}{|c|c|c|}
\hline Location code & Description of sampling location & Volume II section \\
\hline $1-216 \mathrm{H}$ & Building 1, Room 216 hood & Stack Air \\
\hline $1-267 \mathrm{H}$ & Building 1, Room 267 hood & Stack Air \\
\hline $1-373 \mathrm{H}$ & Building 1, Room 373 hood & Stack Air \\
\hline 25 FTU & Building 25 fixed treatment unit & $\begin{array}{l}\text { Fixed Treatment } \\
\text { Units }\end{array}$ \\
\hline $55-128$ & Building 55, Room 128 & Stack Air \\
\hline 69-Storm Drain & Building 69 storm drain inlet & Stormwater \\
\hline $70-103 \mathrm{H}$ & Building 70, Room 103 hood & Stack Air \\
\hline $70-147 A$ & Building 70, Room 147A Berkeley box manifold & Stack Air \\
\hline $70-157 \mathrm{H}$ & Building 70, Room $157 \mathrm{H}$ & Stack Air \\
\hline $70-203 \mathrm{H}$ & Building 70, Room 203 hood & Stack Air \\
\hline $70 A-1129 B$ & Building 70A, Room 1129B & Stack Air \\
\hline $70 \mathrm{~A}-1129 \mathrm{H}$ & Building 70A, Room 1129 hood & Stack Air \\
\hline 70A-1129P & $\begin{array}{l}\text { Building } 70 \mathrm{~A} \text {, Room } 1129 \text { pressurized box } \\
\text { manifold }\end{array}$ & Stack Air \\
\hline $70 A-1145$ & $\begin{array}{l}\text { Building } 70 \mathrm{~A} \text {, Room } 1145 \text { Berkeley box } \\
\text { manifold }\end{array}$ & Stack Air \\
\hline $70 \mathrm{~A}-2211 \mathrm{H}$ & Building 70A, Room 2211 hood & Stack Air \\
\hline $70 \mathrm{~A}-2217 \mathrm{H}$ & Building 70A, Room 2217 hood & Stack Air \\
\hline $70 A-2275$ & $\begin{array}{l}\text { Building } 70 \mathrm{~A} \text {, Room } 2275 \text { Berkeley box } \\
\text { manifold }\end{array}$ & Stack Air \\
\hline 75 NTLF-HTO & $\begin{array}{l}\text { Building } 75 \text {, National Tritium Labeling Facility; } \\
\text { tritiated water vapor (HTO) }\end{array}$ & Stack Air \\
\hline 75 NTLF-Total T & $\begin{array}{l}\text { Building } 75 \text {, National Tritium Labeling Facility, } \\
\text { total tritium (HT + HTO) }\end{array}$ & Stack Air \\
\hline 75 Stack Sump & Sump at the base of the NTLF Stack & Stack Air \\
\hline $75-107 \mathrm{H}$ & Building 75, Room 107 hood & Stack Air \\
\hline 75-Locker & Building 75 , storage locker north of Building & Stack Air \\
\hline 75A-Temp & Building $75 \mathrm{~A}$ temporary hood & Stack Air \\
\hline 75D-SEA & Building 75D sample exchange area & Stack Air \\
\hline 77 FTU & Building 77 fixed treatment unit & $\begin{array}{l}\text { Fixed Treatment } \\
\text { Units }\end{array}$ \\
\hline 85 Glovebox & Building 85 (HWHF) penthouse glovebox & Stack Air \\
\hline 85 Hood & Building 85 (HWHF) penthouse hood & Stack Air \\
\hline
\end{tabular}




\begin{tabular}{|c|c|c|}
\hline Location code & Description of sampling location & Volume II section \\
\hline B75-Tree X & Tree sampling around Building 75 & Vegetation \\
\hline B88 Cave 0 & Building 88, Cave 0 & Stack Air \\
\hline $\mathrm{B} 88-135 \mathrm{H}$ & Building 88, Room 135 hood & Stack Air \\
\hline Banana Creek & Banana Creek & $\begin{array}{l}\text { Supplemental } \\
\text { Monitoring }\end{array}$ \\
\hline $\begin{array}{l}\text { Botanical } \\
\text { Garden Creek }\end{array}$ & Botanical Garden Creek & Creeks; Sediment \\
\hline Building 50 & East of Building 50 & Soil; Sediment \\
\hline Building 69 & North side of Building 69 & Soil; Sediment \\
\hline Building 85 & Northeast of Building 85 & Soil; Sediment \\
\hline Cafeteria Creek & Routine sampling at Cafeteria Creek & Creeks \\
\hline $\begin{array}{l}\text { Cafeteria Creek } \\
\text { (Lower) }\end{array}$ & $\begin{array}{l}\text { Special site at Cafeteria Creek for } \\
\text { supplemental monitoring (see Chapter 10) }\end{array}$ & $\begin{array}{l}\text { Supplemental } \\
\text { Monitoring }\end{array}$ \\
\hline $\begin{array}{l}\text { Cafeteria Creek } \\
\text { (Upper) }\end{array}$ & $\begin{array}{l}\text { Special site at Cafeteria Creek for } \\
\text { supplemental monitoring (see Chapter 10) }\end{array}$ & $\begin{array}{l}\text { Supplemental } \\
\text { Monitoring }\end{array}$ \\
\hline Chicken Creek & Routine sampling at Chicken Creek & $\begin{array}{l}\text { Creeks; } \\
\text { Stormwater }\end{array}$ \\
\hline $\begin{array}{l}\text { Chicken Creek } \\
\text { (Lower) }\end{array}$ & $\begin{array}{l}\text { Special site at Chicken Creek for supplemental } \\
\text { monitoring (see Chapter 10) }\end{array}$ & $\begin{array}{l}\text { Supplemental } \\
\text { Monitoring }\end{array}$ \\
\hline $\begin{array}{l}\text { Chicken Creek- } \\
\text { Main }\end{array}$ & Chicken Creek & Sediment \\
\hline $\begin{array}{l}\text { Chicken Creek- } \\
\text { Trib }\end{array}$ & Chicken Creek Tributary & Sediment \\
\hline $\begin{array}{l}\text { Chicken Creek } \\
\text { (Upper) }\end{array}$ & $\begin{array}{l}\text { Special site at Chicken Creek for supplemental } \\
\text { monitoring (see Chapter 10) }\end{array}$ & $\begin{array}{l}\text { Supplemental } \\
\text { Monitoring }\end{array}$ \\
\hline Claremont Creek & Claremont Creek & Creeks \\
\hline East Canyon & $\begin{array}{l}\text { Between Hazardous Waste Handling Facility } \\
\text { and Centennial Drive }\end{array}$ & Stormwater \\
\hline EEE6-X & $\begin{array}{l}\text { Vegetation sampling in Eucalyptus grove near } \\
\text { Grizzly Peak Gate }\end{array}$ & $\begin{array}{l}\text { Supplemental } \\
\text { Monitoring }\end{array}$ \\
\hline EG-RG-X & $\begin{array}{l}\text { Rain Gauges in Eucalyptus grove between } \\
\text { NTLF Stack and Lawrence Hall of Science }\end{array}$ & Rainwater \\
\hline ENV-31 & Corporation Yard & $\begin{array}{l}\text { Ambient Air; } \\
\text { Supplemental } \\
\text { Monitoring }\end{array}$ \\
\hline ENV-44 & Weather Tower & $\begin{array}{l}\text { Ambient Air; } \\
\text { Supplemental } \\
\text { Monitoring }\end{array}$ \\
\hline
\end{tabular}




\begin{tabular}{|c|c|c|}
\hline Location code & Description of sampling location & Volume II section \\
\hline ENV-69 & Roof of Building 69 & $\begin{array}{l}\text { Ambient Air; } \\
\text { Supplemental } \\
\text { Monitoring }\end{array}$ \\
\hline ENV-75 & Roof of Building 75 & Rainwater \\
\hline ENV-75EG & $\begin{array}{l}\text { Eucalyptus grove between NTLF Stack and } \\
\text { Lawrence Hall of Science }\end{array}$ & $\begin{array}{l}\text { Ambient Air; } \\
\text { Supplemental } \\
\text { Monitoring }\end{array}$ \\
\hline ENV-77 & Between Buildings 77 and 79 & $\begin{array}{l}\text { Ambient Air; } \\
\text { Supplemental } \\
\text { Monitoring }\end{array}$ \\
\hline ENV-78 & East end of Building 78 & $\begin{array}{l}\text { Ambient Air; } \\
\text { Supplemental } \\
\text { Monitoring }\end{array}$ \\
\hline ENV-80 & Roof of Building 80 & $\begin{array}{l}\text { Ambient Air; } \\
\text { Supplemental } \\
\text { Monitoring }\end{array}$ \\
\hline ENV-81 & East of Building 81 & $\begin{array}{l}\text { Ambient Air; } \\
\text { Supplemental } \\
\text { Monitoring }\end{array}$ \\
\hline ENV-85 & East of Building 85 & $\begin{array}{l}\text { Ambient Air; } \\
\text { Supplemental } \\
\text { Monitoring }\end{array}$ \\
\hline ENV-AR & Amito Reservoir & $\begin{array}{l}\text { Ambient Air; } \\
\text { Supplemental } \\
\text { Monitoring }\end{array}$ \\
\hline ENV-B13A & Sampling shelter west of Building 88 & $\begin{array}{l}\text { Ambient Air; } \\
\text { Supplemental } \\
\text { Monitoring }\end{array}$ \\
\hline ENV-B13C & $\begin{array}{l}\text { Background sampling shelter off Panoramic } \\
\text { Way }\end{array}$ & $\begin{array}{l}\text { Ambient Air; } \\
\text { Rainwater; Soil; } \\
\text { Supplemental } \\
\text { Monitoring }\end{array}$ \\
\hline ENV-B13D & $\begin{array}{l}\text { Sampling shelter northwest of Lawrence Hall } \\
\text { of Science }\end{array}$ & $\begin{array}{l}\text { Ambient Air; } \\
\text { Rainwater; } \\
\text { Supplemental } \\
\text { Monitoring }\end{array}$ \\
\hline ENV-LHS & Lawrence Hall of Science & $\begin{array}{l}\text { Ambient Air; } \\
\text { Supplemental } \\
\text { Monitoring }\end{array}$ \\
\hline
\end{tabular}




\begin{tabular}{|c|c|c|}
\hline Location code & Description of sampling location & Volume II section \\
\hline ENV-MSRI & UC Berkeley Math Science Research Institute & $\begin{array}{l}\text { Ambient Air; } \\
\text { Supplemental } \\
\text { Monitoring }\end{array}$ \\
\hline ENV-SSL & UC Berkeley Space Science Laboratory & $\begin{array}{l}\text { Ambient Air; } \\
\text { Supplemental } \\
\text { Monitoring }\end{array}$ \\
\hline ENV-UCBG & UC Berkeley Botanical Gardens & $\begin{array}{l}\text { Ambient Air; } \\
\text { Supplemental } \\
\text { Monitoring }\end{array}$ \\
\hline Field Blank & Blank sample prepared in the field & $\begin{array}{l}\text { Creeks; Fixed } \\
\text { Treatment Units; } \\
\text { Lakes; } \\
\text { Rainwater; } \\
\text { Sewer; } \\
\text { Supplemental } \\
\text { Monitoring }\end{array}$ \\
\hline Hearst Sewer & Hearst sewer station & Sewer \\
\hline Lake Anza & Lake Anza in Tilden Park & $\begin{array}{l}\text { Lakes; } \\
\text { Supplemental } \\
\text { Monitoring }\end{array}$ \\
\hline Lake Temescal & $\begin{array}{l}\text { Lake Temescal near Highways } 13 \text { and } 24 \text { in } \\
\text { Oakland }\end{array}$ & $\begin{array}{l}\text { Lakes; } \\
\text { Supplemental } \\
\text { Monitoring }\end{array}$ \\
\hline $\begin{array}{l}\text { N. Fork } \\
\text { Strawberry } \\
\text { Creek }\end{array}$ & $\begin{array}{l}\text { North Fork of Strawberry Creek outlet near } \\
\text { western boundary of site }\end{array}$ & $\begin{array}{l}\text { Creeks; } \\
\text { Stormwater }\end{array}$ \\
\hline $\begin{array}{l}\text { N. Fork } \\
\text { Strawberry } \\
\text { Creek (Lower) }\end{array}$ & $\begin{array}{l}\text { Special site at North Fork of Strawberry Creek } \\
\text { for supplemental monitoring (see Chapter } \\
\text { 10) }\end{array}$ & $\begin{array}{l}\text { Supplemental } \\
\text { Monitoring }\end{array}$ \\
\hline $\begin{array}{l}\text { N. Fork } \\
\text { Strawberry- } \\
\text { Main }\end{array}$ & $\begin{array}{l}\text { North Fork of Strawberry Creek outlet near } \\
\text { western boundary of site }\end{array}$ & Sediment \\
\hline $\begin{array}{l}\text { N. Fork } \\
\text { Strawberry- } \\
\text { Trib }\end{array}$ & North Fork of Strawberry Creek outlet tributary & Sediment \\
\hline $\begin{array}{l}\text { N. Fork } \\
\text { Strawberry } \\
\text { Creek (Upper) }\end{array}$ & $\begin{array}{l}\text { Special site at North Fork of Strawberry Creek } \\
\text { for supplemental monitoring (see Chapter } \\
\text { 10) }\end{array}$ & $\begin{array}{l}\text { Supplemental } \\
\text { Monitoring }\end{array}$ \\
\hline NEE10-X & Vegetation sampling in Tilden Park & $\begin{array}{l}\text { Supplemental } \\
\text { Monitoring }\end{array}$ \\
\hline
\end{tabular}




\begin{tabular}{|c|c|c|}
\hline Location code & Description of sampling location & Volume II section \\
\hline NNN5-X & $\begin{array}{l}\text { Vegetation sampling in Eucalyptus grove } \\
\text { between NTLF Stack and Lawrence Hall of } \\
\text { Science }\end{array}$ & $\begin{array}{c}\text { Supplemental } \\
\text { Monitoring }\end{array}$ \\
\hline NNW1-X & $\begin{array}{l}\text { Vegetation sampling in Eucalyptus grove } \\
\text { between NTLF Stack and Lawrence Hall of } \\
\text { Science }\end{array}$ & $\begin{array}{l}\text { Supplemental } \\
\text { Monitoring }\end{array}$ \\
\hline NNW2-X & $\begin{array}{l}\text { Vegetation sampling in Eucalyptus grove } \\
\text { between NTLF Stack and Lawrence Hall of } \\
\text { Science }\end{array}$ & $\begin{array}{l}\text { Supplemental } \\
\text { Monitoring }\end{array}$ \\
\hline NNW3-X & Vegetation sampling below LHS parking lot & $\begin{array}{l}\text { Supplemental } \\
\text { Monitoring }\end{array}$ \\
\hline No Name Creek & Routine sampling at No Name Creek & Creeks \\
\hline $\begin{array}{l}\text { No Name Creek } \\
\quad \text { (Lower) }\end{array}$ & $\begin{array}{l}\text { Special site at No Name Creek for } \\
\text { supplemental monitoring (see Chapter 10) }\end{array}$ & $\begin{array}{l}\text { Supplemental } \\
\text { Monitoring }\end{array}$ \\
\hline $\begin{array}{l}\text { No Name Creek } \\
\quad \text { (Upper) }\end{array}$ & $\begin{array}{l}\text { Special site at No Name Creek for } \\
\text { supplemental monitoring (see Chapter 10) }\end{array}$ & $\begin{array}{l}\text { Supplemental } \\
\text { Monitoring }\end{array}$ \\
\hline $\begin{array}{l}\text { NTLF-Hillside } \\
\text { Stack Drain }\end{array}$ & NTLF Stack drain line & Stack Air \\
\hline Pineapple Creek & Pineapple Creek & $\begin{array}{l}\text { Supplemental } \\
\text { Monitoring }\end{array}$ \\
\hline PMB1a1 & $\begin{array}{l}\text { Portion of a core sample from a Eucalyptus } \\
\text { tree approximately } 20 \mathrm{~m} \text { northwest of NTLF } \\
\text { Stack, containing rings approximately pre- } \\
1986\end{array}$ & Vegetation \\
\hline PMB1a2 & $\begin{array}{l}\text { Portion of a core sample from a Eucalyptus } \\
\text { tree approximately } 20 \mathrm{~m} \text { northwest of NTLF } \\
\text { Stack, containing rings approximately } 1986- \\
1994\end{array}$ & Vegetation \\
\hline PMB1a3 & $\begin{array}{l}\text { Portion of a core sample from a Eucalyptus } \\
\text { tree approximately } 20 \mathrm{~m} \text { northwest of NTLF } \\
\text { Stack, containing rings approximately post- } \\
1994\end{array}$ & Vegetation \\
\hline Ravine Creek & Routine sampling at Ravine Creek & Creeks \\
\hline $\begin{array}{l}\text { Ravine Creek } \\
\quad \text { (Lower) }\end{array}$ & $\begin{array}{l}\text { Special site at Ravine Creek for supplemental } \\
\text { monitoring (see Chapter 10) }\end{array}$ & $\begin{array}{l}\text { Supplemental } \\
\text { Monitoring }\end{array}$ \\
\hline $\begin{array}{l}\text { Ravine Creek } \\
\quad \text { (Upper) }\end{array}$ & $\begin{array}{l}\text { Special site at Ravine Creek for supplemental } \\
\text { monitoring (see Chapter 10) }\end{array}$ & $\begin{array}{l}\text { Supplemental } \\
\text { Monitoring }\end{array}$ \\
\hline SEE9-X & Vegetation sampling at Lake Chabot Park & $\begin{array}{l}\text { Supplemental } \\
\text { Monitoring }\end{array}$ \\
\hline SSE7-X & Vegetation sampling below Building 66 & $\begin{array}{l}\text { Supplemental } \\
\text { Monitoring }\end{array}$ \\
\hline
\end{tabular}




\begin{tabular}{|c|c|c|}
\hline Location code & Description of sampling location & Volume II section \\
\hline SSNTLF-01-X & Soil sampling around NTLF & $\begin{array}{l}\text { Supplemental } \\
\text { Monitoring }\end{array}$ \\
\hline $\begin{array}{l}\text { Strawberry } \\
\text { Creek Outfall }\end{array}$ & $\begin{array}{l}\text { Sampling at the point where Strawberry Creek } \\
\text { flows into San Francisco Bay }\end{array}$ & $\begin{array}{l}\text { Supplemental } \\
\text { Monitoring }\end{array}$ \\
\hline $\begin{array}{l}\text { Strawberry } \\
\text { Creek (UC) }\end{array}$ & Upper Strawberry Creek & $\begin{array}{l}\text { Creeks; Sediment; } \\
\text { Supplemental } \\
\text { Monitoring }\end{array}$ \\
\hline $\begin{array}{l}\text { Strawberry } \\
\text { Sewer }\end{array}$ & Strawberry Sewer station & Sewer \\
\hline Ten Inch Creek & Routine sampling at Ten Inch Creek & Creeks; Sediment \\
\hline $\begin{array}{l}\text { Ten Inch Creek } \\
\text { (Lower) }\end{array}$ & $\begin{array}{l}\text { Special site at Ten Inch Creek for supplemental } \\
\text { monitoring (see Chapter 10) }\end{array}$ & $\begin{array}{l}\text { Supplemental } \\
\text { Monitoring }\end{array}$ \\
\hline $\begin{array}{l}\text { Ten Inch Creek } \\
\text { (Upper) }\end{array}$ & $\begin{array}{l}\text { Special site at Ten Inch Creek for supplemental } \\
\text { monitoring (see Chapter 10) }\end{array}$ & $\begin{array}{l}\text { Supplemental } \\
\text { Monitoring }\end{array}$ \\
\hline Travel Blank & $\begin{array}{l}\text { Blank sample prepared prior to field collections } \\
\text { and carried by the sample technician during } \\
\text { collection activities }\end{array}$ & $\begin{array}{l}\text { Ambient Air, Stack } \\
\text { Air }\end{array}$ \\
\hline Wildcat Creek & Wildcat Creek & Creeks \\
\hline WNW4-X & $\begin{array}{l}\text { Vegetation sampling at UC Berkeley radio } \\
\text { antenna near LHS }\end{array}$ & $\begin{array}{l}\text { Supplemental } \\
\text { Monitoring }\end{array}$ \\
\hline WWW8-X & Vegetation Sampling below 88 Cyclotron & $\begin{array}{l}\text { Supplemental } \\
\text { Monitoring }\end{array}$ \\
\hline
\end{tabular}

The following units are used in Volume II:

\begin{tabular}{|c|c|c|}
\hline Unit & Description & Pertains to: \\
\hline$\%$ & percent & moisture content of sample \\
\hline$\mu \mathrm{g} / \mathrm{L}$ & micrograms per liter & $\begin{array}{l}\text { concentration of analyte (nonradioactive) } \\
\text { in liquid }\end{array}$ \\
\hline$\mu \mathrm{mhos} / \mathrm{cm}$ & micromhos per centimeter & specific conductance in liquid \\
\hline $\mathrm{Bq} / \mathrm{g}$ & becquerels per gram & activity of analyte (radioactive) in solid \\
\hline $\mathrm{Bq} / \mathrm{L}$ & becquerels per liter & activity of analyte (radioactive) in liquid \\
\hline $\mathrm{Bq} / \mathrm{m}^{3}$ & becquerels per cubic meter & activity of analyte (radioactive) in air \\
\hline $\mathrm{Bq} / \mathrm{S}$ & becquerels per sample & $\begin{array}{l}\text { activity of analyte (radioactive) in blank } \\
\text { samples }\end{array}$ \\
\hline $\mathrm{mg} / \mathrm{L}$ & milligrams per liter & $\begin{array}{l}\text { concentration of analyte (nonradioactive) } \\
\text { in liquid }\end{array}$ \\
\hline S.U. & standard units & $\mathrm{pH}$ measurement \\
\hline
\end{tabular}


The following stack air data are summarized and discussed in Chapter 4 (Air Quality) of the Site Environmental Report for 2001 (see Volume I):

$\begin{array}{llllll}\text { Analyte } & \text { Location } & \text { Date } & \text { Result } & \text { MDA or PQL Units } & \text { QC Type }\end{array}$

\section{Radiological Activity}

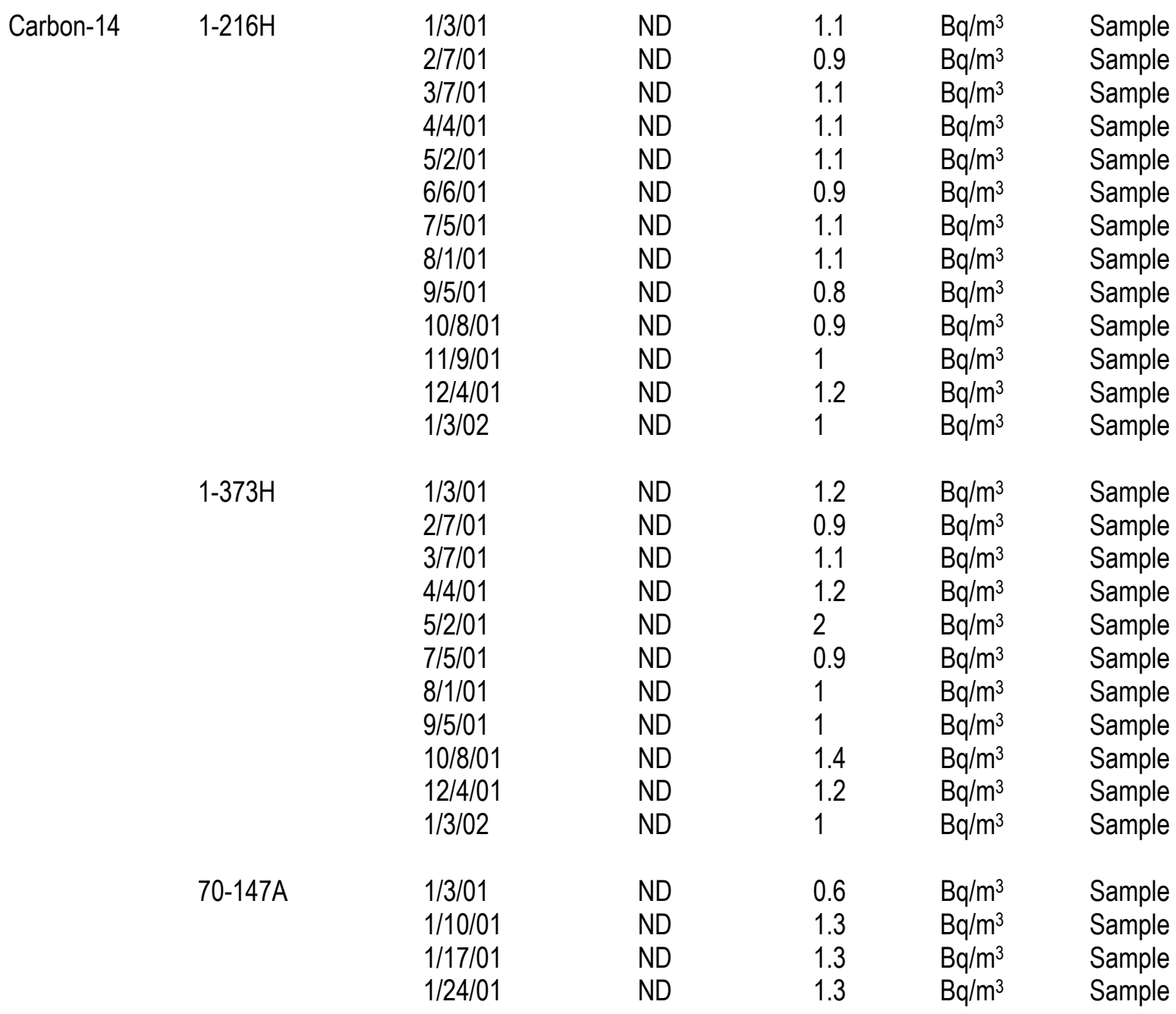


Analyte

Location

Date

Result MDA or PQL

Units

QC Type

\section{Radiological Activity}

\begin{tabular}{|c|c|c|c|c|c|c|}
\hline \multirow{46}{*}{$\begin{array}{l}\text { Carbon-14 } \\
\text { cont. }\end{array}$} & \multirow[t]{45}{*}{$70-147 A$} & $1 / 31 / 01$ & ND & 1.3 & $\mathrm{~Bq} / \mathrm{m}^{3}$ & Sample \\
\hline & & 2/7/01 & ND & 1.3 & $\mathrm{~Bq} / \mathrm{m}^{3}$ & Sample \\
\hline & & $2 / 14 / 01$ & ND & 1.3 & $\mathrm{~Bq} / \mathrm{m}^{3}$ & Sample \\
\hline & & 2/22/01 & ND & 1.1 & $\mathrm{~Bq} / \mathrm{m}^{3}$ & Sample \\
\hline & & 2/28/01 & ND & 1.5 & $\mathrm{~Bq} / \mathrm{m}^{3}$ & Sample \\
\hline & & $3 / 7 / 01$ & ND & 1.3 & $\mathrm{~Bq} / \mathrm{m}^{3}$ & Sample \\
\hline & & $3 / 15 / 01$ & ND & 1.1 & $\mathrm{~Bq} / \mathrm{m}^{3}$ & Sample \\
\hline & & $3 / 21 / 01$ & ND & 1.5 & $\mathrm{Bg} / \mathrm{m}^{3}$ & Sample \\
\hline & & $3 / 28 / 01$ & ND & 1.3 & $\mathrm{~Bq} / \mathrm{m}^{3}$ & Sample \\
\hline & & $4 / 4 / 01$ & ND & 1.3 & $\mathrm{~Bq} / \mathrm{m}^{3}$ & Sample \\
\hline & & $4 / 11 / 01$ & ND & 1.3 & $\mathrm{~Bq} / \mathrm{m}^{3}$ & Sample \\
\hline & & 4/18/01 & ND & 1.3 & $\mathrm{~Bq} / \mathrm{m}^{3}$ & Sample \\
\hline & & $4 / 26 / 01$ & ND & 1.1 & $\mathrm{~Bq} / \mathrm{m}^{3}$ & Sample \\
\hline & & $5 / 2 / 01$ & ND & 1.5 & $\mathrm{~Bq} / \mathrm{m}^{3}$ & Sample \\
\hline & & $5 / 9 / 01$ & ND & 1.3 & $\mathrm{~Bq} / \mathrm{m}^{3}$ & Sample \\
\hline & & $5 / 16 / 01$ & ND & 1.3 & $\mathrm{~Bq} / \mathrm{m}^{3}$ & Sample \\
\hline & & $5 / 23 / 01$ & ND & 1.3 & $\mathrm{~Bq} / \mathrm{m}^{3}$ & Sample \\
\hline & & $5 / 31 / 01$ & ND & 1.1 & $\mathrm{~Bq} / \mathrm{m}^{3}$ & Sample \\
\hline & & 6/6/01 & ND & 1.5 & $\mathrm{~Bq} / \mathrm{m}^{3}$ & Sample \\
\hline & & $6 / 13 / 01$ & ND & 1.3 & $\mathrm{~Bq} / \mathrm{m}^{3}$ & Sample \\
\hline & & $6 / 20 / 01$ & ND & 3 & $\mathrm{~Bq} / \mathrm{m}^{3}$ & Sample \\
\hline & & $6 / 27 / 01$ & ND & 1.3 & $\mathrm{Bg} / \mathrm{m}^{3}$ & Sample \\
\hline & & $7 / 5 / 01$ & ND & 1 & $\mathrm{~Bq} / \mathrm{m}^{3}$ & Sample \\
\hline & & $7 / 11 / 01$ & ND & 1.4 & $\mathrm{~Bq} / \mathrm{m}^{3}$ & Sample \\
\hline & & $7 / 18 / 01$ & ND & 1.1 & $\mathrm{~Bq} / \mathrm{m}^{3}$ & Sample \\
\hline & & $7 / 25 / 01$ & ND & 1.1 & $\mathrm{~Bq} / \mathrm{m}^{3}$ & Sample \\
\hline & & $8 / 1 / 01$ & ND & 1.1 & $\mathrm{~Bq} / \mathrm{m}^{3}$ & Sample \\
\hline & & 8/8/01 & ND & 1.1 & $\mathrm{Bg} / \mathrm{m}^{3}$ & Sample \\
\hline & & $8 / 15 / 01$ & ND & 1.1 & $\mathrm{~Bq} / \mathrm{m}^{3}$ & Sample \\
\hline & & 8/22/01 & ND & 1.1 & $\mathrm{~Bq} / \mathrm{m}^{3}$ & Sample \\
\hline & & 8/29/01 & ND & 1.1 & $\mathrm{~Bq} / \mathrm{m}^{3}$ & Sample \\
\hline & & $9 / 5 / 01$ & ND & 1.1 & $\mathrm{~Bq} / \mathrm{m}^{3}$ & Sample \\
\hline & & $9 / 12 / 01$ & ND & 1.2 & $\mathrm{~Bq} / \mathrm{m}^{3}$ & Sample \\
\hline & & $9 / 19 / 01$ & ND & 1.2 & $\mathrm{~Bq} / \mathrm{m}^{3}$ & Sample \\
\hline & & $9 / 26 / 01$ & ND & 1.6 & $\mathrm{~Bq} / \mathrm{m}^{3}$ & Sample \\
\hline & & $10 / 3 / 01$ & ND & 1.4 & $\mathrm{~Bq} / \mathrm{m}^{3}$ & Sample \\
\hline & & $10 / 9 / 01$ & ND & 4 & $\mathrm{~Bq} / \mathrm{m}^{3}$ & Sample \\
\hline & & $10 / 9 / 01$ & ND & 4 & $\mathrm{~Bq} / \mathrm{m}^{3}$ & Split \\
\hline & & 10/16/01 & ND & 1.1 & $\mathrm{~Bq} / \mathrm{m}^{3}$ & Sample \\
\hline & & $10 / 23 / 01$ & ND & 1.1 & $\mathrm{~Bq} / \mathrm{m}^{3}$ & Sample \\
\hline & & $11 / 8 / 01$ & ND & 1.1 & $\mathrm{~Bq} / \mathrm{m}^{3}$ & Sample \\
\hline & & $11 / 13 / 01$ & ND & 1.5 & $\mathrm{Bg} / \mathrm{m}^{3}$ & Sample \\
\hline & & $11 / 20 / 01$ & ND & 1.1 & $\mathrm{~Bq} / \mathrm{m}^{3}$ & Sample \\
\hline & & $11 / 26 / 01$ & ND & 1.3 & $\mathrm{~Bq} / \mathrm{m}^{3}$ & Sample \\
\hline & & $12 / 4 / 01$ & ND & 1 & $\mathrm{~Bq} / \mathrm{m}^{3}$ & Sample \\
\hline & & $12 / 12 / 01$ & ND & 0.9 & $\mathrm{~Bq} / \mathrm{m}^{3}$ & Sample \\
\hline
\end{tabular}




\begin{tabular}{llllll} 
Analyte & Location & Date & Result & MDA or PQL Units & QC Type \\
\hline
\end{tabular}

\section{Radiological Activity}

\begin{tabular}{|c|c|c|c|c|c|c|}
\hline \multirow[t]{45}{*}{$\begin{array}{l}\text { Carbon-14 } \\
\text { cont. }\end{array}$} & \multirow{2}{*}{$\begin{array}{l}\text { 70-147A } \\
85 \text { Glovebox }\end{array}$} & $\begin{array}{l}12 / 19 / 01 \\
1 / 2 / 02\end{array}$ & $\begin{array}{l}\text { ND } \\
\text { ND }\end{array}$ & $\begin{array}{l}1.1 \\
0.5\end{array}$ & $\begin{array}{l}\mathrm{Bq} / \mathrm{m}^{3} \\
\mathrm{~Bq} / \mathrm{m}^{3}\end{array}$ & $\begin{array}{l}\text { Sample } \\
\text { Sample }\end{array}$ \\
\hline & & $1 / 3 / 01$ & ND & 0.4 & $\mathrm{~Bq} / \mathrm{m}^{3}$ & Sample \\
\hline & & $1 / 10 / 01$ & ND & 0.9 & $\mathrm{~Bq} / \mathrm{m}^{3}$ & Sampl \\
\hline & & $1 / 17 / 01$ & ND & 0.9 & $\mathrm{~Bq} / \mathrm{m}^{3}$ & Samp \\
\hline & & $1 / 24 / 01$ & ND & 0.9 & $\mathrm{~Bq} / \mathrm{m}^{3}$ & Sampl \\
\hline & & $1 / 31 / 01$ & ND & 1 & $\mathrm{Bg} / \mathrm{m}^{3}$ & Sampl \\
\hline & & $2 / 7 / 01$ & ND & 0.9 & $\mathrm{~Bq} / \mathrm{m}^{3}$ & Sampl \\
\hline & & $2 / 14 / 01$ & ND & 0.9 & $\mathrm{~Bq} / \mathrm{m}^{3}$ & Samp \\
\hline & & $2 / 22 / 01$ & ND & 0.8 & $\mathrm{~Bq} / \mathrm{m}^{3}$ & Samp \\
\hline & & $2 / 28 / 01$ & ND & 1.1 & $\mathrm{~Bq} / \mathrm{m}^{3}$ & Samp \\
\hline & & $3 / 7 / 01$ & ND & 1 & $\mathrm{Bg} / \mathrm{m}^{3}$ & Samp \\
\hline & & $3 / 15 / 01$ & ND & 0.8 & $\mathrm{~Bq} / \mathrm{m}^{3}$ & Samp \\
\hline & & $3 / 21 / 01$ & ND & 1.1 & $\mathrm{~Bq} / \mathrm{m}^{3}$ & Samp \\
\hline & & $3 / 28 / 01$ & ND & 0.9 & $\mathrm{~Bq} / \mathrm{m}^{3}$ & Samp \\
\hline & & $4 / 4 / 01$ & ND & 1 & $\mathrm{~Bq} / \mathrm{m}^{3}$ & Samp \\
\hline & & $4 / 11 / 01$ & ND & 0.9 & $\mathrm{~Bq} / \mathrm{m}^{3}$ & Samp \\
\hline & & $4 / 18 / 01$ & ND & 0.9 & $\mathrm{~Bq} / \mathrm{m}^{3}$ & Samp \\
\hline & & $4 / 26 / 01$ & ND & 0.8 & $\mathrm{Bg} / \mathrm{m}^{3}$ & Samp \\
\hline & & $5 / 2 / 01$ & ND & 1.1 & $\mathrm{~Bq} / \mathrm{m}^{3}$ & Samp \\
\hline & & $5 / 9 / 01$ & ND & 0.9 & $\mathrm{~Bq} / \mathrm{m}^{3}$ & Samp \\
\hline & & $5 / 16 / 01$ & ND & 0.9 & $\mathrm{~Bq} / \mathrm{m}^{3}$ & Samp \\
\hline & & $5 / 23 / 01$ & ND & 0.9 & $\mathrm{~Bq} / \mathrm{m}^{3}$ & Samp \\
\hline & & $5 / 31 / 01$ & ND & 0.8 & $\mathrm{~Bq} / \mathrm{m}^{3}$ & Samp \\
\hline & & $6 / 6 / 01$ & ND & 1.1 & $\mathrm{~Bq} / \mathrm{m}^{3}$ & Samp \\
\hline & & $6 / 13 / 01$ & ND & 1.2 & $\mathrm{Bg} / \mathrm{m}^{3}$ & Samp \\
\hline & & $6 / 20 / 01$ & ND & 2 & $\mathrm{~Bq} / \mathrm{m}^{3}$ & Samp \\
\hline & & $6 / 27 / 01$ & ND & 1.2 & $\mathrm{Bg} / \mathrm{m}^{3}$ & Samp \\
\hline & & $7 / 5 / 01$ & ND & 0.9 & $\mathrm{~Bq} / \mathrm{m}^{3}$ & Samp \\
\hline & & $7 / 11 / 01$ & ND & 1.3 & $\mathrm{~Bq} / \mathrm{m}^{3}$ & Samp \\
\hline & & $7 / 18 / 01$ & ND & 1.1 & $\mathrm{~Bq} / \mathrm{m}^{3}$ & Samp \\
\hline & & $7 / 25 / 01$ & ND & 1.1 & $\mathrm{~Bq} / \mathrm{m}^{3}$ & Samp \\
\hline & & $8 / 1 / 01$ & ND & 1.1 & $\mathrm{Bg} / \mathrm{m}^{3}$ & Samp \\
\hline & & $8 / 8 / 01$ & ND & 1.1 & $\mathrm{~Bq} / \mathrm{m}^{3}$ & Samp \\
\hline & & $8 / 15 / 01$ & ND & 1.1 & $\mathrm{~Bq} / \mathrm{m}^{3}$ & Samp \\
\hline & & $8 / 22 / 01$ & ND & 1.1 & $\mathrm{~Bq} / \mathrm{m}^{3}$ & Samp \\
\hline & & $8 / 29 / 01$ & ND & 1.1 & $\mathrm{~Bq} / \mathrm{m}^{3}$ & Samp \\
\hline & & $9 / 5 / 01$ & ND & 1.1 & $\mathrm{~Bq} / \mathrm{m}^{3}$ & Samp \\
\hline & & $9 / 12 / 01$ & ND & 1.1 & $\mathrm{~Bq} / \mathrm{m}^{3}$ & Samp \\
\hline & & $9 / 19 / 01$ & ND & 1.1 & $\mathrm{Bg} / \mathrm{m}^{3}$ & Samp \\
\hline & & $9 / 26 / 01$ & ND & 1.1 & $\mathrm{~Bq} / \mathrm{m}^{3}$ & Samp \\
\hline & & $10 / 3 / 01$ & ND & 1.1 & $\mathrm{~Bq} / \mathrm{m}^{3}$ & Samp \\
\hline & & $10 / 9 / 01$ & ND & 3 & $\mathrm{~Bq} / \mathrm{m}^{3}$ & Samp \\
\hline & & $10 / 16 / 01$ & ND & 1.1 & $\mathrm{~Bq} / \mathrm{m}^{3}$ & Samp \\
\hline & & $10 / 23 / 01$ & ND & 1.1 & $\mathrm{~Bq} / \mathrm{m}^{3}$ & Samp \\
\hline & & $10 / 30 / 01$ & ND & 1.1 & $\mathrm{~Bq} / \mathrm{m}^{3}$ & Samp \\
\hline
\end{tabular}


Analyte

Location

Date

Result MDA or PQL

Units

QC Type

\section{Radiological Activity}

\begin{tabular}{|c|c|c|c|c|c|c|}
\hline \multicolumn{2}{|l|}{$\begin{array}{l}\text { Carbon-14 } \\
\text { cont. }\end{array}$} & $\begin{array}{l}11 / 8 / 01 \\
11 / 13 / 01 \\
11 / 20 / 01 \\
11 / 26 / 01 \\
12 / 4 / 01 \\
12 / 12 / 01 \\
12 / 18 / 01 \\
1 / 2 / 02\end{array}$ & $\begin{array}{l}\text { ND } \\
\text { ND } \\
\text { ND } \\
\text { ND } \\
\text { ND } \\
\text { ND } \\
\text { ND } \\
\text { ND }\end{array}$ & $\begin{array}{l}0.9 \\
1.6 \\
1.1 \\
1.3 \\
1 \\
1 \\
1.3 \\
0.5\end{array}$ & $\begin{array}{l}\mathrm{Bq} / \mathrm{m}^{3} \\
\mathrm{~Bq} / \mathrm{m}^{3} \\
\mathrm{~Bq} / \mathrm{m}^{3} \\
\mathrm{~Bq} / \mathrm{m}^{3} \\
\mathrm{~Bq} / \mathrm{m}^{3} \\
\mathrm{~Bq} / \mathrm{m}^{3} \\
\mathrm{~Bq} / \mathrm{m}^{3} \\
\mathrm{~Bq} / \mathrm{m}^{3}\end{array}$ & $\begin{array}{l}\text { Sample } \\
\text { Sample } \\
\text { Sample } \\
\text { Sample } \\
\text { Sample } \\
\text { Sample } \\
\text { Sample } \\
\text { Sample }\end{array}$ \\
\hline & \multirow{37}{*}{85 Hood } & $1 / 3 / 01$ & ND & 0.5 & $\mathrm{Bg} / \mathrm{m}^{3}$ & Sample \\
\hline & & 1/10/01 & ND & 1.1 & $\mathrm{~Bq} / \mathrm{m}^{3}$ & Sample \\
\hline & & $1 / 17 / 01$ & ND & 1.1 & $\mathrm{~Bq} / \mathrm{m}^{3}$ & Sample \\
\hline & & $1 / 24 / 01$ & ND & 1.1 & $\mathrm{~Bq} / \mathrm{m}^{3}$ & Sample \\
\hline & & $1 / 31 / 01$ & ND & 1.1 & $\mathrm{~Bq} / \mathrm{m}^{3}$ & Sample \\
\hline & & $2 / 7 / 01$ & ND & 1.1 & $\mathrm{~Bq} / \mathrm{m}^{3}$ & Sample \\
\hline & & 2/14/01 & ND & 1.1 & $\mathrm{~Bq} / \mathrm{m}^{3}$ & Sample \\
\hline & & 2/22/01 & ND & 1 & $\mathrm{~Bq} / \mathrm{m}^{3}$ & Sample \\
\hline & & $2 / 28 / 01$ & ND & 1.3 & $\mathrm{~Bq} / \mathrm{m}^{3}$ & Sample \\
\hline & & $3 / 7 / 01$ & ND & 1.1 & $\mathrm{~Bq} / \mathrm{m}^{3}$ & Sample \\
\hline & & $3 / 15 / 01$ & ND & 1 & $\mathrm{~Bq} / \mathrm{m}^{3}$ & Sample \\
\hline & & $3 / 21 / 01$ & ND & 1.3 & $\mathrm{~Bq} / \mathrm{m}^{3}$ & Sample \\
\hline & & $3 / 28 / 01$ & ND & 1.1 & $\mathrm{~Bq} / \mathrm{m}^{3}$ & Sample \\
\hline & & $4 / 4 / 01$ & ND & 1.1 & $\mathrm{~Bq} / \mathrm{m}^{3}$ & Sample \\
\hline & & $4 / 11 / 01$ & ND & 1.1 & $\mathrm{~Bq} / \mathrm{m}^{3}$ & Sample \\
\hline & & $4 / 18 / 01$ & ND & 1.1 & $\mathrm{~Bq} / \mathrm{m}^{3}$ & Sample \\
\hline & & 4/26/01 & 1.1 & 1 & $\mathrm{~Bq} / \mathrm{m}^{3}$ & Sample \\
\hline & & $5 / 2 / 01$ & ND & 1.3 & $\mathrm{~Bq} / \mathrm{m}^{3}$ & Sample \\
\hline & & $5 / 9 / 01$ & ND & 1.1 & $\mathrm{~Bq} / \mathrm{m}^{3}$ & Sample \\
\hline & & $5 / 16 / 01$ & ND & 1.1 & $\mathrm{~Bq} / \mathrm{m}^{3}$ & Sample \\
\hline & & $5 / 23 / 01$ & ND & 1.1 & $\mathrm{~Bq} / \mathrm{m}^{3}$ & Sample \\
\hline & & $5 / 31 / 01$ & 1.6 & 1 & $\mathrm{~Bq} / \mathrm{m}^{3}$ & Sample \\
\hline & & $6 / 6 / 01$ & ND & 1.3 & $\mathrm{~Bq} / \mathrm{m}^{3}$ & Sample \\
\hline & & $6 / 13 / 01$ & ND & 1.1 & $\mathrm{~Bq} / \mathrm{m}^{3}$ & Sample \\
\hline & & $6 / 20 / 01$ & ND & 2 & $\mathrm{~Bq} / \mathrm{m}^{3}$ & Sample \\
\hline & & $6 / 20 / 01$ & ND & 2 & $\mathrm{~Bq} / \mathrm{m}^{3}$ & Split \\
\hline & & $6 / 27 / 01$ & ND & 1.1 & $\mathrm{~Bq} / \mathrm{m}^{3}$ & Sample \\
\hline & & $7 / 5 / 01$ & 1.4 & 0.9 & $\mathrm{~Bq} / \mathrm{m}^{3}$ & Sample \\
\hline & & $7 / 11 / 01$ & ND & 1.3 & $\mathrm{~Bq} / \mathrm{m}^{3}$ & Sample \\
\hline & & $7 / 18 / 01$ & ND & 1.1 & $\mathrm{Bg} / \mathrm{m}^{3}$ & Sample \\
\hline & & $7 / 25 / 01$ & ND & 1.1 & $\mathrm{~Bq} / \mathrm{m}^{3}$ & Sampl \\
\hline & & $8 / 1 / 01$ & ND & 1.1 & $\mathrm{~Bq} / \mathrm{m}^{3}$ & Samp \\
\hline & & $8 / 8 / 01$ & ND & 1.1 & $\mathrm{~Bq} / \mathrm{m}^{3}$ & Sample \\
\hline & & $8 / 15 / 01$ & ND & 1.1 & $\mathrm{~Bq} / \mathrm{m}^{3}$ & Sample \\
\hline & & $8 / 22 / 01$ & ND & 1.1 & $\mathrm{~Bq} / \mathrm{m}^{3}$ & Sample \\
\hline & & $8 / 29 / 01$ & ND & 1.1 & $\mathrm{~Bq} / \mathrm{m}^{3}$ & Sample \\
\hline & & $9 / 5 / 01$ & ND & 1.1 & $\mathrm{Bg} / \mathrm{m}^{3}$ & Sample \\
\hline
\end{tabular}




\begin{tabular}{llllll} 
Analyte & Location & Date & Result & MDA or PQL Units & QC Type \\
\hline
\end{tabular}

\section{Radiological Activity}

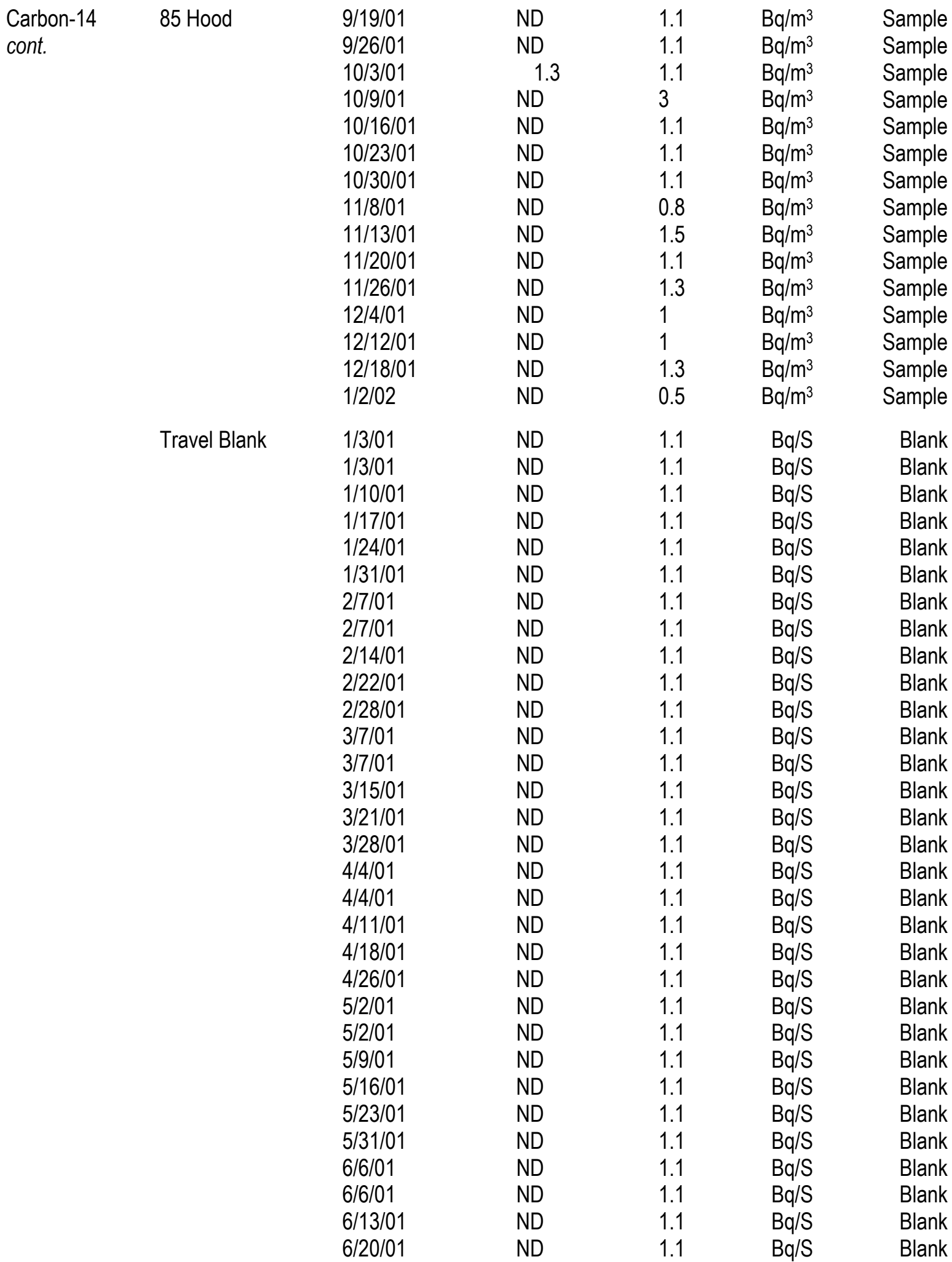


Analyte

Location

Date

Result MDA or PQL

Units QC Type

\section{Radiological Activity}

\begin{tabular}{|c|c|c|c|c|c|c|}
\hline \multirow{34}{*}{$\begin{array}{l}\text { Carbon-14 } \\
\text { cont. }\end{array}$} & \multirow[t]{34}{*}{ Travel Blank } & $6 / 27 / 01$ & ND & 1.1 & $\mathrm{~Bq} / \mathrm{S}$ & Blank \\
\hline & & $7 / 5 / 01$ & ND & 1.1 & $\mathrm{~Bq} / \mathrm{S}$ & Blank \\
\hline & & $7 / 5 / 01$ & ND & 1.1 & $\mathrm{~Bq} / \mathrm{S}$ & Blank \\
\hline & & $7 / 11 / 01$ & ND & 1.1 & $\mathrm{~Bq} / \mathrm{S}$ & Blank \\
\hline & & $7 / 18 / 01$ & ND & 1.1 & $\mathrm{~Bq} / \mathrm{S}$ & Blank \\
\hline & & $7 / 25 / 01$ & ND & 1.1 & $\mathrm{~Bq} / \mathrm{S}$ & Blank \\
\hline & & $8 / 1 / 01$ & ND & 1.1 & $\mathrm{~Bq} / \mathrm{S}$ & Blank \\
\hline & & $8 / 1 / 01$ & ND & 1.1 & $\mathrm{~Bq} / \mathrm{S}$ & Blank \\
\hline & & $8 / 8 / 01$ & ND & 1.1 & $\mathrm{~Bq} / \mathrm{S}$ & Blank \\
\hline & & $8 / 15 / 01$ & ND & 1.1 & $\mathrm{~Bq} / \mathrm{S}$ & Blank \\
\hline & & $8 / 22 / 01$ & ND & 1.1 & $\mathrm{~Bq} / \mathrm{S}$ & Blank \\
\hline & & $8 / 29 / 01$ & ND & 1.1 & $\mathrm{~Bq} / \mathrm{S}$ & Blank \\
\hline & & $9 / 5 / 01$ & ND & 1.1 & $\mathrm{~Bq} / \mathrm{S}$ & Blank \\
\hline & & $9 / 5 / 01$ & ND & 1.1 & $\mathrm{~Bq} / \mathrm{S}$ & Blank \\
\hline & & $9 / 12 / 01$ & ND & 1.1 & $\mathrm{~Bq} / \mathrm{S}$ & Blank \\
\hline & & $9 / 19 / 01$ & ND & 1.1 & $\mathrm{~Bq} / \mathrm{S}$ & Blank \\
\hline & & $9 / 26 / 01$ & ND & 1.1 & $\mathrm{~Bq} / \mathrm{S}$ & Blank \\
\hline & & $10 / 3 / 01$ & ND & 1.1 & $\mathrm{~Bq} / \mathrm{S}$ & Blank \\
\hline & & 10/8/01 & ND & 1.1 & $\mathrm{~Bq} / \mathrm{S}$ & Blank \\
\hline & & 10/9/01 & ND & 1.1 & $\mathrm{~Bq} / \mathrm{S}$ & Blank \\
\hline & & 10/16/01 & ND & 1.1 & $\mathrm{~Bq} / \mathrm{S}$ & Blank \\
\hline & & $10 / 24 / 01$ & ND & 1.1 & $\mathrm{~Bq} / \mathrm{S}$ & Blank \\
\hline & & $10 / 30 / 01$ & ND & 1.1 & $\mathrm{~Bq} / \mathrm{S}$ & Blank \\
\hline & & $11 / 8 / 01$ & ND & 1.1 & $\mathrm{~Bq} / \mathrm{S}$ & Blank \\
\hline & & $11 / 9 / 01$ & ND & 1.1 & $\mathrm{~Bq} / \mathrm{S}$ & Blank \\
\hline & & $11 / 13 / 01$ & ND & 1.1 & $\mathrm{~Bq} / \mathrm{S}$ & Blank \\
\hline & & $11 / 20 / 01$ & ND & 1.1 & $\mathrm{~Bq} / \mathrm{S}$ & Blank \\
\hline & & $11 / 26 / 01$ & ND & 1.1 & $\mathrm{~Bq} / \mathrm{S}$ & Blank \\
\hline & & $12 / 4 / 01$ & ND & 1.1 & $\mathrm{~Bq} / \mathrm{S}$ & Blank \\
\hline & & $12 / 4 / 01$ & ND & 1.1 & $\mathrm{~Bq} / \mathrm{S}$ & Blank \\
\hline & & $12 / 12 / 01$ & ND & 1.1 & $\mathrm{~Bq} / \mathrm{S}$ & Blank \\
\hline & & $12 / 19 / 01$ & ND & 1.1 & $\mathrm{~Bq} / \mathrm{S}$ & Blank \\
\hline & & $1 / 2 / 02$ & ND & 1.1 & $\mathrm{~Bq} / \mathrm{S}$ & Blank \\
\hline & & $1 / 3 / 02$ & ND & 1.1 & $\mathrm{~Bq} / \mathrm{S}$ & Blank \\
\hline \multirow[t]{11}{*}{ Gross alpha } & \multirow[t]{11}{*}{$1-216 \mathrm{H}$} & $1 / 3 / 01$ & 0.0001 & 0.00009 & $\mathrm{~Bq} / \mathrm{m}^{3}$ & Sample \\
\hline & & $2 / 7 / 01$ & 0.0001 & 0.00007 & $\mathrm{~Bq} / \mathrm{m}^{3}$ & Sample \\
\hline & & $3 / 7 / 01$ & ND & 0.00009 & $\mathrm{~Bq} / \mathrm{m}^{3}$ & Sample \\
\hline & & $4 / 4 / 01$ & ND & 0.0002 & $\mathrm{~Bq} / \mathrm{m}^{3}$ & Sample \\
\hline & & $5 / 2 / 01$ & ND & 0.0003 & $\mathrm{~Bq} / \mathrm{m}^{3}$ & Sample \\
\hline & & $6 / 6 / 01$ & ND & 0.00018 & $\mathrm{~Bq} / \mathrm{m}^{3}$ & Sample \\
\hline & & $7 / 5 / 01$ & ND & 0.00009 & $\mathrm{~Bq} / \mathrm{m}^{3}$ & Sample \\
\hline & & $8 / 1 / 01$ & ND & 0.0002 & $\mathrm{~Bq} / \mathrm{m}^{3}$ & Sample \\
\hline & & $9 / 5 / 01$ & ND & 0.00007 & $\mathrm{~Bq} / \mathrm{m}^{3}$ & Sample \\
\hline & & 10/8/01 & 0.0002 & 0.00008 & $\mathrm{~Bq} / \mathrm{m}^{3}$ & Sample \\
\hline & & $11 / 9 / 01$ & 0.0002 & 0.00008 & $\mathrm{~Bq} / \mathrm{m}^{3}$ & Sample \\
\hline
\end{tabular}




\section{Analyte \\ Location \\ Radiological Activity}

Date

Result

MDA or PQL

Units

QC Type

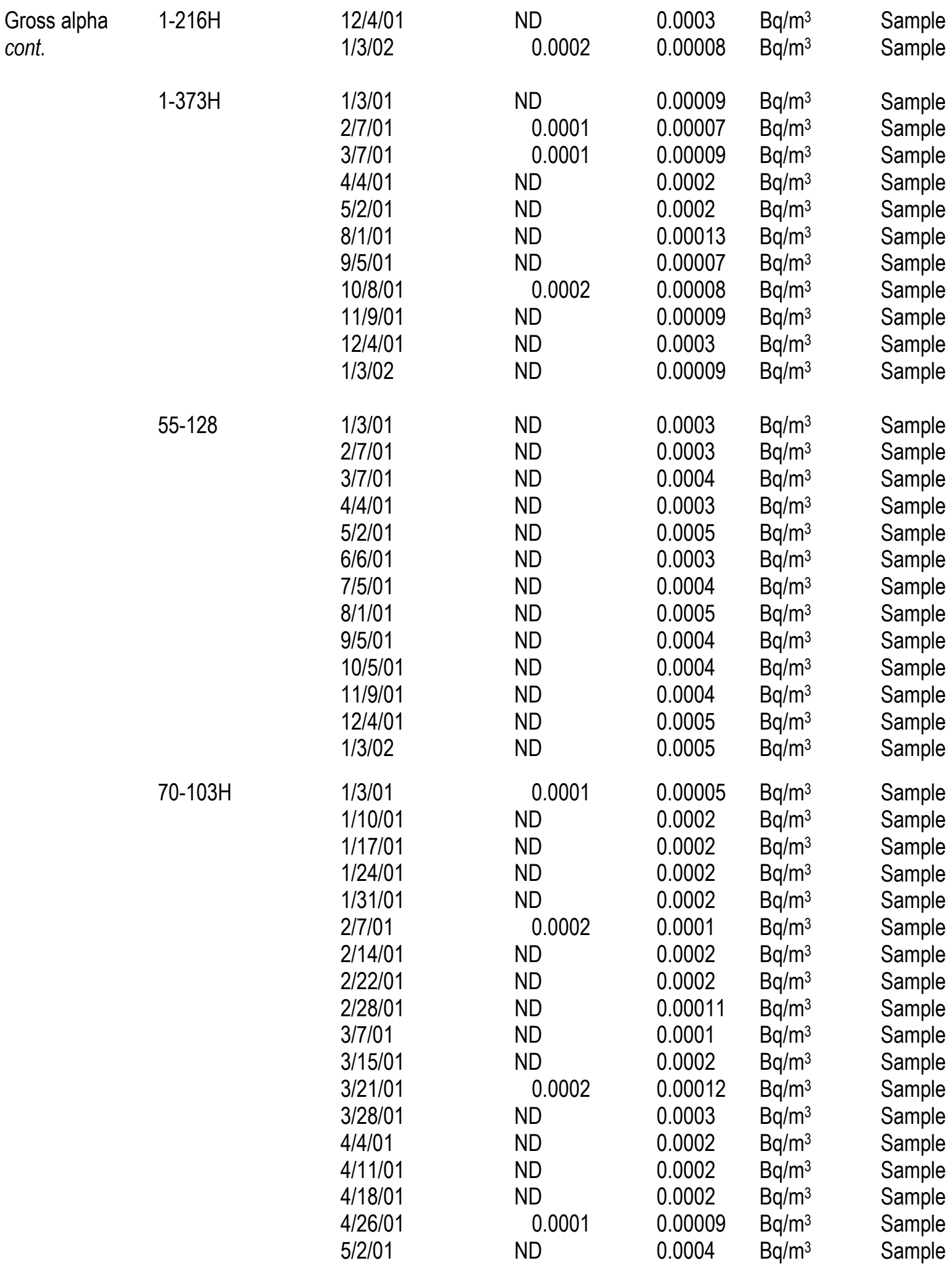


Analyte

Location

Radiological Activity

Gross alpha $\quad 70-103 \mathrm{H}$

cont.
Date

Result

MDA or PQL

Units

QC Type

ND

ND

ND

ND

ND

0.0001

$\mathrm{ND}$

ND

$7 / 5 / 01$

$7 / 11 / 01$

$7 / 18 / 01$

$7 / 25 / 01$

$8 / 1 / 01$

$8 / 8 / 01$

$8 / 15 / 01$

$8 / 29 / 01$

$9 / 5 / 01$

9/12/01

9/19/01

9/26/01

10/3/01

10/9/01

10/16/01

10/23/01

10/30/01

$11 / 8 / 01$

$11 / 13 / 01$

$11 / 20 / 01$

$11 / 26 / 01$

$12 / 4 / 01$

$12 / 12 / 01$

$12 / 19 / 01$

70-147A

$1 / 2 / 02$

$1 / 3 / 01$

$1 / 10 / 01$

$1 / 17 / 01$

$1 / 24 / 01$

$1 / 31 / 01$

2/7/01

2/14/01

2/22/01

$2 / 28 / 01$

$3 / 7 / 01$

$3 / 15 / 01$

$3 / 21 / 01$
ND

0.00009
ND

ND

0.00009

ND

0.00009

ND

0.0001

ND

ND

ND

ND

0.00009
ND

0.00007

ND

0.0001

ND

0.0001

ND

ND

0.00005
ND
ND
ND
ND
ND
0.0001
ND
ND
0.0001
ND
ND
ND

$\begin{array}{lll}0.0001 & \mathrm{~Bq} / \mathrm{m}^{3} & \text { Sample } \\ 0.0003 & \mathrm{~Bq} / \mathrm{m}^{3} & \text { Sample } \\ 0.0002 & \mathrm{~Bq} / \mathrm{m}^{3} & \text { Sample } \\ 0.0002 & \mathrm{~Bq} / \mathrm{m}^{3} & \text { Sample } \\ 0.0003 & \mathrm{~Bq} / \mathrm{m}^{3} & \text { Sample } \\ 0.0002 & \mathrm{~Bq} / \mathrm{m}^{3} & \text { Sample } \\ 0.00008 & \mathrm{~Bq} / \mathrm{m}^{3} & \text { Sample } \\ 0.0002 & \mathrm{~Bq} / \mathrm{m}^{3} & \text { Sample } \\ 0.00017 & \mathrm{~Bq} / \mathrm{m}^{3} & \text { Sample } \\ 0.0003 & \mathrm{~Bq} / \mathrm{m}^{3} & \text { Sample } \\ 0.00008 & \mathrm{~Bq} / \mathrm{m}^{3} & \text { Sample } \\ 0.00008 & \mathrm{~Bq} / \mathrm{m}^{3} & \text { Sample } \\ 0.0002 & \mathrm{~Bq} / \mathrm{m}^{3} & \text { Sample } \\ 0.00008 & \mathrm{~Bq} / \mathrm{m}^{3} & \text { Sample } \\ 0.00008 & \mathrm{~Bq} / \mathrm{m}^{3} & \text { Sample } \\ 0.0003 & \mathrm{~Bq} / \mathrm{m}^{3} & \text { Sample } \\ 0.00008 & \mathrm{~Bq} / \mathrm{m}^{3} & \text { Sample } \\ 0.0002 & \mathrm{~Bq} / \mathrm{m}^{3} & \text { Sample } \\ 0.00009 & \mathrm{~Bq} / \mathrm{m}^{3} & \text { Sample } \\ 0.0002 & \mathrm{~Bq} / \mathrm{m}^{3} & \text { Sample } \\ 0.0002 & \mathrm{~Bq} / \mathrm{m}^{3} & \text { Sample } \\ 0.00011 & \mathrm{~Bq} / \mathrm{m}^{3} & \text { Sample } \\ 0.00009 & \mathrm{~Bq} / \mathrm{m}^{3} & \text { Sample } \\ 0.00009 & \mathrm{~Bq} / \mathrm{m}^{3} & \text { Sample } \\ 0.0002 & \mathrm{~Bq} / \mathrm{m}^{3} & \text { Sample } \\ 0.00007 & \mathrm{~Bq} / \mathrm{m}^{3} & \text { Sample } \\ 0.0003 & \mathrm{~Bq} / \mathrm{m}^{3} & \text { Sample } \\ 0.00009 & \mathrm{~Bq} / \mathrm{m}^{3} & \text { Sample } \\ 0.0001 & \mathrm{~Bq} / \mathrm{m}^{3} & \text { Sample } \\ 0.00008 & \mathrm{~Bq} / \mathrm{m}^{3} & \text { Sample } \\ 0.00019 & \mathrm{~Bq} / \mathrm{m}^{3} & \text { Sample } \\ 0.00009 & \mathrm{~Bq} / \mathrm{m}^{3} & \text { Sample }\end{array}$

$0.00004 \mathrm{~Bq} / \mathrm{m}^{3}$

$0.00004 \mathrm{~Bq} / \mathrm{m}^{3}$

$0.0002 \mathrm{~Bq} / \mathrm{m}^{3}$

$0.0002 \mathrm{~Bq} / \mathrm{m}^{3}$

$0.0002 \mathrm{~Bq} / \mathrm{m}^{3}$

$0.0002 \mathrm{~Bq} / \mathrm{m}^{3}$

$0.00009 \mathrm{~Bq} / \mathrm{m}^{3}$

$0.0002 \mathrm{~Bq} / \mathrm{m}^{3}$

$0.0002 \mathrm{~Bq} / \mathrm{m}^{3}$

$0.0001 \quad \mathrm{~Bq} / \mathrm{m}^{3}$

$0.00009 \mathrm{~Bq} / \mathrm{m}^{3}$

$0.00019 \mathrm{~Bq} / \mathrm{m}^{3}$

$0.00011 \mathrm{~Bq} / \mathrm{m}^{3}$
Sample

Sample

Sample

Sample

Sample

Sample

Sample

Sample

Sample

Sample

Sample

Sample

Sample 


\section{Analyte \\ Location \\ Radiological Activity}

Date

Result

MDA or PQL

Units

QC Type

\begin{tabular}{|c|c|c|c|c|c|c|}
\hline \multirow{39}{*}{\multicolumn{2}{|c|}{$\begin{array}{l}\text { Gross alpha } \\
\text { cont. }\end{array}$}} & $3 / 28 / 01$ & ND & 0.0003 & $\mathrm{~Bq} / \mathrm{m}^{3}$ & Sample \\
\hline & & $4 / 4 / 01$ & ND & 0.0002 & $\mathrm{~Bq} / \mathrm{m}^{3}$ & Sample \\
\hline & & $4 / 11 / 01$ & ND & 0.0002 & $\mathrm{~Bq} / \mathrm{m}^{3}$ & Sample \\
\hline & & $4 / 18 / 01$ & ND & 0.0002 & $\mathrm{~Bq} / \mathrm{m}^{3}$ & Sample \\
\hline & & $4 / 26 / 01$ & 0.0001 & 0.00008 & $\mathrm{~Bq} / \mathrm{m}^{3}$ & Sample \\
\hline & & $5 / 2 / 01$ & ND & 0.0003 & $\mathrm{~Bq} / \mathrm{m}^{3}$ & Sample \\
\hline & & $5 / 9 / 01$ & 0.0001 & 0.00009 & $\mathrm{~Bq} / \mathrm{m}^{3}$ & Sample \\
\hline & & $5 / 16 / 01$ & ND & 0.0003 & $\mathrm{~Bq} / \mathrm{m}^{3}$ & Sample \\
\hline & & $5 / 23 / 01$ & ND & 0.0002 & $\mathrm{~Bq} / \mathrm{m}^{3}$ & Sample \\
\hline & & $5 / 31 / 01$ & ND & 0.0002 & $\mathrm{~Bq} / \mathrm{m}^{3}$ & Sample \\
\hline & & $6 / 6 / 01$ & ND & 0.0003 & $\mathrm{~Bq} / \mathrm{m}^{3}$ & Sample \\
\hline & & $6 / 13 / 01$ & ND & 0.0002 & $\mathrm{~Bq} / \mathrm{m}^{3}$ & Sample \\
\hline & & 6/20/01 & ND & 0.00009 & $\mathrm{~Bq} / \mathrm{m}^{3}$ & Sample \\
\hline & & $6 / 27 / 01$ & ND & 0.0002 & $\mathrm{~Bq} / \mathrm{m}^{3}$ & Sample \\
\hline & & $7 / 5 / 01$ & ND & 0.00019 & $\mathrm{~Bq} / \mathrm{m}^{3}$ & Sample \\
\hline & & $7 / 11 / 01$ & ND & 0.0004 & $\mathrm{~Bq} / \mathrm{m}^{3}$ & Sample \\
\hline & & $7 / 18 / 01$ & ND & 0.00009 & $\mathrm{~Bq} / \mathrm{m}^{3}$ & Sample \\
\hline & & $7 / 25 / 01$ & 0.0001 & 0.00009 & $\mathrm{~Bq} / \mathrm{m}^{3}$ & Sample \\
\hline & & $8 / 1 / 01$ & ND & 0.0002 & $\mathrm{~Bq} / \mathrm{m}^{3}$ & Sample \\
\hline & & $8 / 8 / 01$ & 0.0001 & 0.00009 & $\mathrm{~Bq} / \mathrm{m}^{3}$ & Sample \\
\hline & & $8 / 15 / 01$ & ND & 0.00009 & $\mathrm{~Bq} / \mathrm{m}^{3}$ & Sample \\
\hline & & $8 / 22 / 01$ & 0.0001 & 0.00009 & $\mathrm{~Bq} / \mathrm{m}^{3}$ & Sample \\
\hline & & $8 / 29 / 01$ & ND & 0.0003 & $\mathrm{~Bq} / \mathrm{m}^{3}$ & Sample \\
\hline & & $9 / 5 / 01$ & ND & 0.00009 & $\mathrm{~Bq} / \mathrm{m}^{3}$ & Sample \\
\hline & & $9 / 12 / 01$ & ND & 0.0002 & $\mathrm{~Bq} / \mathrm{m}^{3}$ & Sample \\
\hline & & $9 / 19 / 01$ & 0.0002 & 0.00009 & $\mathrm{~Bq} / \mathrm{m}^{3}$ & Sample \\
\hline & & 9/26/01 & ND & 0.0002 & $\mathrm{~Bq} / \mathrm{m}^{3}$ & Sample \\
\hline & & $10 / 3 / 01$ & ND & 0.0002 & $\mathrm{~Bq} / \mathrm{m}^{3}$ & Sample \\
\hline & & $10 / 9 / 01$ & ND & 0.0001 & $\mathrm{~Bq} / \mathrm{m}^{3}$ & Sample \\
\hline & & $10 / 16 / 01$ & ND & 0.00009 & $\mathrm{~Bq} / \mathrm{m}^{3}$ & Sample \\
\hline & & $10 / 23 / 01$ & ND & 0.00009 & $\mathrm{~Bq} / \mathrm{m}^{3}$ & Sample \\
\hline & & $11 / 8 / 01$ & 0.0002 & 0.00009 & $\mathrm{~Bq} / \mathrm{m}^{3}$ & Sample \\
\hline & & $11 / 13 / 01$ & ND & 0.0003 & $\mathrm{~Bq} / \mathrm{m}^{3}$ & Sample \\
\hline & & $11 / 20 / 01$ & 0.0002 & 0.00009 & $\mathrm{~Bq} / \mathrm{m}^{3}$ & Sample \\
\hline & & $11 / 26 / 01$ & 0.0001 & 0.0001 & $\mathrm{~Bq} / \mathrm{m}^{3}$ & Sample \\
\hline & & $12 / 4 / 01$ & 0.0001 & 0.00008 & $\mathrm{~Bq} / \mathrm{m}^{3}$ & Sample \\
\hline & & $12 / 12 / 01$ & ND & 0.00019 & $\mathrm{~Bq} / \mathrm{m}^{3}$ & Sample \\
\hline & & $12 / 19 / 01$ & 0.0002 & 0.00009 & $\mathrm{~Bq} / \mathrm{m}^{3}$ & Sampl \\
\hline & & $1 / 2 / 02$ & ND & 0.00004 & $\mathrm{~Bq} / \mathrm{m}^{3}$ & Sample \\
\hline & $70-157 \mathrm{H}$ & $1 / 3 / 01$ & 0.0001 & 0.0001 & $\mathrm{~Bq} / \mathrm{m}^{3}$ & Sample \\
\hline & & $2 / 7 / 01$ & 0.00009 & 0.00008 & $\mathrm{~Bq} / \mathrm{m}^{3}$ & Sample \\
\hline & & $3 / 7 / 01$ & ND & 0.0001 & $\mathrm{~Bq} / \mathrm{m}^{3}$ & Sample \\
\hline & & $4 / 4 / 01$ & ND & 0.0003 & $\mathrm{~Bq} / \mathrm{m}^{3}$ & Sample \\
\hline & & $5 / 2 / 01$ & ND & 0.0003 & $\mathrm{~Bq} / \mathrm{m}^{3}$ & Sample \\
\hline & & $6 / 6 / 01$ & ND & 0.0002 & $\mathrm{~Bq} / \mathrm{m}^{3}$ & Sample \\
\hline & & $7 / 5 / 01$ & 0.0001 & 0.00009 & $\mathrm{~Bq} / \mathrm{m}^{3}$ & Sample \\
\hline
\end{tabular}


Analyte

Location

Radiological Activity

Gross alpha $\quad 70-157 \mathrm{H}$

cont.
Date

Result

MDA or PQL

Units

QC Type

ND

ND

0.0001

0.0002

ND

ND

70-203H

$1 / 3 / 01$

$2 / 7 / 01$

$3 / 7 / 01$

$4 / 4 / 01$

$5 / 2 / 01$

$6 / 6 / 01$

$7 / 5 / 01$

$8 / 1 / 01$

9/5/01

10/5/01

$11 / 8 / 01$

$12 / 4 / 01$

$1 / 3 / 02$

70A-1129B

$1 / 3 / 01$

$1 / 10 / 01$

$1 / 17 / 01$

1/24/01

$1 / 31 / 01$

2/7/01

2/14/01

2/22/01

2/28/01

$3 / 7 / 01$

$3 / 15 / 01$

$3 / 21 / 01$

$3 / 28 / 01$

$4 / 4 / 01$

$4 / 11 / 01$

$4 / 18 / 01$

$4 / 26 / 01$

$5 / 2 / 01$

$5 / 9 / 01$

$5 / 16 / 01$

$5 / 23 / 01$

$5 / 31 / 01$

$6 / 6 / 01$

$6 / 13 / 01$

$6 / 20 / 01$

$6 / 27 / 01$

0.0001

ND

0.0001

0.0003

ND

ND

0.00009

ND

\subsection{7}

0.0002

0.0001

ND

0.0002

0.0001

0.0002

ND

ND

ND

0.0003

ND

ND

ND

0.0002

ND

ND

ND

0.0003

ND

ND

0.0003

ND

0.0002

ND

ND

ND

ND

ND

0.0002

ND
$0.0002 \mathrm{~Bq} / \mathrm{m}^{3}$

$0.00007 \mathrm{~Bq} / \mathrm{m}^{3}$

$0.00008 \mathrm{~Bq} / \mathrm{m}^{3}$

$0.00009 \mathrm{~Bq} / \mathrm{m}^{3}$

$0.0002 \mathrm{~Bq} / \mathrm{m}^{3}$

$0.00008 \mathrm{~Bq} / \mathrm{m}^{3}$

$0.00009 \mathrm{~Bq} / \mathrm{m}^{3}$

$0.00007 \mathrm{~Bq} / \mathrm{m}^{3}$

$0.00009 \mathrm{~Bq} / \mathrm{m}^{3}$

$0.0002 \mathrm{~Bq} / \mathrm{m}^{3}$

$0.0003 \mathrm{~Bq} / \mathrm{m}^{3}$

$0.00018 \mathrm{Bg} / \mathrm{m}^{3}$

$0.00009 \mathrm{Bg} / \mathrm{m}^{3}$

$0.0002 \mathrm{~Bq} / \mathrm{m}^{3}$

$0.00007 \mathrm{Bg} / \mathrm{m}^{3}$

$0.00011 \mathrm{Bg} / \mathrm{m}^{3}$

$0.00009 \mathrm{Bg} / \mathrm{m}^{3}$

$0.0002 \mathrm{Bg} / \mathrm{m}^{3}$

$0.00008 \mathrm{~Bq} / \mathrm{m}^{3}$

$0.00005 \mathrm{~Bq} / \mathrm{m}^{3}$

0.0002

0.0002

0.0002

0.0003

0.0001

0.0003

0.0002

0.00012

0.00011

0.0002

0.00013

0.0003

0.0003

0.0003

0.0003

0.00009

0.0004

0.00011

0.0003

0.0003

0.0002

0.0003

0.0003

0.00011

0.0003
$\mathrm{Bq} / \mathrm{m}^{3}$

$\mathrm{Bq} / \mathrm{m}^{3}$

$\mathrm{Bg} / \mathrm{m}^{3}$

$\mathrm{Bg} / \mathrm{m}^{3}$

$\mathrm{Bq} / \mathrm{m}^{3}$

$\mathrm{Bq} / \mathrm{m}^{3}$

$\mathrm{Bg} / \mathrm{m}^{3}$

$\mathrm{Bq} / \mathrm{m}^{3}$

$\mathrm{Bq} / \mathrm{m}^{3}$

$\mathrm{Bq} / \mathrm{m}^{3}$

$\mathrm{Bq} / \mathrm{m}^{3}$

$\mathrm{Bq} / \mathrm{m}^{3}$

$\mathrm{Bq} / \mathrm{m}^{3}$

$\mathrm{Bq} / \mathrm{m}^{3}$

$\mathrm{Bq} / \mathrm{m}^{3}$

$\mathrm{Bq} / \mathrm{m}^{3}$

$\mathrm{Bq} / \mathrm{m}^{3}$

$\mathrm{Bq} / \mathrm{m}^{3}$

$\mathrm{Bg} / \mathrm{m}^{3}$

$\mathrm{Bq} / \mathrm{m}^{3}$

$\mathrm{Bq} / \mathrm{m}^{3}$

$\mathrm{Bq} / \mathrm{m}^{3}$

$\mathrm{Bq} / \mathrm{m}^{3}$

$\mathrm{Bq} / \mathrm{m}^{3}$

$\mathrm{Bq} / \mathrm{m}^{3}$
Sample

Sample

Sample

Sample

Sample

Sample

Sample

Sample

Sample

Sample

Sample

Sample

Sample

Sample

Sample

Sample

Sample

Sample

Sample

Sample

Sample

Sample

Sample

Sample

Sample

Sample

Sample

Sample

Sample

Sample

Sample

Sample

Sample

Sample

Sample

Sample

Sample

Sample

Sample

Sample

Sample

Sample

Sample

Sample

Sample 


\section{Analyte \\ Location \\ Radiological Activity}

Date

Result

MDA or PQL

Units

QC Type

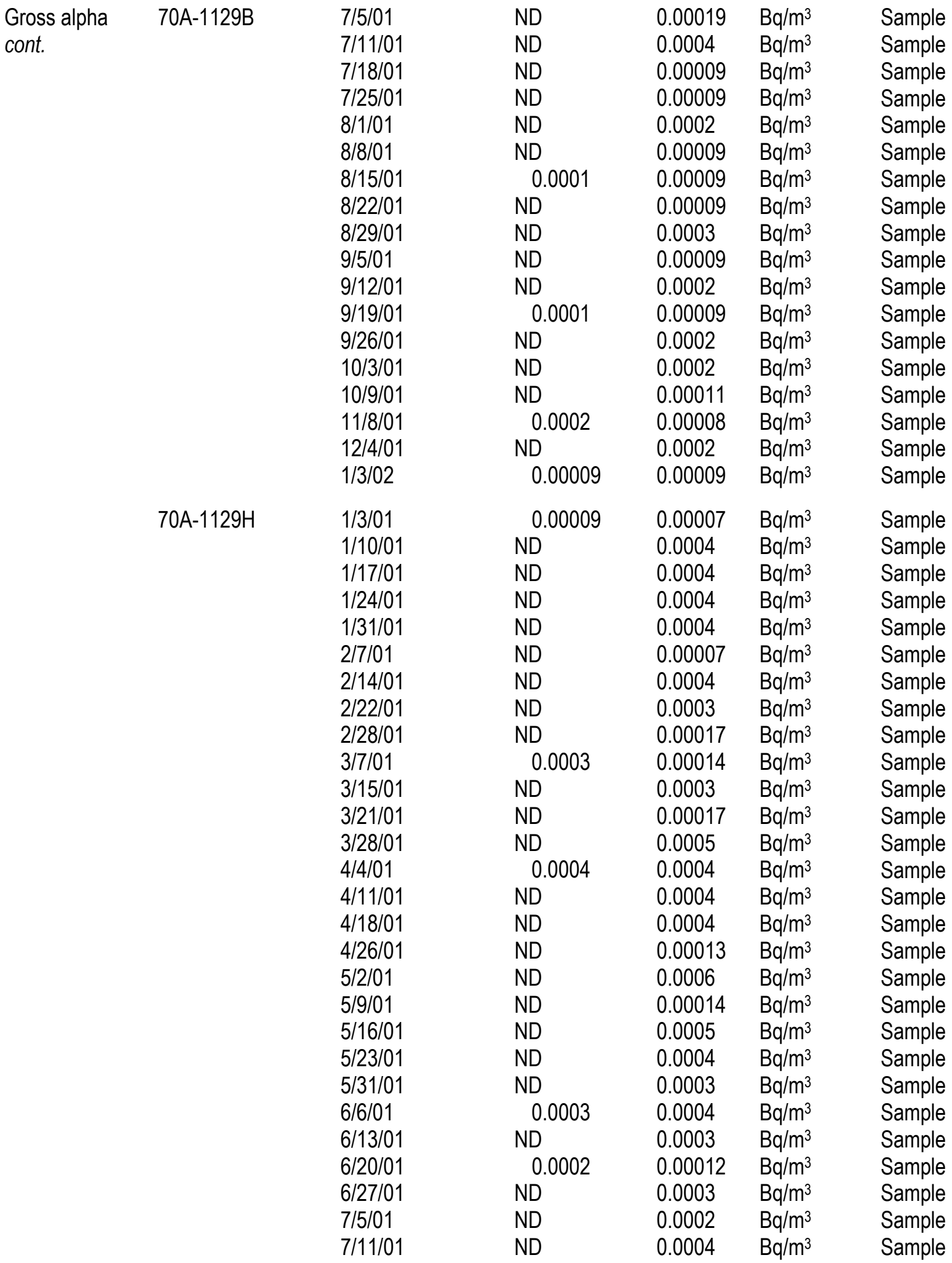


Analyte

\section{Radiological Activity}

Gross alpha $\quad 70 \mathrm{~A}-1129 \mathrm{H}$ cont.
Date

Result

MDA or PQL

Units QC Type

$\begin{array}{clll}0.0001 & 0.0001 & \mathrm{~Bq} / \mathrm{m}^{3} & \text { Sample } \\ 0.0002 & 0.0001 & \mathrm{~Bq} / \mathrm{m}^{3} & \text { Sample } \\ \text { ND } & 0.0002 & \mathrm{~Bq} / \mathrm{m}^{3} & \text { Sample } \\ \text { ND } & 0.00013 & \mathrm{~Bq} / \mathrm{m}^{3} & \text { Sample } \\ 0.0002 & 0.00013 & \mathrm{~Bq} / \mathrm{m}^{3} & \text { Sample } \\ 0.0002 & 0.00012 & \mathrm{~Bq} / \mathrm{m}^{3} & \text { Sample } \\ \text { ND } & 0.0002 & \mathrm{~Bq} / \mathrm{m}^{3} & \text { Sample } \\ \text { ND } & 0.00011 & \mathrm{~Bq} / \mathrm{m}^{3} & \text { Sample } \\ \text { ND } & 0.0003 & \mathrm{~Bq} / \mathrm{m}^{3} & \text { Sample } \\ \text { ND } & 0.00011 & \mathrm{~Bq} / \mathrm{m}^{3} & \text { Sample } \\ \text { ND } & 0.0002 & \mathrm{~Bq} / \mathrm{m}^{3} & \text { Sample } \\ \text { ND } & 0.0003 & \mathrm{~Bq} / \mathrm{m}^{3} & \text { Sample } \\ \text { ND } & 0.00015 & \mathrm{~Bq} / \mathrm{m}^{3} & \text { Sample } \\ \text { ND } & 0.00013 & \mathrm{~Bq} / \mathrm{m}^{3} & \text { Sample } \\ 0.0002 & 0.00012 & \mathrm{~Bq} / \mathrm{m}^{3} & \text { Sample } \\ \text { ND } & 0.0003 & \mathrm{~Bq} / \mathrm{m}^{3} & \text { Sample } \\ 0.00008 & 0.00008 & \mathrm{~Bq} / \mathrm{m}^{3} & \text { Sample } \\ \text { ND } & 0.0003 & \mathrm{~Bq} / \mathrm{m}^{3} & \text { Sample } \\ 0.0001 & 0.00009 & \mathrm{~Bq} / \mathrm{m}^{3} & \text { Sample } \\ \text { ND } & 0.00011 & \mathrm{~Bq} / \mathrm{m}^{3} & \text { Sample } \\ 0.0001 & 0.0001 & \mathrm{~Bq} / \mathrm{m}^{3} & \text { Sample } \\ \text { ND } & 0.0003 & \mathrm{~Bq} / \mathrm{m}^{3} & \text { Sample } \\ 0.0001 & 0.00012 & \mathrm{~Bq} / \mathrm{m}^{3} & \text { Sample } \\ 0.0002 & 0.00006 & \mathrm{~Bq} / \mathrm{m}^{3} & \text { Sample } \\ & & & \end{array}$

70A-1129P $\quad 1 / 3 / 01$

$2 / 7 / 01$

$3 / 7 / 01$

$4 / 4 / 01$

$5 / 2 / 01$

$6 / 6 / 01$

$7 / 5 / 01$

$8 / 1 / 01$

$9 / 5 / 01$

10/5/01

$11 / 8 / 01$

$12 / 4 / 01$

$1 / 3 / 02$

70A-1129RT

$1 / 10 / 01$
$1 / 17 / 01$
$1 / 24 / 01$
$1 / 31 / 01$
$2 / 7 / 01$
$2 / 14 / 01$
$2 / 22 / 01$

$\begin{array}{llll}\text { ND } & 0.00016 & \mathrm{~Bq} / \mathrm{m}^{3} & \text { Sample } \\ \text { 0.00009 } & 0.00008 & \mathrm{~Bq} / \mathrm{m}^{3} & \text { Sample } \\ \text { ND } & 0.0001 & \mathrm{~Bq} / \mathrm{m}^{3} & \text { Sample } \\ \text { ND } & 0.0003 & \mathrm{~Bq} / \mathrm{m}^{3} & \text { Sample } \\ \text { ND } & 0.0004 & \mathrm{~Bq} / \mathrm{m}^{3} & \text { Sample } \\ \text { ND } & 0.0002 & \mathrm{~Bq} / \mathrm{m}^{3} & \text { Sample } \\ \text { ND } & 0.0001 & \mathrm{~Bq} / \mathrm{m}^{3} & \text { Sample } \\ \text { ND } & 0.00013 & \mathrm{~Bq} / \mathrm{m}^{3} & \text { Sample } \\ \text { ND } & 0.00009 & \mathrm{~Bq} / \mathrm{m}^{3} & \text { Sample } \\ \text { ND } & 0.00008 & \mathrm{~Bq} / \mathrm{m}^{3} & \text { Sample } \\ \text { ND } & 0.00007 & \mathrm{~Bq} / \mathrm{m}^{3} & \text { Sample } \\ \text { ND } & 0.0002 & \mathrm{~Bq} / \mathrm{m}^{3} & \text { Sample } \\ \text { ND } & 0.00008 & \mathrm{~Bq} / \mathrm{m}^{3} & \text { Sample }\end{array}$

ND

ND

ND

ND

ND

ND

ND

$\begin{array}{lll}0.00015 & \mathrm{~Bq} / \mathrm{m}^{3} & \text { Sample } \\ 0.0002 & \mathrm{~Bq} / \mathrm{m}^{3} & \text { Sample } \\ 0.00011 & \mathrm{~Bq} / \mathrm{m}^{3} & \text { Sample } \\ 0.0003 & \mathrm{~Bq} / \mathrm{m}^{3} & \text { Sample } \\ 0.0003 & \mathrm{~Bq} / \mathrm{m}^{3} & \text { Sample } \\ 0.0003 & \mathrm{~Bq} / \mathrm{m}^{3} & \text { Sample } \\ 0.0002 & \mathrm{~Bq} / \mathrm{m}^{3} & \text { Sample }\end{array}$




$\begin{array}{llllll}\text { Analyte } & \text { Location } & \text { Date } & \text { Result } & \text { MDA or PQL Units } & \text { QC Type }\end{array}$

\section{Radiological Activity}

\begin{tabular}{|c|c|c|c|c|c|c|}
\hline \multirow{42}{*}{\multicolumn{2}{|c|}{$\begin{array}{l}\text { Gross alpha } \\
\text { cont. }\end{array}$}} & $2 / 28 / 01$ & ND & 0.00013 & $\mathrm{~Bq} / \mathrm{m}^{3}$ & Sample \\
\hline & & $3 / 7 / 01$ & ND & 0.00012 & $\mathrm{~Bq} / \mathrm{m}^{3}$ & Sample \\
\hline & & $3 / 15 / 01$ & ND & 0.0003 & $\mathrm{~Bq} / \mathrm{m}^{3}$ & Sample \\
\hline & & $3 / 21 / 01$ & ND & 0.00014 & $\mathrm{~Bq} / \mathrm{m}^{3}$ & Sample \\
\hline & & $3 / 28 / 01$ & ND & 0.0003 & $\mathrm{~Bq} / \mathrm{m}^{3}$ & Sample \\
\hline & & $4 / 4 / 01$ & ND & 0.00012 & $\mathrm{~Bq} / \mathrm{m}^{3}$ & Sample \\
\hline & & 4/11/01 & ND & 0.0003 & $\mathrm{~Bq} / \mathrm{m}^{3}$ & Sample \\
\hline & & 4/18/01 & ND & 0.00012 & $\mathrm{~Bq} / \mathrm{m}^{3}$ & Sample \\
\hline & & $4 / 26 / 01$ & ND & 0.0006 & $\mathrm{~Bq} / \mathrm{m}^{3}$ & Sample \\
\hline & & $5 / 2 / 01$ & ND & 0.0005 & $\mathrm{~Bq} / \mathrm{m}^{3}$ & Sample \\
\hline & & $5 / 16 / 01$ & ND & 0.0004 & $\mathrm{~Bq} / \mathrm{m}^{3}$ & Sample \\
\hline & & $5 / 23 / 01$ & 0.0002 & 0.00016 & $\mathrm{~Bq} / \mathrm{m}^{3}$ & Sample \\
\hline & & $5 / 31 / 01$ & ND & 0.0004 & $\mathrm{~Bq} / \mathrm{m}^{3}$ & Sample \\
\hline & & 6/6/01 & ND & 0.0005 & $\mathrm{~Bq} / \mathrm{m}^{3}$ & Sample \\
\hline & & $6 / 13 / 01$ & ND & 0.00018 & $\mathrm{~Bq} / \mathrm{m}^{3}$ & Sample \\
\hline & & $6 / 20 / 01$ & ND & 0.0004 & $\mathrm{Bg} / \mathrm{m}^{3}$ & Sample \\
\hline & & $6 / 27 / 01$ & ND & 0.00017 & $\mathrm{~Bq} / \mathrm{m}^{3}$ & Sample \\
\hline & & $7 / 5 / 01$ & ND & 0.0004 & $\mathrm{~Bq} / \mathrm{m}^{3}$ & Sample \\
\hline & & $7 / 11 / 01$ & ND & 0.0002 & $\mathrm{~Bq} / \mathrm{m}^{3}$ & Sample \\
\hline & & $7 / 18 / 01$ & ND & 0.00016 & $\mathrm{~Bq} / \mathrm{m}^{3}$ & Sample \\
\hline & & $7 / 25 / 01$ & ND & 0.00016 & $\mathrm{~Bq} / \mathrm{m}^{3}$ & Sample \\
\hline & & $8 / 1 / 01$ & ND & 0.0004 & $\mathrm{~Bq} / \mathrm{m}^{3}$ & Sample \\
\hline & & $8 / 8 / 01$ & ND & 0.00016 & $\mathrm{Bg} / \mathrm{m}^{3}$ & Sample \\
\hline & & $8 / 15 / 01$ & ND & 0.0004 & $\mathrm{~Bq} / \mathrm{m}^{3}$ & Sample \\
\hline & & 8/22/01 & ND & 0.0004 & $\mathrm{~Bq} / \mathrm{m}^{3}$ & Sample \\
\hline & & $8 / 29 / 01$ & ND & 0.0004 & $\mathrm{~Bq} / \mathrm{m}^{3}$ & Sample \\
\hline & & $9 / 5 / 01$ & 0.0002 & 0.00016 & $\mathrm{~Bq} / \mathrm{m}^{3}$ & Sample \\
\hline & & $9 / 12 / 01$ & ND & 0.00015 & $\mathrm{~Bq} / \mathrm{m}^{3}$ & Sample \\
\hline & & 9/19/01 & ND & 0.0004 & $\mathrm{~Bq} / \mathrm{m}^{3}$ & Sample \\
\hline & & 9/26/01 & ND & 0.0005 & $\mathrm{~Bq} / \mathrm{m}^{3}$ & Sample \\
\hline & & $10 / 3 / 01$ & ND & 0.00017 & $\mathrm{~Bq} / \mathrm{m}^{3}$ & Sample \\
\hline & & 10/9/01 & ND & 0.0005 & $\mathrm{~Bq} / \mathrm{m}^{3}$ & Sample \\
\hline & & $10 / 16 / 01$ & ND & 0.0006 & $\mathrm{~Bq} / \mathrm{m}^{3}$ & Sample \\
\hline & & $10 / 23 / 01$ & ND & 0.0004 & $\mathrm{~Bq} / \mathrm{m}^{3}$ & Sample \\
\hline & & $10 / 30 / 01$ & ND & 0.00017 & $\mathrm{~Bq} / \mathrm{m}^{3}$ & Sample \\
\hline & & 11/8/01 & ND & 0.0003 & $\mathrm{~Bq} / \mathrm{m}^{3}$ & Sample \\
\hline & & $11 / 13 / 01$ & ND & 0.0005 & $\mathrm{~Bq} / \mathrm{m}^{3}$ & Sample \\
\hline & & $11 / 20 / 01$ & ND & 0.0002 & $\mathrm{~Bq} / \mathrm{m}^{3}$ & Sample \\
\hline & & $11 / 26 / 01$ & ND & 0.00018 & $\mathrm{~Bq} / \mathrm{m}^{3}$ & Sample \\
\hline & & $12 / 4 / 01$ & ND & 0.0003 & $\mathrm{~Bq} / \mathrm{m}^{3}$ & Sample \\
\hline & & $12 / 19 / 01$ & ND & 0.00016 & $\mathrm{~Bq} / \mathrm{m}^{3}$ & Sample \\
\hline & & $1 / 2 / 02$ & ND & 0.00008 & $\mathrm{~Bq} / \mathrm{m}^{3}$ & Sample \\
\hline & $70 A-1145$ & $1 / 3 / 01$ & 0.00006 & 0.00004 & $\mathrm{~Bq} / \mathrm{m}^{3}$ & Sample \\
\hline & & $1 / 10 / 01$ & ND & 0.0002 & $\mathrm{~Bq} / \mathrm{m}^{3}$ & Samp \\
\hline & & 1/17/01 & ND & 0.0002 & $\mathrm{~Bq} / \mathrm{m}^{3}$ & Sampl \\
\hline
\end{tabular}


Analyte

Location

Radiological Activity

\begin{tabular}{|c|c|c|c|c|c|c|}
\hline \multirow{41}{*}{\multicolumn{2}{|c|}{$\begin{array}{l}\text { Gross alpha } \\
\text { cont. }\end{array}$}} & $1 / 24 / 01$ & ND & 0.0002 & $\mathrm{~Bq} / \mathrm{m}^{3}$ & Sample \\
\hline & & $1 / 31 / 01$ & ND & 0.0002 & $\mathrm{~Bq} / \mathrm{m}^{3}$ & Sample \\
\hline & & $2 / 7 / 01$ & ND & 0.0001 & $\mathrm{~Bq} / \mathrm{m}^{3}$ & Sample \\
\hline & & $2 / 14 / 01$ & ND & 0.0002 & $\mathrm{~Bq} / \mathrm{m}^{3}$ & Sample \\
\hline & & $2 / 22 / 01$ & ND & 0.0002 & $\mathrm{~Bq} / \mathrm{m}^{3}$ & Sample \\
\hline & & $2 / 28 / 01$ & 0.0002 & 0.00011 & $\mathrm{~Bq} / \mathrm{m}^{3}$ & Sample \\
\hline & & $3 / 7 / 01$ & ND & 0.0001 & $\mathrm{~Bq} / \mathrm{m}^{3}$ & Sample \\
\hline & & $3 / 15 / 01$ & ND & 0.0002 & $\mathrm{~Bq} / \mathrm{m}^{3}$ & Sample \\
\hline & & $3 / 21 / 01$ & ND & 0.00011 & $\mathrm{~Bq} / \mathrm{m}^{3}$ & Sample \\
\hline & & $3 / 28 / 01$ & ND & 0.0003 & $\mathrm{~Bq} / \mathrm{m}^{3}$ & Sample \\
\hline & & $4 / 4 / 01$ & ND & 0.0002 & $\mathrm{~Bq} / \mathrm{m}^{3}$ & Sample \\
\hline & & $4 / 11 / 01$ & ND & 0.0002 & $\mathrm{~Bq} / \mathrm{m}^{3}$ & Sample \\
\hline & & $4 / 18 / 01$ & ND & 0.0002 & $\mathrm{~Bq} / \mathrm{m}^{3}$ & Sample \\
\hline & & $4 / 26 / 01$ & ND & 0.00016 & $\mathrm{~Bq} / \mathrm{m}^{3}$ & Sample \\
\hline & & $5 / 2 / 01$ & ND & 0.0004 & $\mathrm{~Bq} / \mathrm{m}^{3}$ & Sample \\
\hline & & $5 / 9 / 01$ & ND & 0.0001 & $\mathrm{~Bq} / \mathrm{m}^{3}$ & Sample \\
\hline & & $5 / 16 / 01$ & ND & 0.0003 & $\mathrm{~Bq} / \mathrm{m}^{3}$ & Sample \\
\hline & & $5 / 23 / 01$ & ND & 0.0002 & $\mathrm{~Bq} / \mathrm{m}^{3}$ & Sample \\
\hline & & $5 / 31 / 01$ & ND & 0.0002 & $\mathrm{~Bq} / \mathrm{m}^{3}$ & Sample \\
\hline & & $6 / 6 / 01$ & ND & 0.0003 & $\mathrm{~Bq} / \mathrm{m}^{3}$ & Sample \\
\hline & & $6 / 13 / 01$ & ND & 0.0003 & $\mathrm{~Bq} / \mathrm{m}^{3}$ & Sample \\
\hline & & $6 / 20 / 01$ & 0.0002 & 0.0001 & $\mathrm{~Bq} / \mathrm{m}^{3}$ & Sample \\
\hline & & $6 / 27 / 01$ & ND & 0.0002 & $\mathrm{~Bq} / \mathrm{m}^{3}$ & Sample \\
\hline & & $7 / 5 / 01$ & ND & 0.00019 & $\mathrm{~Bq} / \mathrm{m}^{3}$ & Sample \\
\hline & & $7 / 11 / 01$ & ND & 0.0004 & $\mathrm{~Bq} / \mathrm{m}^{3}$ & Sample \\
\hline & & $7 / 18 / 01$ & ND & 0.00009 & $\mathrm{~Bq} / \mathrm{m}^{3}$ & Sample \\
\hline & & $7 / 25 / 01$ & ND & 0.00009 & $\mathrm{~Bq} / \mathrm{m}^{3}$ & Sample \\
\hline & & $8 / 1 / 01$ & ND & 0.0002 & $\mathrm{~Bq} / \mathrm{m}^{3}$ & Sample \\
\hline & & $8 / 8 / 01$ & 0.0001 & 0.00009 & $\mathrm{~Bq} / \mathrm{m}^{3}$ & Sample \\
\hline & & $8 / 15 / 01$ & 0.0002 & 0.00009 & $\mathrm{~Bq} / \mathrm{m}^{3}$ & Sample \\
\hline & & $8 / 22 / 01$ & ND & 0.00009 & $\mathrm{~Bq} / \mathrm{m}^{3}$ & Sample \\
\hline & & $8 / 29 / 01$ & ND & 0.0003 & $\mathrm{~Bq} / \mathrm{m}^{3}$ & Sample \\
\hline & & $9 / 5 / 01$ & 0.0001 & 0.00009 & $\mathrm{~Bq} / \mathrm{m}^{3}$ & Sample \\
\hline & & $9 / 12 / 01$ & ND & 0.0002 & $\mathrm{~Bq} / \mathrm{m}^{3}$ & Sample \\
\hline & & $9 / 19 / 01$ & ND & 0.00009 & $\mathrm{~Bq} / \mathrm{m}^{3}$ & Sample \\
\hline & & $9 / 26 / 01$ & ND & 0.0002 & $\mathrm{~Bq} / \mathrm{m}^{3}$ & Sample \\
\hline & & $10 / 3 / 01$ & ND & 0.0002 & $\mathrm{~Bq} / \mathrm{m}^{3}$ & Sample \\
\hline & & $10 / 9 / 01$ & ND & 0.0001 & $\mathrm{~Bq} / \mathrm{m}^{3}$ & Sample \\
\hline & & $11 / 8 / 01$ & ND & 0.00008 & $\mathrm{~Bq} / \mathrm{m}^{3}$ & Sample \\
\hline & & $12 / 4 / 01$ & ND & 0.0002 & $\mathrm{~Bq} / \mathrm{m}^{3}$ & Sample \\
\hline & & $1 / 3 / 02$ & 0.00009 & 0.00009 & $\mathrm{~Bq} / \mathrm{m}^{3}$ & Sample \\
\hline & $70 \mathrm{~A}-2211 \mathrm{H}$ & $1 / 3 / 01$ & ND & 0.00009 & $\mathrm{~Bq} / \mathrm{m}^{3}$ & Sample \\
\hline & & $2 / 7 / 01$ & 0.0001 & 0.00007 & $\mathrm{~Bq} / \mathrm{m}^{3}$ & Sample \\
\hline & & $3 / 7 / 01$ & ND & 0.00008 & $\mathrm{~Bq} / \mathrm{m}^{3}$ & Sample \\
\hline & & $4 / 4 / 01$ & ND & 0.0002 & $\mathrm{~Bq} / \mathrm{m}^{3}$ & Sample \\
\hline
\end{tabular}




$\begin{array}{llllll}\text { Analyte } & \text { Location } & \text { Date } & \text { Result } & \text { MDA or PQL Units } & \text { QC Type }\end{array}$

\section{Radiological Activity}

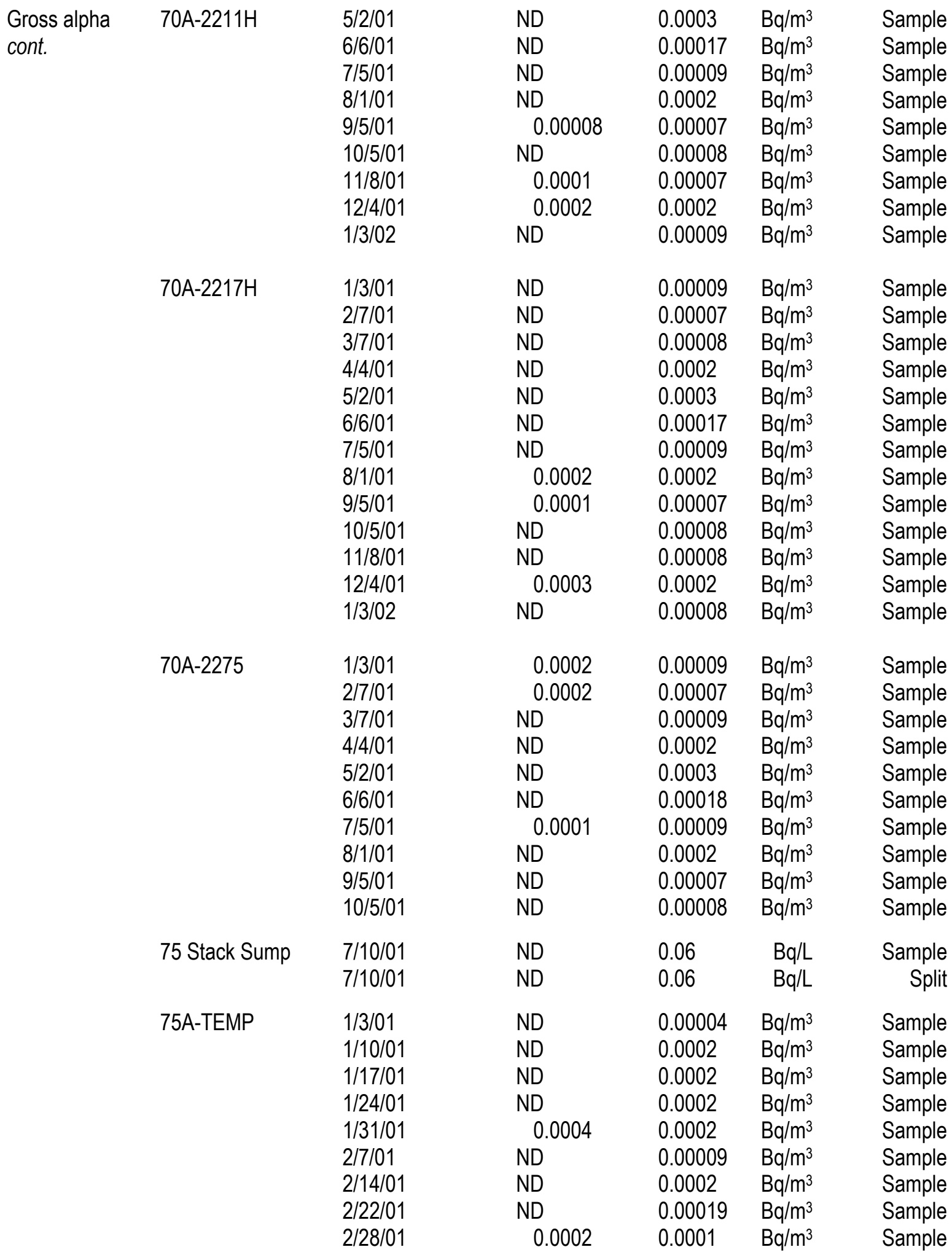


Analyte

Radiological Activity

Gross alpha 75A-TEMP

cont.

Date

Result

MDA or PQL

Units

QC Type

$3 / 7 / 01$

$3 / 15 / 01$

$3 / 21 / 01$

$3 / 28 / 01$

$4 / 4 / 01$

$4 / 11 / 01$

$4 / 18 / 01$

$4 / 26 / 01$

$5 / 2 / 01$

$5 / 9 / 01$

$5 / 16 / 01$

$5 / 23 / 01$

$5 / 31 / 01$

$6 / 6 / 01$

$6 / 13 / 01$

$6 / 20 / 01$

$6 / 27 / 01$

$7 / 5 / 01$

$7 / 11 / 01$

$7 / 18 / 01$

$7 / 25 / 01$

$8 / 1 / 01$

$8 / 8 / 01$

$8 / 15 / 01$

$8 / 22 / 01$

$8 / 29 / 01$

$9 / 5 / 01$

9/12/01

9/19/01

9/26/01

10/3/01

10/9/01

$10 / 16 / 01$

$10 / 24 / 01$

10/30/01

$11 / 8 / 01$

$11 / 13 / 01$

$11 / 20 / 01$

$11 / 26 / 01$

$12 / 4 / 01$

$12 / 12 / 01$

85 Glovebox

\section{$1 / 3 / 01$ \\ $1 / 10 / 01$ \\ $1 / 17 / 01$ \\ $1 / 24 / 01$ \\ $1 / 31 / 01$}

$\begin{array}{clll}0.0002 & 0.00009 & \mathrm{~Bq} / \mathrm{m}^{3} & \text { Sample } \\ \text { ND } & 0.00019 & \mathrm{~Bq} / \mathrm{m}^{3} & \text { Sample } \\ 0.0001 & 0.0001 & \mathrm{~Bq} / \mathrm{m}^{3} & \text { Sample }\end{array}$

ND $\quad 0.0003 \quad \mathrm{~Bq} / \mathrm{m}^{3} \quad$ Sample

ND $\quad 0.0002 \quad \mathrm{~Bq} / \mathrm{m}^{3} \quad$ Sample

ND $\quad 0.0002 \quad \mathrm{~Bq} / \mathrm{m}^{3} \quad$ Sample

ND $\quad 0.0002 \quad \mathrm{~Bq} / \mathrm{m}^{3} \quad$ Sample

ND $\quad 0.00008 \quad \mathrm{~Bq} / \mathrm{m}^{3} \quad$ Sample

ND $\quad 0.0003 \quad \mathrm{~Bq} / \mathrm{m}^{3} \quad$ Sample

$\begin{array}{llll}0.0001 & 0.00009 & \mathrm{~Bq} / \mathrm{m}^{3} & \text { Sample }\end{array}$

ND $\quad 0.0003 \quad \mathrm{~Bq} / \mathrm{m}^{3} \quad$ Sample

ND $\quad 0.0002 \quad \mathrm{~Bq} / \mathrm{m}^{3} \quad$ Sample

ND $\quad 0.0002 \quad \mathrm{~Bq} / \mathrm{m}^{3} \quad$ Sample

ND $\quad 0.0003 \quad \mathrm{~Bq} / \mathrm{m}^{3} \quad$ Sample

ND $\quad 0.0002 \quad \mathrm{~Bq} / \mathrm{m}^{3} \quad$ Sample

$\begin{array}{llll}0.00009 & 0.00009 & \mathrm{~Bq} / \mathrm{m}^{3} & \text { Sample }\end{array}$

ND $\quad 0.0002 \quad \mathrm{~Bq} / \mathrm{m}^{3} \quad$ Sample

ND $\quad 0.00019 \mathrm{~Bq} / \mathrm{m}^{3} \quad$ Sample

ND $\quad 0.0004 \quad \mathrm{~Bq} / \mathrm{m}^{3} \quad$ Sample

ND $\quad 0.00009 \quad \mathrm{~Bq} / \mathrm{m}^{3} \quad$ Sample

ND $\quad 0.00009 \quad \mathrm{~Bq} / \mathrm{m}^{3} \quad$ Sample

ND $\quad 0.0002 \quad \mathrm{~Bq} / \mathrm{m}^{3} \quad$ Sample

ND $\quad 0.00009 \quad \mathrm{~Bq} / \mathrm{m}^{3} \quad$ Sample

$\begin{array}{llll}0.0001 & 0.00009 & \mathrm{~Bq} / \mathrm{m}^{3} & \text { Sample }\end{array}$

ND $\quad 0.00009 \quad \mathrm{~Bq} / \mathrm{m}^{3} \quad$ Sample

ND $\quad 0.0003 \quad \mathrm{~Bq} / \mathrm{m}^{3} \quad$ Sample

ND $\quad 0.00009 \mathrm{~Bq} / \mathrm{m}^{3} \quad$ Sample

ND $\quad 0.0002 \quad \mathrm{~Bq} / \mathrm{m}^{3} \quad$ Sample

$0.00009 \quad 0.00009 \quad \mathrm{~Bq} / \mathrm{m}^{3} \quad$ Sample

ND $\quad 0.0002 \quad \mathrm{~Bq} / \mathrm{m}^{3} \quad$ Sample

ND $\quad 0.0002 \quad \mathrm{~Bq} / \mathrm{m}^{3} \quad$ Sample

ND $\quad 0.0001 \quad \mathrm{~Bq} / \mathrm{m}^{3} \quad$ Sample

ND $\quad 0.0001 \quad \mathrm{~Bq} / \mathrm{m}^{3} \quad$ Sample

$\begin{array}{llll}0.0001 & 0.00008 & \mathrm{~Bq} / \mathrm{m}^{3} & \text { Sample }\end{array}$

$\begin{array}{llll}\text { ND } & 0.0002 & \mathrm{~Bq} / \mathrm{m}^{3} & \text { Sample }\end{array}$

$\begin{array}{llll}0.0001 & 0.00007 & \mathrm{~Bq} / \mathrm{m}^{3} & \text { Sample }\end{array}$

ND $\quad 0.0003 \quad \mathrm{~Bq} / \mathrm{m}^{3} \quad$ Sample

ND $\quad 0.00009 \quad \mathrm{~Bq} / \mathrm{m}^{3} \quad$ Sample

$0.0001 \quad 0.0001 \quad \mathrm{~Bq} / \mathrm{m}^{3} \quad$ Sample

ND $\quad 0.00008 \quad \mathrm{~Bq} / \mathrm{m}^{3} \quad$ Sample

ND $\quad 0.00019 \quad \mathrm{~Bq} / \mathrm{m}^{3} \quad$ Sample

ND $\quad 0.00004 \quad \mathrm{~Bq} / \mathrm{m}^{3} \quad$ Sample

ND $\quad 0.00011 \quad \mathrm{~Bq} / \mathrm{m}^{3} \quad$ Sample

ND $\quad 0.00011 \quad \mathrm{~Bq} / \mathrm{m}^{3} \quad$ Sample

ND $\quad 0.0000012 \mathrm{~Bq} / \mathrm{m}^{3} \quad$ Sample

ND $\quad 0.00013 \quad \mathrm{~Bq} / \mathrm{m}^{3} \quad$ Sample 


$\begin{array}{llllll}\text { Analyte } & \text { Location } & \text { Date } & \text { Result } & \text { MDA or PQL Units } & \text { QC Type }\end{array}$

\section{Radiological Activity}

\begin{tabular}{|c|c|c|c|c|c|c|}
\hline Gross alpha & 85 Glovebox & $2 / 7 / 01$ & ND & 0.00011 & $\mathrm{~Bq} / \mathrm{m}^{3}$ & $\begin{array}{l}\text { Sample } \\
\text { Sample }\end{array}$ \\
\hline cont. & & $2 / 14 / 01$ & ND & 0.00011 & $\mathrm{~Bq} / \mathrm{m}^{3}$ & Sample \\
\hline & & $2 / 22 / 01$ & ND & 0.0001 & $\mathrm{~Bq} / \mathrm{m}^{3}$ & Sample \\
\hline & & $2 / 28 / 01$ & ND & 0.00016 & $\mathrm{~Bq} / \mathrm{m}^{3}$ & Sample \\
\hline & & $3 / 7 / 01$ & ND & 0.00011 & $\mathrm{~Bq} / \mathrm{m}^{3}$ & Sample \\
\hline & & $3 / 15 / 01$ & ND & 0.0001 & $\mathrm{~Bq} / \mathrm{m}^{3}$ & Sample \\
\hline & & $3 / 21 / 01$ & ND & 0.00016 & $\mathrm{~Bq} / \mathrm{m}^{3}$ & Sample \\
\hline & & $3 / 28 / 01$ & ND & 0.00014 & $\mathrm{~Bq} / \mathrm{m}^{3}$ & Sample \\
\hline & & $4 / 4 / 01$ & ND & 0.00009 & $\mathrm{~Bq} / \mathrm{m}^{3}$ & Sample \\
\hline & & $4 / 11 / 01$ & ND & 0.00009 & $\mathrm{~Bq} / \mathrm{m}^{3}$ & Sample \\
\hline & & $4 / 18 / 01$ & ND & 0.000011 & $\mathrm{~Bq} / \mathrm{m}^{3}$ & Sample \\
\hline & & $4 / 26 / 01$ & ND & 0.0001 & $\mathrm{Bg} / \mathrm{m}^{3}$ & Sample \\
\hline & & $5 / 2 / 01$ & ND & 0.00016 & $\mathrm{~Bq} / \mathrm{m}^{3}$ & Sample \\
\hline & & $5 / 9 / 01$ & ND & 0.00011 & $\mathrm{~Bq} / \mathrm{m}^{3}$ & Sample \\
\hline & & $5 / 16 / 01$ & ND & 0.00012 & $\mathrm{~Bq} / \mathrm{m}^{3}$ & Sample \\
\hline & & $5 / 23 / 01$ & ND & 0.00012 & $\mathrm{~Bq} / \mathrm{m}^{3}$ & Sample \\
\hline & & $5 / 31 / 01$ & ND & 0.00008 & $\mathrm{Bg} / \mathrm{m}^{3}$ & Sample \\
\hline & & $6 / 6 / 01$ & ND & 0.00014 & $\mathrm{~Bq} / \mathrm{m}^{3}$ & Sample \\
\hline & & $6 / 13 / 01$ & ND & 0.00012 & $\mathrm{Bg} / \mathrm{m}^{3}$ & Sample \\
\hline & & $6 / 20 / 01$ & ND & 0.00016 & $\mathrm{~Bq} / \mathrm{m}^{3}$ & Sample \\
\hline & & $6 / 27 / 01$ & ND & 0.00012 & $\mathrm{~Bq} / \mathrm{m}^{3}$ & Sample \\
\hline & & $7 / 5 / 01$ & ND & 0.00012 & $\mathrm{~Bq} / \mathrm{m}^{3}$ & Sample \\
\hline & & $7 / 11 / 01$ & ND & 0.00014 & $\mathrm{~Bq} / \mathrm{m}^{3}$ & Sample \\
\hline & & $7 / 18 / 01$ & ND & 0.00009 & $\mathrm{Bg} / \mathrm{m}^{3}$ & Sample \\
\hline & & $7 / 25 / 01$ & ND & 0.00011 & $\mathrm{~Bq} / \mathrm{m}^{3}$ & Sample \\
\hline & & $8 / 1 / 01$ & ND & 0.00016 & $\mathrm{Bg} / \mathrm{m}^{3}$ & Sample \\
\hline & & $8 / 8 / 01$ & ND & 0.0009 & $\mathrm{~Bq} / \mathrm{m}^{3}$ & Sample \\
\hline & & $8 / 15 / 01$ & ND & 0.00008 & $\mathrm{~Bq} / \mathrm{m}^{3}$ & Sample \\
\hline & & $8 / 22 / 01$ & ND & 0.00011 & $\mathrm{~Bq} / \mathrm{m}^{3}$ & Sample \\
\hline & & $8 / 29 / 01$ & ND & 0.00014 & $\mathrm{~Bq} / \mathrm{m}^{3}$ & Sample \\
\hline & & $9 / 5 / 01$ & ND & 0.00014 & $\mathrm{~Bq} / \mathrm{m}^{3}$ & Sample \\
\hline & & $9 / 12 / 01$ & ND & 0.00007 & $\mathrm{~Bq} / \mathrm{m}^{3}$ & Sample \\
\hline & & $9 / 19 / 01$ & ND & 0.00011 & $\mathrm{~Bq} / \mathrm{m}^{3}$ & Sample \\
\hline & & $9 / 26 / 01$ & ND & 0.00012 & $\mathrm{~Bq} / \mathrm{m}^{3}$ & Sample \\
\hline & & $10 / 3 / 01$ & ND & 0.00009 & $\mathrm{~Bq} / \mathrm{m}^{3}$ & Sample \\
\hline & & $10 / 9 / 01$ & ND & 0.00016 & $\mathrm{~Bq} / \mathrm{m}^{3}$ & Sample \\
\hline & & $10 / 16 / 01$ & ND & 0.00014 & $\mathrm{~Bq} / \mathrm{m}^{3}$ & Sample \\
\hline & & $10 / 23 / 01$ & ND & 0.00014 & $\mathrm{~Bq} / \mathrm{m}^{3}$ & Sample \\
\hline & & $11 / 8 / 01$ & ND & 0.00011 & $\mathrm{~Bq} / \mathrm{m}^{3}$ & Sample \\
\hline & & $11 / 13 / 01$ & ND & 0.0002 & $\mathrm{~Bq} / \mathrm{m}^{3}$ & Sample \\
\hline & & $11 / 20 / 01$ & ND & 0.00013 & $\mathrm{~Bq} / \mathrm{m}^{3}$ & Sample \\
\hline & & $11 / 26 / 01$ & ND & 0.001 & $\mathrm{~Bq} / \mathrm{m}^{3}$ & Sample \\
\hline & & $12 / 4 / 01$ & ND & 0.00012 & $\mathrm{~Bq} / \mathrm{m}^{3}$ & Sample \\
\hline & & $12 / 12 / 01$ & ND & 0.00008 & $\mathrm{~Bq} / \mathrm{m}^{3}$ & Sample \\
\hline & & $12 / 18 / 01$ & ND & 0.0001 & $\mathrm{~Bq} / \mathrm{m}^{3}$ & Sample \\
\hline & & $1 / 2 / 02$ & ND & 0.00007 & $\mathrm{~Bq} / \mathrm{m}^{3}$ & Samp \\
\hline
\end{tabular}


Analyte

Location

Radiological Activity

\begin{tabular}{|c|c|c|c|c|c|c|}
\hline \multirow[t]{45}{*}{$\begin{array}{l}\text { Gross alpha } \\
\text { cont. }\end{array}$} & \multirow[t]{45}{*}{$85 \mathrm{Hood}$} & $\begin{array}{l}1 / 3 / 01 \\
1 / 10 / 01\end{array}$ & $\begin{array}{l}0.00008 \\
\text { ND }\end{array}$ & $\begin{array}{l}0.00008 \\
0.0004\end{array}$ & $\begin{array}{l}\mathrm{Bq} / \mathrm{m}^{3} \\
\mathrm{~Bq} / \mathrm{m}^{3}\end{array}$ & $\begin{array}{l}\text { Sample } \\
\text { Sample }\end{array}$ \\
\hline & & $1 / 17 / 01$ & ND & 0.0004 & $\mathrm{Bg} / \mathrm{m}^{3}$ & Sample \\
\hline & & $1 / 24 / 01$ & ND & 0.0004 & $\mathrm{~Bq} / \mathrm{m}^{3}$ & Sample \\
\hline & & 1/31/01 & ND & 0.0004 & $\mathrm{~Bq} / \mathrm{m}^{3}$ & Sample \\
\hline & & $2 / 7 / 01$ & ND & 0.00016 & $\mathrm{~Bq} / \mathrm{m}^{3}$ & Sample \\
\hline & & 2/14/01 & ND & 0.0004 & $\mathrm{~Bq} / \mathrm{m}^{3}$ & Sample \\
\hline & & 2/22/01 & ND & 0.0003 & $\mathrm{~Bq} / \mathrm{m}^{3}$ & Sample \\
\hline & & 2/28/01 & 0.0002 & 0.00018 & $\mathrm{~Bq} / \mathrm{m}^{3}$ & Sample \\
\hline & & $3 / 7 / 01$ & 0.0004 & 0.00016 & $\mathrm{~Bq} / \mathrm{m}^{3}$ & Sample \\
\hline & & $3 / 15 / 01$ & ND & 0.0003 & $\mathrm{~Bq} / \mathrm{m}^{3}$ & Sample \\
\hline & & $3 / 21 / 01$ & ND & 0.00016 & $\mathrm{~Bq} / \mathrm{m}^{3}$ & Sample \\
\hline & & $3 / 28 / 01$ & ND & 0.0005 & $\mathrm{~Bq} / \mathrm{m}^{3}$ & Sample \\
\hline & & $4 / 4 / 01$ & ND & 0.0004 & $\mathrm{~Bq} / \mathrm{m}^{3}$ & Sample \\
\hline & & $4 / 11 / 01$ & ND & 0.0004 & $\mathrm{~Bq} / \mathrm{m}^{3}$ & Sample \\
\hline & & 4/18/01 & ND & 0.0004 & $\mathrm{~Bq} / \mathrm{m}^{3}$ & Sample \\
\hline & & 4/26/01 & ND & 0.00014 & $\mathrm{~Bq} / \mathrm{m}^{3}$ & Sample \\
\hline & & $5 / 2 / 01$ & ND & 0.0003 & $\mathrm{~Bq} / \mathrm{m}^{3}$ & Sample \\
\hline & & $5 / 9 / 01$ & ND & 0.00015 & $\mathrm{~Bq} / \mathrm{m}^{3}$ & Sample \\
\hline & & 5/16/01 & ND & 0.0005 & $\mathrm{~Bq} / \mathrm{m}^{3}$ & Sample \\
\hline & & $5 / 23 / 01$ & ND & 0.0004 & $\mathrm{~Bq} / \mathrm{m}^{3}$ & Sample \\
\hline & & $5 / 31 / 01$ & ND & 0.0004 & $\mathrm{~Bq} / \mathrm{m}^{3}$ & Sample \\
\hline & & $6 / 6 / 01$ & ND & 0.0005 & $\mathrm{Bg} / \mathrm{m}^{3}$ & Sample \\
\hline & & $6 / 13 / 01$ & ND & 0.0004 & $\mathrm{~Bq} / \mathrm{m}^{3}$ & Sample \\
\hline & & $6 / 20 / 01$ & ND & 0.00016 & $\mathrm{~Bq} / \mathrm{m}^{3}$ & Sample \\
\hline & & $6 / 27 / 01$ & ND & 0.0004 & $\mathrm{~Bq} / \mathrm{m}^{3}$ & Sample \\
\hline & & 7/11/01 & ND & 0.00006 & $\mathrm{~Bq} / \mathrm{m}^{3}$ & Sample \\
\hline & & 7/18/01 & ND & 0.00017 & $\mathrm{~Bq} / \mathrm{m}^{3}$ & Sample \\
\hline & & 7/25/01 & 0.0001 & 0.00011 & $\mathrm{~Bq} / \mathrm{m}^{3}$ & Sample \\
\hline & & $8 / 1 / 01$ & ND & 0.0004 & $\mathrm{~Bq} / \mathrm{m}^{3}$ & Sample \\
\hline & & $8 / 8 / 01$ & ND & 0.0009 & $\mathrm{~Bq} / \mathrm{m}^{3}$ & Sample \\
\hline & & $8 / 15 / 01$ & ND & 0.00015 & $\mathrm{~Bq} / \mathrm{m}^{3}$ & Sample \\
\hline & & 8/29/01 & ND & 0.0005 & $\mathrm{~Bq} / \mathrm{m}^{3}$ & Sample \\
\hline & & $9 / 5 / 01$ & ND & 0.00014 & $\mathrm{~Bq} / \mathrm{m}^{3}$ & Sample \\
\hline & & $9 / 12 / 01$ & ND & 0.0004 & $\mathrm{~Bq} / \mathrm{m}^{3}$ & Sample \\
\hline & & 9/19/01 & ND & 0.00014 & $\mathrm{~Bq} / \mathrm{m}^{3}$ & Sample \\
\hline & & $9 / 26 / 01$ & ND & 0.0004 & $\mathrm{~Bq} / \mathrm{m}^{3}$ & Sample \\
\hline & & $10 / 3 / 01$ & ND & 0.0004 & $\mathrm{~Bq} / \mathrm{m}^{3}$ & Sample \\
\hline & & 10/9/01 & 0.0002 & 0.00016 & $\mathrm{~Bq} / \mathrm{m}^{3}$ & Sample \\
\hline & & 10/16/01 & 0.0002 & 0.00014 & $\mathrm{~Bq} / \mathrm{m}^{3}$ & Sample \\
\hline & & 10/23/01 & ND & 0.00015 & $\mathrm{~Bq} / \mathrm{m}^{3}$ & Sample \\
\hline & & $10 / 30 / 01$ & ND & 0.0003 & $\mathrm{~Bq} / \mathrm{m}^{3}$ & Sample \\
\hline & & 11/8/01 & ND & 0.00012 & $\mathrm{~Bq} / \mathrm{m}^{3}$ & Sample \\
\hline & & $11 / 13 / 01$ & ND & 0.0006 & $\mathrm{~Bq} / \mathrm{m}^{3}$ & Sample \\
\hline & & $11 / 20 / 01$ & ND & 0.00015 & $\mathrm{~Bq} / \mathrm{m}^{3}$ & Sample \\
\hline & & $11 / 26 / 01$ & 0.00003 & 0.00003 & $\mathrm{~Bq} / \mathrm{m}^{3}$ & Sample \\
\hline
\end{tabular}




\section{Analyte \\ Location \\ Radiological Activity}

Date

Result MDA or PQL Units

QC Type

\begin{tabular}{|c|c|c|c|c|c|c|}
\hline \multicolumn{2}{|l|}{$\begin{array}{l}\text { Gross alpha } \\
\text { cont. }\end{array}$} & $\begin{array}{l}12 / 4 / 01 \\
12 / 12 / 01 \\
12 / 18 / 01 \\
1 / 2 / 02\end{array}$ & $\begin{array}{l}\text { ND } \\
\text { ND } \\
0.0002 \\
\text { ND }\end{array}$ & $\begin{array}{l}0.00012 \\
0.0003 \\
0.00018 \\
0.00007\end{array}$ & $\begin{array}{l}\mathrm{Bq} / \mathrm{m}^{3} \\
\mathrm{~Bq} / \mathrm{m}^{3} \\
\mathrm{~Bq} / \mathrm{m}^{3} \\
\mathrm{~Bq} / \mathrm{m}^{3}\end{array}$ & $\begin{array}{l}\text { Sample } \\
\text { Sample } \\
\text { Sample } \\
\text { Sample }\end{array}$ \\
\hline & \multirow[t]{42}{*}{ B88 Cave 0} & $1 / 3 / 01$ & 0.00008 & 0.00004 & $\mathrm{~Bq} / \mathrm{m}^{3}$ & Sample \\
\hline & & $1 / 10 / 01$ & ND & 0.0002 & $\mathrm{~Bq} / \mathrm{m}^{3}$ & Sample \\
\hline & & $1 / 17 / 01$ & ND & 0.0007 & $\mathrm{~Bq} / \mathrm{m}^{3}$ & Sample \\
\hline & & $1 / 24 / 01$ & ND & 0.0006 & $\mathrm{~Bq} / \mathrm{m}^{3}$ & Sample \\
\hline & & $1 / 31 / 01$ & ND & 0.0007 & $\mathrm{~Bq} / \mathrm{m}^{3}$ & Sample \\
\hline & & $2 / 7 / 01$ & 0.0003 & 0.0003 & $\mathrm{Bg} / \mathrm{m}^{3}$ & Sample \\
\hline & & $2 / 14 / 01$ & ND & 0.0006 & $\mathrm{~Bq} / \mathrm{m}^{3}$ & Sample \\
\hline & & $2 / 22 / 01$ & ND & 0.0005 & $\mathrm{~Bq} / \mathrm{m}^{3}$ & Sample \\
\hline & & $2 / 28 / 01$ & 0.0002 & 0.0002 & $\mathrm{~Bq} / \mathrm{m}^{3}$ & Sample \\
\hline & & $3 / 7 / 01$ & ND & 0.00004 & $\mathrm{~Bq} / \mathrm{m}^{3}$ & Sample \\
\hline & & $3 / 15 / 01$ & ND & 0.0002 & $\mathrm{~Bq} / \mathrm{m}^{3}$ & Sample \\
\hline & & $3 / 21 / 01$ & 0.0004 & 0.0002 & $\mathrm{~Bq} / \mathrm{m}^{3}$ & Sample \\
\hline & & $3 / 28 / 01$ & ND & 0.0008 & $\mathrm{Bg} / \mathrm{m}^{3}$ & Sample \\
\hline & & $4 / 4 / 01$ & ND & 0.0005 & $\mathrm{~Bq} / \mathrm{m}^{3}$ & Sample \\
\hline & & $4 / 11 / 01$ & ND & 0.0005 & $\mathrm{~Bq} / \mathrm{m}^{3}$ & Sample \\
\hline & & 4/18/01 & ND & 0.0007 & $\mathrm{~Bq} / \mathrm{m}^{3}$ & Sample \\
\hline & & 4/26/01 & ND & 0.0002 & $\mathrm{~Bq} / \mathrm{m}^{3}$ & Sample \\
\hline & & $5 / 2 / 01$ & ND & 0.0007 & $\mathrm{~Bq} / \mathrm{m}^{3}$ & Sample \\
\hline & & $5 / 9 / 01$ & 0.0004 & 0.0002 & $\mathrm{~Bq} / \mathrm{m}^{3}$ & Sample \\
\hline & & $5 / 16 / 01$ & ND & 0.0006 & $\mathrm{Bg} / \mathrm{m}^{3}$ & Sample \\
\hline & & $5 / 23 / 01$ & ND & 0.0006 & $\mathrm{~Bq} / \mathrm{m}^{3}$ & Sample \\
\hline & & $5 / 31 / 01$ & ND & 0.0005 & $\mathrm{~Bq} / \mathrm{m}^{3}$ & Sample \\
\hline & & $6 / 6 / 01$ & ND & 0.0007 & $\mathrm{~Bq} / \mathrm{m}^{3}$ & Sample \\
\hline & & $6 / 13 / 01$ & ND & 0.0004 & $\mathrm{~Bq} / \mathrm{m}^{3}$ & Sample \\
\hline & & $6 / 20 / 01$ & 0.0002 & 0.00009 & $\mathrm{~Bq} / \mathrm{m}^{3}$ & Sample \\
\hline & & $6 / 27 / 01$ & ND & 0.0002 & $\mathrm{~Bq} / \mathrm{m}^{3}$ & Sample \\
\hline & & $7 / 5 / 01$ & ND & 0.0003 & $\mathrm{Bg} / \mathrm{m}^{3}$ & Sample \\
\hline & & $7 / 11 / 01$ & ND & 0.002 & $\mathrm{~Bq} / \mathrm{m}^{3}$ & Sample \\
\hline & & $7 / 18 / 01$ & ND & 0.00016 & $\mathrm{~Bq} / \mathrm{m}^{3}$ & Sample \\
\hline & & $7 / 25 / 01$ & ND & 0.00015 & $\mathrm{~Bq} / \mathrm{m}^{3}$ & Sample \\
\hline & & $8 / 1 / 01$ & ND & 0.0004 & $\mathrm{~Bq} / \mathrm{m}^{3}$ & Sample \\
\hline & & $8 / 8 / 01$ & ND & 0.00016 & $\mathrm{~Bq} / \mathrm{m}^{3}$ & Sample \\
\hline & & $8 / 15 / 01$ & ND & 0.00019 & $\mathrm{Bg} / \mathrm{m}^{3}$ & Sample \\
\hline & & $8 / 22 / 01$ & ND & 0.00017 & $\mathrm{Bg} / \mathrm{m}^{3}$ & Sample \\
\hline & & $8 / 29 / 01$ & ND & 0.0007 & $\mathrm{Bg} / \mathrm{m}^{3}$ & Sample \\
\hline & & $9 / 5 / 01$ & 0.0002 & 0.00018 & $\mathrm{~Bq} / \mathrm{m}^{3}$ & Sample \\
\hline & & $9 / 12 / 01$ & ND & 0.0005 & $\mathrm{Bg} / \mathrm{m}^{3}$ & Sample \\
\hline & & 9/19/01 & ND & 0.00018 & $\mathrm{~Bq} / \mathrm{m}^{3}$ & Sample \\
\hline & & $9 / 26 / 01$ & ND & 0.0004 & $\mathrm{~Bq} / \mathrm{m}^{3}$ & Sample \\
\hline & & $10 / 3 / 01$ & ND & 0.0005 & $\mathrm{Bg} / \mathrm{m}^{3}$ & Sample \\
\hline & & $10 / 9 / 01$ & ND & 0.00017 & $\mathrm{~Bq} / \mathrm{m}^{3}$ & Sampl \\
\hline & & $10 / 16 / 01$ & ND & 0.00018 & $\mathrm{Bg} / \mathrm{m}^{3}$ & Sample \\
\hline
\end{tabular}


Analyte

\section{Radiological Activity}

Gross alpha B88 Cave 0 cont.
Date

$10 / 23 / 01$
$11 / 8 / 01$
$11 / 13 / 01$
$11 / 20 / 01$
$11 / 26 / 01$
$12 / 4 / 01$
$12 / 12 / 01$
$12 / 19 / 01$
$1 / 2 / 02$

B88-135H

$1 / 3 / 01$

2/7/01

$3 / 7 / 01$

$4 / 4 / 01$

$5 / 2 / 01$

$6 / 6 / 01$

$7 / 5 / 01$

$8 / 1 / 01$

$9 / 5 / 01$

$10 / 5 / 01$

$11 / 8 / 01$

$12 / 4 / 01$

$1 / 3 / 02$

Travel Blank
$1 / 3 / 01$
$1 / 3 / 01$
$1 / 3 / 01$

$1 / 3 / 01$

$1 / 3 / 01$

$1 / 10 / 01$

$1 / 10 / 01$

$1 / 10 / 01$

$1 / 17 / 01$

$1 / 17 / 01$

$1 / 17 / 01$

$1 / 24 / 01$

$1 / 24 / 01$

$1 / 24 / 01$

$1 / 31 / 01$

$1 / 31 / 01$

$1 / 31 / 01$

$2 / 7 / 01$

$2 / 7 / 01$

$2 / 7 / 01$

2/7/01

$2 / 7 / 01$

Result MDA or PQL Units QC Type

$\begin{array}{clll}0.0003 & 0.00015 & \mathrm{~Bq} / \mathrm{m}^{3} & \text { Sample } \\ 0.0005 & 0.00013 & \mathrm{~Bq} / \mathrm{m}^{3} & \text { Sample } \\ \text { ND } & 0.0004 & \mathrm{~Bq} / \mathrm{m}^{3} & \text { Sample } \\ 0.0001 & 0.00013 & \mathrm{~Bq} / \mathrm{m}^{3} & \text { Sample } \\ \text { ND } & 0.00014 & \mathrm{~Bq} / \mathrm{m}^{3} & \text { Sample } \\ \text { ND } & 0.0001 & \mathrm{~Bq} / \mathrm{m}^{3} & \text { Sample } \\ \text { ND } & 0.0002 & \mathrm{~Bq} / \mathrm{m}^{3} & \text { Sample } \\ 0.0001 & 0.00011 & \mathrm{~Bq} / \mathrm{m}^{3} & \text { Sample } \\ \text { ND } & 0.00006 & \mathrm{~Bq} / \mathrm{m}^{3} & \text { Sample }\end{array}$

$\begin{array}{llll}\text { ND } & 0.00009 & \mathrm{~Bq} / \mathrm{m}^{3} & \text { Sample } \\ \text { ND } & 0.00007 & \mathrm{~Bq} / \mathrm{m}^{3} & \text { Sample } \\ \text { ND } & 0.00009 & \mathrm{~Bq} / \mathrm{m}^{3} & \text { Sample } \\ \text { ND } & 0.0002 & \mathrm{~Bq} / \mathrm{m}^{3} & \text { Sample } \\ \text { ND } & 0.0003 & \mathrm{~Bq} / \mathrm{m}^{3} & \text { Sample } \\ \text { ND } & 0.00018 & \mathrm{~Bq} / \mathrm{m}^{3} & \text { Sample } \\ 0.0001 & 0.00009 & \mathrm{~Bq} / \mathrm{m}^{3} & \text { Sample } \\ \text { ND } & 0.0002 & \mathrm{~Bq} / \mathrm{m}^{3} & \text { Sample } \\ 0.0001 & 0.00007 & \mathrm{~Bq} / \mathrm{m}^{3} & \text { Sample } \\ \text { ND } & 0.00009 & \mathrm{~Bq} / \mathrm{m}^{3} & \text { Sample } \\ \text { ND } & 0.00009 & \mathrm{~Bq} / \mathrm{m}^{3} & \text { Sample } \\ \text { ND } & 0.0003 & \mathrm{~Bq} / \mathrm{m}^{3} & \text { Sample } \\ \text { ND } & 0.00009 & \mathrm{~Bq} / \mathrm{m}^{3} & \text { Sample }\end{array}$

$\begin{array}{llll}\text { ND } & 0.04 & \mathrm{~Bq} / \mathrm{S} & \text { Blank }\end{array}$

$\begin{array}{llll}N D & 0.15 & \mathrm{~Bq} / \mathrm{S} & \text { Blank }\end{array}$

$\begin{array}{llll}N D & 0.04 & \mathrm{~Bq} / \mathrm{S} & \text { Blank }\end{array}$

$\begin{array}{llll}\text { ND } & 0.04 & \mathrm{~Bq} / \mathrm{S} & \text { Blank }\end{array}$

ND $\quad 0.15 \quad \mathrm{~Bq} / \mathrm{S} \quad$ Blank

ND $\quad 0.11 \quad \mathrm{~Bq} / \mathrm{S} \quad$ Blank

ND $\quad 0.19 \quad \mathrm{~Bq} / \mathrm{S} \quad$ Blank

ND $\quad 0.04 \quad \mathrm{~Bq} / \mathrm{S} \quad$ Blank

ND $\quad 0.11 \quad \mathrm{~Bq} / \mathrm{S} \quad$ Blank

ND $\quad 0.11 \quad \mathrm{~Bq} / \mathrm{S} \quad$ Blank

ND $\quad 0.19 \quad \mathrm{~Bq} / \mathrm{S} \quad$ Blank

ND $\quad 0.11 \quad \mathrm{~Bq} / \mathrm{S} \quad$ Blank

$\begin{array}{llll}\text { ND } & 0.04 & \mathrm{~Bq} / \mathrm{S} & \text { Blank }\end{array}$

$\begin{array}{llll}N D & 0.19 & B q / S & B l a n k\end{array}$

ND $\quad 0.11 \quad B q / S \quad$ Blank

$\begin{array}{llll}\text { ND } & 0.2 & \mathrm{~Bq} / \mathrm{S} & \text { Blank }\end{array}$

ND $\quad 0.11 \quad B q / S \quad B l a n k$

$\begin{array}{llll}N D & 0.19 & B q / S & B l a n k\end{array}$

$\begin{array}{llll}0.05 & 0.04 & \mathrm{~Bq} / \mathrm{S} & \text { Blank }\end{array}$

$\begin{array}{llll}\text { ND } & 0.19 & \mathrm{~Bq} / \mathrm{S} & \text { Blank }\end{array}$

ND $\quad 0.04 \quad \mathrm{~Bq} / \mathrm{S} \quad$ Blank

ND $\quad 0.11 \quad B q / S \quad$ Blank




$\begin{array}{llllll}\text { Analyte } & \text { Location } & \text { Date } & \text { Result } & \text { MDA or PQL Units } & \text { QC Type }\end{array}$

\section{Radiological Activity}

\begin{tabular}{|c|c|c|c|c|c|c|}
\hline Gross alpha & Travel Blank & $2 / 14 / 01$ & ND & 0.11 & $\mathrm{~Bq} / \mathrm{S}$ & Blank \\
\hline cont. & & $2 / 14 / 01$ & ND & 0.11 & $\mathrm{~Bq} / \mathrm{S}$ & Blank \\
\hline & & $2 / 14 / 01$ & ND & 0.19 & $\mathrm{~Bq} / \mathrm{S}$ & Blank \\
\hline & & $2 / 22 / 01$ & ND & 0.11 & $\mathrm{~Bq} / \mathrm{S}$ & Blank \\
\hline & & $2 / 22 / 01$ & ND & 0.19 & $\mathrm{~Bq} / \mathrm{S}$ & Blank \\
\hline & & $2 / 22 / 01$ & ND & 0.11 & $\mathrm{~Bq} / \mathrm{S}$ & Blank \\
\hline & & $2 / 28 / 01$ & ND & 0.04 & $\mathrm{~Bq} / \mathrm{S}$ & Blank \\
\hline & & $2 / 28 / 01$ & 0.05 & 0.04 & $\mathrm{~Bq} / \mathrm{S}$ & Blank \\
\hline & & $2 / 28 / 01$ & ND & 0.2 & $\mathrm{~Bq} / \mathrm{S}$ & Bla \\
\hline & & $3 / 7 / 01$ & ND & 0.19 & $\mathrm{~Bq} / \mathrm{S}$ & Blar \\
\hline & & $3 / 7 / 01$ & ND & 0.04 & $\mathrm{~Bq} / \mathrm{S}$ & Blar \\
\hline & & $3 / 7 / 01$ & ND & 0.04 & $\mathrm{~Bq} / \mathrm{S}$ & Bla \\
\hline & & $3 / 7 / 01$ & ND & 0.19 & $\mathrm{~Bq} / \mathrm{S}$ & Bla \\
\hline & & $3 / 7 / 01$ & ND & 0.04 & $\mathrm{~Bq} / \mathrm{S}$ & Bla \\
\hline & & $3 / 15 / 01$ & ND & 0.11 & $\mathrm{~Bq} / \mathrm{S}$ & Bla \\
\hline & & $3 / 15 / 01$ & ND & 0.11 & $\mathrm{~Bq} / \mathrm{S}$ & Bla \\
\hline & & $3 / 15 / 01$ & ND & 0.19 & $\mathrm{~Bq} / \mathrm{S}$ & Bla \\
\hline & & $3 / 21 / 01$ & ND & 0.2 & $\mathrm{~Bq} / \mathrm{S}$ & Bla \\
\hline & & $3 / 21 / 01$ & ND & 0.04 & $\mathrm{~Bq} / \mathrm{S}$ & Bla \\
\hline & & $3 / 21 / 01$ & ND & 0.04 & $\mathrm{~Bq} / \mathrm{S}$ & \\
\hline & & $3 / 28 / 01$ & ND & 0.2 & $\mathrm{~Bq} / \mathrm{S}$ & $\mathrm{Bla}$ \\
\hline & & $3 / 28 / 01$ & ND & 0.15 & $\mathrm{~Bq} / \mathrm{S}$ & \\
\hline & & $3 / 28 / 01$ & ND & 0.11 & $\mathrm{~Bq} / \mathrm{S}$ & Bla \\
\hline & & $4 / 4 / 01$ & ND & 0.11 & $\mathrm{~Bq} / \mathrm{S}$ & Bla \\
\hline & & $4 / 4 / 01$ & ND & 0.15 & $\mathrm{~Bq} / \mathrm{S}$ & Bla \\
\hline & & $4 / 4 / 01$ & ND & 0.15 & $\mathrm{~Bq} / \mathrm{S}$ & \\
\hline & & $4 / 4 / 01$ & ND & 0.11 & $\mathrm{~Bq} / \mathrm{S}$ & \\
\hline & & $4 / 4 / 01$ & ND & 0.04 & $\mathrm{~Bq} / \mathrm{S}$ & $\mathrm{Bla}$ \\
\hline & & $4 / 11 / 01$ & ND & 0.11 & $\mathrm{~Bq} / \mathrm{S}$ & $\mathrm{Bl}$ \\
\hline & & $4 / 11 / 01$ & ND & 0.15 & $\mathrm{~Bq} / \mathrm{S}$ & Bla \\
\hline & & $4 / 11 / 01$ & ND & 0.11 & $\mathrm{~Bq} / \mathrm{S}$ & \\
\hline & & $4 / 18 / 01$ & ND & 0.19 & $\mathrm{Bg} / \mathrm{S}$ & Bla \\
\hline & & $4 / 18 / 01$ & ND & 0.11 & $\mathrm{~Bq} / \mathrm{S}$ & \\
\hline & & 4/18/01 & ND & 0.04 & $\mathrm{~Bq} / \mathrm{S}$ & Bla \\
\hline & & $4 / 26 / 01$ & ND & 0.11 & $\mathrm{~Bq} / \mathrm{S}$ & Bla \\
\hline & & $4 / 26 / 01$ & ND & 0.19 & $\mathrm{~Bq} / \mathrm{S}$ & $\mathrm{Bla}$ \\
\hline & & $4 / 26 / 01$ & 0.05 & 0.04 & $\mathrm{~Bq} / \mathrm{S}$ & \\
\hline & & $5 / 2 / 01$ & ND & 0.2 & $\mathrm{~Bq} / \mathrm{S}$ & \\
\hline & & $5 / 2 / 01$ & ND & 0.15 & $\mathrm{~Bq} / \mathrm{S}$ & Bla \\
\hline & & $5 / 2 / 01$ & ND & 0.11 & $\mathrm{~Bq} / \mathrm{S}$ & Bla \\
\hline & & $5 / 2 / 01$ & ND & 0.2 & $\mathrm{~Bq} / \mathrm{S}$ & Bla \\
\hline & & $5 / 2 / 01$ & ND & 0.15 & $\mathrm{~Bq} / \mathrm{S}$ & Bla \\
\hline & & $5 / 9 / 01$ & ND & 0.11 & $\mathrm{~Bq} / \mathrm{S}$ & Bla \\
\hline & & $5 / 9 / 01$ & ND & 0.19 & $\mathrm{Bg} / \mathrm{S}$ & \\
\hline & & $5 / 9 / 01$ & ND & 0.04 & $\mathrm{~Bq} / \mathrm{S}$ & \\
\hline & & $5 / 16 / 01$ & ND & 0.19 & $\mathrm{Bg} / \mathrm{S}$ & \\
\hline
\end{tabular}


Analyte

Location

Date

Result MDA or PQL

Units QC Type

\section{Radiological Activity}

Gross alpha Travel Blank cont.

\begin{tabular}{|c|c|c|c|c|}
\hline $5 / 16 / 01$ & ND & 0.11 & $\mathrm{~Bq} / \mathrm{S}$ & Blank \\
\hline $5 / 16 / 01$ & ND & 0.15 & $\mathrm{~Bq} / \mathrm{S}$ & Blank \\
\hline $5 / 23 / 01$ & ND & 0.11 & $\mathrm{~Bq} / \mathrm{S}$ & Blank \\
\hline $5 / 23 / 01$ & ND & 0.19 & $\mathrm{~Bq} / \mathrm{S}$ & Blank \\
\hline $5 / 23 / 01$ & ND & 0.04 & $\mathrm{~Bq} / \mathrm{S}$ & Blank \\
\hline $5 / 31 / 01$ & ND & 0.11 & $\mathrm{~Bq} / \mathrm{S}$ & Blank \\
\hline $5 / 31 / 01$ & ND & 0.15 & $\mathrm{~Bq} / \mathrm{S}$ & Blank \\
\hline $5 / 31 / 01$ & ND & 0.11 & $\mathrm{~Bq} / \mathrm{S}$ & Blank \\
\hline 6/6/01 & ND & 0.19 & $\mathrm{~Bq} / \mathrm{S}$ & Blank \\
\hline 6/6/01 & ND & 0.11 & $\mathrm{~Bq} / \mathrm{S}$ & Blank \\
\hline 6/6/01 & ND & 0.11 & $\mathrm{~Bq} / \mathrm{S}$ & Blank \\
\hline 6/6/01 & ND & 0.11 & $\mathrm{~Bq} / \mathrm{S}$ & Blank \\
\hline 6/6/01 & ND & 0.19 & $\mathrm{~Bq} / \mathrm{S}$ & Blank \\
\hline $6 / 13 / 01$ & ND & 0.04 & $\mathrm{~Bq} / \mathrm{S}$ & Blank \\
\hline $6 / 13 / 01$ & ND & 0.11 & $\mathrm{~Bq} / \mathrm{S}$ & Blank \\
\hline $6 / 13 / 01$ & ND & 0.19 & $\mathrm{~Bq} / \mathrm{S}$ & Blank \\
\hline $6 / 20 / 01$ & ND & 0.11 & $\mathrm{~Bq} / \mathrm{S}$ & Blank \\
\hline $6 / 20 / 01$ & ND & 0.04 & $\mathrm{~Bq} / \mathrm{S}$ & Blank \\
\hline $6 / 20 / 01$ & ND & 0.3 & $\mathrm{~Bq} / \mathrm{S}$ & Blank \\
\hline $6 / 27 / 01$ & ND & 0.19 & $\mathrm{~Bq} / \mathrm{S}$ & Blank \\
\hline $6 / 27 / 01$ & ND & 0.04 & $\mathrm{~Bq} / \mathrm{S}$ & Blank \\
\hline $6 / 27 / 01$ & ND & 0.11 & $\mathrm{~Bq} / \mathrm{S}$ & Blank \\
\hline $7 / 5 / 01$ & 0.05 & 0.04 & $\mathrm{~Bq} / \mathrm{S}$ & Blank \\
\hline $7 / 5 / 01$ & ND & 0.2 & $\mathrm{~Bq} / \mathrm{S}$ & Blank \\
\hline $7 / 5 / 01$ & ND & 0.11 & $\mathrm{~Bq} / \mathrm{S}$ & Blank \\
\hline $7 / 5 / 01$ & ND & 0.2 & $\mathrm{~Bq} / \mathrm{S}$ & Blank \\
\hline $7 / 5 / 01$ & ND & 0.11 & $\mathrm{~Bq} / \mathrm{S}$ & Blank \\
\hline $7 / 11 / 01$ & ND & 0.04 & $\mathrm{~Bq} / \mathrm{S}$ & Blank \\
\hline $7 / 11 / 01$ & ND & 0.19 & $\mathrm{~Bq} / \mathrm{S}$ & Blank \\
\hline $7 / 11 / 01$ & ND & 0.15 & $\mathrm{~Bq} / \mathrm{S}$ & Blank \\
\hline $7 / 18 / 01$ & ND & 0.04 & $\mathrm{~Bq} / \mathrm{S}$ & Blank \\
\hline $7 / 18 / 01$ & 0.05 & 0.04 & $\mathrm{~Bq} / \mathrm{S}$ & Blank \\
\hline $7 / 18 / 01$ & ND & 0.15 & $\mathrm{~Bq} / \mathrm{S}$ & Blank \\
\hline $7 / 25 / 01$ & ND & 0.04 & $\mathrm{~Bq} / \mathrm{S}$ & Blank \\
\hline $7 / 25 / 01$ & ND & 0.04 & $\mathrm{~Bq} / \mathrm{S}$ & Blank \\
\hline $7 / 25 / 01$ & ND & 0.19 & $\mathrm{~Bq} / \mathrm{S}$ & Blank \\
\hline $8 / 1 / 01$ & ND & 0.11 & $\mathrm{~Bq} / \mathrm{S}$ & Blank \\
\hline $8 / 1 / 01$ & ND & 0.3 & $\mathrm{~Bq} / \mathrm{S}$ & Blank \\
\hline $8 / 1 / 01$ & ND & 0.11 & $\mathrm{~Bq} / \mathrm{S}$ & Blank \\
\hline $8 / 1 / 01$ & ND & 0.11 & $\mathrm{~Bq} / \mathrm{S}$ & Blank \\
\hline $8 / 1 / 01$ & ND & 0.3 & $\mathrm{~Bq} / \mathrm{S}$ & Blank \\
\hline 8/8/01 & ND & 0.19 & $\mathrm{~Bq} / \mathrm{S}$ & Blank \\
\hline 8/8/01 & ND & 0.04 & $\mathrm{~Bq} / \mathrm{S}$ & Blank \\
\hline 8/8/01 & ND & 0.04 & $\mathrm{Bg} / \mathrm{S}$ & Blank \\
\hline $8 / 15 / 01$ & ND & 0.11 & $\mathrm{~Bq} / \mathrm{S}$ & Blank \\
\hline $8 / 15 / 01$ & ND & 0.15 & $\mathrm{~Bq} / \mathrm{S}$ & Blank \\
\hline
\end{tabular}




$\begin{array}{llllll}\text { Analyte } & \text { Location } & \text { Date } & \text { Result } & \text { MDA or PQL Units } & \text { QC Type }\end{array}$

\section{Radiological Activity}

\begin{tabular}{|c|c|c|c|c|c|c|}
\hline Gross alpha & Travel Blank & $8 / 15 / 01$ & ND & 0.04 & $\mathrm{~Bq} / \mathrm{S}$ & Blank \\
\hline cont. & & $8 / 22 / 01$ & ND & 0.04 & $\mathrm{~Bq} / \mathrm{S}$ & Blank \\
\hline & & $8 / 22 / 01$ & ND & 0.11 & $\mathrm{~Bq} / \mathrm{S}$ & Blank \\
\hline & & $8 / 22 / 01$ & ND & 0.19 & $\mathrm{~Bq} / \mathrm{S}$ & Blan \\
\hline & & $8 / 29 / 01$ & ND & 0.11 & $\mathrm{~Bq} / \mathrm{S}$ & Blar \\
\hline & & $8 / 29 / 01$ & ND & 0.2 & $\mathrm{~Bq} / \mathrm{S}$ & Blar \\
\hline & & $8 / 29 / 01$ & ND & 0.15 & $\mathrm{~Bq} / \mathrm{S}$ & Blar \\
\hline & & $9 / 5 / 01$ & 0.05 & 0.04 & $\mathrm{Bg} / \mathrm{S}$ & Blar \\
\hline & & $9 / 5 / 01$ & ND & 0.2 & $\mathrm{~Bq} / \mathrm{S}$ & Blar \\
\hline & & $9 / 5 / 01$ & ND & 0.04 & $\mathrm{~Bq} / \mathrm{S}$ & Blar \\
\hline & & $9 / 5 / 01$ & ND & 0.2 & $\mathrm{~Bq} / \mathrm{S}$ & Blar \\
\hline & & $9 / 5 / 01$ & ND & 0.04 & $\mathrm{~Bq} / \mathrm{S}$ & Blar \\
\hline & & $9 / 12 / 01$ & ND & 0.11 & $\mathrm{~Bq} / \mathrm{S}$ & Bla \\
\hline & & $9 / 12 / 01$ & ND & 0.15 & $\mathrm{~Bq} / \mathrm{S}$ & Blar \\
\hline & & $9 / 12 / 01$ & ND & 0.04 & $\mathrm{~Bq} / \mathrm{S}$ & Blar \\
\hline & & $9 / 19 / 01$ & ND & 0.19 & $\mathrm{~Bq} / \mathrm{S}$ & Blar \\
\hline & & 9/19/01 & ND & 0.11 & $\mathrm{~Bq} / \mathrm{S}$ & Blar \\
\hline & & 9/19/01 & 0.06 & 0.04 & $\mathrm{~Bq} / \mathrm{S}$ & Blar \\
\hline & & 9/26/01 & ND & 0.19 & $\mathrm{~Bq} / \mathrm{S}$ & Blar \\
\hline & & 9/26/01 & ND & 0.15 & $\mathrm{~Bq} / \mathrm{S}$ & Blar \\
\hline & & $9 / 26 / 01$ & ND & 0.11 & $\mathrm{Bg} / \mathrm{S}$ & Blar \\
\hline & & $10 / 3 / 01$ & ND & 0.15 & $\mathrm{Bg} / \mathrm{S}$ & Blar \\
\hline & & $10 / 3 / 01$ & ND & 0.04 & $\mathrm{Bg} / \mathrm{S}$ & Blar \\
\hline & & $10 / 3 / 01$ & ND & 0.11 & $\mathrm{~Bq} / \mathrm{S}$ & Blar \\
\hline & & $10 / 8 / 01$ & 0.1 & 0.04 & $\mathrm{~Bq} / \mathrm{S}$ & Blar \\
\hline & & 10/8/01 & ND & 0.2 & $\mathrm{~Bq} / \mathrm{S}$ & Blar \\
\hline & & 10/9/01 & ND & 0.2 & $\mathrm{~Bq} / \mathrm{S}$ & Blar \\
\hline & & $10 / 9 / 01$ & ND & 0.11 & $\mathrm{Bg} / \mathrm{S}$ & Blar \\
\hline & & $10 / 9 / 01$ & 0.05 & 0.04 & $\mathrm{Bg} / \mathrm{S}$ & Blar \\
\hline & & $10 / 16 / 01$ & ND & 0.2 & $\mathrm{~Bq} / \mathrm{S}$ & Blaı \\
\hline & & $10 / 16 / 01$ & ND & 0.15 & $\mathrm{~Bq} / \mathrm{S}$ & Blar \\
\hline & & 10/16/01 & 0.06 & 0.04 & $\mathrm{~Bq} / \mathrm{S}$ & Blar \\
\hline & & $10 / 24 / 01$ & ND & 0.11 & $\mathrm{~Bq} / \mathrm{S}$ & Blar \\
\hline & & $10 / 24 / 01$ & ND & 0.04 & $\mathrm{~Bq} / \mathrm{S}$ & Blar \\
\hline & & $10 / 24 / 01$ & ND & 0.2 & $\mathrm{~Bq} / \mathrm{S}$ & Blar \\
\hline & & $10 / 30 / 01$ & ND & 0.11 & $\mathrm{Bg} / \mathrm{S}$ & Blal \\
\hline & & $10 / 30 / 01$ & ND & 0.11 & $\mathrm{Bg} / \mathrm{S}$ & Blar \\
\hline & & $10 / 30 / 01$ & ND & 0.04 & $\mathrm{~Bq} / \mathrm{S}$ & Blar \\
\hline & & 11/8/01 & ND & 0.11 & $\mathrm{~Bq} / \mathrm{S}$ & Blar \\
\hline & & $11 / 8 / 01$ & ND & 0.2 & $\mathrm{Bg} / \mathrm{S}$ & Blar \\
\hline & & 11/8/01 & ND & 0.04 & $\mathrm{~Bq} / \mathrm{S}$ & Blar \\
\hline & & $11 / 9 / 01$ & ND & 0.2 & $\mathrm{Bg} / \mathrm{S}$ & Blar \\
\hline & & $11 / 9 / 01$ & 0.05 & 0.04 & $\mathrm{Ba} / \mathrm{S}$ & Blan \\
\hline & & $11 / 13 / 01$ & ND & 0.11 & $\mathrm{~Bq} / \mathrm{S}$ & Blaı \\
\hline & & $11 / 13 / 01$ & ND & 0.11 & $\mathrm{Bg} / \mathrm{S}$ & Blar \\
\hline & & $11 / 13 / 01$ & ND & 0.3 & $\mathrm{Bg} / \mathrm{S}$ & \\
\hline
\end{tabular}


Analyte

Location

Radiological Activity

Gross alpha Travel Blank cont.

Gross beta

$\begin{array}{cc}1-216 \mathrm{H} & 1 / 3 / 01 \\ & 2 / 7 / 01 \\ 3 / 7 / 01 \\ 4 / 4 / 01 \\ 5 / 2 / 01 \\ 6 / 6 / 01 \\ 7 / 5 / 01 \\ 8 / 1 / 01 \\ 9 / 5 / 01 \\ 10 / 8 / 01 \\ 11 / 9 / 01 \\ 12 / 4 / 01 \\ 1 / 3 / 02 \\ \\ 1 / 3 / 01 \\ & 2 / 7 / 01 \\ & 3 / 7 / 01 \\ & 4 / 4 / 01 \\ 573 H & 8 / 2 / 01 \\ & 9 / 5 / 01 \\ 10 / 8 / 01 \\ 11 / 9 / 01 \\ 12 / 4 / 01 \\ 1 / 3 / 02\end{array}$

\begin{tabular}{|c|c|c|c|c|}
\hline $11 / 20 / 01$ & ND & 0.2 & $\mathrm{~Bq} / \mathrm{S}$ & Blank \\
\hline $11 / 20 / 01$ & ND & 0.04 & $\mathrm{~Bq} / \mathrm{S}$ & Blank \\
\hline $11 / 20 / 01$ & ND & 0.04 & $\mathrm{~Bq} / \mathrm{S}$ & Blank \\
\hline $11 / 26 / 01$ & ND & 0.3 & $\mathrm{~Bq} / \mathrm{S}$ & Blank \\
\hline $11 / 26 / 01$ & 0.06 & 0.04 & $\mathrm{~Bq} / \mathrm{S}$ & Blank \\
\hline $11 / 26 / 01$ & ND & 0.04 & $\mathrm{~Bq} / \mathrm{S}$ & Blank \\
\hline $12 / 4 / 01$ & 0.1 & 0.04 & $\mathrm{Bg} / \mathrm{S}$ & Blank \\
\hline $12 / 4 / 01$ & ND & 0.2 & $\mathrm{~Bq} / \mathrm{S}$ & Blank \\
\hline $12 / 4 / 01$ & ND & 0.11 & $\mathrm{~Bq} / \mathrm{S}$ & Blank \\
\hline $12 / 4 / 01$ & ND & 0.11 & $\mathrm{~Bq} / \mathrm{S}$ & Blank \\
\hline 12/4/01 & ND & 0.2 & $\mathrm{~Bq} / \mathrm{S}$ & Blank \\
\hline $12 / 12 / 01$ & ND & 0.11 & $\mathrm{~Bq} / \mathrm{S}$ & Blank \\
\hline $12 / 12 / 01$ & ND & 0.15 & $\mathrm{~Bq} / \mathrm{S}$ & Blank \\
\hline $12 / 19 / 01$ & ND & 0.15 & $\mathrm{~Bq} / \mathrm{S}$ & Blank \\
\hline $12 / 19 / 01$ & ND & 0.04 & $\mathrm{~Bq} / \mathrm{S}$ & Blank \\
\hline $12 / 19 / 01$ & ND & 0.04 & $\mathrm{~Bq} / \mathrm{S}$ & Blank \\
\hline $1 / 2 / 02$ & ND & 0.3 & $\mathrm{~Bq} / \mathrm{S}$ & Blank \\
\hline $1 / 2 / 02$ & ND & 0.04 & $\mathrm{~Bq} / \mathrm{S}$ & Blank \\
\hline $1 / 2 / 02$ & ND & 0.04 & $\mathrm{~Bq} / \mathrm{S}$ & Blank \\
\hline $1 / 3 / 02$ & 0.05 & 0.04 & $\mathrm{~Bq} / \mathrm{S}$ & Blank \\
\hline $1 / 3 / 02$ & ND & 0.3 & $\mathrm{~Bq} / \mathrm{S}$ & Blank \\
\hline $1 / 3 / 01$ & 0.0014 & 0.0005 & $\mathrm{~Bq} / \mathrm{m}^{3}$ & Sample \\
\hline $2 / 7 / 01$ & 0.0012 & 0.0003 & $\mathrm{~Bq} / \mathrm{m}^{3}$ & Sample \\
\hline $3 / 7 / 01$ & ND & 0.0005 & $\mathrm{~Bq} / \mathrm{m}^{3}$ & Sample \\
\hline $4 / 4 / 01$ & ND & 0.0005 & $\mathrm{~Bq} / \mathrm{m}^{3}$ & Sample \\
\hline $5 / 2 / 01$ & ND & 0.0005 & $\mathrm{~Bq} / \mathrm{m}^{3}$ & Sample \\
\hline $6 / 6 / 01$ & ND & 0.0004 & $\mathrm{~Bq} / \mathrm{m}^{3}$ & Sample \\
\hline $7 / 5 / 01$ & 0.00065 & 0.0004 & $\mathrm{~Bq} / \mathrm{m}^{3}$ & Sample \\
\hline $8 / 1 / 01$ & ND & 0.0005 & $\mathrm{~Bq} / \mathrm{m}^{3}$ & Sample \\
\hline $9 / 5 / 01$ & ND & 0.0004 & $\mathrm{~Bq} / \mathrm{m}^{3}$ & Sample \\
\hline 10/8/01 & 0.00084 & 0.0003 & $\mathrm{~Bq} / \mathrm{m}^{3}$ & Sample \\
\hline $11 / 9 / 01$ & 0.0011 & 0.0004 & $\mathrm{~Bq} / \mathrm{m}^{3}$ & Sample \\
\hline $12 / 4 / 01$ & ND & 0.0005 & $\mathrm{~Bq} / \mathrm{m}^{3}$ & Sample \\
\hline $1 / 3 / 02$ & ND & 0.0004 & $\mathrm{~Bq} / \mathrm{m}^{3}$ & Sample \\
\hline $1 / 3 / 01$ & 0.00053 & 0.0004 & $\mathrm{~Bq} / \mathrm{m}^{3}$ & Sample \\
\hline $2 / 7 / 01$ & ND & 0.0003 & $\mathrm{~Bq} / \mathrm{m}^{3}$ & Sample \\
\hline $3 / 7 / 01$ & ND & 0.0005 & $\mathrm{~Bq} / \mathrm{m}^{3}$ & Sample \\
\hline $4 / 4 / 01$ & ND & 0.0005 & $\mathrm{~Bq} / \mathrm{m}^{3}$ & Sample \\
\hline $5 / 2 / 01$ & ND & 0.0004 & $\mathrm{~Bq} / \mathrm{m}^{3}$ & Sample \\
\hline $8 / 1 / 01$ & ND & 0.0003 & $\mathrm{~Bq} / \mathrm{m}^{3}$ & Sample \\
\hline $9 / 5 / 01$ & 0.00044 & 0.0004 & $\mathrm{~Bq} / \mathrm{m}^{3}$ & Sample \\
\hline $10 / 8 / 01$ & 0.00078 & 0.0003 & $\mathrm{~Bq} / \mathrm{m}^{3}$ & Sample \\
\hline $11 / 9 / 01$ & 0.0007 & 0.0004 & $\mathrm{~Bq} / \mathrm{m}^{3}$ & Sample \\
\hline $12 / 4 / 01$ & ND & 0.0005 & $\mathrm{~Bq} / \mathrm{m}^{3}$ & Sample \\
\hline $1 / 3 / 02$ & 0.00043 & 0.0004 & $\mathrm{~Bq} / \mathrm{m}^{3}$ & Sample \\
\hline
\end{tabular}




$\begin{array}{llllll}\text { Analyte } & \text { Location } & \text { Date } & \text { Result } & \text { MDA or PQL Units } & \text { QC Type }\end{array}$

\section{Radiological Activity}

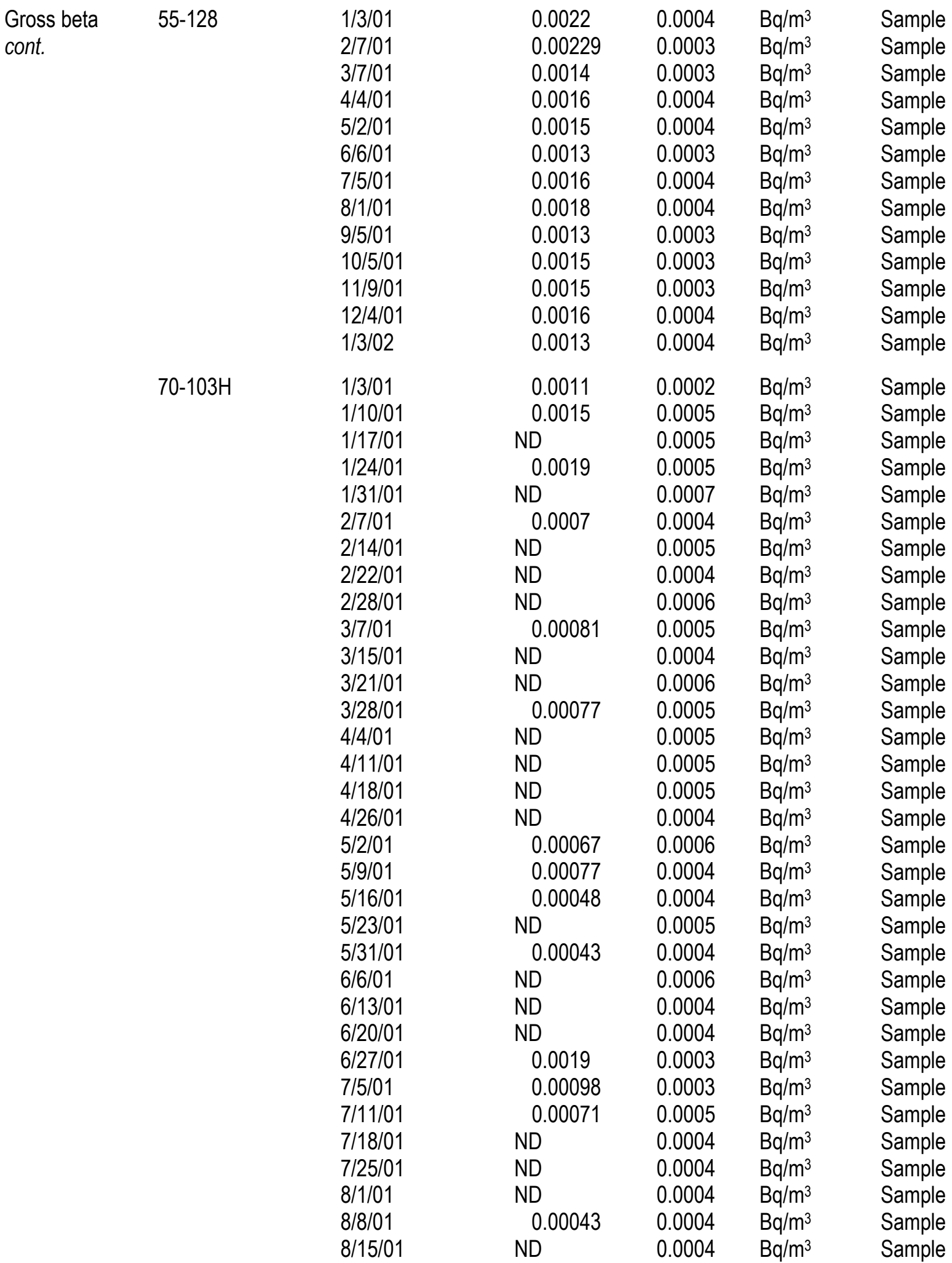


Analyte

\section{Radiological Activity}

\begin{tabular}{|c|c|c|c|c|c|c|}
\hline \multirow{18}{*}{\multicolumn{2}{|c|}{$\begin{array}{l}\text { Gross beta } \\
\text { cont. }\end{array}$}} & $8 / 29 / 01$ & ND & 0.0004 & $\mathrm{~Bq} / \mathrm{m}^{3}$ & Sample \\
\hline & & $9 / 5 / 01$ & ND & 0.0004 & $\mathrm{~Bq} / \mathrm{m}^{3}$ & Sample \\
\hline & & $9 / 12 / 01$ & ND & 0.0004 & $\mathrm{~Bq} / \mathrm{m}^{3}$ & Sample \\
\hline & & $9 / 19 / 01$ & ND & 0.0004 & $\mathrm{~Bq} / \mathrm{m}^{3}$ & Sample \\
\hline & & $9 / 26 / 01$ & 0.00068 & 0.0004 & $\mathrm{~Bq} / \mathrm{m}^{3}$ & Sample \\
\hline & & $10 / 3 / 01$ & 0.00073 & 0.0004 & $\mathrm{~Bq} / \mathrm{m}^{3}$ & Sample \\
\hline & & $10 / 9 / 01$ & 0.00056 & 0.0004 & $\mathrm{~Bq} / \mathrm{m}^{3}$ & Sample \\
\hline & & $10 / 16 / 01$ & 0.00061 & 0.0004 & $\mathrm{~Bq} / \mathrm{m}^{3}$ & Sample \\
\hline & & $10 / 23 / 01$ & 0.00079 & 0.0004 & $\mathrm{~Bq} / \mathrm{m}^{3}$ & Sample \\
\hline & & $10 / 30 / 01$ & 0.00072 & 0.0004 & $\mathrm{~Bq} / \mathrm{m}^{3}$ & Sample \\
\hline & & $11 / 8 / 01$ & 0.00085 & 0.0003 & $\mathrm{~Bq} / \mathrm{m}^{3}$ & Sample \\
\hline & & $11 / 13 / 01$ & 0.0015 & 0.0006 & $\mathrm{~Bq} / \mathrm{m}^{3}$ & Sample \\
\hline & & $11 / 20 / 01$ & 0.00044 & 0.0004 & $\mathrm{~Bq} / \mathrm{m}^{3}$ & Sample \\
\hline & & $11 / 26 / 01$ & ND & 0.0005 & $\mathrm{~Bq} / \mathrm{m}^{3}$ & Sample \\
\hline & & $12 / 4 / 01$ & 0.00046 & 0.0004 & $\mathrm{~Bq} / \mathrm{m}^{3}$ & Sample \\
\hline & & $12 / 12 / 01$ & 0.00054 & 0.0004 & $\mathrm{~Bq} / \mathrm{m}^{3}$ & Sample \\
\hline & & $12 / 19 / 01$ & ND & 0.0004 & $\mathrm{~Bq} / \mathrm{m}^{3}$ & Sample \\
\hline & & $1 / 2 / 02$ & ND & 0.0002 & $\mathrm{~Bq} / \mathrm{m}^{3}$ & Sample \\
\hline & $70-147 A$ & $1 / 3 / 01$ & 0.00073 & 0.0002 & $\mathrm{~Bq} / \mathrm{m}^{3}$ & Sample \\
\hline & & $1 / 10 / 01$ & 0.0009 & 0.0005 & $\mathrm{~Bq} / \mathrm{m}^{3}$ & Sample \\
\hline & & $1 / 17 / 01$ & ND & 0.0004 & $\mathrm{~Bq} / \mathrm{m}^{3}$ & Sample \\
\hline & & $1 / 24 / 01$ & 0.00055 & 0.0004 & $\mathrm{~Bq} / \mathrm{m}^{3}$ & Sample \\
\hline & & $1 / 31 / 01$ & ND & 0.0007 & $\mathrm{~Bq} / \mathrm{m}^{3}$ & Sample \\
\hline & & $2 / 7 / 01$ & 0.00053 & 0.0004 & $\mathrm{~Bq} / \mathrm{m}^{3}$ & Sample \\
\hline & & $2 / 14 / 01$ & ND & 0.0005 & $\mathrm{~Bq} / \mathrm{m}^{3}$ & Sample \\
\hline & & $2 / 22 / 01$ & ND & 0.0004 & $\mathrm{~Bq} / \mathrm{m}^{3}$ & Sample \\
\hline & & $2 / 28 / 01$ & ND & 0.0005 & $\mathrm{~Bq} / \mathrm{m}^{3}$ & Sample \\
\hline & & $3 / 7 / 01$ & ND & 0.0005 & $\mathrm{~Bq} / \mathrm{m}^{3}$ & Sample \\
\hline & & $3 / 15 / 01$ & ND & 0.0004 & $\mathrm{~Bq} / \mathrm{m}^{3}$ & Sample \\
\hline & & $3 / 21 / 01$ & ND & 0.0005 & $\mathrm{~Bq} / \mathrm{m}^{3}$ & Sample \\
\hline & & $3 / 28 / 01$ & ND & 0.0004 & $\mathrm{~Bq} / \mathrm{m}^{3}$ & Sample \\
\hline & & $4 / 4 / 01$ & ND & 0.0005 & $\mathrm{Bg} / \mathrm{m}^{3}$ & Sample \\
\hline & & $4 / 11 / 01$ & ND & 0.0004 & $\mathrm{~Bq} / \mathrm{m}^{3}$ & Sample \\
\hline & & 4/18/01 & ND & 0.0005 & $\mathrm{~Bq} / \mathrm{m}^{3}$ & Sample \\
\hline & & $4 / 26 / 01$ & ND & 0.0004 & $\mathrm{~Bq} / \mathrm{m}^{3}$ & Sample \\
\hline & & $5 / 2 / 01$ & ND & 0.0005 & $\mathrm{Bg} / \mathrm{m}^{3}$ & Sample \\
\hline & & $5 / 9 / 01$ & ND & 0.0004 & $\mathrm{~Bq} / \mathrm{m}^{3}$ & Sample \\
\hline & & $5 / 16 / 01$ & ND & 0.0004 & $\mathrm{Bg} / \mathrm{m}^{3}$ & Sample \\
\hline & & $5 / 23 / 01$ & ND & 0.0005 & $\mathrm{~Bq} / \mathrm{m}^{3}$ & Sample \\
\hline & & $5 / 31 / 01$ & ND & 0.0004 & $\mathrm{~Bq} / \mathrm{m}^{3}$ & Sample \\
\hline & & $6 / 6 / 01$ & ND & 0.0005 & $\mathrm{~Bq} / \mathrm{m}^{3}$ & Sample \\
\hline & & $6 / 13 / 01$ & ND & 0.0005 & $\mathrm{~Bq} / \mathrm{m}^{3}$ & Sample \\
\hline & & $6 / 20 / 01$ & ND & 0.0004 & $\mathrm{~Bq} / \mathrm{m}^{3}$ & Sample \\
\hline & & $6 / 27 / 01$ & ND & 0.0004 & $\mathrm{~Bq} / \mathrm{m}^{3}$ & Sample \\
\hline & & $7 / 5 / 01$ & ND & 0.0004 & $\mathrm{~Bq} / \mathrm{m}^{3}$ & Sample \\
\hline
\end{tabular}




\section{Analyte \\ Location \\ Radiological Activity}

Date

Result

MDA or PQL

Units

QC Type

\begin{tabular}{|c|c|c|c|c|c|c|}
\hline \multirow{24}{*}{\multicolumn{2}{|c|}{$\begin{array}{l}\text { Gross beta } \\
\text { cont. }\end{array}$}} & $7 / 11 / 01$ & ND & 0.0005 & $\mathrm{~Bq} / \mathrm{m}^{3}$ & Sample \\
\hline & & 7/18/01 & ND & 0.0005 & $\mathrm{~Bq} / \mathrm{m}^{3}$ & Sample \\
\hline & & $7 / 25 / 01$ & ND & 0.0004 & $\mathrm{~Bq} / \mathrm{m}^{3}$ & Sample \\
\hline & & $8 / 1 / 01$ & ND & 0.0004 & $\mathrm{~Bq} / \mathrm{m}^{3}$ & Sample \\
\hline & & $8 / 8 / 01$ & ND & 0.0005 & $\mathrm{~Bq} / \mathrm{m}^{3}$ & Sample \\
\hline & & $8 / 15 / 01$ & ND & 0.0005 & $\mathrm{~Bq} / \mathrm{m}^{3}$ & Sample \\
\hline & & 8/22/01 & ND & 0.0004 & $\mathrm{~Bq} / \mathrm{m}^{3}$ & Sample \\
\hline & & 8/29/01 & ND & 0.0004 & $\mathrm{~Bq} / \mathrm{m}^{3}$ & Sample \\
\hline & & $9 / 5 / 01$ & ND & 0.0004 & $\mathrm{~Bq} / \mathrm{m}^{3}$ & Sample \\
\hline & & $9 / 12 / 01$ & ND & 0.0004 & $\mathrm{~Bq} / \mathrm{m}^{3}$ & Samp \\
\hline & & $9 / 19 / 01$ & ND & 0.0004 & $\mathrm{~Bq} / \mathrm{m}^{3}$ & Samp \\
\hline & & $9 / 26 / 01$ & 0.00041 & 0.0004 & $\mathrm{~Bq} / \mathrm{m}^{3}$ & Sample \\
\hline & & $10 / 3 / 01$ & 0.00049 & 0.0004 & $\mathrm{~Bq} / \mathrm{m}^{3}$ & Sample \\
\hline & & $10 / 9 / 01$ & 0.00061 & 0.0004 & $\mathrm{~Bq} / \mathrm{m}^{3}$ & Sample \\
\hline & & $10 / 16 / 01$ & ND & 0.0004 & $\mathrm{~Bq} / \mathrm{m}^{3}$ & Samp \\
\hline & & $10 / 23 / 01$ & 0.00049 & 0.0004 & $\mathrm{~Bq} / \mathrm{m}^{3}$ & Samp \\
\hline & & $11 / 8 / 01$ & 0.00087 & 0.0004 & $\mathrm{~Bq} / \mathrm{m}^{3}$ & Samp \\
\hline & & $11 / 13 / 01$ & ND & 0.0006 & $\mathrm{~Bq} / \mathrm{m}^{3}$ & Samp \\
\hline & & $11 / 20 / 01$ & ND & 0.0004 & $\mathrm{~Bq} / \mathrm{m}^{3}$ & Samp \\
\hline & & $11 / 26 / 01$ & ND & 0.0005 & $\mathrm{~Bq} / \mathrm{m}^{3}$ & Samp \\
\hline & & $12 / 4 / 01$ & ND & 0.0004 & $\mathrm{~Bq} / \mathrm{m}^{3}$ & Samp \\
\hline & & $12 / 12 / 01$ & ND & 0.0004 & $\mathrm{~Bq} / \mathrm{m}^{3}$ & Samp \\
\hline & & 12/19/01 & ND & 0.0004 & $\mathrm{~Bq} / \mathrm{m}^{3}$ & Samp \\
\hline & & $1 / 2 / 02$ & 0.00026 & 0.0002 & $\mathrm{~Bq} / \mathrm{m}^{3}$ & Samp \\
\hline & \multirow[t]{13}{*}{$70-157 \mathrm{H}$} & $1 / 3 / 01$ & 0.0011 & 0.0005 & $\mathrm{~Bq} / \mathrm{m}^{3}$ & Sample \\
\hline & & $2 / 7 / 01$ & 0.00081 & 0.0003 & $\mathrm{~Bq} / \mathrm{m}^{3}$ & Samp \\
\hline & & $3 / 7 / 01$ & 0.00062 & 0.0005 & $\mathrm{~Bq} / \mathrm{m}^{3}$ & Samp \\
\hline & & $4 / 4 / 01$ & 0.00063 & 0.0005 & $\mathrm{~Bq} / \mathrm{m}^{3}$ & Samp \\
\hline & & $5 / 2 / 01$ & ND & 0.0005 & $\mathrm{~Bq} / \mathrm{m}^{3}$ & Samp \\
\hline & & $6 / 6 / 01$ & ND & 0.0004 & $\mathrm{Bg} / \mathrm{m}^{3}$ & Samp \\
\hline & & $7 / 5 / 01$ & ND & 0.0004 & $\mathrm{~Bq} / \mathrm{m}^{3}$ & Samp \\
\hline & & $8 / 1 / 01$ & ND & 0.0005 & $\mathrm{~Bq} / \mathrm{m}^{3}$ & Samp \\
\hline & & $9 / 5 / 01$ & ND & 0.0003 & $\mathrm{~Bq} / \mathrm{m}^{3}$ & Samp \\
\hline & & $10 / 5 / 01$ & 0.00064 & 0.0004 & $\mathrm{~Bq} / \mathrm{m}^{3}$ & Samp \\
\hline & & $11 / 8 / 01$ & 0.0012 & 0.0005 & $\mathrm{~Bq} / \mathrm{m}^{3}$ & Samp \\
\hline & & $12 / 4 / 01$ & ND & 0.0005 & $\mathrm{~Bq} / \mathrm{m}^{3}$ & Samp \\
\hline & & $1 / 3 / 02$ & 0.00046 & 0.0004 & $\mathrm{~Bq} / \mathrm{m}^{3}$ & Samp \\
\hline & \multirow[t]{8}{*}{$70-203 \mathrm{H}$} & $1 / 3 / 01$ & 0.00073 & 0.0004 & $\mathrm{Bg} / \mathrm{m}^{3}$ & Samp \\
\hline & & $2 / 7 / 01$ & 0.00083 & 0.0003 & $\mathrm{Bg} / \mathrm{m}^{3}$ & Samp \\
\hline & & $3 / 7 / 01$ & ND & 0.0004 & $\mathrm{~Bq} / \mathrm{m}^{3}$ & Samp \\
\hline & & $4 / 4 / 01$ & ND & 0.0004 & $\mathrm{Bg} / \mathrm{m}^{3}$ & Samp \\
\hline & & $5 / 2 / 01$ & ND & 0.0004 & $\mathrm{~Bq} / \mathrm{m}^{3}$ & Samp \\
\hline & & $6 / 6 / 01$ & 0.00037 & 0.0004 & $\mathrm{Bg} / \mathrm{m}^{3}$ & Sampl \\
\hline & & $7 / 5 / 01$ & 0.00063 & 0.0004 & $\mathrm{~Bq} / \mathrm{m}^{3}$ & Samp \\
\hline & & $8 / 1 / 01$ & ND & 0.0005 & $\mathrm{~Bq} / \mathrm{m}^{3}$ & Sample \\
\hline
\end{tabular}


Analyte

\section{Radiological Activity}

Gross beta $\quad 70-203 \mathrm{H}$

cont.
Date

Result

MDA or PQL

Units

QC Type

$\begin{array}{llll}\text { ND } & 0.0003 & \mathrm{~Bq} / \mathrm{m}^{3} & \text { Sample } \\ 0.00071 & 0.0005 & \mathrm{~Bq} / \mathrm{m}^{3} & \text { Sample } \\ 0.0011 & 0.0005 & \mathrm{~Bq} / \mathrm{m}^{3} & \text { Sample } \\ \text { ND } & 0.0005 & \mathrm{~Bq} / \mathrm{m}^{3} & \text { Sample } \\ \text { ND } & 0.0004 & \mathrm{~Bq} / \mathrm{m}^{3} & \text { Sample }\end{array}$

$70 \mathrm{~A}-1129 \mathrm{~B}$

\section{$\mathrm{Bq} / \mathrm{m}^{3} \quad$ Sample}

\section{9/5/01 \\ $10 / 5 / 01$ \\ $11 / 8 / 01$ \\ $12 / 4 / 01$ \\ $1 / 3 / 02$ \\ $1 / 3 / 01$ \\ $1 / 10 / 01$ \\ $1 / 17 / 01$ \\ $1 / 24 / 01$ \\ $1 / 31 / 01$ \\ $2 / 7 / 01$}

$2 / 14 / 01$

$2 / 22 / 01$

$2 / 28 / 01$

$3 / 7 / 01$

$3 / 15 / 01$

$3 / 21 / 01$

$3 / 28 / 01$

$4 / 4 / 01$

$4 / 11 / 01$

$4 / 18 / 01$

$4 / 26 / 01$

$5 / 2 / 01$

$5 / 9 / 01$

$5 / 16 / 01$

$5 / 23 / 01$

$5 / 31 / 01$

$6 / 6 / 01$

$6 / 13 / 01$

$6 / 20 / 01$

$6 / 27 / 01$

$7 / 5 / 01$

$7 / 11 / 01$

$7 / 18 / 01$

$7 / 25 / 01$

$8 / 1 / 01$

$8 / 8 / 01$

$8 / 15 / 01$

$8 / 22 / 01$

$8 / 29 / 01$

$9 / 5 / 01$

$9 / 12 / 01$

$9 / 19 / 01$

9/26/01

10/3/01

$$
0.00
$$

0.00199
0.0027
$N D$
0.002
$N D$$$
\mathrm{ND}
$$

0.0008

$\begin{array}{cc}0.0018 & 0.0004 \\ \text { ND } & 0.0005 \\ 0.00047 & 0.0004\end{array}$

$\begin{array}{cc}0.00047 & 0.0004 \\ \mathrm{ND} & 0.0006\end{array}$

$\begin{array}{cc}0.0012 & 0.0005 \\ \mathrm{ND} & 0.0005\end{array}$

$\begin{array}{cc}0.00066 & 0.0006 \\ \text { ND } & 0.0005\end{array}$

ND $\quad 0.0005$

$0.00056 \quad 0.0005$

$0.00058 \quad 0.0005$

$0.00054 \quad 0.0005$

ND

\begin{tabular}{ll}
0.00098 & 0.0006 \\
0.00071 & 0.0004 \\
\hline & 0.0004
\end{tabular}

$\begin{array}{cc}\text { ND } & 0.0004 \\ 0.00064 & 0.0005 \\ 0.00063 & 0.0005\end{array}$

$\begin{array}{cc}0.00063 & 0.0006 \\ \text { ND } & 0.0006\end{array}$

$\begin{array}{ll}0.0011 & 0.0006 \\ 0.00075 & 0.0005 \\ 0.00052 & 0.0005\end{array}$

$\begin{array}{ll}0.00075 & 0.0005 \\ 0.00052 & 0.0004\end{array}$

ND $\quad 0.0005$

ND $\quad 0.0005$

ND $\quad 0.0004$

ND $\quad 0.0004$

ND $\quad 0.0005$

ND $\quad 0.0005$

ND $\quad 0.0004$

$\begin{array}{ll}\text { ND } & 0.0004 \\ \text { ND } & 0.0004\end{array}$

$\begin{array}{ll}\text { ND } & 0.0004 \\ \text { ND } & 0.0004\end{array}$

$\begin{array}{ll}0.00049 & 0.0004 \\ 0.00042 & 0.0005\end{array}$

$0.00042 \quad 0.0004$

$0.00055 \quad 0.0004$

$0.00082 \quad 0.0004$
$\mathrm{Bq} / \mathrm{m}^{3} \quad$ Sample

$\mathrm{Bq} / \mathrm{m}^{3} \quad$ Sample

$\mathrm{Bq} / \mathrm{m}^{3} \quad$ Sample

$\mathrm{Bq} / \mathrm{m}^{3} \quad$ Sample

$\mathrm{Bq} / \mathrm{m}^{3} \quad$ Sample

$\mathrm{Bq} / \mathrm{m}^{3} \quad$ Sample

$\mathrm{Bq} / \mathrm{m}^{3} \quad$ Sample

$\mathrm{Bq} / \mathrm{m}^{3} \quad$ Sample

$\mathrm{Bq} / \mathrm{m}^{3} \quad$ Sample

$\mathrm{Bq} / \mathrm{m}^{3} \quad$ Sample

$\mathrm{Bq} / \mathrm{m}^{3} \quad$ Sample

$\mathrm{Bq} / \mathrm{m}^{3} \quad$ Sample

$\mathrm{Bq} / \mathrm{m}^{3} \quad$ Sample

$\mathrm{Bq} / \mathrm{m}^{3} \quad$ Sample

$\mathrm{Bq} / \mathrm{m}^{3} \quad$ Sample

$\mathrm{Bq} / \mathrm{m}^{3} \quad$ Sample

$\mathrm{Bq} / \mathrm{m}^{3} \quad$ Sample

$\mathrm{Bq} / \mathrm{m}^{3} \quad$ Sample

$\mathrm{Bq} / \mathrm{m}^{3} \quad$ Sample

$\mathrm{Bq} / \mathrm{m}^{3} \quad$ Sample

$\mathrm{Bq} / \mathrm{m}^{3} \quad$ Sample

$\mathrm{Bq} / \mathrm{m}^{3} \quad$ Sample

$\mathrm{Bq} / \mathrm{m}^{3} \quad$ Sample

$\mathrm{Bq} / \mathrm{m}^{3} \quad$ Sample

$\mathrm{Bq} / \mathrm{m}^{3} \quad$ Sample

$\mathrm{Bq} / \mathrm{m}^{3} \quad$ Sample

$\mathrm{Bq} / \mathrm{m}^{3} \quad$ Sample

$\mathrm{Bq} / \mathrm{m}^{3} \quad$ Sample

$\mathrm{Bq} / \mathrm{m}^{3} \quad$ Sample

$\mathrm{Bq} / \mathrm{m}^{3} \quad$ Sample

$\mathrm{Bq} / \mathrm{m}^{3} \quad$ Sample

$\mathrm{Bq} / \mathrm{m}^{3} \quad$ Sample

$\mathrm{Bq} / \mathrm{m}^{3} \quad$ Sample

$\mathrm{Bq} / \mathrm{m}^{3} \quad$ Sample

$\mathrm{Bq} / \mathrm{m}^{3} \quad$ Sample

$\mathrm{Bq} / \mathrm{m}^{3} \quad$ Sample

$\mathrm{Bq} / \mathrm{m}^{3} \quad$ Sample

$\mathrm{Bq} / \mathrm{m}^{3} \quad$ Sample

$\mathrm{Bq} / \mathrm{m}^{3} \quad$ Sample 


\section{Analyte \\ Location \\ Radiological Activity}

Date

Result MDA or PQL

Units

QC Type

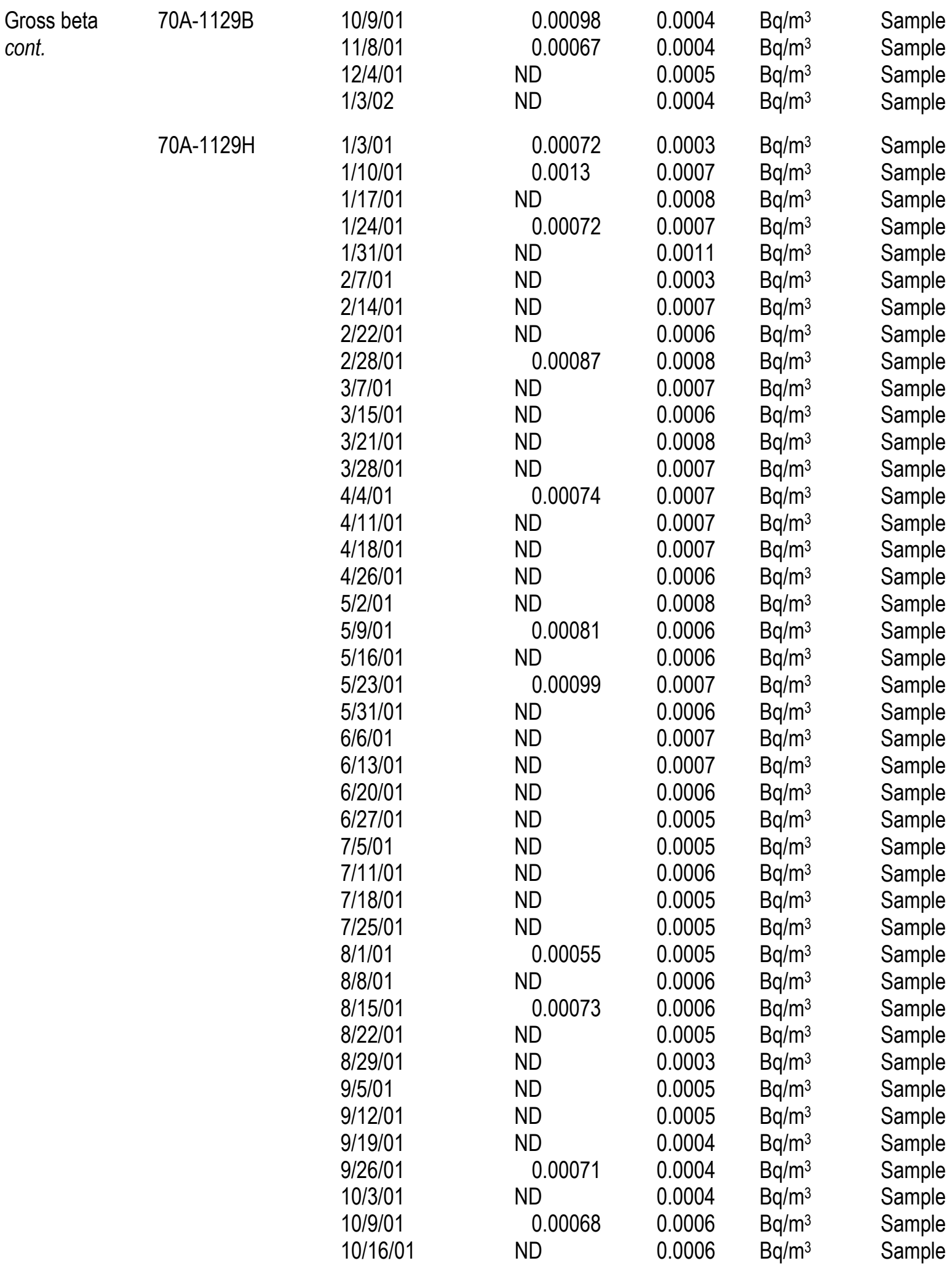


Analyte

\section{Radiological Activity}

Gross beta 70A-1129H cont.
Date

Result

MDA or PQL

Units

QC Type

$\begin{array}{llll}\text { ND } & 0.0005 & \mathrm{~Bq} / \mathrm{m}^{3} & \text { Sample } \\ \text { ND } & 0.0005 & \mathrm{~Bq} / \mathrm{m}^{3} & \text { Sample } \\ \text { 0.00037 } & 0.0003 & \mathrm{~Bq} / \mathrm{m}^{3} & \text { Sample } \\ \text { ND } & 0.0007 & \mathrm{~Bq} / \mathrm{m}^{3} & \text { Sample } \\ \text { ND } & 0.0005 & \mathrm{~Bq} / \mathrm{m}^{3} & \text { Sample } \\ \text { ND } & 0.0006 & \mathrm{~Bq} / \mathrm{m}^{3} & \text { Sample } \\ \text { ND } & 0.0005 & \mathrm{~Bq} / \mathrm{m}^{3} & \text { Sample } \\ \text { ND } & 0.0005 & \mathrm{~Bq} / \mathrm{m}^{3} & \text { Sample } \\ \text { ND } & 0.0006 & \mathrm{~Bq} / \mathrm{m}^{3} & \text { Sample } \\ \text { ND } & 0.0003 & \mathrm{~Bq} / \mathrm{m}^{3} & \text { Sample } \\ \text { ND } & 0.0008 & \mathrm{~Bq} / \mathrm{m}^{3} & \\ \text { ND } & 0.0003 & \mathrm{~Bq} / \mathrm{m}^{3} & \text { Sample } \\ \text { ND } & 0.0005 & \mathrm{~Bq} / \mathrm{m}^{3} & \text { Sample } \\ \text { ND } & 0.0005 & \mathrm{~Bq} / \mathrm{m}^{3} & \text { Sample } \\ \text { ND } & 0.0005 & \mathrm{~Bq} / \mathrm{m}^{3} & \text { Sample } \\ \text { ND } & 0.0004 & \mathrm{~Bq} / \mathrm{m}^{3} & \text { Sample } \\ \text { ND } & 0.0005 & \mathrm{~Bq} / \mathrm{m}^{3} & \text { Sample } \\ \text { ND } & 0.0003 & \mathrm{~Bq} / \mathrm{m}^{3} & \text { Sample } \\ \text { ND } & 0.0005 & \mathrm{~Bq} / \mathrm{m}^{3} & \text { Sample } \\ \text { ND } & 0.0003 & \mathrm{~Bq} / \mathrm{m}^{3} & \text { Sample } \\ \text { ND } & 0.0004 & \mathrm{~Bq} / \mathrm{m}^{3} & \text { Sample } \\ \text { ND } & 0.0005 & \mathrm{~Bq} / \mathrm{m}^{3} & \text { Sample } \\ \text { ND } & 0.0004 & \mathrm{~Bq} / \mathrm{m}^{3} & \text { Sample }\end{array}$

70A-1129RT

$10 / 23 / 01$

$10 / 30 / 01$

$11 / 8 / 01$

$11 / 13 / 01$

$11 / 20 / 01$

$11 / 26 / 01$

$12 / 4 / 01$

$12 / 12 / 01$

$12 / 19 / 01$

$1 / 2 / 02$

70A-1129P

$1 / 3 / 01$

2/7/01

$3 / 7 / 01$

$4 / 4 / 01$

$5 / 2 / 01$

$6 / 6 / 01$

7/5/01

$8 / 1 / 01$

9/5/01

10/5/01

$11 / 8 / 01$

$12 / 4 / 01$

$1 / 3 / 02$

$1 / 10 / 01$

$1 / 17 / 01$

1/24/01

$1 / 31 / 01$

2/7/01

$2 / 14 / 01$

2/22/01

$2 / 28 / 01$

$3 / 7 / 01$

$3 / 15 / 01$

$3 / 21 / 01$

$3 / 28 / 01$

$4 / 4 / 01$

$4 / 11 / 01$

$4 / 18 / 01$

$4 / 26 / 01$

$5 / 2 / 01$

$5 / 16 / 01$

$5 / 23 / 01$

$5 / 31 / 01$

6/6/01
ND

ND

ND

ND

ND

ND

ND

ND

ND

ND

ND

ND

ND

ND

ND

ND

ND

ND

ND

ND

ND
0.0006

0.0004

0.0005

0.0008

0.0005

0.0006

0.0004

0.0006

0.0006

0.0005

0.0007

0.0005

0.0005

0.0006

0.0006

0.0011

0.0008

0.0008

0.0008

0.0008

0.0008
$\mathrm{Bq} / \mathrm{m}^{3}$

$\mathrm{Bg} / \mathrm{m}^{3}$

$\mathrm{Bq} / \mathrm{m}^{3}$

$\mathrm{Bq} / \mathrm{m}^{3}$

$\mathrm{Bq} / \mathrm{m}^{3}$

$\mathrm{Bg} / \mathrm{m}^{3}$

$\mathrm{Bg} / \mathrm{m}^{3}$

$\mathrm{Bg} / \mathrm{m}^{3}$

$\mathrm{Bq} / \mathrm{m}^{3}$

$\mathrm{Bq} / \mathrm{m}^{3}$

$\mathrm{Bq} / \mathrm{m}^{3}$

$\mathrm{Bq} / \mathrm{m}^{3}$

$\mathrm{Bq} / \mathrm{m}^{3}$

$\mathrm{Bq} / \mathrm{m}^{3}$

$\mathrm{Bq} / \mathrm{m}^{3}$

$\mathrm{Bg} / \mathrm{m}^{3}$

$\mathrm{Bq} / \mathrm{m}^{3}$

$\mathrm{Bq} / \mathrm{m}^{3}$

$\mathrm{Bq} / \mathrm{m}^{3}$

$\mathrm{Bq} / \mathrm{m}^{3}$

$\mathrm{Bq} / \mathrm{m}^{3}$
Sample

Sample

Sample

Sample

Sample

Sample

Sample

Sample

Sample

Sample

Sample

Sample

Sample

Sample

Sample

Sample

Sample

Sample

Sample

Sample

Sample 


\section{Analyte \\ Location \\ Radiological Activity}

Date

Result

MDA or PQL

Units

QC Type

\begin{tabular}{|c|c|c|c|c|c|c|}
\hline Gross beta & 70A-1129RT & $6 / 13 / 01$ & ND & 0.0008 & $\mathrm{~Bq} / \mathrm{m}^{3}$ & Sample \\
\hline cont. & & $6 / 20 / 01$ & ND & 0.0008 & $\mathrm{~Bq} / \mathrm{m}^{3}$ & Sample \\
\hline & & $6 / 27 / 01$ & ND & 0.0008 & $\mathrm{~Bq} / \mathrm{m}^{3}$ & Sample \\
\hline & & $7 / 5 / 01$ & ND & 0.0006 & $\mathrm{~Bq} / \mathrm{m}^{3}$ & Sample \\
\hline & & 7/11/01 & ND & 0.0008 & $\mathrm{~Bq} / \mathrm{m}^{3}$ & Sample \\
\hline & & $7 / 18 / 01$ & ND & 0.0008 & $\mathrm{~Bq} / \mathrm{m}^{3}$ & Sample \\
\hline & & $7 / 25 / 01$ & ND & 0.0007 & $\mathrm{~Bq} / \mathrm{m}^{3}$ & Sample \\
\hline & & $8 / 1 / 01$ & ND & 0.0007 & $\mathrm{~Bq} / \mathrm{m}^{3}$ & Sample \\
\hline & & $8 / 8 / 01$ & ND & 0.0008 & $\mathrm{~Bq} / \mathrm{m}^{3}$ & Sample \\
\hline & & $8 / 15 / 01$ & ND & 0.0007 & $\mathrm{~Bq} / \mathrm{m}^{3}$ & Sample \\
\hline & & $8 / 22 / 01$ & ND & 0.0007 & $\mathrm{~Bq} / \mathrm{m}^{3}$ & Sample \\
\hline & & $8 / 29 / 01$ & ND & 0.0007 & $\mathrm{~Bq} / \mathrm{m}^{3}$ & Sample \\
\hline & & $9 / 5 / 01$ & ND & 0.0007 & $\mathrm{~Bq} / \mathrm{m}^{3}$ & Sample \\
\hline & & $9 / 12 / 01$ & ND & 0.0006 & $\mathrm{~Bq} / \mathrm{m}^{3}$ & Sample \\
\hline & & $9 / 19 / 01$ & ND & 0.0007 & $\mathrm{Bg} / \mathrm{m}^{3}$ & Sample \\
\hline & & $9 / 26 / 01$ & ND & 0.0008 & $\mathrm{~Bq} / \mathrm{m}^{3}$ & Sample \\
\hline & & $10 / 3 / 01$ & ND & 0.0007 & $\mathrm{~Bq} / \mathrm{m}^{3}$ & Sample \\
\hline & & 10/9/01 & ND & 0.0008 & $\mathrm{~Bq} / \mathrm{m}^{3}$ & Sample \\
\hline & & 10/16/01 & ND & 0.0009 & $\mathrm{~Bq} / \mathrm{m}^{3}$ & Sample \\
\hline & & $10 / 23 / 01$ & ND & 0.0007 & $\mathrm{~Bq} / \mathrm{m}^{3}$ & Sample \\
\hline & & 10/30/01 & ND & 0.0007 & $\mathrm{~Bq} / \mathrm{m}^{3}$ & Sample \\
\hline & & $11 / 8 / 01$ & ND & 0.0005 & $\mathrm{~Bq} / \mathrm{m}^{3}$ & Sample \\
\hline & & $11 / 13 / 01$ & ND & 0.0009 & $\mathrm{~Bq} / \mathrm{m}^{3}$ & Sample \\
\hline & & $11 / 20 / 01$ & ND & 0.0011 & $\mathrm{~Bq} / \mathrm{m}^{3}$ & Sample \\
\hline & & $11 / 26 / 01$ & ND & 0.0009 & $\mathrm{~Bq} / \mathrm{m}^{3}$ & Sample \\
\hline & & $12 / 4 / 01$ & ND & 0.0006 & $\mathrm{~Bq} / \mathrm{m}^{3}$ & Sample \\
\hline & & $12 / 19 / 01$ & ND & 0.0008 & $\mathrm{~Bq} / \mathrm{m}^{3}$ & Sample \\
\hline & & $1 / 2 / 02$ & ND & 0.0004 & $\mathrm{~Bq} / \mathrm{m}^{3}$ & Sample \\
\hline & $70 A-1145$ & $1 / 3 / 01$ & 0.00025 & 0.0002 & $\mathrm{~Bq} / \mathrm{m}^{3}$ & Sample \\
\hline & & $1 / 10 / 01$ & 0.00066 & 0.0005 & $\mathrm{~Bq} / \mathrm{m}^{3}$ & Sample \\
\hline & & $1 / 17 / 01$ & ND & 0.0005 & $\mathrm{~Bq} / \mathrm{m}^{3}$ & Sample \\
\hline & & 1/24/01 & ND & 0.0005 & $\mathrm{~Bq} / \mathrm{m}^{3}$ & Sample \\
\hline & & $1 / 31 / 01$ & ND & 0.0007 & $\mathrm{~Bq} / \mathrm{m}^{3}$ & Sample \\
\hline & & $2 / 7 / 01$ & ND & 0.0004 & $\mathrm{~Bq} / \mathrm{m}^{3}$ & Sample \\
\hline & & $2 / 14 / 01$ & ND & 0.0005 & $\mathrm{~Bq} / \mathrm{m}^{3}$ & Sample \\
\hline & & $2 / 22 / 01$ & ND & 0.0004 & $\mathrm{~Bq} / \mathrm{m}^{3}$ & Sample \\
\hline & & $2 / 28 / 01$ & ND & 0.0006 & $\mathrm{~Bq} / \mathrm{m}^{3}$ & Sample \\
\hline & & $3 / 7 / 01$ & ND & 0.0005 & $\mathrm{~Bq} / \mathrm{m}^{3}$ & Sample \\
\hline & & $3 / 15 / 01$ & ND & 0.0004 & $\mathrm{~Bq} / \mathrm{m}^{3}$ & Sample \\
\hline & & $3 / 21 / 01$ & ND & 0.0006 & $\mathrm{~Bq} / \mathrm{m}^{3}$ & Sample \\
\hline & & $3 / 28 / 01$ & ND & 0.0005 & $\mathrm{~Bq} / \mathrm{m}^{3}$ & Sample \\
\hline & & $4 / 4 / 01$ & ND & 0.0005 & $\mathrm{~Bq} / \mathrm{m}^{3}$ & Sample \\
\hline & & $4 / 11 / 01$ & ND & 0.0005 & $\mathrm{~Bq} / \mathrm{m}^{3}$ & Sample \\
\hline & & $4 / 18 / 01$ & ND & 0.0005 & $\mathrm{~Bq} / \mathrm{m}^{3}$ & Sampl \\
\hline & & $4 / 26 / 01$ & ND & 0.0008 & $\mathrm{~Bq} / \mathrm{m}^{3}$ & Sampl \\
\hline & & $5 / 2 / 01$ & ND & 0.0006 & $\mathrm{~Bq} / \mathrm{m}^{3}$ & Sam \\
\hline
\end{tabular}


Analyte

Radiological Activity

Gross beta 70A-1145

cont.

Date

Result

MDA or PQL

Units

QC Type

\begin{tabular}{|c|c|c|c|c|c|}
\hline \multirow[t]{26}{*}{$70 \mathrm{~A}-1145$} & $5 / 9 / 01$ & ND & 0.0004 & $\mathrm{~Bq} / \mathrm{m}^{3}$ & Sample \\
\hline & $5 / 16 / 01$ & ND & 0.0004 & $\mathrm{~Bq} / \mathrm{m}^{3}$ & Sample \\
\hline & $5 / 23 / 01$ & ND & 0.0005 & $\mathrm{~Bq} / \mathrm{m}^{3}$ & Sample \\
\hline & $5 / 31 / 01$ & ND & 0.0004 & $\mathrm{~Bq} / \mathrm{m}^{3}$ & Sample \\
\hline & 6/6/01 & ND & 0.0006 & $\mathrm{~Bq} / \mathrm{m}^{3}$ & Sample \\
\hline & $6 / 13 / 01$ & ND & 0.0005 & $\mathrm{~Bq} / \mathrm{m}^{3}$ & Sample \\
\hline & $6 / 20 / 01$ & ND & 0.0005 & $\mathrm{~Bq} / \mathrm{m}^{3}$ & Sample \\
\hline & $6 / 27 / 01$ & ND & 0.0004 & $\mathrm{Bg} / \mathrm{m}^{3}$ & Sample \\
\hline & $7 / 5 / 01$ & ND & 0.0004 & $\mathrm{~Bq} / \mathrm{m}^{3}$ & Sample \\
\hline & $7 / 11 / 01$ & ND & 0.0005 & $\mathrm{~Bq} / \mathrm{m}^{3}$ & Sample \\
\hline & $7 / 18 / 01$ & ND & 0.0005 & $\mathrm{~Bq} / \mathrm{m}^{3}$ & Sample \\
\hline & $7 / 25 / 01$ & ND & 0.0004 & $\mathrm{~Bq} / \mathrm{m}^{3}$ & Sample \\
\hline & $8 / 1 / 01$ & ND & 0.0004 & $\mathrm{~Bq} / \mathrm{m}^{3}$ & Sample \\
\hline & $8 / 8 / 01$ & ND & 0.0005 & $\mathrm{~Bq} / \mathrm{m}^{3}$ & Sample \\
\hline & $8 / 15 / 01$ & ND & 0.0004 & $\mathrm{~Bq} / \mathrm{m}^{3}$ & Sample \\
\hline & $8 / 22 / 01$ & ND & 0.0004 & $\mathrm{~Bq} / \mathrm{m}^{3}$ & Sample \\
\hline & $8 / 29 / 01$ & ND & 0.0004 & $\mathrm{~Bq} / \mathrm{m}^{3}$ & Sample \\
\hline & 9/5/01 & ND & 0.0004 & $\mathrm{~Bq} / \mathrm{m}^{3}$ & Sample \\
\hline & $9 / 12 / 01$ & ND & 0.0004 & $\mathrm{~Bq} / \mathrm{m}^{3}$ & Sample \\
\hline & $9 / 19 / 01$ & ND & 0.0004 & $\mathrm{~Bq} / \mathrm{m}^{3}$ & Sample \\
\hline & $9 / 26 / 01$ & ND & 0.0004 & $\mathrm{~Bq} / \mathrm{m}^{3}$ & Sample \\
\hline & $10 / 3 / 01$ & ND & 0.0004 & $\mathrm{~Bq} / \mathrm{m}^{3}$ & Sample \\
\hline & $10 / 9 / 01$ & 0.00044 & 0.0004 & $\mathrm{~Bq} / \mathrm{m}^{3}$ & Sample \\
\hline & $11 / 8 / 01$ & ND & 0.0004 & $\mathrm{~Bq} / \mathrm{m}^{3}$ & Sample \\
\hline & $12 / 4 / 01$ & ND & 0.0005 & $\mathrm{~Bq} / \mathrm{m}^{3}$ & Sample \\
\hline & $1 / 3 / 02$ & ND & 0.0004 & $\mathrm{~Bq} / \mathrm{m}^{3}$ & Sample \\
\hline \multirow[t]{13}{*}{$70 \mathrm{~A}-2211 \mathrm{H}$} & $1 / 3 / 01$ & 0.00079 & 0.0004 & $\mathrm{~Bq} / \mathrm{m}^{3}$ & Sample \\
\hline & $2 / 7 / 01$ & 0.00096 & 0.0003 & $\mathrm{~Bq} / \mathrm{m}^{3}$ & Sample \\
\hline & $3 / 7 / 01$ & 0.00048 & 0.0004 & $\mathrm{~Bq} / \mathrm{m}^{3}$ & Sample \\
\hline & $4 / 4 / 01$ & 0.00049 & 0.0004 & $\mathrm{~Bq} / \mathrm{m}^{3}$ & Sample \\
\hline & $5 / 2 / 01$ & ND & 0.0005 & $\mathrm{~Bq} / \mathrm{m}^{3}$ & Sample \\
\hline & 6/6/01 & 0.00037 & 0.0003 & $\mathrm{~Bq} / \mathrm{m}^{3}$ & Sample \\
\hline & $7 / 5 / 01$ & ND & 0.0005 & $\mathrm{~Bq} / \mathrm{m}^{3}$ & Sample \\
\hline & $8 / 1 / 01$ & ND & 0.0005 & $\mathrm{Bg} / \mathrm{m}^{3}$ & Sample \\
\hline & $9 / 5 / 01$ & ND & 0.0004 & $\mathrm{~Bq} / \mathrm{m}^{3}$ & Sample \\
\hline & $10 / 5 / 01$ & 0.0005 & 0.0003 & $\mathrm{~Bq} / \mathrm{m}^{3}$ & Sample \\
\hline & $11 / 8 / 01$ & 0.00072 & 0.0004 & $\mathrm{~Bq} / \mathrm{m}^{3}$ & Sample \\
\hline & $12 / 4 / 01$ & 0.00059 & 0.0005 & $\mathrm{~Bq} / \mathrm{m}^{3}$ & Sample \\
\hline & $1 / 3 / 02$ & ND & 0.0004 & $\mathrm{~Bq} / \mathrm{m}^{3}$ & Sample \\
\hline \multirow[t]{5}{*}{$70 \mathrm{~A}-2217 \mathrm{H}$} & $1 / 3 / 01$ & 0.00079 & 0.0004 & $\mathrm{~Bq} / \mathrm{m}^{3}$ & Sample \\
\hline & $2 / 7 / 01$ & 0.00073 & 0.0003 & $\mathrm{~Bq} / \mathrm{m}^{3}$ & Sample \\
\hline & $3 / 7 / 01$ & ND & 0.0004 & $\mathrm{~Bq} / \mathrm{m}^{3}$ & Sample \\
\hline & $4 / 4 / 01$ & ND & 0.0004 & $\mathrm{~Bq} / \mathrm{m}^{3}$ & Sample \\
\hline & $5 / 2 / 01$ & ND & 0.0005 & $\mathrm{~Bq} / \mathrm{m}^{3}$ & Sample \\
\hline
\end{tabular}




$\begin{array}{llllll}\text { Analyte } & \text { Location } & \text { Date } & \text { Result } & \text { MDA or PQL Units } & \text { QC Type }\end{array}$

\section{Radiological Activity}

\begin{tabular}{|c|c|c|c|c|c|c|}
\hline $\begin{array}{l}\text { Gross beta } \\
\text { cont. }\end{array}$ & $70 \mathrm{~A}-2217 \mathrm{H}$ & $\begin{array}{l}6 / 6 / 01 \\
7 / 5 / 01 \\
8 / 1 / 01 \\
9 / 5 / 01 \\
10 / 5 / 01 \\
11 / 8 / 01 \\
12 / 4 / 01 \\
1 / 3 / 02\end{array}$ & $\begin{array}{l}0.0004 \\
0.00049 \\
\text { ND } \\
\text { ND } \\
0.00057 \\
\text { ND } \\
\text { ND } \\
\text { ND }\end{array}$ & $\begin{array}{l}0.0003 \\
0.0004 \\
0.0005 \\
0.0004 \\
0.0004 \\
0.0004 \\
0.0005 \\
0.0004\end{array}$ & $\begin{array}{l}\mathrm{Bq} / \mathrm{m}^{3} \\
\mathrm{~Bq} / \mathrm{m}^{3} \\
\mathrm{~Bq} / \mathrm{m}^{3} \\
\mathrm{~Bq} / \mathrm{m}^{3} \\
\mathrm{~Bq} / \mathrm{m}^{3} \\
\mathrm{~Bq} / \mathrm{m}^{3} \\
\mathrm{~Bq} / \mathrm{m}^{3} \\
\mathrm{~Bq} / \mathrm{m}^{3}\end{array}$ & $\begin{array}{l}\text { Sample } \\
\text { Sample } \\
\text { Sample } \\
\text { Sample } \\
\text { Sample } \\
\text { Sample } \\
\text { Sample } \\
\text { Sample }\end{array}$ \\
\hline & $70 A-2275$ & $\begin{array}{l}1 / 3 / 01 \\
2 / 7 / 01 \\
3 / 7 / 01 \\
4 / 4 / 01 \\
5 / 2 / 01 \\
6 / 6 / 01 \\
7 / 5 / 01 \\
8 / 1 / 01 \\
9 / 5 / 01 \\
10 / 5 / 01\end{array}$ & $\begin{array}{l}0.001 \\
0.0011 \\
0.0005 \\
0.00055 \\
\text { ND } \\
0.00047 \\
0.0005 \\
\text { ND } \\
\text { ND } \\
0.00075\end{array}$ & $\begin{array}{l}0.0004 \\
0.0003 \\
0.0004 \\
0.0004 \\
0.0005 \\
0.0004 \\
0.0005 \\
0.0005 \\
0.0004 \\
0.0003\end{array}$ & $\begin{array}{l}\mathrm{Bq} / \mathrm{m}^{3} \\
\mathrm{~Bq} / \mathrm{m}^{3} \\
\mathrm{~Bq} / \mathrm{m}^{3} \\
\mathrm{~Bq} / \mathrm{m}^{3} \\
\mathrm{~Bq} / \mathrm{m}^{3} \\
\mathrm{~Bq} / \mathrm{m}^{3} \\
\mathrm{~Bq} / \mathrm{m}^{3} \\
\mathrm{~Bq} / \mathrm{m}^{3} \\
\mathrm{~Bq} / \mathrm{m}^{3} \\
\mathrm{~Bq} / \mathrm{m}^{3}\end{array}$ & $\begin{array}{l}\text { Sample } \\
\text { Sample } \\
\text { Sample } \\
\text { Sample } \\
\text { Sample } \\
\text { Sample } \\
\text { Sample } \\
\text { Sample } \\
\text { Sample } \\
\text { Sample }\end{array}$ \\
\hline & 75 Stack Sump & $\begin{array}{l}7 / 10 / 01 \\
7 / 10 / 01\end{array}$ & $\begin{array}{l}0.263 \\
0.18\end{array}$ & $\begin{array}{l}0.07 \\
0.07\end{array}$ & $\begin{array}{l}\mathrm{Bq} / \mathrm{L} \\
\mathrm{Bq} / \mathrm{L}\end{array}$ & $\begin{array}{l}\text { Sample } \\
\text { Split }\end{array}$ \\
\hline & 75A-TEMP & $\begin{array}{l}1 / 3 / 01 \\
1 / 10 / 01 \\
1 / 17 / 01 \\
1 / 24 / 01 \\
1 / 31 / 01 \\
2 / 7 / 01 \\
2 / 14 / 01 \\
2 / 22 / 01 \\
2 / 28 / 01 \\
3 / 7 / 01 \\
3 / 15 / 01 \\
3 / 21 / 01 \\
3 / 28 / 01 \\
4 / 4 / 01 \\
4 / 11 / 01 \\
4 / 18 / 01 \\
4 / 26 / 01 \\
5 / 2 / 01 \\
5 / 9 / 01 \\
5 / 16 / 01 \\
5 / 23 / 01 \\
5 / 31 / 01 \\
6 / 6 / 01 \\
6 / 13 / 01\end{array}$ & $\begin{array}{l}0.00031 \\
\text { ND } \\
\text { ND } \\
0.00052 \\
\text { ND } \\
\text { ND } \\
\text { ND } \\
\text { ND } \\
\text { ND } \\
\text { ND } \\
\text { ND } \\
\text { ND } \\
\text { ND } \\
\text { ND } \\
\text { ND } \\
\text { ND } \\
\text { ND } \\
\text { ND } \\
\text { ND } \\
\text { ND } \\
\text { ND } \\
\text { ND } \\
\text { ND } \\
\text { ND }\end{array}$ & $\begin{array}{l}0.0002 \\
0.0004 \\
0.0004 \\
0.0004 \\
0.0007 \\
0.0004 \\
0.0004 \\
0.0004 \\
0.0005 \\
0.0004 \\
0.0004 \\
0.0005 \\
0.0004 \\
0.0004 \\
0.0004 \\
0.0004 \\
0.0004 \\
0.0005 \\
0.0004 \\
0.0004 \\
0.0004 \\
0.0004 \\
0.0005 \\
0.0005\end{array}$ & $\begin{array}{l}\mathrm{Bq} / \mathrm{m}^{3} \\
\mathrm{~Bq} / \mathrm{m}^{3} \\
\mathrm{~Bq} / \mathrm{m}^{3} \\
\mathrm{~Bq} / \mathrm{m}^{3} \\
\mathrm{~Bq} / \mathrm{m}^{3} \\
\mathrm{~Bq} / \mathrm{m}^{3} \\
\mathrm{~Bq} / \mathrm{m}^{3} \\
\mathrm{~Bq} / \mathrm{m}^{3} \\
\mathrm{~Bq} / \mathrm{m}^{3} \\
\mathrm{~Bq} / \mathrm{m}^{3} \\
\mathrm{~Bq} / \mathrm{m}^{3} \\
\mathrm{~Bq} / \mathrm{m}^{3} \\
\mathrm{~Bq} / \mathrm{m}^{3} \\
\mathrm{~Bq} / \mathrm{m}^{3} \\
\mathrm{~Bq} / \mathrm{m}^{3} \\
\mathrm{~Bq} / \mathrm{m}^{3} \\
\mathrm{~Bq} / \mathrm{m}^{3} \\
\mathrm{~Bq} / \mathrm{m}^{3} \\
\mathrm{~Bq} / \mathrm{m}^{3} \\
\mathrm{~Bq} / \mathrm{m}^{3} \\
\mathrm{~Bq} / \mathrm{m}^{3} \\
\mathrm{~Bq} / \mathrm{m}^{3} \\
\mathrm{~Bq} / \mathrm{m}^{3} \\
\mathrm{~Bq} / \mathrm{m}^{3}\end{array}$ & $\begin{array}{l}\text { Sample } \\
\text { Sample } \\
\text { Sample } \\
\text { Sample } \\
\text { Sample } \\
\text { Sample } \\
\text { Sample } \\
\text { Sample } \\
\text { Sample } \\
\text { Sample } \\
\text { Sample } \\
\text { Sample } \\
\text { Sample } \\
\text { Sample } \\
\text { Sample } \\
\text { Sample } \\
\text { Sample } \\
\text { Sample } \\
\text { Sample } \\
\text { Sample } \\
\text { Sample } \\
\text { Sample } \\
\text { Sample } \\
\text { Sample }\end{array}$ \\
\hline
\end{tabular}


Analyte

\section{Radiological Activity}

Gross beta 75A-TEMP cont.

$\begin{array}{ll}\text { 75A-TEMP } & 6 / 20 / 01 \\ 6 / 27 / 01 \\ 7 / 5 / 01 \\ 7 / 11 / 01 \\ 7 / 18 / 01 \\ 7 / 25 / 01 \\ 8 / 1 / 01 \\ 8 / 8 / 01 \\ 8 / 15 / 01 \\ 8 / 22 / 01 \\ 8 / 29 / 01 \\ 9 / 5 / 01 \\ 9 / 12 / 01 \\ 9 / 19 / 01 \\ 9 / 26 / 01 \\ 10 / 3 / 01 \\ 10 / 9 / 01 \\ 10 / 16 / 01 \\ 10 / 24 / 01 \\ 10 / 30 / 01 \\ 11 / 8 / 01 \\ 11 / 13 / 01 \\ 11 / 20 / 01 \\ 11 / 26 / 01 \\ 12 / 4 / 01 \\ 12 / 12 / 01\end{array}$

85 Glovebox
Date

Result

MDA or PQL Units

QC Type

$\begin{array}{llll}\text { ND } & 0.0004 & \mathrm{~Bq} / \mathrm{m}^{3} & \text { Sample } \\ \text { ND } & 0.0004 & \mathrm{~Bq} / \mathrm{m}^{3} & \text { Sample } \\ \text { ND } & 0.0004 & \mathrm{~Bq} / \mathrm{m}^{3} & \text { Sample } \\ \text { ND } & 0.0005 & \mathrm{~Bq} / \mathrm{m}^{3} & \text { Sample } \\ \text { ND } & 0.0004 & \mathrm{~Bq} / \mathrm{m}^{3} & \text { Sample } \\ \text { ND } & 0.0004 & \mathrm{~Bq} / \mathrm{m}^{3} & \text { Sample } \\ \text { ND } & 0.0004 & \mathrm{~Bq} / \mathrm{m}^{3} & \text { Sample } \\ \text { ND } & 0.0004 & \mathrm{~Bq} / \mathrm{m}^{3} & \text { Sample } \\ \text { ND } & 0.0004 & \mathrm{~Bq} / \mathrm{m}^{3} & \text { Sample } \\ \text { ND } & 0.0004 & \mathrm{~Bq} / \mathrm{m}^{3} & \text { Sample } \\ \text { ND } & 0.0004 & \mathrm{~Bq} / \mathrm{m}^{3} & \text { Sample } \\ \text { ND } & 0.0004 & \mathrm{~Bq} / \mathrm{m}^{3} & \text { Sample } \\ \text { ND } & 0.0004 & \mathrm{~Bq} / \mathrm{m}^{3} & \text { Sample } \\ \text { ND } & 0.0004 & \mathrm{~Bq} / \mathrm{m}^{3} & \text { Sample } \\ \text { ND } & 0.0004 & \mathrm{~Bq} / \mathrm{m}^{3} & \text { Sample } \\ \text { 0.00043 } & 0.0004 & \mathrm{~Bq} / \mathrm{m}^{3} & \text { Sample } \\ \text { ND } & 0.0004 & \mathrm{~Bq} / \mathrm{m}^{3} & \text { Sample } \\ \text { ND } & 0.0005 & \mathrm{~Bq} / \mathrm{m}^{3} & \text { Sample } \\ 0.00037 & 0.0003 & \mathrm{~Bq} / \mathrm{m}^{3} & \text { Sample } \\ \text { 0.00062 } & 0.0004 & \mathrm{~Bq} / \mathrm{m}^{3} & \text { Sample } \\ \text { ND } & 0.0003 & \mathrm{~Bq} / \mathrm{m}^{3} & \text { Sample } \\ \text { 0.00069 } & 0.0006 & \mathrm{~Bq} / \mathrm{m}^{3} & \text { Sample } \\ \text { ND } & 0.0004 & \mathrm{~Bq} / \mathrm{m}^{3} & \text { Sample } \\ \text { ND } & 0.0005 & \mathrm{~Bq} / \mathrm{m}^{3} & \text { Sample } \\ \text { ND } & 0.0004 & \mathrm{~Bq} / \mathrm{m}^{3} & \text { Sample } \\ \text { ND } & 0.0004 & \mathrm{~Bq} / \mathrm{m}^{3} & \text { Sample }\end{array}$

$\begin{array}{llll}0.0003 & 0.00005 & \mathrm{~Bq} / \mathrm{m}^{3} & \text { Sample } \\ 0.00057 & 0.00013 & \mathrm{~Bq} / \mathrm{m}^{3} & \text { Sample } \\ 0.00032 & 0.00011 & \mathrm{~Bq} / \mathrm{m}^{3} & \text { Sample } \\ 0.0000041 & 0.0000012 & \mathrm{~Bq} / \mathrm{m}^{3} & \text { Sample } \\ 0.0003 & 0.00011 & \mathrm{~Bq} / \mathrm{m}^{3} & \text { Sample } \\ 0.00029 & 0.00011 & \mathrm{~Bq} / \mathrm{m}^{3} & \text { Sample } \\ 0.00018 & 0.00011 & \mathrm{~Bq} / \mathrm{m}^{3} & \text { Sample } \\ 0.00027 & 0.0001 & \mathrm{~Bq} / \mathrm{m}^{3} & \text { Sample } \\ 0.00042 & 0.00013 & \mathrm{~Bq} / \mathrm{m}^{3} & \text { Sample } \\ 0.00034 & 0.00009 & \mathrm{~Bq} / \mathrm{m}^{3} & \text { Sample } \\ 0.00038 & 0.0001 & \mathrm{~Bq} / \mathrm{m}^{3} & \text { Sample } \\ 0.00043 & 0.00013 & \mathrm{~Bq} / \mathrm{m}^{3} & \text { Sample } \\ 0.00041 & 0.00011 & \mathrm{~Bq} / \mathrm{m}^{3} & \text { Sample } \\ 0.00035 & 0.00012 & \mathrm{~Bq} / \mathrm{m}^{3} & \text { Sample } \\ 0.00038 & 0.00011 & \mathrm{~Bq} / \mathrm{m}^{3} & \text { Sample } \\ 0.00004 & 0.000011 & \mathrm{~Bq} / \mathrm{m}^{3} & \text { Sample } \\ 0.00032 & 0.0001 & \mathrm{~Bq} / \mathrm{m}^{3} & \text { Sample } \\ 0.00045 & 0.00013 & \mathrm{~Bq} / \mathrm{m}^{3} & \text { Sample } \\ 0.0003 & 0.00011 & \mathrm{~Bq} / \mathrm{m}^{3} & \text { Sample }\end{array}$




\begin{tabular}{llllll} 
Analyte & Location & Date & Result & MDA or PQL Units & QC Type \\
\hline
\end{tabular}

\section{Radiological Activity}

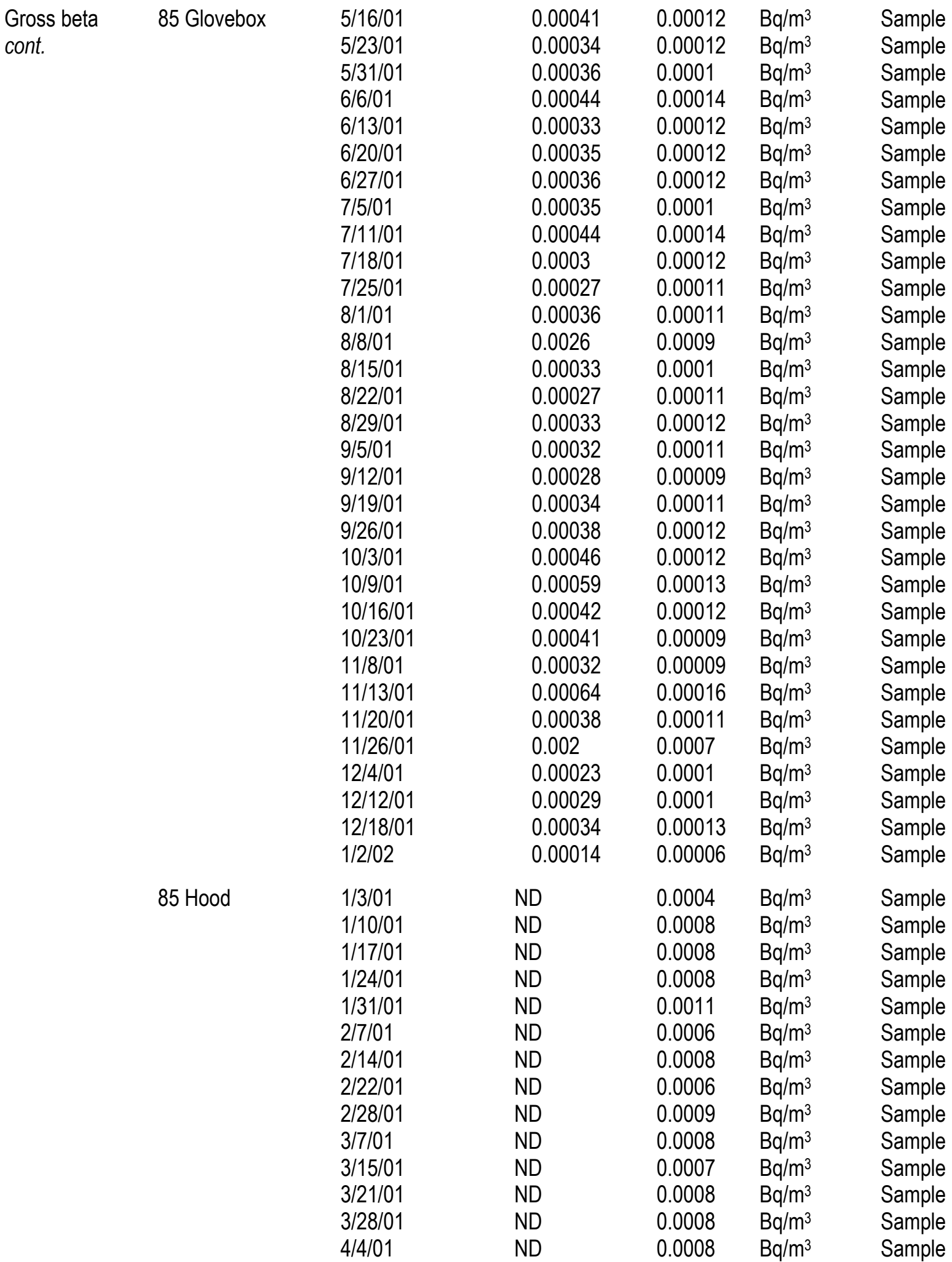


Analyte

Radiological Activity

\begin{tabular}{|c|c|c|c|c|c|c|}
\hline \multirow{36}{*}{\multicolumn{2}{|c|}{$\begin{array}{l}\text { Gross beta } \\
\text { cont. }\end{array}$}} & $4 / 11 / 01$ & ND & 0.0008 & $\mathrm{~Bq} / \mathrm{m}^{3}$ & Sample \\
\hline & & $4 / 18 / 01$ & ND & 0.0008 & $\mathrm{~Bq} / \mathrm{m}^{3}$ & Sample \\
\hline & & $4 / 26 / 01$ & ND & 0.0007 & $\mathrm{~Bq} / \mathrm{m}^{3}$ & Sample \\
\hline & & $5 / 2 / 01$ & ND & 0.0004 & $\mathrm{~Bq} / \mathrm{m}^{3}$ & Sample \\
\hline & & $5 / 9 / 01$ & ND & 0.0006 & $\mathrm{~Bq} / \mathrm{m}^{3}$ & Sample \\
\hline & & $5 / 16 / 01$ & ND & 0.0006 & $\mathrm{~Bq} / \mathrm{m}^{3}$ & Sample \\
\hline & & $5 / 23 / 01$ & ND & 0.0008 & $\mathrm{~Bq} / \mathrm{m}^{3}$ & Sample \\
\hline & & $5 / 31 / 01$ & ND & 0.0008 & $\mathrm{~Bq} / \mathrm{m}^{3}$ & Sample \\
\hline & & $6 / 6 / 01$ & ND & 0.001 & $\mathrm{~Bq} / \mathrm{m}^{3}$ & Sample \\
\hline & & $6 / 13 / 01$ & ND & 0.0008 & $\mathrm{~Bq} / \mathrm{m}^{3}$ & Sample \\
\hline & & $6 / 20 / 01$ & ND & 0.0008 & $\mathrm{~Bq} / \mathrm{m}^{3}$ & Sampl \\
\hline & & $6 / 27 / 01$ & ND & 0.0007 & $\mathrm{~Bq} / \mathrm{m}^{3}$ & Sample \\
\hline & & $7 / 11 / 01$ & ND & 0.0001 & $\mathrm{~Bq} / \mathrm{m}^{3}$ & Sample \\
\hline & & $7 / 18 / 01$ & ND & 0.0008 & $\mathrm{~Bq} / \mathrm{m}^{3}$ & Sample \\
\hline & & $7 / 25 / 01$ & ND & 0.0006 & $\mathrm{~Bq} / \mathrm{m}^{3}$ & Sample \\
\hline & & $8 / 1 / 01$ & ND & 0.0008 & $\mathrm{~Bq} / \mathrm{m}^{3}$ & Sample \\
\hline & & $8 / 8 / 01$ & ND & 0.005 & $\mathrm{~Bq} / \mathrm{m}^{3}$ & Sample \\
\hline & & $8 / 15 / 01$ & ND & 0.0008 & $\mathrm{~Bq} / \mathrm{m}^{3}$ & Samp \\
\hline & & $8 / 29 / 01$ & ND & 0.0006 & $\mathrm{~Bq} / \mathrm{m}^{3}$ & Sample \\
\hline & & $9 / 5 / 01$ & ND & 0.0007 & $\mathrm{~Bq} / \mathrm{m}^{3}$ & Sample \\
\hline & & $9 / 12 / 01$ & ND & 0.0008 & $\mathrm{~Bq} / \mathrm{m}^{3}$ & Sample \\
\hline & & 9/19/01 & ND & 0.0006 & $\mathrm{~Bq} / \mathrm{m}^{3}$ & Samp \\
\hline & & $9 / 26 / 01$ & ND & 0.0007 & $\mathrm{~Bq} / \mathrm{m}^{3}$ & Sampl \\
\hline & & $10 / 3 / 01$ & ND & 0.0006 & $\mathrm{~Bq} / \mathrm{m}^{3}$ & Sample \\
\hline & & 10/9/01 & 0.00096 & 0.0007 & $\mathrm{~Bq} / \mathrm{m}^{3}$ & Samp \\
\hline & & $10 / 16 / 01$ & ND & 0.0007 & $\mathrm{~Bq} / \mathrm{m}^{3}$ & Sample \\
\hline & & $10 / 23 / 01$ & ND & 0.0006 & $\mathrm{~Bq} / \mathrm{m}^{3}$ & Sample \\
\hline & & $10 / 30 / 01$ & 0.00058 & 0.0005 & $\mathrm{~Bq} / \mathrm{m}^{3}$ & Samp \\
\hline & & 11/8/01 & ND & 0.0005 & $\mathrm{~Bq} / \mathrm{m}^{3}$ & Samp \\
\hline & & $11 / 13 / 01$ & ND & 0.0011 & $\mathrm{~Bq} / \mathrm{m}^{3}$ & Sample \\
\hline & & $11 / 20 / 01$ & ND & 0.0007 & $\mathrm{~Bq} / \mathrm{m}^{3}$ & Sample \\
\hline & & $11 / 26 / 01$ & ND & 0.00016 & $\mathrm{~Bq} / \mathrm{m}^{3}$ & Sample \\
\hline & & $12 / 4 / 01$ & ND & 0.0006 & $\mathrm{~Bq} / \mathrm{m}^{3}$ & Sample \\
\hline & & $12 / 12 / 01$ & ND & 0.0006 & $\mathrm{~Bq} / \mathrm{m}^{3}$ & Samp \\
\hline & & 12/18/01 & ND & 0.0009 & $\mathrm{~Bq} / \mathrm{m}^{3}$ & Sample \\
\hline & & $1 / 2 / 02$ & ND & 0.0004 & $\mathrm{~Bq} / \mathrm{m}^{3}$ & Sampl \\
\hline & B88 Cave 0 & $1 / 3 / 01$ & 0.00051 & 0.0002 & $\mathrm{~Bq} / \mathrm{m}^{3}$ & Sample \\
\hline & & $1 / 10 / 01$ & 0.00064 & 0.0004 & $\mathrm{~Bq} / \mathrm{m}^{3}$ & Sample \\
\hline & & 1/17/01 & ND & 0.0013 & $\mathrm{~Bq} / \mathrm{m}^{3}$ & Sample \\
\hline & & 1/24/01 & ND & 0.0013 & $\mathrm{~Bq} / \mathrm{m}^{3}$ & Samp \\
\hline & & 1/31/01 & ND & 0.002 & $\mathrm{~Bq} / \mathrm{m}^{3}$ & Sample \\
\hline & & $2 / 7 / 01$ & ND & 0.0011 & $\mathrm{~Bq} / \mathrm{m}^{3}$ & Sampl \\
\hline & & $2 / 14 / 01$ & ND & 0.0011 & $\mathrm{~Bq} / \mathrm{m}^{3}$ & Samp \\
\hline & & 2/22/01 & ND & 0.001 & $\mathrm{~Bq} / \mathrm{m}^{3}$ & Sampl \\
\hline & & $2 / 28 / 01$ & ND & 0.001 & $\mathrm{~Bq} / \mathrm{m}^{3}$ & Sample \\
\hline
\end{tabular}




\section{Analyte \\ Location \\ Radiological Activity}

Date

Result

MDA or PQL

Units

QC Type

\begin{tabular}{|c|c|c|c|c|c|c|}
\hline Gross beta & B88 Cave 0 & $3 / 7 / 01$ & ND & 0.00019 & $\mathrm{~Bq} / \mathrm{m}^{3}$ & $\begin{array}{l}\text { Sample } \\
\text { Sample }\end{array}$ \\
\hline cont. & & $3 / 15 / 01$ & ND & 0.0005 & $\mathrm{~Bq} / \mathrm{m}^{3}$ & Sample \\
\hline & & $3 / 21 / 01$ & ND & 0.0012 & $\mathrm{~Bq} / \mathrm{m}^{3}$ & Sample \\
\hline & & $3 / 28 / 01$ & ND & 0.0012 & $\mathrm{~Bq} / \mathrm{m}^{3}$ & Sample \\
\hline & & $4 / 4 / 01$ & ND & 0.001 & $\mathrm{~Bq} / \mathrm{m}^{3}$ & Sample \\
\hline & & $4 / 11 / 01$ & ND & 0.001 & $\mathrm{~Bq} / \mathrm{m}^{3}$ & Sample \\
\hline & & $4 / 18 / 01$ & ND & 0.0015 & $\mathrm{~Bq} / \mathrm{m}^{3}$ & Sample \\
\hline & & $4 / 26 / 01$ & ND & 0.0011 & $\mathrm{~Bq} / \mathrm{m}^{3}$ & Sample \\
\hline & & $5 / 2 / 01$ & ND & 0.0011 & $\mathrm{~Bq} / \mathrm{m}^{3}$ & Sample \\
\hline & & $5 / 9 / 01$ & ND & 0.0009 & $\mathrm{~Bq} / \mathrm{m}^{3}$ & Sample \\
\hline & & $5 / 16 / 01$ & ND & 0.0007 & $\mathrm{~Bq} / \mathrm{m}^{3}$ & Sample \\
\hline & & $5 / 23 / 01$ & ND & 0.0013 & $\mathrm{~Bq} / \mathrm{m}^{3}$ & Sample \\
\hline & & $5 / 31 / 01$ & 0.0012 & 0.001 & $\mathrm{~Bq} / \mathrm{m}^{3}$ & Sample \\
\hline & & $6 / 6 / 01$ & ND & 0.0014 & $\mathrm{~Bq} / \mathrm{m}^{3}$ & Sample \\
\hline & & $6 / 13 / 01$ & ND & 0.0007 & $\mathrm{~Bq} / \mathrm{m}^{3}$ & Sample \\
\hline & & $6 / 20 / 01$ & ND & 0.0004 & $\mathrm{~Bq} / \mathrm{m}^{3}$ & Sample \\
\hline & & $6 / 27 / 01$ & 0.00059 & 0.0004 & $\mathrm{Bg} / \mathrm{m}^{3}$ & Sample \\
\hline & & $7 / 5 / 01$ & ND & 0.0007 & $\mathrm{~Bq} / \mathrm{m}^{3}$ & Sample \\
\hline & & $7 / 11 / 01$ & ND & 0.003 & $\mathrm{Bg} / \mathrm{m}^{3}$ & Sample \\
\hline & & $7 / 18 / 01$ & ND & 0.0008 & $\mathrm{~Bq} / \mathrm{m}^{3}$ & Sample \\
\hline & & $7 / 25 / 01$ & ND & 0.0008 & $\mathrm{Bg} / \mathrm{m}^{3}$ & Sample \\
\hline & & $8 / 1 / 01$ & ND & 0.0008 & $\mathrm{~Bq} / \mathrm{m}^{3}$ & Sample \\
\hline & & $8 / 8 / 01$ & ND & 0.0008 & $\mathrm{~Bq} / \mathrm{m}^{3}$ & Sample \\
\hline & & $8 / 15 / 01$ & ND & 0.0009 & $\mathrm{~Bq} / \mathrm{m}^{3}$ & Sample \\
\hline & & $8 / 22 / 01$ & ND & 0.0007 & $\mathrm{~Bq} / \mathrm{m}^{3}$ & Sample \\
\hline & & $8 / 29 / 01$ & ND & 0.0008 & $\mathrm{~Bq} / \mathrm{m}^{3}$ & Sample \\
\hline & & $9 / 5 / 01$ & ND & 0.0009 & $\mathrm{~Bq} / \mathrm{m}^{3}$ & Sample \\
\hline & & $9 / 12 / 01$ & ND & 0.0009 & $\mathrm{~Bq} / \mathrm{m}^{3}$ & Sample \\
\hline & & $9 / 19 / 01$ & ND & 0.0008 & $\mathrm{~Bq} / \mathrm{m}^{3}$ & Sample \\
\hline & & $9 / 26 / 01$ & ND & 0.0007 & $\mathrm{~Bq} / \mathrm{m}^{3}$ & Sample \\
\hline & & $10 / 3 / 01$ & 0.001 & 0.0008 & $\mathrm{Bg} / \mathrm{m}^{3}$ & Sample \\
\hline & & 10/9/01 & ND & 0.0007 & $\mathrm{~Bq} / \mathrm{m}^{3}$ & Sample \\
\hline & & $10 / 16 / 01$ & ND & 0.0009 & $\mathrm{~Bq} / \mathrm{m}^{3}$ & Sample \\
\hline & & $10 / 23 / 01$ & 0.0012 & 0.0006 & $\mathrm{Bg} / \mathrm{m}^{3}$ & Sample \\
\hline & & $11 / 8 / 01$ & 0.00086 & 0.0005 & $\mathrm{~Bq} / \mathrm{m}^{3}$ & Sample \\
\hline & & $11 / 13 / 01$ & 0.0014 & 0.0009 & $\mathrm{~Bq} / \mathrm{m}^{3}$ & Sample \\
\hline & & $11 / 20 / 01$ & ND & 0.0006 & $\mathrm{~Bq} / \mathrm{m}^{3}$ & Sample \\
\hline & & $11 / 26 / 01$ & ND & 0.0007 & $\mathrm{Bg} / \mathrm{m}^{3}$ & Sample \\
\hline & & $12 / 4 / 01$ & ND & 0.0005 & $\mathrm{Ba} / \mathrm{m}^{3}$ & Sample \\
\hline & & $12 / 12 / 01$ & ND & 0.0005 & $\mathrm{~Bq} / \mathrm{m}^{3}$ & Sample \\
\hline & & $12 / 19 / 01$ & ND & 0.0005 & $\mathrm{Bg} / \mathrm{m}^{3}$ & Sample \\
\hline & & $1 / 2 / 02$ & 0.00046 & 0.0003 & $\mathrm{~Bq} / \mathrm{m}^{3}$ & Sample \\
\hline & B88-135H & $1 / 3 / 01$ & ND & 0.0005 & $\mathrm{Bg} / \mathrm{m}^{3}$ & Sample \\
\hline & & $2 / 7 / 01$ & ND & 0.0003 & $\mathrm{Bg} / \mathrm{m}^{3}$ & Sample \\
\hline & & $3 / 7 / 01$ & ND & 0.0005 & $\mathrm{Bg} / \mathrm{m}^{3}$ & Sample \\
\hline & & $4 / 4 / 01$ & ND & 0.0005 & $\mathrm{Bg} / \mathrm{m}^{3}$ & Sample \\
\hline
\end{tabular}


Analyte

Radiological Activity

Gross beta B88-135H

cont.
Date

Result

MDA or PQL

Units

QC Type

$\begin{array}{llll}\text { ND } & 0.0005 & \mathrm{~Bq} / \mathrm{m}^{3} & \text { Sample } \\ \text { ND } & 0.0004 & \mathrm{~Bq} / \mathrm{m}^{3} & \text { Sample } \\ \text { ND } & 0.0004 & \mathrm{~Bq} / \mathrm{m}^{3} & \text { Sample } \\ \text { ND } & 0.0005 & \mathrm{~Bq} / \mathrm{m}^{3} & \text { Sample } \\ \text { ND } & 0.0004 & \mathrm{~Bq} / \mathrm{m}^{3} & \text { Sample } \\ \text { ND } & 0.0004 & \mathrm{~Bq} / \mathrm{m}^{3} & \text { Sample } \\ \text { ND } & 0.0004 & \mathrm{~Bq} / \mathrm{m}^{3} & \text { Sample } \\ \text { ND } & 0.0006 & \mathrm{~Bq} / \mathrm{m}^{3} & \text { Sample } \\ \text { ND } & 0.0004 & \mathrm{~Bq} / \mathrm{m}^{3} & \text { Sample }\end{array}$

Travel Blank

$1 / 3 / 01$

$1 / 3 / 01$

$1 / 3 / 01$

$1 / 3 / 01$

$1 / 3 / 01$

$1 / 10 / 01$

$1 / 10 / 01$

$1 / 10 / 01$

$1 / 17 / 01$

$1 / 17 / 01$

$1 / 17 / 01$

$1 / 24 / 01$

$1 / 24 / 01$

$1 / 24 / 01$

$1 / 31 / 01$

$1 / 31 / 01$

$1 / 31 / 01$

2/7/01

2/7/01

2/7/01

2/7/01

2/7/01

$2 / 14 / 01$

2/14/01

$2 / 14 / 01$

2/22/01

2/22/01

2/22/01

2/28/01

2/28/01

$2 / 28 / 01$

$3 / 7 / 01$

$3 / 7 / 01$

$3 / 7 / 01$

$3 / 7 / 01$

$3 / 7 / 01$
ND

ND

ND

0.36

0.52

0.37

ND

ND

ND

ND

0.44

ND

0.44

ND

ND

0.44

ND

ND

ND

0.44

0.36

ND

ND

0.48

ND

ND

ND

0.48

ND

ND

0.44

ND

0.59

ND

0.52

ND
0.2

0.19

0.2

0.19

0.19

0.2

0.19

0.2

0.19

0.2

0.19

0.2

0.19

0.19

0.3

0.19

0.3

0.19

0.19

0.19

0.19

0.19

0.2

0.19

0.19

0.19

0.2

0.19

0.2

0.2

0.19

0.2

0.15

0.2

0.15

0.2
$\mathrm{Bq} / \mathrm{S}$

$\mathrm{Bq} / \mathrm{S}$

$\mathrm{Bq} / \mathrm{S}$

$\mathrm{Bq} / \mathrm{S}$

$\mathrm{Bq} / \mathrm{S}$

$\mathrm{Bq} / \mathrm{S}$

$\mathrm{Bq} / \mathrm{S}$

$\mathrm{Bq} / \mathrm{S}$

$\mathrm{Bq} / \mathrm{S}$

$\mathrm{Bq} / \mathrm{S}$

$\mathrm{Bq} / \mathrm{S}$

$\mathrm{Bq} / \mathrm{S}$

$\mathrm{Bq} / \mathrm{S}$

$\mathrm{Bq} / \mathrm{S}$

$\mathrm{Bq} / \mathrm{S}$

$\mathrm{Bq} / \mathrm{S}$

$\mathrm{Bq} / \mathrm{S}$

$\mathrm{Bq} / \mathrm{S}$

$\mathrm{Bq} / \mathrm{S}$

$\mathrm{Bq} / \mathrm{S}$

$\mathrm{Bq} / \mathrm{S}$

$\mathrm{Bq} / \mathrm{S}$

$\mathrm{Bq} / \mathrm{S}$

$\mathrm{Bq} / \mathrm{S}$

$\mathrm{Bq} / \mathrm{S}$

$\mathrm{Bq} / \mathrm{S}$

$\mathrm{Bq} / \mathrm{S}$

$\mathrm{Bq} / \mathrm{S}$

$\mathrm{Bq} / \mathrm{S}$

$\mathrm{Bq} / \mathrm{S}$

$\mathrm{Bq} / \mathrm{S}$

$\mathrm{Bq} / \mathrm{S}$

$\mathrm{Bq} / \mathrm{S}$

$\mathrm{Bq} / \mathrm{S}$
$\mathrm{Bq} / \mathrm{S}$

$\mathrm{Bq} / \mathrm{S}$
Blank

Blank

Blank

Blank

Blank

Blank

Blank

Blank

Blank

Blank

Blank

Blank

Blank

Blank

Blank

Blank

Blank

Blank

Blank

Blank

Blank

Blank

Blank

Blank

Blank

Blank

Blank

Blank

Blank

Blank

Blank

Blank

Blank

Blank

Blank

Blank 


$\begin{array}{llllll}\text { Analyte } & \text { Location } & \text { Date } & \text { Result } & \text { MDA or PQL Units } & \text { QC Type }\end{array}$

\section{Radiological Activity}

\begin{tabular}{|c|c|c|c|c|c|c|}
\hline \multirow{45}{*}{$\begin{array}{l}\text { Gross beta } \\
\text { cont. }\end{array}$} & \multirow[t]{45}{*}{ Travel Blank } & $\begin{array}{l}3 / 15 / 01 \\
3 / 15 / 01\end{array}$ & $\begin{array}{l}0.44 \\
N D\end{array}$ & $\begin{array}{l}0.19 \\
0 ?\end{array}$ & $\begin{array}{l}\mathrm{Bq} / \mathrm{S} \\
\mathrm{Bg} / \mathrm{S}\end{array}$ & $\begin{array}{l}\text { Blank } \\
\text { Blank }\end{array}$ \\
\hline & & $\begin{array}{l}3 / 15 / 01 \\
3 / 15 / 01\end{array}$ & $\begin{array}{l}\text { ND } \\
\text { ND }\end{array}$ & $\begin{array}{l}0.2 \\
0.2\end{array}$ & $\begin{array}{l}\mathrm{Bq} / \mathrm{S} \\
\mathrm{Bg} / \mathrm{S}\end{array}$ & $\begin{array}{l}\text { Blank } \\
\text { Blank }\end{array}$ \\
\hline & & $\begin{array}{l}3 / 15 / 01 \\
3 / 21 / 01\end{array}$ & ND & $\begin{array}{l}0.2 \\
02\end{array}$ & $\begin{array}{l}\mathrm{Bq} / \mathrm{S} \\
\mathrm{Ba} / \mathrm{S}\end{array}$ & $\begin{array}{l}\text { Blank } \\
\text { Blank }\end{array}$ \\
\hline & & $\begin{array}{l}3 / 21 / 01 \\
3 / 21 / 01\end{array}$ & ND & $\begin{array}{l}0.2 \\
02\end{array}$ & $\mathrm{~Bq} / \mathrm{S}$ & $\begin{array}{l}\text { Blank } \\
\text { Blank }\end{array}$ \\
\hline & & $\begin{array}{l}3 / 21 / 01 \\
3 / 21 / 01\end{array}$ & 056 & $\begin{array}{l}0.2 \\
0.19\end{array}$ & $\begin{array}{l}\mathrm{Bq} / \mathrm{S} \\
\mathrm{Bg} / \mathrm{S}\end{array}$ & $\begin{array}{l}\text { Blan } \\
\text { Blan }\end{array}$ \\
\hline & & $3 / 28 / 01$ & 0.56 & 0.19 & $\mathrm{Ba} / \mathrm{S}$ & Blan \\
\hline & & $3 / 28 / 01$ & ND & 0.2 & $\mathrm{Bg} / \mathrm{S}$ & Blar \\
\hline & & $3 / 28 / 01$ & ND & 0.19 & $\mathrm{~Bq} / \mathrm{S}$ & Blar \\
\hline & & $4 / 4 / 01$ & 0.41 & 0.19 & $\mathrm{~Bq} / \mathrm{S}$ & Blar \\
\hline & & $4 / 4 / 01$ & ND & 0.2 & $\mathrm{~Bq} / \mathrm{S}$ & Blar \\
\hline & & $4 / 4 / 01$ & ND & 0.19 & $\mathrm{~Bq} / \mathrm{S}$ & Blar \\
\hline & & $4 / 4 / 01$ & ND & 0.2 & $\mathrm{~Bq} / \mathrm{S}$ & Bla \\
\hline & & $4 / 4 / 01$ & 0.41 & 0.19 & $\mathrm{~Bq} / \mathrm{S}$ & Bla \\
\hline & & $4 / 11 / 01$ & ND & 0.2 & $\mathrm{Bg} / \mathrm{S}$ & Blar \\
\hline & & $4 / 11 / 01$ & 0.52 & 0.19 & $\mathrm{~Bq} / \mathrm{S}$ & Blaı \\
\hline & & $4 / 11 / 01$ & ND & 0.2 & $\mathrm{Bg} / \mathrm{S}$ & Blar \\
\hline & & 4/18/01 & ND & 0.2 & $\mathrm{~Bq} / \mathrm{S}$ & Bla \\
\hline & & $4 / 18 / 01$ & 0.52 & 0.19 & $\mathrm{~Bq} / \mathrm{S}$ & Bla \\
\hline & & $4 / 18 / 01$ & ND & 0.2 & $\mathrm{~Bq} / \mathrm{S}$ & Bla \\
\hline & & $4 / 26 / 01$ & 0.52 & 0.19 & $\mathrm{~Bq} / \mathrm{S}$ & Blal \\
\hline & & $4 / 26 / 01$ & ND & 0.2 & $\mathrm{~Bq} / \mathrm{S}$ & Blar \\
\hline & & $4 / 26 / 01$ & ND & 0.2 & $\mathrm{~Bq} / \mathrm{S}$ & Blar \\
\hline & & $5 / 2 / 01$ & ND & 0.19 & $\mathrm{Bg} / \mathrm{S}$ & Blar \\
\hline & & $5 / 2 / 01$ & ND & 0.2 & $\mathrm{~Bq} / \mathrm{S}$ & Bla \\
\hline & & $5 / 2 / 01$ & 0.41 & 0.19 & $\mathrm{~Bq} / \mathrm{S}$ & Blaı \\
\hline & & $5 / 2 / 01$ & ND & 0.2 & $\mathrm{~Bq} / \mathrm{S}$ & Blar \\
\hline & & $5 / 2 / 01$ & 0.41 & 0.19 & $\mathrm{~Bq} / \mathrm{S}$ & Blar \\
\hline & & $5 / 9 / 01$ & ND & 0.2 & $\mathrm{~Bq} / \mathrm{S}$ & Blar \\
\hline & & $5 / 9 / 01$ & 0.41 & 0.19 & $\mathrm{Bg} / \mathrm{S}$ & Blar \\
\hline & & $5 / 9 / 01$ & ND & 0.19 & $\mathrm{~Bq} / \mathrm{S}$ & Blar \\
\hline & & $5 / 16 / 01$ & ND & 0.2 & $\mathrm{~Bq} / \mathrm{S}$ & Blar \\
\hline & & $5 / 16 / 01$ & 0.44 & 0.19 & $\mathrm{~Bq} / \mathrm{S}$ & Blar \\
\hline & & $5 / 16 / 01$ & ND & 0.19 & $\mathrm{~Bq} / \mathrm{S}$ & Blar \\
\hline & & $5 / 23 / 01$ & ND & 0.2 & $\mathrm{Bg} / \mathrm{S}$ & Blar \\
\hline & & $5 / 23 / 01$ & 0.52 & 0.19 & $\mathrm{~Bq} / \mathrm{S}$ & Blar \\
\hline & & $5 / 23 / 01$ & ND & 0.2 & $\mathrm{Bg} / \mathrm{S}$ & Bla \\
\hline & & $5 / 31 / 01$ & ND & 0.2 & $\mathrm{~Bq} / \mathrm{S}$ & Bla \\
\hline & & $5 / 31 / 01$ & 0.7 & 0.19 & $\mathrm{~Bq} / \mathrm{S}$ & Blar \\
\hline & & $5 / 31 / 01$ & ND & 0.2 & $\mathrm{Bg} / \mathrm{S}$ & Blar \\
\hline & & 6/6/01 & 0.44 & 0.19 & $\mathrm{~Bq} / \mathrm{S}$ & Blal \\
\hline & & $6 / 6 / 01$ & ND & 0.2 & $\mathrm{Bg} / \mathrm{S}$ & Blar \\
\hline & & 6/6/01 & ND & 0.19 & $\mathrm{~Bq} / \mathrm{S}$ & Blar \\
\hline & & $6 / 6 / 01$ & 0.52 & 0.19 & $\mathrm{Bg} / \mathrm{S}$ & Blar \\
\hline & & $6 / 6 / 01$ & ND & 0.2 & $\mathrm{~Bq} / \mathrm{S}$ & Blar \\
\hline & & $6 / 13 / 01$ & ND & 0.19 & $\mathrm{~Bq} / \mathrm{S}$ & \\
\hline
\end{tabular}


Analyte

Location

Date

Result MDA or PQL

Units QC Type

\section{Radiological Activity}

\begin{tabular}{|c|c|c|c|c|c|c|}
\hline \multirow{45}{*}{$\begin{array}{l}\text { Gross beta } \\
\text { cont. }\end{array}$} & \multirow[t]{45}{*}{ Travel Blank } & $\begin{array}{l}6 / 13 / 01 \\
6 / 13 / 01\end{array}$ & $\begin{array}{l}\text { ND } \\
036\end{array}$ & $\begin{array}{l}0.2 \\
0.19\end{array}$ & $\begin{array}{l}\mathrm{Bq} / \mathrm{S} \\
\mathrm{Bg} / \mathrm{S}\end{array}$ & $\begin{array}{l}\text { Blank } \\
\text { Blank }\end{array}$ \\
\hline & & $6 / 20 / 01$ & ND & 0.2 & $\mathrm{Bg} / \mathrm{S}$ & Blank \\
\hline & & $6 / 20 / 01$ & 0.52 & 0.19 & $\mathrm{~Bq} / \mathrm{S}$ & Blank \\
\hline & & $6 / 20 / 01$ & ND & 0.2 & $\mathrm{~Bq} / \mathrm{S}$ & Blank \\
\hline & & $6 / 27 / 01$ & ND & 0.19 & $\mathrm{~Bq} / \mathrm{S}$ & Blank \\
\hline & & $6 / 27 / 01$ & 0.48 & 0.19 & $\mathrm{~Bq} / \mathrm{S}$ & Blank \\
\hline & & $6 / 27 / 01$ & ND & 0.2 & $\mathrm{~Bq} / \mathrm{S}$ & Blank \\
\hline & & $7 / 5 / 01$ & 0.52 & 0.19 & $\mathrm{~Bq} / \mathrm{S}$ & Blank \\
\hline & & $7 / 5 / 01$ & ND & 0.2 & $\mathrm{~Bq} / \mathrm{S}$ & Blank \\
\hline & & $7 / 5 / 01$ & ND & 0.2 & $\mathrm{~Bq} / \mathrm{S}$ & Blank \\
\hline & & $7 / 5 / 01$ & ND & 0.19 & $\mathrm{~Bq} / \mathrm{S}$ & Blank \\
\hline & & $7 / 5 / 01$ & 0.56 & 0.19 & $\mathrm{~Bq} / \mathrm{S}$ & Blank \\
\hline & & $7 / 11 / 01$ & 0.7 & 0.19 & $\mathrm{~Bq} / \mathrm{S}$ & Blank \\
\hline & & $7 / 11 / 01$ & ND & 0.2 & $\mathrm{~Bq} / \mathrm{S}$ & Blank \\
\hline & & $7 / 11 / 01$ & ND & 0.19 & $\mathrm{~Bq} / \mathrm{S}$ & Blank \\
\hline & & $7 / 18 / 01$ & ND & 0.2 & $\mathrm{~Bq} / \mathrm{S}$ & Blank \\
\hline & & $7 / 18 / 01$ & 0.44 & 0.19 & $\mathrm{~Bq} / \mathrm{S}$ & Blank \\
\hline & & $7 / 18 / 01$ & ND & 0.2 & $\mathrm{~Bq} / \mathrm{S}$ & Blank \\
\hline & & $7 / 25 / 01$ & ND & 0.2 & $\mathrm{~Bq} / \mathrm{S}$ & Blank \\
\hline & & $7 / 25 / 01$ & ND & 0.19 & $\mathrm{~Bq} / \mathrm{S}$ & Blank \\
\hline & & $7 / 25 / 01$ & 0.56 & 0.19 & $\mathrm{~Bq} / \mathrm{S}$ & Blank \\
\hline & & $8 / 1 / 01$ & ND & 0.19 & $\mathrm{~Bq} / \mathrm{S}$ & Blank \\
\hline & & $8 / 1 / 01$ & ND & 0.2 & $\mathrm{~Bq} / \mathrm{S}$ & Blank \\
\hline & & $8 / 1 / 01$ & 0.63 & 0.19 & $\mathrm{~Bq} / \mathrm{S}$ & Blank \\
\hline & & $8 / 1 / 01$ & 0.59 & 0.19 & $\mathrm{~Bq} / \mathrm{S}$ & Blank \\
\hline & & $8 / 1 / 01$ & ND & 0.2 & $\mathrm{~Bq} / \mathrm{S}$ & Blank \\
\hline & & $8 / 8 / 01$ & 0.56 & 0.19 & $\mathrm{~Bq} / \mathrm{S}$ & Blank \\
\hline & & $8 / 8 / 01$ & ND & 0.2 & $\mathrm{~Bq} / \mathrm{S}$ & Blank \\
\hline & & $8 / 8 / 01$ & ND & 0.2 & $\mathrm{~Bq} / \mathrm{S}$ & Blank \\
\hline & & $8 / 15 / 01$ & ND & 0.2 & $\mathrm{~Bq} / \mathrm{S}$ & Blank \\
\hline & & $8 / 15 / 01$ & 0.48 & 0.19 & $\mathrm{~Bq} / \mathrm{S}$ & Blank \\
\hline & & $8 / 15 / 01$ & ND & 0.19 & $\mathrm{~Bq} / \mathrm{S}$ & Blank \\
\hline & & $8 / 22 / 01$ & ND & 0.19 & $\mathrm{~Bq} / \mathrm{S}$ & Blank \\
\hline & & $8 / 22 / 01$ & ND & 0.19 & $\mathrm{~Bq} / \mathrm{S}$ & Blank \\
\hline & & $8 / 22 / 01$ & 0.48 & 0.19 & $\mathrm{~Bq} / \mathrm{S}$ & Blank \\
\hline & & $8 / 29 / 01$ & ND & 0.19 & $\mathrm{~Bq} / \mathrm{S}$ & Blank \\
\hline & & 8/29/01 & 0.44 & 0.19 & $\mathrm{~Bq} / \mathrm{S}$ & Blank \\
\hline & & $8 / 29 / 01$ & ND & 0.19 & $\mathrm{~Bq} / \mathrm{S}$ & Blank \\
\hline & & $9 / 5 / 01$ & 0.52 & 0.19 & $\mathrm{~Bq} / \mathrm{S}$ & Blank \\
\hline & & $9 / 5 / 01$ & ND & 0.2 & $\mathrm{~Bq} / \mathrm{S}$ & Blank \\
\hline & & $9 / 5 / 01$ & ND & 0.2 & $\mathrm{~Bq} / \mathrm{S}$ & Blank \\
\hline & & $9 / 5 / 01$ & 0.48 & 0.19 & $\mathrm{~Bq} / \mathrm{S}$ & Blank \\
\hline & & $9 / 5 / 01$ & ND & 0.19 & $\mathrm{~Bq} / \mathrm{S}$ & Blank \\
\hline & & $9 / 12 / 01$ & ND & 0.19 & $\mathrm{~Bq} / \mathrm{S}$ & Blank \\
\hline & & $9 / 12 / 01$ & ND & 0.2 & $\mathrm{Bg} / \mathrm{S}$ & Blank \\
\hline
\end{tabular}




$\begin{array}{llllll}\text { Analyte } & \text { Location } & \text { Date } & \text { Result } & \text { MDA or PQL Units } & \text { QC Type }\end{array}$

\section{Radiological Activity}

\begin{tabular}{|c|c|c|c|c|c|c|}
\hline \multirow{45}{*}{$\begin{array}{l}\text { Gross beta } \\
\text { cont. }\end{array}$} & \multirow[t]{44}{*}{ Travel Blank } & $9 / 12 / 01$ & 0.48 & 0.19 & $\mathrm{~Bq} / \mathrm{S}$ & $\begin{array}{l}\text { Blank } \\
\text { Blank }\end{array}$ \\
\hline & & $\begin{array}{l}9 / 19 / 01 \\
9 / 19 / 01\end{array}$ & $\begin{array}{l}\text { ND } \\
N D\end{array}$ & $\begin{array}{l}0.19 \\
0.2\end{array}$ & $\begin{array}{l}\mathrm{Bq} / \mathrm{S} \\
\mathrm{Bq} / \mathrm{S}\end{array}$ & $\begin{array}{l}\text { Blank } \\
\text { Blank }\end{array}$ \\
\hline & & $9 / 19 / 01$ & 0.56 & 0.19 & $\mathrm{~Bq} / \mathrm{S}$ & Blan \\
\hline & & 9/26/01 & ND & 0.19 & $\mathrm{~Bq} / \mathrm{S}$ & Blal \\
\hline & & $9 / 26 / 01$ & ND & 0.2 & $\mathrm{~Bq} / \mathrm{S}$ & Blal \\
\hline & & 9/26/01 & 0.52 & 0.19 & $\mathrm{~Bq} / \mathrm{S}$ & Bla \\
\hline & & $10 / 3 / 01$ & 0.48 & 0.19 & $\mathrm{~Bq} / \mathrm{S}$ & Blar \\
\hline & & 10/3/01 & ND & 0.19 & $\mathrm{~Bq} / \mathrm{S}$ & Blar \\
\hline & & $10 / 3 / 01$ & ND & 0.19 & $\mathrm{~Bq} / \mathrm{S}$ & Blar \\
\hline & & 10/8/01 & 0.36 & 0.19 & $\mathrm{~Bq} / \mathrm{S}$ & Bla \\
\hline & & 10/8/01 & ND & 0.19 & $\mathrm{~Bq} / \mathrm{S}$ & Bla \\
\hline & & 10/9/01 & ND & 0.19 & $\mathrm{~Bq} / \mathrm{S}$ & Bla \\
\hline & & 10/9/01 & 0.48 & 0.19 & $\mathrm{~Bq} / \mathrm{S}$ & Bla \\
\hline & & $10 / 9 / 01$ & ND & 0.19 & $\mathrm{~Bq} / \mathrm{S}$ & Blar \\
\hline & & $10 / 16 / 01$ & ND & 0.2 & $\mathrm{~Bq} / \mathrm{S}$ & Blaı \\
\hline & & $10 / 16 / 01$ & 0.48 & 0.19 & $\mathrm{~Bq} / \mathrm{S}$ & \\
\hline & & 10/16/01 & ND & 0.2 & $\mathrm{~Bq} / \mathrm{S}$ & Bla \\
\hline & & $10 / 24 / 01$ & 0.44 & 0.15 & $\mathrm{~Bq} / \mathrm{S}$ & Bla \\
\hline & & $10 / 24 / 01$ & ND & 0.19 & $\mathrm{~Bq} / \mathrm{S}$ & \\
\hline & & $10 / 24 / 01$ & ND & 0.19 & $\mathrm{~Bq} / \mathrm{S}$ & Bla \\
\hline & & 10/30/01 & ND & 0.19 & $\mathrm{~Bq} / \mathrm{S}$ & Blar \\
\hline & & $10 / 30 / 01$ & ND & 0.19 & $\mathrm{~Bq} / \mathrm{S}$ & \\
\hline & & $10 / 30 / 01$ & 0.44 & 0.19 & $\mathrm{~Bq} / \mathrm{S}$ & \\
\hline & & 11/8/01 & 0.52 & 0.19 & $\mathrm{~Bq} / \mathrm{S}$ & \\
\hline & & 11/8/01 & ND & 0.19 & $\mathrm{~Bq} / \mathrm{S}$ & \\
\hline & & 11/8/01 & ND & 0.19 & $\mathrm{~Bq} / \mathrm{S}$ & \\
\hline & & $11 / 9 / 01$ & ND & 0.2 & $\mathrm{~Bq} / \mathrm{S}$ & Blar \\
\hline & & $11 / 9 / 01$ & 0.44 & 0.19 & $\mathrm{~Bq} / \mathrm{S}$ & \\
\hline & & $11 / 13 / 01$ & ND & 0.19 & $\mathrm{~Bq} / \mathrm{S}$ & \\
\hline & & $11 / 13 / 01$ & ND & 0.2 & $\mathrm{~Bq} / \mathrm{S}$ & \\
\hline & & $11 / 13 / 01$ & 0.52 & 0.19 & $\mathrm{~Bq} / \mathrm{S}$ & Bla \\
\hline & & $11 / 20 / 01$ & 0.52 & 0.19 & $\mathrm{~Bq} / \mathrm{S}$ & Bla \\
\hline & & $11 / 20 / 01$ & ND & 0.2 & $\mathrm{~Bq} / \mathrm{S}$ & \\
\hline & & $11 / 20 / 01$ & ND & 0.2 & $\mathrm{~Bq} / \mathrm{S}$ & \\
\hline & & $11 / 26 / 01$ & ND & 0.2 & $\mathrm{~Bq} / \mathrm{S}$ & \\
\hline & & $11 / 26 / 01$ & 0.41 & 0.19 & $\mathrm{~Bq} / \mathrm{S}$ & \\
\hline & & $11 / 26 / 01$ & ND & 0.2 & $\mathrm{~Bq} / \mathrm{S}$ & Bla \\
\hline & & $12 / 4 / 01$ & ND & 0.19 & $\mathrm{~Bq} / \mathrm{S}$ & Bla \\
\hline & & 12/4/01 & 0.48 & 0.19 & $\mathrm{~Bq} / \mathrm{S}$ & \\
\hline & & $12 / 4 / 01$ & ND & 0.2 & $\mathrm{~Bq} / \mathrm{S}$ & \\
\hline & & $12 / 4 / 01$ & ND & 0.2 & $\mathrm{~Bq} / \mathrm{S}$ & \\
\hline & & $12 / 4 / 01$ & 0.63 & 0.19 & $\mathrm{~Bq} / \mathrm{S}$ & \\
\hline & & $12 / 12 / 01$ & 0.44 & 0.19 & $\mathrm{~Bq} / \mathrm{S}$ & Bla \\
\hline & & $12 / 12 / 01$ & ND & 0.2 & $\mathrm{~Bq} / \mathrm{S}$ & \\
\hline & & $12 / 19 / 01$ & ND & 0.2 & $\mathrm{~Bq} / \mathrm{S}$ & \\
\hline
\end{tabular}


Analyte

\section{Radiological Activity}

Gross beta Travel Blank cont.

\section{lodine-125}

$\begin{array}{cc}1-216 \mathrm{H} & 1 / 3 / 01 \\ 2 / 7 / 01 \\ 3 / 7 / 01 \\ 4 / 4 / 01 \\ 5 / 2 / 01 \\ 6 / 6 / 01 \\ 7 / 5 / 01 \\ 8 / 1 / 01 \\ 9 / 5 / 01 \\ 10 / 8 / 01 \\ 11 / 9 / 01 \\ 12 / 4 / 01 \\ 1 / 3 / 02\end{array}$

1-267H

$1 / 3 / 01$

$2 / 7 / 01$

$3 / 7 / 01$

$4 / 4 / 01$

$5 / 2 / 01$

$6 / 6 / 01$

$7 / 5 / 01$

$8 / 1 / 01$

$9 / 5 / 01$

$10 / 8 / 01$

$11 / 9 / 01$

$12 / 4 / 01$

$1 / 3 / 02$

$1-373 \mathrm{H}$

$1 / 3 / 01$
$2 / 7 / 01$
$3 / 7 / 01$
$4 / 4 / 01$
$5 / 2 / 01$
$8 / 1 / 01$
$9 / 5 / 01$
$10 / 8 / 01$
$11 / 9 / 01$
$12 / 4 / 01$
$1 / 3 / 02$

Result MDA or PQL Units QC Type

$\begin{array}{clll}0.63 & 0.19 & \mathrm{~Bq} / \mathrm{S} & \text { Blank } \\ \mathrm{ND} & 0.2 & \mathrm{~Bq} / \mathrm{S} & \text { Blank } \\ 0.41 & 0.2 & \mathrm{~Bq} / \mathrm{S} & \text { Blank } \\ \mathrm{ND} & 0.2 & \mathrm{~Bq} / \mathrm{S} & \text { Blank } \\ \mathrm{ND} & 0.2 & \mathrm{~Bq} / \mathrm{S} & \text { Blank } \\ \mathrm{ND} & 0.2 & \mathrm{~Bq} / \mathrm{S} & \text { Blank } \\ 0.37 & 0.2 & \mathrm{~Bq} / \mathrm{S} & \text { Blank }\end{array}$

\begin{tabular}{|c|c|c|c|}
\hline ND & 0.0002 & $\mathrm{~Bq} / \mathrm{m}^{3}$ & Sample \\
\hline 0.00027 & 0.0002 & $\mathrm{~Bq} / \mathrm{m}^{3}$ & Sample \\
\hline ND & 0.0002 & $\mathrm{~Bq} / \mathrm{m}^{3}$ & Sample \\
\hline ND & 0.0002 & $\mathrm{~Bq} / \mathrm{m}^{3}$ & Sample \\
\hline ND & 0.0002 & $\mathrm{~Bq} / \mathrm{m}^{3}$ & Sample \\
\hline ND & 0.0002 & $\mathrm{~Bq} / \mathrm{m}^{3}$ & Sample \\
\hline 0.00038 & 0.0003 & $\mathrm{~Bq} / \mathrm{m}^{3}$ & Sample \\
\hline ND & 0.0002 & $\mathrm{~Bq} / \mathrm{m}^{3}$ & Sample \\
\hline ND & 0.00018 & $\mathrm{~Bq} / \mathrm{m}^{3}$ & Sample \\
\hline ND & 0.00019 & $\mathrm{~Bq} / \mathrm{m}^{3}$ & Sample \\
\hline ND & 0.0003 & $\mathrm{~Bq} / \mathrm{m}^{3}$ & Sample \\
\hline ND & 0.0003 & $\mathrm{~Bq} / \mathrm{m}^{3}$ & Sample \\
\hline ND & 0.0003 & $\mathrm{~Bq} / \mathrm{m}^{3}$ & Sample \\
\hline 0.139 & 0.0002 & $\mathrm{~Bq} / \mathrm{m}^{3}$ & Sample \\
\hline 1.82 & 0.0002 & $\mathrm{~Bq} / \mathrm{m}^{3}$ & Sample \\
\hline 2.28 & 0.0002 & $\mathrm{~Bq} / \mathrm{m}^{3}$ & Sample \\
\hline 0.149 & 0.0002 & $\mathrm{~Bq} / \mathrm{m}^{3}$ & Sample \\
\hline 1.09 & 0.0002 & $\mathrm{~Bq} / \mathrm{m}^{3}$ & Sample \\
\hline 0.702 & 0.0002 & $\mathrm{~Bq} / \mathrm{m}^{3}$ & Sample \\
\hline 0.834 & 0.0003 & $\mathrm{~Bq} / \mathrm{m}^{3}$ & Sample \\
\hline 0.0668 & 0.0002 & $\mathrm{~Bq} / \mathrm{m}^{3}$ & Sample \\
\hline 0.0173 & 0.00018 & $\mathrm{~Bq} / \mathrm{m}^{3}$ & Sample \\
\hline 1.23 & 0.00019 & $\mathrm{~Bq} / \mathrm{m}^{3}$ & Sample \\
\hline 0.534 & 0.0003 & $\mathrm{~Bq} / \mathrm{m}^{3}$ & Sample \\
\hline 0.00959 & 0.0003 & $\mathrm{~Bq} / \mathrm{m}^{3}$ & Sample \\
\hline 0.0036 & 0.0003 & $\mathrm{Bg} / \mathrm{m}^{3}$ & Sample \\
\hline
\end{tabular}

$\begin{array}{clll}0.00026 & 0.0002 & \mathrm{~Bq} / \mathrm{m}^{3} & \text { Sample } \\ 0.00071 & 0.0002 & \mathrm{~Bq} / \mathrm{m}^{3} & \text { Sample } \\ 0.00074 & 0.0002 & \mathrm{~Bq} / \mathrm{m}^{3} & \text { Sample } \\ 0.0004 & 0.0002 & \mathrm{~Bq} / \mathrm{m}^{3} & \text { Sample } \\ 0.0002 & 0.00018 & \mathrm{~Bq} / \mathrm{m}^{3} & \text { Sample } \\ \text { ND } & 0.00013 & \mathrm{~Bq} / \mathrm{m}^{3} & \text { Sample } \\ \text { ND } & 0.00019 & \mathrm{~Bq} / \mathrm{m}^{3} & \text { Sample } \\ 0.00031 & 0.00019 & \mathrm{~Bq} / \mathrm{m}^{3} & \text { Sample } \\ 0.00032 & 0.0003 & \mathrm{~Bq} / \mathrm{m}^{3} & \text { Sample } \\ \text { ND } & 0.0003 & \mathrm{~Bq} / \mathrm{m}^{3} & \text { Sample } \\ \text { ND } & 0.0003 & \mathrm{~Bq} / \mathrm{m}^{3} & \text { Sample }\end{array}$




\section{Analyte \\ Location \\ Radiological Activity}

Date

Result MDA or PQL

QC Type

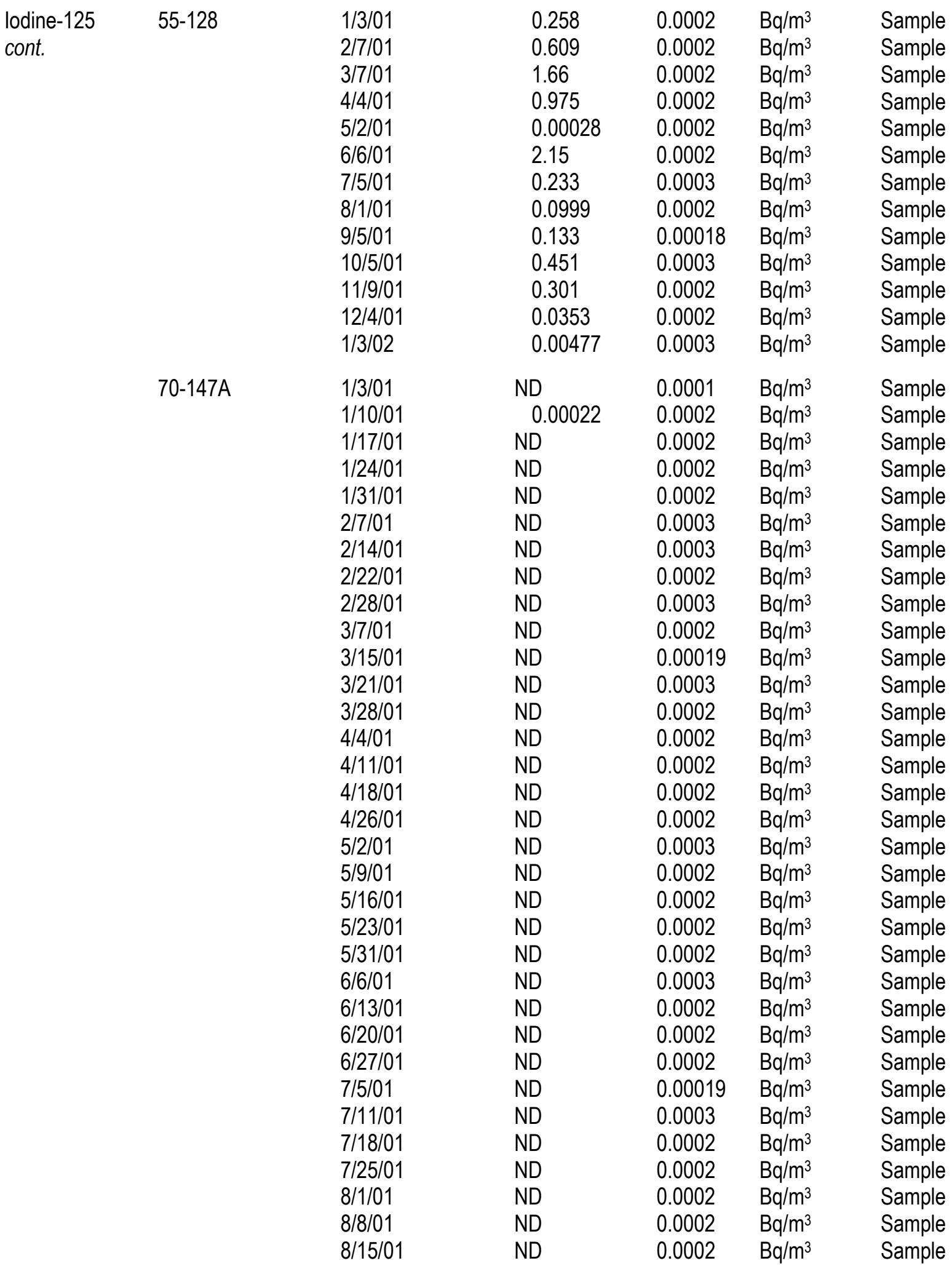


Analyte

\section{Radiological Activity}

lodine-125 70-147A

cont.
Date

$8 / 22 / 01$

$8 / 29 / 01$

$9 / 5 / 01$

$9 / 12 / 01$

$9 / 19 / 01$

9/26/01

$10 / 3 / 01$

10/9/01

$10 / 16 / 01$

$10 / 23 / 01$

$11 / 8 / 01$

$11 / 13 / 01$

$11 / 20 / 01$

$11 / 26 / 01$

$12 / 4 / 01$

$12 / 12 / 01$

$12 / 19 / 01$

$1 / 2 / 02$

$85 \mathrm{Hood}$
$1 / 3 / 01$

$1 / 10 / 01$

$1 / 17 / 01$

$1 / 24 / 01$

$1 / 31 / 01$

2/7/01

$2 / 14 / 01$

2/22/01

2/28/01

$3 / 7 / 01$

$3 / 15 / 01$

$3 / 21 / 01$

$3 / 28 / 01$

$4 / 4 / 01$

$4 / 11 / 01$

$4 / 18 / 01$

$4 / 26 / 01$

$5 / 2 / 01$

$5 / 9 / 01$

$5 / 16 / 01$

$5 / 23 / 01$

$5 / 31 / 01$

$6 / 6 / 01$

$6 / 13 / 01$

6/20/01

$6 / 27 / 01$

$7 / 5 / 01$
Result MDA or PQL Units QC Type

$\begin{array}{clll}\text { ND } & 0.0002 & \mathrm{~Bq} / \mathrm{m}^{3} & \text { Sample } \\ \text { ND } & 0.0002 & \mathrm{~Bq} / \mathrm{m}^{3} & \text { Sample } \\ \text { ND } & 0.0002 & \mathrm{~Bq} / \mathrm{m}^{3} & \text { Sample } \\ \text { ND } & 0.0002 & \mathrm{~Bq} / \mathrm{m}^{3} & \text { Sample } \\ \text { 0.00024 } & 0.0002 & \mathrm{~Bq} / \mathrm{m}^{3} & \text { Sample } \\ \text { ND } & 0.0002 & \mathrm{~Bq} / \mathrm{m}^{3} & \text { Sample } \\ \text { ND } & 0.0002 & \mathrm{~Bq} / \mathrm{m}^{3} & \text { Sample } \\ \text { ND } & 0.0003 & \mathrm{~Bq} / \mathrm{m}^{3} & \text { Sample } \\ \text { ND } & 0.0002 & \mathrm{~Bq} / \mathrm{m}^{3} & \text { Sample } \\ \text { ND } & 0.0002 & \mathrm{~Bq} / \mathrm{m}^{3} & \text { Sample } \\ \text { ND } & 0.0002 & \mathrm{~Bq} / \mathrm{m}^{3} & \text { Sample } \\ \text { ND } & 0.0003 & \mathrm{~Bq} / \mathrm{m}^{3} & \text { Sample } \\ \text { ND } & 0.0002 & \mathrm{~Bq} / \mathrm{m}^{3} & \text { Sample } \\ \text { ND } & 0.0003 & \mathrm{~Bq} / \mathrm{m}^{3} & \text { Sample } \\ 0.00022 & 0.00019 & \mathrm{~Bq} / \mathrm{m}^{3} & \text { Sample } \\ \text { ND } & 0.00019 & \mathrm{~Bq} / \mathrm{m}^{3} & \text { Sample } \\ \text { ND } & 0.0002 & \mathrm{~Bq} / \mathrm{m}^{3} & \text { Sample } \\ 0.00011 & 0.00011 & \mathrm{~Bq} / \mathrm{m}^{3} & \text { Sample }\end{array}$

$\begin{array}{llll}0.00148 & 0.00019 & \mathrm{~Bq} / \mathrm{m}^{3} & \text { Sample }\end{array}$

ND $\quad 0.0004 \quad \mathrm{~Bq} / \mathrm{m}^{3} \quad$ Sample

ND $\quad 0.0004 \quad \mathrm{~Bq} / \mathrm{m}^{3} \quad$ Sample

ND $\quad 0.0004 \quad \mathrm{~Bq} / \mathrm{m}^{3} \quad$ Sample

ND $\quad 0.0004 \quad \mathrm{~Bq} / \mathrm{m}^{3} \quad$ Sample

ND $\quad 0.0005 \quad \mathrm{~Bq} / \mathrm{m}^{3} \quad$ Sample

ND $\quad 0.0005 \quad \mathrm{~Bq} / \mathrm{m}^{3} \quad$ Sample

$\begin{array}{llll}0.0006 & 0.0003 \quad \mathrm{~Bq} / \mathrm{m}^{3} & \text { Sample }\end{array}$

$0.00065 \quad 0.0004 \quad \mathrm{~Bq} / \mathrm{m}^{3} \quad$ Sample

ND $\quad 0.0004 \quad \mathrm{~Bq} / \mathrm{m}^{3} \quad$ Sample

ND $\quad 0.0003 \quad \mathrm{~Bq} / \mathrm{m}^{3} \quad$ Sample

ND $\quad 0.0004 \quad \mathrm{~Bq} / \mathrm{m}^{3} \quad$ Sample

$0.00055 \quad 0.0004 \quad \mathrm{~Bq} / \mathrm{m}^{3} \quad$ Sample

ND $\quad 0.0004 \quad \mathrm{~Bq} / \mathrm{m}^{3} \quad$ Sample

ND $\quad 0.0004 \quad \mathrm{~Bq} / \mathrm{m}^{3} \quad$ Sample

ND $\quad 0.0004 \quad \mathrm{~Bq} / \mathrm{m}^{3} \quad$ Sample

ND $\quad 0.0003 \quad \mathrm{~Bq} / \mathrm{m}^{3} \quad$ Sample

ND $\quad 0.0002 \quad \mathrm{~Bq} / \mathrm{m}^{3} \quad$ Sample

$\begin{array}{llll}0.0236 & 0.0004 & \mathrm{~Bq} / \mathrm{m}^{3} & \text { Sample }\end{array}$

$0.00169 \quad 0.0004 \quad \mathrm{~Bq} / \mathrm{m}^{3} \quad$ Sample

$0.0013 \quad 0.0004 \quad \mathrm{~Bq} / \mathrm{m}^{3} \quad$ Sample

$\begin{array}{llll}0.01 & 0.0004 \quad \mathrm{~Bq} / \mathrm{m}^{3} & \text { Sample }\end{array}$

$0.00637 \quad 0.0005 \quad \mathrm{~Bq} / \mathrm{m}^{3} \quad$ Sample

$0.00334 \quad 0.0004 \quad \mathrm{~Bq} / \mathrm{m}^{3} \quad$ Sample

$\begin{array}{llll}0.0011 & 0.0004 \quad \mathrm{~Bq} / \mathrm{m}^{3} & \text { Sample }\end{array}$

$0.00041 \quad 0.0004 \quad \mathrm{~Bq} / \mathrm{m}^{3} \quad$ Sample

$0.000069 \quad 0.00003 \quad \mathrm{~Bq} / \mathrm{m}^{3} \quad$ Sample 


\section{Analyte \\ Location \\ Radiological Activity}

Date

Result MDA or PQL Units

QC Type

\begin{tabular}{|c|c|c|c|c|c|}
\hline $85 \mathrm{Hood}$ & $\begin{array}{l}7 / 11 / 01 \\
7 / 18 / 01 \\
7 / 25 / 01 \\
8 / 1 / 01 \\
8 / 8 / 01 \\
8 / 15 / 01 \\
8 / 22 / 01 \\
8 / 29 / 01 \\
9 / 5 / 01 \\
9 / 12 / 01 \\
9 / 19 / 01 \\
9 / 26 / 01 \\
10 / 3 / 01 \\
10 / 9 / 01 \\
10 / 16 / 01 \\
10 / 23 / 01 \\
10 / 30 / 01 \\
11 / 8 / 01 \\
11 / 13 / 01 \\
11 / 20 / 01 \\
11 / 26 / 01 \\
12 / 4 / 01 \\
12 / 12 / 01 \\
12 / 18 / 01 \\
1 / 2 / 02\end{array}$ & $\begin{array}{l}0.00017 \\
0.0012 \\
0.001 \\
0.00189 \\
0.003 \\
0.00068 \\
0.00049 \\
\text { ND } \\
0.00038 \\
\text { ND } \\
0.00035 \\
\text { ND } \\
\text { ND } \\
0.00977 \\
0.00806 \\
0.00097 \\
0.00043 \\
0.001 \\
\text { ND } \\
\text { ND } \\
\text { ND } \\
\text { ND } \\
\text { ND } \\
\text { ND } \\
0.00018\end{array}$ & $\begin{array}{l}0.00005 \\
0.0004 \\
0.0003 \\
0.0004 \\
0.002 \\
0.0004 \\
0.0004 \\
0.0004 \\
0.0003 \\
0.0004 \\
0.0003 \\
0.0004 \\
0.0004 \\
0.0004 \\
0.0003 \\
0.0004 \\
0.0003 \\
0.0003 \\
0.0006 \\
0.0004 \\
0.00008 \\
0.0003 \\
0.0003 \\
0.0004 \\
0.00018\end{array}$ & $\begin{array}{l}\mathrm{Bq} / \mathrm{m}^{3} \\
\mathrm{~Bq} / \mathrm{m}^{3} \\
\mathrm{~Bq} / \mathrm{m}^{3} \\
\mathrm{~Bq} / \mathrm{m}^{3} \\
\mathrm{~Bq} / \mathrm{m}^{3} \\
\mathrm{~Bq} / \mathrm{m}^{3} \\
\mathrm{~Bq} / \mathrm{m}^{3} \\
\mathrm{~Bq} / \mathrm{m}^{3} \\
\mathrm{~Bq} / \mathrm{m}^{3} \\
\mathrm{~Bq} / \mathrm{m}^{3} \\
\mathrm{~Bq} / \mathrm{m}^{3} \\
\mathrm{~Bq} / \mathrm{m}^{3} \\
\mathrm{~Bq} / \mathrm{m}^{3} \\
\mathrm{~Bq} / \mathrm{m}^{3} \\
\mathrm{~Bq} / \mathrm{m}^{3} \\
\mathrm{~Bq} / \mathrm{m}^{3} \\
\mathrm{~Bq} / \mathrm{m}^{3} \\
\mathrm{~Bq} / \mathrm{m}^{3} \\
\mathrm{~Bq} / \mathrm{m}^{3} \\
\mathrm{~Bq} / \mathrm{m}^{3} \\
\mathrm{~Bq} / \mathrm{m}^{3} \\
\mathrm{~Bq} / \mathrm{m}^{3} \\
\mathrm{~Bq} / \mathrm{m}^{3} \\
\mathrm{~Bq} / \mathrm{m}^{3} \\
\mathrm{~Bq} / \mathrm{m}^{3}\end{array}$ & $\begin{array}{l}\text { Sample } \\
\text { Sample } \\
\text { Sample } \\
\text { Sample } \\
\text { Sample } \\
\text { Sample } \\
\text { Sample } \\
\text { Sample } \\
\text { Sample } \\
\text { Sample } \\
\text { Sample } \\
\text { Sample } \\
\text { Sample } \\
\text { Sample } \\
\text { Sample } \\
\text { Sample } \\
\text { Sample } \\
\text { Sample } \\
\text { Sample } \\
\text { Sample } \\
\text { Sample } \\
\text { Sample } \\
\text { Sample } \\
\text { Sample } \\
\text { Sample }\end{array}$ \\
\hline Travel Blank & $\begin{array}{l}1 / 3 / 01 \\
1 / 3 / 01 \\
1 / 10 / 01 \\
1 / 17 / 01 \\
1 / 24 / 01 \\
1 / 31 / 01 \\
2 / 7 / 01 \\
2 / 7 / 01 \\
2 / 14 / 01 \\
2 / 22 / 01 \\
2 / 28 / 01 \\
3 / 7 / 01 \\
3 / 7 / 01 \\
3 / 15 / 01 \\
3 / 21 / 01 \\
3 / 28 / 01 \\
4 / 4 / 01 \\
4 / 4 / 01 \\
4 / 11 / 01 \\
4 / 18 / 01 \\
4 / 26 / 01\end{array}$ & $\begin{array}{l}\text { ND } \\
\text { ND } \\
0.1 \\
\text { ND } \\
\text { ND } \\
\text { ND } \\
0.36 \\
\text { ND } \\
\text { ND } \\
\text { ND } \\
\text { ND } \\
0.34 \\
\text { ND } \\
\text { ND } \\
\text { ND } \\
\text { ND } \\
\text { ND } \\
\text { ND } \\
\text { ND } \\
\text { ND } \\
\text { ND }\end{array}$ & $\begin{array}{l}0.11 \\
0.11 \\
0.11 \\
0.11 \\
0.11 \\
0.11 \\
0.15 \\
0.15 \\
0.11 \\
0.11 \\
0.11 \\
0.11 \\
0.11 \\
0.11 \\
0.11 \\
0.11 \\
0.11 \\
0.11 \\
0.11 \\
0.11 \\
0.11\end{array}$ & $\begin{array}{l}\mathrm{Bq} / \mathrm{S} \\
\mathrm{Bq} / \mathrm{S} \\
\mathrm{Bq} / \mathrm{S} \\
\mathrm{Bq} / \mathrm{S} \\
\mathrm{Bq} / \mathrm{S} \\
\mathrm{Bq} / \mathrm{S} \\
\mathrm{Bq} / \mathrm{S} \\
\mathrm{Bq} / \mathrm{S} \\
\mathrm{Bq} / \mathrm{S} \\
\mathrm{Bq} / \mathrm{S} \\
\mathrm{Bq} / \mathrm{S} \\
\mathrm{Bq} / \mathrm{S} \\
\mathrm{Bq} / \mathrm{S} \\
\mathrm{Bq} / \mathrm{S} \\
\mathrm{Bq} / \mathrm{S} \\
\mathrm{Bq} / \mathrm{S} \\
\mathrm{Bq} / \mathrm{S} \\
\mathrm{Bq} / \mathrm{S} \\
\mathrm{Bq} / \mathrm{S} \\
\mathrm{Bq} / \mathrm{S} \\
\mathrm{Bq} / \mathrm{S}\end{array}$ & $\begin{array}{l}\text { Blank } \\
\text { Blank } \\
\text { Blank } \\
\text { Blank } \\
\text { Blank } \\
\text { Blank } \\
\text { Blank } \\
\text { Blank } \\
\text { Blank } \\
\text { Blank } \\
\text { Blank } \\
\text { Blank } \\
\text { Blank } \\
\text { Blank } \\
\text { Blank } \\
\text { Blank } \\
\text { Blank } \\
\text { Blank } \\
\text { Blank } \\
\text { Blank } \\
\text { Blank }\end{array}$ \\
\hline
\end{tabular}


Analyte

Location

Date

Result MDA or PQL

Units QC Type

\section{Radiological Activity}

lodine-125 Travel Blank

cont.
$5 / 2 / 01$

$5 / 9 / 01$

$5 / 16 / 01$

$5 / 23 / 01$

$5 / 31 / 01$

$6 / 6 / 01$

$6 / 6 / 01$

$6 / 13 / 01$

$6 / 20 / 01$

$6 / 27 / 01$

$7 / 5 / 01$

$7 / 5 / 01$

$7 / 11 / 01$

$7 / 18 / 01$

$7 / 25 / 01$

$8 / 1 / 01$

$8 / 1 / 01$

$8 / 8 / 01$

$8 / 15 / 01$

$8 / 22 / 01$

$8 / 29 / 01$

$9 / 5 / 01$

9/5/01

9/12/01

9/19/01

9/26/01

$10 / 3 / 01$

10/8/01

10/9/01

10/16/01

10/24/01

10/30/01

$11 / 8 / 01$

$11 / 9 / 01$

$11 / 13 / 01$

$11 / 20 / 01$

$11 / 26 / 01$

$12 / 4 / 01$

$12 / 4 / 01$

$12 / 12 / 01$

$12 / 19 / 01$

$1 / 2 / 02$

$1 / 3 / 02$
ND

0.17

ND

ND

ND

ND

ND

0.15

ND

ND

ND

0.26

ND

ND

ND

ND

ND

ND

ND

ND

ND

ND

ND

ND

ND

ND

ND

ND

0.3

ND

ND

ND

ND

ND

ND

ND

ND

ND

ND

ND

ND

ND

ND

ND
0.11

0.11

0.11

0.11

0.11

0.11

0.11

0.11

0.11

0.11

0.11

0.11

0.11

0.11

0.11

0.11

0.11

0.11

0.11

0.11

0.11

0.11

0.11

0.11

0.11

0.11

0.11

0.11

0.11

0.11

0.11

0.11

0.11

0.11

0.11

0.11

0.11

0.11

0.11

0.11

0.11

0.11

0.11

0.11
$\mathrm{Bq} / \mathrm{S}$

$\mathrm{Bq} / \mathrm{S}$

$\mathrm{Bq} / \mathrm{S}$

$\mathrm{Bq} / \mathrm{S}$

$\mathrm{Bq} / \mathrm{S}$

$\mathrm{Bq} / \mathrm{S}$

$\mathrm{Bq} / \mathrm{S}$

$\mathrm{Bq} / \mathrm{S}$

$\mathrm{Bq} / \mathrm{S}$

$\mathrm{Bq} / \mathrm{S}$

$\mathrm{Bq} / \mathrm{S}$

$\mathrm{Bq} / \mathrm{S}$

$\mathrm{Bq} / \mathrm{S}$

$\mathrm{Bq} / \mathrm{S}$

$\mathrm{Bq} / \mathrm{S}$

$\mathrm{Bq} / \mathrm{S}$

$\mathrm{Bq} / \mathrm{S}$

$\mathrm{Bq} / \mathrm{S}$

$\mathrm{Bq} / \mathrm{S}$

$\mathrm{Bq} / \mathrm{S}$

$\mathrm{Bq} / \mathrm{S}$

$\mathrm{Bq} / \mathrm{S}$

$\mathrm{Bq} / \mathrm{S}$

$\mathrm{Bq} / \mathrm{S}$

$\mathrm{Bq} / \mathrm{S}$

$\mathrm{Bq} / \mathrm{S}$

$\mathrm{Bq} / \mathrm{S}$

$\mathrm{Bq} / \mathrm{S}$

$\mathrm{Bq} / \mathrm{S}$

$\mathrm{Bq} / \mathrm{S}$

$\mathrm{Bq} / \mathrm{S}$

$\mathrm{Bq} / \mathrm{S}$

$\mathrm{Bq} / \mathrm{S}$

$\mathrm{Bq} / \mathrm{S}$

$\mathrm{Bq} / \mathrm{S}$

$\mathrm{Bq} / \mathrm{S}$

$\mathrm{Bq} / \mathrm{S}$

$\mathrm{Bq} / \mathrm{S}$

$\mathrm{Bq} / \mathrm{S}$

$\mathrm{Bq} / \mathrm{S}$

$\mathrm{Bq} / \mathrm{S}$

$\mathrm{Bq} / \mathrm{S}$

$\mathrm{Bq} / \mathrm{S}$

$\mathrm{Bq} / \mathrm{S}$
Blank

Blank

Blank

Blank

Blank

Blank

Blank

Blank

Blank

Blank

Blank

Blank

Blank

Blank

Blank

Blank

Blank

Blank

Blank

Blank

Blank

Blank

Blank

Blank

Blank

Blank

Blank

Blank

Blank

Blank

Blank

Blank

Blank

Blank

Blank

Blank

Blank

Blank

Blank

Blank

Blank

Blank

Blank

Blank 


$\begin{array}{llllll}\text { Analyte } & \text { Location } & \text { Date } & \text { Result } & \text { MDA or PQL Units } & \text { QC Type }\end{array}$

\section{Radiological Activity}

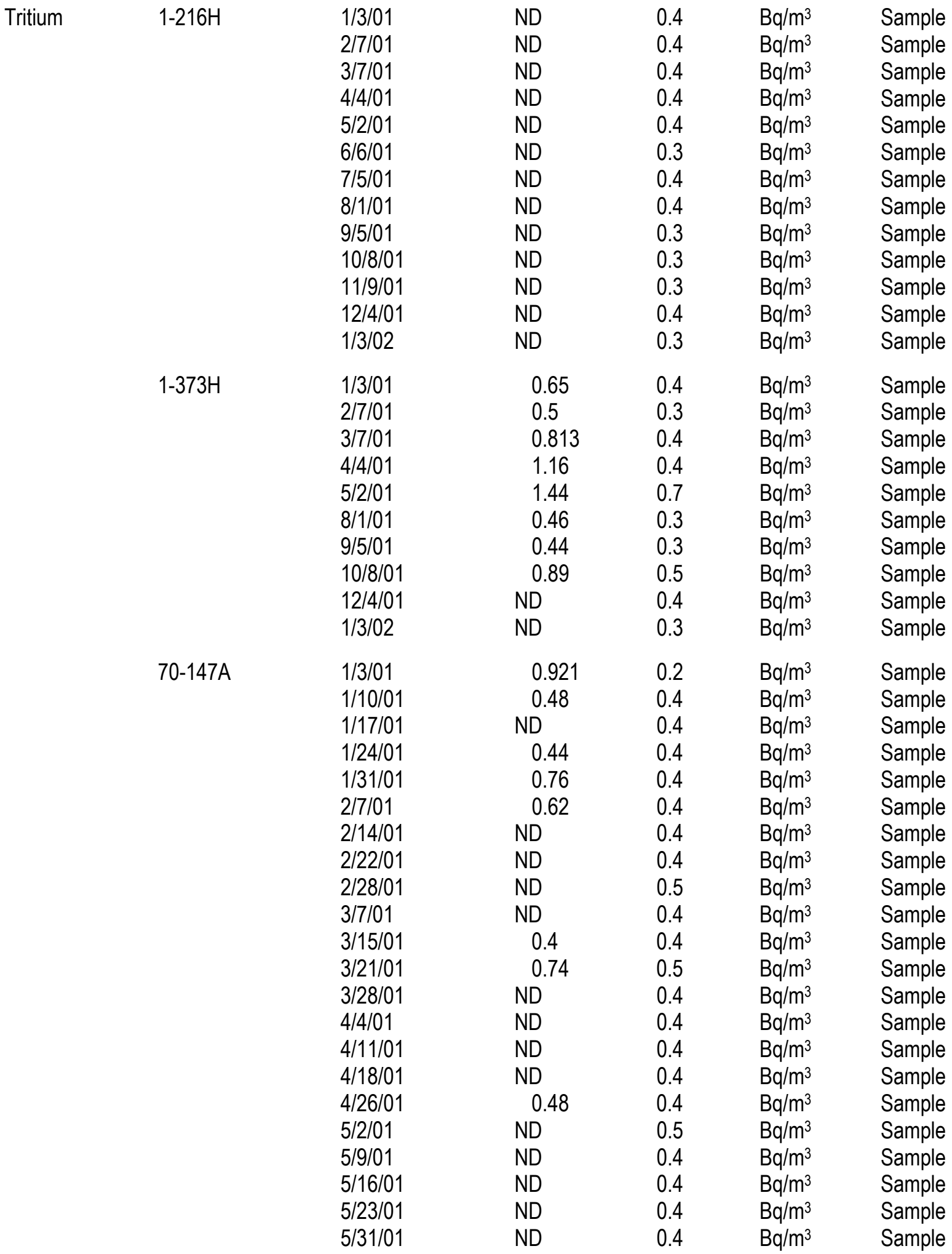


Analyte

Location

Date

Result MDA or PQL

Units

QC Type

\section{Radiological Activity}

Tritium

cont.
70-147A

$6 / 13 / 01$
$6 / 20 / 01$
$6 / 27 / 01$
$7 / 5 / 01$
$7 / 11 / 01$
$7 / 18 / 01$
$7 / 25 / 01$
$8 / 1 / 01$
$8 / 8 / 01$
$8 / 15 / 01$
$8 / 22 / 01$
$8 / 29 / 01$
$9 / 5 / 01$
$9 / 12 / 01$
$9 / 19 / 01$
$9 / 26 / 01$
$10 / 3 / 01$
$10 / 9 / 01$
$10 / 16 / 01$
$10 / 23 / 01$
$11 / 8 / 01$
$11 / 13 / 01$
$11 / 20 / 01$
$11 / 26 / 01$
$12 / 4 / 01$
$12 / 12 / 01$
$12 / 19 / 01$
$1 / 2 / 02$
$1 / 2 / 01$

75 NTLF-HTO

$\begin{array}{ll}1 / 3 / 01 & 62100 \\ 1 / 10 / 01 & 43300 \\ 1 / 17 / 01 & 74200 \\ 1 / 24 / 01 & 21800 \\ 1 / 31 / 01 & 41200 \\ 2 / 7 / 01 & 24600 \\ 2 / 14 / 01 & 22700 \\ 2 / 22 / 01 & 17100 \\ 2 / 28 / 01 & 12600 \\ 3 / 7 / 01 & 33100 \\ 3 / 8 / 01 & 17300 \\ 3 / 15 / 01 & 18000 \\ 3 / 21 / 01 & 28600 \\ 3 / 28 / 01 & 10200 \\ 4 / 4 / 01 & 22200 \\ 4 / 11 / 01 & 10900\end{array}$

$\begin{array}{clll}\text { ND } & 0.5 & \mathrm{~Bq} / \mathrm{m}^{3} & \text { Sample } \\ \text { ND } & 0.4 & \mathrm{~Bq} / \mathrm{m}^{3} & \text { Sample } \\ \text { ND } & 0.4 & \mathrm{~Bq} / \mathrm{m}^{3} & \text { Sample } \\ \text { ND } & 0.4 & \mathrm{~Bq} / \mathrm{m}^{3} & \text { Sample } \\ \text { ND } & 0.3 & \mathrm{~Bq} / \mathrm{m}^{3} & \text { Sample } \\ \text { ND } & 0.5 & \mathrm{~Bq} / \mathrm{m}^{3} & \text { Sample } \\ \text { ND } & 0.4 & \mathrm{~Bq} / \mathrm{m}^{3} & \text { Sample } \\ \text { ND } & 0.4 & \mathrm{~Bq} / \mathrm{m}^{3} & \text { Sample } \\ \text { ND } & 0.4 & \mathrm{~Bq} / \mathrm{m}^{3} & \text { Sample } \\ 2.37 & 0.4 & \mathrm{~Bq} / \mathrm{m}^{3} & \text { Sample } \\ 0.39 & 0.4 & \mathrm{~Bq} / \mathrm{m}^{3} & \text { Sample } \\ 0.41 & 0.4 & \mathrm{~Bq} / \mathrm{m}^{3} & \text { Sample } \\ \text { ND } & 0.4 & \mathrm{~Bq} / \mathrm{m}^{3} & \text { Sample } \\ 0.55 & 0.4 & \mathrm{~Bq} / \mathrm{m}^{3} & \text { Sample } \\ \text { ND } & 0.4 & \mathrm{~Bq} / \mathrm{m}^{3} & \text { Sample } \\ \text { ND } & 0.4 & \mathrm{~Bq} / \mathrm{m}^{3} & \text { Sample } \\ \text { ND } & 0.5 & \mathrm{~Bq} / \mathrm{m}^{3} & \text { Sample } \\ 0.68 & 0.5 & \mathrm{~Bq} / \mathrm{m}^{3} & \text { Sample } \\ \text { ND } & 0.6 & \mathrm{~Bq} / \mathrm{m}^{3} & \text { Sample } \\ \text { ND } & 0.4 & \mathrm{~Bq} / \mathrm{m}^{3} & \text { Sample } \\ \text { ND } & 0.4 & \mathrm{~Bq} / \mathrm{m}^{3} & \text { Sample } \\ \text { ND } & 0.4 & \mathrm{~Bq} / \mathrm{m}^{3} & \text { Sample } \\ \text { ND } & 0.5 & \mathrm{~Bq} / \mathrm{m}^{3} & \text { Sample } \\ \text { 0.37 } & 0.4 & \mathrm{~Bq} / \mathrm{m}^{3} & \text { Sample } \\ \text { ND } & 0.4 & \mathrm{~Bq} / \mathrm{m}^{3} & \text { Sample } \\ \text { ND } & 0.3 & \mathrm{~Bq} / \mathrm{m}^{3} & \text { Sample } \\ \text { ND } & 0.3 & \mathrm{~Bq} / \mathrm{m}^{3} & \text { Sample } \\ \text { ND } & 0.4 & \mathrm{~Bq} / \mathrm{m}^{3} & \text { Sample } \\ 0.31 & 0.18 & \mathrm{~Bq} / \mathrm{m}^{3} & \text { Sample } \\ & 11 & \mathrm{~Bq} / \mathrm{m}^{3} & \text { Sample }\end{array}$

$62100 \quad 11 \quad \mathrm{~Bq} / \mathrm{m}^{3} \quad$ Sample

$10 \mathrm{~Bq} / \mathrm{m}^{3} \quad$ Sample

$15 \quad \mathrm{~Bq} / \mathrm{m}^{3} \quad$ Sample

$8 \quad \mathrm{~Bq} / \mathrm{m}^{3} \quad$ Sample

$11 \quad \mathrm{~Bq} / \mathrm{m}^{3} \quad$ Sample

$7 \quad \mathrm{~Bq} / \mathrm{m}^{3} \quad$ Sample

$6 \quad \mathrm{~Bq} / \mathrm{m}^{3} \quad$ Sample

$5 \quad \mathrm{~Bq} / \mathrm{m}^{3} \quad$ Sample

$5 \quad \mathrm{~Bq} / \mathrm{m}^{3} \quad$ Sample

$10 \quad \mathrm{~Bq} / \mathrm{m}^{3} \quad$ Sample

$14 \quad \mathrm{~Bq} / \mathrm{m}^{3} \quad$ Sample

$7 \quad \mathrm{~Bq} / \mathrm{m}^{3} \quad$ Sample

$9 \mathrm{~Bq} / \mathrm{m}^{3} \quad$ Sample

$5 \quad \mathrm{~Bq} / \mathrm{m}^{3} \quad$ Sample

$7 \quad \mathrm{~Bq} / \mathrm{m}^{3} \quad$ Sample

$4 \mathrm{~Bq} / \mathrm{m}^{3} \quad$ Sample 


$\begin{array}{llllll}\text { Analyte } & \text { Location } & \text { Date } & \text { Result } & \text { MDA or PQL Units } & \text { QC Type }\end{array}$

\section{Radiological Activity}

Tritium cont.

75 NTLF-HTO

75

$4 / 26 / 01$

$5 / 2 / 01$

$5 / 9 / 01$

$5 / 16 / 01$

$5 / 23 / 01$

$5 / 31 / 01$

$6 / 6 / 01$

$6 / 13 / 01$

$6 / 20 / 01$

$6 / 27 / 01$

$7 / 5 / 01$

$7 / 11 / 01$

$7 / 18 / 01$

$7 / 25 / 01$

$8 / 1 / 01$

$8 / 8 / 01$

$8 / 15 / 01$

$8 / 22 / 01$

$8 / 29 / 01$

$9 / 5 / 01$

$9 / 19 / 01$

$9 / 26 / 01$

$10 / 3 / 01$

$10 / 9 / 01$

10/9/01

$10 / 16 / 01$

$10 / 23 / 01$

$10 / 30 / 01$

$11 / 8 / 01$

$11 / 13 / 01$

$11 / 20 / 01$

$11 / 26 / 01$

$12 / 4 / 01$

$12 / 12 / 01$

$12 / 19 / 01$

$1 / 2 / 02$

75 NTLF-Total T

$1 / 3 / 01$

$1 / 10 / 01$

$1 / 17 / 01$

$1 / 24 / 01$

$1 / 31 / 01$

2/7/01

$2 / 14 / 01$

2/22/01

2/28/01

17400
36300
28600
721
8120
6020
8610
6100
9800

57800

11300

24000

15400

15800

15900

40600

38000

29400

14500

12800

20300

51300

48500

26100

18100

19300

15900

13100

14300

11100

12900

12600

11600

11400

17400

32800

24700

54800

25300

84200

21500

67600

30300

20400

25600

14400

\begin{tabular}{|c|c|c|}
\hline 7 & $\mathrm{~Bq} / \mathrm{m}^{3}$ & Sample \\
\hline 11 & $\mathrm{~Bq} / \mathrm{m}^{3}$ & Sample \\
\hline 9 & $\mathrm{Bg} / \mathrm{m}^{3}$ & Sample \\
\hline 1.1 & $\mathrm{~Bq} / \mathrm{m}^{3}$ & Sample \\
\hline 3 & $\mathrm{~Bq} / \mathrm{m}^{3}$ & Sample \\
\hline 3 & $\mathrm{~Bq} / \mathrm{m}^{3}$ & Sample \\
\hline 4 & $\mathrm{~Bq} / \mathrm{m}^{3}$ & Sample \\
\hline 4 & $\mathrm{~Bq} / \mathrm{m}^{3}$ & Sample \\
\hline 5 & $\mathrm{~Bq} / \mathrm{m}^{3}$ & Sample \\
\hline 14 & $\mathrm{~Bq} / \mathrm{m}^{3}$ & Sample \\
\hline 5 & $\mathrm{~Bq} / \mathrm{m}^{3}$ & Sample \\
\hline 8 & $\mathrm{~Bq} / \mathrm{m}^{3}$ & Sample \\
\hline 11 & $\mathrm{~Bq} / \mathrm{m}^{3}$ & Sample \\
\hline 4 & $\mathrm{~Bq} / \mathrm{m}^{3}$ & Sample \\
\hline 7 & $\mathrm{~Bq} / \mathrm{m}^{3}$ & Sample \\
\hline 17 & $\mathrm{~Bq} / \mathrm{m}^{3}$ & Sample \\
\hline 19 & $\mathrm{~Bq} / \mathrm{m}^{3}$ & Sample \\
\hline 15 & $\mathrm{~Bq} / \mathrm{m}^{3}$ & Sample \\
\hline 6 & $\mathrm{~Bq} / \mathrm{m}^{3}$ & Sample \\
\hline 7 & $\mathrm{~Bq} / \mathrm{m}^{3}$ & Sample \\
\hline 9 & $\mathrm{~Bq} / \mathrm{m}^{3}$ & Sample \\
\hline 8 & $\mathrm{~Bq} / \mathrm{m}^{3}$ & Sample \\
\hline 13 & $\mathrm{~Bq} / \mathrm{m}^{3}$ & Sample \\
\hline 7 & $\mathrm{~Bq} / \mathrm{m}^{3}$ & Sample \\
\hline 8 & $\mathrm{~Bq} / \mathrm{m}^{3}$ & Sample \\
\hline 8 & $\mathrm{~Bq} / \mathrm{m}^{3}$ & Split \\
\hline 5 & $\mathrm{~Bq} / \mathrm{m}^{3}$ & Sample \\
\hline 5 & $\mathrm{~Bq} / \mathrm{m}^{3}$ & Sample \\
\hline 5 & $\mathrm{~Bq} / \mathrm{m}^{3}$ & Sample \\
\hline 4 & $\mathrm{~Bq} / \mathrm{m}^{3}$ & Sample \\
\hline 6 & $\mathrm{~Bq} / \mathrm{m}^{3}$ & Sample \\
\hline 5 & $\mathrm{~Bq} / \mathrm{m}^{3}$ & Sample \\
\hline 5 & $\mathrm{Bg} / \mathrm{m}^{3}$ & Sample \\
\hline 4 & $\mathrm{~Bq} / \mathrm{m}^{3}$ & Sample \\
\hline 6 & $\mathrm{~Bq} / \mathrm{m}^{3}$ & Sample \\
\hline 10 & $\mathrm{Bg} / \mathrm{m}^{3}$ & Sample \\
\hline 7 & $\mathrm{~Bq} / \mathrm{m}^{3}$ & Sample \\
\hline 11 & $\mathrm{~Bq} / \mathrm{m}^{3}$ & Sample \\
\hline 10 & $\mathrm{~Bq} / \mathrm{m}^{3}$ & Sample \\
\hline 18 & $\mathrm{~Bq} / \mathrm{m}^{3}$ & Sample \\
\hline 10 & $\mathrm{~Bq} / \mathrm{m}^{3}$ & Sample \\
\hline 16 & $\mathrm{~Bq} / \mathrm{m}^{3}$ & Sample \\
\hline 8 & $\mathrm{Bg} / \mathrm{m}^{3}$ & Sample \\
\hline 6 & $\mathrm{Bg} / \mathrm{m}^{3}$ & Sample \\
\hline 7 & $\mathrm{~Bq} / \mathrm{m}^{3}$ & Sample \\
\hline 5 & $\mathrm{Bg} / \mathrm{m}^{3}$ & Sample \\
\hline
\end{tabular}


Analyte

Location

Date

Result MDA or PQL

Units

QC Type

\section{Radiological Activity}

Tritium cont.

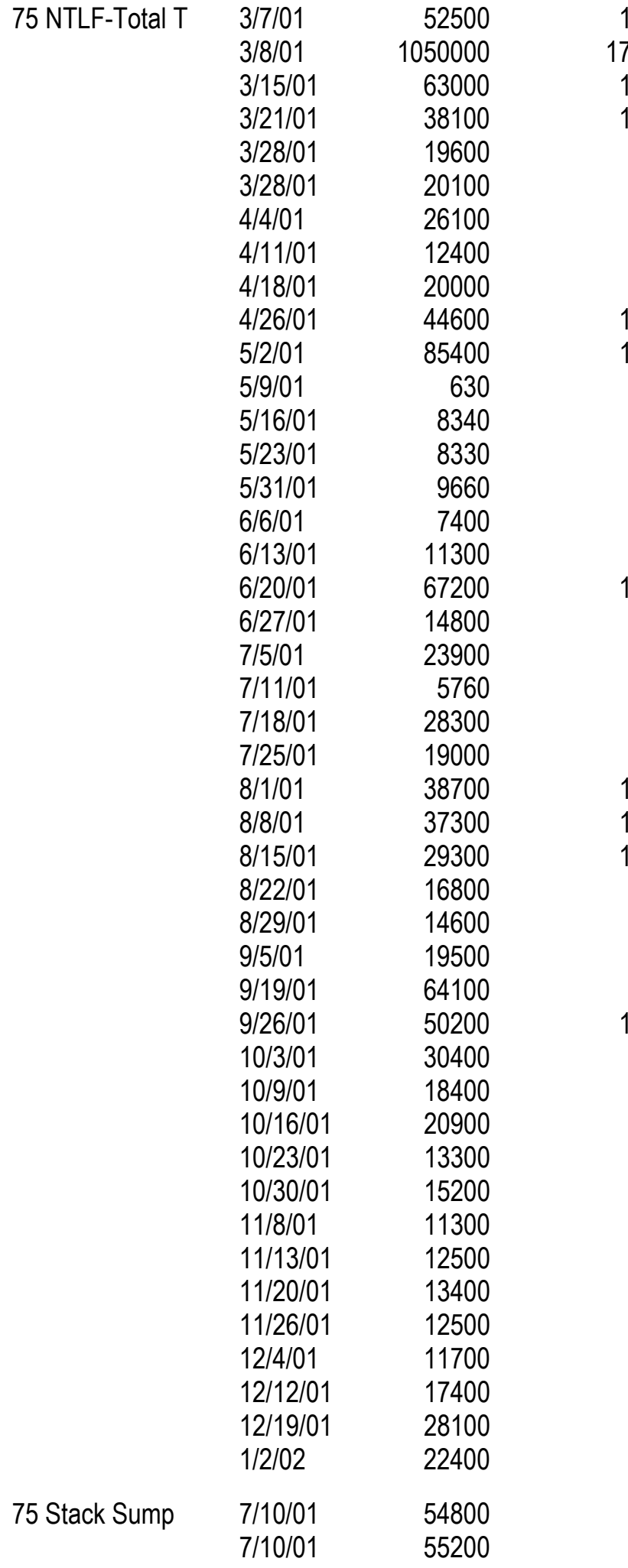

\begin{tabular}{|c|c|c|}
\hline 13 & $\mathrm{~Bq} / \mathrm{m}^{3}$ & Sample \\
\hline 170 & $\mathrm{~Bq} / \mathrm{m}^{3}$ & Sample \\
\hline 14 & $\mathrm{~Bq} / \mathrm{m}^{3}$ & Sample \\
\hline 12 & $\mathrm{Bg} / \mathrm{m}^{3}$ & Sample \\
\hline 7 & $\mathrm{~Bq} / \mathrm{m}^{3}$ & Sample \\
\hline 7 & $\mathrm{~Bq} / \mathrm{m}^{3}$ & Split \\
\hline 8 & $\mathrm{~Bq} / \mathrm{m}^{3}$ & Sample \\
\hline 5 & $\mathrm{~Bq} / \mathrm{m}^{3}$ & Sample \\
\hline 7 & $\mathrm{~Bq} / \mathrm{m}^{3}$ & Sample \\
\hline 13 & $\mathrm{~Bq} / \mathrm{m}^{3}$ & Sample \\
\hline 18 & $\mathrm{~Bq} / \mathrm{m}^{3}$ & Sample \\
\hline 1 & $\mathrm{~Bq} / \mathrm{m}^{3}$ & Sample \\
\hline 3 & $\mathrm{~Bq} / \mathrm{m}^{3}$ & Sample \\
\hline 4 & $\mathrm{~Bq} / \mathrm{m}^{3}$ & Sample \\
\hline 4 & $\mathrm{~Bq} / \mathrm{m}^{3}$ & Sample \\
\hline 4 & $\mathrm{~Bq} / \mathrm{m}^{3}$ & Sample \\
\hline 5 & $\mathrm{Bg} / \mathrm{m}^{3}$ & Sample \\
\hline 16 & $\mathrm{~Bq} / \mathrm{m}^{3}$ & Sample \\
\hline 6 & $\mathrm{~Bq} / \mathrm{m}^{3}$ & Sample \\
\hline 8 & $\mathrm{~Bq} / \mathrm{m}^{3}$ & Sample \\
\hline 4 & $\mathrm{~Bq} / \mathrm{m}^{3}$ & Sample \\
\hline 5 & $\mathrm{~Bq} / \mathrm{m}^{3}$ & Sample \\
\hline 8 & $\mathrm{~Bq} / \mathrm{m}^{3}$ & Sample \\
\hline 16 & $\mathrm{~Bq} / \mathrm{m}^{3}$ & Sample \\
\hline 19 & $\mathrm{~Bq} / \mathrm{m}^{3}$ & Sample \\
\hline 16 & $\mathrm{~Bq} / \mathrm{m}^{3}$ & Sample \\
\hline 6 & $\mathrm{~Bq} / \mathrm{m}^{3}$ & Sample \\
\hline 8 & $\mathrm{~Bq} / \mathrm{m}^{3}$ & Sample \\
\hline 8 & $\mathrm{~Bq} / \mathrm{m}^{3}$ & Sample \\
\hline 9 & $\mathrm{~Bq} / \mathrm{m}^{3}$ & Sample \\
\hline 13 & $\mathrm{Bg} / \mathrm{m}^{3}$ & Sample \\
\hline 8 & $\mathrm{Bg} / \mathrm{m}^{3}$ & Sample \\
\hline 7 & $\mathrm{~Bq} / \mathrm{m}^{3}$ & Sample \\
\hline 6 & $\mathrm{~Bq} / \mathrm{m}^{3}$ & Sample \\
\hline 5 & $\mathrm{~Bq} / \mathrm{m}^{3}$ & Sample \\
\hline 5 & $\mathrm{~Bq} / \mathrm{m}^{3}$ & Sample \\
\hline 4 & $\mathrm{~Bq} / \mathrm{m}^{3}$ & Sample \\
\hline 6 & $\mathrm{~Bq} / \mathrm{m}^{3}$ & Sample \\
\hline 6 & $\mathrm{Bg} / \mathrm{m}^{3}$ & Sample \\
\hline 6 & $\mathrm{~Bq} / \mathrm{m}^{3}$ & Sample \\
\hline 5 & $\mathrm{Bg} / \mathrm{m}^{3}$ & Sample \\
\hline 6 & $\mathrm{~Bq} / \mathrm{m}^{3}$ & Sample \\
\hline 8 & $\mathrm{~Bq} / \mathrm{m}^{3}$ & Sample \\
\hline 7 & $\mathrm{~Bq} / \mathrm{m}^{3}$ & Sample \\
\hline 5 & $\mathrm{~Bq} / \mathrm{L}$ & Sample \\
\hline 5 & $\mathrm{~Bq} / \mathrm{L}$ & Split \\
\hline
\end{tabular}




$\begin{array}{llllll}\text { Analyte } & \text { Location } & \text { Date } & \text { Result } & \text { MDA or PQL Units } & \text { QC Type }\end{array}$

\section{Radiological Activity}

Tritium cont.
75 Stack Sump

10/12/01

$11 / 13 / 01$

$11 / 13 / 01$

75-107H
$1 / 3 / 01$

$1 / 10 / 01$

$1 / 17 / 01$

$1 / 24 / 01$

$1 / 31 / 01$

$2 / 7 / 01$

2/14/01

2/22/01

$2 / 28 / 01$

$3 / 7 / 01$

$3 / 15 / 01$

$3 / 21 / 01$

$3 / 28 / 01$

$4 / 4 / 01$

$4 / 11 / 01$

$4 / 18 / 01$

$4 / 26 / 01$

$5 / 2 / 01$

$5 / 9 / 01$

$5 / 16 / 01$

$5 / 23 / 01$

$5 / 31 / 01$

$6 / 6 / 01$

$6 / 13 / 01$

$6 / 20 / 01$

$6 / 20 / 01$

$6 / 27 / 01$

$7 / 5 / 01$

$7 / 11 / 01$

$7 / 18 / 01$

$7 / 25 / 01$

$8 / 1 / 01$

$8 / 8 / 01$

$8 / 15 / 01$

$8 / 22 / 01$

$8 / 29 / 01$

$9 / 5 / 01$

9/12/01

$9 / 19 / 01$

9/26/01

$10 / 3 / 01$

10/9/01

10/16/01
9780

2440

662

571

696

530

473

916

772

525

3780

1530

1200

623

438

497

533

424

622

700

310

335

6630

259

634

620

327

616

472

390

431

475

535

462

512

429

559

386

890

1000

706

602

1170
6

0.7

$$
1.8
$$

1

\section{1}

0.8

0.7

\section{1}

1

$$
1
$$

0.9

$$
3
$$

1.8

1.3

0.9

0.7

0.9

$\begin{array}{ll}\mathrm{Bq} / \mathrm{L} & \text { Sample } \\ \mathrm{Bq} / \mathrm{L} & \text { Sample } \\ \mathrm{Bq} / \mathrm{L} & \text { Sample }\end{array}$

$\mathrm{Bq} / \mathrm{m}^{3} \quad$ Sample

$\mathrm{Bq} / \mathrm{m}^{3} \quad$ Sample

$\mathrm{Bq} / \mathrm{m}^{3} \quad$ Sample

$\mathrm{Bq} / \mathrm{m}^{3} \quad$ Sample

$\mathrm{Bq} / \mathrm{m}^{3} \quad$ Sample

$\mathrm{Bq} / \mathrm{m}^{3} \quad$ Sample

$\mathrm{Bq} / \mathrm{m}^{3} \quad$ Sample

$\mathrm{Bq} / \mathrm{m}^{3} \quad$ Sample

$\mathrm{Bq} / \mathrm{m}^{3} \quad$ Sample

$\mathrm{Bq} / \mathrm{m}^{3} \quad$ Sample

$\mathrm{Bq} / \mathrm{m}^{3} \quad$ Sample

$\mathrm{Bq} / \mathrm{m}^{3} \quad$ Sample

$\mathrm{Bq} / \mathrm{m}^{3} \quad$ Sample

$\mathrm{Bq} / \mathrm{m}^{3} \quad$ Sample

$\mathrm{Bq} / \mathrm{m}^{3} \quad$ Sample

$\mathrm{Bq} / \mathrm{m}^{3} \quad$ Sample

$\mathrm{Bq} / \mathrm{m}^{3} \quad$ Sample

$\mathrm{Bq} / \mathrm{m}^{3} \quad$ Sample

$\mathrm{Bq} / \mathrm{m}^{3} \quad$ Sample

$\mathrm{Bq} / \mathrm{m}^{3} \quad$ Sample

$\mathrm{Bq} / \mathrm{m}^{3} \quad$ Sample

$\mathrm{Bq} / \mathrm{m}^{3} \quad$ Sample

$\mathrm{Bq} / \mathrm{m}^{3} \quad$ Sample

$\mathrm{Bq} / \mathrm{m}^{3} \quad$ Sample

$\mathrm{Bq} / \mathrm{m}^{3} \quad$ Sample

$\mathrm{Bq} / \mathrm{m}^{3} \quad$ Split

$\mathrm{Bq} / \mathrm{m}^{3} \quad$ Sample

$\mathrm{Bq} / \mathrm{m}^{3} \quad$ Sample

$\mathrm{Bq} / \mathrm{m}^{3} \quad$ Sample

$\mathrm{Bq} / \mathrm{m}^{3} \quad$ Sample

$\mathrm{Bq} / \mathrm{m}^{3} \quad$ Sample

$\mathrm{Bq} / \mathrm{m}^{3} \quad$ Sample

$\mathrm{Bq} / \mathrm{m}^{3} \quad$ Sample

$\mathrm{Bq} / \mathrm{m}^{3} \quad$ Sample

$\mathrm{Bq} / \mathrm{m}^{3} \quad$ Sample

$\mathrm{Bq} / \mathrm{m}^{3} \quad$ Sample

$\mathrm{Bq} / \mathrm{m}^{3} \quad$ Sample

$\mathrm{Bq} / \mathrm{m}^{3} \quad$ Sample

$\mathrm{Bq} / \mathrm{m}^{3} \quad$ Sample

$\mathrm{Bq} / \mathrm{m}^{3} \quad$ Sample

$\mathrm{Bq} / \mathrm{m}^{3} \quad$ Sample

$\mathrm{Bq} / \mathrm{m}^{3} \quad$ Sample

$\mathrm{Bq} / \mathrm{m}^{3} \quad$ Sample 
Analyte

Location

Date

Result MDA or PQL

Units QC Type

\section{Radiological Activity}

Tritium

cont.
75-107H

(1)

75-Locker

85 Glovebox

$\begin{array}{lr}10 / 23 / 01 & 748 \\ 10 / 30 / 01 & 987 \\ 11 / 8 / 01 & 780 \\ 11 / 13 / 01 & 2190 \\ 11 / 20 / 01 & 1110 \\ 11 / 26 / 01 & 592 \\ 12 / 4 / 01 & 1030 \\ 12 / 12 / 01 & 817 \\ 12 / 19 / 01 & 1180 \\ 1 / 2 / 02 & 6090\end{array}$

$1 / 3 / 01$

$2 / 7 / 01$

$3 / 7 / 01$

$4 / 4 / 01$

$5 / 2 / 01$

$6 / 6 / 01$

$7 / 5 / 01$

$8 / 1 / 01$

$9 / 5 / 01$

$10 / 5 / 01$

$11 / 8 / 01$

$12 / 4 / 01$

$1 / 3 / 02$

$1 / 3 / 01$

$1 / 10 / 01$

$1 / 17 / 01$

$1 / 24 / 01$

$1 / 31 / 01$

2/7/01

2/14/01

2/22/01

$2 / 28 / 01$

$3 / 7 / 01$

$3 / 15 / 01$

$3 / 21 / 01$

$3 / 28 / 01$

$4 / 4 / 01$

$4 / 11 / 01$

$4 / 18 / 01$

$4 / 26 / 01$

$5 / 2 / 01$

$5 / 9 / 01$

$5 / 16 / 01$

$5 / 23 / 01$
748

87

2190

1110

592

1030

817

1180
6090

40.8

91.3

65.5

122

72.2

58.7

87.3

67.9

52.7

50.7

56.8

65.7

47.8

1.78

1.9

3.12

1.6

3.17

4.72

1.98

2.3

1.8

2.93

2.3

1.7

1.3

2.98

1.4

1.6

1.5

1.9

2.02

4.11

2.71
1.1

1.2

1

2

1.4

1

1.2

1.2

1.3

3

0.4

0.2

0.4

0.3

0.4

0.3

0.3

0.4

0.3

0.3

0.3

0.4

0.3

0.3

0.5

0.5

0.5

0.6

0.4

0.4

0.4

0.5

0.7

0.6

0.7

0.6

0.6

0.6

0.6

0.7

0.8

0.4

0.5

0.5
$\mathrm{Bq} / \mathrm{m}^{3}$

$\mathrm{Bq} / \mathrm{m}^{3}$

$\mathrm{Bq} / \mathrm{m}^{3}$

$\mathrm{Bq} / \mathrm{m}^{3}$

$\mathrm{Bq} / \mathrm{m}^{3}$

$\mathrm{Bq} / \mathrm{m}^{3}$

$\mathrm{Bq} / \mathrm{m}^{3}$

$\mathrm{Bq} / \mathrm{m}^{3}$

$\mathrm{Bq} / \mathrm{m}^{3}$

$\mathrm{Bq} / \mathrm{m}^{3}$

$\mathrm{Bq} / \mathrm{m}^{3}$

$\mathrm{Bq} / \mathrm{m}^{3}$

$\mathrm{Bq} / \mathrm{m}^{3}$

$\mathrm{Bq} / \mathrm{m}^{3}$

$\mathrm{Bg} / \mathrm{m}^{3}$

$\mathrm{Bq} / \mathrm{m}^{3}$

$\mathrm{Bq} / \mathrm{m}^{3}$

$\mathrm{Bq} / \mathrm{m}^{3}$

$\mathrm{Bq} / \mathrm{m}^{3}$

$\mathrm{Bq} / \mathrm{m}^{3}$

$\mathrm{Bq} / \mathrm{m}^{3}$

$\mathrm{Bq} / \mathrm{m}^{3}$

$\mathrm{Bq} / \mathrm{m}^{3}$

$\mathrm{Bq} / \mathrm{m}^{3}$

$\mathrm{Bq} / \mathrm{m}^{3}$

$\mathrm{Bq} / \mathrm{m}^{3}$

$\mathrm{Bg} / \mathrm{m}^{3}$

$\mathrm{Bq} / \mathrm{m}^{3}$

$\mathrm{Bq} / \mathrm{m}^{3}$

$\mathrm{Bq} / \mathrm{m}^{3}$

$\mathrm{Bq} / \mathrm{m}^{3}$

$\mathrm{Bg} / \mathrm{m}^{3}$

$\mathrm{Bq} / \mathrm{m}^{3}$

$\mathrm{Bq} / \mathrm{m}^{3}$

$\mathrm{Bq} / \mathrm{m}^{3}$

$\mathrm{Bq} / \mathrm{m}^{3}$

$\mathrm{Bq} / \mathrm{m}^{3}$

$\mathrm{Bq} / \mathrm{m}^{3}$

$\mathrm{Bq} / \mathrm{m}^{3}$

$\mathrm{Bq} / \mathrm{m}^{3}$

$\mathrm{Bq} / \mathrm{m}^{3}$

$\mathrm{Bq} / \mathrm{m}^{3}$

$\mathrm{Bq} / \mathrm{m}^{3}$

$\mathrm{Bq} / \mathrm{m}^{3}$
Sample

Sample

Sample

Sample

Sample

Sample

Sample

Sample

Sample

Sample

Sample

Sample

Sample

Sample

Sample

Sample

Sample

Sample

Sample

Sample

Sample

Sample

Sample

Sample

Sample

Sample

Sample

Sample

Sample

Sample

Sample

Sample

Sample

Sample

Sample

Sample

Sample

Sample

Sample

Sample

Sample

Sample

Sample

Sample 


\begin{tabular}{llllll} 
Analyte & Location & Date & Result & MDA or PQL Units & QC Type \\
\hline
\end{tabular}

\section{Radiological Activity}

Tritium cont.
85 Glovebox

8

$5 / 31 / 01$

$6 / 6 / 01$

$6 / 13 / 01$

$6 / 20 / 01$

$6 / 27 / 01$

$7 / 5 / 01$

$7 / 11 / 01$

$7 / 18 / 01$

$7 / 25 / 01$

$8 / 1 / 01$

$8 / 8 / 01$

$8 / 15 / 01$

$8 / 22 / 01$

$8 / 29 / 01$

$9 / 5 / 01$

$9 / 12 / 01$

$9 / 19 / 01$

$9 / 26 / 01$

$10 / 3 / 01$

$10 / 9 / 01$

$10 / 16 / 01$

$10 / 23 / 01$

$10 / 30 / 01$

$11 / 8 / 01$

$11 / 13 / 01$

$11 / 20 / 01$

$11 / 26 / 01$

$12 / 4 / 01$

$12 / 12 / 01$

$12 / 18 / 01$

$1 / 2 / 02$

85 Hood

$1 / 3 / 01$

$1 / 10 / 01$

$1 / 17 / 01$

$1 / 24 / 01$

$1 / 31 / 01$

2/7/01

2/14/01

2/22/01

$2 / 28 / 01$

$3 / 7 / 01$

$3 / 15 / 01$

$3 / 21 / 01$

$3 / 28 / 01$

$4 / 4 / 01$

$4 / 11 / 01$
1.8

2.8

2.6

7.26

2.7

3.77

1.6

1.74

4.03

38.6

73.4

77.3

62.8

174

96.6

12.8

15.4

2.7

4.99

0.92

0.85

1.38

1.31

1.63

1.8

1.79

1.47

1.56

1.61

1.2

1.61

6.04

8.43

4.38

5.08

4.25

5.28

3.61

4.29

5.85

4.76

7.75

7.47

5.84

5.77

4.17
0.4

0.9

0.8

0.7

0.8

0.7

1

0.4

0.9

1.1

1.5

0.7

0.7

\section{1}

\section{1}

0.4

0.4

0.8

0.6

0.4

0.4

0.4

0.4

0.3

0.5

0.4

0.4

0.3

0.3

0.5

0.17

0.3

0.6

0.6

0.6

0.6

0.5

0.4

0.4

0.7

0.7

0.6

0.7

0.7

0.6

0.6
$\mathrm{Bq} / \mathrm{m}^{3}$

$\mathrm{Bq} / \mathrm{m}^{3}$

$\mathrm{Bq} / \mathrm{m}^{3}$

$\mathrm{Bq} / \mathrm{m}^{3}$

$\mathrm{Bq} / \mathrm{m}^{3}$

$\mathrm{Bq} / \mathrm{m}^{3}$

$\mathrm{Bg} / \mathrm{m}^{3}$

$\mathrm{Bq} / \mathrm{m}^{3}$

$\mathrm{Bq} / \mathrm{m}^{3}$

$\mathrm{Bq} / \mathrm{m}^{3}$

$\mathrm{Bq} / \mathrm{m}^{3}$

$\mathrm{Bq} / \mathrm{m}^{3}$

$\mathrm{Bq} / \mathrm{m}^{3}$

$\mathrm{Bq} / \mathrm{m}^{3}$

$\mathrm{Bq} / \mathrm{m}^{3}$

$\mathrm{Bq} / \mathrm{m}^{3}$

$\mathrm{Bq} / \mathrm{m}^{3}$

$\mathrm{Bq} / \mathrm{m}^{3}$

$\mathrm{Bq} / \mathrm{m}^{3}$

$\mathrm{Bq} / \mathrm{m}^{3}$

$\mathrm{Bq} / \mathrm{m}^{3}$

$\mathrm{Bq} / \mathrm{m}^{3}$

$\mathrm{Bq} / \mathrm{m}^{3}$

$\mathrm{Bq} / \mathrm{m}^{3}$

$\mathrm{Bq} / \mathrm{m}^{3}$

$\mathrm{Bq} / \mathrm{m}^{3}$

$\mathrm{Bq} / \mathrm{m}^{3}$

$\mathrm{Bq} / \mathrm{m}^{3}$

$\mathrm{Bq} / \mathrm{m}^{3}$

$\mathrm{Bq} / \mathrm{m}^{3}$

$\mathrm{Bq} / \mathrm{m}^{3}$

$\mathrm{Bq} / \mathrm{m}^{3}$

$\mathrm{Bq} / \mathrm{m}^{3}$

$\mathrm{Bq} / \mathrm{m}^{3}$

$\mathrm{Bq} / \mathrm{m}^{3}$

$\mathrm{Bq} / \mathrm{m}^{3}$

$\mathrm{Bq} / \mathrm{m}^{3}$

$\mathrm{Bq} / \mathrm{m}^{3}$

$\mathrm{Bq} / \mathrm{m}^{3}$

$\mathrm{Bq} / \mathrm{m}^{3}$

$\mathrm{Bq} / \mathrm{m}^{3}$

$\mathrm{Bq} / \mathrm{m}^{3}$

$\mathrm{Bq} / \mathrm{m}^{3}$

$\mathrm{Bq} / \mathrm{m}^{3}$

$\mathrm{Bq} / \mathrm{m}^{3}$

$\mathrm{Bq} / \mathrm{m}^{3}$
Sample

Sample

Sample

Sample

Sample

Sample

Sample

Sample

Sample

Sample

Sample

Sample

Sample

Sample

Sample

Sample

Sample

Sample

Sample

Sample

Sample

Sample

Sample

Sample

Sample

Sample

Sample

Sample

Sample

Sample

Sample

Sample

Sample

Sample

Sample

Sample

Sample

Sample

Sample

Sample

Sample

Sample

Sample

Sample

Sample

Sample 
Analyte

Location

Date

Result MDA or PQL

Units

QC Type

\section{Radiological Activity}

Tritium cont.
$85 \mathrm{Hood}$

$4 / 26 / 01$
$5 / 2 / 01$
$5 / 9 / 01$
$5 / 16 / 01$
$5 / 23 / 01$
$5 / 31 / 01$
$6 / 6 / 01$
$6 / 13 / 01$
$6 / 20 / 01$
$6 / 27 / 01$
$7 / 5 / 01$
$7 / 11 / 01$
$7 / 18 / 01$
$7 / 25 / 01$
$8 / 1 / 01$
$8 / 8 / 01$
$8 / 15 / 01$
$8 / 22 / 01$
$8 / 29 / 01$
$9 / 5 / 01$
$9 / 19 / 01$
$9 / 26 / 01$
$10 / 3 / 01$
$10 / 9 / 01$
$10 / 16 / 01$
$10 / 23 / 01$
$10 / 30 / 01$
$11 / 8 / 01$
$11 / 13 / 01$
$11 / 20 / 01$
$11 / 26 / 01$
$12 / 4 / 01$
$12 / 12 / 01$
$12 / 18 / 01$
$1 / 2 / 02$

$91 / 100$

NTLF Hillside Stack 11/13/01

Drain

$11 / 29 / 01$

$12 / 3 / 01$

Travel Blank

$1 / 3 / 01$
$1 / 3 / 01$
$1 / 3 / 01$
$1 / 10 / 01$
$1 / 10 / 01$
$1 / 17 / 01$

\section{3}

4.82

75

5.27

6.55

8.64

6.18

5.44

8.85

11.3

10.7

12.2

8.7

7.44

10.1

7.81

26.7

33.3

28.3

66.5

29.3

7.79

7.51

7.4

6.9

8.4

6.77

6.3

5.86

6.53

6.96

5.26

28.7

4.64

3.92

4.17

223000

177000

133000

ND

ND

ND

ND

ND

ND
0.6

0.7

0.8

0.6

0.5

0.5

0.4

0.9

0.8

0.7

0.8

0.8

1

0.4

0.8

1.1

1.5

0.5

0.6

1

1

0.4

0.7

0.6

0.4

0.4

0.4

0.4

0.3

0.5

0.4

0.4

0.3

0.3

0.4

0.17

80

70

60

0.5

0.4

0.4

0.6

0.4

0.4
$\mathrm{Bq} / \mathrm{m}^{3}$

$\mathrm{Bq} / \mathrm{m}^{3}$

$\mathrm{Bq} / \mathrm{m}^{3}$

$\mathrm{Bq} / \mathrm{m}^{3}$

$\mathrm{Bq} / \mathrm{m}^{3}$

$\mathrm{Bq} / \mathrm{m}^{3}$

$\mathrm{Bq} / \mathrm{m}^{3}$

$\mathrm{Bq} / \mathrm{m}^{3}$

$\mathrm{Bq} / \mathrm{m}^{3}$

$\mathrm{Bq} / \mathrm{m}^{3}$

$\mathrm{Bq} / \mathrm{m}^{3}$

$\mathrm{Bq} / \mathrm{m}^{3}$

$\mathrm{Bq} / \mathrm{m}^{3}$

$\mathrm{Bq} / \mathrm{m}^{3}$

$\mathrm{Bq} / \mathrm{m}^{3}$

$\mathrm{Bq} / \mathrm{m}^{3}$

$\mathrm{Bg} / \mathrm{m}^{3}$

$\mathrm{Bq} / \mathrm{m}^{3}$

$\mathrm{Bq} / \mathrm{m}^{3}$

$\mathrm{Bq} / \mathrm{m}^{3}$

$\mathrm{Bq} / \mathrm{m}^{3}$

$\mathrm{Bq} / \mathrm{m}^{3}$

$\mathrm{Bq} / \mathrm{m}^{3}$

$\mathrm{Bq} / \mathrm{m}^{3}$

$\mathrm{Bq} / \mathrm{m}^{3}$

$\mathrm{Bg} / \mathrm{m}^{3}$

$\mathrm{Bq} / \mathrm{m}^{3}$

$\mathrm{Bq} / \mathrm{m}^{3}$

$\mathrm{Bq} / \mathrm{m}^{3}$

$\mathrm{Bq} / \mathrm{m}^{3}$

$\mathrm{Bq} / \mathrm{m}^{3}$

$\mathrm{Bq} / \mathrm{m}^{3}$

$\mathrm{Bq} / \mathrm{m}^{3}$

$\mathrm{Bq} / \mathrm{m}^{3}$

$\mathrm{Bq} / \mathrm{m}^{3}$

$\mathrm{Bq} / \mathrm{m}^{3}$

$\mathrm{Bq} / \mathrm{L}$

$\mathrm{Bq} / \mathrm{L} \quad$ Sample

$\mathrm{Bq} / \mathrm{L} \quad$ Sample

\section{$\mathrm{Bq} / \mathrm{S}$}

$\mathrm{Bq} / \mathrm{S}$

$\mathrm{Bq} / \mathrm{S}$

$\mathrm{Bq} / \mathrm{S}$

$\mathrm{Bq} / \mathrm{S}$

$\mathrm{Bq} / \mathrm{S}$
Sample

Sample

Sample

Sample

Sample

Sample

Sample

Sample

Sample

Sample

Sample

Sample

Sample

Sample

Sample

Sample

Sample

Sample

Sample

Sample

Sample

Sample

Sample

Sample

Sample

Sample

Sample

Sample

Sample

Sample

Sample

Sample

Sample

Sample

Sample

Sample

Sample

Blank

Blank

Blank

Blank

Blank

Blank 


$\begin{array}{llllll}\text { Analyte } & \text { Location } & \text { Date } & \text { Result } & \text { MDA or PQL Units } & \text { QC Type }\end{array}$

\section{Radiological Activity}

Tritium cont.
Travel Blank

$1 / 17 / 01$

$1 / 24 / 01$

$1 / 24 / 01$

$1 / 31 / 01$

$1 / 31 / 01$

2/7/01

2/7/01

2/7/01

$2 / 14 / 01$

$2 / 14 / 01$

$2 / 22 / 01$

$2 / 22 / 01$

$2 / 28 / 01$

$2 / 28 / 01$

$3 / 7 / 01$

$3 / 7 / 01$

$3 / 7 / 01$

$3 / 15 / 01$

$3 / 15 / 01$

$3 / 21 / 01$

$3 / 21 / 01$

$3 / 28 / 01$

$3 / 28 / 01$

$4 / 4 / 01$

$4 / 4 / 01$

$4 / 4 / 01$

$4 / 11 / 01$

$4 / 11 / 01$

$4 / 18 / 01$

$4 / 18 / 01$

$4 / 26 / 01$

$4 / 26 / 01$

$5 / 2 / 01$

$5 / 2 / 01$

$5 / 2 / 01$

$5 / 9 / 01$

$5 / 9 / 01$

$5 / 16 / 01$

$5 / 16 / 01$

$5 / 23 / 01$

$5 / 23 / 01$

$5 / 31 / 01$

$5 / 31 / 01$

$6 / 6 / 01$

6/6/01

6/6/01
ND

ND

0.57

ND

ND

ND

ND

ND

ND

ND

ND

ND

ND

ND

ND

0.4

ND

ND

ND

ND

ND

ND

ND

ND

ND

ND

ND

ND

ND

ND

ND

ND

ND

ND

ND

ND

ND

ND

ND

ND

ND

ND

ND

ND

ND

ND
0.4

0.4

0.6

0.4

0.5

0.4

0.4

0.5

0.4

0.4

0.4

0.6

0.4

0.4

0.4

0.6

0.9

0.4

0.6

0.4

0.6

0.6

0.4

0.4

0.4

0.6

0.4

0.6

0.7

0.4

0.7

0.4

0.4

0.4

0.5

0.4

0.4

0.4

0.4

0.4

0.4

0.4

0.7

0.4
$\mathrm{Bq} / \mathrm{S}$

$\mathrm{Bq} / \mathrm{S}$

$\mathrm{Bq} / \mathrm{S}$

$\mathrm{Bq} / \mathrm{S}$

$\mathrm{Bq} / \mathrm{S}$

$\mathrm{Bq} / \mathrm{S}$

$\mathrm{Bq} / \mathrm{S}$

$\mathrm{Bq} / \mathrm{S}$

$\mathrm{Bq} / \mathrm{S}$

$\mathrm{Bq} / \mathrm{S}$

$\mathrm{Bq} / \mathrm{S}$

$\mathrm{Bq} / \mathrm{S}$

$\mathrm{Bq} / \mathrm{S}$

$\mathrm{Bq} / \mathrm{S}$

$\mathrm{Bq} / \mathrm{S}$

$\mathrm{Bq} / \mathrm{S}$

$\mathrm{Bq} / \mathrm{S}$

$\mathrm{Bq} / \mathrm{S}$

$\mathrm{Bq} / \mathrm{S}$

$\mathrm{Bq} / \mathrm{S}$

$\mathrm{Bq} / \mathrm{S}$

$\mathrm{Bq} / \mathrm{S}$

$\mathrm{Bq} / \mathrm{S}$

$\mathrm{Bq} / \mathrm{S}$

$\mathrm{Bq} / \mathrm{S}$

$\mathrm{Bq} / \mathrm{S}$

$\mathrm{Bq} / \mathrm{S}$

$\mathrm{Bq} / \mathrm{S}$

$\mathrm{Bq} / \mathrm{S}$

$\mathrm{Bq} / \mathrm{S}$

$\mathrm{Bq} / \mathrm{S}$

$\mathrm{Bq} / \mathrm{S}$

$\mathrm{Bq} / \mathrm{S}$

$\mathrm{Bq} / \mathrm{S}$

$\mathrm{Bq} / \mathrm{S}$

$\mathrm{Bq} / \mathrm{S}$

$\mathrm{Bq} / \mathrm{S}$

$\mathrm{Bq} / \mathrm{S}$

$\mathrm{Bq} / \mathrm{S}$

$\mathrm{Bq} / \mathrm{S}$

$\mathrm{Bq} / \mathrm{S}$

$\mathrm{Bq} / \mathrm{S}$

$\mathrm{Bq} / \mathrm{S}$

$\mathrm{Bq} / \mathrm{S}$

$\mathrm{Bq} / \mathrm{S}$

$\mathrm{Bq} / \mathrm{S}$
Blank

Blank

Blank

Blank

Blank

Blank

Blank

Blank

Blank

Blank

Blank

Blank

Blank

Blank

Blank

Blank

Blank

Blank

Blank

Blank

Blank

Blank

Blank

Blank

Blank

Blank

Blank

Blank

Blank

Blank

Blank

Blank

Blank

Blank

Blank

Blank

Blank

Blank

Blank

Blank

Blank

Blank

Blank

Blank

Blank

Blank 
Analyte

Location

Date

Result MDA or PQL

Units QC Type

\section{Radiological Activity}

Tritium

Travel Blank

$6 / 13 / 01$

$6 / 13 / 01$

$6 / 20 / 01$

$6 / 20 / 01$

$6 / 27 / 01$

$6 / 27 / 01$

$7 / 5 / 01$

$7 / 5 / 01$

$7 / 5 / 01$

$7 / 11 / 01$

$7 / 11 / 01$

$7 / 18 / 01$

$7 / 18 / 01$

$7 / 25 / 01$

$7 / 25 / 01$

$8 / 1 / 01$

$8 / 1 / 01$

$8 / 1 / 01$

$8 / 8 / 01$

$8 / 8 / 01$

$8 / 15 / 01$

$8 / 15 / 01$

$8 / 22 / 01$

$8 / 22 / 01$

$8 / 29 / 01$

$8 / 29 / 01$

$9 / 5 / 01$

9/5/01

9/5/01

$9 / 12 / 01$

9/12/01

9/19/01

9/19/01

9/26/01

9/26/01

$10 / 3 / 01$

10/3/01

10/8/01

10/9/01

10/9/01

10/16/01

10/16/01

$10 / 24 / 01$

$10 / 24 / 01$

$10 / 30 / 01$

$10 / 30 / 01$

ND

ND

ND

0.46

0.66

ND

ND

ND

ND

ND

ND

ND

ND

ND

ND

ND

ND

1.2

ND

11.9

11.7

ND

11

ND

ND

44.4

ND

4

ND

ND

ND

ND

ND

ND

ND

ND

ND

ND

ND

ND

ND

ND

ND

ND

ND

ND
0.7

0.4

0.4

0.4

0.6

0.4

0.4

0.7

0.4

0.7

0.4

0.4

0.4

0.4

0.7

0.4

0.4

0.4

0.4

0.6

0.8

0.4

0.4

0.4

0.4

0.4

0.4

0.4

0.4

0.4

0.4

0.4

0.4

0.4

0.4

0.4

0.4

0.6

0.4

0.4

0.4

0.4

0.4

0.4

0.4

0.4
$\mathrm{Bq} / \mathrm{S}$

$\mathrm{Bq} / \mathrm{S}$

$\mathrm{Bq} / \mathrm{S}$

$\mathrm{Bq} / \mathrm{S}$

$\mathrm{Bq} / \mathrm{S}$

$\mathrm{Bq} / \mathrm{S}$

$\mathrm{Bq} / \mathrm{S}$

$\mathrm{Bq} / \mathrm{S}$

$\mathrm{Bq} / \mathrm{S}$

$\mathrm{Bq} / \mathrm{S}$

$\mathrm{Bq} / \mathrm{S}$

$\mathrm{Bq} / \mathrm{S}$

$\mathrm{Bq} / \mathrm{S}$

$\mathrm{Bq} / \mathrm{S}$

$\mathrm{Bq} / \mathrm{S}$

$\mathrm{Bq} / \mathrm{S}$

$\mathrm{Bq} / \mathrm{S}$

$\mathrm{Bq} / \mathrm{S}$

$\mathrm{Bq} / \mathrm{S}$

$\mathrm{Bq} / \mathrm{S}$

$\mathrm{Bq} / \mathrm{S}$

$\mathrm{Bq} / \mathrm{S}$

$\mathrm{Bq} / \mathrm{S}$

$\mathrm{Bq} / \mathrm{S}$

$\mathrm{Bq} / \mathrm{S}$

$\mathrm{Bq} / \mathrm{S}$

$\mathrm{Bq} / \mathrm{S}$

$\mathrm{Bq} / \mathrm{S}$

$\mathrm{Bq} / \mathrm{S}$

$\mathrm{Bq} / \mathrm{S}$

$\mathrm{Bq} / \mathrm{S}$

$\mathrm{Bq} / \mathrm{S}$

$\mathrm{Bq} / \mathrm{S}$

$\mathrm{Bq} / \mathrm{S}$

$\mathrm{Bq} / \mathrm{S}$

$\mathrm{Bq} / \mathrm{S}$

$\mathrm{Bq} / \mathrm{S}$

$\mathrm{Bq} / \mathrm{S}$

$\mathrm{Bq} / \mathrm{S}$

$\mathrm{Bq} / \mathrm{S}$

$\mathrm{Bq} / \mathrm{S}$

$\mathrm{Bq} / \mathrm{S}$

$\mathrm{Bq} / \mathrm{S}$

$\mathrm{Bq} / \mathrm{S}$

$\mathrm{Bq} / \mathrm{S}$

$\mathrm{Bq} / \mathrm{S}$
Blank

Blank

Blank

Blank

Blank

Blank

Blank

Blank

Blank

Blank

Blank

Blank

Blank

Blank

Blank

Blank

Blank

Blank

Blank

Blank

Blank

Blank

Blank

Blank

Blank

Blank

Blank

Blank

Blank

Blank

Blank

Blank

Blank

Blank

Blank

Blank

Blank

Blank

Blank

Blank

Blank

Blank

Blank

Blank

Blank

Blank 


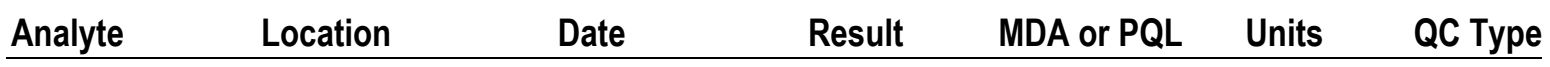

\section{Radiological Activity}

\begin{tabular}{|c|c|c|c|c|c|c|}
\hline \multirow{19}{*}{$\begin{array}{l}\text { Tritium } \\
\text { cont. }\end{array}$} & \multirow[t]{19}{*}{ Travel Blank } & $11 / 8 / 01$ & ND & 0.4 & $\mathrm{~Bq} / \mathrm{S}$ & Blank \\
\hline & & $11 / 8 / 01$ & ND & 0.4 & $\mathrm{~Bq} / \mathrm{S}$ & Blank \\
\hline & & $11 / 9 / 01$ & ND & 0.4 & $\mathrm{~Bq} / \mathrm{S}$ & Blar \\
\hline & & $11 / 13 / 01$ & ND & 0.4 & $\mathrm{Bg} / \mathrm{S}$ & Blar \\
\hline & & $11 / 13 / 01$ & ND & 0.4 & $\mathrm{~Bq} / \mathrm{S}$ & Blan \\
\hline & & $11 / 20 / 01$ & ND & 0.4 & $\mathrm{~Bq} / \mathrm{S}$ & Blar \\
\hline & & $11 / 20 / 01$ & ND & 0.4 & $\mathrm{~Bq} / \mathrm{S}$ & Blar \\
\hline & & $11 / 26 / 01$ & ND & 0.4 & $\mathrm{~Bq} / \mathrm{S}$ & Bla \\
\hline & & $11 / 26 / 01$ & ND & 0.4 & $\mathrm{~Bq} / \mathrm{S}$ & Bla \\
\hline & & $12 / 4 / 01$ & ND & 0.4 & $\mathrm{~Bq} / \mathrm{S}$ & Bla \\
\hline & & $12 / 4 / 01$ & ND & 0.4 & $\mathrm{~Bq} / \mathrm{S}$ & Blar \\
\hline & & $12 / 4 / 01$ & ND & 0.4 & $\mathrm{~Bq} / \mathrm{S}$ & Blar \\
\hline & & $12 / 12 / 01$ & ND & 0.4 & $\mathrm{~Bq} / \mathrm{S}$ & Blar \\
\hline & & $12 / 12 / 01$ & ND & 0.4 & $\mathrm{~Bq} / \mathrm{S}$ & $\mathrm{Bla}$ \\
\hline & & $12 / 19 / 01$ & ND & 0.4 & $\mathrm{~Bq} / \mathrm{S}$ & Bla \\
\hline & & 12/19/01 & ND & 0.4 & $\mathrm{~Bq} / \mathrm{S}$ & Bla \\
\hline & & $1 / 2 / 02$ & ND & 0.4 & $\mathrm{~Bq} / \mathrm{S}$ & I0 \\
\hline & & $1 / 2 / 02$ & ND & 0.4 & $\mathrm{~Bq} / \mathrm{S}$ & \\
\hline & & $1 / 3 / 02$ & ND & 0.4 & $\mathrm{~Bq} / \mathrm{S}$ & \\
\hline
\end{tabular}


The following ambient air data are summarized and discussed in Chapter 4 (Air Quality) of the Site Environmental Report for 2001 (see Volume I). Some of the results reported below are also reported in the Supplemental Monitoring section of this volume and discussed in Chapter 10 (Supplemental Monitoring) of Volume I:

$\begin{array}{llllll}\text { Analyte } & \text { Location } & \text { Date } & \text { Result } & \text { MDA or PQL Units } & \text { QC Type }\end{array}$

\section{Radiological Activity}

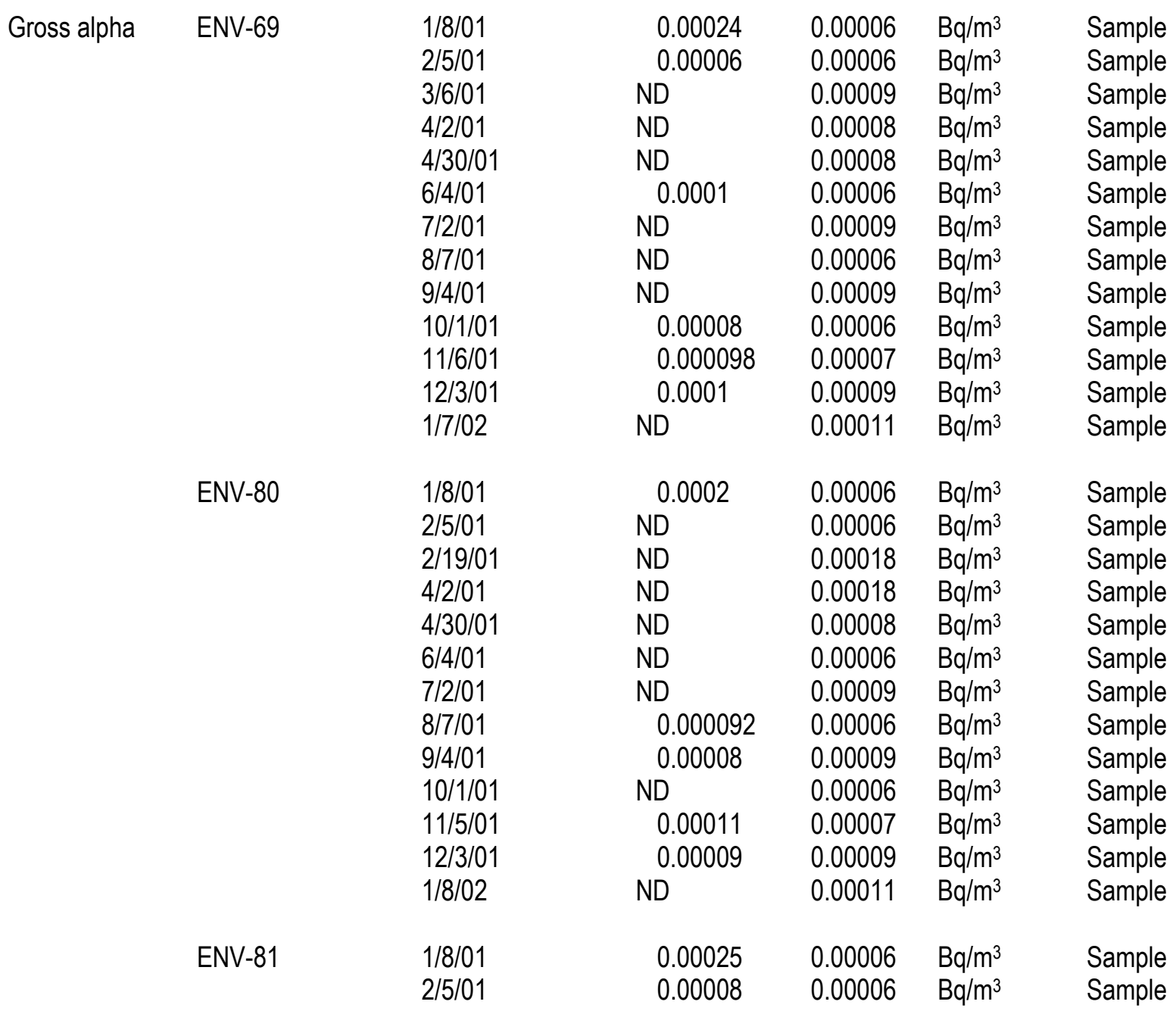




\section{Analyte \\ Radiological Activity}

\begin{tabular}{|c|c|c|c|c|c|c|}
\hline \multirow{37}{*}{$\begin{array}{l}\text { Gross alpha } \\
\text { cont. }\end{array}$} & \multirow[t]{11}{*}{ ENV-81 } & $3 / 6 / 01$ & ND & 0.00009 & $\mathrm{~Bq} / \mathrm{m}^{3}$ & Sample \\
\hline & & $4 / 2 / 01$ & ND & 0.00008 & $\mathrm{~Bq} / \mathrm{m}^{3}$ & Sample \\
\hline & & $4 / 30 / 01$ & ND & 0.00008 & $\mathrm{~Bq} / \mathrm{m}^{3}$ & Sample \\
\hline & & $6 / 5 / 01$ & 0.00009 & 0.00006 & $\mathrm{~Bq} / \mathrm{m}^{3}$ & Sample \\
\hline & & $7 / 2 / 01$ & ND & 0.0001 & $\mathrm{~Bq} / \mathrm{m}^{3}$ & Sample \\
\hline & & $8 / 7 / 01$ & ND & 0.00006 & $\mathrm{~Bq} / \mathrm{m}^{3}$ & Sample \\
\hline & & $9 / 4 / 01$ & ND & 0.00009 & $\mathrm{~Bq} / \mathrm{m}^{3}$ & Sample \\
\hline & & $10 / 1 / 01$ & ND & 0.00006 & $\mathrm{~Bq} / \mathrm{m}^{3}$ & Sample \\
\hline & & $11 / 5 / 01$ & 0.00014 & 0.00008 & $\mathrm{~Bq} / \mathrm{m}^{3}$ & Sample \\
\hline & & $12 / 3 / 01$ & ND & 0.0001 & $\mathrm{~Bq} / \mathrm{m}^{3}$ & Sample \\
\hline & & $1 / 7 / 02$ & ND & 0.00011 & $\mathrm{~Bq} / \mathrm{m}^{3}$ & Sample \\
\hline & \multirow[t]{13}{*}{ ENV-B13C } & $1 / 8 / 01$ & 0.00016 & 0.00006 & $\mathrm{~Bq} / \mathrm{m}^{3}$ & Sample \\
\hline & & $2 / 6 / 01$ & 0.00007 & 0.00006 & $\mathrm{~Bq} / \mathrm{m}^{3}$ & Sample \\
\hline & & $3 / 5 / 01$ & ND & 0.00009 & $\mathrm{~Bq} / \mathrm{m}^{3}$ & Sample \\
\hline & & $4 / 2 / 01$ & ND & 0.00008 & $\mathrm{~Bq} / \mathrm{m}^{3}$ & Sample \\
\hline & & $4 / 30 / 01$ & ND & 0.00008 & $\mathrm{~Bq} / \mathrm{m}^{3}$ & Sample \\
\hline & & $6 / 5 / 01$ & 0.00008 & 0.00006 & $\mathrm{~Bq} / \mathrm{m}^{3}$ & Sample \\
\hline & & $7 / 3 / 01$ & $N D$ & 0.00009 & $\mathrm{~Bq} / \mathrm{m}^{3}$ & Sample \\
\hline & & $8 / 7 / 01$ & 0.00006 & 0.00006 & $\mathrm{~Bq} / \mathrm{m}^{3}$ & Sample \\
\hline & & $9 / 4 / 01$ & ND & 0.00009 & $\mathrm{~Bq} / \mathrm{m}^{3}$ & Sample \\
\hline & & $10 / 1 / 01$ & ND & 0.00006 & $\mathrm{~Bq} / \mathrm{m}^{3}$ & Sample \\
\hline & & $11 / 5 / 01$ & 0.00011 & 0.00008 & $\mathrm{~Bq} / \mathrm{m}^{3}$ & Sample \\
\hline & & $12 / 3 / 01$ & ND & 0.00009 & $\mathrm{~Bq} / \mathrm{m}^{3}$ & Sample \\
\hline & & $1 / 7 / 02$ & ND & 0.00011 & $\mathrm{~Bq} / \mathrm{m}^{3}$ & Sample \\
\hline & \multirow[t]{13}{*}{ Travel Blank } & $1 / 9 / 01$ & ND & 0.19 & $\mathrm{~Bq} / \mathrm{S}$ & Blank \\
\hline & & $2 / 5 / 01$ & ND & 0.15 & $\mathrm{~Bq} / \mathrm{S}$ & Blank \\
\hline & & $3 / 6 / 01$ & ND & 0.2 & $\mathrm{~Bq} / \mathrm{S}$ & Blank \\
\hline & & $4 / 2 / 01$ & ND & 0.19 & $\mathrm{~Bq} / \mathrm{S}$ & Blank \\
\hline & & $5 / 1 / 01$ & ND & 0.19 & $\mathrm{~Bq} / \mathrm{S}$ & Blank \\
\hline & & $6 / 5 / 01$ & ND & 0.19 & $\mathrm{~Bq} / \mathrm{S}$ & Blank \\
\hline & & $7 / 2 / 01$ & ND & 0.2 & $\mathrm{~Bq} / \mathrm{S}$ & Blank \\
\hline & & $8 / 7 / 01$ & ND & 0.19 & $\mathrm{~Bq} / \mathrm{S}$ & Blank \\
\hline & & $9 / 4 / 01$ & ND & 0.2 & $\mathrm{~Bq} / \mathrm{S}$ & Blank \\
\hline & & $10 / 2 / 01$ & ND & 0.15 & $\mathrm{~Bq} / \mathrm{S}$ & Blank \\
\hline & & 11/7/01 & ND & 0.2 & $\mathrm{~Bq} / \mathrm{S}$ & Blank \\
\hline & & $12 / 4 / 01$ & ND & 0.2 & $\mathrm{~Bq} / \mathrm{S}$ & Blank \\
\hline & & $1 / 8 / 02$ & ND & 0.3 & $\mathrm{~Bq} / \mathrm{S}$ & Blank \\
\hline \multirow[t]{6}{*}{ Gross beta } & \multirow[t]{6}{*}{ ENV-69 } & $1 / 8 / 01$ & 0.00132 & 0.00007 & $\mathrm{~Bq} / \mathrm{m}^{3}$ & Sample \\
\hline & & $2 / 5 / 01$ & 0.00051 & 0.00008 & $\mathrm{~Bq} / \mathrm{m}^{3}$ & Sample \\
\hline & & $3 / 6 / 01$ & 0.00032 & 0.00008 & $\mathrm{~Bq} / \mathrm{m}^{3}$ & Sample \\
\hline & & $4 / 2 / 01$ & 0.00043 & 0.00008 & $\mathrm{~Bq} / \mathrm{m}^{3}$ & Sample \\
\hline & & $4 / 30 / 01$ & 0.00037 & 0.00008 & $\mathrm{~Bq} / \mathrm{m}^{3}$ & Sample \\
\hline & & $6 / 4 / 01$ & 0.000425 & 0.00006 & $\mathrm{~Bq} / \mathrm{m}^{3}$ & Sample \\
\hline
\end{tabular}




\begin{tabular}{|c|c|c|c|c|c|c|}
\hline \multirow{2}{*}{\multicolumn{2}{|c|}{$\begin{array}{l}\text { Analyte Location } \\
\text { Radiological Activity }\end{array}$}} & \multirow[t]{2}{*}{ Date } & \multirow[t]{2}{*}{ Result } & MDA or PQL & \multirow[t]{2}{*}{ Units } & \multirow[t]{2}{*}{ QC Type } \\
\hline & & & & & & \\
\hline $\begin{array}{l}\text { Gross beta } \\
\text { cont. }\end{array}$ & ENV-69 & $\begin{array}{l}7 / 2 / 01 \\
8 / 7 / 01 \\
9 / 4 / 01 \\
10 / 1 / 01 \\
11 / 6 / 01 \\
12 / 3 / 01 \\
1 / 7 / 02 \\
\\
1 / 8 / 01 \\
2 / 5 / 01 \\
2 / 19 / 01 \\
4 / 2 / 01 \\
4 / 30 / 01 \\
6 / 4 / 01 \\
7 / 2 / 01 \\
8 / 7 / 01 \\
9 / 4 / 01 \\
10 / 1 / 01 \\
11 / 5 / 01 \\
12 / 3 / 01 \\
1 / 8 / 02\end{array}$ & $\begin{array}{l}0.0004 \\
0.00032 \\
0.0004 \\
0.00049 \\
0.000803 \\
0.000684 \\
0.00032 \\
\\
0.00147 \\
0.000702 \\
0.00042 \\
0.0006 \\
0.00048 \\
0.000519 \\
0.00044 \\
0.00033 \\
0.0003 \\
0.000547 \\
0.000803 \\
0.000776 \\
0.00036\end{array}$ & $\begin{array}{l}0.00008 \\
0.00006 \\
0.00008 \\
0.00008 \\
0.00006 \\
0.00008 \\
0.00007 \\
\\
0.00007 \\
0.00008 \\
0.00015 \\
0.00018 \\
0.00008 \\
0.00006 \\
0.00007 \\
0.00006 \\
0.00007 \\
0.00007 \\
0.00006 \\
0.00008 \\
0.00007\end{array}$ & $\begin{array}{l}\mathrm{Bq} / \mathrm{m}^{3} \\
\mathrm{~Bq} / \mathrm{m}^{3} \\
\mathrm{~Bq} / \mathrm{m}^{3} \\
\mathrm{~Bq} / \mathrm{m}^{3} \\
\mathrm{~Bq} / \mathrm{m}^{3} \\
\mathrm{~Bq} / \mathrm{m}^{3} \\
\mathrm{~Bq} / \mathrm{m}^{3} \\
\\
\mathrm{~Bq} / \mathrm{m}^{3} \\
\mathrm{~Bq} / \mathrm{m}^{3} \\
\mathrm{~Bq} / \mathrm{m}^{3} \\
\mathrm{~Bq} / \mathrm{m}^{3} \\
\mathrm{~Bq} / \mathrm{m}^{3} \\
\mathrm{~Bq} / \mathrm{m}^{3} \\
\mathrm{~Bq} / \mathrm{m}^{3} \\
\mathrm{~Bq} / \mathrm{m}^{3} \\
\mathrm{~Bq} / \mathrm{m}^{3} \\
\mathrm{~Bq} / \mathrm{m}^{3} \\
\mathrm{~Bq} / \mathrm{m}^{3} \\
\mathrm{~Bq} / \mathrm{m}^{3} \\
\mathrm{~Bq} / \mathrm{m}^{3}\end{array}$ & $\begin{array}{l}\text { Sample } \\
\text { Sample } \\
\text { Sample } \\
\text { Sample } \\
\text { Sample } \\
\text { Sample } \\
\text { Sample } \\
\\
\text { Sample } \\
\text { Sample } \\
\text { Sample } \\
\text { Sample } \\
\text { Sample } \\
\text { Sample } \\
\text { Sample } \\
\text { Sample } \\
\text { Sample } \\
\text { Sample } \\
\text { Sample } \\
\text { Sample } \\
\text { Sample }\end{array}$ \\
\hline & ENV-81 & $\begin{array}{l}1 / 8 / 01 \\
2 / 5 / 01 \\
3 / 6 / 01 \\
4 / 2 / 01 \\
4 / 30 / 01 \\
6 / 5 / 01 \\
7 / 2 / 01 \\
8 / 7 / 01 \\
9 / 4 / 01 \\
10 / 1 / 01 \\
11 / 5 / 01 \\
12 / 3 / 01 \\
1 / 7 / 02\end{array}$ & $\begin{array}{l}0.00144 \\
0.0007 \\
0.00042 \\
0.00043 \\
0.00042 \\
0.000476 \\
0.00045 \\
0.00033 \\
0.00035 \\
0.000539 \\
0.000881 \\
0.000741 \\
0.000467\end{array}$ & $\begin{array}{l}0.00007 \\
0.00008 \\
0.00007 \\
0.00008 \\
0.00008 \\
0.00006 \\
0.00008 \\
0.00006 \\
0.00008 \\
0.00008 \\
0.00006 \\
0.00009 \\
0.00008\end{array}$ & $\begin{array}{l}\mathrm{Bq} / \mathrm{m}^{3} \\
\mathrm{~Bq} / \mathrm{m}^{3} \\
\mathrm{~Bq} / \mathrm{m}^{3} \\
\mathrm{~Bq} / \mathrm{m}^{3} \\
\mathrm{~Bq} / \mathrm{m}^{3} \\
\mathrm{~Bq} / \mathrm{m}^{3} \\
\mathrm{~Bq} / \mathrm{m}^{3} \\
\mathrm{~Bq} / \mathrm{m}^{3} \\
\mathrm{~Bq} / \mathrm{m}^{3} \\
\mathrm{~Bq} / \mathrm{m}^{3} \\
\mathrm{~Bq} / \mathrm{m}^{3} \\
\mathrm{~Bq} / \mathrm{m}^{3} \\
\mathrm{~Bq} / \mathrm{m}^{3}\end{array}$ & $\begin{array}{l}\text { Sample } \\
\text { Sample } \\
\text { Sample } \\
\text { Sample } \\
\text { Sample } \\
\text { Sample } \\
\text { Sample } \\
\text { Sample } \\
\text { Sample } \\
\text { Sample } \\
\text { Sample } \\
\text { Sample } \\
\text { Sample }\end{array}$ \\
\hline & ENV-B13C & $\begin{array}{l}1 / 8 / 01 \\
2 / 6 / 01 \\
3 / 5 / 01 \\
4 / 2 / 01 \\
4 / 30 / 01 \\
6 / 5 / 01 \\
7 / 3 / 01 \\
8 / 7 / 01 \\
9 / 4 / 01 \\
10 / 1 / 01\end{array}$ & $\begin{array}{l}0.00151 \\
0.000642 \\
0.00039 \\
0.00048 \\
0.00037 \\
0.000464 \\
0.0004 \\
0.00035 \\
0.00041 \\
0.000545\end{array}$ & $\begin{array}{l}0.00007 \\
0.00008 \\
0.00008 \\
0.00008 \\
0.00008 \\
0.00006 \\
0.00008 \\
0.00006 \\
0.00008 \\
0.00008\end{array}$ & $\begin{array}{l}\mathrm{Bq} / \mathrm{m}^{3} \\
\mathrm{~Bq} / \mathrm{m}^{3} \\
\mathrm{~Bq} / \mathrm{m}^{3} \\
\mathrm{~Bq} / \mathrm{m}^{3} \\
\mathrm{~Bq} / \mathrm{m}^{3} \\
\mathrm{~Bq} / \mathrm{m}^{3} \\
\mathrm{~Bq} / \mathrm{m}^{3} \\
\mathrm{~Bq} / \mathrm{m}^{3} \\
\mathrm{~Bq} / \mathrm{m}^{3} \\
\mathrm{~Bq} / \mathrm{m}^{3}\end{array}$ & $\begin{array}{l}\text { Sample } \\
\text { Sample } \\
\text { Sample } \\
\text { Sample } \\
\text { Sample } \\
\text { Sample } \\
\text { Sample } \\
\text { Sample } \\
\text { Sample } \\
\text { Sample }\end{array}$ \\
\hline
\end{tabular}




\section{Analyte \\ Radiological Activity}

Gross beta ENV-B13C cont.

Tritium

\section{(1)$$
\text { T }
$$

Travel Blank

$1 / 9 / 01$
$2 / 5 / 01$
$3 / 6 / 01$
$4 / 2 / 01$
$5 / 1 / 01$
$6 / 5 / 01$
$7 / 2 / 01$
$8 / 7 / 01$
$9 / 4 / 01$
$10 / 2 / 01$
$11 / 7 / 01$
$12 / 4 / 01$
$1 / 8 / 02$

ENV-31

$5 / 1 / 01$

$5 / 1 / 01$

$6 / 5 / 01$

$6 / 5 / 01$

$7 / 3 / 01$

$7 / 3 / 01$

$8 / 7 / 01$

$9 / 4 / 01$

$10 / 2 / 01$

$10 / 2 / 01$

$11 / 6 / 01$

$12 / 4 / 01$

$12 / 4 / 01$

$1 / 8 / 02$

$1 / 8 / 02$

ENV-44

$5 / 1 / 01$
$6 / 5 / 01$
$6 / 5 / 01$
$7 / 3 / 01$
$8 / 7 / 01$
$9 / 4 / 01$
$10 / 2 / 01$
$10 / 2 / 01$
$11 / 6 / 01$
$11 / 6 / 01$
$12 / 4 / 01$
$1 / 8 / 02$

Result MDA or PQL Units QC Type

$\begin{array}{llll}0.000782 & 0.00006 & \mathrm{~Bq} / \mathrm{m}^{3} & \text { Sample } \\ 0.000615 & 0.00008 & \mathrm{~Bq} / \mathrm{m}^{3} & \text { Sample } \\ 0.00029 & 0.00007 & \mathrm{~Bq} / \mathrm{m}^{3} & \text { Sample }\end{array}$

$\begin{array}{llll}0.34 & 0.2 & \mathrm{~Bq} / \mathrm{S} & \text { Blank }\end{array}$

$0.44 \quad 0.19 \quad \mathrm{~Bq} / \mathrm{S} \quad$ Blank

$0.28 \quad 0.19 \quad \mathrm{~Bq} / \mathrm{S} \quad$ Blank

$\begin{array}{llll}0.41 & 0.19 & \mathrm{~Bq} / \mathrm{S} & \text { Blank }\end{array}$

$\begin{array}{llll}0.31 & 0.19 & B q / S & B l a n k\end{array}$

$\begin{array}{llll}0.35 & 0.19 & B q / S & B l a n k\end{array}$

$0.44 \quad 0.19 \quad \mathrm{~Bq} / \mathrm{S} \quad$ Blank

$0.31 \quad 0.19 \quad \mathrm{~Bq} / \mathrm{S} \quad$ Blank

$0.35 \quad 0.19 \quad \mathrm{~Bq} / \mathrm{S} \quad$ Blank

$0.31 \quad 0.19 \quad \mathrm{~Bq} / \mathrm{S} \quad$ Blank

$\begin{array}{llll}0.41 & 0.19 & \mathrm{~Bq} / \mathrm{S} & \text { Blank }\end{array}$

$0.33 \quad 0.19 \quad \mathrm{~Bq} / \mathrm{S} \quad$ Blank

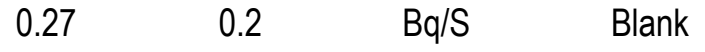

$\begin{array}{llll}0.3 & 0.18 & \mathrm{~Bq} / \mathrm{m}^{3} & \text { Sample }\end{array}$

$\begin{array}{lllr}0.326 & 0.08 & \mathrm{~Bq} / \mathrm{m}^{3} & \text { Split } \\ \text { ND } & 0.15 & \mathrm{~Bq} / \mathrm{m}^{3} & \text { Sample }\end{array}$

ND $\quad 0.15 \quad \mathrm{~Bq} / \mathrm{m}^{3} \quad$ Split

ND $\quad 0.18 \quad \mathrm{~Bq} / \mathrm{m}^{3} \quad$ Sample

$\begin{array}{lllr}0.166 & 0.013 & \mathrm{~Bq} / \mathrm{m}^{3} & \text { Split } \\ \text { ND } & 0.15 & \mathrm{~Bq} / \mathrm{m}^{3} & \text { Sample }\end{array}$

$\begin{array}{llll}0.32 & 0.18 & \mathrm{~Bq} / \mathrm{m}^{3} & \text { Sample }\end{array}$

ND $\quad 0.18 \quad \mathrm{~Bq} / \mathrm{m}^{3} \quad$ Sample

$\begin{array}{llll}\text { ND } & 0.18 & \mathrm{~Bq} / \mathrm{m}^{3} & \text { Split }\end{array}$

ND $\quad 0.15 \quad B q / \mathrm{m}^{3} \quad$ Sample

$\begin{array}{llll}\mathrm{ND} & 0.19 & \mathrm{~Bq} / \mathrm{m}^{3} & \text { Sample }\end{array}$

$\begin{array}{cllr}0.0871 & 0.013 & \mathrm{~Bq} / \mathrm{m}^{3} & \text { Split } \\ \mathrm{ND} & 0.15 & \mathrm{~Bq} / \mathrm{m}^{3} & \text { Sample } \\ 0.115 & 0.011 & \mathrm{~Bq} / \mathrm{m}^{3} & \text { Split }\end{array}$

$\begin{array}{cllr}0.479 & 0.18 & \mathrm{~Bq} / \mathrm{m}^{3} & \text { Sample } \\ 0.16 & 0.15 & \mathrm{~Bq} / \mathrm{m}^{3} & \text { Sample } \\ 0.19 & 0.15 & \mathrm{~Bq} / \mathrm{m}^{3} & \text { Split } \\ 0.509 & 0.18 & \mathrm{~Bq} / \mathrm{m}^{3} & \text { Sample } \\ \mathrm{ND} & 0.15 & \mathrm{~Bq} / \mathrm{m}^{3} & \text { Sample } \\ 0.21 & 0.18 & \mathrm{~Bq} / \mathrm{m}^{3} & \text { Sample } \\ 0.32 & 0.18 & \mathrm{~Bq} / \mathrm{m}^{3} & \text { Sample } \\ 0.419 & 0.09 & \mathrm{~Bq} / \mathrm{m}^{3} & \text { Split } \\ 0.29 & 0.15 & \mathrm{~Bq} / \mathrm{m}^{3} & \text { Sample } \\ 0.29 & 0.15 & \mathrm{~Bq} / \mathrm{m}^{3} & \text { Split } \\ \text { ND } & 0.2 & \mathrm{~Bq} / \mathrm{m}^{3} & \text { Sample } \\ 0.283 & 0.15 & \mathrm{~Bq} / \mathrm{m}^{3} & \text { Sample }\end{array}$




\section{Analyte Location
Radiological Activity}

Tritium

cont.

\begin{abstract}
ENV-69
\end{abstract}

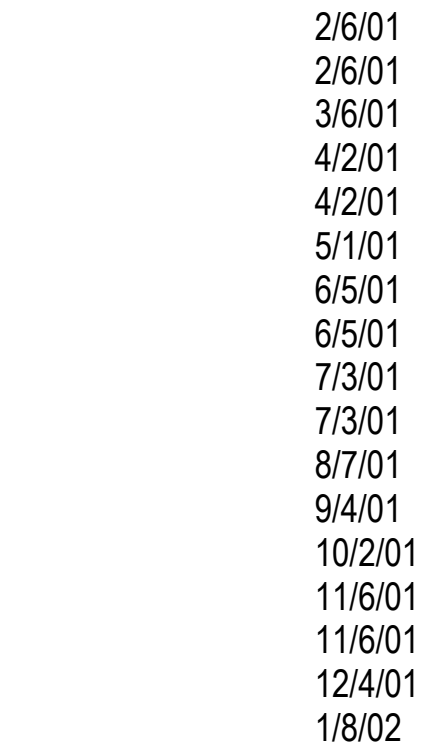

ENV-75EG
$1 / 9 / 01$

$1 / 9 / 01$

2/6/01

2/6/01

$3 / 6 / 01$

$3 / 6 / 01$

$4 / 3 / 01$

$4 / 3 / 01$

$5 / 1 / 01$

$5 / 1 / 01$

$6 / 5 / 01$

$6 / 5 / 01$

$7 / 3 / 01$

$7 / 3 / 01$

8/7/01

$8 / 7 / 01$

9/4/01

9/4/01

$10 / 2 / 01$

$10 / 2 / 01$

$11 / 6 / 01$

$11 / 6 / 01$

$12 / 4 / 01$

$12 / 4 / 01$

$1 / 8 / 02$

$1 / 8 / 02$

\begin{tabular}{|c|c|c|c|}
\hline 1.51 & 0.15 & $\mathrm{~Bq} / \mathrm{m}^{3}$ & Sample \\
\hline 1.23 & 0.18 & $\mathrm{~Bq} / \mathrm{m}^{3}$ & Sample \\
\hline 1.22 & 0.18 & $\mathrm{~Bq} / \mathrm{m}^{3}$ & Split \\
\hline 0.24 & 0.18 & $\mathrm{~Bq} / \mathrm{m}^{3}$ & Sample \\
\hline 0.894 & 0.19 & $\mathrm{~Bq} / \mathrm{m}^{3}$ & Sample \\
\hline 0.775 & 0.09 & $\mathrm{~Bq} / \mathrm{m}^{3}$ & Split \\
\hline 0.717 & 0.2 & $\mathrm{~Bq} / \mathrm{m}^{3}$ & Sample \\
\hline 0.395 & 0.15 & $\mathrm{~Bq} / \mathrm{m}^{3}$ & Sample \\
\hline 0.506 & 0.08 & $\mathrm{~Bq} / \mathrm{m}^{3}$ & Split \\
\hline 0.614 & 0.18 & $\mathrm{~Bq} / \mathrm{m}^{3}$ & Sample \\
\hline 0.588 & 0.18 & $\mathrm{~Bq} / \mathrm{m}^{3}$ & Split \\
\hline 0.682 & 0.15 & $\mathrm{~Bq} / \mathrm{m}^{3}$ & Sample \\
\hline 0.51 & 0.18 & $\mathrm{~Bq} / \mathrm{m}^{3}$ & Sample \\
\hline 0.883 & 0.18 & $\mathrm{~Bq} / \mathrm{m}^{3}$ & Sample \\
\hline 0.29 & 0.15 & $\mathrm{~Bq} / \mathrm{m}^{3}$ & Sample \\
\hline 0.25 & 0.15 & $\mathrm{~Bq} / \mathrm{m}^{3}$ & Split \\
\hline 0.29 & 0.18 & $\mathrm{~Bq} / \mathrm{m}^{3}$ & Sample \\
\hline 0.456 & 0.15 & $\mathrm{~Bq} / \mathrm{m}^{3}$ & Sample \\
\hline
\end{tabular}

$\begin{array}{llll}5.86 & 0.15 & \mathrm{~Bq} / \mathrm{m}^{3} & \text { Sample }\end{array}$

$3.67 \quad 0.01 \quad \mathrm{~Bq} / \mathrm{m}^{3} \quad$ Split

$\begin{array}{llll}4.67 & 0.18 & \mathrm{~Bq} / \mathrm{m}^{3} & \text { Sample }\end{array}$

$\begin{array}{llll}5.74 & 0.013 \quad \mathrm{~Bq} / \mathrm{m}^{3} & \text { Split }\end{array}$

$\begin{array}{llll}3.58 & 0.18 & \mathrm{~Bq} / \mathrm{m}^{3} & \text { Sample }\end{array}$

$\begin{array}{llll}4.27 & 0.013 \quad \mathrm{~Bq} / \mathrm{m}^{3} \quad \text { Split }\end{array}$

$2.69 \quad 0.18 \quad \mathrm{~Bq} / \mathrm{m}^{3} \quad$ Sample

$2.84 \quad 0.013 \quad \mathrm{~Bq} / \mathrm{m}^{3} \quad$ Split

$\begin{array}{llll}1.31 & 0.18 & \mathrm{~Bq} / \mathrm{m}^{3} & \text { Sample }\end{array}$

$\begin{array}{llll}1.63 & 0.08 & \mathrm{~Bq} / \mathrm{m}^{3} & \text { Split }\end{array}$

$1.29 \quad 0.15 \quad \mathrm{~Bq} / \mathrm{m}^{3} \quad$ Sample

$\begin{array}{llll}1.51 & 0.08 & \mathrm{~Bq} / \mathrm{m}^{3} & \text { Split }\end{array}$

$3.34 \quad 0.18 \quad \mathrm{~Bq} / \mathrm{m}^{3} \quad$ Sample

$\begin{array}{llll}4.41 & 0.09 & \mathrm{~Bq} / \mathrm{m}^{3} & \text { Split }\end{array}$

$1.53 \quad 0.15 \quad \mathrm{~Bq} / \mathrm{m}^{3} \quad$ Sample

$\begin{array}{llll}2.12 & 0.09 & \mathrm{~Bq} / \mathrm{m}^{3} & \text { Split }\end{array}$

$2.3 \quad 0.18 \quad \mathrm{~Bq} / \mathrm{m}^{3} \quad$ Sample

$\begin{array}{llll}2.19 & 0.1 & \mathrm{~Bq} / \mathrm{m}^{3} & \text { Split }\end{array}$

$2.85 \quad 0.18 \quad \mathrm{~Bq} / \mathrm{m}^{3} \quad$ Sample

$\begin{array}{llll}2.88 & 0.09 & \mathrm{~Bq} / \mathrm{m}^{3} & \text { Split }\end{array}$

$\begin{array}{llll}1.25 & 0.15 & \mathrm{~Bq} / \mathrm{m}^{3} & \text { Sample }\end{array}$

$\begin{array}{llll}1.62 & 0.08 & \mathrm{~Bq} / \mathrm{m}^{3} & \text { Split }\end{array}$

$\begin{array}{llll}1.17 & 0.18 & \mathrm{~Bq} / \mathrm{m}^{3} & \text { Sample }\end{array}$

$\begin{array}{llll}1.36 & 0.08 & \mathrm{~Bq} / \mathrm{m}^{3} & \text { Split }\end{array}$

$2.88 \quad 0.15 \quad \mathrm{~Bq} / \mathrm{m}^{3} \quad$ Sample

$\begin{array}{llll}2.73 & 0.06 \quad \mathrm{~Bq} / \mathrm{m}^{3} & \text { Split }\end{array}$ 
Analyte

\section{Radiological Activity}

Tritium cont.

ENV-77
$\quad 5 / 1 / 01$
$6 / 5 / 01$
$7 / 3 / 01$
$7 / 3 / 01$
$8 / 7 / 01$
$9 / 4 / 01$
$10 / 2 / 01$
$11 / 6 / 01$
$11 / 6 / 01$
$12 / 4 / 01$
$12 / 4 / 01$
$1 / 8 / 02$
$1 / 8 / 02$

ENV-78

ENV-85

$5 / 1 / 01$

$6 / 5 / 01$

$7 / 3 / 01$

$7 / 3 / 01$

$8 / 7 / 01$

$8 / 7 / 01$

$9 / 4 / 01$

$10 / 2 / 01$

$11 / 6 / 01$

$12 / 4 / 01$

$12 / 4 / 01$

$1 / 8 / 02$

$1 / 8 / 02$

$1 / 9 / 01$
Date

1.35

0.679

1.95

1.67

0.22

0.31

0.893

0.557

0.714

0.354

0.345

0.462

0.603

2/6/01

$3 / 6 / 01$

$3 / 6 / 01$

$4 / 3 / 01$

$5 / 1 / 01$

$6 / 5 / 01$

$7 / 3 / 01$

$8 / 7 / 01$

$8 / 7 / 01$

$9 / 4 / 01$

10/2/01

$11 / 6 / 01$

$12 / 4 / 01$

$12 / 4 / 01$

$1 / 8 / 02$

$1 / 8 / 02$
1.83

0.8

2.2

2.84

0.42

0.499

1.05

1.33

0.755

0.732

0.716

0.809

0.872

0.16

ND

ND

ND

ND

ND

0.27

ND

ND

0.0921

0.31

ND

ND

ND

0.13

ND

ND
0.18

0.15

0.18

0.18

0.15

0.18

0.18

0.15

0.08

0.18

0.18

0.15

0.15

$0.18 \mathrm{~Bq} / \mathrm{m}^{3}$

0.15

0.18

0.09

0.17

0.17

0.18

0.18

0.15

0.2

0.2

0.15

0.15

0.15

0.18

0.18

0.18

0.18

0.18

0.15

0.18

0.15

0.013

0.18

0.19

0.15

0.19

0.07

0.15

0.06

\section{$\mathrm{Bq} / \mathrm{m}^{3}$}

$\mathrm{Bq} / \mathrm{m}^{3}$

$\mathrm{Bg} / \mathrm{m}^{3}$

$\mathrm{Bq} / \mathrm{m}^{3}$

$\mathrm{Bq} / \mathrm{m}^{3}$

$\mathrm{Bq} / \mathrm{m}^{3}$

$\mathrm{Bg} / \mathrm{m}^{3}$

$\mathrm{Bg} / \mathrm{m}^{3}$

$\mathrm{Bq} / \mathrm{m}^{3}$

$\mathrm{Bg} / \mathrm{m}^{3}$

$\mathrm{Bq} / \mathrm{m}^{3}$

$\mathrm{Bg} / \mathrm{m}^{3}$

$\mathrm{Bq} / \mathrm{m}^{3}$

$\mathrm{Bq} / \mathrm{m}^{3}$

$\mathrm{Bg} / \mathrm{m}^{3}$

$\mathrm{Bg} / \mathrm{m}^{3}$

$\mathrm{Bg} / \mathrm{m}^{3}$

$\mathrm{Bg} / \mathrm{m}^{3}$

$\mathrm{Bq} / \mathrm{m}^{3}$

$\mathrm{Bg} / \mathrm{m}^{3}$

$\mathrm{Bq} / \mathrm{m}^{3}$

$\mathrm{Bq} / \mathrm{m}^{3}$

$\mathrm{Bq} / \mathrm{m}^{3}$

$\mathrm{Bq} / \mathrm{m}^{3}$

$\mathrm{Bq} / \mathrm{m}^{3}$

$\mathrm{Bq} / \mathrm{m}^{3}$

$\mathrm{Bq} / \mathrm{m}^{3}$

$\mathrm{Bg} / \mathrm{m}^{3}$

$\mathrm{Bq} / \mathrm{m}^{3}$

$\mathrm{Bg} / \mathrm{m}^{3}$

$\mathrm{Bq} / \mathrm{m}^{3}$

$\mathrm{Bq} / \mathrm{m}^{3}$

$\mathrm{Bq} / \mathrm{m}^{3}$

$\mathrm{Bq} / \mathrm{m}^{3}$

$\mathrm{Bq} / \mathrm{m}^{3}$

$\mathrm{Bq} / \mathrm{m}^{3}$

$\mathrm{Bq} / \mathrm{m}^{3}$

$\mathrm{Bq} / \mathrm{m}^{3}$

$\mathrm{Bq} / \mathrm{m}^{3}$

$\mathrm{Bq} / \mathrm{m}^{3}$

$\mathrm{Bg} / \mathrm{m}^{3}$

$\mathrm{Bq} / \mathrm{m}^{3}$
Sample
Sample
Sample
Split
Sample
Sample
Sample
Sample
Split
Sample
Split
Sample
Split

Sample

Sample

Sample

Split

Sample

Split

Sample

Sample

Sample

Sample

Split

Sample

Split

Sample

Sample

Sample

Split

Sample

Sample

Sample

Sample

Sample

Split

Sample

Sample

Sample

Sample

Split

Sample

Split 


\section{Analyte Location
Radiological Activity}

Tritium

cont.

ENV-AR $\quad 6 / 5 / 01$
$6 / 5 / 01$
$7 / 3 / 01$
$7 / 3 / 01$
$8 / 7 / 01$
$8 / 7 / 01$
$9 / 5 / 01$
$9 / 5 / 01$
$10 / 2 / 01$
$10 / 2 / 01$
$11 / 6 / 01$
$11 / 6 / 01$
$12 / 4 / 01$
$12 / 4 / 01$
$1 / 8 / 02$
$1 / 8 / 02$

ENV-B13A

ENB

$1 / 9 / 01$

$1 / 9 / 01$

$2 / 6 / 01$

$3 / 6 / 01$

$4 / 3 / 01$

$4 / 3 / 01$

$5 / 1 / 01$

$5 / 1 / 01$

$6 / 5 / 01$

$7 / 3 / 01$

8/7/01

9/4/01

9/4/01

$10 / 2 / 01$

$10 / 2 / 01$

$11 / 6 / 01$

$12 / 4 / 01$

$1 / 8 / 02$

ENV-B13C

$1 / 9 / 01$

$1 / 9 / 01$

$2 / 6 / 01$

$2 / 6 / 01$

$3 / 6 / 01$

$3 / 6 / 01$

$4 / 3 / 01$

$4 / 3 / 01$

$5 / 1 / 01$

$5 / 1 / 01$
Result MDA or PQL Units

QC Type

$\begin{array}{cllr}0.2 & 0.14 & \mathrm{~Bq} / \mathrm{m}^{3} & \text { Sample } \\ 0.237 & 0.01 & \mathrm{~Bq} / \mathrm{m}^{3} & \text { Split } \\ \mathrm{ND} & 0.18 & \mathrm{~Bq} / \mathrm{m}^{3} & \text { Sample } \\ 0.202 & 0.013 & \mathrm{~Bq} / \mathrm{m}^{3} & \text { Split } \\ \mathrm{ND} & 0.15 & \mathrm{~Bq} / \mathrm{m}^{3} & \text { Sample } \\ 0.0874 & 0.013 & \mathrm{~Bq} / \mathrm{m}^{3} & \text { Split } \\ 0.25 & 0.18 & \mathrm{~Bq} / \mathrm{m}^{3} & \text { Sample } \\ 0.0902 & 0.014 & \mathrm{~Bq} / \mathrm{m}^{3} & \text { Split } \\ \mathrm{ND} & 0.19 & \mathrm{~Bq} / \mathrm{m}^{3} & \text { Sample } \\ 0.0945 & 0.015 & \mathrm{~Bq} / \mathrm{m}^{3} & \text { Split } \\ \mathrm{ND} & 0.15 & \mathrm{~Bq} / \mathrm{m}^{3} & \text { Sample } \\ 0.0474 & 0.01 & \mathrm{~Bq} / \mathrm{m}^{3} & \text { Split } \\ \mathrm{ND} & 0.18 & \mathrm{~Bq} / \mathrm{m}^{3} & \text { Sample } \\ 0.0548 & 0.013 & \mathrm{~Bq} / \mathrm{m}^{3} & \text { Split } \\ 0.88 & 0.15 & \mathrm{~Bq} / \mathrm{m}^{3} & \text { Sample } \\ 0.817 & 0.011 & \mathrm{~Bq} / \mathrm{m}^{3} & \text { Split }\end{array}$

$\begin{array}{llll}0.16 & 0.15 & \mathrm{~Bq} / \mathrm{m}^{3} & \text { Sample }\end{array}$

$\begin{array}{llll}0.15 & 0.15 & \mathrm{~Bq} / \mathrm{m}^{3} & \text { Split }\end{array}$

ND

ND

0.18

$\mathrm{Bq} / \mathrm{m}^{3}$

$0.18 \quad \mathrm{~Bq} / \mathrm{m}^{3}$

0.18

$\mathrm{Bq} / \mathrm{m}^{3}$

$0.18 \quad \mathrm{~Bq} / \mathrm{m}^{3}$

$0.18 \mathrm{~Bq} / \mathrm{m}^{3}$

$0.013 \mathrm{~Bq} / \mathrm{m}^{3}$

$0.15 \mathrm{~Bq} / \mathrm{m}^{3}$

$0.18 \quad \mathrm{~Bq} / \mathrm{m}^{3}$

$0.15 \mathrm{~Bq} / \mathrm{m}^{3}$

$0.18 \mathrm{~Bq} / \mathrm{m}^{3}$

$0.18 \mathrm{~Bq} / \mathrm{m}^{3}$

$0.18 \mathrm{~Bq} / \mathrm{m}^{3}$

$0.014 \mathrm{~Bq} / \mathrm{m}^{3}$

$0.15 \mathrm{~Bq} / \mathrm{m}^{3}$

$0.18 \mathrm{~Bq} / \mathrm{m}^{3}$

0.15

$\mathrm{Bq} / \mathrm{m}^{3}$

Sample

Sample

Sample

Split

Sample

Split

Sample

Sample

Sample

Sample

Split

Sample

Split

Sample

Sample

Sample

$\begin{array}{cllr}\text { ND } & 0.15 & \mathrm{~Bq} / \mathrm{m}^{3} & \text { Sample } \\ 0.0466 & 0.01 & \mathrm{~Bq} / \mathrm{m}^{3} & \text { Split } \\ \text { ND } & 0.18 & \mathrm{~Bq} / \mathrm{m}^{3} & \text { Sample } \\ 0.0608 & 0.013 & \mathrm{~Bq} / \mathrm{m}^{3} & \text { Split } \\ \text { ND } & 0.18 & \mathrm{~Bq} / \mathrm{m}^{3} & \text { Sample } \\ 0.0285 & 0.013 & \mathrm{~Bq} / \mathrm{m}^{3} & \text { Split } \\ \text { ND } & 0.18 & \mathrm{~Bq} / \mathrm{m}^{3} & \text { Sample } \\ 0.0439 & 0.013 & \mathrm{~Bq} / \mathrm{m}^{3} & \text { Split } \\ \text { ND } & 0.18 & \mathrm{~Bq} / \mathrm{m}^{3} & \text { Sample } \\ \text { ND } & 0.18 & \mathrm{~Bq} / \mathrm{m}^{3} & \text { Split }\end{array}$




\section{Analyte \\ Radiological Activity}

Tritium cont.

\begin{tabular}{|c|c|c|c|c|c|}
\hline & $6 / 5 / 01$ & 0.32 & 0.15 & $\mathrm{~Bq} / \mathrm{m}^{3}$ & Sample \\
\hline & $6 / 5 / 01$ & 0.311 & 0.011 & $\mathrm{~Bq} / \mathrm{m}^{3}$ & Split \\
\hline & $7 / 3 / 01$ & ND & 0.18 & $\mathrm{~Bq} / \mathrm{m}^{3}$ & Sample \\
\hline & $8 / 7 / 01$ & ND & 0.15 & $\mathrm{~Bq} / \mathrm{m}^{3}$ & Sample \\
\hline & $9 / 4 / 01$ & ND & 0.18 & $\mathrm{~Bq} / \mathrm{m}^{3}$ & Sample \\
\hline & $9 / 4 / 01$ & ND & 0.18 & $\mathrm{~Bq} / \mathrm{m}^{3}$ & Split \\
\hline & $10 / 2 / 01$ & ND & 0.18 & $\mathrm{~Bq} / \mathrm{m}^{3}$ & Sample \\
\hline & $11 / 6 / 01$ & ND & 0.15 & $\mathrm{~Bq} / \mathrm{m}^{3}$ & Sample \\
\hline & $11 / 6 / 01$ & 0.037 & 0.011 & $\mathrm{~Bq} / \mathrm{m}^{3}$ & Split \\
\hline & $12 / 4 / 01$ & ND & 0.18 & $\mathrm{~Bq} / \mathrm{m}^{3}$ & Sample \\
\hline & $1 / 8 / 02$ & ND & 0.15 & $\mathrm{~Bq} / \mathrm{m}^{3}$ & Sample \\
\hline ENV-B13D & $1 / 9 / 01$ & ND & 0.15 & $\mathrm{~Bq} / \mathrm{m}^{3}$ & Sample \\
\hline & $1 / 9 / 01$ & 0.14 & 0.06 & $\mathrm{~Bq} / \mathrm{m}^{3}$ & Split \\
\hline & $2 / 6 / 01$ & ND & 0.18 & $\mathrm{~Bq} / \mathrm{m}^{3}$ & Sample \\
\hline & $2 / 6 / 01$ & 0.076 & 0.07 & $\mathrm{~Bq} / \mathrm{m}^{3}$ & Split \\
\hline & $3 / 6 / 01$ & ND & 0.18 & $\mathrm{~Bq} / \mathrm{m}^{3}$ & Sample \\
\hline & $3 / 6 / 01$ & 0.12 & 0.07 & $\mathrm{~Bq} / \mathrm{m}^{3}$ & Split \\
\hline & $4 / 3 / 01$ & ND & 0.18 & $\mathrm{~Bq} / \mathrm{m}^{3}$ & Sample \\
\hline & $4 / 3 / 01$ & 0.13 & 0.08 & $\mathrm{~Bq} / \mathrm{m}^{3}$ & Split \\
\hline & $5 / 1 / 01$ & ND & 0.18 & $\mathrm{~Bq} / \mathrm{m}^{3}$ & Sample \\
\hline & $5 / 1 / 01$ & ND & 0.18 & $\mathrm{~Bq} / \mathrm{m}^{3}$ & Split \\
\hline & $6 / 5 / 01$ & ND & 0.18 & $\mathrm{~Bq} / \mathrm{m}^{3}$ & Sample \\
\hline & $7 / 3 / 01$ & ND & 0.18 & $\mathrm{~Bq} / \mathrm{m}^{3}$ & Sample \\
\hline & $8 / 7 / 01$ & 0.16 & 0.15 & $\mathrm{~Bq} / \mathrm{m}^{3}$ & Sample \\
\hline & $9 / 4 / 01$ & 0.22 & 0.18 & $\mathrm{~Bq} / \mathrm{m}^{3}$ & Sample \\
\hline & $9 / 4 / 01$ & 0.23 & 0.09 & $\mathrm{~Bq} / \mathrm{m}^{3}$ & Split \\
\hline & $10 / 2 / 01$ & 0.22 & 0.18 & $\mathrm{~Bq} / \mathrm{m}^{3}$ & Sample \\
\hline & $10 / 2 / 01$ & 0.23 & 0.18 & $\mathrm{~Bq} / \mathrm{m}^{3}$ & Split \\
\hline & $11 / 6 / 01$ & ND & 0.15 & $\mathrm{~Bq} / \mathrm{m}^{3}$ & Sample \\
\hline & $12 / 4 / 01$ & 0.421 & 0.19 & $\mathrm{~Bq} / \mathrm{m}^{3}$ & Sample \\
\hline & $1 / 8 / 02$ & ND & 0.15 & $\mathrm{~Bq} / \mathrm{m}^{3}$ & Sample \\
\hline ENV-LHS & $1 / 9 / 01$ & 1.42 & 0.15 & $\mathrm{~Bq} / \mathrm{m}^{3}$ & Sample \\
\hline & $1 / 9 / 01$ & 1.36 & 0.06 & $\mathrm{Bg} / \mathrm{m}^{3}$ & Split \\
\hline & $2 / 6 / 01$ & 1.29 & 0.18 & $\mathrm{~Bq} / \mathrm{m}^{3}$ & Sample \\
\hline & $2 / 6 / 01$ & 1.21 & 0.07 & $\mathrm{~Bq} / \mathrm{m}^{3}$ & Split \\
\hline & $3 / 6 / 01$ & 2.27 & 0.18 & $\mathrm{~Bq} / \mathrm{m}^{3}$ & Sample \\
\hline & $3 / 6 / 01$ & 2.3 & 0.07 & $\mathrm{~Bq} / \mathrm{m}^{3}$ & Split \\
\hline & $4 / 3 / 01$ & 1.05 & 0.18 & $\mathrm{~Bq} / \mathrm{m}^{3}$ & Sample \\
\hline & $4 / 3 / 01$ & 1.05 & 0.08 & $\mathrm{~Bq} / \mathrm{m}^{3}$ & Split \\
\hline & $5 / 1 / 01$ & 0.775 & 0.18 & $\mathrm{~Bq} / \mathrm{m}^{3}$ & Sample \\
\hline & $5 / 1 / 01$ & 0.797 & 0.07 & $\mathrm{~Bq} / \mathrm{m}^{3}$ & Split \\
\hline & $6 / 5 / 01$ & 0.449 & 0.15 & $\mathrm{~Bq} / \mathrm{m}^{3}$ & Sample \\
\hline & $6 / 5 / 01$ & 0.572 & 0.08 & $\mathrm{Bg} / \mathrm{m}^{3}$ & Split \\
\hline & $7 / 3 / 01$ & 0.95 & 0.18 & $\mathrm{~Bq} / \mathrm{m}^{3}$ & Sample \\
\hline
\end{tabular}




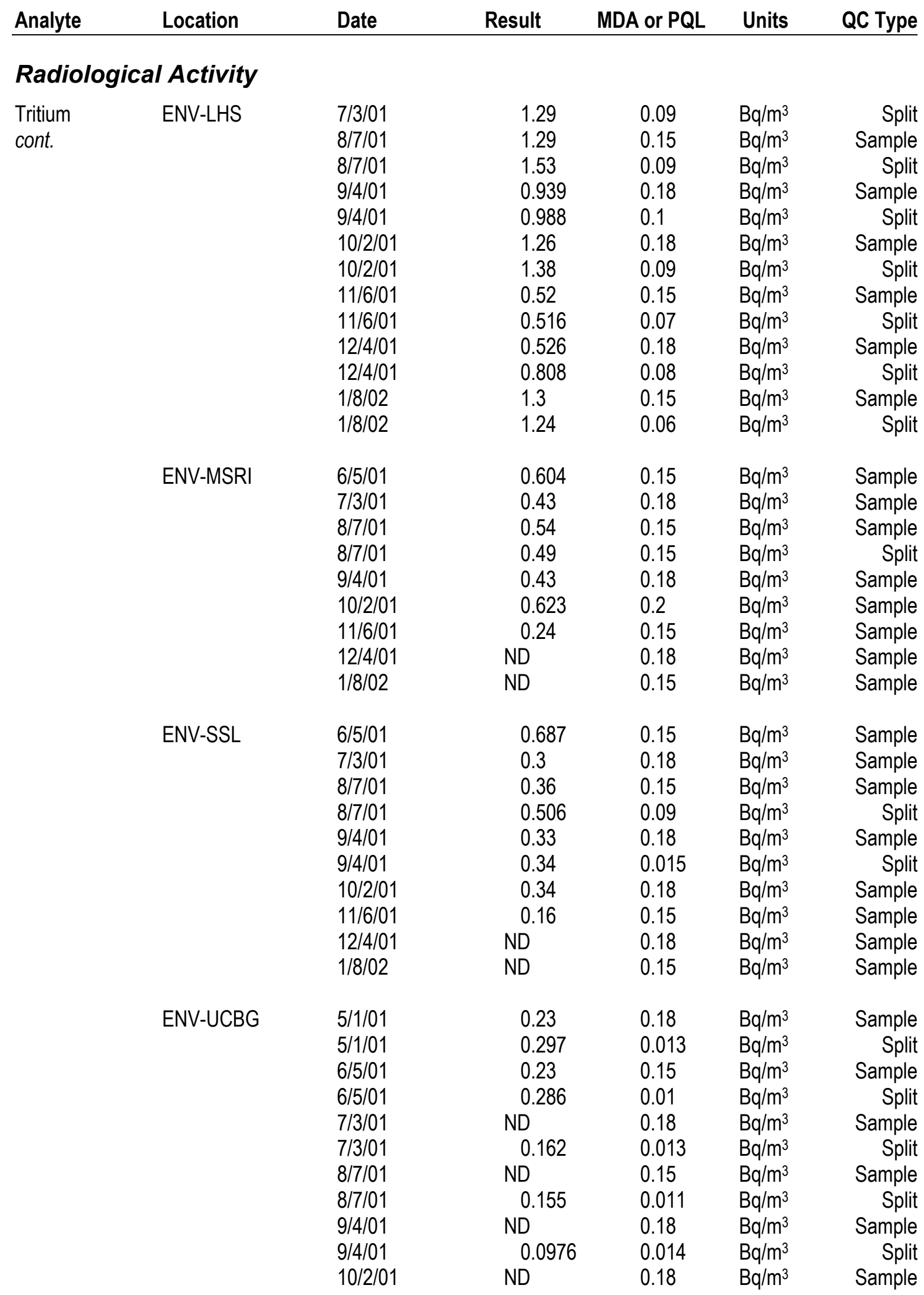




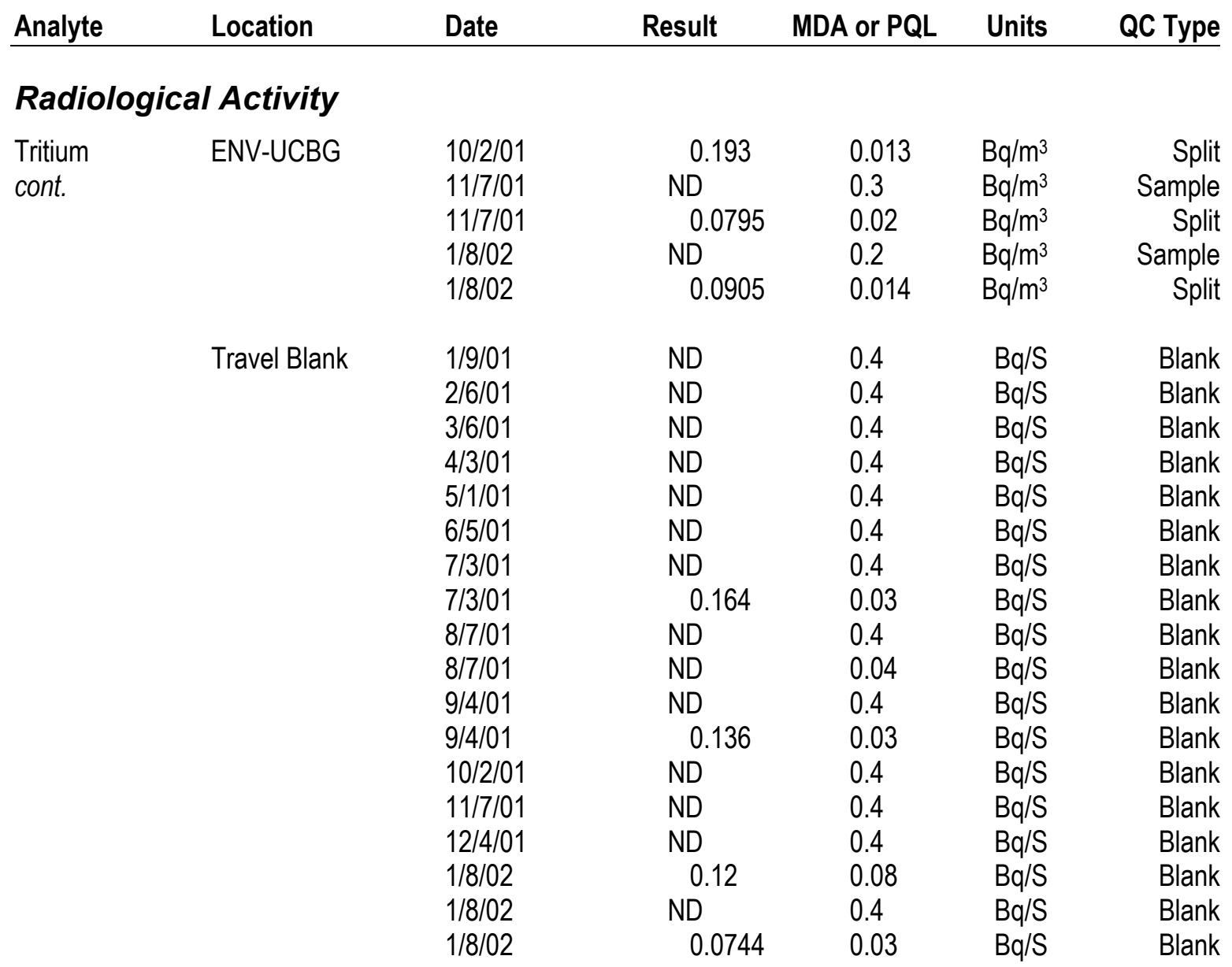


The following rainwater data are summarized and discussed in Chapter 5 (Surface Waters and Wastewater) of the Site Environmental Report for 2001 (see Volume I):

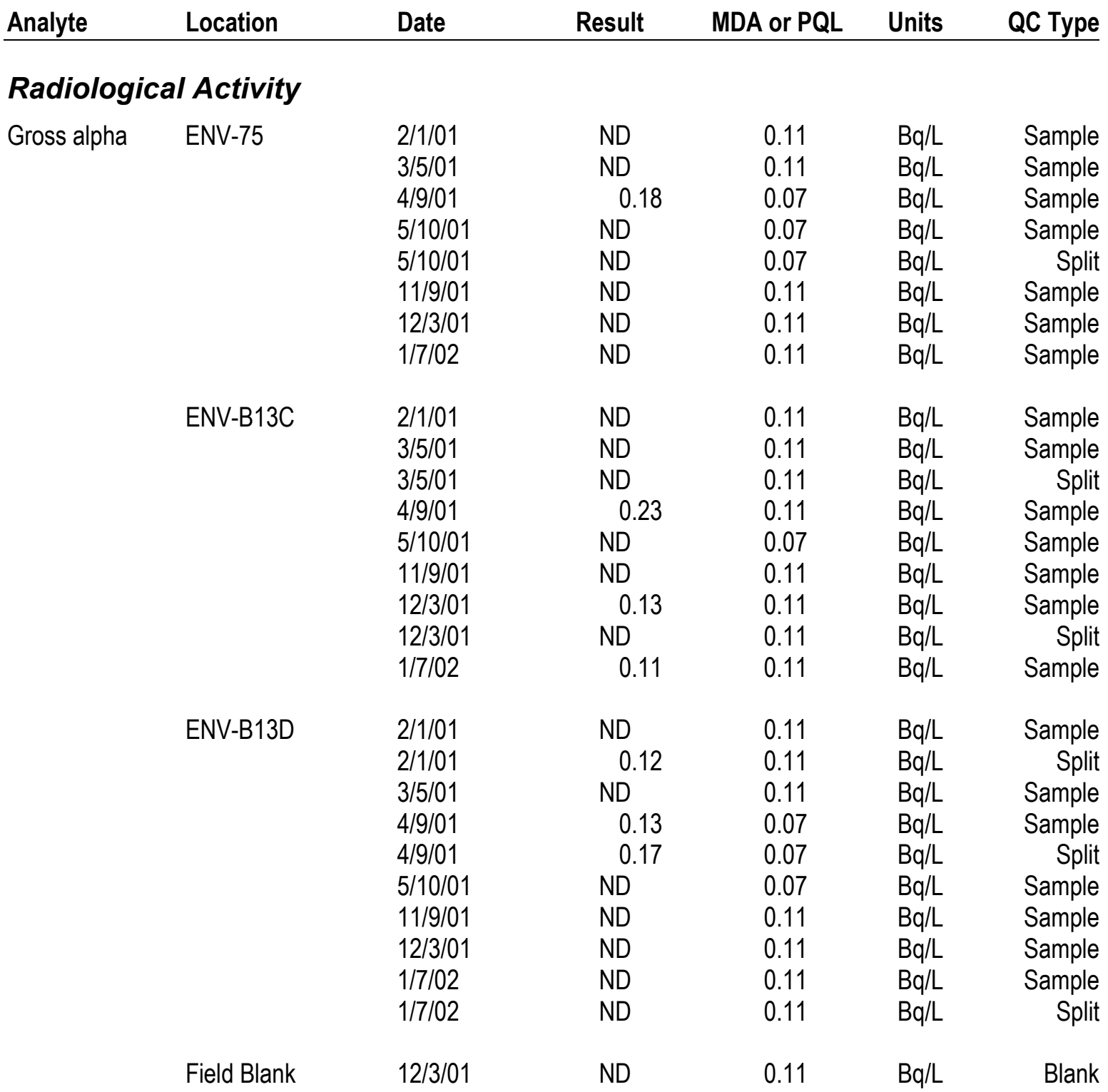


Analyte

Location

Date

Result MDA or PQL

Units QC Type

\section{Radiological Activity}

\begin{tabular}{|c|c|c|c|c|c|c|}
\hline \multirow[t]{2}{*}{ Gross beta } & ENV-75 & $\begin{array}{l}2 / 1 / 01 \\
3 / 5 / 01 \\
4 / 9 / 01 \\
5 / 10 / 01 \\
5 / 10 / 01 \\
11 / 9 / 01 \\
12 / 3 / 01 \\
1 / 7 / 02\end{array}$ & $\begin{array}{c}0.26 \\
\text { ND } \\
0.11 \\
\text { ND } \\
\text { ND } \\
0.2 \\
0.11 \\
\text { ND }\end{array}$ & $\begin{array}{l}0.11 \\
0.11 \\
0.11 \\
0.11 \\
0.11 \\
0.07 \\
0.07 \\
0.11\end{array}$ & $\begin{array}{l}\mathrm{Bq} / \mathrm{L} \\
\mathrm{Bq} / \mathrm{L} \\
\mathrm{Bq} / \mathrm{L} \\
\mathrm{Bq} / \mathrm{L} \\
\mathrm{Bq} / \mathrm{L} \\
\mathrm{Bq} / \mathrm{L} \\
\mathrm{Bq} / \mathrm{L} \\
\mathrm{Bq} / \mathrm{L}\end{array}$ & $\begin{array}{r}\text { Sample } \\
\text { Sample } \\
\text { Sample } \\
\text { Sample } \\
\text { Split } \\
\text { Sample } \\
\text { Sample } \\
\text { Sample }\end{array}$ \\
\hline & ENV-B13C & $\begin{array}{l}2 / 1 / 01 \\
3 / 5 / 01 \\
3 / 5 / 01 \\
4 / 9 / 01 \\
5 / 10 / 01 \\
11 / 9 / 01 \\
12 / 3 / 01 \\
12 / 3 / 01 \\
1 / 7 / 02\end{array}$ & $\begin{array}{l}0.13 \\
0.11 \\
N D \\
0.33 \\
0.13 \\
0.28 \\
0.21 \\
0.14 \\
0.15\end{array}$ & $\begin{array}{l}0.11 \\
0.11 \\
0.11 \\
0.11 \\
0.11 \\
0.07 \\
0.11 \\
0.11 \\
0.11\end{array}$ & $\begin{array}{l}\mathrm{Bq} / \mathrm{L} \\
\mathrm{Bq} / \mathrm{L} \\
\mathrm{Bq} / \mathrm{L} \\
\mathrm{Bq} / \mathrm{L} \\
\mathrm{Bq} / \mathrm{L} \\
\mathrm{Bq} / \mathrm{L} \\
\mathrm{Bq} / \mathrm{L} \\
\mathrm{Bq} / \mathrm{L} \\
\mathrm{Bq} / \mathrm{L}\end{array}$ & $\begin{array}{r}\text { Sample } \\
\text { Sample } \\
\text { Split } \\
\text { Sample } \\
\text { Sample } \\
\text { Sample } \\
\text { Sample } \\
\text { Split } \\
\text { Sample }\end{array}$ \\
\hline & ENV-B13D & $\begin{array}{l}2 / 1 / 01 \\
2 / 1 / 01 \\
3 / 5 / 01 \\
4 / 9 / 01 \\
4 / 9 / 01 \\
5 / 10 / 01 \\
11 / 9 / 01 \\
12 / 3 / 01 \\
1 / 7 / 02 \\
1 / 7 / 02\end{array}$ & $\begin{array}{l}\text { ND } \\
\text { ND } \\
\text { ND } \\
0.11 \\
0.14 \\
N D \\
N D \\
N D \\
N D \\
N D\end{array}$ & $\begin{array}{l}0.11 \\
0.11 \\
0.11 \\
0.11 \\
0.11 \\
0.11 \\
0.07 \\
0.07 \\
0.11 \\
0.11\end{array}$ & $\begin{array}{l}\mathrm{Bq} / \mathrm{L} \\
\mathrm{Bq} / \mathrm{L} \\
\mathrm{Bq} / \mathrm{L} \\
\mathrm{Bq} / \mathrm{L} \\
\mathrm{Bq} / \mathrm{L} \\
\mathrm{Bq} / \mathrm{L} \\
\mathrm{Bq} / \mathrm{L} \\
\mathrm{Bq} / \mathrm{L} \\
\mathrm{Bq} / \mathrm{L} \\
\mathrm{Bq} / \mathrm{L}\end{array}$ & $\begin{array}{r}\text { Sample } \\
\text { Split } \\
\text { Sample } \\
\text { Sample } \\
\text { Split } \\
\text { Sample } \\
\text { Sample } \\
\text { Sample } \\
\text { Sample } \\
\text { Split }\end{array}$ \\
\hline & Field Blank & $12 / 3 / 01$ & ND & 0.07 & $\mathrm{~Bq} / \mathrm{L}$ & Blank \\
\hline \multirow[t]{2}{*}{ Tritium } & EG-RG-2M & $\begin{array}{l}1 / 24 / 01 \\
1 / 26 / 01 \\
1 / 29 / 01 \\
2 / 13 / 01 \\
2 / 23 / 01 \\
3 / 1 / 01 \\
3 / 2 / 01 \\
3 / 9 / 01 \\
4 / 10 / 01 \\
5 / 8 / 01\end{array}$ & $\begin{array}{r}125 \\
186 \\
1050 \\
221 \\
204 \\
110 \\
4670 \\
118 \\
67 \\
280\end{array}$ & $\begin{array}{l}7 \\
7 \\
7 \\
7 \\
7 \\
7 \\
8 \\
7 \\
7 \\
7\end{array}$ & $\begin{array}{l}\mathrm{Bq} / \mathrm{L} \\
\mathrm{Bq} / \mathrm{L} \\
\mathrm{Bq} / \mathrm{L} \\
\mathrm{Bq} / \mathrm{L} \\
\mathrm{Bq} / \mathrm{L} \\
\mathrm{Bq} / \mathrm{L} \\
\mathrm{Bq} / \mathrm{L} \\
\mathrm{Bq} / \mathrm{L} \\
\mathrm{Bq} / \mathrm{L} \\
\mathrm{Bq} / \mathrm{L}\end{array}$ & $\begin{array}{l}\text { Sample } \\
\text { Sample } \\
\text { Sample } \\
\text { Sample } \\
\text { Sample } \\
\text { Sample } \\
\text { Sample } \\
\text { Sample } \\
\text { Sample } \\
\text { Sample }\end{array}$ \\
\hline & EG-RG-7M & $\begin{array}{l}1 / 24 / 01 \\
1 / 26 / 01 \\
1 / 29 / 01\end{array}$ & $\begin{array}{r}271 \\
726 \\
6070\end{array}$ & $\begin{array}{l}7 \\
7 \\
9\end{array}$ & $\begin{array}{l}\mathrm{Bq} / \mathrm{L} \\
\mathrm{Bq} / \mathrm{L} \\
\mathrm{Bq} / \mathrm{L}\end{array}$ & $\begin{array}{l}\text { Sample } \\
\text { Sample } \\
\text { Sample }\end{array}$ \\
\hline
\end{tabular}




\section{Analyte Location
Radiological Activity}

Tritium

EG-RG-7M

$2 / 13 / 01$

2/23/01

$3 / 1 / 01$

$3 / 2 / 01$

$3 / 9 / 01$

$4 / 10 / 01$

$5 / 8 / 01$

EG-RG-AA

$1 / 24 / 01$

$1 / 26 / 01$

$1 / 29 / 01$

$2 / 13 / 01$

2/23/01

$3 / 1 / 01$

$3 / 2 / 01$

$3 / 9 / 01$

$4 / 10 / 01$

$5 / 8 / 01$

EG-RG-FG

$1 / 24 / 01$

$1 / 26 / 01$

$1 / 29 / 01$

$2 / 13 / 01$

2/23/01

$3 / 1 / 01$

$3 / 2 / 01$

$3 / 9 / 01$

$4 / 10 / 01$

$5 / 8 / 01$

ENV-75

$2 / 1 / 01$
$2 / 1 / 01$
$3 / 5 / 01$
$3 / 5 / 01$
$4 / 9 / 01$
$4 / 9 / 01$
$5 / 10 / 01$
$5 / 10 / 01$
$11 / 9 / 01$
$11 / 9 / 01$
$12 / 3 / 01$
$12 / 3 / 01$
$1 / 7 / 02$
$1 / 7 / 02$

2/1/01

$2 / 1 / 01$

$3 / 5 / 01$

$4 / 9 / 01$

$4 / 9 / 01$

$5 / 10 / 01$

$5 / 10 / 01$

$11 / 9 / 01$

$11 / 9 / 01$

$12 / 3 / 01$

$12 / 3 / 01$

$1 / 7 / 02$

$\begin{array}{rr}526 & 8 \\ 863 & 8 \\ 250 & 7 \\ 35600 & 20 \\ 289 & 7 \\ 312 & 7 \\ 1290 & 7\end{array}$

$\begin{array}{rll}8 & \mathrm{~Bq} / \mathrm{L} & \text { Sample } \\ 8 & \mathrm{~Bq} / \mathrm{L} & \text { Sample } \\ 7 & \mathrm{~Bq} / \mathrm{L} & \text { Sample } \\ 20 & \mathrm{~Bq} / \mathrm{L} & \text { Sample } \\ 7 & \mathrm{~Bq} / \mathrm{L} & \text { Sample } \\ 7 & \mathrm{~Bq} / \mathrm{L} & \text { Sample } \\ 7 & \mathrm{~Bq} / \mathrm{L} & \text { Sample }\end{array}$

$\begin{array}{rr}511 \\ 293 \\ 2090 \\ 1060 \\ 522 \\ 199 & 7 \\ 20800 & 18 \\ 243 & 7 \\ 181 & 7 \\ 937 & \end{array}$

$\begin{array}{ll}\mathrm{Bq} / \mathrm{L} & \text { Sample } \\ \mathrm{Bq} / \mathrm{L} & \text { Sample } \\ \mathrm{Bq} / \mathrm{L} & \text { Sample } \\ \mathrm{Bq} / \mathrm{L} & \text { Sample } \\ \mathrm{Bq} / \mathrm{L} & \text { Sample } \\ \mathrm{Bq} / \mathrm{L} & \text { Sample } \\ \mathrm{Bq} / \mathrm{L} & \text { Sample } \\ \mathrm{Bq} / \mathrm{L} & \text { Sample } \\ \mathrm{Bq} / \mathrm{L} & \text { Sample } \\ \mathrm{Bq} / \mathrm{L} & \text { Sample }\end{array}$

$\begin{array}{cccc}59.3 & 7 & \mathrm{~Bq} / \mathrm{L} & \text { Sample } \\ 85.2 & 7 & \mathrm{~Bq} / \mathrm{L} & \text { Sample } \\ 1290 & 7 & \mathrm{~Bq} / \mathrm{L} & \text { Sample } \\ 220 & 8 & \mathrm{~Bq} / \mathrm{L} & \text { Sample } \\ 151 & 8 & \mathrm{~Bq} / \mathrm{L} & \text { Sample } \\ 123 & 7 & \mathrm{~Bq} / \mathrm{L} & \text { Sample } \\ 6410 & 9 & \mathrm{~Bq} / \mathrm{L} & \text { Sample } \\ 188 & 7 & \mathrm{~Bq} / \mathrm{L} & \text { Sample } \\ 43.7 & 7 & \mathrm{~Bq} / \mathrm{L} & \text { Sample } \\ 253 & 7 & \mathrm{~Bq} / \mathrm{L} & \text { Sample }\end{array}$

ND 8

$8 \quad 8$

ND

ND

9.9

9.6

ND

ND

$\mathrm{Bq} / \mathrm{L} \quad$ Sample

$\mathrm{Bq} / \mathrm{L} \quad$ Split

$\mathrm{Bq} / \mathrm{L} \quad$ Sample

$\mathrm{Bq} / \mathrm{L} \quad$ Split

$\mathrm{Bq} / \mathrm{L} \quad$ Sample

$\mathrm{Bq} / \mathrm{L} \quad$ Split

Bq/L Sample

$\mathrm{Bq} / \mathrm{L} \quad$ Split

$\begin{array}{llll}\text { ND } & 7 & \mathrm{~Bq} / \mathrm{L} & \text { Sample }\end{array}$

$\begin{array}{llll}\text { ND } & 7 & \mathrm{~Bq} / \mathrm{L} & \text { Split }\end{array}$

ND $\quad 7$

ND $\quad 7$

ND $\quad 7$

ND $\quad 7$

$\mathrm{Bq} / \mathrm{L}$

$\mathrm{Bq} / \mathrm{L}$

$\mathrm{Bq} / \mathrm{L}$

$\mathrm{Bq} / \mathrm{L}$

Sample Split

Sample Split 
Analyte

\section{Radiological Activity}

Tritium

cont.
Date

2/1/01

$2 / 1 / 01$

$3 / 5 / 01$

$3 / 5 / 01$

$4 / 9 / 01$

$4 / 9 / 01$

$5 / 10 / 01$

$5 / 10 / 01$

$11 / 9 / 01$

$11 / 9 / 01$

$12 / 3 / 01$

$12 / 3 / 01$

$1 / 7 / 02$

$1 / 7 / 02$

ENV-B13D

2/1/01

2/1/01

$3 / 5 / 01$

$3 / 5 / 01$

$4 / 9 / 01$

$4 / 9 / 01$

$5 / 10 / 01$

$5 / 10 / 01$

11/9/01

$11 / 9 / 01$

$12 / 3 / 01$

$12 / 3 / 01$

$1 / 7 / 02$

$1 / 7 / 02$

12/3/01
ND

ND

ND

ND

9.3

ND

ND

ND

ND

ND

ND

ND

ND

ND

ND

ND

ND

ND

ND

ND

ND

ND

ND

ND

ND

ND

ND

ND

ND
MDA or PQL

Units

QC Type

3

8

8

7

7

7

7

7

7

7

7

7

7

7

7

7

8

7

7

7

7

7

7

7

7

7

7

7

7

7

$\begin{array}{lr}\mathrm{Bq} / \mathrm{L} & \text { Sample } \\ \mathrm{Bq} / \mathrm{L} & \text { Split } \\ \mathrm{Bq} / \mathrm{L} & \text { Sample } \\ \mathrm{Bq} / \mathrm{L} & \text { Split } \\ \mathrm{Bq} / \mathrm{L} & \text { Sample } \\ \mathrm{Bq} / \mathrm{L} & \text { Split } \\ \mathrm{Bq} / \mathrm{L} & \text { Sample } \\ \mathrm{Bq} / \mathrm{L} & \text { Split } \\ \mathrm{Bq} / \mathrm{L} & \text { Sample } \\ \mathrm{Bq} / \mathrm{L} & \text { Split } \\ \mathrm{Bq} / \mathrm{L} & \text { Sample } \\ \mathrm{Bq} / \mathrm{L} & \text { Split } \\ \mathrm{Bq} / \mathrm{L} & \text { Sample } \\ \mathrm{Bq} / \mathrm{L} & \text { Split }\end{array}$

Bq/L Sample

$\mathrm{Bq} / \mathrm{L} \quad$ Split

$\mathrm{Bq} / \mathrm{L} \quad$ Sample

$\mathrm{Bq} / \mathrm{L} \quad$ Split

$\mathrm{Bq} / \mathrm{L} \quad$ Sample

$\mathrm{Bq} / \mathrm{L} \quad$ Split

$\mathrm{Bq} / \mathrm{L} \quad$ Sample

$\mathrm{Bq} / \mathrm{L} \quad$ Split

$\mathrm{Bq} / \mathrm{L} \quad$ Sample

$\mathrm{Bq} / \mathrm{L} \quad$ Split

$\mathrm{Bq} / \mathrm{L} \quad$ Sample

$\mathrm{Bq} / \mathrm{L} \quad$ Split

$\mathrm{Bq} / \mathrm{L} \quad$ Sample

$\mathrm{Bq} / \mathrm{L} \quad$ Split

$\mathrm{Bq} / \mathrm{L} \quad$ Blank 


\section{Creeks}

The following creeks data are summarized and discussed in Chapter 5 (Surface Waters and Wastewater) of the Site Environmental Report for 2001 (see Volume I). Supplemental sampling data are included in the Supplemental Monitoring section of this volume and discussed in Chapter 10 (Supplemental Monitoring) of Volume I:

\begin{tabular}{|c|c|c|c|c|c|c|}
\hline Analyte & Location & Date & Result & $\begin{array}{r}\text { MDA } \\
\text { or PQL } \\
\end{array}$ & Units & QC Type \\
\hline \multicolumn{7}{|c|}{ Radiological Activity } \\
\hline \multirow[t]{6}{*}{ Gross alpha } & Chicken Creek & $\begin{array}{l}2 / 15 / 01 \\
5 / 25 / 01 \\
5 / 25 / 01 \\
8 / 30 / 01 \\
12 / 21 / 01 \\
12 / 21 / 01\end{array}$ & $\begin{array}{l}\text { ND } \\
\text { ND } \\
\text { ND } \\
0.074 \\
0.1 \\
N D\end{array}$ & $\begin{array}{l}0.15 \\
0.11 \\
0.11 \\
0.07 \\
0.11 \\
0.11\end{array}$ & $\begin{array}{l}\mathrm{Bq} / \mathrm{L} \\
\mathrm{Bq} / \mathrm{L} \\
\mathrm{Bq} / \mathrm{L} \\
\mathrm{Bq} / \mathrm{L} \\
\mathrm{Bq} / \mathrm{L} \\
\mathrm{Bq} / \mathrm{L}\end{array}$ & $\begin{array}{r}\text { Sample } \\
\text { Sample } \\
\text { Split } \\
\text { Sample } \\
\text { Sample } \\
\text { Split }\end{array}$ \\
\hline & Claremont Creek & $\begin{array}{l}2 / 14 / 01 \\
5 / 25 / 01 \\
8 / 30 / 01 \\
12 / 21 / 01\end{array}$ & $\begin{array}{l}N D \\
N D \\
0.085 \\
0.13\end{array}$ & $\begin{array}{l}0.11 \\
0.11 \\
0.07 \\
0.07\end{array}$ & $\begin{array}{l}\mathrm{Bq} / \mathrm{L} \\
\mathrm{Bq} / \mathrm{L} \\
\mathrm{Bq} / \mathrm{L} \\
\mathrm{Bq} / \mathrm{L}\end{array}$ & $\begin{array}{l}\text { Sample } \\
\text { Sample } \\
\text { Sample } \\
\text { Sample }\end{array}$ \\
\hline & N. Fork Strawberry Creek & $\begin{array}{l}2 / 15 / 01 \\
2 / 15 / 01 \\
5 / 25 / 01 \\
8 / 30 / 01 \\
8 / 30 / 01 \\
12 / 21 / 01\end{array}$ & $\begin{array}{l}\text { ND } \\
\text { ND } \\
\text { ND } \\
0.11 \\
\text { ND } \\
\text { ND }\end{array}$ & $\begin{array}{l}0.11 \\
0.15 \\
0.11 \\
0.07 \\
0.07 \\
0.07\end{array}$ & $\begin{array}{l}\mathrm{Bq} / \mathrm{L} \\
\mathrm{Bq} / \mathrm{L} \\
\mathrm{Bq} / \mathrm{L} \\
\mathrm{Bq} / \mathrm{L} \\
\mathrm{Bq} / \mathrm{L} \\
\mathrm{Bq} / \mathrm{L}\end{array}$ & $\begin{array}{r}\text { Sample } \\
\text { Split } \\
\text { Sample } \\
\text { Sample } \\
\text { Split } \\
\text { Sample }\end{array}$ \\
\hline & Strawberry Creek (UC) & $\begin{array}{l}2 / 14 / 01 \\
5 / 25 / 01 \\
8 / 30 / 01 \\
12 / 21 / 01\end{array}$ & $\begin{array}{l}\text { ND } \\
\text { ND } \\
\text { ND } \\
\text { ND }\end{array}$ & $\begin{array}{l}0.11 \\
0.11 \\
0.07 \\
0.07\end{array}$ & $\begin{array}{l}\mathrm{Bq} / \mathrm{L} \\
\mathrm{Bq} / \mathrm{L} \\
\mathrm{Bq} / \mathrm{L} \\
\mathrm{Bq} / \mathrm{L}\end{array}$ & $\begin{array}{l}\text { Sample } \\
\text { Sample } \\
\text { Sample } \\
\text { Sample }\end{array}$ \\
\hline & Wildcat Creek & $\begin{array}{l}2 / 14 / 01 \\
5 / 25 / 01 \\
8 / 30 / 01 \\
12 / 21 / 01\end{array}$ & $\begin{array}{l}\text { ND } \\
\text { ND } \\
\text { ND } \\
\text { ND }\end{array}$ & $\begin{array}{l}0.11 \\
0.11 \\
0.07 \\
0.07\end{array}$ & $\begin{array}{l}\mathrm{Bq} / \mathrm{L} \\
\mathrm{Bq} / \mathrm{L} \\
\mathrm{Bq} / \mathrm{L} \\
\mathrm{Bq} / \mathrm{L}\end{array}$ & $\begin{array}{l}\text { Sample } \\
\text { Sample } \\
\text { Sample } \\
\text { Sample }\end{array}$ \\
\hline & Field Blank & $\begin{array}{l}2 / 15 / 01 \\
5 / 25 / 01\end{array}$ & $\begin{array}{l}\text { ND } \\
\text { ND }\end{array}$ & $\begin{array}{l}0.11 \\
0.07\end{array}$ & $\begin{array}{l}\mathrm{Bq} / \mathrm{L} \\
\mathrm{Bq} / \mathrm{L}\end{array}$ & $\begin{array}{l}\text { Blank } \\
\text { Blank }\end{array}$ \\
\hline
\end{tabular}




\begin{tabular}{|c|c|c|c|c|c|c|}
\hline Analyte & Location & Date & Result & $\begin{array}{r}\text { MDA } \\
\text { or PQL }\end{array}$ & Units & QC Type \\
\hline \multicolumn{7}{|c|}{ Radiological Activity } \\
\hline $\begin{array}{l}\text { Gross alpha } \\
\text { cont. }\end{array}$ & Field Blank & $\begin{array}{l}8 / 30 / 01 \\
12 / 21 / 01\end{array}$ & $\begin{array}{l}\text { ND } \\
\text { ND }\end{array}$ & $\begin{array}{l}0.07 \\
0.07\end{array}$ & $\begin{array}{l}\mathrm{Bq} / \mathrm{L} \\
\mathrm{Bq} / \mathrm{L}\end{array}$ & $\begin{array}{l}\text { Blank } \\
\text { Blank }\end{array}$ \\
\hline \multirow[t]{6}{*}{ Gross beta } & Chicken Creek & $\begin{array}{l}2 / 15 / 01 \\
5 / 25 / 01 \\
5 / 25 / 01 \\
8 / 30 / 01 \\
12 / 21 / 01 \\
12 / 21 / 01\end{array}$ & $\begin{array}{l}\text { ND } \\
\text { ND } \\
\text { ND } \\
0.11 \\
\text { ND } \\
\text { ND }\end{array}$ & $\begin{array}{l}0.11 \\
0.11 \\
0.11 \\
0.07 \\
0.11 \\
0.15\end{array}$ & $\begin{array}{l}\mathrm{Bq} / \mathrm{L} \\
\mathrm{Bq} / \mathrm{L} \\
\mathrm{Bq} / \mathrm{L} \\
\mathrm{Bq} / \mathrm{L} \\
\mathrm{Bq} / \mathrm{L} \\
\mathrm{Bq} / \mathrm{L}\end{array}$ & $\begin{array}{r}\text { Sample } \\
\text { Sample } \\
\text { Split } \\
\text { Sample } \\
\text { Sample } \\
\text { Split }\end{array}$ \\
\hline & Claremont Creek & $\begin{array}{l}2 / 14 / 01 \\
5 / 25 / 01 \\
8 / 30 / 01 \\
12 / 21 / 01\end{array}$ & $\begin{array}{l}\text { ND } \\
\text { ND } \\
\text { ND } \\
\text { ND }\end{array}$ & $\begin{array}{l}0.11 \\
0.15 \\
0.07 \\
0.11\end{array}$ & $\begin{array}{l}\mathrm{Bq} / \mathrm{L} \\
\mathrm{Bq} / \mathrm{L} \\
\mathrm{Bq} / \mathrm{L} \\
\mathrm{Bq} / \mathrm{L}\end{array}$ & $\begin{array}{l}\text { Sample } \\
\text { Sample } \\
\text { Sample } \\
\text { Sample }\end{array}$ \\
\hline & N. Fork Strawberry Creek & $\begin{array}{l}2 / 15 / 01 \\
2 / 15 / 01 \\
5 / 25 / 01 \\
8 / 30 / 01 \\
8 / 30 / 01 \\
12 / 21 / 01\end{array}$ & $\begin{array}{l}\text { ND } \\
\text { ND } \\
\text { ND } \\
\text { ND } \\
0.16 \\
\text { ND }\end{array}$ & $\begin{array}{l}0.11 \\
0.11 \\
0.11 \\
0.07 \\
0.11 \\
0.11\end{array}$ & $\begin{array}{l}\mathrm{Bq} / \mathrm{L} \\
\mathrm{Bq} / \mathrm{L} \\
\mathrm{Bq} / \mathrm{L} \\
\mathrm{Bq} / \mathrm{L} \\
\mathrm{Bq} / \mathrm{L} \\
\mathrm{Bq} / \mathrm{L}\end{array}$ & $\begin{array}{r}\text { Sample } \\
\text { Split } \\
\text { Sample } \\
\text { Sample } \\
\text { Split } \\
\text { Sample }\end{array}$ \\
\hline & Strawberry Creek (UC) & $\begin{array}{l}2 / 14 / 01 \\
5 / 25 / 01 \\
8 / 30 / 01 \\
12 / 21 / 01\end{array}$ & $\begin{array}{l}\mathrm{ND} \\
\mathrm{ND} \\
0.11 \\
\mathrm{ND}\end{array}$ & $\begin{array}{l}0.11 \\
0.11 \\
0.07 \\
0.11\end{array}$ & $\begin{array}{l}\mathrm{Bq} / \mathrm{L} \\
\mathrm{Bq} / \mathrm{L} \\
\mathrm{Bq} / \mathrm{L} \\
\mathrm{Bq} / \mathrm{L}\end{array}$ & $\begin{array}{l}\text { Sample } \\
\text { Sample } \\
\text { Sample } \\
\text { Sample }\end{array}$ \\
\hline & Wildcat Creek & $\begin{array}{l}2 / 14 / 01 \\
5 / 25 / 01 \\
8 / 30 / 01 \\
12 / 21 / 01\end{array}$ & $\begin{array}{l}\text { ND } \\
\text { ND } \\
\text { ND } \\
\text { ND }\end{array}$ & $\begin{array}{l}0.11 \\
0.11 \\
0.07 \\
0.11\end{array}$ & $\begin{array}{l}\mathrm{Bq} / \mathrm{L} \\
\mathrm{Bq} / \mathrm{L} \\
\mathrm{Bq} / \mathrm{L} \\
\mathrm{Bq} / \mathrm{L}\end{array}$ & $\begin{array}{l}\text { Sample } \\
\text { Sample } \\
\text { Sample } \\
\text { Sample }\end{array}$ \\
\hline & Field Blank & $\begin{array}{l}5 / 25 / 01 \\
8 / 30 / 01 \\
12 / 21 / 01\end{array}$ & $\begin{array}{l}\text { ND } \\
\text { ND } \\
\text { ND }\end{array}$ & $\begin{array}{l}0.11 \\
0.07 \\
0.11\end{array}$ & $\begin{array}{l}\mathrm{Bq} / \mathrm{L} \\
\mathrm{Bq} / \mathrm{L} \\
\mathrm{Bq} / \mathrm{L}\end{array}$ & $\begin{array}{l}\text { Blank } \\
\text { Blank } \\
\text { Blank }\end{array}$ \\
\hline \multirow[t]{2}{*}{ Tritium } & $\begin{array}{l}\text { Botanical Garden Creek } \\
\text { Cafeteria Creek }\end{array}$ & $\begin{array}{l}2 / 14 / 01 \\
2 / 14 / 01\end{array}$ & $\begin{array}{l}\text { ND } \\
\text { ND }\end{array}$ & $\begin{array}{l}11 \\
11\end{array}$ & $\begin{array}{l}\mathrm{Bq} / \mathrm{L} \\
\mathrm{Bq} / \mathrm{L}\end{array}$ & $\begin{array}{l}\text { Sample } \\
\text { Sample }\end{array}$ \\
\hline & Chicken Creek & $\begin{array}{l}1 / 8 / 01 \\
1 / 8 / 01 \\
2 / 14 / 01 \\
2 / 15 / 01 \\
2 / 15 / 01\end{array}$ & $\begin{array}{l}24 \\
24 \\
32 \\
28 \\
26\end{array}$ & $\begin{array}{r}7 \\
7 \\
11 \\
7 \\
7\end{array}$ & $\begin{array}{l}\mathrm{Bq} / \mathrm{L} \\
\mathrm{Bq} / \mathrm{L} \\
\mathrm{Bq} / \mathrm{L} \\
\mathrm{Bq} / \mathrm{L} \\
\mathrm{Bq} / \mathrm{L}\end{array}$ & $\begin{array}{r}\text { Sample } \\
\text { Duplicate } \\
\text { Sample } \\
\text { Sample } \\
\text { Split }\end{array}$ \\
\hline
\end{tabular}




\begin{tabular}{llrl} 
MDA & \multicolumn{4}{c}{ MDalyte Location } & Date & Result & or PQL Units QC Type
\end{tabular}

\section{Radiological Activity}

Tritium cont.
Chicken Creek

Claremont Creek

N. Fork Strawberry Creek

No Name Creek

Ravine Creek

Strawberry Creek (UC)

Ten Inch Creek

Wildcat Creek
2/14/01

2/14/01

$2 / 14 / 01$

$5 / 25 / 01$

$5 / 25 / 01$

$8 / 30 / 01$

$8 / 30 / 01$

$12 / 21 / 01$

$12 / 21 / 01$

2/14/01

$5 / 25 / 01$
$5 / 25 / 01$
$8 / 30 / 01$
$8 / 30 / 01$
$12 / 21 / 01$
$12 / 21 / 01$

2/14/01

2/14/01
8.7

9.2

8.7

7.6

28.5

26

ND

ND

ND

ND

ND

ND

ND

ND

ND

ND

ND

ND

ND

ND

ND

ND

ND

ND

10

ND

11

ND

11

ND

ND

ND

ND

ND

ND

ND

ND

ND

7

7

7

7

7

7

$\mathrm{Bq} / \mathrm{L}$

$\mathrm{Bq} / \mathrm{L}$

$\mathrm{Bq} / \mathrm{L}$

$\mathrm{Bq} / \mathrm{L}$

$\mathrm{Bq} / \mathrm{L}$

$\mathrm{Bq} / \mathrm{L}$

$\mathrm{Bq} / \mathrm{L}$

$\mathrm{Bq} / \mathrm{L}$

$\mathrm{Bq} / \mathrm{L}$

$\mathrm{Bq} / \mathrm{L}$

$\mathrm{Bq} / \mathrm{L}$

$\mathrm{Bq} / \mathrm{L}$

$\mathrm{Bq} / \mathrm{L}$

$\mathrm{Bq} / \mathrm{L}$

$\mathrm{Bq} / \mathrm{L}$

$\mathrm{Bq} / \mathrm{L}$

$\mathrm{Bq} / \mathrm{L}$

$\mathrm{Bq} / \mathrm{L}$

$\mathrm{Bq} / \mathrm{L}$

$\mathrm{Bq} / \mathrm{L}$

$\mathrm{Bq} / \mathrm{L}$

$\mathrm{Bq} / \mathrm{L}$

$\mathrm{Bq} / \mathrm{L}$

$\mathrm{Bq} / \mathrm{L}$

$\mathrm{Bq} / \mathrm{L}$

$\mathrm{Bq} / \mathrm{L}$

$\mathrm{Bq} / \mathrm{L}$

$\mathrm{Bq} / \mathrm{L}$

$\mathrm{Bq} / \mathrm{L}$

$\mathrm{Bq} / \mathrm{L}$

$\mathrm{Bq} / \mathrm{L}$

$\mathrm{Bq} / \mathrm{L}$

$\mathrm{Bq} / \mathrm{L}$

$\mathrm{Bq} / \mathrm{L}$

$\mathrm{Bq} / \mathrm{L}$

$\mathrm{Bq} / \mathrm{L}$

11

ND

ND
$\mathrm{Bq} / \mathrm{L}$

$\mathrm{Bq} / \mathrm{L}$
Sample
Split
Sample
Split
Sample
Split

Sample

Split

Sample

Split

Sample

Split

Sample

Split

Sample

Duplicate

Sample

Sample

Split

Sample

Split

Sample

Split

Sample

Split

Sample

Sample

Sample

Split

Sample

Split

Sample

Split

Sample

Split

Sample

Sample

Split 


\begin{tabular}{|c|c|c|c|c|c|c|}
\hline Analyte & Location & Date & Result & $\begin{array}{r}\text { MDA } \\
\text { or PQL } \\
\end{array}$ & Units & QC Type \\
\hline \multicolumn{7}{|c|}{ Radiological Activity } \\
\hline $\begin{array}{l}\text { Tritium } \\
\text { cont. }\end{array}$ & Wildcat Creek & $\begin{array}{l}5 / 25 / 01 \\
5 / 25 / 01 \\
8 / 30 / 01 \\
8 / 30 / 01 \\
12 / 21 / 01 \\
12 / 21 / 01\end{array}$ & $\begin{array}{l}\text { ND } \\
\text { ND } \\
\text { ND } \\
\text { ND } \\
\text { ND } \\
\text { ND }\end{array}$ & $\begin{array}{l}7 \\
7 \\
7 \\
7 \\
7 \\
7\end{array}$ & $\begin{array}{l}\mathrm{Bq} / \mathrm{L} \\
\mathrm{Bq} / \mathrm{L} \\
\mathrm{Bq} / \mathrm{L} \\
\mathrm{Bq} / \mathrm{L} \\
\mathrm{Bq} / \mathrm{L} \\
\mathrm{Bq} / \mathrm{L}\end{array}$ & $\begin{array}{r}\text { Sample } \\
\text { Split } \\
\text { Sample } \\
\text { Split } \\
\text { Sample } \\
\text { Split }\end{array}$ \\
\hline & Field Blank & $\begin{array}{l}2 / 15 / 01 \\
5 / 25 / 01 \\
8 / 30 / 01 \\
12 / 21 / 01\end{array}$ & $\begin{array}{l}\text { ND } \\
\text { ND } \\
\text { ND } \\
\text { ND }\end{array}$ & $\begin{array}{l}7 \\
7 \\
7 \\
7\end{array}$ & $\begin{array}{l}\mathrm{Bq} / \mathrm{L} \\
\mathrm{Bq} / \mathrm{L} \\
\mathrm{Bq} / \mathrm{L} \\
\mathrm{Bq} / \mathrm{L}\end{array}$ & $\begin{array}{l}\text { Blank } \\
\text { Blank } \\
\text { Blank } \\
\text { Blank }\end{array}$ \\
\hline
\end{tabular}

\section{Metals and/or Minerals}

\begin{tabular}{|c|c|c|c|c|c|c|}
\hline \multirow[t]{7}{*}{ Antimony } & Botanical Garden Creek & $2 / 14 / 01$ & ND & 4 & $\mu \mathrm{g} / \mathrm{L}$ & Sample \\
\hline & Cafeteria Creek & 2/14/01 & ND & 4 & $\mu \mathrm{g} / \mathrm{L}$ & Sample \\
\hline & Chicken Creek & $2 / 14 / 01$ & ND & 4 & $\mu \mathrm{g} / \mathrm{L}$ & Sample \\
\hline & No Name Creek & 2/14/01 & ND & 4 & $\mu \mathrm{g} / \mathrm{L}$ & Sample \\
\hline & N. Fork Strawberry Creek & $2 / 14 / 01$ & ND & 4 & $\mu \mathrm{g} / \mathrm{L}$ & Sample \\
\hline & Ravine Creek & 2/14/01 & ND & 4 & $\mu \mathrm{g} / \mathrm{L}$ & Sample \\
\hline & Ten Inch Creek & $2 / 14 / 01$ & ND & 4 & $\mu \mathrm{g} / \mathrm{L}$ & Sample \\
\hline \multirow[t]{7}{*}{ Arsenic } & Botanical Garden Creek & $2 / 14 / 01$ & ND & 2 & $\mu \mathrm{g} / \mathrm{L}$ & Sample \\
\hline & Cafeteria Creek & 2/14/01 & ND & 2 & $\mu \mathrm{g} / \mathrm{L}$ & Sample \\
\hline & Chicken Creek & $2 / 14 / 01$ & 4 & 2 & $\mu \mathrm{g} / \mathrm{L}$ & Sample \\
\hline & No Name Creek & $2 / 14 / 01$ & ND & 2 & $\mu \mathrm{g} / \mathrm{L}$ & Sample \\
\hline & N. Fork Strawberry Creek & $2 / 14 / 01$ & 2 & 2 & $\mu \mathrm{g} / \mathrm{L}$ & Sample \\
\hline & Ravine Creek & 2/14/01 & ND & 2 & $\mu \mathrm{g} / \mathrm{L}$ & Sample \\
\hline & Ten Inch Creek & $2 / 14 / 01$ & ND & 2 & $\mu \mathrm{g} / \mathrm{L}$ & Sample \\
\hline \multirow[t]{7}{*}{ Barium } & Botanical Garden Creek & $2 / 14 / 01$ & ND & 100 & $\mu \mathrm{g} / \mathrm{L}$ & Sample \\
\hline & Cafeteria Creek & $2 / 14 / 01$ & ND & 100 & $\mu \mathrm{g} / \mathrm{L}$ & Sample \\
\hline & Chicken Creek & 2/14/01 & 120 & 100 & $\mu \mathrm{g} / \mathrm{L}$ & Sample \\
\hline & No Name Creek & $2 / 14 / 01$ & 100 & 100 & $\mu \mathrm{g} / \mathrm{L}$ & Sample \\
\hline & N. Fork Strawberry Creek & $2 / 14 / 01$ & ND & 100 & $\mu \mathrm{g} / \mathrm{L}$ & Sample \\
\hline & Ravine Creek & $2 / 14 / 01$ & ND & 100 & $\mu \mathrm{g} / \mathrm{L}$ & Sample \\
\hline & Ten Inch Creek & $2 / 14 / 01$ & ND & 100 & $\mu \mathrm{g} / \mathrm{L}$ & Sample \\
\hline \multirow[t]{7}{*}{ Beryllium } & Botanical Garden Creek & $2 / 14 / 01$ & ND & 0.2 & $\mu \mathrm{g} / \mathrm{L}$ & Sample \\
\hline & Cafeteria Creek & $2 / 14 / 01$ & ND & 0.2 & $\mu \mathrm{g} / \mathrm{L}$ & Sample \\
\hline & Chicken Creek & $2 / 14 / 01$ & ND & 0.2 & $\mu g / L$ & Sample \\
\hline & No Name Creek & $2 / 14 / 01$ & ND & 0.2 & $\mu \mathrm{g} / \mathrm{L}$ & Sample \\
\hline & N. Fork Strawberry Creek & $2 / 14 / 01$ & ND & 0.2 & $\mu \mathrm{g} / \mathrm{L}$ & Sample \\
\hline & Ravine Creek & $2 / 14 / 01$ & ND & 0.2 & $\mu \mathrm{g} / \mathrm{L}$ & Sample \\
\hline & Ten Inch Creek & $2 / 14 / 01$ & ND & 0.2 & $\mu \mathrm{g} / \mathrm{L}$ & Sample \\
\hline
\end{tabular}


MDA

$\begin{array}{llllll}\text { Analyte Location } & \text { Date } & \text { Result } & \text { or PQL Units QC Type }\end{array}$

\section{Metals and/or Minerals}

\begin{tabular}{|c|c|c|c|c|c|c|}
\hline \multirow[t]{7}{*}{ Cadmium } & Botanical Garden Creek & $2 / 14 / 01$ & ND & 1 & $\mu \mathrm{g} / \mathrm{L}$ & Sample \\
\hline & Cafeteria Creek & $2 / 14 / 01$ & ND & 1 & $\mu \mathrm{g} / \mathrm{L}$ & Sample \\
\hline & Chicken Creek & $2 / 14 / 01$ & ND & 1 & $\mu \mathrm{g} / \mathrm{L}$ & Sample \\
\hline & No Name Creek & $2 / 14 / 01$ & ND & 1 & $\mu \mathrm{g} / \mathrm{L}$ & Sample \\
\hline & N. Fork Strawberry Creek & $2 / 14 / 01$ & ND & 1 & $\mu \mathrm{g} / \mathrm{L}$ & Sample \\
\hline & Ravine Creek & $2 / 14 / 01$ & ND & 1 & $\mu \mathrm{g} / \mathrm{L}$ & Sample \\
\hline & Ten Inch Creek & $2 / 14 / 01$ & ND & 1 & $\mu \mathrm{g} / \mathrm{L}$ & Sample \\
\hline \multirow[t]{7}{*}{ Chromium } & Botanical Garden Creek & $2 / 14 / 01$ & ND & 10 & $\mu \mathrm{g} / \mathrm{L}$ & Sample \\
\hline & Cafeteria Creek & $2 / 14 / 01$ & ND & 10 & $\mu \mathrm{g} / \mathrm{L}$ & Sample \\
\hline & Chicken Creek & $2 / 14 / 01$ & ND & 10 & $\mu \mathrm{g} / \mathrm{L}$ & Sample \\
\hline & No Name Creek & $2 / 14 / 01$ & ND & 10 & $\mu \mathrm{g} / \mathrm{L}$ & Sample \\
\hline & N. Fork Strawberry Creek & $2 / 14 / 01$ & ND & 10 & $\mu \mathrm{g} / \mathrm{L}$ & Sample \\
\hline & Ravine Creek & $2 / 14 / 01$ & ND & 10 & $\mu \mathrm{g} / \mathrm{L}$ & Sample \\
\hline & Ten Inch Creek & $2 / 14 / 01$ & ND & 10 & $\mu \mathrm{g} / \mathrm{L}$ & Sample \\
\hline \multirow[t]{7}{*}{ Cobalt } & Botanical Garden Creek & $2 / 14 / 01$ & ND & 50 & $\mu \mathrm{g} / \mathrm{L}$ & Sample \\
\hline & Cafeteria Creek & $2 / 14 / 01$ & ND & 50 & $\mu \mathrm{g} / \mathrm{L}$ & Sample \\
\hline & Chicken Creek & $2 / 14 / 01$ & ND & 50 & $\mu \mathrm{g} / \mathrm{L}$ & Sample \\
\hline & No Name Creek & $2 / 14 / 01$ & ND & 50 & $\mu \mathrm{g} / \mathrm{L}$ & Sample \\
\hline & N. Fork Strawberry Creek & $2 / 14 / 01$ & ND & 50 & $\mu \mathrm{g} / \mathrm{L}$ & Sample \\
\hline & Ravine Creek & $2 / 14 / 01$ & ND & 50 & $\mu \mathrm{g} / \mathrm{L}$ & Sample \\
\hline & Ten Inch Creek & $2 / 14 / 01$ & ND & 50 & $\mu \mathrm{g} / \mathrm{L}$ & Sample \\
\hline \multirow{7}{*}{ Copper } & Botanical Garden Creek & $2 / 14 / 01$ & ND & 10 & $\mu \mathrm{g} / \mathrm{L}$ & Sample \\
\hline & Cafeteria Creek & $2 / 14 / 01$ & ND & 10 & $\mu \mathrm{g} / \mathrm{L}$ & Sample \\
\hline & Chicken Creek & $2 / 14 / 01$ & ND & 10 & $\mu \mathrm{g} / \mathrm{L}$ & Sample \\
\hline & No Name Creek & $2 / 14 / 01$ & ND & 10 & $\mu \mathrm{g} / \mathrm{L}$ & Sample \\
\hline & N. Fork Strawberry Creek & $2 / 14 / 01$ & ND & 10 & $\mu \mathrm{g} / \mathrm{L}$ & Sample \\
\hline & Ravine Creek & $2 / 14 / 01$ & ND & 10 & $\mu \mathrm{g} / \mathrm{L}$ & Sample \\
\hline & Ten Inch Creek & $2 / 14 / 01$ & ND & 10 & $\mu \mathrm{g} / \mathrm{L}$ & Sample \\
\hline \multirow[t]{7}{*}{ Lead } & Botanical Garden Creek & $2 / 14 / 01$ & ND & 5 & $\mu \mathrm{g} / \mathrm{L}$ & Sample \\
\hline & Cafeteria Creek & $2 / 14 / 01$ & ND & 5 & $\mu \mathrm{g} / \mathrm{L}$ & Sample \\
\hline & Chicken Creek & $2 / 14 / 01$ & ND & 5 & $\mu \mathrm{g} / \mathrm{L}$ & Sample \\
\hline & No Name Creek & $2 / 14 / 01$ & ND & 5 & $\mu \mathrm{g} / \mathrm{L}$ & Sample \\
\hline & N. Fork Strawberry Creek & $2 / 14 / 01$ & ND & 5 & $\mu \mathrm{g} / \mathrm{L}$ & Sample \\
\hline & Ravine Creek & $2 / 14 / 01$ & ND & 5 & $\mu \mathrm{g} / \mathrm{L}$ & Sample \\
\hline & Ten Inch Creek & $2 / 14 / 01$ & ND & 5 & $\mu \mathrm{g} / \mathrm{L}$ & Sample \\
\hline \multirow[t]{5}{*}{ Mercury } & Botanical Garden Creek & $2 / 14 / 01$ & ND & 0.2 & $\mu \mathrm{g} / \mathrm{L}$ & Sample \\
\hline & Cafeteria Creek & $2 / 14 / 01$ & ND & 0.2 & $\mu \mathrm{g} / \mathrm{L}$ & Sample \\
\hline & Chicken Creek & $2 / 14 / 01$ & ND & 0.2 & $\mu \mathrm{g} / \mathrm{L}$ & Sample \\
\hline & No Name Creek & $2 / 14 / 01$ & ND & 0.2 & $\mu \mathrm{g} / \mathrm{L}$ & Sample \\
\hline & N. Fork Strawberry Creek & $2 / 14 / 01$ & ND & 0.2 & $\mu \mathrm{g} / \mathrm{L}$ & Sampl \\
\hline
\end{tabular}




\begin{tabular}{|c|c|c|c|c|c|c|}
\hline Analyte & Location & Date & Result & $\begin{array}{r}\text { MDA } \\
\text { or PQL }\end{array}$ & Units & QC Type \\
\hline \multicolumn{7}{|c|}{ Metals and/or Minerals } \\
\hline \multirow{2}{*}{$\begin{array}{l}\text { Mercury } \\
\text { cont. }\end{array}$} & Ravine Creek & $2 / 14 / 01$ & ND & 0.2 & $\mu \mathrm{g} / \mathrm{L}$ & Sample \\
\hline & Ten Inch Creek & $2 / 14 / 01$ & ND & 0.2 & $\mu \mathrm{g} / \mathrm{L}$ & Sample \\
\hline \multirow[t]{7}{*}{ Molybdenum } & Botanical Garden Creek & $2 / 14 / 01$ & ND & 50 & $\mu \mathrm{g} / \mathrm{L}$ & Sample \\
\hline & Cafeteria Creek & $2 / 14 / 01$ & ND & 50 & $\mu \mathrm{g} / \mathrm{L}$ & Sample \\
\hline & Chicken Creek & $2 / 14 / 01$ & ND & 50 & $\mu \mathrm{g} / \mathrm{L}$ & Sample \\
\hline & No Name Creek & $2 / 14 / 01$ & ND & 50 & $\mu \mathrm{g} / \mathrm{L}$ & Sample \\
\hline & N. Fork Strawberry Creek & $2 / 14 / 01$ & ND & 50 & $\mu \mathrm{g} / \mathrm{L}$ & Sample \\
\hline & Ravine Creek & $2 / 14 / 01$ & ND & 50 & $\mu \mathrm{g} / \mathrm{L}$ & Sample \\
\hline & Ten Inch Creek & $2 / 14 / 01$ & ND & 50 & $\mu \mathrm{g} / \mathrm{L}$ & Sample \\
\hline \multirow[t]{7}{*}{ Nickel } & Botanical Garden Creek & $2 / 14 / 01$ & ND & 10 & $\mu \mathrm{g} / \mathrm{L}$ & Sample \\
\hline & Cafeteria Creek & $2 / 14 / 01$ & ND & 10 & $\mu \mathrm{g} / \mathrm{L}$ & Sample \\
\hline & Chicken Creek & $2 / 14 / 01$ & ND & 10 & $\mu \mathrm{g} / \mathrm{L}$ & Sample \\
\hline & No Name Creek & $2 / 14 / 01$ & ND & 10 & $\mu \mathrm{g} / \mathrm{L}$ & Sample \\
\hline & N. Fork Strawberry Creek & $2 / 14 / 01$ & ND & 10 & $\mu \mathrm{g} / \mathrm{L}$ & Sample \\
\hline & Ravine Creek & $2 / 14 / 01$ & ND & 10 & $\mu \mathrm{g} / \mathrm{L}$ & Sample \\
\hline & Ten Inch Creek & $2 / 14 / 01$ & ND & 10 & $\mu \mathrm{g} / \mathrm{L}$ & Sample \\
\hline \multirow[t]{7}{*}{ Selenium } & Botanical Garden Creek & $2 / 14 / 01$ & 5 & 2 & $\mu \mathrm{g} / \mathrm{L}$ & Sample \\
\hline & Cafeteria Creek & $2 / 14 / 01$ & ND & 2 & $\mu \mathrm{g} / \mathrm{L}$ & Sample \\
\hline & Chicken Creek & $2 / 14 / 01$ & ND & 2 & $\mu \mathrm{g} / \mathrm{L}$ & Sample \\
\hline & No Name Creek & $2 / 14 / 01$ & ND & 2 & $\mu \mathrm{g} / \mathrm{L}$ & Sample \\
\hline & N. Fork Strawberry Creek & $2 / 14 / 01$ & ND & 2 & $\mu \mathrm{g} / \mathrm{L}$ & Sample \\
\hline & Ravine Creek & $2 / 14 / 01$ & ND & 2 & $\mu \mathrm{g} / \mathrm{L}$ & Sample \\
\hline & Ten Inch Creek & $2 / 14 / 01$ & ND & 2 & $\mu \mathrm{g} / \mathrm{L}$ & Sample \\
\hline \multirow[t]{7}{*}{ Silver } & Botanical Garden Creek & $2 / 14 / 01$ & ND & 10 & $\mu \mathrm{g} / \mathrm{L}$ & Sample \\
\hline & Cafeteria Creek & $2 / 14 / 01$ & ND & 10 & $\mu \mathrm{g} / \mathrm{L}$ & Sample \\
\hline & Chicken Creek & $2 / 14 / 01$ & ND & 10 & $\mu \mathrm{g} / \mathrm{L}$ & Sample \\
\hline & No Name Creek & $2 / 14 / 01$ & ND & 10 & $\mu \mathrm{g} / \mathrm{L}$ & Sample \\
\hline & N. Fork Strawberry Creek & $2 / 14 / 01$ & ND & 10 & $\mu \mathrm{g} / \mathrm{L}$ & Sample \\
\hline & Ravine Creek & $2 / 14 / 01$ & ND & 10 & $\mu \mathrm{g} / \mathrm{L}$ & Sample \\
\hline & Ten Inch Creek & $2 / 14 / 01$ & ND & 10 & $\mu \mathrm{g} / \mathrm{L}$ & Sample \\
\hline \multirow[t]{7}{*}{ Thallium } & Botanical Garden Creek & $2 / 14 / 01$ & ND & 1 & $\mu \mathrm{g} / \mathrm{L}$ & Sample \\
\hline & Cafeteria Creek & $2 / 14 / 01$ & ND & 1 & $\mu \mathrm{g} / \mathrm{L}$ & Sample \\
\hline & Chicken Creek & $2 / 14 / 01$ & ND & 1 & $\mu \mathrm{g} / \mathrm{L}$ & Sample \\
\hline & No Name Creek & $2 / 14 / 01$ & ND & 1 & $\mu \mathrm{g} / \mathrm{L}$ & Sample \\
\hline & N. Fork Strawberry Creek & $2 / 14 / 01$ & ND & 1 & $\mu \mathrm{g} / \mathrm{L}$ & Sample \\
\hline & Ravine Creek & $2 / 14 / 01$ & ND & 1 & $\mu \mathrm{g} / \mathrm{L}$ & Sample \\
\hline & Ten Inch Creek & $2 / 14 / 01$ & ND & 1 & $\mu \mathrm{g} / \mathrm{L}$ & Sample \\
\hline \multirow[t]{3}{*}{ Vanadium } & Botanical Garden Creek & $2 / 14 / 01$ & ND & 10 & $\mu \mathrm{g} / \mathrm{L}$ & Sample \\
\hline & Cafeteria Creek & $2 / 14 / 01$ & ND & 10 & $\mu \mathrm{g} / \mathrm{L}$ & Sample \\
\hline & Chicken Creek & $2 / 14 / 01$ & 10 & 10 & $\mu \mathrm{g} / \mathrm{L}$ & Sample \\
\hline
\end{tabular}


MDA

Analyte Location Date Result or PQL Units QC Type

\section{Metals and/or Minerals}

Vanadium

cont.

No Name Creek

N. Fork Strawberry Creek 2/14/01

Ravine Creek

Ten Inch Creek

$2 / 14 / 01$

$2 / 14 / 01$

Zinc

$\begin{array}{ll}\text { Botanical Garden Creek } & \text { 2/14/01 } \\ \text { Cafeteria Creek } & 2 / 14 / 01 \\ \text { Chicken Creek } & 2 / 14 / 01 \\ \text { No Name Creek } & 2 / 14 / 01 \\ \text { N. Fork Strawberry Creek } & 2 / 14 / 01 \\ \text { Ravine Creek } & 2 / 14 / 01 \\ \text { Ten Inch Creek } & 2 / 14 / 01\end{array}$

\section{Volatile Organic Compounds}

1,1,1,2-Tetrachloroethane

Botanical Garden Creek

Cafeteria Creek

2/14/01

2/14/01

Chicken Creek

No Name Creek

$2 / 14 / 01$

$2 / 14 / 01$

N. Fork Strawberry Creek

Ravine Creek

Ten Inch Creek

2/14/01

2/14/01

$2 / 14 / 01$

1,1,1-Trichloroethane

Botanical Garden Creek 2/14/0

Cafeteria Creek 2/14/01

Chicken Creek 2/14/01

No Name Creek 2/14/01

N. Fork Strawberry Creek 2/14/01

Ravine Creek

Ten Inch Creek

2/14/01

2/14/01

1,1,2,2-Tetrachloroethane

Botanical Garden Creek 2/14/01

Cafeteria Creek 2/14/01

Chicken Creek 2/14/01

No Name Creek 2/14/01

N. Fork Strawberry Creek

2/14/01

Ravine Creek

2/14/01

Ten Inch Creek

2/14/01

1,1,2-Trichloroethane

2/14/01

Cafeteria Creek

2/14/01

Chicken Creek

2/14/01
ND $\quad 10$

ND $\quad 10$

ND $\quad 10$

ND $\quad 10$

10

40

34

30

20

20

ND

10

10

10

or PQ

QC Type

$\mu \mathrm{g} / \mathrm{L} \quad$ Sample

$\mu \mathrm{g} / \mathrm{L} \quad$ Sample

$\mu \mathrm{g} / \mathrm{L} \quad$ Sample

$\mu \mathrm{g} / \mathrm{L} \quad$ Sample

$\mu \mathrm{g} / \mathrm{L} \quad$ Sample

$\mu \mathrm{g} / \mathrm{L} \quad$ Sample

$\mu \mathrm{g} / \mathrm{L} \quad$ Sample

$\mu \mathrm{g} / \mathrm{L} \quad$ Sample

$\mu \mathrm{g} / \mathrm{L} \quad$ Sample

$\mu \mathrm{g} / \mathrm{L} \quad$ Sample

$\mu \mathrm{g} / \mathrm{L} \quad$ Sample

$\begin{array}{llll}\text { ND } & 2 & \mu \mathrm{g} / \mathrm{L} & \text { Sample } \\ \text { ND } & 2 & \mu \mathrm{g} / \mathrm{L} & \text { Sample } \\ \text { ND } & 2 & \mu \mathrm{g} / \mathrm{L} & \text { Sample } \\ \text { ND } & 2 & \mu \mathrm{g} / \mathrm{L} & \text { Sample } \\ \text { ND } & 2 & \mu \mathrm{g} / \mathrm{L} & \text { Sample } \\ \text { ND } & 2 & \mu \mathrm{g} / \mathrm{L} & \text { Sample } \\ \text { ND } & 2 & \mu \mathrm{g} / \mathrm{L} & \text { Sample }\end{array}$

$\begin{array}{llll}\text { ND } & 1 & \mu \mathrm{g} / \mathrm{L} & \text { Sample }\end{array}$

ND $\quad 1 \quad \mu g / L \quad$ Sample

ND $\quad 1 \quad \mu g / L \quad$ Sample

ND $\quad 1 \quad \mu g / L \quad$ Sample

ND $\quad 1 \quad \mu g / L \quad$ Sample

ND $\quad 1 \quad \mu g / L \quad$ Sample

ND $\quad 1 \quad \mu g / L \quad$ Sample

$\begin{array}{llll}N D & 1 & \mu g / L & \text { Sample }\end{array}$

ND $\quad 1 \quad \mu g / L \quad$ Sample

ND $\quad 1 \quad \mu g / L \quad$ Sample

ND $\quad 1 \quad \mu g / L \quad$ Sample

ND $\quad 1 \quad \mu g / L \quad$ Sample

ND $\quad 1 \quad \mu g / L \quad$ Sample

ND $\quad 1 \quad \mu g / L \quad$ Sample

$\begin{array}{llll}\text { ND } & 1 & \mu g / L & \text { Sample } \\ \text { ND } & 1 & \mu g / L & \text { Sample } \\ \text { ND } & 1 & \mu g / L & \text { Sample }\end{array}$




\begin{tabular}{llll} 
Analyte Location & Date & Result & or PQL \\
\hline
\end{tabular}

\section{Volatile Organic Compounds}

1,1,2-Trichloroethane

cont.

$\begin{array}{ll}\text { No Name Creek } & 2 / 14 / 01 \\ \text { N. Fork Strawberry Creek } & 2 / 14 / 01 \\ \text { Ravine Creek } & 2 / 14 / 01 \\ \text { Ten Inch Creek } & 2 / 14 / 01\end{array}$

1,1-Dichloroethane

$\begin{array}{ll}\text { Botanical Garden Creek } & \text { 2/14/01 } \\ \text { Cafeteria Creek } & 2 / 14 / 01 \\ \text { Chicken Creek } & 2 / 14 / 01 \\ \text { No Name Creek } & 2 / 14 / 01 \\ \text { N. Fork Strawberry Creek } & 2 / 14 / 01 \\ \text { Ravine Creek } & 2 / 14 / 01 \\ \text { Ten Inch Creek } & 2 / 14 / 01\end{array}$

1,1-Dichloroethene

Botanical Garden Creek 2/14/0

Cafeteria Creek 2/14/01

Chicken Creek 2/14/01

No Name Creek 2/14/01

N. Fork Strawberry Creek 2/14/01

Ravine Creek

Ten Inch Creek

1,1-Dichloropropene

$\begin{array}{ll}\text { Botanical Garden Creek } & 2 / 14 / 01 \\ \text { Cafeteria Creek } & 2 / 14 / 01 \\ \text { Chicken Creek } & 2 / 14 / 01 \\ \text { No Name Creek } & 2 / 14 / 01 \\ \text { N. Fork Strawberry Creek } & 2 / 14 / 01 \\ \text { Ravine Creek } & 2 / 14 / 01 \\ \text { Ten Inch Creek } & 2 / 14 / 01\end{array}$

1,2,3-Trichlorobenzene

$\begin{array}{ll}\text { Botanical Garden Creek } & \text { 2/14/01 } \\ \text { Cafeteria Creek } & 2 / 14 / 01 \\ \text { Chicken Creek } & 2 / 14 / 01 \\ \text { No Name Creek } & 2 / 14 / 01 \\ \text { N. Fork Strawberry Creek } & 2 / 14 / 01 \\ \text { Ravine Creek } & 2 / 14 / 01 \\ \text { Ten Inch Creek } & 2 / 14 / 01\end{array}$

1,2,3-Trichloropropane

$\begin{array}{ll}\text { Botanical Garden Creek } & \text { 2/14/01 } \\ \text { Cafeteria Creek } & 2 / 14 / 01 \\ \text { Chicken Creek } & 2 / 14 / 01\end{array}$

ND

ND

ND

ND

ND

ND

ND

ND

ND

ND

ND

ND

ND

ND

ND

ND

ND

ND

ND

ND

ND

ND

ND

ND

ND

ND

ND

ND

ND

ND

ND

ND

ND

ND

ND $\mu \mathrm{g} / \mathrm{L} \quad$ Sample

$\mu \mathrm{g} / \mathrm{L} \quad$ Sample

$\mu \mathrm{g} / \mathrm{L} \quad$ Sample

$\mu \mathrm{g} / \mathrm{L} \quad$ Sample

$\mu \mathrm{g} / \mathrm{L} \quad$ Sample

$\mu \mathrm{g} / \mathrm{L} \quad$ Sample

$\mu \mathrm{g} / \mathrm{L} \quad$ Sample

$\mu \mathrm{g} / \mathrm{L} \quad$ Sample

$\mu \mathrm{g} / \mathrm{L} \quad$ Sample

$\mu \mathrm{g} / \mathrm{L} \quad$ Sample

$\mu \mathrm{g} / \mathrm{L} \quad$ Sample

$\mu \mathrm{g} / \mathrm{L} \quad$ Sample

$\mu \mathrm{g} / \mathrm{L} \quad$ Sample

$\mu \mathrm{g} / \mathrm{L} \quad$ Sample

$\mu \mathrm{g} / \mathrm{L} \quad$ Sample

$\mu \mathrm{g} / \mathrm{L} \quad$ Sample

$\mu \mathrm{g} / \mathrm{L} \quad$ Sample

$\mu \mathrm{g} / \mathrm{L} \quad$ Sample

$\mu \mathrm{g} / \mathrm{L} \quad$ Sample

$\mu g / L \quad$ Sample

$\mu g / L \quad$ Sample

$\mu \mathrm{g} / \mathrm{L} \quad$ Sample

$\mu g / L \quad$ Sample

$\mu \mathrm{g} / \mathrm{L} \quad$ Sample

$\mu \mathrm{g} / \mathrm{L} \quad$ Sample

$\begin{array}{ll}\mu \mathrm{g} / \mathrm{L} & \text { Sample } \\ \mu \mathrm{g} / \mathrm{L} & \text { Sample } \\ \mu \mathrm{g} / \mathrm{L} & \text { Sample } \\ \mu \mathrm{g} / \mathrm{L} & \text { Sample } \\ \mu \mathrm{g} / \mathrm{L} & \text { Sample } \\ \mu \mathrm{g} / \mathrm{L} & \text { Sample } \\ \mu \mathrm{g} / \mathrm{L} & \text { Sample }\end{array}$

$\mu \mathrm{g} / \mathrm{L} \quad$ Sample

$\mu \mathrm{g} / \mathrm{L} \quad$ Sample

$\mu g / L \quad$ Sample 
MDA

$\begin{array}{llllll}\text { Analyte Location } & \text { Date } & \text { Result } & \text { or PQL Units QC Type }\end{array}$

\section{Volatile Organic Compounds}

1,2,3-Trichloropropane

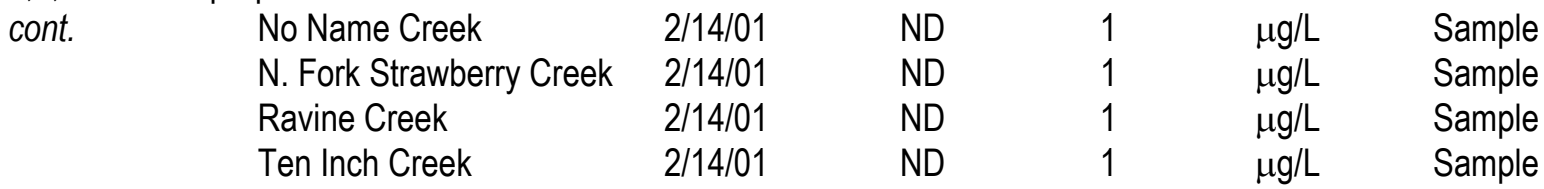

1,2,4-Trichlorobenzene

$\begin{array}{llllll}\text { Botanical Garden Creek } & 2 / 14 / 01 & \text { ND } & 1 & \mu \mathrm{g} / \mathrm{L} & \text { Sample } \\ \text { Cafeteria Creek } & 2 / 14 / 01 & \text { ND } & 1 & \mu \mathrm{g} / \mathrm{L} & \text { Sample } \\ \text { Chicken Creek } & 2 / 14 / 01 & \text { ND } & 1 & \mu \mathrm{g} / \mathrm{L} & \text { Sample } \\ \text { No Name Creek } & 2 / 14 / 01 & \text { ND } & 1 & \mu \mathrm{g} / \mathrm{L} & \text { Sample } \\ \text { N. Fork Strawberry Creek } & 2 / 14 / 01 & \text { ND } & 1 & \mu \mathrm{g} / \mathrm{L} & \text { Sample } \\ \text { Ravine Creek } & 2 / 14 / 01 & \text { ND } & 1 & \mu \mathrm{g} / \mathrm{L} & \text { Sample } \\ \text { Ten Inch Creek } & 2 / 14 / 01 & \text { ND } & 1 & \mu \mathrm{g} / \mathrm{L} & \text { Sample }\end{array}$

1,2,4-Trimethylbenzene

$\begin{array}{llllll}\text { Botanical Garden Creek } & 2 / 14 / 01 & \text { ND } & 1 & \mu \mathrm{g} / \mathrm{L} & \text { Sample } \\ \text { Cafeteria Creek } & 2 / 14 / 01 & \text { ND } & 1 & \mu \mathrm{g} / \mathrm{L} & \text { Sample } \\ \text { Chicken Creek } & 2 / 14 / 01 & \text { ND } & 1 & \mu \mathrm{g} / \mathrm{L} & \text { Sample } \\ \text { No Name Creek } & 2 / 14 / 01 & \text { ND } & 1 & \mu \mathrm{g} / \mathrm{L} & \text { Sample } \\ \text { N. Fork Strawberry Creek } & 2 / 14 / 01 & \text { ND } & 1 & \mu \mathrm{g} / \mathrm{L} & \text { Sample } \\ \text { Ravine Creek } & 2 / 14 / 01 & \text { ND } & 1 & \mu \mathrm{g} / \mathrm{L} & \text { Sample } \\ \text { Ten Inch Creek } & 2 / 14 / 01 & \text { ND } & 1 & \mu \mathrm{g} / \mathrm{L} & \text { Sample }\end{array}$

1,2-Dibromo-3-chloropropane

$\begin{array}{ll}\text { Botanical Garden Creek } & 2 / 14 / 01 \\ \text { Cafeteria Creek } & 2 / 14 / 01 \\ \text { Chicken Creek } & 2 / 14 / 01 \\ \text { No Name Creek } & 2 / 14 / 01 \\ \text { N. Fork Strawberry Creek } & 2 / 14 / 01 \\ \text { Ravine Creek } & 2 / 14 / 01 \\ \text { Ten Inch Creek } & 2 / 14 / 01\end{array}$

$\begin{array}{llll}\text { ND } & 2 & \mu \mathrm{g} / \mathrm{L} & \text { Sample } \\ \text { ND } & 2 & \mu \mathrm{g} / \mathrm{L} & \text { Sample } \\ \text { ND } & 2 & \mu \mathrm{g} / \mathrm{L} & \text { Sample } \\ \text { ND } & 2 & \mu \mathrm{g} / \mathrm{L} & \text { Sample } \\ \text { ND } & 2 & \mu \mathrm{g} / \mathrm{L} & \text { Sample } \\ \text { ND } & 2 & \mu \mathrm{g} / \mathrm{L} & \text { Sample } \\ \text { ND } & 2 & \mu \mathrm{g} / \mathrm{L} & \text { Sample }\end{array}$

1,2-Dibromoethane

$\begin{array}{llllll}\text { Botanical Garden Creek } & 2 / 14 / 01 & \text { ND } & 2 & \mu \mathrm{g} / \mathrm{L} & \text { Sample } \\ \text { Cafeteria Creek } & 2 / 14 / 01 & \text { ND } & 2 & \mu \mathrm{g} / \mathrm{L} & \text { Sample } \\ \text { Chicken Creek } & 2 / 14 / 01 & \text { ND } & 2 & \mu \mathrm{g} / \mathrm{L} & \text { Sample } \\ \text { No Name Creek } & 2 / 14 / 01 & \text { ND } & 2 & \mu \mathrm{g} / \mathrm{L} & \text { Sample } \\ \text { N. Fork Strawberry Creek } & 2 / 14 / 01 & \text { ND } & 2 & \mu \mathrm{g} / \mathrm{L} & \text { Sample } \\ \text { Ravine Creek } & 2 / 14 / 01 & \text { ND } & 2 & \mu \mathrm{g} / \mathrm{L} & \text { Sample } \\ \text { Ten Inch Creek } & 2 / 14 / 01 & \text { ND } & 2 & \mu \mathrm{g} / \mathrm{L} & \text { Sample }\end{array}$

1,2-Dichlorobenzene

$\begin{array}{llllll}\text { Botanical Garden Creek } & 2 / 14 / 01 & \text { ND } & 1 & \mu \mathrm{g} / \mathrm{L} & \text { Sample } \\ \text { Cafeteria Creek } & 2 / 14 / 01 & \text { ND } & 1 & \mu \mathrm{g} / \mathrm{L} & \text { Sample } \\ \text { Chicken Creek } & 2 / 14 / 01 & \text { ND } & 1 & \mu \mathrm{g} / \mathrm{L} & \text { Sample }\end{array}$




$\begin{array}{lllll}\text { Analyte Location } & \text { Date } & \text { Result } & \text { or PQL Units } & \text { QC Type }\end{array}$

\section{Volatile Organic Compounds}

1,2-Dichlorobenzene cont.

$\begin{array}{ll}\text { No Name Creek } & 2 / 14 / 01 \\ \text { N. Fork Strawberry Creek } & 2 / 14 / 01 \\ \text { Ravine Creek } & 2 / 14 / 01 \\ \text { Ten Inch Creek } & 2 / 14 / 01\end{array}$

ND

ND

ND

ND

ND

ND

ND

ND

ND

ND

ND

Ten Inch Creek

1,2-Dichloropropane

$\begin{array}{ll}\text { Botanical Garden Creek } & 2 / 14 / 01 \\ \text { Cafeteria Creek } & 2 / 14 / 01 \\ \text { Chicken Creek } & 2 / 14 / 01 \\ \text { No Name Creek } & 2 / 14 / 01 \\ \text { N. Fork Strawberry Creek } & 2 / 14 / 01 \\ \text { Ravine Creek } & 2 / 14 / 01 \\ \text { Ten Inch Creek } & 2 / 14 / 01\end{array}$

1,3,5-Trimethylbenzene

$\begin{array}{ll}\text { Botanical Garden Creek } & 2 / 14 / 01 \\ \text { Cafeteria Creek } & 2 / 14 / 01 \\ \text { Chicken Creek } & 2 / 14 / 01 \\ \text { No Name Creek } & 2 / 14 / 01 \\ \text { N. Fork Strawberry Creek } & 2 / 14 / 01 \\ \text { Ravine Creek } & 2 / 14 / 01 \\ \text { Ten Inch Creek } & 2 / 14 / 01\end{array}$

1,3-Dichlorobenzene

$\begin{array}{ll}\text { Botanical Garden Creek } & 2 / 14 / 01 \\ \text { Cafeteria Creek } & 2 / 14 / 01 \\ \text { Chicken Creek } & 2 / 14 / 01 \\ \text { No Name Creek } & 2 / 14 / 01 \\ \text { N. Fork Strawberry Creek } & 2 / 14 / 01 \\ \text { Ravine Creek } & 2 / 14 / 01 \\ \text { Ten Inch Creek } & 2 / 14 / 01\end{array}$

1,3-Dichloropropane

Botanical Garden Creek
Cafeteria Creek
Chicken Creek

$2 / 14 / 01$

2/14/01

2/14/01

$\begin{array}{clll}\text { ND } & 1 & \mu \mathrm{g} / \mathrm{L} & \text { Sample } \\ \text { ND } & 1 & \mu \mathrm{g} / \mathrm{L} & \text { Sample } \\ \text { ND } & 1 & \mu \mathrm{g} / \mathrm{L} & \text { Sample } \\ \text { ND } & 1 & \mu \mathrm{g} / \mathrm{L} & \text { Sample } \\ \text { ND } & 1 & \mu \mathrm{g} / \mathrm{L} & \text { Sample } \\ \text { ND } & 1 & \mu \mathrm{g} / \mathrm{L} & \text { Sample } \\ \text { ND } & 1 & \mu \mathrm{g} / \mathrm{L} & \text { Sample }\end{array}$

ND

ND

ND

ND

ND

ND

ND

ND

ND

ND

ND

ND

ND

ND

$\mu \mathrm{g} / \mathrm{L} \quad$ Sample

$\mu \mathrm{g} / \mathrm{L} \quad$ Sample

$\mu g / L \quad$ Sample

$\mu \mathrm{g} / \mathrm{L} \quad$ Sample

$\mu \mathrm{g} / \mathrm{L} \quad$ Sample

$\mu \mathrm{g} / \mathrm{L} \quad$ Sample

$\mu \mathrm{g} / \mathrm{L} \quad$ Sample

$\begin{array}{ll}\mu \mathrm{g} / \mathrm{L} & \text { Sample } \\ \mu \mathrm{g} / \mathrm{L} & \text { Sample } \\ \mu \mathrm{g} / \mathrm{L} & \text { Sample } \\ \mu \mathrm{g} / \mathrm{L} & \text { Sample } \\ \mu \mathrm{g} / \mathrm{L} & \text { Sample } \\ \mu \mathrm{g} / \mathrm{L} & \text { Sample } \\ \mu \mathrm{g} / \mathrm{L} & \text { Sample }\end{array}$

ND

ND

$\mu \mathrm{g} / \mathrm{L} \quad$ Sample $\mu \mathrm{g} / \mathrm{L} \quad$ Sample $\mu \mathrm{g} / \mathrm{L} \quad$ Sample 
MDA

$\begin{array}{llllll}\text { Analyte Location } & \text { Date } & \text { Result } & \text { or PQL Units QC Type }\end{array}$

\section{Volatile Organic Compounds}

1,3-Dichloropropane

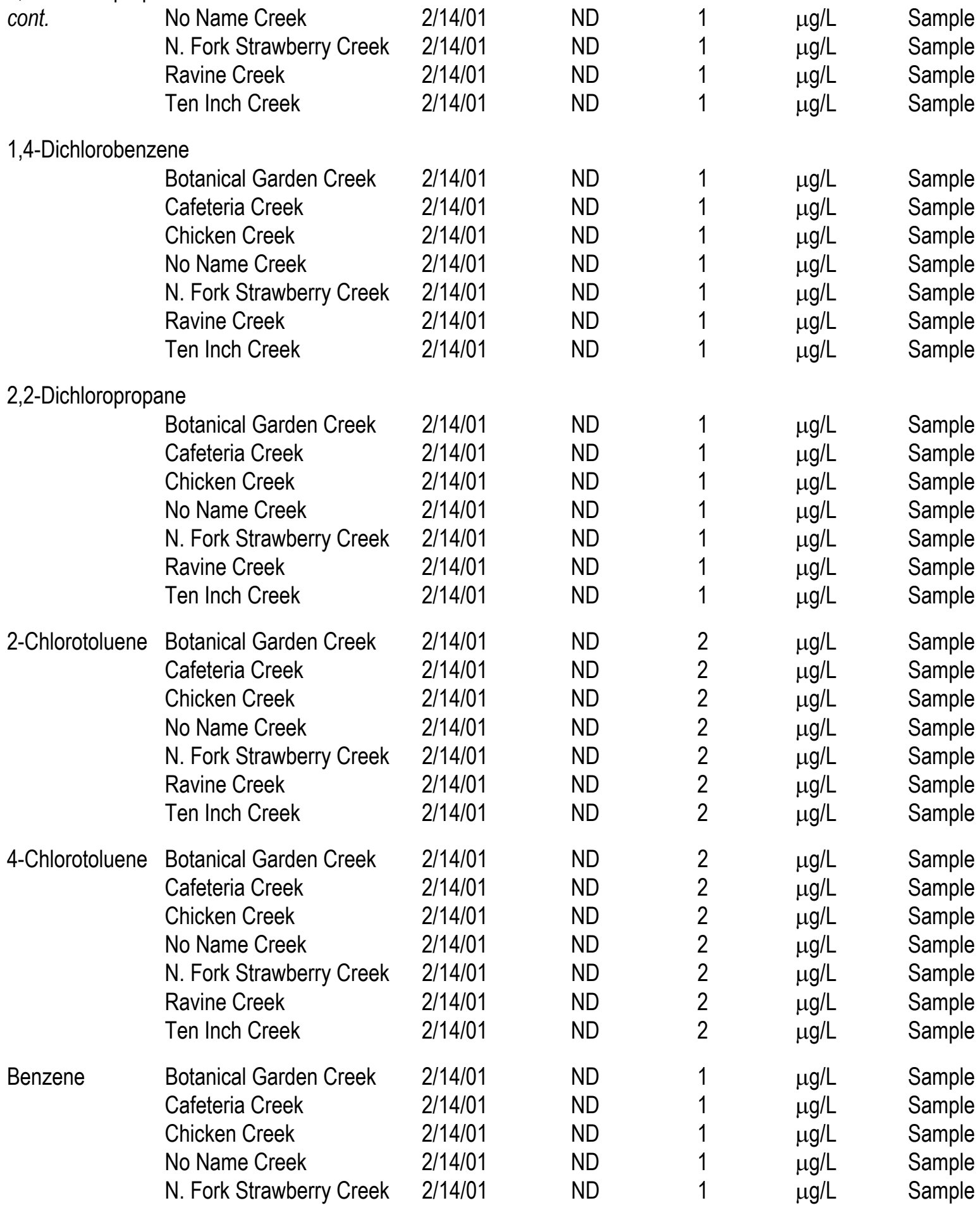




\section{Analyte Location}

\begin{tabular}{|c|c|c|c|c|c|c|}
\hline $\begin{array}{l}\text { Benzene } \\
\text { cont. }\end{array}$ & $\begin{array}{l}\text { Ravine Creek } \\
\text { Ten Inch Creek }\end{array}$ & $\begin{array}{l}2 / 14 / 01 \\
2 / 14 / 01\end{array}$ & $\begin{array}{l}\text { ND } \\
\text { ND }\end{array}$ & $\begin{array}{l}1 \\
1\end{array}$ & $\begin{array}{l}\mu \mathrm{g} / \mathrm{L} \\
\mu \mathrm{g} / \mathrm{L}\end{array}$ & $\begin{array}{l}\text { Sample } \\
\text { Sample }\end{array}$ \\
\hline Bromobenzene & $\begin{array}{l}\text { Botanical Garden Creek } \\
\text { Cafeteria Creek } \\
\text { Chicken Creek } \\
\text { No Name Creek } \\
\text { N. Fork Strawberry Creek } \\
\text { Ravine Creek } \\
\text { Ten Inch Creek }\end{array}$ & $\begin{array}{l}2 / 14 / 01 \\
2 / 14 / 01 \\
2 / 14 / 01 \\
2 / 14 / 01 \\
2 / 14 / 01 \\
2 / 14 / 01 \\
2 / 14 / 01\end{array}$ & $\begin{array}{l}\text { ND } \\
\text { ND } \\
\text { ND } \\
\text { ND } \\
\text { ND } \\
\text { ND } \\
\text { ND }\end{array}$ & $\begin{array}{l}1 \\
1 \\
1 \\
1 \\
1 \\
1 \\
1\end{array}$ & $\begin{array}{l}\mu \mathrm{g} / \mathrm{L} \\
\mu \mathrm{g} / \mathrm{L} \\
\mu \mathrm{g} / \mathrm{L} \\
\mu \mathrm{g} / \mathrm{L} \\
\mu \mathrm{g} / \mathrm{L} \\
\mu \mathrm{g} / \mathrm{L} \\
\mu \mathrm{g} / \mathrm{L}\end{array}$ & $\begin{array}{l}\text { Sample } \\
\text { Sample } \\
\text { Sample } \\
\text { Sample } \\
\text { Sample } \\
\text { Sample } \\
\text { Sample }\end{array}$ \\
\hline Bromochlorome & $\begin{array}{l}\text { hane } \\
\text { Botanical Garden Creek } \\
\text { Cafeteria Creek } \\
\text { Chicken Creek } \\
\text { No Name Creek } \\
\text { N. Fork Strawberry Creek } \\
\text { Ravine Creek } \\
\text { Ten Inch Creek }\end{array}$ & $\begin{array}{l}2 / 14 / 01 \\
2 / 14 / 01 \\
2 / 14 / 01 \\
2 / 14 / 01 \\
2 / 14 / 01 \\
2 / 14 / 01 \\
2 / 14 / 01\end{array}$ & $\begin{array}{l}\text { ND } \\
\text { ND } \\
\text { ND } \\
\text { ND } \\
\text { ND } \\
\text { ND } \\
\text { ND }\end{array}$ & $\begin{array}{l}2 \\
2 \\
2 \\
2 \\
2 \\
2 \\
2\end{array}$ & $\begin{array}{l}\mu \mathrm{g} / \mathrm{L} \\
\mu \mathrm{g} / \mathrm{L} \\
\mu \mathrm{g} / \mathrm{L} \\
\mu \mathrm{g} / \mathrm{L} \\
\mu \mathrm{g} / \mathrm{L} \\
\mu \mathrm{g} / \mathrm{L} \\
\mu \mathrm{g} / \mathrm{L}\end{array}$ & $\begin{array}{l}\text { Sample } \\
\text { Sample } \\
\text { Sample } \\
\text { Sample } \\
\text { Sample } \\
\text { Sample } \\
\text { Sample }\end{array}$ \\
\hline Bromodichlorom & $\begin{array}{l}\text { ethane } \\
\text { Botanical Garden Creek } \\
\text { Cafeteria Creek } \\
\text { Chicken Creek } \\
\text { No Name Creek } \\
\text { N. Fork Strawberry Creek } \\
\text { Ravine Creek } \\
\text { Ten Inch Creek }\end{array}$ & $\begin{array}{l}2 / 14 / 01 \\
2 / 14 / 01 \\
2 / 14 / 01 \\
2 / 14 / 01 \\
2 / 14 / 01 \\
2 / 14 / 01 \\
2 / 14 / 01\end{array}$ & $\begin{array}{l}\text { ND } \\
\text { ND } \\
\text { ND } \\
\text { ND } \\
\text { ND } \\
\text { ND } \\
\text { ND }\end{array}$ & $\begin{array}{l}1 \\
1 \\
1 \\
1 \\
1 \\
1 \\
1\end{array}$ & $\begin{array}{l}\mu \mathrm{g} / \mathrm{L} \\
\mu \mathrm{g} / \mathrm{L} \\
\mu \mathrm{g} / \mathrm{L} \\
\mu \mathrm{g} / \mathrm{L} \\
\mu \mathrm{g} / \mathrm{L} \\
\mu \mathrm{g} / \mathrm{L} \\
\mu \mathrm{g} / \mathrm{L}\end{array}$ & $\begin{array}{l}\text { Sample } \\
\text { Sample } \\
\text { Sample } \\
\text { Sample } \\
\text { Sample } \\
\text { Sample } \\
\text { Sample }\end{array}$ \\
\hline Bromoform & $\begin{array}{l}\text { Botanical Garden Creek } \\
\text { Cafeteria Creek } \\
\text { Chicken Creek } \\
\text { No Name Creek } \\
\text { N. Fork Strawberry Creek } \\
\text { Ravine Creek } \\
\text { Ten Inch Creek }\end{array}$ & $\begin{array}{l}2 / 14 / 01 \\
2 / 14 / 01 \\
2 / 14 / 01 \\
2 / 14 / 01 \\
2 / 14 / 01 \\
2 / 14 / 01 \\
2 / 14 / 01\end{array}$ & $\begin{array}{l}\text { ND } \\
\text { ND } \\
\text { ND } \\
\text { ND } \\
\text { ND } \\
\text { ND } \\
\text { ND }\end{array}$ & $\begin{array}{l}2 \\
2 \\
2 \\
2 \\
2 \\
2 \\
2\end{array}$ & $\begin{array}{l}\mu \mathrm{g} / \mathrm{L} \\
\mu \mathrm{g} / \mathrm{L} \\
\mu \mathrm{g} / \mathrm{L} \\
\mu \mathrm{g} / \mathrm{L} \\
\mu \mathrm{g} / \mathrm{L} \\
\mu \mathrm{g} / \mathrm{L} \\
\mu \mathrm{g} / \mathrm{L}\end{array}$ & $\begin{array}{l}\text { Sample } \\
\text { Sample } \\
\text { Sample } \\
\text { Sample } \\
\text { Sample } \\
\text { Sample } \\
\text { Sample }\end{array}$ \\
\hline Bromomethane & $\begin{array}{l}\text { Botanical Garden Creek } \\
\text { Cafeteria Creek } \\
\text { Chicken Creek } \\
\text { No Name Creek } \\
\text { N. Fork Strawberry Creek } \\
\text { Ravine Creek } \\
\text { Ten Inch Creek }\end{array}$ & $\begin{array}{l}2 / 14 / 01 \\
2 / 14 / 01 \\
2 / 14 / 01 \\
2 / 14 / 01 \\
2 / 14 / 01 \\
2 / 14 / 01 \\
2 / 14 / 01\end{array}$ & $\begin{array}{l}\text { ND } \\
\text { ND } \\
\text { ND } \\
\text { ND } \\
\text { ND } \\
\text { ND } \\
\text { ND }\end{array}$ & $\begin{array}{l}4 \\
4 \\
4 \\
4 \\
4 \\
4 \\
4\end{array}$ & $\begin{array}{l}\mu \mathrm{g} / \mathrm{L} \\
\mu \mathrm{g} / \mathrm{L} \\
\mu \mathrm{g} / \mathrm{L} \\
\mu \mathrm{g} / \mathrm{L} \\
\mu \mathrm{g} / \mathrm{L} \\
\mu \mathrm{g} / \mathrm{L} \\
\mu \mathrm{g} / \mathrm{L}\end{array}$ & $\begin{array}{l}\text { Sample } \\
\text { Sample } \\
\text { Sample } \\
\text { Sample } \\
\text { Sample } \\
\text { Sample } \\
\text { Sample }\end{array}$ \\
\hline
\end{tabular}


MDA

$\begin{array}{llllll}\text { Analyte Location } & \text { Date } & \text { Result } & \text { or PQL Units QC Type }\end{array}$

\section{Volatile Organic Compounds}

Carbon Tetrachloride

\begin{tabular}{|c|c|c|c|c|c|c|}
\hline & Botanical Garden Creek & $2 / 14 / 01$ & ND & 1 & $\mu \mathrm{g} / \mathrm{L}$ & Sample \\
\hline & Cafeteria Creek & $2 / 14 / 01$ & ND & 1 & $\mu \mathrm{g} / \mathrm{L}$ & Sample \\
\hline & Chicken Creek & $2 / 14 / 01$ & ND & 1 & $\mu \mathrm{g} / \mathrm{L}$ & Sample \\
\hline & No Name Creek & $2 / 14 / 01$ & ND & 1 & $\mu \mathrm{g} / \mathrm{L}$ & Sample \\
\hline & N. Fork Strawberry Creek & $2 / 14 / 01$ & ND & 1 & $\mu \mathrm{g} / \mathrm{L}$ & Sample \\
\hline & Ravine Creek & $2 / 14 / 01$ & ND & 1 & $\mu \mathrm{g} / \mathrm{L}$ & Sample \\
\hline & Ten Inch Creek & $2 / 14 / 01$ & ND & 1 & $\mu \mathrm{g} / \mathrm{L}$ & Sample \\
\hline Chlorobenzene & Botanical Garden Creek & $2 / 14 / 01$ & ND & 1 & $\mu \mathrm{g} / \mathrm{L}$ & Sample \\
\hline & Cafeteria Creek & $2 / 14 / 01$ & ND & 1 & $\mu \mathrm{g} / \mathrm{L}$ & Sample \\
\hline & Chicken Creek & $2 / 14 / 01$ & ND & 1 & $\mu \mathrm{g} / \mathrm{L}$ & Sample \\
\hline & No Name Creek & $2 / 14 / 01$ & ND & 1 & $\mu \mathrm{g} / \mathrm{L}$ & Samp \\
\hline & N. Fork Strawberry Creek & $2 / 14 / 01$ & ND & 1 & $\mu \mathrm{g} / \mathrm{L}$ & Sample \\
\hline & Ravine Creek & $2 / 14 / 01$ & ND & 1 & $\mu \mathrm{g} / \mathrm{L}$ & Sample \\
\hline & Ten Inch Creek & $2 / 14 / 01$ & ND & 1 & $\mu \mathrm{g} / \mathrm{L}$ & Samp \\
\hline Chloroethane & Botanical Garden Creek & $2 / 14 / 01$ & ND & 30 & $\mu \mathrm{g} / \mathrm{L}$ & Sample \\
\hline & Cafeteria Creek & $2 / 14 / 01$ & ND & 30 & $\mu \mathrm{g} / \mathrm{L}$ & Sample \\
\hline & Chicken Creek & $2 / 14 / 01$ & ND & 30 & $\mu \mathrm{g} / \mathrm{L}$ & Sample \\
\hline & No Name Creek & $2 / 14 / 01$ & ND & 30 & $\mu \mathrm{g} / \mathrm{L}$ & Sampl \\
\hline & N. Fork Strawberry Creek & $2 / 14 / 01$ & ND & 30 & $\mu \mathrm{g} / \mathrm{L}$ & Samp \\
\hline & Ravine Creek & $2 / 14 / 01$ & ND & 30 & $\mu \mathrm{g} / \mathrm{L}$ & Sampl \\
\hline & Ten Inch Creek & $2 / 14 / 01$ & ND & 30 & $\mu \mathrm{g} / \mathrm{L}$ & Samp \\
\hline Chloroform & Botanical Garden Creek & $2 / 14 / 01$ & ND & 3 & $\mu \mathrm{g} / \mathrm{L}$ & Sample \\
\hline & Cafeteria Creek & $2 / 14 / 01$ & ND & 3 & $\mu \mathrm{g} / \mathrm{L}$ & Samp \\
\hline & Chicken Creek & $2 / 14 / 01$ & ND & 3 & $\mu \mathrm{g} / \mathrm{L}$ & Sampl \\
\hline & No Name Creek & $2 / 14 / 01$ & ND & 3 & $\mu \mathrm{g} / \mathrm{L}$ & Sampl \\
\hline & N. Fork Strawberry Creek & $2 / 14 / 01$ & ND & 3 & $\mu \mathrm{g} / \mathrm{L}$ & Samp \\
\hline & Ravine Creek & $2 / 14 / 01$ & ND & 3 & $\mu \mathrm{g} / \mathrm{L}$ & Samp \\
\hline & Ten Inch Creek & $2 / 14 / 01$ & ND & 3 & $\mu \mathrm{g} / \mathrm{L}$ & Sample \\
\hline Chloromethane & Botanical Garden Creek & $2 / 14 / 01$ & ND & 1 & $\mu \mathrm{g} / \mathrm{L}$ & Sample \\
\hline & Cafeteria Creek & $2 / 14 / 01$ & ND & 1 & $\mu \mathrm{g} / \mathrm{L}$ & Samp \\
\hline & Chicken Creek & $2 / 14 / 01$ & ND & 1 & $\mu \mathrm{g} / \mathrm{L}$ & Samp \\
\hline & No Name Creek & $2 / 14 / 01$ & ND & 1 & $\mu \mathrm{g} / \mathrm{L}$ & Samp \\
\hline & N. Fork Strawberry Creek & $2 / 14 / 01$ & ND & 1 & $\mu \mathrm{g} / \mathrm{L}$ & Samp \\
\hline & Ravine Creek & $2 / 14 / 01$ & ND & 1 & $\mu \mathrm{g} / \mathrm{L}$ & Samp \\
\hline & Ten Inch Creek & $2 / 14 / 01$ & ND & 1 & $\mu \mathrm{g} / \mathrm{L}$ & Sampl \\
\hline -1,2-Dichloroe & thene & & & & & \\
\hline & Botanical Garden Creek & $2 / 14 / 01$ & ND & 1 & $\mu \mathrm{g} / \mathrm{L}$ & Sample \\
\hline & Cafeteria Creek & $2 / 14 / 01$ & ND & 1 & $\mu \mathrm{g} / \mathrm{L}$ & Sampl \\
\hline & Chicken Creek & $2 / 14 / 01$ & ND & 1 & $\mu \mathrm{g} / \mathrm{L}$ & Sampl \\
\hline & No Name Creek & $2 / 14 / 01$ & ND & 1 & $\mu \mathrm{g} / \mathrm{L}$ & Sampl \\
\hline
\end{tabular}




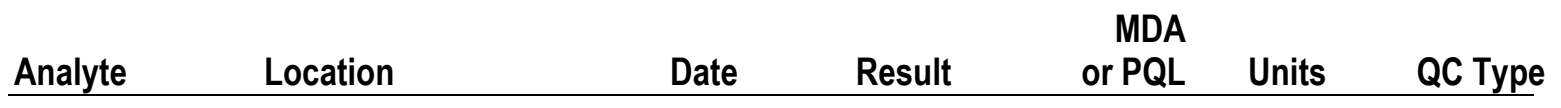

\section{Volatile Organic Compounds}

cis-1,2-Dichloroethene

cont.

$\begin{array}{ll}\text { N. Fork Strawberry Creek } & 2 / 14 / 01 \\ \text { Ravine Creek } & 2 / 14 / 01 \\ \text { Ten Inch Creek } & 2 / 14 / 01\end{array}$

cis-1,3-Dichloropropene

$\begin{array}{ll}\text { Botanical Garden Creek } & 2 / 14 / 01 \\ \text { Cafeteria Creek } & 2 / 14 / 01 \\ \text { Chicken Creek } & 2 / 14 / 01 \\ \text { No Name Creek } & 2 / 14 / 01 \\ \text { N. Fork Strawberry Creek } & 2 / 14 / 01 \\ \text { Ravine Creek } & 2 / 14 / 01 \\ \text { Ten Inch Creek } & 2 / 14 / 01\end{array}$

Dibromochloromethane

$\begin{array}{ll}\text { Botanical Garden Creek } & 2 / 14 / 01 \\ \text { Cafeteria Creek } & 2 / 14 / 01 \\ \text { Chicken Creek } & 2 / 14 / 01 \\ \text { No Name Creek } & 2 / 14 / 01 \\ \text { N. Fork Strawberry Creek } & 2 / 14 / 01 \\ \text { Ravine Creek } & 2 / 14 / 01 \\ \text { Ten Inch Creek } & 2 / 14 / 01\end{array}$

Dibromomethane

Botanical Garden Creek

Cafeteria Creek

$2 / 14 / 01$

Chicken Creek

No Name Creek

N. Fork Strawberry Creek

Ravine Creek

Ten Inch Creek

Ethylbenzene

Botanical Garden Creek
Cafeteria Creek
Chicken Creek
No Name Creek
N. Fork Strawberry Creek
Ravine Creek
Ten Inch Creek

Freon 113-1,1,2-Trichlorotrifluoroethane

Botanical Garden Creek

Cafeteria Creek

Chicken Creek

$2 / 14 / 01$

No Name Creek

$2 / 14 / 01$

2/14/01

N. Fork Strawberry Creek
2/14/01

2/14/01

2/14/01

$2 / 14 / 01$

2/14/01

2/14/01

2/14/01

2/14/01

$2 / 14 / 01$

2/14/01

$2 / 14 / 01$

2/14/01

2/14/01
ND

ND

ND

ND

ND

ND

ND

ND

ND

ND

ND

ND

ND

ND

ND

ND

ND

ND

ND

ND

ND

ND

ND

ND

ND

ND

ND

ND

ND

ND

ND

ND

ND

ND

ND

ND $\mu \mathrm{g} / \mathrm{L}$

$\mu \mathrm{g} / \mathrm{L} \quad$ Sample

$\mu \mathrm{g} / \mathrm{L} \quad$ Sample

$\mu \mathrm{g} / \mathrm{L} \quad$ Sample

$\mu \mathrm{g} / \mathrm{L} \quad$ Sample

$\mu \mathrm{g} / \mathrm{L} \quad$ Sample

$\mu \mathrm{g} / \mathrm{L} \quad$ Sample

$\mu g / L \quad$ Sample

$\mu \mathrm{g} / \mathrm{L} \quad$ Sample

$\mu \mathrm{g} / \mathrm{L} \quad$ Sample

$\begin{array}{ll}\mu \mathrm{g} / \mathrm{L} & \text { Sample } \\ \mu \mathrm{g} / \mathrm{L} & \text { Sample } \\ \mu \mathrm{g} / \mathrm{L} & \text { Sample } \\ \mu \mathrm{g} / \mathrm{L} & \text { Sample } \\ \mu \mathrm{g} / \mathrm{L} & \text { Sample } \\ \mu \mathrm{g} / \mathrm{L} & \text { Sample } \\ \mu \mathrm{g} / \mathrm{L} & \text { Sample }\end{array}$

$\mu \mathrm{g} / \mathrm{L} \quad$ Sample

$\mu \mathrm{g} / \mathrm{L} \quad$ Sample

$\mu \mathrm{g} / \mathrm{L} \quad$ Sample

$\mu \mathrm{g} / \mathrm{L} \quad$ Sample

$\mu \mathrm{g} / \mathrm{L} \quad$ Sample

$\mu \mathrm{g} / \mathrm{L} \quad$ Sample

$\mu \mathrm{g} / \mathrm{L} \quad$ Sample

$\mu \mathrm{g} / \mathrm{L} \quad$ Sample

$\mu \mathrm{g} / \mathrm{L} \quad$ Sample

$\mu \mathrm{g} / \mathrm{L} \quad$ Sample

$\mu \mathrm{g} / \mathrm{L} \quad$ Sample

$\mu \mathrm{g} / \mathrm{L} \quad$ Sample

$\mu \mathrm{g} / \mathrm{L} \quad$ Sample

$\mu \mathrm{g} / \mathrm{L} \quad$ Sample

$\mu \mathrm{g} / \mathrm{L} \quad$ Sample

$\mu \mathrm{g} / \mathrm{L} \quad$ Sample

$\mu \mathrm{g} / \mathrm{L} \quad$ Sample

$\mu \mathrm{g} / \mathrm{L} \quad$ Sample

$\mu \mathrm{g} / \mathrm{L} \quad$ Sample 


\section{MDA}

$\begin{array}{llllll}\text { Analyte Location } & \text { Date } & \text { Result } & \text { or PQL Units QC Type }\end{array}$

\section{Volatile Organic Compounds}

Freon 113-1,1,2-Trichlorotrifluoroethane

cont.

Ravine Creek

Ten Inch Creek

Freon 114-1,2-Dichlorotetrafluoroethane

Botanical Garden Creek

Cafeteria Creek

Chicken Creek

No Name Creek

N. Fork Strawberry Creek

Ravine Creek

Ten Inch Creek

2/14/01

$2 / 14 / 01$

2/14/01

$2 / 14 / 01$

$2 / 14 / 01$

$2 / 14 / 01$

$2 / 14 / 01$

Freon 11-Trichlorofluoromethane

Botanical Garden Creek

Cafeteria Creek

Chicken Creek

No Name Creek

N. Fork Strawberry Creek

Ravine Creek

Ten Inch Creek

2/14/01

2/14/01

$2 / 14 / 01$

2/14/01

$2 / 14 / 01$

$2 / 14 / 01$

2/14/01

Freon 123A-1,2-Dichlorotrifluoroethane

Botanical Garden Creek

Cafeteria Creek

Chicken Creek

No Name Creek

N. Fork Strawberry Creek

Ravine Creek

Ten Inch Creek

2/14/01

$2 / 14 / 01$

$2 / 14 / 01$

2/14/01

$2 / 14 / 01$

2/14/01

2/14/01

Freon 123-Dichlorotrifluoroethane

Botanical Garden Creek

Cafeteria Creek

Chicken Creek

No Name Creek

N. Fork Strawberry Creek

Ravine Creek

Ten Inch Creek

2/14/01

2/14/01

2/14/01

$2 / 14 / 01$

2/14/01

$2 / 14 / 01$

$2 / 14 / 01$

Freon 12-Dichlorodifluoromethane

Botanical Garden Creek 2/14/0

Cafeteria Creek

$2 / 14 / 01$

Chicken Creek

No Name Creek

2/14/01

2/14/01

N. Fork Strawberry Creek
ND

ND

1
1

$\mu \mathrm{g} / \mathrm{L}$

$\mu \mathrm{g} / \mathrm{L}$

Sample

Sample

ND

ND

ND

ND

ND

ND

ND

ND

ND

ND

ND

ND

ND

ND

ND

ND

ND

ND

ND

ND

ND

ND

ND

ND

ND

ND

ND

ND

ND

ND

ND

ND

ND $\mu \mathrm{g} / \mathrm{L}$

$\mu \mathrm{g} / \mathrm{L}$

$\mu \mathrm{g} / \mathrm{L}$

$\mu \mathrm{g} / \mathrm{L}$

$\mu \mathrm{g} / \mathrm{L}$

$\mu \mathrm{g} / \mathrm{L}$

$\mu \mathrm{g} / \mathrm{L}$

Sample

Sample

Sample

Sample

Sample

Sample

Sample

$\mu \mathrm{g} / \mathrm{L} \quad$ Sample

$\mu \mathrm{g} / \mathrm{L} \quad$ Sample

$\mu \mathrm{g} / \mathrm{L} \quad$ Sample

$\mu \mathrm{g} / \mathrm{L} \quad$ Sample

$\mu \mathrm{g} / \mathrm{L} \quad$ Sample

$\mu \mathrm{g} / \mathrm{L} \quad$ Sample

$\mu \mathrm{g} / \mathrm{L} \quad$ Sample

$\mu \mathrm{g} / \mathrm{L} \quad$ Sample

$\mu \mathrm{g} / \mathrm{L} \quad$ Sample

$\mu \mathrm{g} / \mathrm{L} \quad$ Sample

$\mu \mathrm{g} / \mathrm{L} \quad$ Sample

$\mu \mathrm{g} / \mathrm{L} \quad$ Sample

$\mu \mathrm{g} / \mathrm{L} \quad$ Sample

$\mu \mathrm{g} / \mathrm{L} \quad$ Sample

$\mu g / L \quad$ Sample

$\mu \mathrm{g} / \mathrm{L} \quad$ Sample

$\mu \mathrm{g} / \mathrm{L} \quad$ Sample

$\mu \mathrm{g} / \mathrm{L} \quad$ Sample

$\mu \mathrm{g} / \mathrm{L} \quad$ Sample

$\mu \mathrm{g} / \mathrm{L} \quad$ Sample

$\mu \mathrm{g} / \mathrm{L} \quad$ Sample

$\mu \mathrm{g} / \mathrm{L} \quad$ Sample

$\mu \mathrm{g} / \mathrm{L} \quad$ Sample

$\mu \mathrm{g} / \mathrm{L} \quad$ Sample

$\mu \mathrm{g} / \mathrm{L} \quad$ Sample $\mu \mathrm{g} / \mathrm{L} \quad$ Sample 


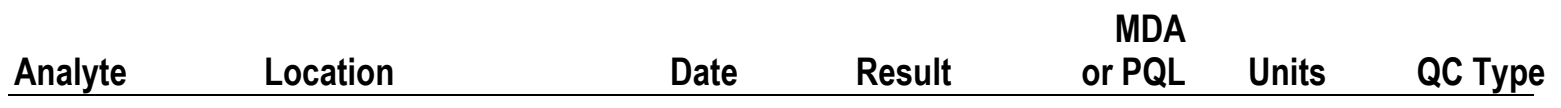

\section{Volatile Organic Compounds}

Freon 12-Dichlorodifluoromethane

cont.

Ravine Creek

Ten Inch Creek

Freon 21-Dichlorofluoromethane

Botanical Garden Creek

Cafeteria Creek

Chicken Creek

No Name Creek

N. Fork Strawberry Creek

Ravine Creek

Ten Inch Creek

Freon 22-Chlorodifluoromethane

Botanical Garden Creek

Cafeteria Creek

Chicken Creek

No Name Creek

N. Fork Strawberry Creek

Ravine Creek

Ten Inch Creek

2/14/01

2/14/01

2/14/01

2/14/01

2/14/01

$2 / 14 / 01$

2/14/01

Hexachlorobutadiene

Botanical Garden Creek

Cafeteria Creek

Chicken Creek

No Name Creek

N. Fork Strawberry Creek

Ravine Creek

Ten Inch Creek

2/14/01

2/14/01

2/14/01

2/14/01

$2 / 14 / 01$

2/14/01

2/14/01

Isopropylbenzene

Botanical Garden Creek

Cafeteria Creek

Chicken Creek

No Name Creek

N. Fork Strawberry Creek

Ravine Creek

Ten Inch Creek

2/14/01

2/14/01

2/14/01

2/14/01

$2 / 14 / 01$

$2 / 14 / 01$

2/14/01

Methyl tert-Butyl Ether

$\begin{array}{ll}\text { Botanical Garden Creek } & \text { 2/14/01 } \\ \text { Cafeteria Creek } & 2 / 14 / 01 \\ \text { Chicken Creek } & 2 / 14 / 01 \\ \text { No Name Creek } & 2 / 14 / 01 \\ \text { N. Fork Strawberry Creek } & 2 / 14 / 01\end{array}$

ND

ND

3

$\mu \mathrm{g} / \mathrm{L} \quad$ Sample

$\mu \mathrm{g} / \mathrm{L} \quad$ Sample

$\begin{array}{llll}\text { ND } & 3 & \mu g / L & \text { Sample } \\ \text { ND } & 3 & \mu g / L & \text { Sample } \\ \text { ND } & 3 & \mu g / L & \text { Sample } \\ \text { ND } & 3 & \mu g / L & \text { Sample } \\ \text { ND } & 3 & \mu g / L & \text { Sample } \\ \text { ND } & 3 & \mu g / L & \text { Sample } \\ \text { ND } & 3 & \mu g / L & \text { Sample }\end{array}$

ND

30

30

ND

$\begin{array}{ll}\text { ND } & 30 \\ \text { ND } & 30\end{array}$

ND $\quad 30$

ND $\quad 30$

ND $\quad 30$

ND

ND

ND

ND

ND

ND

ND

ND

ND

ND

ND

ND

ND

ND

ND

ND

ND

ND

ND $\mu \mathrm{g} / \mathrm{L} \quad$ Sample

$\mu \mathrm{g} / \mathrm{L} \quad$ Sample

$\mu \mathrm{g} / \mathrm{L} \quad$ Sample

$\mu \mathrm{g} / \mathrm{L} \quad$ Sample

$\mu \mathrm{g} / \mathrm{L} \quad$ Sample

$\mu \mathrm{g} / \mathrm{L} \quad$ Sample

$\mu \mathrm{g} / \mathrm{L} \quad$ Sample

$\mu \mathrm{g} / \mathrm{L} \quad$ Sample

$\mu \mathrm{g} / \mathrm{L} \quad$ Sample

$\mu \mathrm{g} / \mathrm{L} \quad$ Sample

$\mu \mathrm{g} / \mathrm{L} \quad$ Sample

$\mu \mathrm{g} / \mathrm{L} \quad$ Sample

$\mu \mathrm{g} / \mathrm{L} \quad$ Sample

$\mu \mathrm{g} / \mathrm{L} \quad$ Sample

$\mu \mathrm{g} / \mathrm{L} \quad$ Sample

$\mu \mathrm{g} / \mathrm{L} \quad$ Sample

$\mu \mathrm{g} / \mathrm{L} \quad$ Sample

$\mu \mathrm{g} / \mathrm{L} \quad$ Sample

$\mu \mathrm{g} / \mathrm{L} \quad$ Sample

$\mu \mathrm{g} / \mathrm{L} \quad$ Sample

$\mu \mathrm{g} / \mathrm{L} \quad$ Sample

$\mu \mathrm{g} / \mathrm{L} \quad$ Sample

$\mu \mathrm{g} / \mathrm{L} \quad$ Sample

$\mu \mathrm{g} / \mathrm{L} \quad$ Sample

$\mu \mathrm{g} / \mathrm{L} \quad$ Sample

$\mu \mathrm{g} / \mathrm{L} \quad$ Sample 
MDA

$\begin{array}{llllll}\text { Analyte Location } & \text { Date } & \text { Result } & \text { or PQL Units QC Type }\end{array}$

\section{Volatile Organic Compounds}

Methyl tert-Butyl Ether

cont.

Ravine Creek

Ten Inch Creek

Methylene Chloride

$\begin{array}{ll}\text { Botanical Garden Creek } & \text { 2/14/01 } \\ \text { Cafeteria Creek } & 2 / 14 / 01 \\ \text { Chicken Creek } & 2 / 14 / 01 \\ \text { No Name Creek } & 2 / 14 / 01 \\ \text { N. Fork Strawberry Creek } & 2 / 14 / 01 \\ \text { Ravine Creek } & 2 / 14 / 01 \\ \text { Ten Inch Creek } & 2 / 14 / 01\end{array}$

Naphthalene

Botanical Garden Creek

Cafeteria Creek

Chicken Creek

No Name Creek

N. Fork Strawberry Creek

Ravine Creek

Ten Inch Creek

n-Butylbenzene

Botanical Garden Creek

Cafeteria Creek

Chicken Creek

No Name Creek

N. Fork Strawberry Creek

Ravine Creek

Ten Inch Creek

2/14/01

2/14/01

2/14/01

2/14/01

2/14/01

2/14/01

2/14/01

2/14/01

2/14/01

2/14/01

2/14/01

2/14/01

2/14/01

2/14/01

n-Propylbenzene

Botanical Garden Creek

Cafeteria Creek

Chicken Creek

No Name Creek

N. Fork Strawberry Creek

Ravine Creek

Ten Inch Creek

$2 / 14 / 01$

2/14/01

2/14/01

2/14/01

2/14/01

2/14/01

2/14/01
ND

ND

ND

ND

$\mathrm{ND}$

$\mathrm{ND}$

ND

ND

ND

ND

ND

ND

ND

ND

$\mathrm{ND}$

ND

ND

ND

ND

ND

ND

ND

ND

ND

ND

ND

$\mathrm{ND}$

ND

ND

ND

ND

$\mathrm{ND}$

ND

ND

ND

ND

ND
5

$5 \mu \mathrm{g} / \mathrm{L}$

$\begin{array}{ll}\mu \mathrm{g} / \mathrm{L} & \text { Sample } \\ \mu \mathrm{g} / \mathrm{L} & \text { Sample }\end{array}$

$\begin{array}{ll}\mu \mathrm{g} / \mathrm{L} & \text { Sample } \\ \mu \mathrm{g} / \mathrm{L} & \text { Sample }\end{array}$

$\mu \mathrm{g} / \mathrm{L}$

Sample

$\mu \mathrm{g} / \mathrm{L} \quad$ Sample

$\mu \mathrm{g} / \mathrm{L} \quad$ Sample

$\mu \mathrm{g} / \mathrm{L} \quad$ Sample

$\mu \mathrm{g} / \mathrm{L} \quad$ Sample

$\mu \mathrm{g} / \mathrm{L} \quad$ Sample

$\mu \mathrm{g} / \mathrm{L} \quad$ Sample

$\mu g / L \quad$ Sample

$\mu \mathrm{g} / \mathrm{L} \quad$ Sample

$\mu \mathrm{g} / \mathrm{L} \quad$ Sample

$\mu \mathrm{g} / \mathrm{L} \quad$ Sample

$\mu \mathrm{g} / \mathrm{L} \quad$ Sample

$\mu \mathrm{g} / \mathrm{L} \quad$ Sample

$\mu \mathrm{g} / \mathrm{L} \quad$ Sample

$\mu g / L \quad$ Sample

$\mu \mathrm{g} / \mathrm{L} \quad$ Sample

$\mu \mathrm{g} / \mathrm{L} \quad$ Sample

$\mu \mathrm{g} / \mathrm{L} \quad$ Sample

$\mu \mathrm{g} / \mathrm{L} \quad$ Sample

$\mu \mathrm{g} / \mathrm{L} \quad$ Sample

$\mu \mathrm{g} / \mathrm{L} \quad$ Sample

$\mu \mathrm{g} / \mathrm{L} \quad$ Sample

$\mu \mathrm{g} / \mathrm{L} \quad$ Sample

$\mu \mathrm{g} / \mathrm{L} \quad$ Sample

$\mu \mathrm{g} / \mathrm{L} \quad$ Sample

$\mu \mathrm{g} / \mathrm{L} \quad$ Sample

$\mu \mathrm{g} / \mathrm{L} \quad$ Sample

$\mu \mathrm{g} / \mathrm{L} \quad$ Sample

Botanical Garden Creek 2/14/01

Chicken Creek 2/14/01

$2 / 14 / 01$

2/14/01

$2 / 14 / 01$

$2 / 14 / 01$

Ten Inch Creek 


$\begin{array}{lllll}\text { Analyte Location } & \text { Date } & \text { Result } & \text { or PQL Units } & \text { QC Type }\end{array}$

\section{Volatile Organic Compounds}

sec-Butylbenzene

$\begin{array}{llllll}\text { Botanical Garden Creek } & 2 / 14 / 01 & \text { ND } & 1 & \mu \mathrm{g} / \mathrm{L} & \text { Sample } \\ \text { Cafeteria Creek } & 2 / 14 / 01 & \text { ND } & 1 & \mu \mathrm{g} / \mathrm{L} & \text { Sample } \\ \text { Chicken Creek } & 2 / 14 / 01 & \text { ND } & 1 & \mu \mathrm{g} / \mathrm{L} & \text { Sample } \\ \text { No Name Creek } & 2 / 14 / 01 & \text { ND } & 1 & \mu \mathrm{g} / & \text { Sample } \\ \text { N. Fork Strawberry Creek } & 2 / 14 / 01 & \mathrm{ND} & 1 & \mu \mathrm{g} / \mathrm{L} & \text { Sample } \\ \text { Ravine Creek } & 2 / 14 / 01 & \mathrm{ND} & 1 & \mu \mathrm{g} / \mathrm{L} & \text { Sample } \\ \text { Ten Inch Creek } & 2 / 14 / 01 & \mathrm{ND} & 1 & \mu \mathrm{g} / \mathrm{L} & \text { Sample } \\ \text { Botanical Garden Creek } & 2 / 14 / 01 & \mathrm{ND} & 1 & \mu \mathrm{g} / \mathrm{L} & \text { Sample } \\ \text { Cafeteria Creek } & 2 / 14 / 01 & \mathrm{ND} & 1 & \mu \mathrm{g} / \mathrm{L} & \text { Sample } \\ \text { Chicken Creek } & 2 / 14 / 01 & \mathrm{ND} & 1 & \mu \mathrm{g} / \mathrm{L} & \text { Sample } \\ \text { No Name Creek } & 2 / 14 / 01 & \mathrm{ND} & 1 & \mu \mathrm{g} / \mathrm{L} & \text { Sample } \\ \text { N. Fork Strawberry Creek } & 2 / 14 / 01 & \mathrm{ND} & 1 & \mu \mathrm{g} / \mathrm{L} & \text { Sample } \\ \text { Ravine Creek } & 2 / 14 / 01 & \mathrm{ND} & 1 & \mu \mathrm{g} / \mathrm{L} & \text { Sample } \\ \text { Ten Inch Creek } & 2 / 14 / 01 & \mathrm{ND} & 1 & \mu \mathrm{g} / \mathrm{L} & \text { Sample }\end{array}$

tert-Butylbenzene

$\begin{array}{ll}\text { Botanical Garden Creek } & \text { 2/14/01 } \\ \text { Cafeteria Creek } & 2 / 14 / 01 \\ \text { Chicken Creek } & 2 / 14 / 01 \\ \text { No Name Creek } & 2 / 14 / 01 \\ \text { N. Fork Strawberry Creek } & 2 / 14 / 01 \\ \text { Ravine Creek } & 2 / 14 / 01 \\ \text { Ten Inch Creek } & 2 / 14 / 01\end{array}$

ND

$\mu \mathrm{g} / \mathrm{L} \quad$ Sample

$\begin{array}{llll}\text { ND } & 1 & \mu \mathrm{g} / \mathrm{L} & \text { Sample }\end{array}$

ND $\quad 1 \quad \mu g / L \quad$ Sample

$\begin{array}{llll}\text { ND } & 1 & \mu \mathrm{g} / \mathrm{L} & \text { Sample }\end{array}$

ND $\quad 1 \quad \mu g / L \quad$ Sample

ND $\quad 1 \quad \mu g / L \quad$ Sample

ND $\quad 1 \quad \mu g / L \quad$ Sample

Tetrachloroethene

Botanical Garden Creek $\quad$ 2/14/01

Cafeteria Creek 2/14/01

Chicken Creek 2/14/01

No Name Creek 2/14/01

N. Fork Strawberry Creek $\quad 2 / 14 / 01$

Ravine Creek 2/14/01

Ten Inch Creek 2/14/01

Toluene Botanical Garden Creek 2/14/01

Cafeteria Creek 2/14/01

Chicken Creek 2/14/01

No Name Creek 2/14/01

N. Fork Strawberry Creek $\quad$ 2/14/01

Ravine Creek 2/14/01

Ten Inch Creek 2/14/01
ND

ND

ND

ND

ND

ND

ND

ND

ND

ND

ND

ND

ND

ND

$\begin{array}{ll}\mu \mathrm{g} / \mathrm{L} & \text { Sample } \\ \mu \mathrm{g} / \mathrm{L} & \text { Sample } \\ \mu \mathrm{g} / \mathrm{L} & \text { Sample } \\ \mu \mathrm{g} / \mathrm{L} & \text { Sample } \\ \mu \mathrm{g} / \mathrm{L} & \text { Sample } \\ \mu \mathrm{g} / \mathrm{L} & \text { Sample } \\ \mu \mathrm{g} / \mathrm{L} & \text { Sample } \\ \mu \mathrm{g} / \mathrm{L} & \text { Sample } \\ \mu \mathrm{g} / \mathrm{L} & \text { Sample } \\ \mu \mathrm{g} / \mathrm{L} & \text { Sample } \\ \mu \mathrm{g} / \mathrm{L} & \text { Sample } \\ \mu \mathrm{g} / \mathrm{L} & \text { Sample } \\ \mu \mathrm{g} / \mathrm{L} & \text { Sample } \\ \mu \mathrm{g} / \mathrm{L} & \text { Sample }\end{array}$

trans-1,2-Dichloroethene

Botanical Garden Creek $\quad$ 2/14/01

Cafeteria Creek

2/14/01

$\mu \mathrm{g} / \mathrm{L} \quad$ Sample

$\mu \mathrm{g} / \mathrm{L} \quad$ Sample 


\section{MDA}

$\begin{array}{llllll}\text { Analyte Location } & \text { Date } & \text { Result } & \text { or PQL Units QC Type }\end{array}$

\section{Volatile Organic Compounds}

trans-1,2-Dichloroethene

\begin{tabular}{|c|c|c|c|c|c|c|}
\hline \multirow[t]{5}{*}{ cont. } & Chicken Creek & $2 / 14 / 01$ & ND & 1 & $\mu \mathrm{g} / \mathrm{L}$ & Sample \\
\hline & No Name Creek & $2 / 14 / 01$ & ND & 1 & $\mu \mathrm{g} / \mathrm{L}$ & Sample \\
\hline & N. Fork Strawberry Creek & $2 / 14 / 01$ & ND & 1 & $\mu \mathrm{g} / \mathrm{L}$ & Sample \\
\hline & Ravine Creek & $2 / 14 / 01$ & ND & 1 & $\mu \mathrm{g} / \mathrm{L}$ & Sample \\
\hline & Ten Inch Creek & $2 / 14 / 01$ & ND & 1 & $\mu \mathrm{g} / \mathrm{L}$ & Sample \\
\hline \multicolumn{7}{|c|}{ trans-1,3-Dichloropropene } \\
\hline & Botanical Garden Creek & $2 / 14 / 01$ & ND & 1 & $\mu \mathrm{g} / \mathrm{L}$ & Sample \\
\hline & Cafeteria Creek & $2 / 14 / 01$ & ND & 1 & $\mu \mathrm{g} / \mathrm{L}$ & Sample \\
\hline & Chicken Creek & $2 / 14 / 01$ & ND & 1 & $\mu \mathrm{g} / \mathrm{L}$ & Sample \\
\hline & No Name Creek & $2 / 14 / 01$ & ND & 1 & $\mu \mathrm{g} / \mathrm{L}$ & Sample \\
\hline & N. Fork Strawberry Creek & $2 / 14 / 01$ & ND & 1 & $\mu \mathrm{g} / \mathrm{L}$ & Sample \\
\hline & Ravine Creek & $2 / 14 / 01$ & ND & 1 & $\mu \mathrm{g} / \mathrm{L}$ & Sample \\
\hline & Ten Inch Creek & $2 / 14 / 01$ & ND & 1 & $\mu \mathrm{g} / \mathrm{L}$ & Sample \\
\hline \multirow[t]{7}{*}{ Trichloroethene } & Botanical Garden Creek & $2 / 14 / 01$ & ND & 1 & $\mu \mathrm{g} / \mathrm{L}$ & Sample \\
\hline & Cafeteria Creek & $2 / 14 / 01$ & ND & 1 & $\mu \mathrm{g} / \mathrm{L}$ & Samp \\
\hline & Chicken Creek & $2 / 14 / 01$ & ND & 1 & $\mu \mathrm{g} / \mathrm{L}$ & Samp \\
\hline & No Name Creek & $2 / 14 / 01$ & ND & 1 & $\mu \mathrm{g} / \mathrm{L}$ & Samp \\
\hline & N. Fork Strawberry Creek & $2 / 14 / 01$ & ND & 1 & $\mu \mathrm{g} / \mathrm{L}$ & Samp \\
\hline & Ravine Creek & $2 / 14 / 01$ & ND & 1 & $\mu \mathrm{g} / \mathrm{L}$ & Samp \\
\hline & Ten Inch Creek & $2 / 14 / 01$ & ND & 1 & $\mu \mathrm{g} / \mathrm{L}$ & Sampl \\
\hline \multirow[t]{7}{*}{ Vinyl Chloride } & Botanical Garden Creek & $2 / 14 / 01$ & ND & 1 & $\mu \mathrm{g} / \mathrm{L}$ & Sample \\
\hline & Cafeteria Creek & $2 / 14 / 01$ & ND & 1 & $\mu \mathrm{g} / \mathrm{L}$ & Samp \\
\hline & Chicken Creek & $2 / 14 / 01$ & ND & 1 & $\mu \mathrm{g} / \mathrm{L}$ & Samp \\
\hline & No Name Creek & $2 / 14 / 01$ & ND & 1 & $\mu \mathrm{g} / \mathrm{L}$ & Samp \\
\hline & N. Fork Strawberry Creek & $2 / 14 / 01$ & ND & 1 & $\mu \mathrm{g} / \mathrm{L}$ & Samp \\
\hline & Ravine Creek & $2 / 14 / 01$ & ND & 1 & $\mu \mathrm{g} / \mathrm{L}$ & Samp \\
\hline & Ten Inch Creek & $2 / 14 / 01$ & ND & 1 & $\mu \mathrm{g} / \mathrm{L}$ & Sampl \\
\hline \multirow[t]{7}{*}{ Xylenes (total) } & Botanical Garden Creek & $2 / 14 / 01$ & ND & 2 & $\mu \mathrm{g} / \mathrm{L}$ & Sample \\
\hline & Cafeteria Creek & $2 / 14 / 01$ & ND & 2 & $\mu \mathrm{g} / \mathrm{L}$ & Samp \\
\hline & Chicken Creek & $2 / 14 / 01$ & ND & 2 & $\mu \mathrm{g} / \mathrm{L}$ & Samp \\
\hline & No Name Creek & $2 / 14 / 01$ & ND & 2 & $\mu \mathrm{g} / \mathrm{L}$ & Samp \\
\hline & N. Fork Strawberry Creek & $2 / 14 / 01$ & ND & 2 & $\mu \mathrm{g} / \mathrm{L}$ & Samp \\
\hline & Ravine Creek & $2 / 14 / 01$ & ND & 2 & $\mu \mathrm{g} / \mathrm{L}$ & Sampl \\
\hline & Ten Inch Creek & $2 / 14 / 01$ & ND & 2 & $\mu \mathrm{g} / \mathrm{L}$ & Sampl \\
\hline
\end{tabular}


The following lake data are summarized and discussed in Chapter 5 (Surface Waters and Wastewater) of the Site Environmental Report for 2001 (see Volume I):

\begin{tabular}{|c|c|c|c|c|c|c|}
\hline Analyte & Location & Date & Result & MDA or PQL & Units & QC Type \\
\hline \multicolumn{7}{|c|}{ Radiological Activity } \\
\hline \multirow[t]{4}{*}{ Gross alpha } & Lake Anza & $8 / 23 / 01$ & ND & 0.07 & $\mathrm{~Bq} / \mathrm{L}$ & Sample \\
\hline & & $8 / 23 / 01$ & ND & 0.07 & $\mathrm{~Bq} / \mathrm{L}$ & Split \\
\hline & Lake Temescal & $8 / 23 / 01$ & ND & 0.11 & $\mathrm{~Bq} / \mathrm{L}$ & Sample \\
\hline & Field Blank & $8 / 23 / 01$ & ND & 0.07 & $\mathrm{~Bq} / \mathrm{L}$ & Blank \\
\hline \multirow[t]{3}{*}{ Gross beta } & Lake Anza & $8 / 23 / 01$ & ND & 0.11 & $\mathrm{~Bq} / \mathrm{L}$ & Sample \\
\hline & & $8 / 23 / 01$ & ND & 0.11 & $\mathrm{~Bq} / \mathrm{L}$ & Sp \\
\hline & Lake Temescal & $8 / 23 / 01$ & ND & 0.15 & $\mathrm{~Bq} / \mathrm{L}$ & Sample \\
\hline \multirow[t]{5}{*}{ Tritium } & Lake Anza & $8 / 23 / 01$ & ND & 7 & $\mathrm{~Bq} / \mathrm{L}$ & Sample \\
\hline & & $8 / 23 / 01$ & ND & 7 & $\mathrm{~Bq} / \mathrm{L}$ & Sp \\
\hline & Lake Temescal & $8 / 23 / 01$ & ND & 7 & $\mathrm{~Bq} / \mathrm{L}$ & Sample \\
\hline & & $8 / 23 / 01$ & ND & 7 & $\mathrm{~Bq} / \mathrm{L}$ & \\
\hline & Field Blank & $8 / 23 / 01$ & ND & 7 & $\mathrm{~Bq} / \mathrm{L}$ & Blank \\
\hline
\end{tabular}




\section{Stormwater}

The following stormwater data are summarized and discussed in Chapter 5 (Surface Waters and Wastewater) of the Site Environmental Report for 2001 (see Volume 1):

\begin{tabular}{|c|c|c|c|c|c|c|}
\hline Analyte & Location & Date & Result & $\begin{array}{r}\text { MDA } \\
\text { or PQL } \\
\end{array}$ & Units & QC Type \\
\hline \multicolumn{7}{|c|}{ Radiological Activity } \\
\hline \multirow[t]{4}{*}{ Gross alpha } & 69-Storm Drain Manhole & $\begin{array}{l}1 / 23 / 01 \\
12 / 1 / 01\end{array}$ & $\begin{array}{l}\text { ND } \\
\text { ND }\end{array}$ & $\begin{array}{l}0.11 \\
0.11\end{array}$ & $\begin{array}{l}\mathrm{Bq} / \mathrm{L} \\
\mathrm{Bq} / \mathrm{L}\end{array}$ & $\begin{array}{l}\text { Sample } \\
\text { Sample }\end{array}$ \\
\hline & Chicken Creek & $\begin{array}{l}1 / 23 / 01 \\
11 / 10 / 01\end{array}$ & $\begin{array}{l}\text { ND } \\
\text { ND }\end{array}$ & $\begin{array}{l}0.11 \\
0.11\end{array}$ & $\begin{array}{l}\mathrm{Bq} / \mathrm{L} \\
\mathrm{Bq} / \mathrm{L}\end{array}$ & $\begin{array}{l}\text { Sample } \\
\text { Sample }\end{array}$ \\
\hline & East Canyon & $\begin{array}{l}1 / 23 / 01 \\
12 / 1 / 01\end{array}$ & $\begin{array}{l}\text { ND } \\
\text { ND }\end{array}$ & $\begin{array}{l}0.11 \\
0.11\end{array}$ & $\begin{array}{l}\mathrm{Bq} / \mathrm{L} \\
\mathrm{Bq} / \mathrm{L}\end{array}$ & $\begin{array}{l}\text { Sample } \\
\text { Sample }\end{array}$ \\
\hline & N. Fork Strawberry Creek & $\begin{array}{l}1 / 23 / 01 \\
11 / 10 / 01\end{array}$ & $\begin{array}{l}\text { ND } \\
\text { ND }\end{array}$ & $\begin{array}{l}0.11 \\
0.11\end{array}$ & $\begin{array}{l}\mathrm{Bq} / \mathrm{L} \\
\mathrm{Bq} / \mathrm{L}\end{array}$ & $\begin{array}{l}\text { Sample } \\
\text { Sample }\end{array}$ \\
\hline \multirow[t]{4}{*}{ Gross beta } & 69-Storm Drain Manhole & $\begin{array}{l}1 / 23 / 01 \\
12 / 1 / 01\end{array}$ & $\begin{array}{l}\mathrm{ND} \\
0.21\end{array}$ & $\begin{array}{l}0.11 \\
0.11\end{array}$ & $\begin{array}{l}\mathrm{Bq} / \mathrm{L} \\
\mathrm{Bq} / \mathrm{L}\end{array}$ & $\begin{array}{l}\text { Sample } \\
\text { Sample }\end{array}$ \\
\hline & Chicken Creek & $\begin{array}{l}1 / 23 / 01 \\
11 / 10 / 01\end{array}$ & $\begin{array}{l}0.15 \\
0.22\end{array}$ & $\begin{array}{l}0.11 \\
0.07\end{array}$ & $\begin{array}{l}\mathrm{Bq} / \mathrm{L} \\
\mathrm{Bq} / \mathrm{L}\end{array}$ & $\begin{array}{l}\text { Sample } \\
\text { Sample }\end{array}$ \\
\hline & East Canyon & $\begin{array}{l}1 / 23 / 01 \\
12 / 1 / 01\end{array}$ & $\begin{array}{l}\mathrm{ND} \\
0.14\end{array}$ & $\begin{array}{l}0.11 \\
0.11\end{array}$ & $\begin{array}{l}\mathrm{Bq} / \mathrm{L} \\
\mathrm{Bq} / \mathrm{L}\end{array}$ & $\begin{array}{l}\text { Sample } \\
\text { Sample }\end{array}$ \\
\hline & N. Fork Strawberry Creek & $\begin{array}{l}1 / 23 / 01 \\
11 / 10 / 01\end{array}$ & $\begin{array}{l}\text { ND } \\
\text { ND }\end{array}$ & $\begin{array}{l}0.11 \\
0.07\end{array}$ & $\begin{array}{l}\mathrm{Bq} / \mathrm{L} \\
\mathrm{Bq} / \mathrm{L}\end{array}$ & $\begin{array}{l}\text { Sample } \\
\text { Sample }\end{array}$ \\
\hline \multirow[t]{2}{*}{ Tritium } & 69-Storm Drain Manhole & $\begin{array}{l}1 / 23 / 01 \\
12 / 1 / 01\end{array}$ & $\begin{array}{l}57 \\
60.7\end{array}$ & $\begin{array}{l}7 \\
7\end{array}$ & $\begin{array}{l}\mathrm{Bq} / \mathrm{L} \\
\mathrm{Bq} / \mathrm{L}\end{array}$ & $\begin{array}{l}\text { Sample } \\
\text { Sample }\end{array}$ \\
\hline & Chicken Creek & $\begin{array}{l}1 / 23 / 01 \\
11 / 10 / 01\end{array}$ & $\begin{array}{r}16 \\
N D\end{array}$ & $\begin{array}{l}7 \\
7\end{array}$ & $\begin{array}{l}\mathrm{Bq} / \mathrm{L} \\
\mathrm{Bq} / \mathrm{L}\end{array}$ & $\begin{array}{l}\text { Sample } \\
\text { Sample }\end{array}$ \\
\hline
\end{tabular}




\begin{tabular}{|c|c|c|c|c|c|c|}
\hline Analyte & Location & Date & Result & $\begin{array}{r}\text { MDA } \\
\text { or PQL } \\
\end{array}$ & Units & QC Type \\
\hline \multicolumn{7}{|c|}{ Radiological Activity } \\
\hline \multirow[t]{2}{*}{$\begin{array}{l}\text { Tritium } \\
\text { cont. }\end{array}$} & East Canyon & $\begin{array}{l}1 / 23 / 01 \\
12 / 1 / 01\end{array}$ & $\begin{array}{l}N D \\
N D\end{array}$ & $\begin{array}{l}7 \\
7\end{array}$ & $\begin{array}{l}\mathrm{Bq} / \mathrm{L} \\
\mathrm{Bq} / \mathrm{L}\end{array}$ & $\begin{array}{l}\text { Sample } \\
\text { Sample }\end{array}$ \\
\hline & N. Fork Strawberry Creek & $\begin{array}{l}1 / 23 / 01 \\
11 / 10 / 01\end{array}$ & $\begin{array}{l}\text { ND } \\
\text { ND }\end{array}$ & $\begin{array}{l}7 \\
7\end{array}$ & $\begin{array}{l}\mathrm{Bq} / \mathrm{L} \\
\mathrm{Bq} / \mathrm{L}\end{array}$ & $\begin{array}{l}\text { Sample } \\
\text { Sample }\end{array}$ \\
\hline
\end{tabular}

\section{General Indicator Parameters}

Chemical Oxygen Demand

69-Storm Drain Manhole

$1 / 23 / 01$

$12 / 1 / 01$

ND $\quad 20$

$\mathrm{mg} / \mathrm{L} \quad$ Sample

$7120 \quad \mathrm{mg} / \mathrm{L} \quad$ Sample

Chicken Creek

$1 / 23 / 01$

86

$11 / 10 / 01$

170

20

20

$\mathrm{mg} / \mathrm{L} \quad$ Sample

$\mathrm{mg} / \mathrm{L} \quad$ Sample

East Canyon

$1 / 23 / 01$

ND

20

$12 / 1 / 01$

$35 \quad 20$

$\mathrm{mg} / \mathrm{L} \quad$ Sample

$\mathrm{mg} / \mathrm{L} \quad$ Sample

N. Fork Strawberry Creek

$1 / 23 / 01$

$40 \quad 20$

$\mathrm{mg} / \mathrm{L} \quad$ Sample

$11 / 10 / 01$

ND

$\mathrm{mg} / \mathrm{L} \quad$ Sample

$\mathrm{pH}$

69-Storm Drain Manhole

$1 / 23 / 01$

$12 / 1 / 01$

$7.54-$
7.15

S.U.

Sample

$7.15-$

S.U.

Sample

Chicken Creek

$1 / 23 / 01$

$7.27 \quad-$

S.U. Sample

$11 / 10 / 01$

$7.5-$

S.U.

Sample

East Canyon $\quad 1 / 23 / 01$

$7.21 \quad-$

S.U.

Sample

$12 / 1 / 01$

$7.36 \quad-$

S.U.

Sample

N. Fork Strawberry Creek 1/23/01

$11 / 10 / 01$

$\begin{array}{ll}7.17 & - \\ 7.75 & -\end{array}$

S.U.

Sample

$7.75-$

S.U. Sample

Specific Conductance

69-Storm Drain Manhole
Chicken Creek

$1 / 23 / 01$

59

$12 / 1 / 01$

206

$1 / 23 / 01$

78

$11 / 10 / 01$

405

East Canyon

$1 / 23 / 01$

$12 / 1 / 01$

54

85

N. Fork Strawberry Creek

$1 / 23 / 01$

70

35

$\begin{array}{lll}1 & \mu \mathrm{mhos} / \mathrm{cm} & \text { Sample } \\ 1 & \mu \mathrm{mhos} / \mathrm{cm} & \text { Sample } \\ 1 & \mu \mathrm{mhos} / \mathrm{cm} & \text { Sample } \\ 1 & \mu \mathrm{mhos} / \mathrm{cm} & \text { Sample } \\ 1 & \mu \mathrm{mhos} / \mathrm{cm} & \text { Sample } \\ 1 & \mu \mathrm{mhos} / \mathrm{cm} & \text { Sample } \\ 1 & \mu \mathrm{mhos} / \mathrm{cm} & \text { Sample } \\ 1 & \mu \mathrm{mhos} / \mathrm{cm} & \text { Sample }\end{array}$




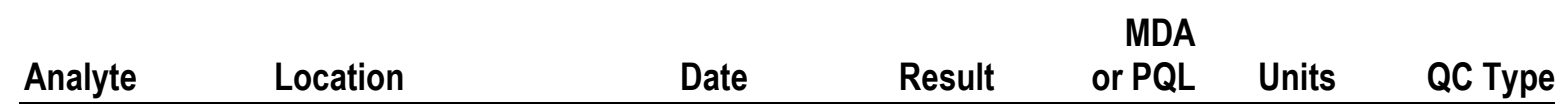

\section{General Indicator Parameters}

Total suspended solids (TSS)

$$
\text { 69-Storm Drain Manhole }
$$

$1 / 23 / 01$

$12 / 1 / 01$

Chicken Creek

$1 / 23 / 01$

$11 / 10 / 01$

East Canyon

N. Fork Strawberry Creek

$1 / 23 / 01$

$12 / 1 / 01$

$1 / 23 / 01$

$11 / 10 / 01$

Metals and/or Minerals

Aluminum

69-Storm Drain Manhole

$1 / 23 / 01$

$12 / 1 / 01$

Chicken Creek

$1 / 23 / 01$

$11 / 10 / 01$

East Canyon

$1 / 23 / 01$

$12 / 1 / 01$

N. Fork Strawberry Creek

$1 / 23 / 01$

$11 / 10 / 01$

Aluminum (Filtered)

69-Storm Drain Manhole

$1 / 23 / 01$

$1 / 23 / 01$

$1 / 23 / 01$

East Canyon

N. Fork Strawberry Creek

$1 / 23 / 01$

69-Storm Drain Manhole 1/23/01

Chicken Creek

$1 / 23 / 01$

East Canyon

$1 / 23 / 01$

N. Fork Strawberry Creek $\quad$ 1/23/01

$\begin{array}{llll}4.7 & 2 & \mathrm{mg} / \mathrm{L} & \text { Sample }\end{array}$

$\begin{array}{llll}7 & 5 & \mathrm{mg} / \mathrm{L} & \text { Sample }\end{array}$

$180 \quad 5 \quad \mathrm{mg} / \mathrm{L} \quad$ Sample

$17 \quad 5 \quad \mathrm{mg} / \mathrm{L} \quad$ Sample

$24 \quad 3 \quad \mathrm{mg} / \mathrm{L} \quad$ Sample

$153 \mathrm{mg} / \mathrm{L} \quad$ Sample

$42 \quad 5 \quad \mathrm{mg} / \mathrm{L} \quad$ Sample

$89 \quad 5 \quad \mathrm{mg} / \mathrm{L} \quad$ Sample

$0.2 \quad 0.05 \quad \mathrm{mg} / \mathrm{L} \quad$ Sample

$\begin{array}{llll}0.1 & 0.05 & \mathrm{mg} / \mathrm{L} & \text { Sample }\end{array}$

$\begin{array}{llll}5 & 0.05 & \mathrm{mg} / \mathrm{L} & \text { Sample }\end{array}$

$\begin{array}{llll}0.5 & 0.05 \quad \mathrm{mg} / \mathrm{L} \quad \text { Sample }\end{array}$

$\begin{array}{llll}0.4 & 0.05 & \mathrm{mg} / \mathrm{L} & \text { Sample }\end{array}$

$1 \quad 0.05 \quad \mathrm{mg} / \mathrm{L} \quad$ Sample

$\begin{array}{llll} & 0.05 & \mathrm{mg} / \mathrm{L} & \text { Sample }\end{array}$

$\begin{array}{llll}4.9 & 0.05 & \mathrm{mg} / \mathrm{L} & \text { Sample }\end{array}$

ND $\quad 0.05 \quad \mathrm{mg} / \mathrm{L} \quad$ Sample

$0.1 \quad 0.05 \quad \mathrm{mg} / \mathrm{L} \quad$ Sample

ND $\quad 0.05 \quad \mathrm{mg} / \mathrm{L} \quad$ Sample

$0.05 \quad 0.05 \quad \mathrm{mg} / \mathrm{L} \quad$ Sample

ND $\quad 0.3 \quad \mathrm{mg} / \mathrm{L} \quad$ Sample

ND $\quad 0.3 \quad \mathrm{mg} / \mathrm{L} \quad$ Sample

ND $\quad 0.3 \quad \mathrm{mg} / \mathrm{L} \quad$ Sample

ND $\quad 0.3 \quad \mathrm{mg} / \mathrm{L} \quad$ Sample




\begin{tabular}{|c|c|c|c|c|c|c|}
\hline Analyte & Location & Date & Result & $\begin{array}{r}\text { MDA } \\
\text { or PQL }\end{array}$ & Units & QC Type \\
\hline
\end{tabular}

\section{Metals and/or Minerals}

Antimony (Filtered)

69-Storm Drain Manhol

Chicken Creek

East Canyon

N. Fork Strawberry Creek

Arsenic

69-Storm Drain Manhole

Chicken Creek

East Canyon

N. Fork Strawberry Creek

Arsenic (Filtered) 69-Storm Drain Manhole

Chicken Creek

East Canyon

N. Fork Strawberry Creek

$1 / 23 / 01$

Barium

69-Storm Drain Manhole

Chicken Creek

East Canyon

N. Fork Strawberry Creek

$1 / 23 / 01$

Barium (Filtered) 69-Storm Drain Manhole

$1 / 23 / 01$

Chicken Creek

$1 / 23 / 01$

East Canyon

$1 / 23 / 01$

N. Fork Strawberry Creek

$1 / 23 / 01$

Beryllium

$1 / 23 / 01$
$1 / 23 / 01$
ND

ND

ND

ND

ND

ND

ND

ND

ND

ND

ND

ND

ND

ND

ND

ND

ND

ND

ND

ND

ND

ND
$0.3 \quad \mathrm{mg} / \mathrm{L}$

Sample

$0.3 \quad \mathrm{mg} / \mathrm{L} \quad$ Sample

$0.3 \quad \mathrm{mg} / \mathrm{L} \quad$ Sample

$0.3 \quad \mathrm{mg} / \mathrm{L} \quad$ Sample

$0.05 \mathrm{mg} / \mathrm{L} \quad$ Sample

$0.05 \mathrm{mg} / \mathrm{L} \quad$ Sample

$0.05 \mathrm{mg} / \mathrm{L} \quad$ Sample

$0.05 \mathrm{mg} / \mathrm{L} \quad$ Sample

$0.05 \mathrm{mg} / \mathrm{L} \quad$ Sample

$0.05 \mathrm{mg} / \mathrm{L} \quad$ Sample

$0.05 \mathrm{mg} / \mathrm{L} \quad$ Sample

$0.05 \mathrm{mg} / \mathrm{L} \quad$ Sample

$0.5 \mathrm{mg} / \mathrm{L} \quad$ Sample

$0.5 \quad \mathrm{mg} / \mathrm{L} \quad$ Sample

$0.5 \quad \mathrm{mg} / \mathrm{L} \quad$ Sample

$0.5 \quad \mathrm{mg} / \mathrm{L} \quad$ Sample

$0.5 \quad \mathrm{mg} / \mathrm{L} \quad$ Sample

$0.5 \quad \mathrm{mg} / \mathrm{L} \quad$ Sample

$0.5 \quad \mathrm{mg} / \mathrm{L} \quad$ Sample

$0.5 \quad \mathrm{mg} / \mathrm{L} \quad$ Sample

$0.05 \mathrm{mg} / \mathrm{L} \quad$ Sample

Chicken Creek

0.05

$\mathrm{mg} / \mathrm{L}$

Sample 


\begin{tabular}{|c|c|c|c|c|c|c|}
\hline Analyte & Location & Date & Result & $\begin{array}{r}\text { MDA } \\
\text { or } P Q L \\
\end{array}$ & Units & QC Type \\
\hline \multicolumn{7}{|c|}{ Metals and/or Minerals } \\
\hline \multirow{2}{*}{$\begin{array}{l}\text { Beryllium } \\
\text { cont. }\end{array}$} & East Canyon & $1 / 23 / 01$ & ND & 0.05 & $\mathrm{mg} / \mathrm{L}$ & Sample \\
\hline & N. Fork Strawberry Creek & $1 / 23 / 01$ & ND & 0.05 & $\mathrm{mg} / \mathrm{L}$ & Sample \\
\hline
\end{tabular}

Beryllium (Filtered)

69-Storm Drain Manhole

Chicken Creek

$1 / 23 / 01$

$1 / 23 / 01$

East Canyon

N. Fork Strawberry Creek

$1 / 23 / 01$

$1 / 23 / 01$

Boron

69-Storm Drain Manhole

$1 / 23 / 01$

Chicken Creek

$1 / 23 / 01$

East Canyon

$1 / 23 / 01$

N. Fork Strawberry Creek 1/23/01

Boron (Filtered)

69-Storm Drain Manhole

$1 / 23 / 01$

Chicken Creek

$1 / 23 / 01$

East Canyon

$1 / 23 / 01$

N. Fork Strawberry Creek

$1 / 23 / 01$

Cadmium

69-Storm Drain Manhole

$1 / 23 / 01$

Chicken Creek

$1 / 23 / 01$

$1 / 23 / 01$

N. Fork Strawberry Creek $\quad 1 / 23 / 01$

$\begin{array}{lc}\text { 69-Storm Drain Manhole } & 1 / 23 / 01 \\ \text { Chicken Creek } & 1 / 23 / 01 \\ \text { East Canyon } & 1 / 23 / 01 \\ \text { N. Fork Strawberry Creek } & 1 / 23 / 0\end{array}$

Chromium 69-Storm Drain Manhole
$1 / 23 / 01$

$1 / 23 / 01$

Cog

East Canyon

Cadmium (Filtered)

ND

$0.05 \mathrm{mg} / \mathrm{L} \quad$ Sample

ND

$0.05 \mathrm{mg} / \mathrm{L} \quad$ Sample

ND

$0.05 \mathrm{mg} / \mathrm{L}$

Sample

ND

$0.05 \mathrm{mg} / \mathrm{L}$

Sample

ND

$0.5 \quad \mathrm{mg} / \mathrm{L}$

Sample

ND

$0.5 \mathrm{mg} / \mathrm{L}$

Sample

ND

$0.5 \mathrm{mg} / \mathrm{L}$

Sample

ND

$0.5 \mathrm{mg} / \mathrm{L}$

Sample

ND

$0.5 \mathrm{mg} / \mathrm{L}$

Sample

ND

$0.5 \mathrm{mg} / \mathrm{L}$

Sample

ND

$0.5 \quad \mathrm{mg} / \mathrm{L}$

Sample

ND

$0.5 \mathrm{mg} / \mathrm{L}$

Sample

ND

$0.1 \mathrm{mg} / \mathrm{L}$

Sample

ND

$0.1 \mathrm{mg} / \mathrm{L}$

Sample

ND

$0.1 \quad \mathrm{mg} / \mathrm{L}$

Sample

ND

$0.1 \quad \mathrm{mg} / \mathrm{L}$

Sample

ND

$0.1 \quad \mathrm{mg} / \mathrm{L} \quad$ Sample

ND

$0.1 \mathrm{mg} / \mathrm{L}$

Sample

ND

$0.1 \mathrm{mg} / \mathrm{L}$

Sample

ND

ND

$0.1 \mathrm{mg} / \mathrm{L}$

$0.5 \mathrm{mg} / \mathrm{L}$

Sample

Sample 


\begin{tabular}{llllll} 
Lnalyte & \multicolumn{4}{c}{ MDA } \\
Location & Date & Result & or PQL Units & QC Type
\end{tabular}

Metals and/or Minerals

$\begin{array}{lccccc}\text { Chicken Creek } & 1 / 23 / 01 & \text { ND } & 0.5 & \mathrm{mg} / \mathrm{L} & \text { Sample } \\ \text { East Canyon } & 1 / 23 / 01 & \text { ND } & 0.5 & \mathrm{mg} / \mathrm{L} & \text { Sample } \\ \text { N. Fork Strawberry Creek } & 1 / 23 / 01 & \text { ND } & 0.5 & \mathrm{mg} / \mathrm{L} & \text { Sample }\end{array}$

Chromium (Filtered)

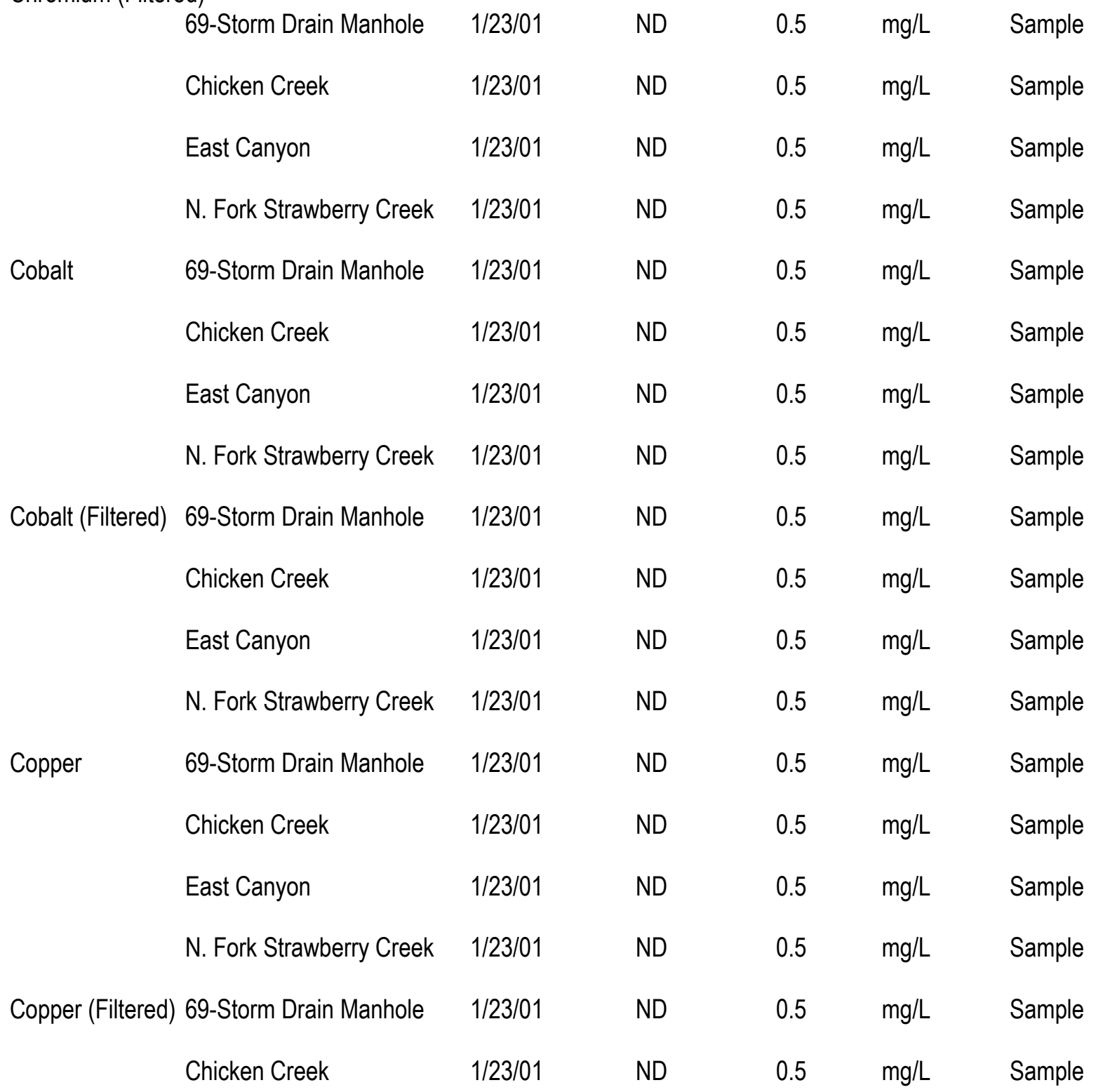




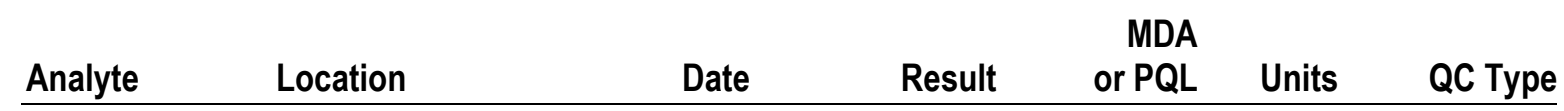

\section{Metals and/or Minerals}

Copper (Filtered)

cont.

East Canyon

N. Fork Strawberry Creek $\quad$ 1/23/01

Iron

69-Storm Drain Manhole $\quad$ 1/23/01

$12 / 1 / 01$

Chicken Creek

East Canyon

N. Fork Strawberry Creek

$1 / 23 / 01$

$11 / 10 / 01$

$1 / 23 / 01$

$12 / 1 / 01$

$1 / 23 / 01$

$11 / 10 / 01$

Iron (Filtered)

69-Storm Drain Manhole 1/23/01

Chicken Creek

$1 / 23 / 01$

East Canyon

N. Fork Strawberry Creek

$1 / 23 / 01$

$1 / 23 / 01$

Lead

69-Storm Drain Manhole

$1 / 23 / 01$

Chicken Creek

$1 / 23 / 01$

East Canyon

$1 / 23 / 01$

N. Fork Strawberry Creek

$1 / 23 / 01$

Lead (Filtered)

69-Storm Drain Manhol

Chicken Creek

$1 / 23 / 01$

$1 / 23 / 01$

East Canyon

N. Fork Strawberry Creek

$1 / 23 / 01$

Magnesium
69-Storm Drain Manhole 12/1/01

Chicken Creek

$11 / 10 / 01$
ND

ND

ND $\quad 0.5 \quad \mathrm{mg} / \mathrm{L}$

$0.2 \quad 0.05 \quad \mathrm{mg} / \mathrm{L}$

$\begin{array}{lll}5.8 & 0.5 \quad \mathrm{mg} / \mathrm{L}\end{array}$

$\begin{array}{lll}0.72 & 0.05 \quad \mathrm{mg} / \mathrm{L}\end{array}$

$0.5 \quad 0.5 \quad \mathrm{mg} / \mathrm{L}$

$1.3 \quad 0.05 \quad \mathrm{mg} / \mathrm{L}$

$\begin{array}{llll}1.5 & 0.5 & \mathrm{mg} / \mathrm{L} & \text { Sample }\end{array}$

$\begin{array}{llll}5.9 & 0.05 \quad \mathrm{mg} / \mathrm{L} & \text { Sample }\end{array}$

ND

$0.5 \mathrm{mg} / \mathrm{L}$

Sample

ND

$0.5 \quad \mathrm{mg} / \mathrm{L}$

Sample

ND

$0.5 \quad \mathrm{mg} / \mathrm{L}$

Sample

ND

$0.5 \quad \mathrm{mg} / \mathrm{L}$

Sample

ND

$0.5 \mathrm{mg} / \mathrm{L}$

Sample

ND

$0.5 \quad \mathrm{mg} / \mathrm{L}$

Sample

ND

$0.5 \mathrm{mg} / \mathrm{L}$

Sample

ND

$0.5 \mathrm{mg} / \mathrm{L}$

Sample

ND

$0.5 \mathrm{mg} / \mathrm{L}$

Sample

ND

$0.5 \quad \mathrm{mg} / \mathrm{L}$

Sample

ND

$0.5 \quad \mathrm{mg} / \mathrm{L}$

Sample

ND

$0.5 \quad \mathrm{mg} / \mathrm{L}$

Sample

1.4

0.05

15

1.4

$\mathrm{mg} / \mathrm{L}$

Sample

$0.05 \mathrm{mg} / \mathrm{L}$

Sample 


\begin{tabular}{|c|c|c|c|c|c|c|}
\hline Analyte & Location & Date & Result & $\begin{array}{r}\text { MDA } \\
\text { or PQL } \\
\end{array}$ & Units & QC Type \\
\hline \multicolumn{7}{|c|}{ Metals and/or Minerals } \\
\hline \multirow{2}{*}{$\begin{array}{l}\text { Magnesium } \\
\text { cont. }\end{array}$} & East Canyon & $12 / 1 / 01$ & 2.7 & 0.05 & $\mathrm{mg} / \mathrm{L}$ & Sample \\
\hline & N. Fork Strawberry Creek & $11 / 10 / 01$ & 2.6 & 0.05 & $\mathrm{mg} / \mathrm{L}$ & Sample \\
\hline \multirow[t]{4}{*}{ Manganese } & 69-Storm Drain Manhole & $1 / 23 / 01$ & ND & 0.15 & $\mathrm{mg} / \mathrm{L}$ & Sample \\
\hline & Chicken Creek & $1 / 23 / 01$ & ND & 0.15 & $\mathrm{mg} / \mathrm{L}$ & Sample \\
\hline & East Canyon & $1 / 23 / 01$ & ND & 0.15 & $\mathrm{mg} / \mathrm{L}$ & Sample \\
\hline & N. Fork Strawberry Creek & $1 / 23 / 01$ & ND & 0.15 & $\mathrm{mg} / \mathrm{L}$ & Sample \\
\hline \multicolumn{7}{|c|}{ Manganese (Filtered) } \\
\hline & 69-Storm Drain Manhole & $1 / 23 / 01$ & ND & 0.15 & $\mathrm{mg} / \mathrm{L}$ & Sample \\
\hline & Chicken Creek & $1 / 23 / 01$ & ND & 0.15 & $\mathrm{mg} / \mathrm{L}$ & Sample \\
\hline & East Canyon & $1 / 23 / 01$ & ND & 0.15 & $\mathrm{mg} / \mathrm{L}$ & Sample \\
\hline & N. Fork Strawberry Creek & $1 / 23 / 01$ & ND & 0.15 & $\mathrm{mg} / \mathrm{L}$ & Sample \\
\hline \multirow[t]{4}{*}{ Mercury } & 69-Storm Drain Manhole & $1 / 23 / 01$ & ND & 0.005 & $\mathrm{mg} / \mathrm{L}$ & Sample \\
\hline & Chicken Creek & $1 / 23 / 01$ & ND & 0.005 & $\mathrm{mg} / \mathrm{L}$ & Sample \\
\hline & East Canyon & $1 / 23 / 01$ & ND & 0.005 & $\mathrm{mg} / \mathrm{L}$ & Sample \\
\hline & N. Fork Strawberry Creek & $1 / 23 / 01$ & ND & 0.005 & $\mathrm{mg} / \mathrm{L}$ & Sample \\
\hline \multicolumn{7}{|c|}{ Mercury (Filtered) } \\
\hline & 69-Storm Drain Manhole & $1 / 23 / 01$ & ND & 0.005 & $\mathrm{mg} / \mathrm{L}$ & Sample \\
\hline & Chicken Creek & $1 / 23 / 01$ & ND & 0.005 & $\mathrm{mg} / \mathrm{L}$ & Sample \\
\hline & East Canyon & $1 / 23 / 01$ & ND & 0.005 & $\mathrm{mg} / \mathrm{L}$ & Sample \\
\hline & N. Fork Strawberry Creek & $1 / 23 / 01$ & ND & 0.005 & $\mathrm{mg} / \mathrm{L}$ & Sample \\
\hline \multirow[t]{4}{*}{ Molybdenum } & 69-Storm Drain Manhole & $1 / 23 / 01$ & ND & 0.5 & $\mathrm{mg} / \mathrm{L}$ & Sample \\
\hline & Chicken Creek & $1 / 23 / 01$ & ND & 0.5 & $\mathrm{mg} / \mathrm{L}$ & Sample \\
\hline & East Canyon & $1 / 23 / 01$ & ND & 0.5 & $\mathrm{mg} / \mathrm{L}$ & Sample \\
\hline & N. Fork Strawberry Creek & $1 / 23 / 01$ & ND & 0.5 & $\mathrm{mg} / \mathrm{L}$ & Sample \\
\hline
\end{tabular}




\begin{tabular}{llllll} 
Mnalyte & Location & Date & Result & or PQL Units & QC Type \\
\hline
\end{tabular}

\section{Metals and/or Minerals}

Molybdenum (Filtered)

$$
\text { 69-Storm Drain Manhole }
$$

Chicken Creek

East Canyon

N. Fork Strawberry Creek $\quad 1 / 23 / 01$

Nickel

69-Storm Drain Manhole

Chicken Creek

East Canyon

N. Fork Strawberry Creek $\quad$ 1/23/01

Nickel (Filtered)

69-Storm Drain Manhole

Chicken Creek

East Canyon

N. Fork Strawberry Creek

Selenium

69-Storm Drain Manhole

Chicken Creek

East Canyon

N. Fork Strawberry Creek $\quad 1 / 23 / 01$

Selenium (Filtered)

69-Storm Drain Manhole

Chicken Creek

East Canyon

N. Fork Strawberry Creek

Silver

Silver

69-Storm Drain Manhole 1/23/0

Chicken Creek

East Canyon
$1 / 23 / 01$

$1 / 23 / 01$

$1 / 23 / 01$

$1 / 23 / 01$

$1 / 23 / 01$

$1 / 23 / 01$

$1 / 23 / 01$

$1 / 23 / 01$

$1 / 23 / 01$

$1 / 23 / 01$

1/23/01

1/23/01

$1 / 23 / 01$

$1 / 23 / 01$

$1 / 23 / 01$

$1 / 23 / 01$

$1 / 23 / 01$

ND

ND

ND

ND

ND

ND

ND

ND

ND

ND

ND

ND

ND

ND

ND

ND

ND

ND

ND

ND

ND

$1 / 23 / 01$

$1 / 23 / 01$
D

$0.5 \mathrm{mg} / \mathrm{L}$

Sample

$0.5 \quad \mathrm{mg} / \mathrm{L} \quad$ Sample

$0.5 \quad \mathrm{mg} / \mathrm{L} \quad$ Sample

$0.5 \quad \mathrm{mg} / \mathrm{L} \quad$ Sample

$0.5 \quad \mathrm{mg} / \mathrm{L} \quad$ Sample

$0.5 \quad \mathrm{mg} / \mathrm{L} \quad$ Sample

$0.5 \quad \mathrm{mg} / \mathrm{L} \quad$ Sample

$0.5 \quad \mathrm{mg} / \mathrm{L} \quad$ Sample

$0.5 \quad \mathrm{mg} / \mathrm{L} \quad$ Sample

$0.5 \quad \mathrm{mg} / \mathrm{L} \quad$ Sample

$0.5 \quad \mathrm{mg} / \mathrm{L} \quad$ Sample

$0.5 \quad \mathrm{mg} / \mathrm{L} \quad$ Sample

$0.05 \mathrm{mg} / \mathrm{L} \quad$ Sample

$0.05 \quad \mathrm{mg} / \mathrm{L} \quad$ Sample

$0.05 \quad \mathrm{mg} / \mathrm{L} \quad$ Sample

$0.05 \mathrm{mg} / \mathrm{L} \quad$ Sample

$0.05 \quad \mathrm{mg} / \mathrm{L} \quad$ Sample

$0.05 \mathrm{mg} / \mathrm{L} \quad$ Sample

$0.05 \mathrm{mg} / \mathrm{L} \quad$ Sample

$0.05 \mathrm{mg} / \mathrm{L} \quad$ Sample

$0.5 \quad \mathrm{mg} / \mathrm{L} \quad$ Sample

ND $\quad 0.5 \quad \mathrm{mg} / \mathrm{L} \quad$ Sample

ND $\quad 0.5 \quad \mathrm{mg} / \mathrm{L} \quad$ Sample 


\begin{tabular}{llllll} 
Mnalyte & Location & Date & Result & or PQL Units & QC Type \\
\hline
\end{tabular}

\section{Metals and/or Minerals}

cont.

\begin{tabular}{|c|c|c|c|c|c|c|}
\hline & N. Fork Strawberry Creek & $1 / 23 / 01$ & ND & 0.5 & $\mathrm{mg} / \mathrm{L}$ & Sample \\
\hline \multirow[t]{4}{*}{ Silver (Filtered) } & 69-Storm Drain Manhole & $1 / 23 / 01$ & ND & 0.5 & $\mathrm{mg} / \mathrm{L}$ & Sample \\
\hline & Chicken Creek & $1 / 23 / 01$ & ND & 0.5 & $\mathrm{mg} / \mathrm{L}$ & Sample \\
\hline & East Canyon & $1 / 23 / 01$ & ND & 0.5 & $\mathrm{mg} / \mathrm{L}$ & Sample \\
\hline & N. Fork Strawberry Creek & $1 / 23 / 01$ & ND & 0.5 & $\mathrm{mg} / \mathrm{L}$ & Sample \\
\hline \multirow[t]{4}{*}{ Thallium } & 69-Storm Drain Manhole & $1 / 23 / 01$ & ND & 0.05 & $\mathrm{mg} / \mathrm{L}$ & Sample \\
\hline & Chicken Creek & $1 / 23 / 01$ & ND & 0.05 & $\mathrm{mg} / \mathrm{L}$ & Sample \\
\hline & East Canyon & $1 / 23 / 01$ & ND & 0.05 & $\mathrm{mg} / \mathrm{L}$ & Sample \\
\hline & N. Fork Strawberry Creek & $1 / 23 / 01$ & ND & 0.05 & $\mathrm{mg} / \mathrm{L}$ & Sample \\
\hline \multicolumn{7}{|c|}{ Thallium (Filtered) } \\
\hline & 69-Storm Drain Manhole & $1 / 23 / 01$ & ND & 0.05 & $\mathrm{mg} / \mathrm{L}$ & Sample \\
\hline & Chicken Creek & $1 / 23 / 01$ & ND & 0.05 & $\mathrm{mg} / \mathrm{L}$ & Sample \\
\hline & East Canyon & $1 / 23 / 01$ & ND & 0.05 & $\mathrm{mg} / \mathrm{L}$ & Sample \\
\hline & N. Fork Strawberry Creek & $1 / 23 / 01$ & ND & 0.05 & $\mathrm{mg} / \mathrm{L}$ & Sample \\
\hline \multirow[t]{4}{*}{ Vanadium } & 69-Storm Drain Manhole & $1 / 23 / 01$ & ND & 0.5 & $\mathrm{mg} / \mathrm{L}$ & Sample \\
\hline & Chicken Creek & $1 / 23 / 01$ & ND & 0.5 & $\mathrm{mg} / \mathrm{L}$ & Sample \\
\hline & East Canyon & $1 / 23 / 01$ & ND & 0.5 & $\mathrm{mg} / \mathrm{L}$ & Sample \\
\hline & N. Fork Strawberry Creek & $1 / 23 / 01$ & ND & 0.5 & $\mathrm{mg} / \mathrm{L}$ & Sample \\
\hline \multicolumn{7}{|c|}{ Vanadium (Filtered) } \\
\hline & 69-Storm Drain Manhole & $1 / 23 / 01$ & ND & 0.5 & $\mathrm{mg} / \mathrm{L}$ & Sample \\
\hline & Chicken Creek & $1 / 23 / 01$ & ND & 0.5 & $\mathrm{mg} / \mathrm{L}$ & Sample \\
\hline & East Canyon & $1 / 23 / 01$ & ND & 0.5 & $\mathrm{mg} / \mathrm{L}$ & Sample \\
\hline & N. Fork Strawberry Creek & $1 / 23 / 01$ & ND & 0.5 & $\mathrm{mg} / \mathrm{L}$ & Sample \\
\hline \multirow[t]{2}{*}{ Zinc } & 69-Storm Drain Manhole & $\begin{array}{l}1 / 23 / 01 \\
1 / 1 / 01\end{array}$ & ND & 0.5 & $\mathrm{mg} / \mathrm{L}$ & Sample \\
\hline & & 12/1/01 & 0.089 & 0.05 & $\mathrm{mg} / \mathrm{L}$ & Sample \\
\hline
\end{tabular}




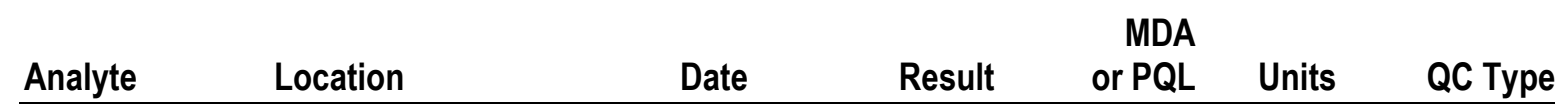

Metals and/or Minerals

\begin{tabular}{|c|c|c|c|c|c|c|}
\hline & Chicken Creek & $\begin{array}{l}1 / 23 / 01 \\
11 / 10 / 01\end{array}$ & $\begin{array}{l}\text { ND } \\
\text { ND }\end{array}$ & $\begin{array}{l}0.5 \\
0.05\end{array}$ & $\begin{array}{l}\mathrm{mg} / \mathrm{L} \\
\mathrm{mg} / \mathrm{L}\end{array}$ & $\begin{array}{l}\text { Sample } \\
\text { Sample }\end{array}$ \\
\hline & East Canyon & $\begin{array}{l}1 / 23 / 01 \\
12 / 1 / 01\end{array}$ & $\begin{array}{l}\text { ND } \\
\text { ND }\end{array}$ & $\begin{array}{l}0.5 \\
0.05\end{array}$ & $\begin{array}{l}\mathrm{mg} / \mathrm{L} \\
\mathrm{mg} / \mathrm{L}\end{array}$ & $\begin{array}{l}\text { Sample } \\
\text { Sample }\end{array}$ \\
\hline & N. Fork Strawberry Creek & $\begin{array}{l}1 / 23 / 01 \\
11 / 10 / 01\end{array}$ & $\begin{array}{l}\text { ND } \\
\text { ND }\end{array}$ & $\begin{array}{l}0.5 \\
0.05\end{array}$ & $\begin{array}{l}\mathrm{mg} / \mathrm{L} \\
\mathrm{mg} / \mathrm{L}\end{array}$ & $\begin{array}{l}\text { Sample } \\
\text { Sample }\end{array}$ \\
\hline Zinc (Filtered) & 69-Storm Drain Manhole & $1 / 23 / 01$ & ND & 0.5 & $\mathrm{mg} / \mathrm{L}$ & Sample \\
\hline & Chicken Creek & $1 / 23 / 01$ & ND & 0.5 & $\mathrm{mg} / \mathrm{L}$ & Sample \\
\hline & East Canyon & $1 / 23 / 01$ & ND & 0.5 & $\mathrm{mg} / \mathrm{L}$ & Sample \\
\hline & N. Fork Strawberry Creek & $1 / 23 / 01$ & ND & 0.5 & $\mathrm{mg} / \mathrm{L}$ & Sample \\
\hline
\end{tabular}

\section{Nutrients}

Ammonia Nitrogen (as $\mathrm{N}$ )

69-Storm Drain Manhole

$1 / 23 / 01$

$12 / 1 / 01$

Chicken Creek

$1 / 23 / 01$

$11 / 10 / 01$

$1 / 23 / 01$

$12 / 1 / 01$

N. Fork Strawberry Creek

$1 / 23 / 01$

$11 / 10 / 01$

Nitrate (as N)

$1 / 23 / 01$

Nitrate plus Nitrite (as N)

$1 / 23 / 01$

$\begin{array}{ll}\text { East Canyon } & 1 / 23 / 01 \\ \text { N. Fork Strawberry Creek } & 1 / 23 / 01\end{array}$

0.28

0.32

$0.1 \mathrm{mg} / \mathrm{L}$

Sample

69-Storm Drain Manhole 12/1/01

$\begin{array}{llll}0.1 & 0.02 & \mathrm{mg} / \mathrm{L} & \text { Sample }\end{array}$

$\begin{array}{llll}0.04 & 0.02 \quad \mathrm{mg} / \mathrm{L} \quad \text { Sample }\end{array}$

$\begin{array}{llll}0.33 & 0.02 & \mathrm{mg} / \mathrm{L} & \text { Sample }\end{array}$

$\begin{array}{llll}0.8 & 0.2 & \mathrm{mg} / \mathrm{L} & \text { Sample }\end{array}$

$\begin{array}{llll}0.3 & 0.02 & \mathrm{mg} / \mathrm{L} & \text { Sample }\end{array}$

$\begin{array}{llll}0.05 & 0.02 \quad \mathrm{mg} / \mathrm{L} \quad \text { Sample }\end{array}$

$0.25 \quad 0.02 \quad \mathrm{mg} / \mathrm{L} \quad$ Sample

$\begin{array}{llll}0.06 & 0.02 & \mathrm{mg} / \mathrm{L} & \text { Sample }\end{array}$

$0.15 \quad 0.1 \quad \mathrm{mg} / \mathrm{L} \quad$ Sample

$0.5 \quad 0.1 \quad \mathrm{mg} / \mathrm{L} \quad$ Sample

0.76

$0.1 \quad \mathrm{mg} / \mathrm{L}$

Sample 


\begin{tabular}{|c|c|c|c|c|c|c|}
\hline Analyte & Location & Date & Result & $\begin{array}{r}\text { MDA } \\
\text { or PQL } \\
\end{array}$ & Units & QC Type \\
\hline \multicolumn{7}{|l|}{ Nutrients } \\
\hline \multicolumn{7}{|c|}{ Nitrate plus Nitrite (as N) } \\
\hline \multirow[t]{3}{*}{ cont. } & Chicken Creek & $11 / 10 / 01$ & ND & 1 & $\mathrm{mg} / \mathrm{L}$ & Sample \\
\hline & East Canyon & $12 / 1 / 01$ & 0.17 & 0.1 & $\mathrm{mg} / \mathrm{L}$ & Sample \\
\hline & N. Fork Strawberry Creek & $11 / 10 / 01$ & 0.38 & 0.1 & $\mathrm{mg} / \mathrm{L}$ & Sample \\
\hline \multirow[t]{4}{*}{ Nitrite (as N) } & 69-Storm Drain Manhole & $1 / 23 / 01$ & 0.029 & 0.02 & $\mathrm{mg} / \mathrm{L}$ & Sample \\
\hline & Chicken Creek & $1 / 23 / 01$ & 0.029 & 0.02 & $\mathrm{mg} / \mathrm{L}$ & Sample \\
\hline & East Canyon & $1 / 23 / 01$ & 0.036 & 0.02 & $\mathrm{mg} / \mathrm{L}$ & Sample \\
\hline & N. Fork Strawberry Creek & $1 / 23 / 01$ & 0.029 & 0.02 & $\mathrm{mg} / \mathrm{L}$ & Sample \\
\hline
\end{tabular}

Petroleum Hydrocarbons

\begin{tabular}{|c|c|c|c|c|c|c|}
\hline \multirow[t]{3}{*}{ Diesel Fuel } & 69-Storm Drain Manhole & $\begin{array}{l}1 / 23 / 01 \\
12 / 1 / 01\end{array}$ & $\begin{array}{l}190 \\
400\end{array}$ & $\begin{array}{r}50 \\
100\end{array}$ & $\begin{array}{l}\mu \mathrm{g} / \mathrm{L} \\
\mu \mathrm{g} / \mathrm{L}\end{array}$ & $\begin{array}{l}\text { Sample } \\
\text { Sample }\end{array}$ \\
\hline & \multirow[t]{2}{*}{ Chicken Creek } & $1 / 23 / 01$ & 570 & 100 & $\mu \mathrm{g} / \mathrm{L}$ & Sample \\
\hline & & $11 / 10 / 01$ & 1100 & 50 & $\mu \mathrm{g} / \mathrm{L}$ & Sample \\
\hline & \multirow[t]{2}{*}{ East Canyon } & $1 / 23 / 01$ & 250 & 50 & $\mu \mathrm{g} / \mathrm{L}$ & Sample \\
\hline & & $12 / 1 / 01$ & ND & 100 & $\mu \mathrm{g} / \mathrm{L}$ & Sample \\
\hline & \multirow[t]{2}{*}{ N. Fork Strawberry Creek } & $1 / 23 / 01$ & 400 & 300 & $\mu \mathrm{g} / \mathrm{L}$ & Sample \\
\hline & & $11 / 10 / 01$ & 200 & 50 & $\mu \mathrm{g} / \mathrm{L}$ & Sample \\
\hline \multirow[t]{4}{*}{ Oil and Grease } & 69-Storm Drain Manhole & $12 / 1 / 01$ & ND & 5 & $\mathrm{mg} / \mathrm{L}$ & Sample \\
\hline & Chicken Creek & $11 / 10 / 01$ & ND & 5 & $\mathrm{mg} / \mathrm{L}$ & Sample \\
\hline & East Canyon & $12 / 1 / 01$ & ND & 5 & $\mathrm{mg} / \mathrm{L}$ & Sample \\
\hline & N. Fork Strawberry Creek & $11 / 10 / 01$ & ND & 5 & $\mathrm{mg} / \mathrm{L}$ & Sample \\
\hline
\end{tabular}


The following sewer data are summarized and discussed in Chapter 5 (Surface Waters and Wastewater) of the Site Environmental Report for 2001 (see Volume I):

\begin{tabular}{lllll} 
Lnalyte & \multicolumn{4}{c}{ MDA } \\
Location & Date & Result & or PQL Units & QC Type
\end{tabular}

Radiological Activity

\begin{tabular}{|c|c|c|c|c|c|c|}
\hline Gross alpha & Hearst Sewer & $1 / 4 / 01$ & ND & 0.11 & $\mathrm{~Bq} / \mathrm{L}$ & Sample \\
\hline & & $1 / 4 / 01$ & ND & 0.11 & $\mathrm{~Bq} / \mathrm{L}$ & Split \\
\hline & & $1 / 18 / 01$ & ND & 0.07 & $\mathrm{~Bq} / \mathrm{L}$ & Sample \\
\hline & & $1 / 30 / 01$ & ND & 0.07 & $\mathrm{~Bq} / \mathrm{L}$ & Sample \\
\hline & & $1 / 30 / 01$ & ND & 0.07 & $\mathrm{~Bq} / \mathrm{L}$ & Split \\
\hline & & $2 / 15 / 01$ & ND & 0.11 & $\mathrm{~Bq} / \mathrm{L}$ & Sample \\
\hline & & $3 / 1 / 01$ & ND & 0.11 & $\mathrm{~Bq} / \mathrm{L}$ & Sample \\
\hline & & $3 / 1 / 01$ & ND & 0.11 & $\mathrm{~Bq} / \mathrm{L}$ & Split \\
\hline & & $3 / 16 / 01$ & ND & 0.11 & $\mathrm{~Bq} / \mathrm{L}$ & Sample \\
\hline & & $3 / 29 / 01$ & ND & 0.07 & $\mathrm{~Bq} / \mathrm{L}$ & Sample \\
\hline & & $3 / 29 / 01$ & ND & 0.07 & $\mathrm{~Bq} / \mathrm{L}$ & Split \\
\hline & & $4 / 12 / 01$ & ND & 0.07 & $\mathrm{~Bq} / \mathrm{L}$ & Sample \\
\hline & & $4 / 12 / 01$ & ND & 0.07 & $\mathrm{~Bq} / \mathrm{L}$ & Split \\
\hline & & 4/26/01 & ND & 0.07 & $\mathrm{~Bq} / \mathrm{L}$ & Sample \\
\hline & & $4 / 26 / 01$ & ND & 0.11 & $\mathrm{~Bq} / \mathrm{L}$ & Split \\
\hline & & $5 / 10 / 01$ & ND & 0.07 & $\mathrm{~Bq} / \mathrm{L}$ & Sample \\
\hline & & $5 / 24 / 01$ & ND & 0.07 & $\mathrm{~Bq} / \mathrm{L}$ & Sample \\
\hline & & $5 / 24 / 01$ & ND & 0.07 & $\mathrm{~Bq} / \mathrm{L}$ & Spl \\
\hline & & $6 / 7 / 01$ & ND & 0.07 & $\mathrm{~Bq} / \mathrm{L}$ & Sample \\
\hline & & $6 / 21 / 01$ & 0.078 & 0.07 & $\mathrm{~Bq} / \mathrm{L}$ & Sample \\
\hline & & $7 / 5 / 01$ & ND & 0.07 & $\mathrm{~Bq} / \mathrm{L}$ & Sample \\
\hline & & $7 / 5 / 01$ & ND & 0.07 & $\mathrm{~Bq} / \mathrm{L}$ & Split \\
\hline & & $7 / 19 / 01$ & ND & 0.07 & $\mathrm{Bg} / \mathrm{L}$ & Sample \\
\hline & & $8 / 2 / 01$ & ND & 0.07 & $\mathrm{~Bq} / \mathrm{L}$ & Sample \\
\hline & & $8 / 2 / 01$ & ND & 0.07 & $\mathrm{~Bq} / \mathrm{L}$ & $\mathrm{Sp}$ \\
\hline & & $8 / 16 / 01$ & 0.078 & 0.07 & $\mathrm{~Bq} / \mathrm{L}$ & Sample \\
\hline & & 8/30/01 & ND & 0.11 & $\mathrm{~Bq} / \mathrm{L}$ & Sample \\
\hline & & $8 / 30 / 01$ & ND & 0.07 & $\mathrm{~Bq} / \mathrm{L}$ & Split \\
\hline & & $9 / 13 / 01$ & ND & 0.07 & $\mathrm{~Bq} / \mathrm{L}$ & Sample \\
\hline & & $9 / 27 / 01$ & ND & 0.07 & $\mathrm{Bg} / \mathrm{L}$ & Sample \\
\hline & & $10 / 10 / 01$ & 0.19 & 0.11 & $\mathrm{Bg} / \mathrm{L}$ & Samp \\
\hline
\end{tabular}




\begin{tabular}{|c|c|c|c|c|c|c|}
\hline Analyte & Location & Date & Result & $\begin{array}{r}\text { MDA } \\
\text { or } P Q L\end{array}$ & Units & QC Type \\
\hline
\end{tabular}

\section{Radiological Activity}

Gross alpha cont.

\begin{tabular}{|c|c|c|c|c|c|}
\hline Hearst Sewer & $10 / 24 / 01$ & ND & 0.07 & $\mathrm{~Bq} / \mathrm{L}$ & Sample \\
\hline & 11/7/01 & ND & 0.11 & $\mathrm{~Bq} / \mathrm{L}$ & Sample \\
\hline & 11/7/01 & ND & 0.11 & $\mathrm{~Bq} / \mathrm{L}$ & Split \\
\hline & $11 / 21 / 01$ & ND & 0.11 & $\mathrm{~Bq} / \mathrm{L}$ & Sample \\
\hline & 12/5/01 & ND & 0.07 & $\mathrm{~Bq} / \mathrm{L}$ & Sample \\
\hline & $12 / 5 / 01$ & ND & 0.07 & $\mathrm{~Bq} / \mathrm{L}$ & Split \\
\hline & $12 / 20 / 01$ & ND & 0.11 & $\mathrm{~Bq} / \mathrm{L}$ & Sample \\
\hline & $1 / 3 / 02$ & ND & 0.08 & $\mathrm{~Bq} / \mathrm{L}$ & Sample \\
\hline Strawberry Sewer & $1 / 4 / 01$ & ND & 0.07 & $\mathrm{~Bq} / \mathrm{L}$ & Sample \\
\hline & $1 / 18 / 01$ & ND & 0.07 & $\mathrm{~Bq} / \mathrm{L}$ & Sample \\
\hline & $1 / 18 / 01$ & ND & 0.07 & $\mathrm{~Bq} / \mathrm{L}$ & Split \\
\hline & $1 / 30 / 01$ & ND & 0.07 & $\mathrm{~Bq} / \mathrm{L}$ & Sample \\
\hline & $2 / 15 / 01$ & ND & 0.11 & $\mathrm{~Bq} / \mathrm{L}$ & Sample \\
\hline & $2 / 15 / 01$ & 0.14 & 0.11 & $\mathrm{~Bq} / \mathrm{L}$ & Split \\
\hline & $3 / 1 / 01$ & ND & 0.11 & $\mathrm{~Bq} / \mathrm{L}$ & Sample \\
\hline & $3 / 16 / 01$ & ND & 0.11 & $\mathrm{~Bq} / \mathrm{L}$ & Sample \\
\hline & $3 / 16 / 01$ & ND & 0.11 & $\mathrm{~Bq} / \mathrm{L}$ & Split \\
\hline & $3 / 29 / 01$ & ND & 0.07 & $\mathrm{~Bq} / \mathrm{L}$ & Sample \\
\hline & $4 / 12 / 01$ & ND & 0.07 & $\mathrm{~Bq} / \mathrm{L}$ & Sample \\
\hline & $4 / 26 / 01$ & ND & 0.11 & $\mathrm{~Bq} / \mathrm{L}$ & Sample \\
\hline & $5 / 10 / 01$ & ND & 0.07 & $\mathrm{~Bq} / \mathrm{L}$ & Sample \\
\hline & $5 / 10 / 01$ & ND & 0.07 & $\mathrm{~Bq} / \mathrm{L}$ & Split \\
\hline & $5 / 24 / 01$ & ND & 0.07 & $\mathrm{~Bq} / \mathrm{L}$ & Sample \\
\hline & $6 / 7 / 01$ & ND & 0.07 & $\mathrm{~Bq} / \mathrm{L}$ & Sample \\
\hline & $6 / 7 / 01$ & ND & 0.07 & $\mathrm{Bg} / \mathrm{L}$ & Split \\
\hline & $6 / 21 / 01$ & ND & 0.07 & $\mathrm{~Bq} / \mathrm{L}$ & Sample \\
\hline & $6 / 21 / 01$ & ND & 0.07 & $\mathrm{~Bq} / \mathrm{L}$ & Split \\
\hline & $7 / 5 / 01$ & ND & 0.07 & $\mathrm{Bg} / \mathrm{L}$ & Sample \\
\hline & $7 / 19 / 01$ & ND & 0.07 & $\mathrm{~Bq} / \mathrm{L}$ & Sample \\
\hline & $7 / 19 / 01$ & ND & 0.07 & $\mathrm{~Bq} / \mathrm{L}$ & Split \\
\hline & $8 / 2 / 01$ & ND & 0.07 & $\mathrm{~Bq} / \mathrm{L}$ & Sample \\
\hline & $8 / 16 / 01$ & ND & 0.07 & $\mathrm{~Bq} / \mathrm{L}$ & Sample \\
\hline & $8 / 16 / 01$ & ND & 0.07 & $\mathrm{~Bq} / \mathrm{L}$ & Split \\
\hline & 8/30/01 & ND & 0.07 & $\mathrm{~Bq} / \mathrm{L}$ & Sample \\
\hline & $9 / 13 / 01$ & ND & 0.07 & $\mathrm{~Bq} / \mathrm{L}$ & Sample \\
\hline & $9 / 13 / 01$ & ND & 0.07 & $\mathrm{~Bq} / \mathrm{L}$ & Split \\
\hline & $9 / 27 / 01$ & ND & 0.07 & $\mathrm{~Bq} / \mathrm{L}$ & Sample \\
\hline & $9 / 27 / 01$ & ND & 0.07 & $\mathrm{~Bq} / \mathrm{L}$ & Split \\
\hline & $10 / 10 / 01$ & 0.16 & 0.11 & $\mathrm{Bg} / \mathrm{L}$ & Sample \\
\hline & $10 / 24 / 01$ & ND & 0.07 & $\mathrm{Bg} / \mathrm{L}$ & Sample \\
\hline & $10 / 24 / 01$ & 0.096 & 0.07 & $\mathrm{~Bq} / \mathrm{L}$ & Split \\
\hline & $11 / 7 / 01$ & ND & 0.11 & $\mathrm{Bg} / \mathrm{L}$ & Sample \\
\hline & $11 / 21 / 01$ & ND & 0.11 & $\mathrm{~Bq} / \mathrm{L}$ & Sample \\
\hline & $11 / 21 / 01$ & ND & 0.11 & $\mathrm{~Bq} / \mathrm{L}$ & Split \\
\hline & $12 / 5 / 01$ & ND & 0.07 & $\mathrm{Bg} / \mathrm{L}$ & Sample \\
\hline
\end{tabular}




\begin{tabular}{|c|c|c|c|c|c|c|}
\hline Analyte & Location & Date & Result & $\begin{array}{r}\text { MDA } \\
\text { or PQL } \\
\end{array}$ & Units & QC Type \\
\hline \multicolumn{7}{|c|}{ Radiological Activity } \\
\hline $\begin{array}{l}\text { Gross alpha } \\
\text { cont. }\end{array}$ & Strawberry Sewer & $\begin{array}{l}12 / 20 / 01 \\
12 / 20 / 01 \\
1 / 3 / 02\end{array}$ & $\begin{array}{l}\mathrm{ND} \\
\mathrm{ND} \\
\mathrm{ND}\end{array}$ & $\begin{array}{l}0.11 \\
0.11 \\
0.08\end{array}$ & $\begin{array}{l}\mathrm{Bq} / \mathrm{L} \\
\mathrm{Bq} / \mathrm{L} \\
\mathrm{Bq} / \mathrm{L}\end{array}$ & $\begin{array}{r}\text { Sample } \\
\text { Split } \\
\text { Sample }\end{array}$ \\
\hline & Field Blank & $\begin{array}{l}5 / 24 / 01 \\
8 / 30 / 01 \\
11 / 7 / 01\end{array}$ & $\begin{array}{l}\mathrm{ND} \\
\mathrm{ND} \\
\mathrm{ND}\end{array}$ & $\begin{array}{l}0.07 \\
0.07 \\
0.11\end{array}$ & $\begin{array}{l}\mathrm{Bq} / \mathrm{L} \\
\mathrm{Bq} / \mathrm{L} \\
\mathrm{Bq} / \mathrm{L}\end{array}$ & $\begin{array}{l}\text { Blank } \\
\text { Blank } \\
\text { Blank }\end{array}$ \\
\hline Gross beta & Hearst Sewer & 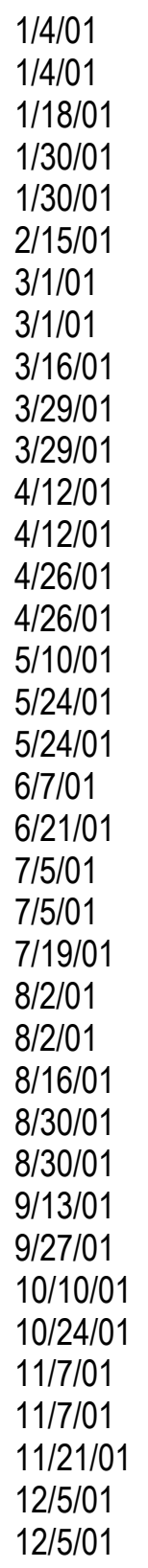 & $\begin{array}{l}0.3 \\
0.21 \\
0.415 \\
0.467 \\
0.474 \\
0.32 \\
0.3 \\
0.23 \\
0.441 \\
0.33 \\
0.35 \\
0.926 \\
0.896 \\
0.385 \\
0.433 \\
0.31 \\
0.32 \\
0.378 \\
0.411 \\
0.522 \\
0.481 \\
0.493 \\
0.53 \\
0.37 \\
0.489 \\
0.744 \\
0.619 \\
0.574 \\
0.637 \\
0.53 \\
0.737 \\
0.522 \\
0.515 \\
0.615 \\
0.448 \\
0.29 \\
0.31\end{array}$ & $\begin{array}{l}0.11 \\
0.11 \\
0.11 \\
0.11 \\
0.11 \\
0.11 \\
0.11 \\
0.11 \\
0.11 \\
0.11 \\
0.11 \\
0.11 \\
0.11 \\
0.11 \\
0.11 \\
0.11 \\
0.11 \\
0.11 \\
0.11 \\
0.07 \\
0.11 \\
0.11 \\
0.11 \\
0.11 \\
0.11 \\
0.11 \\
0.11 \\
0.07 \\
0.07 \\
0.11 \\
0.11 \\
0.07 \\
0.07 \\
0.07 \\
0.07 \\
0.07 \\
0.07\end{array}$ & $\begin{array}{l}\mathrm{Bq} / \mathrm{L} \\
\mathrm{Bq} / \mathrm{L} \\
\mathrm{Bq} / \mathrm{L} \\
\mathrm{Bq} / \mathrm{L} \\
\mathrm{Bq} / \mathrm{L} \\
\mathrm{Bq} / \mathrm{L} \\
\mathrm{Bq} / \mathrm{L} \\
\mathrm{Bq} / \mathrm{L} \\
\mathrm{Bq} / \mathrm{L} \\
\mathrm{Bq} / \mathrm{L} \\
\mathrm{Bq} / \mathrm{L} \\
\mathrm{Bq} / \mathrm{L} \\
\mathrm{Bq} / \mathrm{L} \\
\mathrm{Bq} / \mathrm{L} \\
\mathrm{Bq} / \mathrm{L} \\
\mathrm{Bq} / \mathrm{L} \\
\mathrm{Bq} / \mathrm{L} \\
\mathrm{Bq} / \mathrm{L} \\
\mathrm{Bq} / \mathrm{L} \\
\mathrm{Bq} / \mathrm{L} \\
\mathrm{Bq} / \mathrm{L} \\
\mathrm{Bq} / \mathrm{L} \\
\mathrm{Bq} / \mathrm{L} \\
\mathrm{Bq} / \mathrm{L} \\
\mathrm{Bq} / \mathrm{L} \\
\mathrm{Bq} / \mathrm{L} \\
\mathrm{Bq} / \mathrm{L} \\
\mathrm{Bq} / \mathrm{L} \\
\mathrm{Bq} / \mathrm{L} \\
\mathrm{Bq} / \mathrm{L} \\
\mathrm{Bq} / \mathrm{L} \\
\mathrm{Bq} / \mathrm{L} \\
\mathrm{Bq} / \mathrm{L} \\
\mathrm{Bq} / \mathrm{L} \\
\mathrm{Bq} / \mathrm{L} \\
\mathrm{Bq} / \mathrm{L} \\
\mathrm{Bq} / \mathrm{L}\end{array}$ & $\begin{array}{r}\text { Sample } \\
\text { Split } \\
\text { Sample } \\
\text { Sample } \\
\text { Split } \\
\text { Sample } \\
\text { Sample } \\
\text { Split } \\
\text { Sample } \\
\text { Sample } \\
\text { Split } \\
\text { Sample } \\
\text { Split } \\
\text { Sample } \\
\text { Split } \\
\text { Sample } \\
\text { Sample } \\
\text { Split } \\
\text { Sample } \\
\text { Sample } \\
\text { Sample } \\
\text { Split } \\
\text { Sample } \\
\text { Sample } \\
\text { Split } \\
\text { Sample } \\
\text { Sample } \\
\text { Split } \\
\text { Sample } \\
\text { Sample } \\
\text { Sample } \\
\text { Sample } \\
\text { Sample } \\
\text { Split } \\
\text { Sample } \\
\text { Sample } \\
\text { Split }\end{array}$ \\
\hline
\end{tabular}




\begin{tabular}{|c|c|c|c|c|c|c|}
\hline Analyte & Location & Date & Result & $\begin{array}{r}\text { MDA } \\
\text { or PQL }\end{array}$ & Units & QC Type \\
\hline
\end{tabular}

\section{Radiological Activity}

Gross beta cont.

\begin{tabular}{|c|c|c|c|c|c|}
\hline Hearst Sewer & $\begin{array}{l}12 / 20 / 01 \\
1 / 3 / 02\end{array}$ & $\begin{array}{l}0.511 \\
N D\end{array}$ & $\begin{array}{l}0.11 \\
0.11\end{array}$ & $\begin{array}{l}\mathrm{Bq} / \mathrm{L} \\
\mathrm{Bq} / \mathrm{L}\end{array}$ & $\begin{array}{l}\text { Sample } \\
\text { Sample }\end{array}$ \\
\hline Strawberry Sewer & $1 / 4 / 01$ & 0.526 & 0.11 & $\mathrm{~Bq} / \mathrm{L}$ & Sample \\
\hline & $1 / 18 / 01$ & 0.24 & 0.11 & $\mathrm{~Bq} / \mathrm{L}$ & Sample \\
\hline & $1 / 18 / 01$ & 0.29 & 0.11 & $\mathrm{~Bq} / \mathrm{L}$ & Split \\
\hline & 1/30/01 & 0.19 & 0.11 & $\mathrm{~Bq} / \mathrm{L}$ & Sample \\
\hline & $2 / 15 / 01$ & 0.37 & 0.11 & $\mathrm{~Bq} / \mathrm{L}$ & Sample \\
\hline & $2 / 15 / 01$ & 0.31 & 0.11 & $\mathrm{~Bq} / \mathrm{L}$ & Split \\
\hline & $3 / 1 / 01$ & 0.24 & 0.11 & $\mathrm{~Bq} / \mathrm{L}$ & Sample \\
\hline & $3 / 16 / 01$ & 0.36 & 0.11 & $\mathrm{~Bq} / \mathrm{L}$ & Sample \\
\hline & $3 / 16 / 01$ & 0.32 & 0.11 & $\mathrm{~Bq} / \mathrm{L}$ & Split \\
\hline & $3 / 29 / 01$ & 0.37 & 0.11 & $\mathrm{~Bq} / \mathrm{L}$ & Sample \\
\hline & 4/12/01 & 0.34 & 0.11 & $\mathrm{~Bq} / \mathrm{L}$ & Sample \\
\hline & $4 / 26 / 01$ & 0.37 & 0.11 & $\mathrm{~Bq} / \mathrm{L}$ & Sample \\
\hline & $5 / 10 / 01$ & 0.53 & 0.11 & $\mathrm{~Bq} / \mathrm{L}$ & Sample \\
\hline & $5 / 10 / 01$ & 0.607 & 0.11 & $\mathrm{~Bq} / \mathrm{L}$ & Split \\
\hline & $5 / 24 / 01$ & 0.31 & 0.11 & $\mathrm{~Bq} / \mathrm{L}$ & Sample \\
\hline & $6 / 7 / 01$ & 0.385 & 0.11 & $\mathrm{~Bq} / \mathrm{L}$ & Sample \\
\hline & $6 / 7 / 01$ & 0.381 & 0.11 & $\mathrm{~Bq} / \mathrm{L}$ & Split \\
\hline & $6 / 21 / 01$ & 0.422 & 0.11 & $\mathrm{~Bq} / \mathrm{L}$ & Sample \\
\hline & $6 / 21 / 01$ & 0.4 & 0.11 & $\mathrm{~Bq} / \mathrm{L}$ & Split \\
\hline & $7 / 5 / 01$ & 0.28 & 0.11 & $\mathrm{~Bq} / \mathrm{L}$ & Sample \\
\hline & $7 / 19 / 01$ & 0.32 & 0.11 & $\mathrm{~Bq} / \mathrm{L}$ & Sample \\
\hline & $7 / 19 / 01$ & 0.36 & 0.11 & $\mathrm{~Bq} / \mathrm{L}$ & Split \\
\hline & $8 / 2 / 01$ & 0.385 & 0.11 & $\mathrm{~Bq} / \mathrm{L}$ & Sample \\
\hline & $8 / 16 / 01$ & 0.378 & 0.11 & $\mathrm{~Bq} / \mathrm{L}$ & Sample \\
\hline & 8/16/01 & 0.361 & 0.11 & $\mathrm{~Bq} / \mathrm{L}$ & Split \\
\hline & 8/30/01 & 0.24 & 0.11 & $\mathrm{~Bq} / \mathrm{L}$ & Sample \\
\hline & $9 / 13 / 01$ & 0.23 & 0.07 & $\mathrm{Bg} / \mathrm{L}$ & Sample \\
\hline & $9 / 13 / 01$ & 0.27 & 0.07 & $\mathrm{~Bq} / \mathrm{L}$ & Split \\
\hline & $9 / 27 / 01$ & 0.24 & 0.07 & $\mathrm{~Bq} / \mathrm{L}$ & Sample \\
\hline & $9 / 27 / 01$ & 0.32 & 0.11 & $\mathrm{~Bq} / \mathrm{L}$ & Split \\
\hline & 10/10/01 & 0.567 & 0.11 & $\mathrm{~Bq} / \mathrm{L}$ & Sample \\
\hline & $10 / 24 / 01$ & 0.32 & 0.07 & $\mathrm{~Bq} / \mathrm{L}$ & Sample \\
\hline & $10 / 24 / 01$ & 0.3 & 0.07 & $\mathrm{~Bq} / \mathrm{L}$ & Split \\
\hline & $11 / 7 / 01$ & 0.29 & 0.07 & $\mathrm{~Bq} / \mathrm{L}$ & Sample \\
\hline & $11 / 21 / 01$ & 0.415 & 0.07 & $\mathrm{~Bq} / \mathrm{L}$ & Sample \\
\hline & $11 / 21 / 01$ & 0.27 & 0.07 & $\mathrm{~Bq} / \mathrm{L}$ & Split \\
\hline & $12 / 5 / 01$ & 0.28 & 0.11 & $\mathrm{~Bq} / \mathrm{L}$ & Sample \\
\hline & $12 / 20 / 01$ & 0.25 & 0.11 & $\mathrm{~Bq} / \mathrm{L}$ & Sample \\
\hline & $12 / 20 / 01$ & 0.19 & 0.11 & $\mathrm{~Bq} / \mathrm{L}$ & Split \\
\hline & $1 / 3 / 02$ & ND & 0.11 & $\mathrm{~Bq} / \mathrm{L}$ & Sample \\
\hline Field Blank & $5 / 24 / 01$ & ND & 0.11 & $\mathrm{~Bq} / \mathrm{L}$ & Blank \\
\hline
\end{tabular}




\begin{tabular}{|c|c|c|c|c|c|c|}
\hline Analyte & Location & Date & Result & $\begin{array}{r}\text { MDA } \\
\text { or PQL } \\
\end{array}$ & Units & QC Type \\
\hline \multicolumn{7}{|c|}{ Radiological Activity } \\
\hline $\begin{array}{l}\text { Gross beta } \\
\text { cont. }\end{array}$ & Field Blank & $\begin{array}{l}8 / 30 / 01 \\
11 / 7 / 01\end{array}$ & $\begin{array}{l}\text { ND } \\
\text { ND }\end{array}$ & $\begin{array}{l}0.07 \\
0.07\end{array}$ & $\begin{array}{l}\mathrm{Bq} / \mathrm{L} \\
\mathrm{Bq} / \mathrm{L}\end{array}$ & $\begin{array}{l}\text { Blank } \\
\text { Blank }\end{array}$ \\
\hline lodine-125 & Hearst Sewer & $\begin{array}{l}1 / 4 / 01 \\
1 / 4 / 01 \\
1 / 18 / 01 \\
1 / 30 / 01 \\
1 / 30 / 01 \\
2 / 15 / 01 \\
3 / 1 / 01 \\
3 / 1 / 01 \\
3 / 16 / 01 \\
3 / 29 / 01 \\
3 / 29 / 01 \\
4 / 12 / 01 \\
4 / 12 / 01 \\
4 / 26 / 01 \\
4 / 26 / 01 \\
5 / 10 / 01 \\
5 / 24 / 01 \\
5 / 24 / 01 \\
6 / 7 / 01 \\
6 / 21 / 01 \\
7 / 5 / 01 \\
7 / 5 / 01 \\
7 / 19 / 01 \\
7\end{array}$ & $\begin{array}{l}1.4 \\
1.4 \\
3 \\
2.43 \\
1.81 \\
2.25 \\
1.77 \\
1.69 \\
1.96 \\
1.96 \\
1.8 \\
5 \\
5.52 \\
1.61 \\
2.09 \\
1.91 \\
1.85 \\
1.97 \\
2.4 \\
2.91 \\
5 \\
6.48 \\
2.36 \\
2.37 \\
2.58 \\
3.7 \\
3.59 \\
3.55 \\
3.81 \\
2.6 \\
3.78 \\
3.37 \\
3.28 \\
3.39 \\
2.35 \\
2.23 \\
2.16 \\
3.14 \\
\text { ND }\end{array}$ & $\begin{array}{l}0.4 \\
0.4 \\
0.4 \\
0.4 \\
0.4 \\
0.5 \\
0.4 \\
0.4 \\
0.4 \\
0.4 \\
0.4 \\
0.4 \\
0.4 \\
0.4 \\
0.4 \\
0.4 \\
0.4 \\
0.4 \\
0.4 \\
0.4 \\
0.4 \\
0.4 \\
0.4 \\
0.4 \\
0.4 \\
0.4 \\
0.4 \\
0.4 \\
0.4 \\
0.4 \\
0.7 \\
0.4 \\
0.4 \\
0.4 \\
0.4 \\
0.5 \\
0.5 \\
0.4 \\
1.1\end{array}$ & $\begin{array}{l}\mathrm{Bq} / \mathrm{L} \\
\mathrm{Bq} / \mathrm{L} \\
\mathrm{Bq} / \mathrm{L} \\
\mathrm{Bq} / \mathrm{L} \\
\mathrm{Bq} / \mathrm{L} \\
\mathrm{Bq} / \mathrm{L} \\
\mathrm{Bq} / \mathrm{L} \\
\mathrm{Bq} / \mathrm{L} \\
\mathrm{Bq} / \mathrm{L} \\
\mathrm{Bq} / \mathrm{L} \\
\mathrm{Bq} / \mathrm{L} \\
\mathrm{Bq} / \mathrm{L} \\
\mathrm{Bq} / \mathrm{L} \\
\mathrm{Bq} / \mathrm{L} \\
\mathrm{Bq} / \mathrm{L} \\
\mathrm{Bq} / \mathrm{L} \\
\mathrm{Bq} / \mathrm{L} \\
\mathrm{Bq} / \mathrm{L} \\
\mathrm{Bq} / \mathrm{L} \\
\mathrm{Bq} / \mathrm{L} \\
\mathrm{Bq} / \mathrm{L} \\
\mathrm{Bq} / \mathrm{L} \\
\mathrm{Bq} / \mathrm{L} \\
\mathrm{Bq} / \mathrm{L} \\
\mathrm{Bq} / \mathrm{L} \\
\mathrm{Bq} / \mathrm{L} \\
\mathrm{Bq} / \mathrm{L} \\
\mathrm{Bq} / \mathrm{L} \\
\mathrm{Bq} / \mathrm{L} \\
\mathrm{Bq} / \mathrm{L} \\
\mathrm{Bq} / \mathrm{L} \\
\mathrm{Bq} / \mathrm{L} \\
\mathrm{Bq} / \mathrm{L} \\
\mathrm{Bq} / \mathrm{L} \\
\mathrm{Bq} / \mathrm{L} \\
\mathrm{Bq} / \mathrm{L} \\
\mathrm{Bq} / \mathrm{L} \\
\mathrm{Bq} / \mathrm{L} \\
\mathrm{Bq} / \mathrm{L}\end{array}$ & $\begin{array}{r}\text { Sample } \\
\text { Split } \\
\text { Sample } \\
\text { Sample } \\
\text { Split } \\
\text { Sample } \\
\text { Sample } \\
\text { Split } \\
\text { Sample } \\
\text { Sample } \\
\text { Split } \\
\text { Sample } \\
\text { Split } \\
\text { Sample } \\
\text { Split } \\
\text { Sample } \\
\text { Sample } \\
\text { Split } \\
\text { Sample } \\
\text { Sample } \\
\text { Sample } \\
\text { Split } \\
\text { Sample } \\
\text { Sample } \\
\text { Split } \\
\text { Sample } \\
\text { Sample } \\
\text { Split } \\
\text { Sample } \\
\text { Sample } \\
\text { Sample } \\
\text { Sample } \\
\text { Sample } \\
\text { Split } \\
\text { Sample } \\
\text { Sample } \\
\text { Split } \\
\text { Sample } \\
\text { Sample }\end{array}$ \\
\hline & Strawberry Sewer & $\begin{array}{l}1 / 4 / 01 \\
1 / 18 / 01 \\
1 / 18 / 01\end{array}$ & $\begin{array}{l}2.55 \\
1.89 \\
2.12\end{array}$ & $\begin{array}{l}0.4 \\
0.4 \\
0.4\end{array}$ & $\begin{array}{l}\mathrm{Bq} / \mathrm{L} \\
\mathrm{Bq} / \mathrm{L} \\
\mathrm{Bq} / \mathrm{L}\end{array}$ & $\begin{array}{r}\text { Sample } \\
\text { Sample } \\
\text { Split }\end{array}$ \\
\hline
\end{tabular}




\begin{tabular}{|c|c|c|c|c|c|c|}
\hline Analvt & Location & Date & Result & $\begin{array}{l}\text { MDA } \\
\text { or POL }\end{array}$ & Units & QC Type \\
\hline
\end{tabular}

\section{Radiological Activity}

lodine-125

cont.

\begin{tabular}{|c|c|c|c|c|c|c|}
\hline & & $\begin{array}{l}2 / 15 / 01 \\
3 / 1 / 01\end{array}$ & 2.01 & 0.5 & $\mathrm{~Bq} / \mathrm{L}$ & Split \\
\hline & & $3 / 1 / 01$ & 2.26 & 0.4 & $\mathrm{~Bq} / \mathrm{L}$ & Sample \\
\hline & & $3 / 16 / 01$ & 1.72 & 0.4 & $\mathrm{~Bq} / \mathrm{L}$ & Sample \\
\hline & & $3 / 16 / 01$ & 1.81 & 0.4 & $\mathrm{~Bq} / \mathrm{L}$ & Split \\
\hline & & $3 / 29 / 01$ & 2.26 & 0.4 & $\mathrm{~Bq} / \mathrm{L}$ & Sample \\
\hline & & $4 / 12 / 01$ & 2.16 & 0.4 & $\mathrm{~Bq} / \mathrm{L}$ & Sample \\
\hline & & $4 / 26 / 01$ & 2.33 & 0.4 & $\mathrm{~Bq} / \mathrm{L}$ & Sample \\
\hline & & $5 / 10 / 01$ & 2.99 & 0.4 & $\mathrm{~Bq} / \mathrm{L}$ & Sample \\
\hline & & $5 / 10 / 01$ & 3.53 & 0.4 & $\mathrm{~Bq} / \mathrm{L}$ & Split \\
\hline & & $5 / 24 / 01$ & 1.64 & 0.4 & $\mathrm{~Bq} / \mathrm{L}$ & Sample \\
\hline & & $6 / 7 / 01$ & 1.91 & 0.4 & $\mathrm{~Bq} / \mathrm{L}$ & Sample \\
\hline & & $6 / 7 / 01$ & 2.51 & 0.4 & $\mathrm{~Bq} / \mathrm{L}$ & Split \\
\hline & & $6 / 21 / 01$ & 2.2 & 0.4 & $\mathrm{~Bq} / \mathrm{L}$ & Sample \\
\hline & & $6 / 21 / 01$ & 2.05 & 0.4 & $\mathrm{~Bq} / \mathrm{L}$ & Split \\
\hline & & $7 / 5 / 01$ & 1.5 & 0.4 & $\mathrm{~Bq} / \mathrm{L}$ & Sample \\
\hline & & $7 / 19 / 01$ & 1.99 & 0.4 & $\mathrm{~Bq} / \mathrm{L}$ & Sample \\
\hline & & $7 / 19 / 01$ & 1.66 & 0.4 & $\mathrm{~Bq} / \mathrm{L}$ & Split \\
\hline & & $8 / 2 / 01$ & 2.01 & 0.4 & $\mathrm{~Bq} / \mathrm{L}$ & Sample \\
\hline & & $8 / 16 / 01$ & 1.95 & 0.4 & $\mathrm{~Bq} / \mathrm{L}$ & Sample \\
\hline & & $8 / 16 / 01$ & 2.06 & 0.4 & $\mathrm{~Bq} / \mathrm{L}$ & Split \\
\hline & & $8 / 30 / 01$ & 1.4 & 0.4 & $\mathrm{~Bq} / \mathrm{L}$ & Sample \\
\hline & & $9 / 13 / 01$ & 1.4 & 0.4 & $\mathrm{~Bq} / \mathrm{L}$ & Sample \\
\hline & & $9 / 13 / 01$ & 1.3 & 0.4 & $\mathrm{~Bq} / \mathrm{L}$ & Split \\
\hline & & 9/27/01 & 1.48 & 0.4 & $\mathrm{~Bq} / \mathrm{L}$ & Sample \\
\hline & & 9/27/01 & 2.8 & 0.4 & $\mathrm{~Bq} / \mathrm{L}$ & Split \\
\hline & & $10 / 10 / 01$ & 3.48 & 0.7 & $\mathrm{~Bq} / \mathrm{L}$ & Sample \\
\hline & & $10 / 24 / 01$ & 1.87 & 0.4 & $\mathrm{~Bq} / \mathrm{L}$ & Sample \\
\hline & & $10 / 24 / 01$ & 1.71 & 0.4 & $\mathrm{~Bq} / \mathrm{L}$ & Split \\
\hline & & $11 / 7 / 01$ & 1.56 & 0.4 & $\mathrm{~Bq} / \mathrm{L}$ & Sample \\
\hline & & $11 / 21 / 01$ & 1.81 & 0.4 & $\mathrm{~Bq} / \mathrm{L}$ & Sample \\
\hline & & $11 / 21 / 01$ & 1.78 & 0.4 & $\mathrm{~Bq} / \mathrm{L}$ & Split \\
\hline & & $12 / 5 / 01$ & 1.7 & 0.5 & $\mathrm{~Bq} / \mathrm{L}$ & Sample \\
\hline & & $12 / 20 / 01$ & 2.06 & 0.4 & $\mathrm{~Bq} / \mathrm{L}$ & Sample \\
\hline & & $12 / 20 / 01$ & 1.6 & 0.4 & $\mathrm{~Bq} / \mathrm{L}$ & Split \\
\hline & & $1 / 3 / 02$ & ND & 1.1 & $\mathrm{~Bq} / \mathrm{L}$ & Sample \\
\hline & Field Blank & $5 / 24 / 01$ & ND & 0.4 & $\mathrm{~Bq} / \mathrm{L}$ & Blank \\
\hline & & $8 / 30 / 01$ & ND & 0.4 & $\mathrm{~Bq} / \mathrm{L}$ & Blank \\
\hline & & $11 / 7 / 01$ & ND & 0.3 & $\mathrm{~Bq} / \mathrm{L}$ & Blank \\
\hline Tritium & Hearst Sewer & $1 / 4 / 01$ & ND & 7 & $\mathrm{~Bq} / \mathrm{L}$ & Sample \\
\hline & & $1 / 4 / 01$ & ND & 7 & $\mathrm{~Bq} / \mathrm{L}$ & Split \\
\hline & & $1 / 18 / 01$ & 8.6 & 7 & $\mathrm{~Bq} / \mathrm{L}$ & Sample \\
\hline & & $1 / 18 / 01$ & ND & 7 & $\mathrm{~Bq} / \mathrm{L}$ & Split \\
\hline
\end{tabular}




\begin{tabular}{|c|c|c|c|c|c|c|}
\hline Analyte & Location & Date & Result & $\begin{array}{r}\text { MDA } \\
\text { or PQL }\end{array}$ & Units & QC Type \\
\hline
\end{tabular}

\section{Radiological Activity}

Tritium cont.
Hearst Sewer

\begin{abstract}
1/30/01
\end{abstract}
$1 / 30 / 01$

2/15/01

2/15/01

$3 / 1 / 01$

$3 / 1 / 01$

$3 / 16 / 01$

$3 / 16 / 01$

$3 / 29 / 01$

$3 / 29 / 01$

$4 / 12 / 01$

$4 / 12 / 01$

$4 / 26 / 01$

$4 / 26 / 01$

$5 / 10 / 01$

$5 / 10 / 01$

$5 / 24 / 01$

$5 / 24 / 01$

$6 / 7 / 01$

$6 / 7 / 01$

$6 / 21 / 01$

$6 / 21 / 01$

$7 / 5 / 01$

$7 / 5 / 01$

$7 / 19 / 01$

$7 / 19 / 01$

$8 / 2 / 01$

$8 / 2 / 01$

$8 / 16 / 01$

$8 / 16 / 01$

$8 / 30 / 01$

$8 / 30 / 01$

$9 / 13 / 01$

$9 / 13 / 01$

9/27/01

9/27/01

10/10/01

$10 / 10 / 01$

$10 / 24 / 01$

$10 / 24 / 01$

$11 / 7 / 01$

$11 / 7 / 01$

$11 / 21 / 01$

$11 / 21 / 01$

$12 / 5 / 01$

$\begin{array}{lllr}\text { ND } & 7 & \mathrm{~Bq} / \mathrm{L} & \text { Sample } \\ \text { ND } & 7 & \mathrm{~Bq} / \mathrm{L} & \text { Split } \\ \text { ND } & 7 & \mathrm{~Bq} / \mathrm{L} & \text { Sample } \\ \text { ND } & 7 & \mathrm{~Bq} / \mathrm{L} & \text { Split } \\ \text { ND } & 7 & \mathrm{~Bq} / \mathrm{L} & \text { Sample } \\ \text { ND } & 7 & \mathrm{~Bq} / \mathrm{L} & \text { Split } \\ \text { ND } & 7 & \mathrm{~Bq} / \mathrm{L} & \text { Sample } \\ \text { ND } & 7 & \mathrm{~Bq} / \mathrm{L} & \text { Split } \\ \text { ND } & 7 & \mathrm{~Bq} / \mathrm{L} & \text { Sample } \\ \text { ND } & 7 & \mathrm{~Bq} / \mathrm{L} & \text { Split } \\ \text { ND } & 7 & \mathrm{~Bq} / \mathrm{L} & \text { Sample } \\ \text { ND } & 7 & \mathrm{~Bq} / \mathrm{L} & \text { Split } \\ \text { ND } & 8 & \mathrm{~Bq} / \mathrm{L} & \text { Sample } \\ \text { ND } & 8 & \mathrm{~Bq} / \mathrm{L} & \text { Split } \\ \text { ND } & 7 & \mathrm{~Bq} / \mathrm{L} & \text { Sample } \\ \text { ND } & 8 & \mathrm{~Bq} / \mathrm{L} & \text { Split } \\ \text { ND } & 7 & \mathrm{~Bq} / \mathrm{L} & \text { Sample } \\ \text { ND } & 7 & \mathrm{~Bq} / \mathrm{L} & \text { Split } \\ \text { ND } & 7 & \mathrm{~Bq} / \mathrm{L} & \text { Sample } \\ \text { ND } & 7 & \mathrm{~Bq} / \mathrm{L} & \text { Split } \\ \text { ND } & 7 & \mathrm{~Bq} / \mathrm{L} & \text { Sample } \\ \text { ND } & 7 & \mathrm{~Bq} / \mathrm{L} & \text { Split } \\ \text { ND } & 7 & \mathrm{~Bq} / \mathrm{L} & \text { Sample } \\ \text { ND } & 7 & \mathrm{~Bq} / \mathrm{L} & \text { Split } \\ \text { ND } & 8 & \mathrm{~Bq} / \mathrm{L} & \text { Sample } \\ \text { ND } & 8 & \mathrm{~Bq} / \mathrm{L} & \text { Split } \\ \text { ND } & 7 & \mathrm{~Bq} / \mathrm{L} & \text { Sample } \\ \text { ND } & 7 & \mathrm{~Bq} / \mathrm{L} & \text { Split } \\ \text { ND } & 7 & \mathrm{~Bq} / \mathrm{L} & \text { Sample } \\ \text { ND } & 7 & \mathrm{~Bq} / \mathrm{L} & \text { Split } \\ \text { ND } & 7 & \mathrm{~Bq} / \mathrm{L} & \text { Sample } \\ \text { ND } & 7 & \mathrm{~Bq} / \mathrm{L} & \text { Split } \\ \text { ND } & 7 & \mathrm{~Bq} / \mathrm{L} & \text { Sample } \\ \text { ND } & 7 & \mathrm{~Bq} / \mathrm{L} & \text { Split } \\ \text { ND } & 7 & \mathrm{~Bq} / \mathrm{L} & \text { Sample } \\ \text { ND } & 7 & \mathrm{~Bq} / \mathrm{L} & \text { Split } \\ \text { ND } & 7 & \mathrm{~Bq} / \mathrm{L} & \text { Sample } \\ \text { ND } & 7 & \mathrm{~Bq} / \mathrm{L} & \text { Split } \\ \text { ND } & 8 & \mathrm{~Bq} / \mathrm{L} & \text { Sample } \\ \text { ND } & 8 & \mathrm{~Bq} / \mathrm{L} & \text { Split } \\ \text { ND } & 7 & \mathrm{~Bq} / \mathrm{L} & \text { Sample } \\ \text { ND } & 7 & \mathrm{~Bq} / \mathrm{L} & \text { Split } \\ \text { ND } & 7 & \mathrm{~Bq} / \mathrm{L} & \text { Sample } \\ \text { ND } & 7 & \mathrm{~Bq} / \mathrm{L} & \text { Split } \\ \text { ND } & 7 & \mathrm{~Bq} / \mathrm{L} & \text { Sample }\end{array}$




\begin{tabular}{|c|c|c|c|c|c|c|}
\hline Analyte & Location & Date & Result & $\begin{array}{r}\text { MDA } \\
\text { or PQL }\end{array}$ & Units & QC Type \\
\hline
\end{tabular}

\section{Radiological Activity}

Tritium cont.

$\begin{array}{lllllr}\text { Hearst Sewer } & \text { ND } & 7 & \mathrm{~Bq} / \mathrm{L} & \text { Split } \\ & 12 / 201 & \mathrm{ND} & 7 & \mathrm{~Bq} / \mathrm{L} & \text { Sample } \\ 12 / 20 / 01 & 7.6 & 7 & \mathrm{~Bq} / \mathrm{L} & \text { Split } \\ 1 / 3 / 02 & \mathrm{ND} & 7 & \mathrm{~Bq} / \mathrm{L} & \text { Sample } \\ \text { Strawberry Sewer } & 1 / 3 / 02 & \mathrm{ND} & 7 & \mathrm{~Bq} / \mathrm{L} & \text { Split } \\ & 1 / 4 / 01 & 44.4 & 7 & \mathrm{~Bq} / \mathrm{L} & \text { Sample } \\ 1 / 4 / 01 & 40.4 & 7 & \mathrm{~Bq} / \mathrm{L} & \text { Split } \\ 1 / 18 / 01 & 49.3 & 7 & \mathrm{~Bq} / \mathrm{L} & \text { Sample } \\ 1 / 18 / 01 & 46.7 & 7 & \mathrm{~Bq} / \mathrm{L} & \text { Split } \\ 1 / 30 / 01 & 7.5 & 7 & \mathrm{~Bq} / \mathrm{L} & \text { Sample } \\ 1 / 30 / 01 & \mathrm{ND} & 7 & \mathrm{~Bq} / \mathrm{L} & \text { Split } \\ 2 / 15 / 01 & \mathrm{ND} & 7 & \mathrm{~Bq} / \mathrm{L} & \text { Sample } \\ 2 / 15 / 01 & 9.9 & 7 & \mathrm{~Bq} / \mathrm{L} & \text { Split } \\ 3 / 1 / 01 & 12 & 7 & \mathrm{~Bq} / \mathrm{L} & \text { Sample } \\ 3 / 1 / 01 & 13 & 7 & \mathrm{~Bq} / \mathrm{L} & \text { Split } \\ 3 / 16 / 01 & 15 & 7 & \mathrm{~Bq} / \mathrm{L} & \text { Sample } \\ 3 / 16 / 01 & 17 & 7 & \mathrm{~Bq} / \mathrm{L} & \text { Split } \\ 3 / 29 / 01 & 29 & 7 & \mathrm{~Bq} / \mathrm{L} & \text { Sample } \\ 3 / 29 / 01 & 31 & 7 & \mathrm{~Bq} / \mathrm{L} & \text { Split } \\ 4 / 12 / 01 & 15 & 7 & \mathrm{~Bq} / \mathrm{L} & \text { Sample } \\ 4 / 12 / 01 & 16 & 7 & \mathrm{~Bq} / \mathrm{L} & \text { Split } \\ 4 / 26 / 01 & 9.5 & 8 & \mathrm{~Bq} / \mathrm{L} & \text { Sample } \\ 4 / 26 / 01 & 11 & 8 & \mathrm{~Bq} / \mathrm{L} & \text { Split } \\ 5 / 10 / 01 & 24 & 7 & \mathrm{~Bq} / \mathrm{L} & \text { Sample } \\ 5 / 10 / 01 & 27 & 7 & \mathrm{~Bq} / \mathrm{L} & \text { Split } \\ 5 / 24 / 01 & 21 & 7 & \mathrm{~Bq} / \mathrm{L} & \text { Sample } \\ 5 / 24 / 01 & 21 & 7 & \mathrm{~Bq} / \mathrm{L} & \text { Split } \\ 6 / 7 / 01 & 19 & 7 & \mathrm{~Bq} / \mathrm{L} & \text { Sample } \\ 6 / 7 / 01 & 19 & 7 & \mathrm{~Bq} / \mathrm{L} & \text { Split } \\ 6 / 21 / 01 & 27 & 8 & \mathrm{~Bq} / \mathrm{L} & \text { Sample } \\ 6 / 21 / 01 & 27 & 8 & \mathrm{~Bq} / \mathrm{L} & \text { Split } \\ 7 / 5 / 01 & 27 & 7 & \mathrm{~Bq} / \mathrm{L} & \text { Sample } \\ 7 / 5 / 01 & 28 & 7 & \mathrm{~Bq} / \mathrm{L} & \text { Split } \\ 7 / 19 / 01 & 8.7 & 7 & \mathrm{~Bq} / \mathrm{L} & \text { Sample } \\ 7 / 19 / 01 & 7.8 & 8 & \mathrm{~Bq} / \mathrm{L} & \text { Split } \\ 8 / 2 / 01 & \mathrm{ND} & 7 & \mathrm{~Bq} / \mathrm{L} & \text { Sample } \\ 8 / 2 / 01 & \mathrm{ND} & 7 & \mathrm{~Bq} / \mathrm{L} & \text { Split } \\ 8 / 16 / 01 & 50.7 & 7 & \mathrm{~Bq} / \mathrm{L} & \text { Sample } \\ 8 / 16 / 01 & 55.9 & 7 & \mathrm{~Bq} / \mathrm{L} & \text { Split } \\ 8 / 30 / 01 & 74.8 & 7 & \mathrm{~Bq} / \mathrm{L} & \text { Sample } \\ 8 / 30 / 01 & 80.4 & 7 & \mathrm{~Bq} / \mathrm{L} & \text { Split } \\ 9 / 13 / 01 & 28.7 & 7 & \mathrm{~Bq} / \mathrm{L} & \text { Sample } \\ 9 / 13 / 01 & 29.1 & 7 & \mathrm{~Bq} / \mathrm{L} & \text { Split } \\ 9 / 27 / 01 & 21 & 7 & \mathrm{~Bq} / \mathrm{L} & \text { Sample } \\ 9 / 27 / 01 & 18 & 7 & \mathrm{~Bq} / \mathrm{L} & \text { Split }\end{array}$




\begin{tabular}{lllll} 
Lnalyte & \multicolumn{4}{c}{ MDA } \\
Location & Date & Result & or PQL Units & QC Type
\end{tabular}

\section{Radiological Activity}

Tritium

cont.
Strawberry Sewer

10/10/01

$10 / 10 / 01$

$10 / 24 / 01$

$10 / 24 / 01$

$11 / 7 / 01$

$11 / 7 / 01$

$11 / 21 / 01$

$11 / 21 / 01$

$12 / 5 / 01$

$12 / 5 / 01$

$12 / 20 / 01$

$12 / 20 / 01$

$1 / 3 / 02$

$1 / 3 / 02$

Field Blank

$5 / 24 / 01$

$8 / 30 / 01$

$11 / 7 / 01$

\section{3}

32.6

ND

ND

ND

ND

ND

ND

ND

ND

ND

ND

152

13

ND

ND

ND

7
7
8
8
7
7
7
7
7
7
7
7
7
7

$\begin{array}{lr}\mathrm{Bq} / \mathrm{L} & \text { Sample } \\ \mathrm{Bq} / \mathrm{L} & \text { Split } \\ \mathrm{Bq} / \mathrm{L} & \text { Sample } \\ \mathrm{Bq} / \mathrm{L} & \text { Split } \\ \mathrm{Bq} / \mathrm{L} & \text { Sample } \\ \mathrm{Bq} / \mathrm{L} & \text { Split } \\ \mathrm{Bq} / \mathrm{L} & \text { Sample } \\ \mathrm{Bq} / \mathrm{L} & \text { Split } \\ \mathrm{Bq} / \mathrm{L} & \text { Sample } \\ \mathrm{Bq} / \mathrm{L} & \text { Split } \\ \mathrm{Bq} / \mathrm{L} & \text { Sample } \\ \mathrm{Bq} / \mathrm{L} & \text { Split } \\ \mathrm{Bq} / \mathrm{L} & \text { Sample } \\ \mathrm{Bq} / \mathrm{L} & \text { Split }\end{array}$

$7 \quad \mathrm{~Bq} / \mathrm{L}$

Blank

$\mathrm{Bq} / \mathrm{L} \quad$ Blank

$\mathrm{Bq} / \mathrm{L}$

Blank

\section{General Indicator Parameters}

Chemical Oxygen Demand (Filtered)

$$
\text { Hearst Sewer }
$$

$1 / 18 / 01$

210

40

$4 / 17 / 01$

$7 / 10 / 01$

$10 / 31 / 01$

82

62

7

7

\section{$1 / 18 / 01$}

$4 / 17 / 01$

$7 / 10 / 01$

$10 / 31 / 01$

61

Field pH

Hearst Sewer

$1 / 18 / 01$

$4 / 17 / 01$

$7 / 10 / 01$

10/31/01

8.95

7.34 -

$8.10-$

$8.70-$

$1 / 18 / 01$

$4 / 17 / 01$

$7 / 10 / 01$

$10 / 31 / 01$

$\begin{array}{ll}8.41 & - \\ 8.25 & - \\ 7.99 & - \\ 7.93 & -\end{array}$

Total suspended solids (TSS)

$$
\text { Hearst Sewer }
$$

$1 / 18 / 01$

20

10

$\begin{array}{cc}\mathrm{mg} / \mathrm{L} & \text { Sample } \\ \mathrm{mg} / \mathrm{L} & \text { Sample } \\ \mathrm{mg} / \mathrm{L} & \text { Sample } \\ \mathrm{mg} / \mathrm{L} & \text { Sample } \\ \mathrm{mg} / \mathrm{L} & \text { Sample } \\ \mathrm{mg} / \mathrm{L} & \text { Sample } \\ \mathrm{mg} / \mathrm{L} & \text { Sample } \\ \mathrm{mg} / \mathrm{L} & \text { Sample }\end{array}$

S.U.

S.U.

Sample

S.U.

Sample

S.U.

Sample

Sample

S.U.

S.U.

S.U.

Sample

$4 / 17 / 01$

Sample

Sample

Sample $\mathrm{mg} / \mathrm{L}$ $\mathrm{mg} / \mathrm{L}$
Sample Sample 


$\begin{array}{lllll}\text { Lnalyte } & \text { Location } & \text { Date } & \text { Result } & \text { or PQL Units } \\ \text { AC Type }\end{array}$

\section{General Indicator Parameters}

Total suspended solids (TSS)

cont.

$\begin{array}{llrrrr}\text { Hearst Sewer } & 7 / 10 / 01 & 160 & 5 & \mathrm{mg} / \mathrm{L} & \text { Sample } \\ & 10 / 31 / 01 & 330 & 10 & \mathrm{mg} / \mathrm{L} & \text { Sample } \\ \text { Strawberry Sewer } & 1 / 18 / 01 & 240 & 20 & \mathrm{mg} / \mathrm{L} & \text { Sample } \\ & 4 / 17 / 01 & 190 & 10 & \mathrm{mg} / \mathrm{L} & \text { Sample } \\ & 7 / 10 / 01 & 94 & 5 & \mathrm{mg} / \mathrm{L} & \text { Sample } \\ & 10 / 31 / 01 & 160 & 5 & \mathrm{mg} / \mathrm{L} & \text { Sample }\end{array}$

Metals and/or Minerals

\begin{tabular}{|c|c|c|c|c|c|c|}
\hline \multirow[t]{3}{*}{ Cadmium } & Hearst Sewer & 10/31/01 & ND & 0.01 & $\mathrm{mg} / \mathrm{L}$ & Sample \\
\hline & Strawberry Sewer & $\begin{array}{l}10 / 31 / 01 \\
10 / 31 / 01\end{array}$ & $\begin{array}{l}\text { ND } \\
\text { ND }\end{array}$ & $\begin{array}{l}0.01 \\
0.01\end{array}$ & $\begin{array}{l}\mathrm{mg} / \mathrm{L} \\
\mathrm{mg} / \mathrm{L}\end{array}$ & $\begin{array}{r}\text { Sample } \\
\text { Split }\end{array}$ \\
\hline & Field Blank & $10 / 31 / 01$ & ND & 0.01 & $\mathrm{mg} / \mathrm{L}$ & Blank \\
\hline \multirow[t]{3}{*}{ Chromium } & Hearst Sewer & 10/31/01 & ND & 0.01 & $\mathrm{mg} / \mathrm{L}$ & Sample \\
\hline & Strawberry Sewer & $\begin{array}{l}10 / 31 / 01 \\
10 / 31 / 01\end{array}$ & $\begin{array}{l}\text { ND } \\
\text { ND }\end{array}$ & $\begin{array}{l}0.01 \\
0.01\end{array}$ & $\begin{array}{l}\mathrm{mg} / \mathrm{L} \\
\mathrm{mg} / \mathrm{L}\end{array}$ & $\begin{array}{r}\text { Sample } \\
\text { Split }\end{array}$ \\
\hline & Field Blank & $10 / 31 / 01$ & ND & 0.01 & $\mathrm{mg} / \mathrm{L}$ & Blank \\
\hline \multirow[t]{3}{*}{ Copper } & Hearst Sewer & $10 / 31 / 01$ & 0.1 & 0.01 & $\mathrm{mg} / \mathrm{L}$ & Sample \\
\hline & Strawberry Sewer & $\begin{array}{l}10 / 31 / 01 \\
10 / 31 / 01\end{array}$ & $\begin{array}{l}0.053 \\
0.13\end{array}$ & $\begin{array}{l}0.01 \\
0.01\end{array}$ & $\begin{array}{l}\mathrm{mg} / \mathrm{L} \\
\mathrm{mg} / \mathrm{L}\end{array}$ & $\begin{array}{r}\text { Sample } \\
\text { Split }\end{array}$ \\
\hline & Field Blank & 10/31/01 & 0.13 & 0.01 & $\mathrm{mg} / \mathrm{L}$ & Blank \\
\hline \multirow[t]{3}{*}{ Lead } & Hearst Sewer & $10 / 31 / 01$ & ND & 0.05 & $\mathrm{mg} / \mathrm{L}$ & Sample \\
\hline & Strawberry Sewer & $\begin{array}{l}10 / 31 / 01 \\
10 / 31 / 01\end{array}$ & $\begin{array}{l}\text { ND } \\
\text { ND }\end{array}$ & $\begin{array}{l}0.05 \\
0.05\end{array}$ & $\begin{array}{l}\mathrm{mg} / \mathrm{L} \\
\mathrm{mg} / \mathrm{L}\end{array}$ & $\begin{array}{r}\text { Sample } \\
\text { Split }\end{array}$ \\
\hline & Field Blank & 10/31/01 & ND & 0.05 & $\mathrm{mg} / \mathrm{L}$ & Blank \\
\hline \multirow[t]{3}{*}{ Nickel } & Hearst Sewer & $10 / 31 / 01$ & ND & 0.05 & $\mathrm{mg} / \mathrm{L}$ & Sample \\
\hline & Strawberry Sewer & $\begin{array}{l}10 / 31 / 01 \\
10 / 31 / 01\end{array}$ & $\begin{array}{l}\text { ND } \\
\text { ND }\end{array}$ & $\begin{array}{l}0.05 \\
0.05\end{array}$ & $\begin{array}{l}\mathrm{mg} / \mathrm{L} \\
\mathrm{mg} / \mathrm{L}\end{array}$ & $\begin{array}{r}\text { Sample } \\
\text { Split }\end{array}$ \\
\hline & Field Blank & $10 / 31 / 01$ & ND & 0.05 & $\mathrm{mg} / \mathrm{L}$ & Blank \\
\hline \multirow[t]{3}{*}{ Silver } & Hearst Sewer & $10 / 31 / 01$ & ND & 0.01 & $\mathrm{mg} / \mathrm{L}$ & Sample \\
\hline & Strawberry Sewer & $\begin{array}{l}10 / 31 / 01 \\
10 / 31 / 01\end{array}$ & $\begin{array}{l}N D \\
N D\end{array}$ & $\begin{array}{l}0.01 \\
0.01\end{array}$ & $\begin{array}{l}\mathrm{mg} / \mathrm{L} \\
\mathrm{mg} / \mathrm{L}\end{array}$ & $\begin{array}{r}\text { Sample } \\
\text { Split }\end{array}$ \\
\hline & Field Blank & $10 / 31 / 01$ & ND & 0.01 & $\mathrm{mg} / \mathrm{L}$ & Blank \\
\hline
\end{tabular}




\begin{tabular}{lllll} 
Lnalyte & \multicolumn{4}{c}{ MDA } \\
Location & Date & Result & or PQL Units & QC Type
\end{tabular}

Metals and/or Minerals

Zinc

$\begin{array}{lllllr}\text { Hearst Sewer } & 10 / 31 / 01 & 0.19 & 0.05 & \mathrm{mg} / \mathrm{L} & \text { Sample } \\ \text { Strawberry Sewer } & 10 / 31 / 01 & 0.12 & 0.05 & \mathrm{mg} / \mathrm{L} & \text { Sample } \\ & 10 / 31 / 01 & 0.12 & 0.05 & \mathrm{mg} / \mathrm{L} & \text { Split } \\ \text { Field Blank } & 10 / 31 / 01 & \mathrm{ND} & 0.05 & \mathrm{mg} / \mathrm{L} & \text { Blank }\end{array}$

\section{Volatile Organic Compounds}

\begin{tabular}{|c|c|c|c|c|c|c|}
\hline 1,1,1-Trichloroethane & Hearst Sewer & $1 / 18 / 01$ & ND & 0.5 & $\mu \mathrm{g} / \mathrm{L}$ & Sample \\
\hline & & $1 / 18 / 01$ & ND & 0.5 & $\mu \mathrm{g} / \mathrm{L}$ & Split \\
\hline & & $4 / 17 / 01$ & ND & 0.5 & $\mu \mathrm{g} / \mathrm{L}$ & Sample \\
\hline & & $4 / 17 / 01$ & ND & 0.5 & $\mu \mathrm{g} / \mathrm{L}$ & Split \\
\hline & & $7 / 10 / 01$ & ND & 0.5 & $\mu \mathrm{g} / \mathrm{L}$ & Sample \\
\hline & & $7 / 10 / 01$ & ND & 0.5 & $\mu \mathrm{g} / \mathrm{L}$ & $\mathrm{Sp}$ \\
\hline & & $10 / 31 / 01$ & ND & 0.5 & $\mu \mathrm{g} / \mathrm{L}$ & Sample \\
\hline & & $10 / 31 / 01$ & ND & 0.5 & $\mu \mathrm{g} / \mathrm{L}$ & Split \\
\hline & Strawberry Sewer & $1 / 18 / 01$ & ND & 0.5 & $\mu \mathrm{g} / \mathrm{L}$ & Sample \\
\hline & & $4 / 17 / 01$ & ND & 0.5 & $\mu \mathrm{g} / \mathrm{L}$ & Sample \\
\hline & & $7 / 10 / 01$ & ND & 0.5 & $\mu \mathrm{g} / \mathrm{L}$ & Sample \\
\hline & & $10 / 31 / 01$ & ND & 0.5 & $\mu \mathrm{g} / \mathrm{L}$ & Samp \\
\hline & Field Blank & $4 / 17 / 01$ & ND & 0.5 & $\mu \mathrm{g} / \mathrm{L}$ & Bla \\
\hline & & $4 / 17 / 01$ & ND & 0.5 & $\mu \mathrm{g} / \mathrm{L}$ & Blar \\
\hline 1,1,2,2-Tetrachloroethane & Hearst Sewer & $1 / 18 / 01$ & ND & 0.5 & $\mu \mathrm{g} / \mathrm{L}$ & Sample \\
\hline & & $1 / 18 / 01$ & ND & 0.5 & $\mu \mathrm{g} / \mathrm{L}$ & Split \\
\hline & & $4 / 17 / 01$ & ND & 0.5 & $\mu \mathrm{g} / \mathrm{L}$ & Sampl \\
\hline & & $4 / 17 / 01$ & ND & 0.5 & $\mu \mathrm{g} / \mathrm{L}$ & $\mathrm{Sp}$ \\
\hline & & $7 / 10 / 01$ & ND & 0.5 & $\mu \mathrm{g} / \mathrm{L}$ & Sampl \\
\hline & & $7 / 10 / 01$ & ND & 0.5 & $\mu \mathrm{g} / \mathrm{L}$ & Sp \\
\hline & & $10 / 31 / 01$ & ND & 0.5 & $\mu \mathrm{g} / \mathrm{L}$ & Sampl \\
\hline & & $10 / 31 / 01$ & ND & 0.5 & $\mu \mathrm{g} / \mathrm{L}$ & $S p$ \\
\hline & Strawberry Sewer & $1 / 18 / 01$ & ND & 0.5 & $\mu \mathrm{g} / \mathrm{L}$ & Samp \\
\hline & & $4 / 17 / 01$ & ND & 0.5 & $\mu \mathrm{g} / \mathrm{L}$ & Sampl \\
\hline & & $7 / 10 / 01$ & ND & 0.5 & $\mu \mathrm{g} / \mathrm{L}$ & Sampl \\
\hline & & $10 / 31 / 01$ & ND & 0.5 & $\mu \mathrm{g} / \mathrm{L}$ & Samp \\
\hline & Field Blank & $4 / 17 / 01$ & ND & 0.5 & $\mu \mathrm{g} / \mathrm{L}$ & Bla \\
\hline & & $4 / 17 / 01$ & ND & 0.5 & $\mu \mathrm{g} / \mathrm{L}$ & Blar \\
\hline
\end{tabular}




\section{Analyte \\ Location \\ Volatile Organic Compounds}

MDA

1,1,2-Trichloroethane Hearst Sewer

(1)

1,1-Dichloroethane

1,1-Dichloroethene
$1 / 18 / 01$

$1 / 18 / 01$

$4 / 17 / 01$

$4 / 17 / 01$

$7 / 10 / 01$

$7 / 10 / 01$

$10 / 31 / 01$

$10 / 31 / 01$

Strawberry Sewe

$1 / 18 / 01$

$4 / 17 / 01$

$7 / 10 / 01$

$10 / 31 / 01$

Field Blank

$4 / 17 / 01$

$4 / 17 / 01$

Hearst Sewer

$1 / 18 / 01$

$1 / 18 / 01$

$4 / 17 / 01$

$4 / 17 / 01$

$7 / 10 / 01$

$7 / 10 / 01$

10/31/01

10/31/01

Strawberry Sewer

$1 / 18 / 01$

$4 / 17 / 01$

$7 / 10 / 01$

10/31/01

Field Blank

$4 / 17 / 01$

$4 / 17 / 01$

Hearst Sewer $\quad 1 / 18 / 01$

$1 / 18 / 01$

$4 / 17 / 01$

$4 / 17 / 01$

$7 / 10 / 01$

$7 / 10 / 01$

10/31/01

10/31/01

Strawberry Sewer

$1 / 18 / 01$

$4 / 17 / 01$

$N D$
$N D$
$N D$
$N D$
$N D$
$N D$
$N D$
$N D$
$N D$
$N D$
$N D$
$N D$

ND

ND

ND

ND

ND

ND

ND

ND

ND

ND

ND

ND

ND

ND

ND

ND

ND

ND

ND

ND

ND

ND

ND

ND

ND

ND or PQL

Units QC Type

$\begin{array}{rrr}0.5 & \mu \mathrm{g} / \mathrm{L} & \text { Sample } \\ 0.5 & \mu \mathrm{g} / \mathrm{L} & \text { Split } \\ 0.5 & \mu \mathrm{g} / \mathrm{L} & \text { Sample } \\ 0.5 & \mu \mathrm{g} / \mathrm{L} & \text { Split } \\ 0.5 & \mu \mathrm{g} / \mathrm{L} & \text { Sample } \\ 0.5 & \mu \mathrm{g} / \mathrm{L} & \text { Split } \\ 0.5 & \mu \mathrm{g} / \mathrm{L} & \text { Sample } \\ 0.5 & \mu \mathrm{g} / \mathrm{L} & \text { Split }\end{array}$

$0.5 \mu \mathrm{g} / \mathrm{L} \quad$ Sample

$0.5 \mu \quad \mu g / L \quad$ Sample

$0.5 \mu \mathrm{g} / \mathrm{L} \quad$ Sample

$0.5 \mu \quad \mu g / L \quad$ Sample

$0.5 \mu \mathrm{g} / \mathrm{L} \quad$ Blank

$0.5 \mu \mathrm{g} / \mathrm{L} \quad$ Blank

\section{$0.5 \mu \quad \mu g / L \quad$ Sample}

$0.5 \mu \quad \mu g / L \quad$ Split

$0.5 \mu \quad \mu g / L \quad$ Sample

$0.5 \mu \mathrm{g} / \mathrm{L} \quad$ Split

$0.5 \mu \quad \mu g / L \quad$ Sample

$0.5 \mu \mathrm{gg} / \mathrm{L} \quad$ Split

$0.5 \quad \mu g / L \quad$ Sample

$0.5 \mu \mathrm{g} / \mathrm{L} \quad$ Split

$0.5 \mu \mathrm{g} / \mathrm{L} \quad$ Sample

$0.5 \mu \mathrm{g} / \mathrm{L} \quad$ Sample

$0.5 \mu \mathrm{g} / \mathrm{L} \quad$ Sample

$0.5 \mu \mathrm{g} / \mathrm{L} \quad$ Sample

$0.5 \mu \mathrm{g} / \mathrm{L} \quad$ Blank

$0.5 \mu \mathrm{g} / \mathrm{L} \quad$ Blank

$0.5 \mu \mathrm{g} / \mathrm{L} \quad$ Sample

$0.5 \mu \quad \mu g / L \quad$ Split

$0.5 \mu \quad \mu g / L \quad$ Sample

$0.5 \mu \mathrm{g} / \mathrm{L} \quad$ Split

$0.5 \mu \mathrm{g} / \mathrm{L} \quad$ Sample

$0.5 \mu \mathrm{g} / \mathrm{L} \quad$ Split

$0.5 \mu \mathrm{gg} / \mathrm{L} \quad$ Sample

$0.5 \mu \mathrm{g} / \mathrm{L} \quad$ Split

$0.5 \quad \mu g / L \quad$ Sample

$0.5 \mu \mathrm{g} / \mathrm{L} \quad$ Sample 


$\begin{array}{llllll}\text { Lnalyte } & \text { Location } & \text { Date } & \text { Result } & \text { or PQL Units } & \text { QC Type }\end{array}$

\section{Volatile Organic Compounds}

\begin{tabular}{|c|c|c|c|c|c|c|}
\hline \multirow[t]{3}{*}{$\begin{array}{l}\text { 1,1-Dichloroethene } \\
\text { cont. }\end{array}$} & Strawberry Sewer & $\begin{array}{l}7 / 10 / 01 \\
10 / 31 / 01\end{array}$ & $\begin{array}{l}\text { ND } \\
\text { ND }\end{array}$ & $\begin{array}{l}0.5 \\
0.5\end{array}$ & $\begin{array}{l}\mu \mathrm{g} / \mathrm{L} \\
\mu \mathrm{g} / \mathrm{L}\end{array}$ & $\begin{array}{l}\text { Sample } \\
\text { Sample }\end{array}$ \\
\hline & Field Blank & $4 / 17 / 01$ & ND & 0.5 & $\mu \mathrm{g} / \mathrm{L}$ & Bla \\
\hline & & 榲 & & & & \\
\hline \multirow{14}{*}{ 1,2-Dichlorobenzene } & Hearst Sewer & $1 / 18 / 01$ & ND & 0.5 & $\mu \mathrm{g} / \mathrm{L}$ & Samp \\
\hline & & $1 / 18 / 01$ & ND & 0.5 & $\mu \mathrm{g} / \mathrm{L}$ & \\
\hline & & $4 / 17 / 01$ & ND & 0.5 & $\mu \mathrm{g} / \mathrm{L}$ & Samp \\
\hline & & $4 / 17 / 01$ & ND & 0.5 & $\mu \mathrm{g} / \mathrm{L}$ & S| \\
\hline & & $7 / 10 / 01$ & ND & 0.5 & $\mu \mathrm{g} / \mathrm{L}$ & Samp \\
\hline & & $7 / 10 / 01$ & ND & 0.5 & $\mu \mathrm{g} / \mathrm{L}$ & \\
\hline & & $10 / 31 / 01$ & ND & 0.5 & $\mu \mathrm{g} / \mathrm{L}$ & Samr \\
\hline & & $10 / 31 / 01$ & ND & 0.5 & $\mu \mathrm{g} / \mathrm{L}$ & \\
\hline & Strawberry Sewer & $1 / 18 / 01$ & ND & 0.5 & $\mu \mathrm{g} / \mathrm{L}$ & Sam \\
\hline & & $4 / 17 / 01$ & ND & 0.5 & $\mu \mathrm{g} / \mathrm{L}$ & Samp \\
\hline & & $7 / 10 / 01$ & ND & 0.5 & $\mu \mathrm{g} / \mathrm{L}$ & Samp \\
\hline & & $10 / 31 / 01$ & ND & 0.5 & $\mu \mathrm{g} / \mathrm{L}$ & Samp \\
\hline & Field Blank & $4 / 17 / 01$ & ND & 0.5 & $\mu \mathrm{g} / \mathrm{L}$ & \\
\hline & & $4 / 17 / 01$ & ND & 0.5 & $\mu \mathrm{g} / \mathrm{L}$ & \\
\hline \multirow[t]{14}{*}{ 1,2-Dichloroethane } & Hearst Sewer & $1 / 18 / 01$ & ND & 0.5 & $\mu \mathrm{g} / \mathrm{L}$ & Sam \\
\hline & & $1 / 18 / 01$ & ND & 0.5 & $\mu \mathrm{g} / \mathrm{L}$ & \\
\hline & & $4 / 17 / 01$ & ND & 0.5 & $\mu \mathrm{g} / \mathrm{L}$ & Samp \\
\hline & & $4 / 17 / 01$ & ND & 0.5 & $\mu \mathrm{g} / \mathrm{L}$ & \\
\hline & & $7 / 10 / 01$ & ND & 0.5 & $\mu \mathrm{g} / \mathrm{L}$ & Samp \\
\hline & & $7 / 10 / 01$ & ND & 0.5 & $\mu \mathrm{g} / \mathrm{L}$ & \\
\hline & & $10 / 31 / 01$ & ND & 0.5 & $\mu \mathrm{g} / \mathrm{L}$ & Samp \\
\hline & & $10 / 31 / 01$ & ND & 0.5 & $\mu \mathrm{g} / \mathrm{L}$ & \\
\hline & Strawberry Sewer & $1 / 18 / 01$ & ND & 0.5 & $\mu \mathrm{g} / \mathrm{L}$ & Samp \\
\hline & & $4 / 17 / 01$ & ND & 0.5 & $\mu \mathrm{g} / \mathrm{L}$ & Samp \\
\hline & & $7 / 10 / 01$ & ND & 0.5 & $\mu \mathrm{g} / \mathrm{L}$ & Samp \\
\hline & & $10 / 31 / 01$ & ND & 0.5 & $\mu \mathrm{g} / \mathrm{L}$ & Samp \\
\hline & Field Blank & $4 / 17 / 01$ & ND & 0.5 & $\mu \mathrm{g} / \mathrm{L}$ & $\mathrm{Bla}$ \\
\hline & & $4 / 17 / 01$ & ND & 0.5 & $\mu \mathrm{g} / \mathrm{L}$ & \\
\hline \multirow[t]{6}{*}{ 1,2-Dichloroethene (total) } & Hearst Sewer & $4 / 17 / 01$ & ND & 1 & $\mu \mathrm{g} / \mathrm{L}$ & Sam \\
\hline & & $4 / 17 / 01$ & ND & 1 & $\mu \mathrm{g} / \mathrm{L}$ & \\
\hline & & $7 / 10 / 01$ & ND & 1 & $\mu \mathrm{g} / \mathrm{L}$ & Samp \\
\hline & & $7 / 10 / 01$ & ND & 1 & $\mu \mathrm{g} / \mathrm{L}$ & $\mathrm{S}$ \\
\hline & & $10 / 31 / 01$ & ND & 1 & $\mu \mathrm{g} / \mathrm{L}$ & Samp \\
\hline & & $10 / 31 / 01$ & ND & 1 & $\mu \mathrm{g} / \mathrm{L}$ & \\
\hline
\end{tabular}




\begin{tabular}{lllrl} 
Lnalyte & \multicolumn{4}{c}{ MDA } \\
Location & Date & Result & or PQL Units & QC Type \\
\hline
\end{tabular}

\section{Volatile Organic Compounds}

1,2-Dichloroethene (total) Strawberry Sewer 4/17/01 cont.

1,2-Dichloropropane

Strawberry Sewer

$\begin{array}{ll}1 / 18 / 01 & N D \\ 4 / 17 / 01 & N D \\ 7 / 10 / 01 & N D \\ 10 / 31 / 01 & N D\end{array}$

Field Blank

$\begin{array}{ll}4 / 17 / 01 & \text { ND } \\ 4 / 17 / 01 & \text { ND }\end{array}$

1,3-Dichlorobenzene
$1 / 18 / 01$

$1 / 18 / 01$

$4 / 17 / 01$

$4 / 17 / 01$

$7 / 10 / 01$

$7 / 10 / 01$

$10 / 31 / 01$

$10 / 31 / 01$

Strawberry Sewer $1 / 18 / 01$

$4 / 17 / 01$

$7 / 10 / 01$

$10 / 31 / 01$

Field Blank

$4 / 17 / 01$
$4 / 17 / 01$

Hearst Sewer
$1 / 18 / 01$ $1 / 18 / 01$
ND

ND

ND

ND

ND

ND

ND

ND

ND

ND

ND

ND

ND

$\mathrm{ND}$

$\mathrm{ND}$

$\mathrm{ND}$

ND

ND

ND

ND

ND

ND

ND

ND

ND

ND

ND

ND

ND

ND

ND

ND

ND

ND

ND

$\begin{array}{llr}1 & \mu \mathrm{g} / \mathrm{L} & \text { Sample } \\ 1 & \mu \mathrm{g} / \mathrm{L} & \text { Sample } \\ 1 & \mu \mathrm{g} / \mathrm{L} & \text { Sample } \\ 1 & \mu \mathrm{g} / \mathrm{L} & \text { Blank } \\ 1 & \mu \mathrm{g} / \mathrm{L} & \text { Blank } \\ 0.5 & \mu \mathrm{g} / \mathrm{L} & \text { Sample } \\ 0.5 & \mu \mathrm{g} / \mathrm{L} & \text { Split } \\ 0.5 & \mu \mathrm{g} / \mathrm{L} & \text { Sample } \\ 0.5 & \mu \mathrm{g} / \mathrm{L} & \text { Split } \\ 0.5 & \mu \mathrm{g} / \mathrm{L} & \text { Sample } \\ 0.5 & \mu \mathrm{g} / \mathrm{L} & \text { Split } \\ 0.5 & \mu \mathrm{g} / \mathrm{L} & \text { Sample } \\ 0.5 & \mu \mathrm{g} / \mathrm{L} & \text { Split }\end{array}$

$0.5 \quad \mu \mathrm{g} / \mathrm{L} \quad$ Sample

$0.5 \mu \mathrm{g} / \mathrm{L} \quad$ Sample

$0.5 \mu \mathrm{g} / \mathrm{L} \quad$ Sample

$0.5 \mu \mathrm{g} / \mathrm{L} \quad$ Sample

$\begin{array}{lll}0.5 & \mu \mathrm{g} / \mathrm{L} & \text { Blank } \\ 0.5 & \mu \mathrm{g} / \mathrm{L} & \text { Blank }\end{array}$

\section{$0.5 \mu \mathrm{g} / \mathrm{L} \quad$ Sample}

$0.5 \mu \mathrm{g} / \mathrm{L} \quad$ Split

$0.5 \mu \mathrm{g} / \mathrm{L} \quad$ Sample

$0.5 \mu \mathrm{g} / \mathrm{L} \quad$ Split

$0.5 \mu \quad \mu g / L \quad$ Sample

$0.5 \mu \mathrm{g} / \mathrm{L} \quad$ Split

$0.5 \mu \mathrm{g} / \mathrm{L} \quad$ Sample

$0.5 \mu \mathrm{g} / \mathrm{L} \quad$ Split

$0.5 \mu \mathrm{g} / \mathrm{L} \quad$ Sample

$0.5 \mu \quad \mu g / L \quad$ Sample

$0.5 \mu \mathrm{g} / \mathrm{L} \quad$ Sample

$0.5 \mu \mathrm{g} / \mathrm{L} \quad$ Sample

$0.5 \mu \mathrm{g} / \mathrm{L} \quad$ Blank

$0.5 \mu \mathrm{g} / \mathrm{L} \quad$ Blank

$\begin{array}{llr}0.5 & \mu \mathrm{g} / \mathrm{L} & \text { Sample } \\ 0.5 & \mu \mathrm{g} / \mathrm{L} & \text { Split }\end{array}$




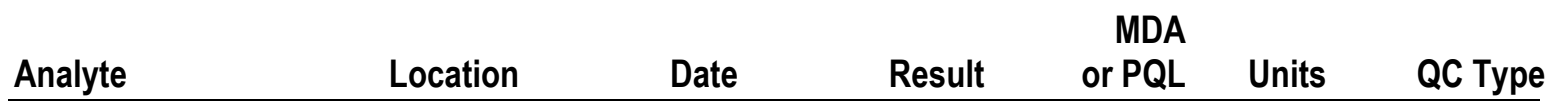

\section{Volatile Organic Compounds}

\begin{tabular}{|c|c|c|c|c|c|c|}
\hline 1,4-Dichlorobenzene & Hearst Sewer & $4 / 17 / 01$ & ND & 0.5 & $\mu \mathrm{g} / \mathrm{L}$ & Sample \\
\hline cont. & & $4 / 17 / 01$ & ND & 0.5 & $\mu \mathrm{g} / \mathrm{L}$ & Split \\
\hline & & $7 / 10 / 01$ & ND & 0.5 & $\mu \mathrm{g} / \mathrm{L}$ & Sample \\
\hline & & $7 / 10 / 01$ & ND & 0.5 & $\mu \mathrm{g} / \mathrm{L}$ & Split \\
\hline & & $10 / 31 / 01$ & ND & 0.5 & $\mu \mathrm{g} / \mathrm{L}$ & Sample \\
\hline & & $10 / 31 / 01$ & ND & 0.5 & $\mu \mathrm{g} / \mathrm{L}$ & Split \\
\hline & Strawberry Sewer & $1 / 18 / 01$ & ND & 0.5 & $\mu \mathrm{g} / \mathrm{L}$ & Sample \\
\hline & & $4 / 17 / 01$ & ND & 0.5 & $\mu \mathrm{g} / \mathrm{L}$ & Sample \\
\hline & & $7 / 10 / 01$ & ND & 0.5 & $\mu \mathrm{g} / \mathrm{L}$ & Sample \\
\hline & & $10 / 31 / 01$ & ND & 0.5 & $\mu \mathrm{g} / \mathrm{L}$ & Sample \\
\hline & Field Blank & $4 / 17 / 01$ & ND & 0.5 & $\mu \mathrm{g} / \mathrm{L}$ & Blank \\
\hline & & $4 / 17 / 01$ & ND & 0.5 & $\mu \mathrm{g} / \mathrm{L}$ & Blank \\
\hline Butanone & Hearst Sewer & 4/17/01 & ND & 20 & $\mu \mathrm{g} / \mathrm{L}$ & Sample \\
\hline & & $4 / 17 / 01$ & ND & 20 & $\mu \mathrm{g} / \mathrm{L}$ & Split \\
\hline & & $7 / 10 / 01$ & ND & 20 & $\mu \mathrm{g} / \mathrm{L}$ & Sample \\
\hline & & $7 / 10 / 01$ & ND & 20 & $\mu \mathrm{g} / \mathrm{L}$ & Split \\
\hline & & 10/31/01 & ND & 20 & $\mu \mathrm{g} / \mathrm{L}$ & Sample \\
\hline & & $10 / 31 / 01$ & ND & 20 & $\mu \mathrm{g} / \mathrm{L}$ & $\mathrm{S}$ \\
\hline & Strawberry Sewer & $4 / 17 / 01$ & ND & 20 & $\mu \mathrm{g} / \mathrm{L}$ & Sample \\
\hline & & $7 / 10 / 01$ & ND & 20 & $\mu \mathrm{g} / \mathrm{L}$ & Sample \\
\hline & & $10 / 31 / 01$ & ND & 20 & $\mu \mathrm{g} / \mathrm{L}$ & Sample \\
\hline & Field Blank & $4 / 17 / 01$ & ND & 20 & $\mu \mathrm{g} / \mathrm{L}$ & Blank \\
\hline & & $4 / 17 / 01$ & ND & 20 & $\mu \mathrm{g} / \mathrm{L}$ & Blank \\
\hline 2-Chloroethylvinylether & Hearst Sewer & $4 / 17 / 01$ & ND & 10 & $\mu \mathrm{g} / \mathrm{L}$ & Sample \\
\hline & & $4 / 17 / 01$ & ND & 10 & $\mu \mathrm{g} / \mathrm{L}$ & Split \\
\hline & & $7 / 10 / 01$ & ND & 10 & $\mu \mathrm{g} / \mathrm{L}$ & Sample \\
\hline & & $7 / 10 / 01$ & ND & 10 & $\mu \mathrm{g} / \mathrm{L}$ & Spl \\
\hline & & $10 / 31 / 01$ & ND & 10 & $\mu \mathrm{g} / \mathrm{L}$ & Sample \\
\hline & & $10 / 31 / 01$ & ND & 10 & $\mu \mathrm{g} / \mathrm{L}$ & $\mathrm{Sp}$ \\
\hline & Strawberry Sewer & $4 / 17 / 01$ & ND & 10 & $\mu \mathrm{g} / \mathrm{L}$ & Sample \\
\hline & & $7 / 10 / 01$ & ND & 10 & $\mu \mathrm{g} / \mathrm{L}$ & Sample \\
\hline & & $10 / 31 / 01$ & ND & 10 & $\mu \mathrm{g} / \mathrm{L}$ & Sample \\
\hline & Field Blank & $4 / 17 / 01$ & ND & 10 & $\mu \mathrm{g} / \mathrm{L}$ & Bla \\
\hline & & $4 / 17 / 01$ & ND & 10 & $\mu \mathrm{g} / \mathrm{L}$ & Bla \\
\hline 2-Hexanone & Hearst Sewer & $4 / 17 / 01$ & ND & 20 & $\mu \mathrm{g} / \mathrm{L}$ & Sample \\
\hline & & $4 / 17 / 01$ & ND & 20 & $\mu \mathrm{g} / \mathrm{L}$ & Split \\
\hline & & 7/10/01 & ND & 20 & $\mu \mathrm{g} / \mathrm{L}$ & Sample \\
\hline
\end{tabular}




\section{Analyte \\ Location \\ Volatile Organic Compounds}

MDA

\begin{tabular}{|c|c|c|c|c|c|c|}
\hline $\begin{array}{l}\text { 2-Hexanone } \\
\text { cont. }\end{array}$ & Hearst Sewer & $\begin{array}{l}7 / 10 / 01 \\
10 / 31 / 01 \\
10 / 31 / 01\end{array}$ & $\begin{array}{l}\text { ND } \\
\text { ND } \\
\text { ND }\end{array}$ & $\begin{array}{l}20 \\
20 \\
20\end{array}$ & $\begin{array}{l}\mu \mathrm{g} / \mathrm{L} \\
\mu \mathrm{g} / \mathrm{L} \\
\mu \mathrm{g} / \mathrm{L}\end{array}$ & $\begin{array}{r}\text { Split } \\
\text { Sample } \\
\text { Split }\end{array}$ \\
\hline & Strawberry Sewer & $\begin{array}{l}4 / 17 / 01 \\
7 / 10 / 01 \\
10 / 31 / 01\end{array}$ & $\begin{array}{l}\text { ND } \\
\text { ND } \\
\text { ND }\end{array}$ & $\begin{array}{l}20 \\
20 \\
20\end{array}$ & $\begin{array}{l}\mu \mathrm{g} / \mathrm{L} \\
\mu \mathrm{g} / \mathrm{L} \\
\mu \mathrm{g} / \mathrm{L}\end{array}$ & $\begin{array}{l}\text { Sample } \\
\text { Sample } \\
\text { Sample }\end{array}$ \\
\hline & Field Blank & $\begin{array}{l}4 / 17 / 01 \\
4 / 17 / 01\end{array}$ & $\begin{array}{l}\text { ND } \\
\text { ND }\end{array}$ & $\begin{array}{l}20 \\
20\end{array}$ & $\begin{array}{l}\mu \mathrm{g} / \mathrm{L} \\
\mu \mathrm{g} / \mathrm{L}\end{array}$ & $\begin{array}{l}\text { Blank } \\
\text { Blank }\end{array}$ \\
\hline 4-Methyl-2-pentanone & Hearst Sewer & $\begin{array}{l}4 / 17 / 01 \\
4 / 17 / 01 \\
7 / 10 / 01 \\
7 / 10 / 01 \\
10 / 31 / 01 \\
10 / 31 / 01\end{array}$ & $\begin{array}{l}\text { ND } \\
\text { ND } \\
\text { ND } \\
\text { ND } \\
\text { ND } \\
\text { ND }\end{array}$ & $\begin{array}{l}20 \\
20 \\
20 \\
20 \\
20 \\
20\end{array}$ & $\begin{array}{l}\mu \mathrm{g} / \mathrm{L} \\
\mu \mathrm{g} / \mathrm{L} \\
\mu \mathrm{g} / \mathrm{L} \\
\mu \mathrm{g} / \mathrm{L} \\
\mu \mathrm{g} / \mathrm{L} \\
\mu \mathrm{g} / \mathrm{L}\end{array}$ & $\begin{array}{r}\text { Sample } \\
\text { Split } \\
\text { Sample } \\
\text { Split } \\
\text { Sample } \\
\text { Split }\end{array}$ \\
\hline & Strawberry Sewer & $\begin{array}{l}4 / 17 / 01 \\
7 / 10 / 01 \\
10 / 31 / 01\end{array}$ & $\begin{array}{l}\text { ND } \\
\text { ND } \\
\text { ND }\end{array}$ & $\begin{array}{l}20 \\
20 \\
20\end{array}$ & $\begin{array}{l}\mu \mathrm{g} / \mathrm{L} \\
\mu \mathrm{g} / \mathrm{L} \\
\mu \mathrm{g} / \mathrm{L}\end{array}$ & $\begin{array}{l}\text { Sample } \\
\text { Sample } \\
\text { Sample }\end{array}$ \\
\hline & Field Blank & $\begin{array}{l}4 / 17 / 01 \\
4 / 17 / 01\end{array}$ & $\begin{array}{l}\text { ND } \\
\text { ND }\end{array}$ & $\begin{array}{l}20 \\
20\end{array}$ & $\begin{array}{l}\mu \mathrm{g} / \mathrm{L} \\
\mu \mathrm{g} / \mathrm{L}\end{array}$ & $\begin{array}{l}\text { Blank } \\
\text { Blank }\end{array}$ \\
\hline Acetone & Hearst Sewer & $\begin{array}{l}4 / 17 / 01 \\
4 / 17 / 01 \\
7 / 10 / 01 \\
7 / 10 / 01 \\
10 / 31 / 01 \\
10 / 31 / 01\end{array}$ & $\begin{array}{l}290 \\
300 \\
150 \\
160 \\
230 \\
230\end{array}$ & $\begin{array}{l}20 \\
20 \\
20 \\
20 \\
20 \\
20\end{array}$ & $\begin{array}{l}\mu \mathrm{g} / \mathrm{L} \\
\mu \mathrm{g} / \mathrm{L} \\
\mu \mathrm{g} / \mathrm{L} \\
\mu \mathrm{g} / \mathrm{L} \\
\mu \mathrm{g} / \mathrm{L} \\
\mu \mathrm{g} / \mathrm{L}\end{array}$ & $\begin{array}{r}\text { Sample } \\
\text { Split } \\
\text { Sample } \\
\text { Split } \\
\text { Sample } \\
\text { Split }\end{array}$ \\
\hline & Strawberry Sewer & $\begin{array}{l}4 / 17 / 01 \\
7 / 10 / 01 \\
10 / 31 / 01\end{array}$ & $\begin{array}{r}6700 \\
800 \\
44\end{array}$ & $\begin{array}{l}20 \\
20 \\
20\end{array}$ & $\begin{array}{l}\mu \mathrm{g} / \mathrm{L} \\
\mu \mathrm{g} / \mathrm{L} \\
\mu \mathrm{g} / \mathrm{L}\end{array}$ & $\begin{array}{l}\text { Sample } \\
\text { Sample } \\
\text { Sample }\end{array}$ \\
\hline & Field Blank & $\begin{array}{l}4 / 17 / 01 \\
4 / 17 / 01\end{array}$ & $\begin{array}{l}140 \\
\text { ND }\end{array}$ & $\begin{array}{l}20 \\
20\end{array}$ & $\begin{array}{l}\mu \mathrm{g} / \mathrm{L} \\
\mu \mathrm{g} / \mathrm{L}\end{array}$ & $\begin{array}{l}\text { Blank } \\
\text { Blank }\end{array}$ \\
\hline Benzene & Hearst Sewer & $\begin{array}{l}1 / 18 / 01 \\
1 / 18 / 01 \\
4 / 17 / 01 \\
4 / 17 / 01 \\
7 / 10 / 01 \\
7 / 10 / 01 \\
10 / 31 / 01 \\
10 / 31 / 01\end{array}$ & $\begin{array}{l}\text { ND } \\
\text { ND } \\
\text { ND } \\
\text { ND } \\
\text { ND } \\
\text { ND } \\
\text { ND } \\
\text { ND }\end{array}$ & $\begin{array}{l}0.5 \\
0.5 \\
0.5 \\
0.5 \\
0.5 \\
0.5 \\
0.5 \\
0.5\end{array}$ & $\begin{array}{l}\mu \mathrm{g} / \mathrm{L} \\
\mu \mathrm{g} / \mathrm{L} \\
\mu \mathrm{g} / \mathrm{L} \\
\mu \mathrm{g} / \mathrm{L} \\
\mu \mathrm{g} / \mathrm{L} \\
\mu \mathrm{g} / \mathrm{L} \\
\mu \mathrm{g} / \mathrm{L} \\
\mu \mathrm{g} / \mathrm{L}\end{array}$ & $\begin{array}{r}\text { Sample } \\
\text { Split } \\
\text { Sample } \\
\text { Split } \\
\text { Sample } \\
\text { Split } \\
\text { Sample } \\
\text { Split }\end{array}$ \\
\hline
\end{tabular}




\begin{tabular}{lllrl} 
Lnalyte & MDA & \multicolumn{4}{c}{ Location } & Date & Result & or PQL & Units & QC Type \\
\hline
\end{tabular}

\section{Volatile Organic Compounds}

\begin{tabular}{|c|c|c|c|c|c|}
\hline $\begin{array}{l}\text { Benzene } \\
\text { cont. }\end{array}$ & Strawberry Sewer & $\begin{array}{l}1 / 18 / 01 \\
4 / 17 / 01 \\
7 / 10 / 01 \\
10 / 31 / 01\end{array}$ & $\begin{array}{l}\text { ND } \\
\text { ND } \\
\text { ND } \\
\text { ND }\end{array}$ & $\begin{array}{l}0.5 \\
0.5 \\
0.5 \\
0.5\end{array}$ & $\begin{array}{l}\mu \mathrm{g} / \mathrm{L} \\
\mu \mathrm{g} / \mathrm{L} \\
\mu \mathrm{g} / \mathrm{L} \\
\mu \mathrm{g} / \mathrm{L}\end{array}$ \\
\hline & Field Blank & $\begin{array}{l}4 / 17 / 01 \\
4 / 17 / 01\end{array}$ & $\begin{array}{l}\text { ND } \\
\text { ND }\end{array}$ & $\begin{array}{l}0.5 \\
0.5\end{array}$ & $\begin{array}{l}\mu \mathrm{g} / \mathrm{L} \\
\mu \mathrm{g} / \mathrm{L}\end{array}$ \\
\hline Bromodichloromethane & Hearst Sewer & $\begin{array}{l}1 / 18 / 01 \\
1 / 18 / 01 \\
4 / 17 / 01 \\
4 / 17 / 01 \\
7 / 10 / 01 \\
7 / 10 / 01 \\
10 / 31 / 01 \\
10 / 31 / 01\end{array}$ & $\begin{array}{l}\text { ND } \\
\text { ND } \\
0.87 \\
0.91 \\
N D \\
N D \\
N D \\
N D\end{array}$ & $\begin{array}{l}0.5 \\
0.5 \\
0.5 \\
0.5 \\
0.5 \\
0.5 \\
0.5 \\
0.5\end{array}$ & $\begin{array}{l}\mu g / L \\
\mu g / L \\
\mu g / L \\
\mu g / L \\
\mu g / L \\
\mu g / L \\
\mu g / L \\
\mu g / L\end{array}$ \\
\hline & Strawberry Sewer & $\begin{array}{l}1 / 18 / 01 \\
4 / 17 / 01 \\
7 / 10 / 01 \\
10 / 31 / 01\end{array}$ & $\begin{array}{l}\mathrm{ND} \\
0.53 \\
\mathrm{ND} \\
\mathrm{ND}\end{array}$ & $\begin{array}{l}0.5 \\
0.5 \\
0.5 \\
0.5\end{array}$ & $\begin{array}{l}\mu \mathrm{g} / \mathrm{L} \\
\mu \mathrm{g} / \mathrm{L} \\
\mu \mathrm{g} / \mathrm{L} \\
\mu \mathrm{g} / \mathrm{L}\end{array}$ \\
\hline & Field Blank & $\begin{array}{l}4 / 17 / 01 \\
4 / 17 / 01\end{array}$ & $\begin{array}{l}\text { ND } \\
\text { ND }\end{array}$ & $\begin{array}{l}0.5 \\
0.5\end{array}$ & $\begin{array}{l}\mu \mathrm{g} / \mathrm{L} \\
\mu \mathrm{g} / \mathrm{L}\end{array}$ \\
\hline Bromoform & Hearst Sewer & $\begin{array}{l}1 / 18 / 01 \\
1 / 18 / 01 \\
4 / 17 / 01 \\
4 / 17 / 01 \\
7 / 10 / 01 \\
7 / 10 / 01 \\
10 / 31 / 01 \\
10 / 31 / 01\end{array}$ & $\begin{array}{l}\text { ND } \\
\text { ND } \\
\text { ND } \\
\text { ND } \\
\text { ND } \\
\text { ND } \\
\text { ND } \\
\text { ND }\end{array}$ & $\begin{array}{l}0.5 \\
0.5 \\
0.5 \\
0.5 \\
0.5 \\
0.5 \\
0.5 \\
0.5\end{array}$ & $\begin{array}{l}\mu \mathrm{g} / \mathrm{L} \\
\mu \mathrm{g} / \mathrm{L} \\
\mu \mathrm{g} / \mathrm{L} \\
\mu \mathrm{g} / \mathrm{L} \\
\mu \mathrm{g} / \mathrm{L} \\
\mu \mathrm{g} / \mathrm{L} \\
\mu \mathrm{g} / \mathrm{L} \\
\mu \mathrm{g} / \mathrm{L}\end{array}$ \\
\hline & Strawberry Sewer & $\begin{array}{l}1 / 18 / 01 \\
4 / 17 / 01 \\
7 / 10 / 01 \\
10 / 31 / 01\end{array}$ & $\begin{array}{l}\text { ND } \\
\text { ND } \\
\text { ND } \\
\text { ND }\end{array}$ & $\begin{array}{l}0.5 \\
0.5 \\
0.5 \\
0.5\end{array}$ & $\begin{array}{l}\mu g / L \\
\mu g / L \\
\mu g / L \\
\mu g / L\end{array}$ \\
\hline & Field Blank & $\begin{array}{l}4 / 17 / 01 \\
4 / 17 / 01\end{array}$ & $\begin{array}{l}\text { ND } \\
\text { ND }\end{array}$ & $\begin{array}{l}0.5 \\
0.5\end{array}$ & $\begin{array}{l}\mu \mathrm{g} / \mathrm{L} \\
\mu \mathrm{g} / \mathrm{L}\end{array}$ \\
\hline Bromomethane & Hearst Sewer & $\begin{array}{l}1 / 18 / 01 \\
1 / 18 / 01 \\
4 / 17 / 01 \\
4 / 17 / 01 \\
7 / 10 / 01\end{array}$ & $\begin{array}{l}\text { ND } \\
\text { ND } \\
\text { ND } \\
\text { ND } \\
\text { ND }\end{array}$ & $\begin{array}{l}0.5 \\
0.5 \\
0.5 \\
0.5 \\
1\end{array}$ & $\begin{array}{l}\mu g / L \\
\mu g / L \\
\mu g / L \\
\mu g / L \\
\mu g / L\end{array}$ \\
\hline
\end{tabular}




\section{Analyte \\ Location \\ Volatile Organic Compounds}

MDA

Bromomethane

cont.

Carbon disulfide

Carbon tetrachloride
Hearst Sewer

Strawberry Sewer

Field Blank

Hearst Sewer

Strawberry Sewer $\quad 4 / 17 / 01$

Field Blank

Hearst Sewer

Strawberry Sewer

Field Blank
$7 / 10 / 01$

$10 / 31 / 01$

$10 / 31 / 01$

$1 / 18 / 01$

$4 / 17 / 01$

$7 / 10 / 01$

10/31/01

$4 / 17 / 01$

$4 / 17 / 01$

4/17/01

$4 / 17 / 01$

$7 / 10 / 01$

$7 / 10 / 01$

10/31/01

10/31/01

$7 / 10 / 01$

10/31/01

$4 / 17 / 01$
$4 / 17 / 01$

1/18/01

$1 / 18 / 01$

$4 / 17 / 01$

$4 / 17 / 01$

$7 / 10 / 01$

$7 / 10 / 01$

10/31/01

10/31/01

$1 / 18 / 01$

$7 / 10 / 01$

$7 / 10 / 01$

10/31/01

ND

ND

ND

ND

ND

ND

ND

ND

ND

ND

ND

ND

ND

ND

ND

ND

ND

ND

ND

ND

ND

ND

ND

ND

ND

ND

ND

ND

ND

ND

ND

ND

$4 / 17 / 01$

$4 / 17 / 01$ or PQL

Units QC Type 


\begin{tabular}{lllll} 
Lnalyte & \multicolumn{4}{c}{ MDA } \\
Location & Date & Result & or PQL Units & QC Type
\end{tabular}

\section{Volatile Organic Compounds}

\begin{tabular}{|c|c|c|c|c|c|c|}
\hline \multirow[t]{14}{*}{ Chlorobenzene } & \multirow[t]{8}{*}{ Hearst Sewer } & $1 / 18 / 01$ & ND & 0.5 & $\mu \mathrm{g} / \mathrm{L}$ & Sample \\
\hline & & $1 / 18 / 01$ & ND & 0.5 & $\mu \mathrm{g} / \mathrm{L}$ & Split \\
\hline & & $4 / 17 / 01$ & ND & 0.5 & $\mu \mathrm{g} / \mathrm{L}$ & Sample \\
\hline & & $4 / 17 / 01$ & ND & 0.5 & $\mu \mathrm{g} / \mathrm{L}$ & \\
\hline & & $7 / 10 / 01$ & ND & 0.5 & $\mu \mathrm{g} / \mathrm{L}$ & Samp \\
\hline & & $7 / 10 / 01$ & ND & 0.5 & $\mu \mathrm{g} / \mathrm{L}$ & \\
\hline & & $10 / 31 / 01$ & ND & 0.5 & $\mu \mathrm{g} / \mathrm{L}$ & Samp \\
\hline & & $10 / 31 / 01$ & ND & 0.5 & $\mu \mathrm{g} / \mathrm{L}$ & \\
\hline & \multirow[t]{4}{*}{ Strawberry Sewer } & $1 / 18 / 01$ & ND & 0.5 & $\mu \mathrm{g} / \mathrm{L}$ & Samr \\
\hline & & 4/17/01 & ND & 0.5 & $\mu \mathrm{g} / \mathrm{L}$ & Samp \\
\hline & & $7 / 10 / 01$ & ND & 0.5 & $\mu \mathrm{g} / \mathrm{L}$ & Samp \\
\hline & & $10 / 31 / 01$ & ND & 0.5 & $\mu \mathrm{g} / \mathrm{L}$ & Samp \\
\hline & \multirow[t]{2}{*}{ Field Blank } & $4 / 17 / 01$ & ND & 0.5 & $\mu \mathrm{g} / \mathrm{L}$ & \\
\hline & & $4 / 17 / 01$ & ND & 0.5 & $\mu \mathrm{g} / \mathrm{L}$ & Bla \\
\hline \multirow{14}{*}{ Chloroethane } & \multirow{8}{*}{ Hearst Sewer } & $1 / 18 / 01$ & ND & 1 & $\mu \mathrm{g} / \mathrm{L}$ & Samp \\
\hline & & $1 / 18 / 01$ & ND & 1 & $\mu \mathrm{g} / \mathrm{L}$ & $\mathrm{Sp}$ \\
\hline & & $4 / 17 / 01$ & ND & 1 & $\mu \mathrm{g} / \mathrm{L}$ & Samp \\
\hline & & $4 / 17 / 01$ & ND & 1 & $\mu \mathrm{g} / \mathrm{L}$ & $\mathrm{Sp}$ \\
\hline & & $7 / 10 / 01$ & ND & 1 & $\mu \mathrm{g} / \mathrm{L}$ & Samp \\
\hline & & $7 / 10 / 01$ & ND & 1 & $\mu \mathrm{g} / \mathrm{L}$ & $\mathrm{Sp}$ \\
\hline & & $10 / 31 / 01$ & ND & 0.5 & $\mu \mathrm{g} / \mathrm{L}$ & Samp \\
\hline & & $10 / 31 / 01$ & ND & 0.5 & $\mu \mathrm{g} / \mathrm{L}$ & \\
\hline & \multirow[t]{4}{*}{ Strawberry Sewer } & $1 / 18 / 01$ & ND & 1 & $\mu \mathrm{g} / \mathrm{L}$ & Samp \\
\hline & & $4 / 17 / 01$ & ND & 1 & $\mu \mathrm{g} / \mathrm{L}$ & Samp \\
\hline & & $7 / 10 / 01$ & ND & 1 & $\mu \mathrm{g} / \mathrm{L}$ & Samp \\
\hline & & $10 / 31 / 01$ & ND & 0.5 & $\mu \mathrm{g} / \mathrm{L}$ & Samp \\
\hline & \multirow[t]{2}{*}{ Field Blank } & 4/17/01 & ND & 1 & $\mu \mathrm{g} / \mathrm{L}$ & Blal \\
\hline & & $4 / 17 / 01$ & ND & 1 & $\mu \mathrm{g} / \mathrm{L}$ & Bla \\
\hline \multirow[t]{12}{*}{ Chloroform } & \multirow[t]{8}{*}{ Hearst Sewer } & 1/18/01 & 9.2 & 0.5 & $\mu \mathrm{g} / \mathrm{L}$ & Samp \\
\hline & & $1 / 18 / 01$ & 9.6 & 0.5 & $\mu \mathrm{g} / \mathrm{L}$ & \\
\hline & & 4/17/01 & 5.8 & 0.5 & $\mu \mathrm{g} / \mathrm{L}$ & Samp \\
\hline & & 4/17/01 & 6 & 0.5 & $\mu \mathrm{g} / \mathrm{L}$ & \\
\hline & & $7 / 10 / 01$ & 4.4 & 0.5 & $\mu \mathrm{g} / \mathrm{L}$ & Samp \\
\hline & & $7 / 10 / 01$ & 4.6 & 0.5 & $\mu \mathrm{g} / \mathrm{L}$ & $\mathrm{Sp}$ \\
\hline & & $10 / 31 / 01$ & 28 & 0.5 & $\mu \mathrm{g} / \mathrm{L}$ & Samp \\
\hline & & $10 / 31 / 01$ & 29 & 0.5 & $\mu \mathrm{g} / \mathrm{L}$ & \\
\hline & \multirow[t]{4}{*}{ Strawberry Sewer } & $1 / 18 / 01$ & 3.8 & 0.5 & $\mu \mathrm{g} / \mathrm{L}$ & Samp \\
\hline & & 4/17/01 & 2.7 & 0.5 & $\mu \mathrm{g} / \mathrm{L}$ & Samp \\
\hline & & $7 / 10 / 01$ & 2.5 & 0.5 & $\mu \mathrm{g} / \mathrm{L}$ & Samp \\
\hline & & 10/31/01 & 4.2 & 0.5 & $\mu \mathrm{g} / \mathrm{L}$ & Samp \\
\hline
\end{tabular}




\section{Analyte \\ Location \\ Volatile Organic Compounds}

MDA

\begin{tabular}{|c|c|c|c|c|c|c|}
\hline $\begin{array}{l}\text { Chloroform } \\
\text { cont. }\end{array}$ & Field Blank & $\begin{array}{l}4 / 17 / 01 \\
4 / 17 / 01\end{array}$ & $\begin{array}{l}\text { ND } \\
\text { ND }\end{array}$ & $\begin{array}{l}0.5 \\
0.5\end{array}$ & $\begin{array}{l}\mu \mathrm{g} / \mathrm{L} \\
\mu \mathrm{g} / \mathrm{L}\end{array}$ & $\begin{array}{l}\text { Blank } \\
\text { Blank }\end{array}$ \\
\hline \multirow[t]{14}{*}{ Chloromethane } & \multirow[t]{8}{*}{ Hearst Sewer } & $1 / 18 / 01$ & ND & 1 & $\mu \mathrm{g} / \mathrm{L}$ & Sample \\
\hline & & $1 / 18 / 01$ & ND & 1 & $\mu \mathrm{g} / \mathrm{L}$ & Split \\
\hline & & $4 / 17 / 01$ & ND & 1 & $\mu \mathrm{g} / \mathrm{L}$ & Sample \\
\hline & & $4 / 17 / 01$ & ND & 1 & $\mu \mathrm{g} / \mathrm{L}$ & Split \\
\hline & & $7 / 10 / 01$ & ND & 1 & $\mu \mathrm{g} / \mathrm{L}$ & Sample \\
\hline & & $7 / 10 / 01$ & ND & 1 & $\mu \mathrm{g} / \mathrm{L}$ & Split \\
\hline & & $10 / 31 / 01$ & ND & 0.5 & $\mu \mathrm{g} / \mathrm{L}$ & Sample \\
\hline & & $10 / 31 / 01$ & ND & 0.5 & $\mu \mathrm{g} / \mathrm{L}$ & Split \\
\hline & \multirow[t]{4}{*}{ Strawberry Sewer } & $1 / 18 / 01$ & ND & 1 & $\mu \mathrm{g} / \mathrm{L}$ & Sample \\
\hline & & $4 / 17 / 01$ & ND & 1 & $\mu \mathrm{g} / \mathrm{L}$ & Sample \\
\hline & & $7 / 10 / 01$ & ND & 1 & $\mu \mathrm{g} / \mathrm{L}$ & Sample \\
\hline & & $10 / 31 / 01$ & ND & 0.5 & $\mu g / L$ & Sample \\
\hline & \multirow[t]{2}{*}{ Field Blank } & $4 / 17 / 01$ & ND & 1 & $\mu \mathrm{g} / \mathrm{L}$ & Blank \\
\hline & & 4/17/01 & ND & 1 & $\mu \mathrm{g} / \mathrm{L}$ & Blank \\
\hline \multirow[t]{11}{*}{ cis-1,2-Dichloroethene } & \multirow[t]{6}{*}{ Hearst Sewer } & $4 / 17 / 01$ & ND & 0.5 & $\mu \mathrm{g} / \mathrm{L}$ & Sample \\
\hline & & $4 / 17 / 01$ & ND & 0.5 & $\mu \mathrm{g} / \mathrm{L}$ & Split \\
\hline & & $7 / 10 / 01$ & ND & 0.5 & $\mu \mathrm{g} / \mathrm{L}$ & Sample \\
\hline & & $7 / 10 / 01$ & ND & 0.5 & $\mu \mathrm{g} / \mathrm{L}$ & Split \\
\hline & & $10 / 31 / 01$ & ND & 0.5 & $\mu g / L$ & Sample \\
\hline & & 10/31/01 & ND & 0.5 & $\mu \mathrm{g} / \mathrm{L}$ & Split \\
\hline & \multirow[t]{3}{*}{ Strawberry Sewer } & $4 / 17 / 01$ & ND & 0.5 & $\mu \mathrm{g} / \mathrm{L}$ & Sample \\
\hline & & $7 / 10 / 01$ & ND & 0.5 & $\mu \mathrm{g} / \mathrm{L}$ & Sample \\
\hline & & 10/31/01 & ND & 0.5 & $\mu \mathrm{g} / \mathrm{L}$ & Sample \\
\hline & \multirow[t]{2}{*}{ Field Blank } & $4 / 17 / 01$ & ND & 0.5 & $\mu \mathrm{g} / \mathrm{L}$ & Blank \\
\hline & & $4 / 17 / 01$ & ND & 0.5 & $\mu \mathrm{g} / \mathrm{L}$ & Blank \\
\hline \multirow[t]{10}{*}{ cis-1,3-Dichloropropene } & \multirow[t]{8}{*}{ Hearst Sewer } & $1 / 18 / 01$ & ND & 0.5 & $\mu g / L$ & Sample \\
\hline & & $1 / 18 / 01$ & ND & 0.5 & $\mu \mathrm{g} / \mathrm{L}$ & Split \\
\hline & & $4 / 17 / 01$ & ND & 0.5 & $\mu \mathrm{g} / \mathrm{L}$ & Sample \\
\hline & & $4 / 17 / 01$ & ND & 0.5 & $\mu \mathrm{g} / \mathrm{L}$ & Split \\
\hline & & $7 / 10 / 01$ & ND & 0.5 & $\mu g / L$ & Sample \\
\hline & & $7 / 10 / 01$ & ND & 0.5 & $\mu \mathrm{g} / \mathrm{L}$ & Split \\
\hline & & 10/31/01 & ND & 0.5 & $\mu g / L$ & Sample \\
\hline & & $10 / 31 / 01$ & ND & 0.5 & $\mu \mathrm{g} / \mathrm{L}$ & Split \\
\hline & \multirow[t]{2}{*}{ Strawberry Sewer } & $1 / 18 / 01$ & ND & 0.5 & $\mu \mathrm{g} / \mathrm{L}$ & Sample \\
\hline & & $4 / 17 / 01$ & ND & 0.5 & $\mu \mathrm{g} / \mathrm{L}$ & Sample \\
\hline
\end{tabular}




\section{Analyte \\ Volatile Organic Compounds}

\begin{tabular}{|c|c|c|c|c|c|c|}
\hline \multirow[t]{3}{*}{$\begin{array}{l}\text { cis-1,3-Dichloropropene } \\
\text { cont. }\end{array}$} & Strawberry Sewer & $\begin{array}{l}7 / 10 / 01 \\
10 / 31 / 01\end{array}$ & $\begin{array}{l}\text { ND } \\
\text { ND }\end{array}$ & $\begin{array}{l}0.5 \\
0.5\end{array}$ & $\begin{array}{l}\mu \mathrm{g} / \mathrm{L} \\
\mu \mathrm{g} / \mathrm{L}\end{array}$ & $\begin{array}{l}\text { Sample } \\
\text { Sample }\end{array}$ \\
\hline & \multirow[t]{2}{*}{ Field Blank } & $4 / 17 / 01$ & ND & 0.5 & $\mu \mathrm{g} / \mathrm{L}$ & Blank \\
\hline & & $4 / 17 / 01$ & ND & 0.5 & $\mu \mathrm{g} / \mathrm{L}$ & Blank \\
\hline \multirow[t]{14}{*}{ Dibromochloromethane } & \multirow[t]{8}{*}{ Hearst Sewer } & $1 / 18 / 01$ & ND & 0.5 & $\mu \mathrm{g} / \mathrm{L}$ & Sample \\
\hline & & $1 / 18 / 01$ & ND & 0.5 & $\mu \mathrm{g} / \mathrm{L}$ & $S_{1}$ \\
\hline & & $4 / 17 / 01$ & ND & 0.5 & $\mu \mathrm{g} / \mathrm{L}$ & Samp \\
\hline & & $4 / 17 / 01$ & ND & 0.5 & $\mu \mathrm{g} / \mathrm{L}$ & $\mathrm{Sp}$ \\
\hline & & $7 / 10 / 01$ & ND & 0.5 & $\mu \mathrm{g} / \mathrm{L}$ & Samp \\
\hline & & $7 / 10 / 01$ & ND & 0.5 & $\mu \mathrm{g} / \mathrm{L}$ & $\mathrm{Sp}$ \\
\hline & & $10 / 31 / 01$ & ND & 0.5 & $\mu \mathrm{g} / \mathrm{L}$ & Samp \\
\hline & & $10 / 31 / 01$ & ND & 0.5 & $\mu \mathrm{g} / \mathrm{L}$ & \\
\hline & \multirow[t]{4}{*}{ Strawberry Sewer } & $1 / 18 / 01$ & ND & 0.5 & $\mu \mathrm{g} / \mathrm{L}$ & Samp \\
\hline & & $4 / 17 / 01$ & ND & 0.5 & $\mu \mathrm{g} / \mathrm{L}$ & Samp \\
\hline & & $7 / 10 / 01$ & ND & 0.5 & $\mu \mathrm{g} / \mathrm{L}$ & Samp \\
\hline & & $10 / 31 / 01$ & ND & 0.5 & $\mu \mathrm{g} / \mathrm{L}$ & Samp \\
\hline & \multirow[t]{2}{*}{ Field Blank } & $4 / 17 / 01$ & ND & 0.5 & $\mu \mathrm{g} / \mathrm{L}$ & $\mathrm{Bla}$ \\
\hline & & $4 / 17 / 01$ & ND & 0.5 & $\mu \mathrm{g} / \mathrm{L}$ & Bla \\
\hline \multirow[t]{11}{*}{ Dibromomethane } & \multirow[t]{6}{*}{ Hearst Sewer } & $4 / 17 / 01$ & ND & 0.5 & $\mu \mathrm{g} / \mathrm{L}$ & Samr \\
\hline & & $4 / 17 / 01$ & ND & 0.5 & $\mu \mathrm{g} / \mathrm{L}$ & $\mathrm{S}$ \\
\hline & & $7 / 10 / 01$ & ND & 0.5 & $\mu \mathrm{g} / \mathrm{L}$ & Samp \\
\hline & & $7 / 10 / 01$ & ND & 0.5 & $\mu \mathrm{g} / \mathrm{L}$ & $\mathrm{Sp}$ \\
\hline & & $10 / 31 / 01$ & ND & 0.5 & $\mu \mathrm{g} / \mathrm{L}$ & Samp \\
\hline & & $10 / 31 / 01$ & ND & 0.5 & $\mu \mathrm{g} / \mathrm{L}$ & \\
\hline & \multirow[t]{3}{*}{ Strawberry Sewer } & $4 / 17 / 01$ & ND & 0.5 & $\mu \mathrm{g} / \mathrm{L}$ & Samp \\
\hline & & $7 / 10 / 01$ & ND & 0.5 & $\mu \mathrm{g} / \mathrm{L}$ & Samp \\
\hline & & 10/31/01 & ND & 0.5 & $\mu \mathrm{g} / \mathrm{L}$ & Samp \\
\hline & \multirow[t]{2}{*}{ Field Blank } & $4 / 17 / 01$ & ND & 0.5 & $\mu \mathrm{g} / \mathrm{L}$ & Bla \\
\hline & & $4 / 17 / 01$ & ND & 0.5 & $\mu \mathrm{g} / \mathrm{L}$ & Bla \\
\hline \multirow[t]{6}{*}{ Dichlorodifluoromethane } & \multirow[t]{6}{*}{ Hearst Sewer } & $4 / 17 / 01$ & ND & 0.5 & $\mu \mathrm{g} / \mathrm{L}$ & Samp \\
\hline & & $4 / 17 / 01$ & ND & 0.5 & $\mu \mathrm{g} / \mathrm{L}$ & \\
\hline & & $7 / 10 / 01$ & ND & 0.5 & $\mu \mathrm{g} / \mathrm{L}$ & Samp \\
\hline & & $7 / 10 / 01$ & ND & 0.5 & $\mu \mathrm{g} / \mathrm{L}$ & \\
\hline & & $10 / 31 / 01$ & ND & 0.5 & $\mu \mathrm{g} / \mathrm{L}$ & Samp \\
\hline & & $10 / 31 / 01$ & ND & 0.5 & $\mu \mathrm{g} / \mathrm{L}$ & \\
\hline
\end{tabular}




\section{Analyte \\ Volatile Organic Compounds}

Dichlorodifluoromethane cont.

Ethanol

Ethylbenzene

Freon 113
Strawberry Sewer 4/17/01

$7 / 10 / 01$

$10 / 31 / 01$

Field Blank

$4 / 17 / 01$

$4 / 17 / 01$

Hearst Sewer

$4 / 17 / 01 \quad 12000$

$4 / 17 / 01$

$7 / 10 / 01$

$7 / 10 / 01$

$10 / 31 / 01$

$10 / 31 / 01$

Strawberry Sewer

4/17/01

$10 / 31 / 01$

Field Blank

$4 / 17 / 01$

$4 / 17 / 01$

Hearst Sewer

$1 / 18 / 01$

$4 / 17 / 01$

$4 / 17 / 01$

$7 / 10 / 01$

$7 / 10 / 01$

10/31/01

10/31/01

Strawberry Sewer

$1 / 18 / 01$

$4 / 17 / 01$

$7 / 10 / 01$

10/31/01

Field Blank

$4 / 17 / 01$

$4 / 17 / 01$

Hearst Sewer
ND

ND

ND

ND

ND

13000

ND

ND

1600

1600

ND

ND

1500

ND

ND

ND

ND

ND

ND

ND

ND

ND

ND

ND

ND

ND

ND

ND

ND

ND

ND

ND

ND

ND

ND

$\begin{array}{ll}1 / 18 / 01 & \text { ND } \\ 1 / 18 / 01 & \text { ND } \\ 4 / 17 / 01 & \text { ND } \\ 4 / 17 / 01 & \text { ND } \\ 7 / 10 / 01 & \text { ND } \\ 7 / 10 / 01 & \text { ND }\end{array}$

MDA

or PQL Units QC Type

$\begin{array}{lll}0.5 & \mu g / L & \text { Sample } \\ 0.5 & \mu g / L & \text { Sample } \\ 0.5 & \mu g / L & \text { Sample }\end{array}$

$0.5 \mu \quad \mu g / L \quad$ Blank

$0.5 \mu \mathrm{g} / \mathrm{L} \quad$ Blank

$\begin{array}{llr}1000 & \mu g / L & \text { Sample } \\ 1000 & \mu g / L & \text { Split } \\ 1000 & \mu g / L & \text { Sample } \\ 1000 & \mu g / L & \text { Split } \\ 1000 & \mu g / L & \text { Sample } \\ 1000 & \mu g / L & \text { Split }\end{array}$

$\begin{array}{lll}1000 & \mu \mathrm{g} / \mathrm{L} & \text { Sample } \\ 1000 & \mu \mathrm{g} / \mathrm{L} & \text { Sample } \\ 1000 & \mu \mathrm{g} / \mathrm{L} & \text { Sample }\end{array}$

$1000 \quad \mu \mathrm{g} / \mathrm{L} \quad$ Blank

$1000 \mu \mathrm{g} / \mathrm{L} \quad$ Blank

$\begin{array}{rlr}0.5 & \mu \mathrm{g} / \mathrm{L} & \text { Sample } \\ 0.5 & \mu \mathrm{g} / \mathrm{L} & \text { Split } \\ 0.5 & \mu \mathrm{g} / \mathrm{L} & \text { Sample } \\ 0.5 & \mu \mathrm{g} / \mathrm{L} & \text { Split } \\ 0.5 & \mu \mathrm{g} / \mathrm{L} & \text { Sample } \\ 0.5 & \mu \mathrm{g} / \mathrm{L} & \text { Split } \\ 0.5 & \mu \mathrm{g} / \mathrm{L} & \text { Sample } \\ 0.5 & \mu \mathrm{g} / \mathrm{L} & \text { Split }\end{array}$

$\begin{array}{lll}0.5 & \mu g / L & \text { Sample } \\ 0.5 & \mu g / L & \text { Sample } \\ 0.5 & \mu g / L & \text { Sample } \\ 0.5 & \mu g / L & \text { Sample }\end{array}$

$\begin{array}{lll}0.5 & \mu \mathrm{g} / \mathrm{L} & \text { Blank } \\ 0.5 & \mu \mathrm{g} / \mathrm{L} & \text { Blank }\end{array}$




\begin{tabular}{|c|c|c|c|c|c|c|}
\hline Analyte & Location & Date & Result & $\begin{array}{r}\text { MDA } \\
\text { or PQL }\end{array}$ & Units & QC Type \\
\hline
\end{tabular}

\section{Volatile Organic Compounds}

\begin{tabular}{|c|c|c|c|c|c|c|}
\hline \multirow[t]{7}{*}{$\begin{array}{l}\text { Freon } 113 \\
\text { cont. }\end{array}$} & Hearst Sewer & $\begin{array}{l}10 / 31 / 01 \\
10 / 31 / 01\end{array}$ & $\begin{array}{l}\text { ND } \\
N D\end{array}$ & $\begin{array}{l}0.5 \\
0.5\end{array}$ & $\begin{array}{l}\mu \mathrm{g} / \mathrm{L} \\
\mu \mathrm{g} / \mathrm{L}\end{array}$ & $\begin{array}{r}\text { Sample } \\
\text { Split }\end{array}$ \\
\hline & Strawberry Sewer & $1 / 18 / 01$ & ND & 0.5 & $\mu \mathrm{g} / \mathrm{L}$ & Sam \\
\hline & & $4 / 17 / 01$ & ND & 0.5 & $\mu \mathrm{g} / \mathrm{L}$ & Sample \\
\hline & & $7 / 10 / 01$ & ND & 0.5 & $\mu \mathrm{g} / \mathrm{L}$ & Samp \\
\hline & & 10/31/01 & ND & 0.5 & $\mu \mathrm{g} / \mathrm{L}$ & Samp \\
\hline & Field Blank & $4 / 17 / 01$ & ND & 0.5 & $\mu \mathrm{g} / \mathrm{L}$ & Bla \\
\hline & & $4 / 17 / 01$ & ND & 0.5 & $\mu \mathrm{g} / \mathrm{L}$ & Bla \\
\hline \multirow[t]{3}{*}{ Methyl tert-Butyl Ether } & Hearst Sewer & $1 / 18 / 01$ & ND & 0.5 & $\mu \mathrm{g} / \mathrm{L}$ & Samp \\
\hline & & $1 / 18 / 01$ & ND & 0.5 & $\mu \mathrm{g} / \mathrm{L}$ & $\mathrm{Sp}$ \\
\hline & Strawberry Sewer & $1 / 18 / 01$ & ND & 0.5 & $\mu \mathrm{g} / \mathrm{L}$ & Samp \\
\hline \multirow[t]{14}{*}{ Methylene chloride } & Hearst Sewer & $1 / 18 / 01$ & ND & 1 & $\mu \mathrm{g} / \mathrm{L}$ & Sam \\
\hline & & $1 / 18 / 01$ & ND & 1 & $\mu \mathrm{g} / \mathrm{L}$ & $\mathrm{S}$ \\
\hline & & $4 / 17 / 01$ & ND & 1 & $\mu \mathrm{g} / \mathrm{L}$ & Samp \\
\hline & & $4 / 17 / 01$ & ND & 1 & $\mu \mathrm{g} / \mathrm{L}$ & $S p$ \\
\hline & & $7 / 10 / 01$ & ND & 1 & $\mu \mathrm{g} / \mathrm{L}$ & Samp \\
\hline & & $7 / 10 / 01$ & ND & 1 & $\mu \mathrm{g} / \mathrm{L}$ & $\mathrm{Sr}$ \\
\hline & & $10 / 31 / 01$ & 5.2 & 1 & $\mu \mathrm{g} / \mathrm{L}$ & Samp \\
\hline & & $10 / 31 / 01$ & 5.1 & 1 & $\mu \mathrm{g} / \mathrm{L}$ & \\
\hline & Strawberry Sewer & $1 / 18 / 01$ & ND & 1 & $\mu \mathrm{g} / \mathrm{L}$ & Samp \\
\hline & & $4 / 17 / 01$ & ND & 1 & $\mu \mathrm{g} / \mathrm{L}$ & Samp \\
\hline & & $7 / 10 / 01$ & 67 & 1 & $\mu \mathrm{g} / \mathrm{L}$ & Samp \\
\hline & & $10 / 31 / 01$ & ND & 1 & $\mu \mathrm{g} / \mathrm{L}$ & Samp \\
\hline & Field Blank & $4 / 17 / 01$ & ND & 1 & $\mu \mathrm{g} / \mathrm{L}$ & Bla \\
\hline & & $4 / 17 / 01$ & ND & 1 & $\mu \mathrm{g} / \mathrm{L}$ & Bla \\
\hline \multirow[t]{9}{*}{ Naphthalene } & Hearst Sewer & $4 / 17 / 01$ & ND & 0.5 & $\mu \mathrm{g} / \mathrm{L}$ & Samp \\
\hline & & $4 / 17 / 01$ & ND & 0.5 & $\mu \mathrm{g} / \mathrm{L}$ & \\
\hline & & $7 / 10 / 01$ & ND & 0.5 & $\mu \mathrm{g} / \mathrm{L}$ & Samp \\
\hline & & $7 / 10 / 01$ & ND & 0.5 & $\mu \mathrm{g} / \mathrm{L}$ & $\mathrm{Sp}$ \\
\hline & & $10 / 31 / 01$ & ND & 0.5 & $\mu \mathrm{g} / \mathrm{L}$ & Samp \\
\hline & & $10 / 31 / 01$ & ND & 0.5 & $\mu \mathrm{g} / \mathrm{L}$ & \\
\hline & Strawberry Sewer & $4 / 17 / 01$ & ND & 0.5 & $\mu \mathrm{g} / \mathrm{L}$ & Samp \\
\hline & & $7 / 10 / 01$ & ND & 0.5 & $\mu \mathrm{g} / \mathrm{L}$ & Samp \\
\hline & & $10 / 31 / 01$ & ND & 0.5 & $\mu \mathrm{g} / \mathrm{L}$ & Samp \\
\hline
\end{tabular}




\section{Analyte \\ Location \\ Volatile Organic Compounds}

MDA

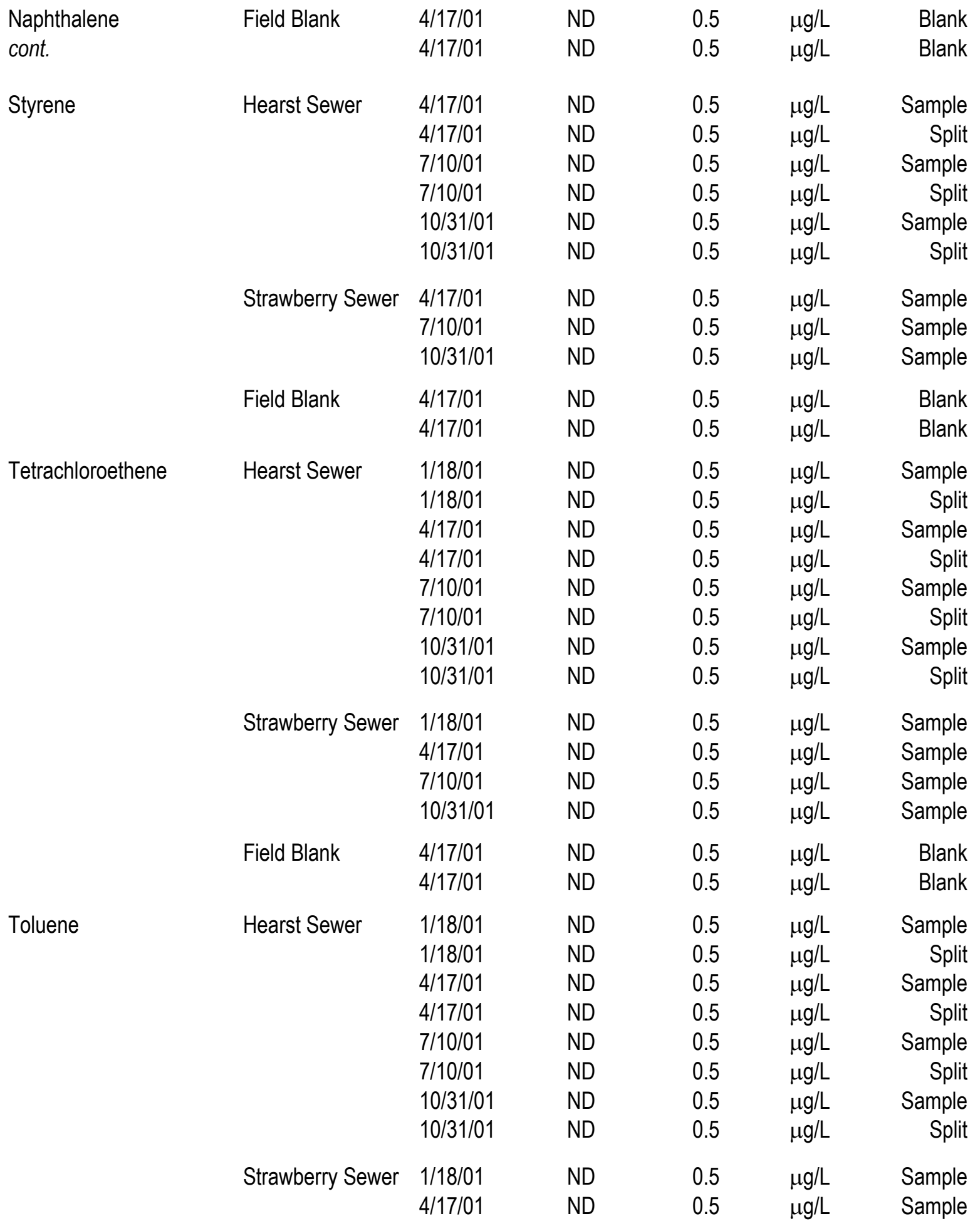




\begin{tabular}{|c|c|c|c|c|c|c|}
\hline Analyte & Location & Date & Result & $\begin{array}{r}\text { MDA } \\
\text { or PQL }\end{array}$ & Units & QC Type \\
\hline
\end{tabular}

\section{Volatile Organic Compounds}

\begin{tabular}{|c|c|c|c|c|c|c|}
\hline \multirow[t]{2}{*}{$\begin{array}{l}\text { Toluene } \\
\text { cont. }\end{array}$} & Strawberry Sewer & $\begin{array}{l}7 / 10 / 01 \\
10 / 31 / 01\end{array}$ & $\begin{array}{l}\mathrm{ND} \\
2.5\end{array}$ & $\begin{array}{l}0.5 \\
0.5\end{array}$ & $\begin{array}{l}\mu \mathrm{g} / \mathrm{L} \\
\mu \mathrm{g} / \mathrm{L}\end{array}$ & $\begin{array}{l}\text { Sample } \\
\text { Sample }\end{array}$ \\
\hline & Field Blank & $4 / 17 / 01$ & ND & 0.5 & $\mu \mathrm{g} / \mathrm{L}$ & Blank \\
\hline \multirow{14}{*}{ Total xylene isomers } & Hearct Sower & $1 / 18 / 01$ & $\mathrm{ND}$ & 1 & ua/l & Samnle \\
\hline & & $1 / 18 / 01$ & ND & 1 & $\mu \mathrm{g} / \mathrm{L}$ & Split \\
\hline & & $4 / 17 / 01$ & ND & 1 & $\mu \mathrm{g} / \mathrm{L}$ & Sample \\
\hline & & $4 / 17 / 01$ & ND & 1 & $\mu \mathrm{g} / \mathrm{L}$ & Split \\
\hline & & $7 / 10 / 01$ & ND & 1 & $\mu \mathrm{g} / \mathrm{L}$ & Sample \\
\hline & & $7 / 10 / 01$ & ND & 1 & $\mu \mathrm{g} / \mathrm{L}$ & Split \\
\hline & & $10 / 31 / 01$ & ND & 1 & $\mu \mathrm{g} / \mathrm{L}$ & Sample \\
\hline & & $10 / 31 / 01$ & ND & 1 & $\mu \mathrm{g} / \mathrm{L}$ & Split \\
\hline & Strawberry Sewer & $1 / 18 / 01$ & ND & 1 & $\mu \mathrm{g} / \mathrm{L}$ & Sample \\
\hline & & $4 / 17 / 01$ & ND & 1 & $\mu \mathrm{g} / \mathrm{L}$ & Sample \\
\hline & & $7 / 10 / 01$ & ND & 1 & $\mu \mathrm{g} / \mathrm{L}$ & Sample \\
\hline & & $10 / 31 / 01$ & ND & 1 & $\mu \mathrm{g} / \mathrm{L}$ & Sample \\
\hline & Field Blank & $4 / 17 / 01$ & ND & 1 & $\mu \mathrm{g} / \mathrm{L}$ & Blank \\
\hline & & $4 / 17 / 01$ & ND & 1 & $\mu \mathrm{g} / \mathrm{L}$ & Blank \\
\hline \multirow[t]{14}{*}{ trans-1,2-Dichloroethene } & Hearst Sewer & $1 / 18 / 01$ & ND & 0.5 & $\mu \mathrm{g} / \mathrm{L}$ & Sample \\
\hline & & $1 / 18 / 01$ & ND & 0.5 & $\mu \mathrm{g} / \mathrm{L}$ & Split \\
\hline & & $4 / 17 / 01$ & ND & 0.5 & $\mu \mathrm{g} / \mathrm{L}$ & Sample \\
\hline & & $4 / 17 / 01$ & ND & 0.5 & $\mu \mathrm{g} / \mathrm{L}$ & Split \\
\hline & & $7 / 10 / 01$ & ND & 0.5 & $\mu \mathrm{g} / \mathrm{L}$ & Sample \\
\hline & & $7 / 10 / 01$ & ND & 0.5 & $\mu \mathrm{g} / \mathrm{L}$ & Split \\
\hline & & $10 / 31 / 01$ & ND & 0.5 & $\mu \mathrm{g} / \mathrm{L}$ & Sample \\
\hline & & $10 / 31 / 01$ & ND & 0.5 & $\mu \mathrm{g} / \mathrm{L}$ & Split \\
\hline & Strawberry Sewer & $1 / 18 / 01$ & ND & 0.5 & $\mu \mathrm{g} / \mathrm{L}$ & Sample \\
\hline & & $4 / 17 / 01$ & ND & 0.5 & $\mu \mathrm{g} / \mathrm{L}$ & Sample \\
\hline & & $7 / 10 / 01$ & ND & 0.5 & $\mu \mathrm{g} / \mathrm{L}$ & Sample \\
\hline & & $10 / 31 / 01$ & ND & 0.5 & $\mu \mathrm{g} / \mathrm{L}$ & Sample \\
\hline & Field Blank & $4 / 17 / 01$ & ND & 0.5 & $\mu \mathrm{g} / \mathrm{L}$ & Blank \\
\hline & & $4 / 17 / 01$ & ND & 0.5 & $\mu \mathrm{g} / \mathrm{L}$ & Bla \\
\hline \multicolumn{7}{|l|}{ trans-1,3-Dichloropropene } \\
\hline & Hearst Sewer & $1 / 18 / 01$ & ND & 0.5 & $\mu \mathrm{g} / \mathrm{L}$ & Sample \\
\hline & & $1 / 18 / 01$ & ND & 0.5 & $\mu \mathrm{g} / \mathrm{L}$ & Split \\
\hline & & $4 / 17 / 01$ & ND & 0.5 & $\mu \mathrm{g} / \mathrm{L}$ & Sample \\
\hline & & $4 / 17 / 01$ & ND & 0.5 & $\mu \mathrm{g} / \mathrm{L}$ & \\
\hline & & $7 / 10 / 01$ & ND & 0.5 & $\mu \mathrm{g} / \mathrm{L}$ & Sample \\
\hline
\end{tabular}




\begin{tabular}{|c|c|c|c|c|c|c|}
\hline Analyte & Location & Date & Result & $\begin{array}{r}\text { MDA } \\
\text { or } P Q L\end{array}$ & Units & QC Type \\
\hline
\end{tabular}

\section{Volatile Organic Compounds}

trans-1,3-Dichloropropene

\begin{tabular}{|c|c|c|c|c|c|c|}
\hline cont. & Hearst Sewer & $\begin{array}{l}7 / 10 / 01 \\
10 / 31 / 01 \\
10 / 31 / 01\end{array}$ & $\begin{array}{l}\text { ND } \\
\text { ND } \\
\text { ND }\end{array}$ & $\begin{array}{l}0.5 \\
0.5 \\
0.5\end{array}$ & $\begin{array}{l}\mu \mathrm{g} / \mathrm{L} \\
\mu \mathrm{g} / \mathrm{L} \\
\mu \mathrm{g} / \mathrm{L}\end{array}$ & $\begin{array}{r}\text { Split } \\
\text { Sample } \\
\text { Split }\end{array}$ \\
\hline & Strawberry Sewer & $\begin{array}{l}1 / 18 / 01 \\
4 / 17 / 01 \\
7 / 10 / 01 \\
10 / 31 / 01\end{array}$ & $\begin{array}{l}\text { ND } \\
\text { ND } \\
\text { ND } \\
\text { ND }\end{array}$ & $\begin{array}{l}0.5 \\
0.5 \\
0.5 \\
0.5\end{array}$ & $\begin{array}{l}\mu \mathrm{g} / \mathrm{L} \\
\mu \mathrm{g} / \mathrm{L} \\
\mu \mathrm{g} / \mathrm{L} \\
\mu \mathrm{g} / \mathrm{L}\end{array}$ & $\begin{array}{l}\text { Sample } \\
\text { Sample } \\
\text { Sample } \\
\text { Sample }\end{array}$ \\
\hline & Field Blank & $\begin{array}{l}4 / 17 / 01 \\
4 / 17 / 01\end{array}$ & $\begin{array}{l}\text { ND } \\
\text { ND }\end{array}$ & $\begin{array}{l}0.5 \\
0.5\end{array}$ & $\begin{array}{l}\mu \mathrm{g} / \mathrm{L} \\
\mu \mathrm{g} / \mathrm{L}\end{array}$ & $\begin{array}{l}\text { Blank } \\
\text { Blank }\end{array}$ \\
\hline Trichloroethene & Hearst Sewer & $\begin{array}{l}1 / 18 / 01 \\
1 / 18 / 01 \\
4 / 17 / 01 \\
4 / 17 / 01 \\
7 / 10 / 01 \\
7 / 10 / 01 \\
10 / 31 / 01 \\
10 / 31 / 01\end{array}$ & $\begin{array}{l}\text { ND } \\
\text { ND } \\
\text { ND } \\
\text { ND } \\
\text { ND } \\
\text { ND } \\
\text { ND } \\
\text { ND }\end{array}$ & $\begin{array}{l}0.5 \\
0.5 \\
0.5 \\
0.5 \\
0.5 \\
0.5 \\
0.5 \\
0.5\end{array}$ & $\begin{array}{l}\mu \mathrm{g} / \mathrm{L} \\
\mu \mathrm{g} / \mathrm{L} \\
\mu \mathrm{g} / \mathrm{L} \\
\mu \mathrm{g} / \mathrm{L} \\
\mu \mathrm{g} / \mathrm{L} \\
\mu \mathrm{g} / \mathrm{L} \\
\mu \mathrm{g} / \mathrm{L} \\
\mu \mathrm{g} / \mathrm{L}\end{array}$ & $\begin{array}{r}\text { Sample } \\
\text { Split } \\
\text { Sample } \\
\text { Split } \\
\text { Sample } \\
\text { Split } \\
\text { Sample } \\
\text { Split }\end{array}$ \\
\hline & Strawberry Sewer & $\begin{array}{l}1 / 18 / 01 \\
4 / 17 / 01 \\
7 / 10 / 01 \\
10 / 31 / 01\end{array}$ & $\begin{array}{l}\text { ND } \\
\text { ND } \\
\text { ND } \\
\text { ND }\end{array}$ & $\begin{array}{l}0.5 \\
0.5 \\
0.5 \\
0.5\end{array}$ & $\begin{array}{l}\mu \mathrm{g} / \mathrm{L} \\
\mu \mathrm{g} / \mathrm{L} \\
\mu \mathrm{g} / \mathrm{L} \\
\mu \mathrm{g} / \mathrm{L}\end{array}$ & $\begin{array}{l}\text { Sample } \\
\text { Sample } \\
\text { Sample } \\
\text { Sample }\end{array}$ \\
\hline & Field Blank & $\begin{array}{l}4 / 17 / 01 \\
4 / 17 / 01\end{array}$ & $\begin{array}{l}\text { ND } \\
\text { ND }\end{array}$ & $\begin{array}{l}0.5 \\
0.5\end{array}$ & $\begin{array}{l}\mu \mathrm{g} / \mathrm{L} \\
\mu \mathrm{g} / \mathrm{L}\end{array}$ & $\begin{array}{l}\text { Blank } \\
\text { Blank }\end{array}$ \\
\hline Trichlorofluoromethane & Hearst Sewer & $\begin{array}{l}1 / 18 / 01 \\
1 / 18 / 01 \\
4 / 17 / 01 \\
4 / 17 / 01 \\
7 / 10 / 01 \\
7 / 10 / 01 \\
10 / 31 / 01 \\
10 / 31 / 01\end{array}$ & $\begin{array}{l}\text { ND } \\
\text { ND } \\
\text { ND } \\
\text { ND } \\
\text { ND } \\
\text { ND } \\
\text { ND } \\
\text { ND }\end{array}$ & $\begin{array}{l}0.5 \\
0.5 \\
0.5 \\
0.5 \\
0.5 \\
0.5 \\
0.5 \\
0.5\end{array}$ & $\begin{array}{l}\mu \mathrm{g} / \mathrm{L} \\
\mu \mathrm{g} / \mathrm{L} \\
\mu \mathrm{g} / \mathrm{L} \\
\mu \mathrm{g} / \mathrm{L} \\
\mu \mathrm{g} / \mathrm{L} \\
\mu \mathrm{g} / \mathrm{L} \\
\mu \mathrm{g} / \mathrm{L} \\
\mu \mathrm{g} / \mathrm{L}\end{array}$ & $\begin{array}{r}\text { Sample } \\
\text { Split } \\
\text { Sample } \\
\text { Split } \\
\text { Sample } \\
\text { Split } \\
\text { Sample } \\
\text { Split }\end{array}$ \\
\hline & Strawberry Sewer & $\begin{array}{l}1 / 18 / 01 \\
4 / 17 / 01 \\
7 / 10 / 01 \\
10 / 31 / 01\end{array}$ & $\begin{array}{l}\text { ND } \\
\text { ND } \\
\text { ND } \\
\text { ND }\end{array}$ & $\begin{array}{l}0.5 \\
0.5 \\
0.5 \\
0.5\end{array}$ & $\begin{array}{l}\mu \mathrm{g} / \mathrm{L} \\
\mu \mathrm{g} / \mathrm{L} \\
\mu \mathrm{g} / \mathrm{L} \\
\mu \mathrm{g} / \mathrm{L}\end{array}$ & $\begin{array}{l}\text { Sample } \\
\text { Sample } \\
\text { Sample } \\
\text { Sample }\end{array}$ \\
\hline & Field Blank & $\begin{array}{l}4 / 17 / 01 \\
4 / 17 / 01\end{array}$ & $\begin{array}{l}\text { ND } \\
\text { ND }\end{array}$ & $\begin{array}{l}0.5 \\
0.5\end{array}$ & $\begin{array}{l}\mu \mathrm{g} / \mathrm{L} \\
\mu \mathrm{g} / \mathrm{L}\end{array}$ & $\begin{array}{l}\text { Blank } \\
\text { Blank }\end{array}$ \\
\hline
\end{tabular}




\begin{tabular}{lllll} 
Lnalyte & \multicolumn{4}{c}{ MDA } \\
Location & Date & Result & or PQL Units & QC Type
\end{tabular}

\section{Volatile Organic Compounds}

\begin{tabular}{|c|c|c|c|c|c|c|}
\hline \multirow{8}{*}{\multicolumn{2}{|c|}{ Vinyl chloride }} & $1 / 18 / 01$ & ND & 0.5 & $\mu \mathrm{g} / \mathrm{L}$ & Sample \\
\hline & & $1 / 18 / 01$ & ND & 0.5 & $\mu \mathrm{g} / \mathrm{L}$ & Split \\
\hline & & $4 / 17 / 01$ & ND & 0.5 & $\mu \mathrm{g} / \mathrm{L}$ & Sample \\
\hline & & $4 / 17 / 01$ & ND & 0.5 & $\mu \mathrm{g} / \mathrm{L}$ & $\mathrm{Sp}$ \\
\hline & & $7 / 10 / 01$ & ND & 0.5 & $\mu \mathrm{g} / \mathrm{L}$ & Sampl \\
\hline & & $7 / 10 / 01$ & ND & 0.5 & $\mu \mathrm{g} / \mathrm{L}$ & $\mathrm{Sp}$ \\
\hline & & $10 / 31 / 01$ & ND & 0.5 & $\mu \mathrm{g} / \mathrm{L}$ & Sample \\
\hline & & $10 / 31 / 01$ & ND & 0.5 & $\mu \mathrm{g} / \mathrm{L}$ & Sp \\
\hline & \multirow[t]{4}{*}{ Strawberry Sewer } & $1 / 18 / 01$ & ND & 0.5 & $\mu \mathrm{g} / \mathrm{L}$ & Samp \\
\hline & & 4/17/01 & ND & 0.5 & $\mu \mathrm{g} / \mathrm{L}$ & Samp \\
\hline & & $7 / 10 / 01$ & ND & 0.5 & $\mu \mathrm{g} / \mathrm{L}$ & Sampl \\
\hline & & $10 / 31 / 01$ & ND & 0.5 & $\mu \mathrm{g} / \mathrm{L}$ & Samp \\
\hline & \multirow[t]{2}{*}{ Field Blank } & $4 / 17 / 01$ & ND & 0.5 & $\mu \mathrm{g} / \mathrm{L}$ & Bla \\
\hline & & $4 / 17 / 01$ & ND & 0.5 & $\mu q / L$ & Blal \\
\hline
\end{tabular}




\section{Fixed Treatment Units}

The following fixed treatment unit data are summarized and discussed in Chapter 5 (Surface Waters and Wastewater) of the Site Environmental Report for 2001 (see Volume I):

\begin{tabular}{|c|c|c|c|c|c|c|}
\hline Analyte & Location & Date & Result & $\begin{array}{l}\text { MDA } \\
\text { or PQL }\end{array}$ & Units & QC Type \\
\hline \multicolumn{7}{|c|}{ General Indicator Parameters } \\
\hline \multirow[t]{2}{*}{ Cyanide } & 25 FTU & $\begin{array}{l}1 / 18 / 01 \\
4 / 18 / 01 \\
12 / 3 / 01\end{array}$ & $\begin{array}{l}\text { ND } \\
\text { ND } \\
\text { ND }\end{array}$ & $\begin{array}{l}0.02 \\
0.02 \\
0.02\end{array}$ & $\begin{array}{l}\mathrm{mg} / \mathrm{L} \\
\mathrm{mg} / \mathrm{L} \\
\mathrm{mg} / \mathrm{L}\end{array}$ & $\begin{array}{l}\text { Sample } \\
\text { Sample } \\
\text { Sample }\end{array}$ \\
\hline & 77 FTU & $\begin{array}{l}1 / 12 / 01 \\
10 / 23 / 01\end{array}$ & $\begin{array}{l}\text { ND } \\
\text { ND }\end{array}$ & $\begin{array}{l}0.02 \\
0.02\end{array}$ & $\begin{array}{l}\mathrm{mg} / \mathrm{L} \\
\mathrm{mg} / \mathrm{L}\end{array}$ & $\begin{array}{l}\text { Sample } \\
\text { Sample }\end{array}$ \\
\hline \multirow[t]{2}{*}{$\mathrm{pH}$ (field tested) } & 25 FTU & $\begin{array}{l}1 / 18 / 01 \\
4 / 18 / 01 \\
4 / 27 / 01 \\
12 / 3 / 01\end{array}$ & $\begin{array}{l}8.71 \\
7.97 \\
7.97 \\
7.61\end{array}$ & $\begin{array}{l}- \\
- \\
-\end{array}$ & $\begin{array}{l}\text { S.U. } \\
\text { S.U. } \\
\text { S.U. } \\
\text { S.U. }\end{array}$ & $\begin{array}{l}\text { Sample } \\
\text { Sample } \\
\text { Sample } \\
\text { Sample }\end{array}$ \\
\hline & 77 FTU & $\begin{array}{l}1 / 12 / 01 \\
4 / 10 / 01 \\
10 / 23 / 01\end{array}$ & $\begin{array}{l}7.94 \\
7.23 \\
7.11\end{array}$ & $\begin{array}{l}- \\
- \\
-\end{array}$ & $\begin{array}{l}\text { S.U. } \\
\text { S.U. } \\
\text { S.U. }\end{array}$ & $\begin{array}{l}\text { Sample } \\
\text { Sample } \\
\text { Sample }\end{array}$ \\
\hline $\begin{array}{l}\mathrm{pH} \text { (laboratory tested) } \\
\text { Metals and/or } \boldsymbol{M}\end{array}$ & $\begin{array}{l}25 \text { FTU } \\
\text { erals }\end{array}$ & $1 / 18 / 01$ & 7.33 & - & S.U. & Sample \\
\hline Cadmium & 25 FTU & $\begin{array}{l}1 / 18 / 01 \\
1 / 18 / 01 \\
4 / 18 / 01 \\
12 / 3 / 01 \\
12 / 3 / 01\end{array}$ & $\begin{array}{l}\text { ND } \\
\text { ND } \\
\text { ND } \\
\text { ND } \\
\text { ND }\end{array}$ & $\begin{array}{l}0.01 \\
0.01 \\
0.01 \\
0.01 \\
0.01\end{array}$ & $\begin{array}{l}\mathrm{mg} / \mathrm{L} \\
\mathrm{mg} / \mathrm{L} \\
\mathrm{mg} / \mathrm{L} \\
\mathrm{mg} / \mathrm{L} \\
\mathrm{mg} / \mathrm{L}\end{array}$ & $\begin{array}{r}\text { Sample } \\
\text { Split } \\
\text { Sample } \\
\text { Sample } \\
\text { Split }\end{array}$ \\
\hline & 77 FTU & $\begin{array}{l}1 / 12 / 01 \\
1 / 12 / 01 \\
4 / 10 / 01 \\
4 / 10 / 01 \\
10 / 23 / 01\end{array}$ & $\begin{array}{l}\text { ND } \\
\text { ND } \\
\text { ND } \\
\text { ND } \\
\text { ND }\end{array}$ & $\begin{array}{l}0.01 \\
0.01 \\
0.01 \\
0.01 \\
0.01\end{array}$ & $\begin{array}{l}\mathrm{mg} / \mathrm{L} \\
\mathrm{mg} / \mathrm{L} \\
\mathrm{mg} / \mathrm{L} \\
\mathrm{mg} / \mathrm{L} \\
\mathrm{mg} / \mathrm{L}\end{array}$ & $\begin{array}{r}\text { Sample } \\
\text { Split } \\
\text { Sample } \\
\text { Split } \\
\text { Sample }\end{array}$ \\
\hline
\end{tabular}




\begin{tabular}{llllll} 
Lnalyte & \multicolumn{4}{c}{ MDA } \\
Location & Date & Result & or PQL & Units & QC Type \\
\hline
\end{tabular}

\section{Metals and/or Minerals}

Cadmium

cont.

Chromium

Copper

Lead
Field Blank

25 FTU

77 FTU

Field Blank

25 FTU

77 FTU

$1 / 12 / 01$

$1 / 12 / 01$

$4 / 10 / 01$

$4 / 10 / 01$

$10 / 23 / 01$

Field Blank

$1 / 12 / 01$

$10 / 23 / 01$

$1 / 18 / 01$

$1 / 18 / 01$

$4 / 18 / 01$

$12 / 3 / 01$

$12 / 3 / 01$

77 FTU
$1 / 12 / 01$

$1 / 12 / 01$

$4 / 10 / 01$

$4 / 10 / 01$

$10 / 23 / 01$

$\begin{array}{cccr}\text { ND } & 0.01 & \mathrm{mg} / \mathrm{L} & \begin{array}{r}\text { Blank } \\ \text { Blank }\end{array} \\ \text { ND } & 0.01 & \mathrm{mg} / \mathrm{L} & \text { Sample } \\ \text { ND } & 0.01 & \mathrm{mg} / \mathrm{L} & \begin{array}{r}\text { Split } \\ \text { ND }\end{array} \\ \text { ND } & 0.01 & \mathrm{mg} / \mathrm{L} & \text { Sample } \\ \text { ND } & 0.01 & \mathrm{mg} / \mathrm{L} & \text { Sample } \\ \text { ND } & 0.01 & \mathrm{mg} / \mathrm{L} & \text { Split } \\ & 0.01 & \mathrm{mg} / \mathrm{L} & \\ \text { ND } & 0.01 & \mathrm{mg} / \mathrm{L} & \text { Sample } \\ \text { ND } & 0.01 & \mathrm{mg} / \mathrm{L} & \text { Split } \\ \text { ND } & 0.01 & \mathrm{mg} / \mathrm{L} & \text { Sample } \\ \text { ND } & 0.01 & \mathrm{mg} / \mathrm{L} & \text { Split } \\ 0.01 & 0.01 & \mathrm{mg} / \mathrm{L} & \text { Sample }\end{array}$

ND $\quad 0.01 \quad \mathrm{mg} / \mathrm{L} \quad$ Blank

ND $\quad 0.01 \quad \mathrm{mg} / \mathrm{L} \quad$ Blank

$\begin{array}{rrrr}0.28 & 0.01 & \mathrm{mg} / \mathrm{L} & \text { Sample } \\ 0.28 & 0.01 & \mathrm{mg} / \mathrm{L} & \text { Split } \\ 0.21 & 0.01 & \mathrm{mg} / \mathrm{L} & \text { Sample } \\ 0.21 & 0.01 & \mathrm{mg} / \mathrm{L} & \text { Sample } \\ 0.18 & 0.01 & \mathrm{mg} / \mathrm{L} & \text { Split }\end{array}$

$\begin{array}{rrrr}0.03 & 0.01 & \mathrm{mg} / \mathrm{L} & \text { Sample } \\ 0.03 & 0.01 & \mathrm{mg} / \mathrm{L} & \text { Split } \\ 0.02 & 0.01 & \mathrm{mg} / \mathrm{L} & \text { Sample } \\ 0.02 & 0.01 & \mathrm{mg} / \mathrm{L} & \text { Split } \\ 0.03 & 0.01 & \mathrm{mg} / \mathrm{L} & \text { Sample }\end{array}$

$\begin{array}{cccc}\mathrm{ND} & 0.01 & \mathrm{mg} / \mathrm{L} & \text { Blank } \\ 0.13 & 0.01 & \mathrm{mg} / \mathrm{L} & \text { Blank }\end{array}$

$\begin{array}{cccr}0.1 & 0.05 & \mathrm{mg} / \mathrm{L} & \text { Sample } \\ 0.09 & 0.05 & \mathrm{mg} / \mathrm{L} & \text { Split } \\ \text { ND } & 0.05 & \mathrm{mg} / \mathrm{L} & \text { Sample } \\ 0.06 & 0.05 & \mathrm{mg} / \mathrm{L} & \text { Sample } \\ \text { ND } & 0.05 & \mathrm{mg} / \mathrm{L} & \text { Split }\end{array}$

$\begin{array}{rrrr}\text { ND } & 0.05 & \mathrm{mg} / \mathrm{L} & \text { Sample } \\ \text { ND } & 0.05 & \mathrm{mg} / \mathrm{L} & \text { Split } \\ \text { ND } & 0.05 & \mathrm{mg} / \mathrm{L} & \text { Sample } \\ \text { ND } & 0.05 & \mathrm{mg} / \mathrm{L} & \text { Split } \\ \text { ND } & 0.05 & \mathrm{mg} / \mathrm{L} & \text { Sample }\end{array}$


MDA

\begin{tabular}{llllll} 
Analyte Location & Date & Result or PQL & Units & QC Type \\
\hline
\end{tabular}

Metals and/or Minerals

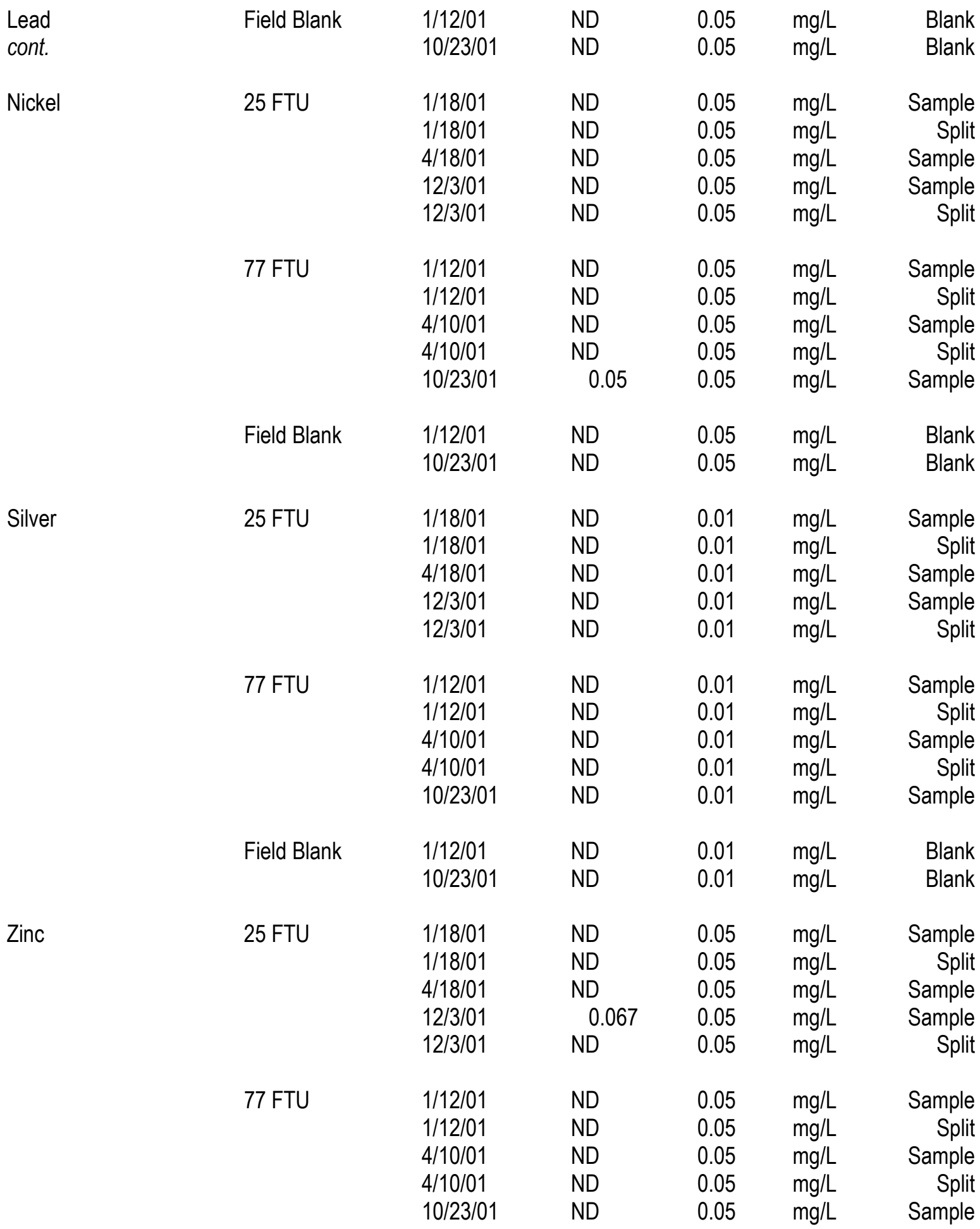




\begin{tabular}{|c|c|c|c|c|c|c|}
\hline Analyte & Location & Date & Result & $\begin{array}{l}\text { MDA } \\
\text { or PQL }\end{array}$ & Units & QC Type \\
\hline
\end{tabular}

Metals and/or Minerals

Zinc

cont.

Field Blank

$1 / 12 / 01$

$10 / 23 / 01$
ND

ND
$0.05 \mathrm{mg} / \mathrm{L}$

$0.05 \mathrm{mg} / \mathrm{L}$
Blank

Blank

\section{Volatile Organic Compounds}

\begin{tabular}{|c|c|c|c|c|c|c|}
\hline \multirow[t]{10}{*}{ 1,1,1-Trichloroethane } & \multirow[t]{5}{*}{25 FTU } & $1 / 18 / 01$ & ND & 0.5 & $\mu \mathrm{g} / \mathrm{L}$ & Sample \\
\hline & & $1 / 18 / 01$ & ND & 0.5 & $\mu \mathrm{g} / \mathrm{L}$ & Split \\
\hline & & $4 / 18 / 01$ & ND & 0.5 & $\mu \mathrm{g} / \mathrm{L}$ & Sample \\
\hline & & $4 / 18 / 01$ & ND & 0.5 & $\mu \mathrm{g} / \mathrm{L}$ & Split \\
\hline & & $12 / 3 / 01$ & ND & 0.5 & $\mu \mathrm{g} / \mathrm{L}$ & Sample \\
\hline & \multirow[t]{3}{*}{77 FTU } & $1 / 12 / 01$ & ND & 0.5 & $\mu \mathrm{g} / \mathrm{L}$ & Sample \\
\hline & & $10 / 23 / 01$ & ND & 0.5 & $\mu \mathrm{g} / \mathrm{L}$ & Sample \\
\hline & & $10 / 23 / 01$ & ND & 0.5 & $\mu \mathrm{g} / \mathrm{L}$ & Split \\
\hline & \multirow[t]{2}{*}{ Field Blank } & $4 / 18 / 01$ & ND & 0.5 & $\mu \mathrm{g} / \mathrm{L}$ & Blank \\
\hline & & $12 / 3 / 01$ & ND & 0.5 & $\mu \mathrm{g} / \mathrm{L}$ & Blank \\
\hline \multirow[t]{10}{*}{ 1,1,2,2-Tetrachloroethane } & \multirow[t]{5}{*}{$25 \mathrm{FTU}$} & $1 / 18 / 01$ & ND & 0.5 & $\mu \mathrm{g} / \mathrm{L}$ & Sample \\
\hline & & $1 / 18 / 01$ & ND & 0.5 & $\mu \mathrm{g} / \mathrm{L}$ & Split \\
\hline & & $4 / 18 / 01$ & ND & 0.5 & $\mu \mathrm{g} / \mathrm{L}$ & Sample \\
\hline & & $4 / 18 / 01$ & ND & 0.5 & $\mu \mathrm{g} / \mathrm{L}$ & Split \\
\hline & & $12 / 3 / 01$ & ND & 0.5 & $\mu \mathrm{g} / \mathrm{L}$ & Sample \\
\hline & \multirow[t]{3}{*}{$77 \mathrm{FTU}$} & $1 / 12 / 01$ & ND & 0.5 & $\mu \mathrm{g} / \mathrm{L}$ & Sample \\
\hline & & $10 / 23 / 01$ & ND & 0.5 & $\mu \mathrm{g} / \mathrm{L}$ & Sample \\
\hline & & $10 / 23 / 01$ & ND & 0.5 & $\mu \mathrm{g} / \mathrm{L}$ & Split \\
\hline & \multirow[t]{2}{*}{ Field Blank } & $4 / 18 / 01$ & ND & 0.5 & $\mu \mathrm{g} / \mathrm{L}$ & Blank \\
\hline & & $12 / 3 / 01$ & ND & 0.5 & $\mu \mathrm{g} / \mathrm{L}$ & Blank \\
\hline \multirow[t]{10}{*}{ 1,1,2-Trichloroethane } & \multirow[t]{5}{*}{$25 \mathrm{FTU}$} & $1 / 18 / 01$ & ND & 0.5 & $\mu \mathrm{g} / \mathrm{L}$ & Sample \\
\hline & & $1 / 18 / 01$ & ND & 0.5 & $\mu \mathrm{g} / \mathrm{L}$ & Split \\
\hline & & $4 / 18 / 01$ & ND & 0.5 & $\mu \mathrm{g} / \mathrm{L}$ & Sample \\
\hline & & $4 / 18 / 01$ & ND & 0.5 & $\mu \mathrm{g} / \mathrm{L}$ & Split \\
\hline & & $12 / 3 / 01$ & ND & 0.5 & $\mu \mathrm{g} / \mathrm{L}$ & Sample \\
\hline & \multirow[t]{3}{*}{77 FTU } & $1 / 12 / 01$ & ND & 0.5 & $\mu \mathrm{g} / \mathrm{L}$ & Sample \\
\hline & & $10 / 23 / 01$ & ND & 0.5 & $\mu \mathrm{g} / \mathrm{L}$ & Sample \\
\hline & & $10 / 23 / 01$ & ND & 0.5 & $\mu \mathrm{g} / \mathrm{L}$ & Split \\
\hline & \multirow[t]{2}{*}{ Field Blank } & $4 / 18 / 01$ & ND & 0.5 & $\mu \mathrm{g} / \mathrm{L}$ & Blank \\
\hline & & $12 / 3 / 01$ & ND & 0.5 & $\mu \mathrm{g} / \mathrm{L}$ & Blank \\
\hline \multirow[t]{2}{*}{ 1,1-Dichloroethane } & \multirow[t]{2}{*}{$25 \mathrm{FTU}$} & $1 / 18 / 01$ & ND & 0.5 & $\mu \mathrm{g} / \mathrm{L}$ & Sample \\
\hline & & $1 / 18 / 01$ & ND & 0.5 & $\mu \mathrm{g} / \mathrm{L}$ & Split \\
\hline
\end{tabular}


MDA

\begin{tabular}{llllll} 
Analyte Location & Date & Result or PQL & Units & QC Type \\
\hline
\end{tabular}

\section{Volatile Organic Compounds}

\begin{tabular}{|c|c|c|c|c|c|c|}
\hline \multirow[t]{3}{*}{$\begin{array}{l}\text { 1,1-Dichloroethane } \\
\text { cont. }\end{array}$} & $25 \mathrm{FTU}$ & $\begin{array}{l}4 / 18 / 01 \\
4 / 18 / 01 \\
12 / 3 / 01\end{array}$ & $\begin{array}{l}\text { ND } \\
\text { ND } \\
\text { ND }\end{array}$ & $\begin{array}{l}0.5 \\
0.5 \\
0.5\end{array}$ & $\begin{array}{l}\mu \mathrm{g} / \mathrm{L} \\
\mu \mathrm{g} / \mathrm{L} \\
\mu \mathrm{g} / \mathrm{L}\end{array}$ & $\begin{array}{r}\text { Sample } \\
\text { Split } \\
\text { Sample }\end{array}$ \\
\hline & 77 FTU & $\begin{array}{l}1 / 12 / 01 \\
10 / 23 / 01 \\
10 / 23 / 01\end{array}$ & $\begin{array}{l}\text { ND } \\
\text { ND } \\
\text { ND }\end{array}$ & $\begin{array}{l}0.5 \\
0.5 \\
0.5\end{array}$ & $\begin{array}{l}\mu \mathrm{g} / \mathrm{L} \\
\mu \mathrm{g} / \mathrm{L} \\
\mu \mathrm{g} / \mathrm{L}\end{array}$ & $\begin{array}{r}\text { Sample } \\
\text { Sample } \\
\text { Split }\end{array}$ \\
\hline & Field Blank & $\begin{array}{l}4 / 18 / 01 \\
12 / 3 / 01\end{array}$ & $\begin{array}{l}\text { ND } \\
\text { ND }\end{array}$ & $\begin{array}{l}0.5 \\
0.5\end{array}$ & $\begin{array}{l}\mu \mathrm{g} / \mathrm{L} \\
\mu \mathrm{g} / \mathrm{L}\end{array}$ & $\begin{array}{l}\text { Blank } \\
\text { Blank }\end{array}$ \\
\hline \multirow[t]{3}{*}{ 1,1-Dichloroethene } & $25 \mathrm{FTU}$ & $\begin{array}{l}1 / 18 / 01 \\
1 / 18 / 01 \\
4 / 18 / 01 \\
4 / 18 / 01 \\
12 / 3 / 01\end{array}$ & $\begin{array}{l}\text { ND } \\
\text { ND } \\
\text { ND } \\
\text { ND } \\
\text { ND }\end{array}$ & $\begin{array}{l}0.5 \\
0.5 \\
0.5 \\
0.5 \\
0.5\end{array}$ & $\begin{array}{l}\mu \mathrm{g} / \mathrm{L} \\
\mu \mathrm{g} / \mathrm{L} \\
\mu \mathrm{g} / \mathrm{L} \\
\mu \mathrm{g} / \mathrm{L} \\
\mu \mathrm{g} / \mathrm{L}\end{array}$ & $\begin{array}{r}\text { Sample } \\
\text { Split } \\
\text { Sample } \\
\text { Split } \\
\text { Sample }\end{array}$ \\
\hline & 77 FTU & $\begin{array}{l}1 / 12 / 01 \\
10 / 23 / 01 \\
10 / 23 / 01\end{array}$ & $\begin{array}{l}\text { ND } \\
\text { ND } \\
\text { ND }\end{array}$ & $\begin{array}{l}0.5 \\
0.5 \\
0.5\end{array}$ & $\begin{array}{l}\mu \mathrm{g} / \mathrm{L} \\
\mu \mathrm{g} / \mathrm{L} \\
\mu \mathrm{g} / \mathrm{L}\end{array}$ & $\begin{array}{r}\text { Sample } \\
\text { Sample } \\
\text { Split }\end{array}$ \\
\hline & Field Blank & $\begin{array}{l}4 / 18 / 01 \\
12 / 3 / 01\end{array}$ & $\begin{array}{l}\text { ND } \\
\text { ND }\end{array}$ & $\begin{array}{l}0.5 \\
0.5\end{array}$ & $\begin{array}{l}\mu \mathrm{g} / \mathrm{L} \\
\mu \mathrm{g} / \mathrm{L}\end{array}$ & $\begin{array}{l}\text { Blar } \\
\text { Blar }\end{array}$ \\
\hline \multirow[t]{3}{*}{ 1,2-Dichlorobenzene } & 25 FTU & $\begin{array}{l}1 / 18 / 01 \\
1 / 18 / 01 \\
4 / 18 / 01 \\
4 / 18 / 01 \\
12 / 3 / 01\end{array}$ & $\begin{array}{l}\text { ND } \\
\text { ND } \\
\text { ND } \\
\text { ND } \\
\text { ND }\end{array}$ & $\begin{array}{l}0.5 \\
0.5 \\
0.5 \\
0.5 \\
0.5\end{array}$ & $\begin{array}{l}\mu \mathrm{g} / \mathrm{L} \\
\mu \mathrm{g} / \mathrm{L} \\
\mu \mathrm{g} / \mathrm{L} \\
\mu \mathrm{g} / \mathrm{L} \\
\mu \mathrm{g} / \mathrm{L}\end{array}$ & $\begin{array}{r}\text { Sample } \\
\text { Split } \\
\text { Sample } \\
\text { Split } \\
\text { Sample }\end{array}$ \\
\hline & 77 FTU & $\begin{array}{l}1 / 12 / 01 \\
10 / 23 / 01 \\
10 / 23 / 01\end{array}$ & $\begin{array}{l}\text { ND } \\
\text { ND } \\
\text { ND }\end{array}$ & $\begin{array}{l}0.5 \\
0.5 \\
0.5\end{array}$ & $\begin{array}{l}\mu \mathrm{g} / \mathrm{L} \\
\mu \mathrm{g} / \mathrm{L} \\
\mu \mathrm{g} / \mathrm{L}\end{array}$ & $\begin{array}{r}\text { Sample } \\
\text { Sample } \\
\text { Split }\end{array}$ \\
\hline & Field Blank & $\begin{array}{l}4 / 18 / 01 \\
12 / 3 / 01\end{array}$ & $\begin{array}{l}\text { ND } \\
\text { ND }\end{array}$ & $\begin{array}{l}0.5 \\
0.5\end{array}$ & $\begin{array}{l}\mu \mathrm{g} / \mathrm{L} \\
\mu \mathrm{g} / \mathrm{L}\end{array}$ & $\begin{array}{l}\text { Blar } \\
\text { Blar }\end{array}$ \\
\hline \multirow[t]{2}{*}{ 1,2-Dichloroethane } & $25 \mathrm{FTU}$ & $\begin{array}{l}1 / 18 / 01 \\
1 / 18 / 01 \\
4 / 18 / 01 \\
4 / 18 / 01 \\
12 / 3 / 01\end{array}$ & $\begin{array}{l}\text { ND } \\
\text { ND } \\
\text { ND } \\
\text { ND } \\
\text { ND }\end{array}$ & $\begin{array}{l}0.5 \\
0.5 \\
0.5 \\
0.5 \\
0.5\end{array}$ & $\begin{array}{l}\mu \mathrm{g} / \mathrm{L} \\
\mu \mathrm{g} / \mathrm{L} \\
\mu \mathrm{g} / \mathrm{L} \\
\mu \mathrm{g} / \mathrm{L} \\
\mu \mathrm{g} / \mathrm{L}\end{array}$ & $\begin{array}{r}\text { Sample } \\
\text { Split } \\
\text { Sample } \\
\text { Split } \\
\text { Sample }\end{array}$ \\
\hline & 77 FTU & $\begin{array}{l}1 / 12 / 01 \\
10 / 23 / 01 \\
10 / 23 / 01\end{array}$ & $\begin{array}{l}\text { ND } \\
\text { ND } \\
\text { ND }\end{array}$ & $\begin{array}{l}0.5 \\
0.5 \\
0.5\end{array}$ & $\begin{array}{l}\mu \mathrm{g} / \mathrm{L} \\
\mu \mathrm{g} / \mathrm{L} \\
\mu \mathrm{g} / \mathrm{L}\end{array}$ & $\begin{array}{r}\text { Samp } \\
\text { Samp } \\
\text { Sp }\end{array}$ \\
\hline
\end{tabular}




\begin{tabular}{|c|c|c|c|c|c|c|}
\hline Analyte & Location & Date & Result & $\begin{array}{r}\text { MDA } \\
\text { or PQL } \\
\end{array}$ & Units & QC Type \\
\hline \multicolumn{7}{|c|}{ Volatile Organic Compounds } \\
\hline \multirow{2}{*}{$\begin{array}{l}\text { 1,2-Dichloroethane } \\
\text { cont. }\end{array}$} & \multirow[t]{2}{*}{ Field Blank } & $4 / 18 / 01$ & ND & 0.5 & $\mu g / L$ & Blank \\
\hline & & $12 / 3 / 01$ & ND & 0.5 & $\mu g / L$ & Blank \\
\hline \multirow[t]{7}{*}{ 1,2-Dichloroethene (total) } & \multirow[t]{3}{*}{25 FTU } & $4 / 18 / 01$ & ND & 1 & $\mu g / L$ & Sample \\
\hline & & $4 / 18 / 01$ & ND & 1 & $\mu \mathrm{g} / \mathrm{L}$ & Split \\
\hline & & $12 / 3 / 01$ & ND & 1 & $\mu g / L$ & Sample \\
\hline & \multirow[t]{2}{*}{77 FTU } & $10 / 23 / 01$ & ND & 1 & $\mu g / L$ & Sample \\
\hline & & $10 / 23 / 01$ & ND & 1 & $\mu \mathrm{g} / \mathrm{L}$ & Split \\
\hline & \multirow[t]{2}{*}{ Field Blank } & $4 / 18 / 01$ & ND & 1 & $\mu g / L$ & Blank \\
\hline & & $12 / 3 / 01$ & ND & 1 & $\mu \mathrm{g} / \mathrm{L}$ & Blank \\
\hline \multirow[t]{10}{*}{ 1,2-Dichloropropane } & \multirow[t]{5}{*}{25 FTU } & $1 / 18 / 01$ & ND & 0.5 & $\mu g / L$ & Sample \\
\hline & & $1 / 18 / 01$ & ND & 0.5 & $\mu \mathrm{g} / \mathrm{L}$ & Split \\
\hline & & $4 / 18 / 01$ & ND & 0.5 & $\mu g / L$ & Sample \\
\hline & & $4 / 18 / 01$ & ND & 0.5 & $\mu \mathrm{g} / \mathrm{L}$ & Split \\
\hline & & $12 / 3 / 01$ & ND & 0.5 & $\mu g / L$ & Sample \\
\hline & \multirow[t]{3}{*}{77 FTU } & $1 / 12 / 01$ & ND & 0.5 & $\mu g / L$ & Sample \\
\hline & & $10 / 23 / 01$ & ND & 0.5 & $\mu \mathrm{g} / \mathrm{L}$ & Sample \\
\hline & & $10 / 23 / 01$ & ND & 0.5 & $\mu \mathrm{g} / \mathrm{L}$ & Split \\
\hline & \multirow[t]{2}{*}{ Field Blank } & $4 / 18 / 01$ & ND & 0.5 & $\mu g / L$ & Blank \\
\hline & & $12 / 3 / 01$ & ND & 0.5 & $\mu \mathrm{g} / \mathrm{L}$ & Blank \\
\hline \multirow[t]{10}{*}{ 1,3-Dichlorobenzene } & \multirow[t]{5}{*}{$25 \mathrm{FTU}$} & $1 / 18 / 01$ & ND & 0.5 & $\mu g / L$ & Sample \\
\hline & & $1 / 18 / 01$ & ND & 0.5 & $\mu \mathrm{g} / \mathrm{L}$ & Split \\
\hline & & $4 / 18 / 01$ & ND & 0.5 & $\mu g / L$ & Sample \\
\hline & & $4 / 18 / 01$ & ND & 0.5 & $\mu g / L$ & Split \\
\hline & & $12 / 3 / 01$ & ND & 0.5 & $\mu \mathrm{g} / \mathrm{L}$ & Sample \\
\hline & \multirow[t]{3}{*}{77 FTU } & $1 / 12 / 01$ & ND & 0.5 & $\mu g / L$ & Sample \\
\hline & & $10 / 23 / 01$ & ND & 0.5 & $\mu \mathrm{g} / \mathrm{L}$ & Sample \\
\hline & & $10 / 23 / 01$ & ND & 0.5 & $\mu \mathrm{g} / \mathrm{L}$ & Split \\
\hline & \multirow[t]{2}{*}{ Field Blank } & $4 / 18 / 01$ & ND & 0.5 & $\mu g / L$ & Blank \\
\hline & & $12 / 3 / 01$ & ND & 0.5 & $\mu \mathrm{g} / \mathrm{L}$ & Blank \\
\hline \multirow[t]{5}{*}{ 1,4-Dichlorobenzene } & \multirow[t]{5}{*}{25 FTU } & $1 / 18 / 01$ & ND & 0.5 & $\mu g / L$ & Sample \\
\hline & & $1 / 18 / 01$ & ND & 0.5 & $\mu \mathrm{g} / \mathrm{L}$ & Split \\
\hline & & $4 / 18 / 01$ & ND & 0.5 & $\mu \mathrm{g} / \mathrm{L}$ & Sample \\
\hline & & $4 / 18 / 01$ & ND & 0.5 & $\mu \mathrm{g} / \mathrm{L}$ & Split \\
\hline & & $12 / 3 / 01$ & ND & 0.5 & $\mu \mathrm{g} / \mathrm{L}$ & Sample \\
\hline
\end{tabular}


MDA

$\begin{array}{llllll}\text { Analyte Location } & \text { Date } & \text { Result or PQL Units } & \text { QC Type }\end{array}$

\section{Volatile Organic Compounds}

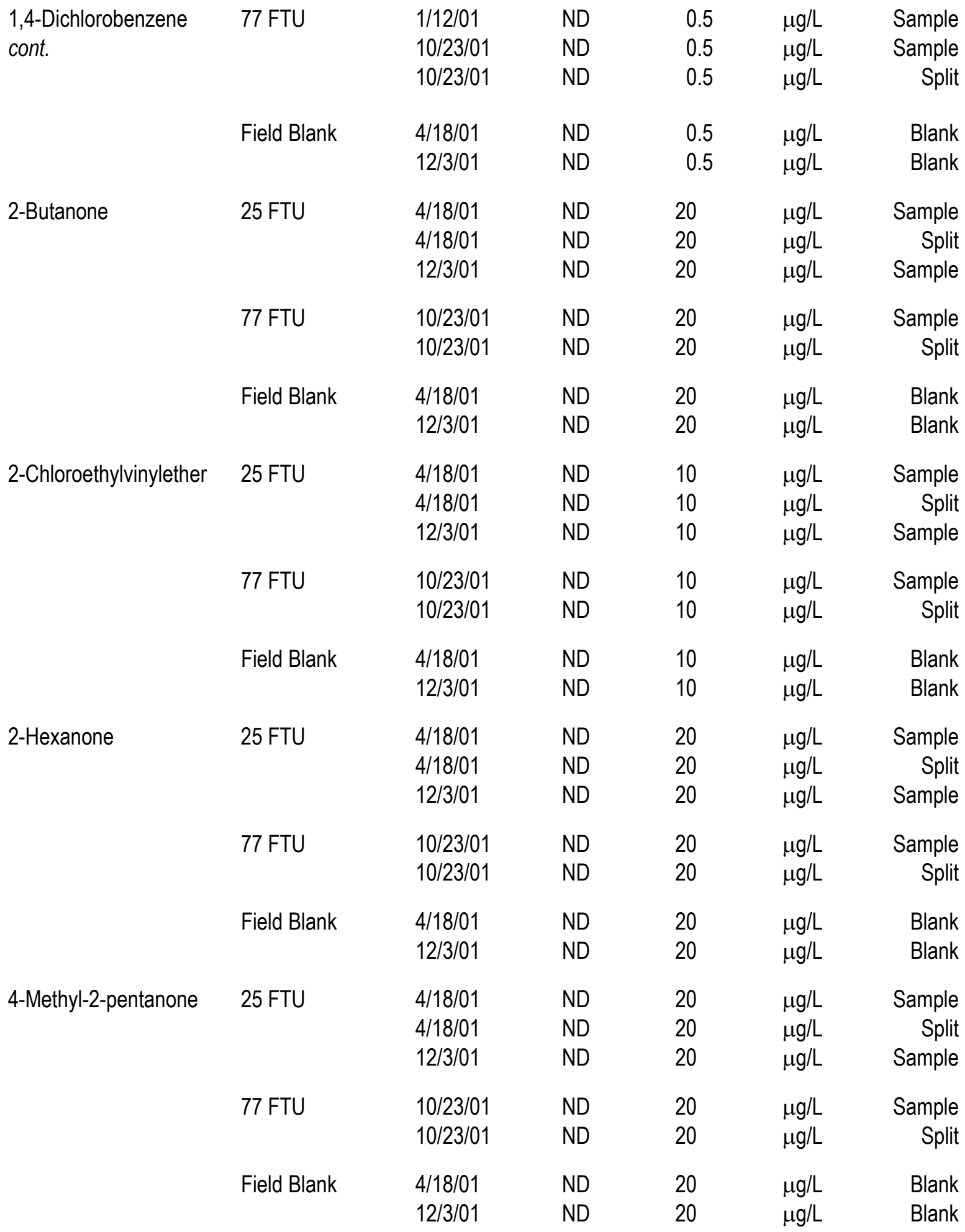




\begin{tabular}{|c|c|c|c|c|c|c|}
\hline Analyte & Location & Date & Result & $\begin{array}{r}\text { MDA } \\
\text { or } P Q L \\
\end{array}$ & Units & QC Type \\
\hline \multicolumn{7}{|c|}{ Volatile Organic Compounds } \\
\hline \multirow[t]{7}{*}{ Acetone } & 25 FTU & $4 / 18 / 01$ & 160 & 20 & $\mu g / L$ & Sample \\
\hline & & $4 / 18 / 01$ & 160 & 20 & $\mu g / L$ & Split \\
\hline & & $12 / 3 / 01$ & 200 & 20 & $\mu \mathrm{g} / \mathrm{L}$ & Sample \\
\hline & 77 FTU & $10 / 23 / 01$ & ND & 20 & $\mu g / L$ & Sample \\
\hline & & $10 / 23 / 01$ & ND & 20 & $\mu g / L$ & Split \\
\hline & Field Blank & $4 / 18 / 01$ & ND & 20 & $\mu g / L$ & Blank \\
\hline & & $12 / 3 / 01$ & ND & 20 & $\mu g / L$ & Blank \\
\hline \multirow[t]{10}{*}{ Benzene } & 25 FTU & $1 / 18 / 01$ & ND & 0.5 & $\mu g / L$ & Sample \\
\hline & & $1 / 18 / 01$ & ND & 0.5 & $\mu \mathrm{g} / \mathrm{L}$ & Split \\
\hline & & $4 / 18 / 01$ & ND & 0.5 & $\mu \mathrm{g} / \mathrm{L}$ & Sample \\
\hline & & $4 / 18 / 01$ & ND & 0.5 & $\mu \mathrm{g} / \mathrm{L}$ & Split \\
\hline & & $12 / 3 / 01$ & ND & 0.5 & $\mu g / L$ & Sample \\
\hline & 77 FTU & $1 / 12 / 01$ & ND & 0.5 & $\mu \mathrm{g} / \mathrm{L}$ & Sample \\
\hline & & $10 / 23 / 01$ & ND & 0.5 & $\mu g / L$ & Sample \\
\hline & & $10 / 23 / 01$ & ND & 0.5 & $\mu \mathrm{g} / \mathrm{L}$ & Split \\
\hline & Field Blank & $4 / 18 / 01$ & ND & 0.5 & $\mu g / L$ & Blank \\
\hline & & $12 / 3 / 01$ & ND & 0.5 & $\mu \mathrm{g} / \mathrm{L}$ & Blank \\
\hline \multirow[t]{10}{*}{ Bromodichloromethane } & $25 \mathrm{FTU}$ & $1 / 18 / 01$ & 0.5 & 0.5 & $\mu g / L$ & Sample \\
\hline & & $1 / 18 / 01$ & 0.54 & 0.5 & $\mu \mathrm{g} / \mathrm{L}$ & Split \\
\hline & & $4 / 18 / 01$ & 1.1 & 0.5 & $\mu \mathrm{g} / \mathrm{L}$ & Sample \\
\hline & & $4 / 18 / 01$ & 1.1 & 0.5 & $\mu \mathrm{g} / \mathrm{L}$ & Split \\
\hline & & $12 / 3 / 01$ & ND & 0.5 & $\mu g / L$ & Sample \\
\hline & 77 FTU & $1 / 12 / 01$ & ND & 0.5 & $\mu g / L$ & Sample \\
\hline & & $10 / 23 / 01$ & ND & 0.5 & $\mu g / L$ & Sample \\
\hline & & $10 / 23 / 01$ & ND & 0.5 & $\mu \mathrm{g} / \mathrm{L}$ & Split \\
\hline & Field Blank & $4 / 18 / 01$ & ND & 0.5 & $\mu g / L$ & Blank \\
\hline & & $12 / 3 / 01$ & ND & 0.5 & $\mu \mathrm{g} / \mathrm{L}$ & Blank \\
\hline \multirow[t]{8}{*}{ Bromoform } & $25 \mathrm{FTU}$ & $1 / 18 / 01$ & ND & 0.5 & $\mu g / L$ & Sample \\
\hline & & $1 / 18 / 01$ & ND & 0.5 & $\mu \mathrm{g} / \mathrm{L}$ & Split \\
\hline & & $4 / 18 / 01$ & ND & 0.5 & $\mu \mathrm{g} / \mathrm{L}$ & Sample \\
\hline & & $4 / 18 / 01$ & ND & 0.5 & $\mu \mathrm{g} / \mathrm{L}$ & Split \\
\hline & & $12 / 3 / 01$ & ND & 0.5 & $\mu g / L$ & Sample \\
\hline & 77 FTU & $1 / 12 / 01$ & ND & 0.5 & $\mu \mathrm{g} / \mathrm{L}$ & Sample \\
\hline & & 10/23/01 & ND & 0.5 & $\mu g / L$ & Sample \\
\hline & & 10/23/01 & ND & 0.5 & $\mu \mathrm{g} / \mathrm{L}$ & Split \\
\hline
\end{tabular}




\section{Analyte \\ Location \\ Volatile Organic Compounds}

Date

MDA

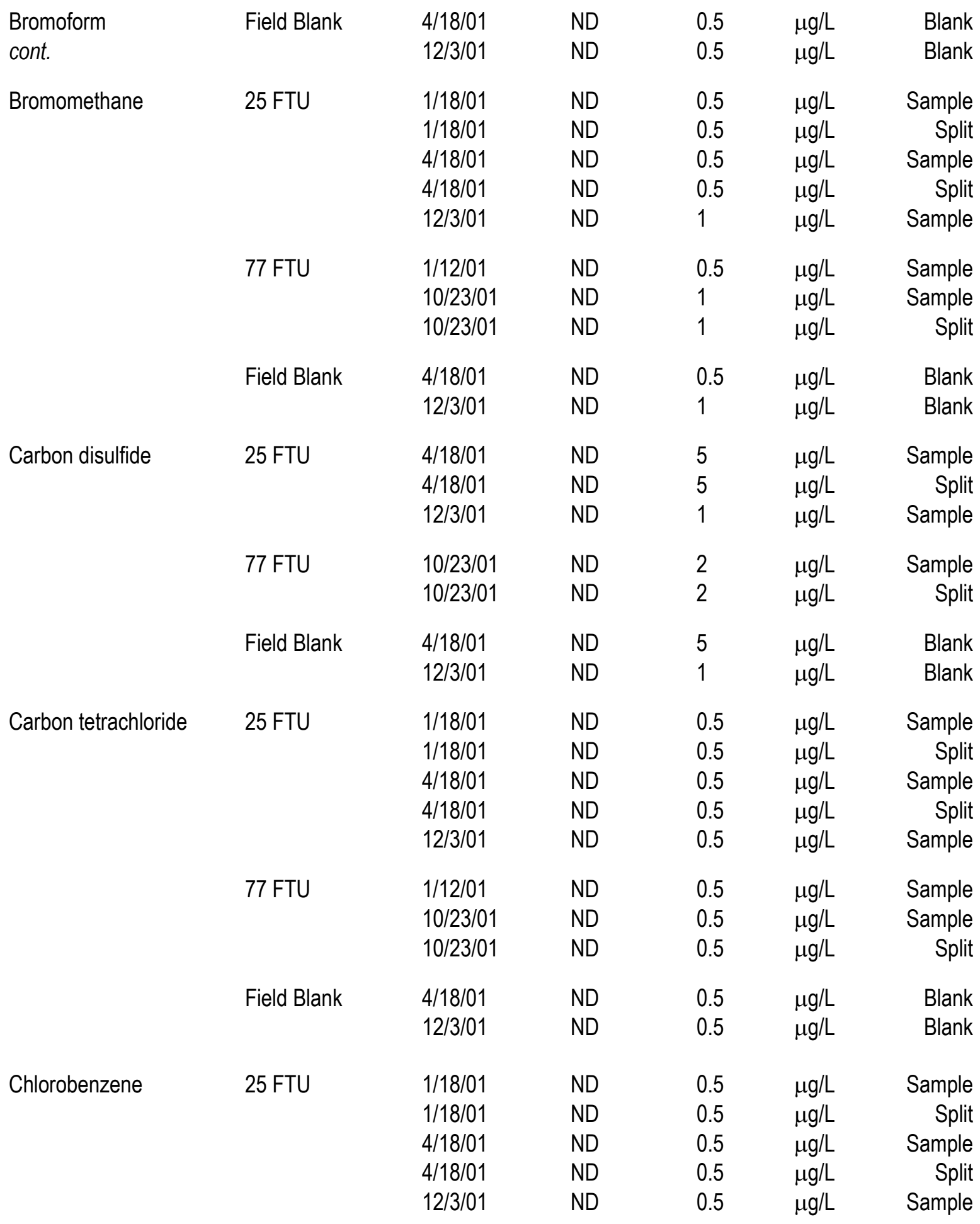




\begin{tabular}{|c|c|c|c|c|c|c|}
\hline Analyte & Location & Date & Result & $\begin{array}{r}\text { MDA } \\
\text { or PQL } \\
\end{array}$ & Units & QC Type \\
\hline \multicolumn{7}{|c|}{ Volatile Organic Compounds } \\
\hline \multirow{5}{*}{$\begin{array}{l}\text { Chlorobenzene } \\
\text { cont. }\end{array}$} & 77 FTU & $1 / 12 / 01$ & ND & 0.5 & $\mu g / L$ & Sample \\
\hline & & $10 / 23 / 01$ & ND & 0.5 & $\mu \mathrm{g} / \mathrm{L}$ & Sample \\
\hline & & $10 / 23 / 01$ & ND & 0.5 & $\mu \mathrm{g} / \mathrm{L}$ & Split \\
\hline & Field Blank & $4 / 18 / 01$ & ND & 0.5 & $\mu \mathrm{g} / \mathrm{L}$ & Blank \\
\hline & & $12 / 3 / 01$ & ND & 0.5 & $\mu \mathrm{g} / \mathrm{L}$ & Blank \\
\hline \multirow[t]{10}{*}{ Chloroethane } & 25 FTU & $1 / 18 / 01$ & ND & 1 & $\mu g / L$ & Sample \\
\hline & & $1 / 18 / 01$ & ND & 1 & $\mu g / L$ & Split \\
\hline & & $4 / 18 / 01$ & ND & 1 & $\mu \mathrm{g} / \mathrm{L}$ & Sample \\
\hline & & $4 / 18 / 01$ & ND & 1 & $\mu \mathrm{g} / \mathrm{L}$ & Split \\
\hline & & $12 / 3 / 01$ & ND & 0.5 & $\mu g / L$ & Sample \\
\hline & 77 FTU & $1 / 12 / 01$ & ND & 1 & $\mu g / L$ & Sample \\
\hline & & $10 / 23 / 01$ & ND & 0.5 & $\mu \mathrm{g} / \mathrm{L}$ & Sample \\
\hline & & $10 / 23 / 01$ & ND & 0.5 & $\mu \mathrm{g} / \mathrm{L}$ & Split \\
\hline & Field Blank & $4 / 18 / 01$ & ND & 1 & $\mu g / L$ & Blank \\
\hline & & $12 / 3 / 01$ & ND & 0.5 & $\mu \mathrm{g} / \mathrm{L}$ & Blank \\
\hline \multirow[t]{10}{*}{ Chloroform } & 25 FTU & $1 / 18 / 01$ & 9.1 & 0.5 & $\mu g / L$ & Sample \\
\hline & & $1 / 18 / 01$ & 9.8 & 0.5 & $\mu \mathrm{g} / \mathrm{L}$ & Split \\
\hline & & $4 / 18 / 01$ & 9.9 & 0.5 & $\mu \mathrm{g} / \mathrm{L}$ & Sample \\
\hline & & $4 / 18 / 01$ & 9.8 & 0.5 & $\mu \mathrm{g} / \mathrm{L}$ & Split \\
\hline & & $12 / 3 / 01$ & 4.2 & 0.5 & $\mu g / L$ & Sample \\
\hline & 77 FTU & $1 / 12 / 01$ & 3.7 & 0.5 & $\mu g / L$ & Sample \\
\hline & & 10/23/01 & 1.9 & 0.5 & $\mu \mathrm{g} / \mathrm{L}$ & Sample \\
\hline & & 10/23/01 & 1.9 & 0.5 & $\mu \mathrm{g} / \mathrm{L}$ & Split \\
\hline & Field Blank & $4 / 18 / 01$ & ND & 0.5 & $\mu g / L$ & Blank \\
\hline & & $12 / 3 / 01$ & ND & 0.5 & $\mu \mathrm{g} / \mathrm{L}$ & Blank \\
\hline \multirow[t]{10}{*}{ Chloromethane } & 25 FTU & $1 / 18 / 01$ & ND & 1 & $\mu g / L$ & Sample \\
\hline & & $1 / 18 / 01$ & ND & 1 & $\mu \mathrm{g} / \mathrm{L}$ & Split \\
\hline & & $4 / 18 / 01$ & ND & 1 & $\mu \mathrm{g} / \mathrm{L}$ & Sample \\
\hline & & $4 / 18 / 01$ & ND & 1 & $\mu \mathrm{g} / \mathrm{L}$ & Split \\
\hline & & $12 / 3 / 01$ & ND & 0.5 & $\mu g / L$ & Sample \\
\hline & 77 FTU & $1 / 12 / 01$ & ND & 1 & $\mu \mathrm{g} / \mathrm{L}$ & Sample \\
\hline & & $10 / 23 / 01$ & ND & 0.5 & $\mu \mathrm{g} / \mathrm{L}$ & Sample \\
\hline & & $10 / 23 / 01$ & ND & 0.5 & $\mu g / L$ & Split \\
\hline & Field Blank & $4 / 18 / 01$ & ND & 1 & $\mu \mathrm{g} / \mathrm{L}$ & Blank \\
\hline & & $12 / 3 / 01$ & ND & 0.5 & $\mu \mathrm{g} / \mathrm{L}$ & Blank \\
\hline
\end{tabular}




\section{Analyte \\ Location \\ Volatile Organic Compounds}

Date

MDA

\begin{tabular}{|c|c|c|c|c|c|c|}
\hline \multirow[t]{7}{*}{ cis-1,2-Dichloroethene } & \multirow[t]{3}{*}{$25 \mathrm{FTU}$} & $4 / 18 / 01$ & ND & 0.5 & $\mu \mathrm{g} / \mathrm{L}$ & Sample \\
\hline & & $4 / 18 / 01$ & ND & 0.5 & $\mu \mathrm{g} / \mathrm{L}$ & Split \\
\hline & & $12 / 3 / 01$ & ND & 0.5 & $\mu \mathrm{g} / \mathrm{L}$ & Sample \\
\hline & \multirow[t]{2}{*}{77 FTU } & $10 / 23 / 01$ & ND & 0.5 & $\mu \mathrm{g} / \mathrm{L}$ & Samr \\
\hline & & $10 / 23 / 01$ & ND & 0.5 & $\mu \mathrm{g} / \mathrm{L}$ & S \\
\hline & \multirow[t]{2}{*}{ Field Blank } & $4 / 18 / 01$ & ND & 0.5 & $\mu \mathrm{g} / \mathrm{L}$ & Bla \\
\hline & & $12 / 3 / 01$ & ND & 0.5 & $\mu \mathrm{g} / \mathrm{L}$ & Bla \\
\hline \multirow[t]{10}{*}{ cis-1,3-Dichloropropene } & \multirow[t]{5}{*}{25 FTU } & $1 / 18 / 01$ & ND & 0.5 & $\mu \mathrm{g} / \mathrm{L}$ & Samp \\
\hline & & $1 / 18 / 01$ & ND & 0.5 & $\mu \mathrm{g} / \mathrm{L}$ & $\mathrm{S}$ \\
\hline & & $4 / 18 / 01$ & ND & 0.5 & $\mu \mathrm{g} / \mathrm{L}$ & Samp \\
\hline & & $4 / 18 / 01$ & ND & 0.5 & $\mu \mathrm{g} / \mathrm{L}$ & $\mathrm{Sr}$ \\
\hline & & $12 / 3 / 01$ & ND & 0.5 & $\mu \mathrm{g} / \mathrm{L}$ & Samp \\
\hline & \multirow[t]{3}{*}{77 FTU } & $1 / 12 / 01$ & ND & 0.5 & $\mu \mathrm{g} / \mathrm{L}$ & Sample \\
\hline & & $10 / 23 / 01$ & ND & 0.5 & $\mu \mathrm{g} / \mathrm{L}$ & Sample \\
\hline & & $10 / 23 / 01$ & ND & 0.5 & $\mu \mathrm{g} / \mathrm{L}$ & $\mathrm{Sp}$ \\
\hline & \multirow[t]{2}{*}{ Field Blank } & $4 / 18 / 01$ & ND & 0.5 & $\mu \mathrm{g} / \mathrm{L}$ & Blank \\
\hline & & $12 / 3 / 01$ & ND & 0.5 & $\mu \mathrm{g} / \mathrm{L}$ & Bla \\
\hline \multirow{10}{*}{ Dibromochloromethane } & \multirow[t]{5}{*}{25 FTU } & $1 / 18 / 01$ & ND & 0.5 & $\mu \mathrm{g} / \mathrm{L}$ & Sample \\
\hline & & $1 / 18 / 01$ & ND & 0.5 & $\mu \mathrm{g} / \mathrm{L}$ & $\mathrm{Sp}$ \\
\hline & & $4 / 18 / 01$ & ND & 0.5 & $\mu \mathrm{g} / \mathrm{L}$ & Sample \\
\hline & & $4 / 18 / 01$ & ND & 0.5 & $\mu \mathrm{g} / \mathrm{L}$ & $\mathrm{Sp}$ \\
\hline & & $12 / 3 / 01$ & ND & 0.5 & $\mu \mathrm{g} / \mathrm{L}$ & Samp \\
\hline & \multirow[t]{3}{*}{77 FTU } & $1 / 12 / 01$ & ND & 0.5 & $\mu \mathrm{g} / \mathrm{L}$ & Sample \\
\hline & & $10 / 23 / 01$ & ND & 0.5 & $\mu \mathrm{g} / \mathrm{L}$ & Samp \\
\hline & & 10/23/01 & ND & 0.5 & $\mu \mathrm{g} / \mathrm{L}$ & $\mathrm{Sp}$ \\
\hline & \multirow[t]{2}{*}{ Field Blank } & $4 / 18 / 01$ & ND & 0.5 & $\mu \mathrm{g} / \mathrm{L}$ & Blank \\
\hline & & $12 / 3 / 01$ & ND & 0.5 & $\mu \mathrm{g} / \mathrm{L}$ & Blank \\
\hline \multirow{7}{*}{ Dibromomethane } & \multirow[t]{3}{*}{$25 \mathrm{FTU}$} & $4 / 18 / 01$ & ND & 0.5 & $\mu \mathrm{g} / \mathrm{L}$ & Sample \\
\hline & & $4 / 18 / 01$ & ND & 0.5 & $\mu \mathrm{g} / \mathrm{L}$ & Split \\
\hline & & $12 / 3 / 01$ & ND & 0.5 & $\mu \mathrm{g} / \mathrm{L}$ & Sample \\
\hline & \multirow{2}{*}{77 FTU } & $10 / 23 / 01$ & ND & 0.5 & $\mu \mathrm{g} / \mathrm{L}$ & Sample \\
\hline & & 10/23/01 & ND & 0.5 & $\mu \mathrm{g} / \mathrm{L}$ & \\
\hline & \multirow[t]{2}{*}{ Field Blank } & $4 / 18 / 01$ & ND & 0.5 & $\mu \mathrm{g} / \mathrm{L}$ & Blank \\
\hline & & $12 / 3 / 01$ & ND & 0.5 & $\mu \mathrm{g} / \mathrm{L}$ & \\
\hline
\end{tabular}




\begin{tabular}{lllll} 
Lnalyte & \multicolumn{4}{c}{ MDA } \\
Location & Date & Result or PQL & Units & QC Type \\
\hline
\end{tabular}

Volatile Organic Compounds

Dichlorodifluoromethane 25 FTU

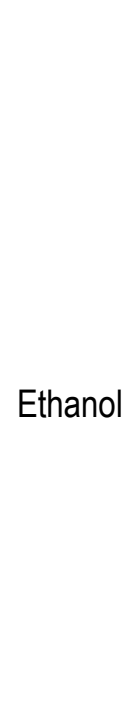

Ethylbenzene

Freon 113

25 FTU

77 FTU

25 FTU

77 FTU

25 FTU

77 FTU

$\begin{array}{ll}4 / 18 / 01 & \text { ND } \\ 4 / 18 / 01 & \text { ND } \\ 12 / 3 / 01 & \text { ND }\end{array}$

$10 / 23 / 01$

$10 / 23 / 01$

Field Blank

$4 / 18 / 01$

$12 / 3 / 01$

$4 / 18 / 01$

$4 / 18 / 01$

$12 / 3 / 01$

$10 / 23 / 01$

$10 / 23 / 01$

Field Blank

$4 / 18 / 01$

$12 / 3 / 01$

$1 / 18 / 01$

$4 / 18 / 01$

$4 / 18 / 01$

$12 / 3 / 01$

$1 / 12 / 01$

$10 / 23 / 01$

$10 / 23 / 01$

Field Blank

4/18/01

$12 / 3 / 01$

$1 / 18 / 01$

$1 / 18 / 01$

$4 / 18 / 01$

$4 / 18 / 01$

$12 / 3 / 01$

77 FTU

$1 / 12 / 01$

$10 / 23 / 01$

$10 / 23 / 01$

Field Blank
ND

ND

ND

ND

ND

ND

ND

ND

ND

ND

ND

ND

ND

ND

ND

ND

ND

ND

ND

ND

ND

ND

ND

ND

ND

ND

ND

ND

ND

ND

ND
$4 / 18 / 01$

$12 / 3 / 01$

$\begin{array}{rrr}0.5 & \mu \mathrm{g} / \mathrm{L} & \text { Sample } \\ 0.5 & \mu \mathrm{g} / \mathrm{L} & \text { Split } \\ 0.5 & \mu \mathrm{g} / \mathrm{L} & \text { Sample } \\ & & \\ 0.5 & \mu \mathrm{g} / \mathrm{L} & \text { Sample } \\ 0.5 & \mu \mathrm{g} / \mathrm{L} & \text { Split }\end{array}$

$0.5 \mu \mathrm{g} / \mathrm{L} \quad$ Blank

$0.5 \mu \mathrm{g} / \mathrm{L} \quad$ Blank

$\begin{array}{rrr}1000 & \mu \mathrm{g} / \mathrm{L} & \text { Sample } \\ 1000 & \mu \mathrm{g} / \mathrm{L} & \text { Split } \\ 1000 & \mu \mathrm{g} / \mathrm{L} & \text { Sample } \\ 1000 & \mu \mathrm{g} / \mathrm{L} & \text { Sample } \\ 1000 & \mu \mathrm{g} / \mathrm{L} & \text { Split }\end{array}$

1000

1000

$\mu \mathrm{g} / \mathrm{L}$

$\mu g / L$

Blank

Blank

$\begin{array}{rrr}0.5 & \mu \mathrm{g} / \mathrm{L} & \text { Sample } \\ 0.5 & \mu \mathrm{g} / \mathrm{L} & \text { Split } \\ 0.5 & \mu \mathrm{g} / \mathrm{L} & \text { Sample } \\ 0.5 & \mu \mathrm{g} / \mathrm{L} & \text { Split } \\ 0.5 & \mu \mathrm{g} / \mathrm{L} & \text { Sample }\end{array}$

$\begin{array}{llr}0.5 & \mu \mathrm{g} / \mathrm{L} & \text { Sample } \\ 0.5 & \mu \mathrm{g} / \mathrm{L} & \text { Sample } \\ 0.5 & \mu \mathrm{g} / \mathrm{L} & \text { Split }\end{array}$

$\begin{array}{lll}0.5 & \mu \mathrm{g} / \mathrm{L} & \text { Blank } \\ 0.5 & \mu \mathrm{g} / \mathrm{L} & \text { Blank }\end{array}$

$\begin{array}{rlr}0.5 & \mu g / L & \text { Sample } \\ 0.5 & \mu g / L & \text { Split } \\ 0.5 & \mu g / L & \text { Sample } \\ 0.5 & \mu g / L & \text { Split } \\ 0.5 & \mu g / L & \text { Sample }\end{array}$

$\begin{array}{rrr}0.5 & \mu \mathrm{g} / \mathrm{L} & \text { Sample } \\ 0.5 & \mu \mathrm{g} / \mathrm{L} & \text { Sample } \\ 0.5 & \mu \mathrm{g} / \mathrm{L} & \text { Split }\end{array}$

$\begin{array}{lll}0.5 & \mu \mathrm{g} / \mathrm{L} & \text { Blank } \\ 0.5 & \mu \mathrm{g} / \mathrm{L} & \text { Blank }\end{array}$




\section{Analyte \\ Volatile Organic Compounds}

\begin{tabular}{|c|c|c|c|c|c|c|}
\hline \multirow[t]{2}{*}{ Methyl tert-Butyl Ether } & $25 \mathrm{FTU}$ & $\begin{array}{l}1 / 18 / 01 \\
1 / 18 / 01\end{array}$ & $\begin{array}{l}\text { ND } \\
\text { ND }\end{array}$ & $\begin{array}{l}0.5 \\
0.5\end{array}$ & $\begin{array}{l}\mu \mathrm{g} / \mathrm{L} \\
\mu \mathrm{g} / \mathrm{L}\end{array}$ & $\begin{array}{r}\text { Sample } \\
\text { Split }\end{array}$ \\
\hline & 77 FTU & $1 / 12 / 01$ & ND & 0.5 & $\mu \mathrm{g} / \mathrm{L}$ & Sample \\
\hline \multirow[t]{10}{*}{ Methylene chloride } & $25 \mathrm{FTU}$ & $1 / 18 / 01$ & ND & 1 & $\mu \mathrm{g} / \mathrm{L}$ & Sample \\
\hline & & $1 / 18 / 01$ & ND & 1 & $\mu \mathrm{g} / \mathrm{L}$ & Split \\
\hline & & $4 / 18 / 01$ & ND & 1 & $\mu \mathrm{g} / \mathrm{L}$ & Sample \\
\hline & & $4 / 18 / 01$ & ND & 1 & $\mu \mathrm{g} / \mathrm{L}$ & Split \\
\hline & & $12 / 3 / 01$ & ND & 1 & $\mu \mathrm{g} / \mathrm{L}$ & Sample \\
\hline & 77 FTU & $1 / 12 / 01$ & ND & 1 & $\mu \mathrm{g} / \mathrm{L}$ & Sample \\
\hline & & $10 / 23 / 01$ & ND & 1 & $\mu \mathrm{g} / \mathrm{L}$ & Sample \\
\hline & & $10 / 23 / 01$ & ND & 1 & $\mu \mathrm{g} / \mathrm{L}$ & Split \\
\hline & Field Blank & $4 / 18 / 01$ & ND & 1 & $\mu \mathrm{g} / \mathrm{L}$ & Blank \\
\hline & & $12 / 3 / 01$ & ND & 1 & $\mu \mathrm{g} / \mathrm{L}$ & Blank \\
\hline \multirow[t]{7}{*}{ Naphthalene } & $25 \mathrm{FTU}$ & $4 / 18 / 01$ & ND & 0.5 & $\mu \mathrm{g} / \mathrm{L}$ & Sample \\
\hline & & $4 / 18 / 01$ & ND & 0.5 & $\mu \mathrm{g} / \mathrm{L}$ & Split \\
\hline & & $12 / 3 / 01$ & ND & 0.5 & $\mu \mathrm{g} / \mathrm{L}$ & Samp \\
\hline & 77 FTU & $10 / 23 / 01$ & ND & 0.5 & $\mu \mathrm{g} / \mathrm{L}$ & Sample \\
\hline & & $10 / 23 / 01$ & ND & 0.5 & $\mu \mathrm{g} / \mathrm{L}$ & Split \\
\hline & Field Blank & $4 / 18 / 01$ & ND & 0.5 & $\mu \mathrm{g} / \mathrm{L}$ & Blank \\
\hline & & $12 / 3 / 01$ & ND & 0.5 & $\mu \mathrm{g} / \mathrm{L}$ & Blank \\
\hline \multirow[t]{7}{*}{ Styrene } & $25 \mathrm{FTU}$ & $4 / 18 / 01$ & ND & 0.5 & $\mu \mathrm{g} / \mathrm{L}$ & Sample \\
\hline & & $4 / 18 / 01$ & ND & 0.5 & $\mu \mathrm{g} / \mathrm{L}$ & Split \\
\hline & & $12 / 3 / 01$ & ND & 0.5 & $\mu \mathrm{g} / \mathrm{L}$ & Sample \\
\hline & 77 FTU & 10/23/01 & ND & 0.5 & $\mu \mathrm{g} / \mathrm{L}$ & Sample \\
\hline & & $10 / 23 / 01$ & ND & 0.5 & $\mu \mathrm{g} / \mathrm{L}$ & \\
\hline & Field Blank & $4 / 18 / 01$ & ND & 0.5 & $\mu \mathrm{g} / \mathrm{L}$ & Blank \\
\hline & & $12 / 3 / 01$ & ND & 0.5 & $\mu \mathrm{g} / \mathrm{L}$ & Blank \\
\hline \multirow[t]{6}{*}{ Tetrachloroethene } & 25 FTU & $1 / 18 / 01$ & ND & 0.5 & $\mu \mathrm{g} / \mathrm{L}$ & Sample \\
\hline & & $1 / 18 / 01$ & ND & 0.5 & $\mu \mathrm{g} / \mathrm{L}$ & Split \\
\hline & & $4 / 18 / 01$ & ND & 0.5 & $\mu \mathrm{g} / \mathrm{L}$ & Sample \\
\hline & & $4 / 18 / 01$ & ND & 0.5 & $\mu \mathrm{g} / \mathrm{L}$ & Split \\
\hline & & $12 / 3 / 01$ & ND & 0.5 & $\mu g / L$ & Sample \\
\hline & 77 FTU & $1 / 12 / 01$ & ND & 0.5 & $\mu$ & Sample \\
\hline
\end{tabular}

MDA

Date Result or PQL

Units QC Type plit Split ple

le

Split

ank

ank ple sin 


\begin{tabular}{|c|c|c|c|c|c|c|}
\hline Analyte & Location & Date & Result & $\begin{array}{r}\text { MDA } \\
\text { or PQL } \\
\end{array}$ & Units & QC Type \\
\hline \multicolumn{7}{|c|}{ Volatile Organic Compounds } \\
\hline \multirow{4}{*}{$\begin{array}{l}\text { Tetrachloroethene } \\
\text { cont. }\end{array}$} & 77 FTU & $10 / 23 / 01$ & ND & 0.5 & $\mu g / L$ & Sample \\
\hline & & $10 / 23 / 01$ & ND & 0.5 & $\mu g / L$ & Split \\
\hline & Field Blank & $4 / 18 / 01$ & ND & 0.5 & $\mu g / L$ & Blank \\
\hline & & $12 / 3 / 01$ & ND & 0.5 & $\mu g / L$ & Blank \\
\hline \multirow{10}{*}{ Toluene } & 25 FTU & $1 / 18 / 01$ & ND & 0.5 & $\mu g / L$ & Sample \\
\hline & & $1 / 18 / 01$ & ND & 0.5 & $\mu g / L$ & Split \\
\hline & & $4 / 18 / 01$ & ND & 0.5 & $\mu \mathrm{g} / \mathrm{L}$ & Sample \\
\hline & & $4 / 18 / 01$ & ND & 0.5 & $\mu g / L$ & Split \\
\hline & & $12 / 3 / 01$ & ND & 0.5 & $\mu g / L$ & Sample \\
\hline & 77 FTU & $1 / 12 / 01$ & ND & 0.5 & $\mu g / L$ & Sample \\
\hline & & $10 / 23 / 01$ & ND & 0.5 & $\mu \mathrm{g} / \mathrm{L}$ & Sample \\
\hline & & $10 / 23 / 01$ & ND & 0.5 & $\mu g / L$ & Split \\
\hline & Field Blank & $4 / 18 / 01$ & ND & 0.5 & $\mu \mathrm{g} / \mathrm{L}$ & Blank \\
\hline & & $12 / 3 / 01$ & ND & 0.5 & $\mu \mathrm{g} / \mathrm{L}$ & Blank \\
\hline \multirow[t]{10}{*}{ Total xylene isomers } & 25 FTU & $1 / 18 / 01$ & ND & 1 & $\mu g / L$ & Sample \\
\hline & & $1 / 18 / 01$ & ND & 1 & $\mu g / L$ & Split \\
\hline & & $4 / 18 / 01$ & ND & 1 & $\mu g / L$ & Sample \\
\hline & & $4 / 18 / 01$ & ND & 1 & $\mu \mathrm{g} / \mathrm{L}$ & Split \\
\hline & & $12 / 3 / 01$ & ND & 1 & $\mu \mathrm{g} / \mathrm{L}$ & Sample \\
\hline & 77 FTU & $1 / 12 / 01$ & ND & 1 & $\mu \mathrm{g} / \mathrm{L}$ & Sample \\
\hline & & $10 / 23 / 01$ & ND & 1 & $\mu g / L$ & Sample \\
\hline & & $10 / 23 / 01$ & ND & 1 & $\mu \mathrm{g} / \mathrm{L}$ & Split \\
\hline & Field Blank & $4 / 18 / 01$ & ND & 1 & $\mu g / L$ & Blank \\
\hline & & $12 / 3 / 01$ & ND & 1 & $\mu \mathrm{g} / \mathrm{L}$ & Blank \\
\hline \multirow[t]{10}{*}{ trans-1,2-Dichloroethene } & 25 FTU & $1 / 18 / 01$ & ND & 0.5 & $\mu g / L$ & Sample \\
\hline & & $1 / 18 / 01$ & ND & 0.5 & $\mu \mathrm{g} / \mathrm{L}$ & Split \\
\hline & & $4 / 18 / 01$ & ND & 0.5 & $\mu g / L$ & Sample \\
\hline & & $4 / 18 / 01$ & ND & 0.5 & $\mu g / L$ & Split \\
\hline & & $12 / 3 / 01$ & ND & 0.5 & $\mu g / L$ & Sample \\
\hline & 77 FTU & $1 / 12 / 01$ & ND & 0.5 & $\mu g / L$ & Sample \\
\hline & & $10 / 23 / 01$ & ND & 0.5 & $\mu \mathrm{g} / \mathrm{L}$ & Sample \\
\hline & & $10 / 23 / 01$ & ND & 0.5 & $\mu g / L$ & Split \\
\hline & Field Blank & 4/18/01 & ND & 0.5 & $\mu \mathrm{g} / \mathrm{L}$ & Blank \\
\hline & & $12 / 3 / 01$ & ND & 0.5 & $\mu g / L$ & Blank \\
\hline
\end{tabular}




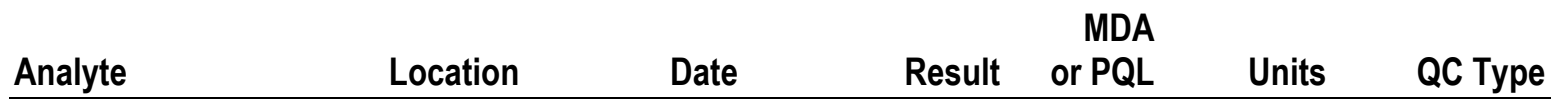

\section{Volatile Organic Compounds}

trans-1,3-Dichloropropene

\begin{tabular}{|c|c|c|c|c|c|c|}
\hline & $25 \mathrm{FTU}$ & $1 / 18 / 01$ & ND & 0.5 & $\mu \mathrm{g} / \mathrm{L}$ & Sample \\
\hline & & $1 / 18 / 01$ & ND & 0.5 & $\mu \mathrm{g} / \mathrm{L}$ & Split \\
\hline & & $4 / 18 / 01$ & ND & 0.5 & $\mu \mathrm{g} / \mathrm{L}$ & Sample \\
\hline & & $4 / 18 / 01$ & ND & 0.5 & $\mu \mathrm{g} / \mathrm{L}$ & Split \\
\hline & & $12 / 3 / 01$ & ND & 0.5 & $\mu \mathrm{g} / \mathrm{L}$ & Sample \\
\hline & 77 FTU & $1 / 12 / 01$ & ND & 0.5 & $\mu \mathrm{g} / \mathrm{L}$ & Sample \\
\hline & & $10 / 23 / 01$ & ND & 0.5 & $\mu \mathrm{g} / \mathrm{L}$ & Sample \\
\hline & & $10 / 23 / 01$ & ND & 0.5 & $\mu \mathrm{g} / \mathrm{L}$ & Split \\
\hline & Field Blank & $4 / 18 / 01$ & ND & 0.5 & $\mu \mathrm{g} / \mathrm{L}$ & Blank \\
\hline & & $12 / 3 / 01$ & ND & 0.5 & $\mu \mathrm{g} / \mathrm{L}$ & Blank \\
\hline Trichloroethene & $25 \mathrm{FTU}$ & $1 / 18 / 01$ & ND & 0.5 & $\mu \mathrm{g} / \mathrm{L}$ & Sample \\
\hline & & $1 / 18 / 01$ & ND & 0.5 & $\mu \mathrm{g} / \mathrm{L}$ & Split \\
\hline & & $4 / 18 / 01$ & ND & 0.5 & $\mu \mathrm{g} / \mathrm{L}$ & Sample \\
\hline & & $4 / 18 / 01$ & ND & 0.5 & $\mu \mathrm{g} / \mathrm{L}$ & Split \\
\hline & & $12 / 3 / 01$ & ND & 0.5 & $\mu \mathrm{g} / \mathrm{L}$ & Sample \\
\hline & 77 FTU & $1 / 12 / 01$ & ND & 0.5 & $\mu \mathrm{g} / \mathrm{L}$ & Sample \\
\hline & & 10/23/01 & ND & 0.5 & $\mu \mathrm{g} / \mathrm{L}$ & Sample \\
\hline & & 10/23/01 & ND & 0.5 & $\mu \mathrm{g} / \mathrm{L}$ & Split \\
\hline & Field Blank & $4 / 18 / 01$ & ND & 0.5 & $\mu \mathrm{g} / \mathrm{L}$ & Blank \\
\hline & & $12 / 3 / 01$ & ND & 0.5 & $\mu \mathrm{g} / \mathrm{L}$ & Blank \\
\hline Trichlorofluoromethane & $25 \mathrm{FTU}$ & $1 / 18 / 01$ & ND & 0.5 & $\mu \mathrm{g} / \mathrm{L}$ & Sample \\
\hline & & $1 / 18 / 01$ & ND & 0.5 & $\mu \mathrm{g} / \mathrm{L}$ & Split \\
\hline & & $4 / 18 / 01$ & ND & 0.5 & $\mu \mathrm{g} / \mathrm{L}$ & Sample \\
\hline & & $4 / 18 / 01$ & ND & 0.5 & $\mu \mathrm{g} / \mathrm{L}$ & Split \\
\hline & & $12 / 3 / 01$ & ND & 0.5 & $\mu \mathrm{g} / \mathrm{L}$ & Sample \\
\hline & 77 FTU & $1 / 12 / 01$ & ND & 0.5 & $\mu \mathrm{g} / \mathrm{L}$ & Sample \\
\hline & & $10 / 23 / 01$ & ND & 0.5 & $\mu \mathrm{g} / \mathrm{L}$ & Sample \\
\hline & & $10 / 23 / 01$ & ND & 0.5 & $\mu \mathrm{g} / \mathrm{L}$ & Split \\
\hline & Field Blank & $4 / 18 / 01$ & ND & 0.5 & $\mu \mathrm{g} / \mathrm{L}$ & Blank \\
\hline & & $12 / 3 / 01$ & ND & 0.5 & $\mu \mathrm{g} / \mathrm{L}$ & Blank \\
\hline Vinyl chloride & $25 \mathrm{FTU}$ & $1 / 18 / 01$ & ND & 0.5 & $\mu \mathrm{g} / \mathrm{L}$ & Sample \\
\hline & & $1 / 18 / 01$ & ND & 0.5 & $\mu \mathrm{g} / \mathrm{L}$ & Split \\
\hline & & $4 / 18 / 01$ & ND & 0.5 & $\mu \mathrm{g} / \mathrm{L}$ & Sample \\
\hline & & $4 / 18 / 01$ & ND & 0.5 & $\mu \mathrm{g} / \mathrm{L}$ & Split \\
\hline & & $12 / 3 / 01$ & ND & 0.5 & $\mu \mathrm{g} / \mathrm{L}$ & Sample \\
\hline
\end{tabular}




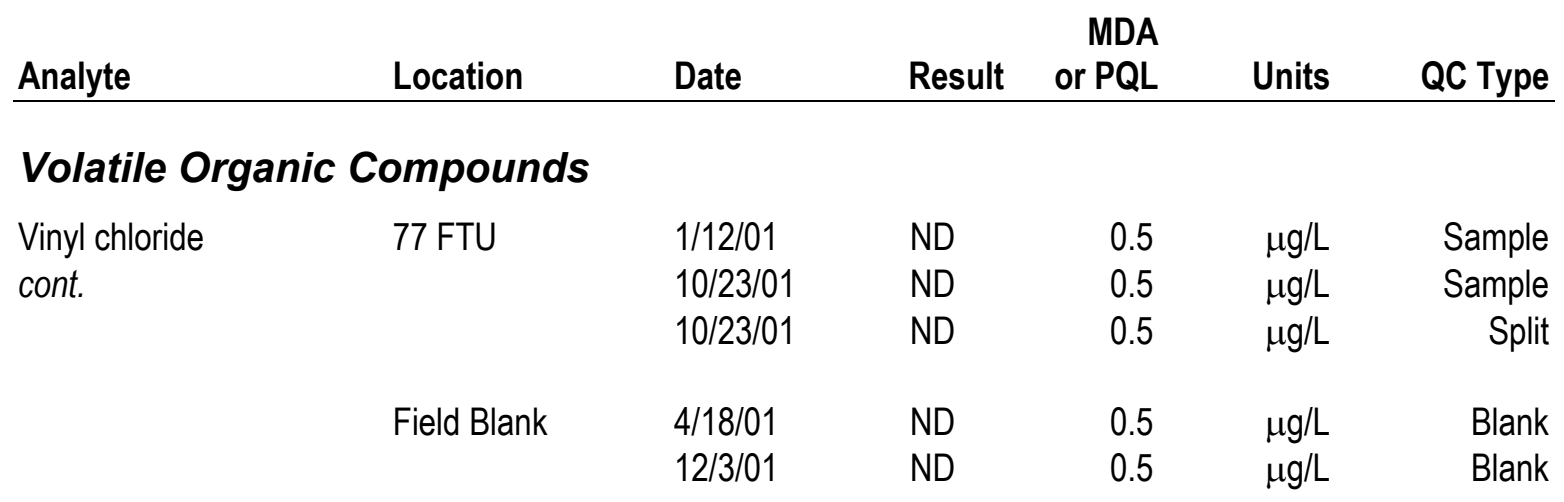


The following routine soil data are summarized and discussed in Chapter 7 (Soil and Sediment) of the Site Environmental Report for 2001 (see Volume I). Supplemental sampling data are included in the Supplemental Monitoring section of this volume and discussed in Chapter 10 (Supplemental Monitoring) of Volume I:

\begin{tabular}{|c|c|c|c|c|c|c|}
\hline Analyte & Location & Date & Result & $\begin{array}{r}\text { MDA } \\
\text { or PQL } \\
\end{array}$ & Units & QC Type \\
\hline \multicolumn{7}{|c|}{ Radiological Activity } \\
\hline \multirow[t]{4}{*}{ Actinium 228} & Building 50 & $\begin{array}{l}10 / 16 / 01 \\
10 / 16 / 01\end{array}$ & $\begin{array}{l}0.026 \\
0.022\end{array}$ & $\begin{array}{l}0.007 \\
0.009\end{array}$ & $\begin{array}{l}\mathrm{Bq} / \mathrm{g} \\
\mathrm{Bq} / \mathrm{g}\end{array}$ & $\begin{array}{r}\text { Sample } \\
\text { Duplicate }\end{array}$ \\
\hline & Building 69 & 10/16/01 & 0.015 & 0.005 & $\mathrm{~Bq} / \mathrm{g}$ & Sample \\
\hline & Building 85 & 10/16/01 & 0.019 & 0.0004 & $\mathrm{~Bq} / \mathrm{g}$ & Sample \\
\hline & ENV-B13C & 10/16/01 & 0.026 & 0.008 & $\mathrm{~Bq} / \mathrm{g}$ & Sample \\
\hline \multirow[t]{2}{*}{ Cesium 137} & Building 50 & $\begin{array}{l}10 / 16 / 01 \\
10 / 16 / 01\end{array}$ & $\begin{array}{l}0.03 \\
0.022\end{array}$ & $\begin{array}{l}0.003 \\
0.003\end{array}$ & $\begin{array}{l}\mathrm{Bq} / \mathrm{g} \\
\mathrm{Bq} / \mathrm{g}\end{array}$ & $\begin{array}{r}\text { Sample } \\
\text { Duplicate }\end{array}$ \\
\hline & ENV-B13C & $10 / 16 / 01$ & 0.015 & 0.003 & $\mathrm{~Bq} / \mathrm{g}$ & Sample \\
\hline \multirow[t]{4}{*}{ Gross alpha } & Building 50 & $\begin{array}{l}10 / 16 / 01 \\
10 / 16 / 01\end{array}$ & $\begin{array}{l}0.28 \\
0.26\end{array}$ & $\begin{array}{l}0.19 \\
0.19\end{array}$ & $\begin{array}{l}\mathrm{Bq} / \mathrm{g} \\
\mathrm{Bq} / \mathrm{g}\end{array}$ & $\begin{array}{r}\text { Sample } \\
\text { Duplicate }\end{array}$ \\
\hline & Building 69 & $10 / 16 / 01$ & ND & 0.19 & $\mathrm{~Bq} / \mathrm{g}$ & Sample \\
\hline & Building 85 & $10 / 16 / 01$ & ND & 0.19 & $\mathrm{~Bq} / \mathrm{g}$ & Sample \\
\hline & ENV-B13C & 10/16/01 & 0.34 & 0.19 & $\mathrm{~Bq} / \mathrm{g}$ & Sample \\
\hline \multirow[t]{2}{*}{ Gross beta } & Building 50 & $\begin{array}{l}10 / 16 / 01 \\
10 / 16 / 01\end{array}$ & $\begin{array}{l}0.841 \\
0.852\end{array}$ & $\begin{array}{l}0.19 \\
0.19\end{array}$ & $\begin{array}{l}\mathrm{Bq} / \mathrm{g} \\
\mathrm{Bq} / \mathrm{g}\end{array}$ & $\begin{array}{l}\text { Sample } \\
\text { Duplicate }\end{array}$ \\
\hline & Building 69 & $10 / 16 / 01$ & 0.44 & 0.19 & $\mathrm{~Bq} / \mathrm{g}$ & Sample \\
\hline
\end{tabular}




\begin{tabular}{|c|c|c|c|c|c|c|}
\hline Analyte & Location & Date & Result & $\begin{array}{r}\text { MDA } \\
\text { or PQL }\end{array}$ & Units & QC Type \\
\hline \multicolumn{7}{|c|}{ Radiological Activity } \\
\hline \multirow{2}{*}{$\begin{array}{l}\text { Gross beta } \\
\text { cont. }\end{array}$} & Building 85 & 10/16/01 & 0.44 & 0.19 & $\mathrm{~Bq} / \mathrm{g}$ & Sample \\
\hline & ENV-B13C & $10 / 16 / 01$ & 0.9 & 0.19 & $\mathrm{~Bq} / \mathrm{g}$ & Sample \\
\hline \multirow[t]{5}{*}{ Lead 214} & Building 50 & 10/16/01 & 0.0041 & 0.0009 & $\mathrm{~Bq} / \mathrm{g}$ & Sample \\
\hline & & & & & & \\
\hline & Building 69 & 10/16/01 & 0.0026 & 0.0007 & $\mathrm{~Bq} / \mathrm{g}$ & Sample \\
\hline & Building 85 & $10 / 16 / 01$ & 0.0041 & 0.0006 & $\mathrm{~Bq} / \mathrm{g}$ & Sample \\
\hline & ENV-B13C & $10 / 16 / 01$ & 0.0052 & 0.001 & $\mathrm{~Bq} / \mathrm{g}$ & Sample \\
\hline \multirow[t]{4}{*}{ Potassium 40} & Building 50 & $\begin{array}{l}10 / 16 / 01 \\
10 / 16 / 01\end{array}$ & $\begin{array}{l}0.63 \\
0.56\end{array}$ & $\begin{array}{l}0.03 \\
0.03\end{array}$ & $\begin{array}{l}\mathrm{Bq} / \mathrm{g} \\
\mathrm{Bq} / \mathrm{g}\end{array}$ & $\begin{array}{r}\text { Sample } \\
\text { Duplicate }\end{array}$ \\
\hline & Building 69 & $10 / 16 / 01$ & 0.26 & 0.015 & $\mathrm{~Bq} / \mathrm{g}$ & Sample \\
\hline & Building 85 & $10 / 16 / 01$ & 0.3 & 0.02 & $\mathrm{~Bq} / \mathrm{g}$ & Sample \\
\hline & ENV-B13C & $10 / 16 / 01$ & 0.63 & 0.04 & $\mathrm{~Bq} / \mathrm{g}$ & Sample \\
\hline \multirow[t]{4}{*}{ Radium 226} & Building 50 & $\begin{array}{l}10 / 16 / 01 \\
10 / 16 / 01\end{array}$ & $\begin{array}{l}0.0011 \\
0.0019\end{array}$ & $\begin{array}{l}0.0003 \\
0.0004\end{array}$ & $\begin{array}{l}\mathrm{Bq} / \mathrm{g} \\
\mathrm{Bq} / \mathrm{g}\end{array}$ & $\begin{array}{r}\text { Sample } \\
\text { Duplicate }\end{array}$ \\
\hline & Building 69 & $10 / 16 / 01$ & 0.00074 & 0.00019 & $\mathrm{~Bq} / \mathrm{g}$ & Sample \\
\hline & Building 85 & $10 / 16 / 01$ & 0.0015 & 0.0002 & $\mathrm{~Bq} / \mathrm{g}$ & Sample \\
\hline & ENV-B13C & $10 / 16 / 01$ & 0.0011 & 0.0004 & $\mathrm{~Bq} / \mathrm{g}$ & Sample \\
\hline \multirow[t]{4}{*}{ Tritium } & Building 50 & $\begin{array}{l}10 / 16 / 01 \\
10 / 16 / 01\end{array}$ & $\begin{array}{l}\text { ND } \\
N D\end{array}$ & $\begin{array}{l}0.007 \\
0.007\end{array}$ & $\begin{array}{l}\mathrm{Bq} / \mathrm{g} \\
\mathrm{Bq} / \mathrm{g}\end{array}$ & $\begin{array}{r}\text { Sample } \\
\text { Duplicate }\end{array}$ \\
\hline & Building 69 & 10/16/01 & ND & 0.007 & $\mathrm{~Bq} / \mathrm{g}$ & Sample \\
\hline & Building 85 & 10/16/01 & ND & 0.007 & $\mathrm{~Bq} / \mathrm{g}$ & Sample \\
\hline & ENV-B13C & 10/16/01 & ND & 0.007 & $\mathrm{~Bq} / \mathrm{g}$ & Sample \\
\hline
\end{tabular}

\section{General Indicator Parameters}

Moisture by weight

$$
\text { Building } 50
$$

$10 / 16 / 01$

$10 / 16 / 01$

$\begin{array}{ll}7.64 & 0.05 \\ 8.99 & 0.05\end{array}$
\% Sample
$\% \quad$ Duplicate




\begin{tabular}{llllll} 
Lnalyte & Location & Date & Result & or PQL & Units \\
\hline
\end{tabular}

\section{General Indicator Parameters}

Moisture by weight

cont.

Building 69

10/16/01

9.27

0.05

$\% \quad$ Sample

Building 85

10/16/01

7.72

0.05

$\% \quad$ Sample

ENV-B13C

10/16/01

12.89

0.05

$\% \quad$ Sample

$\mathrm{pH}$

Building 50

$10 / 16 / 01$

6.39

$-$

S.U.

Sample

10/16/01

6.55

S.U.

Duplicate

Building 69

10/16/01

6.43

S.U.

Sample

Building 85

10/16/01

6.33

S.U.

Sample

ENV-B13C

10/16/01

4.65

S.U.

Sample

\section{Metals and/or Minerals}

\begin{tabular}{|c|c|c|c|c|c|c|}
\hline \multirow[t]{4}{*}{ Aluminum } & Building 50 & $\begin{array}{l}10 / 16 / 01 \\
10 / 16 / 01\end{array}$ & $\begin{array}{l}21000 \\
25000\end{array}$ & $\begin{array}{l}10 \\
10\end{array}$ & $\begin{array}{l}\mathrm{mg} / \mathrm{kg} \\
\mathrm{mg} / \mathrm{kg}\end{array}$ & $\begin{array}{r}\text { Sample } \\
\text { Duplicate }\end{array}$ \\
\hline & Building 69 & $10 / 16 / 01$ & 38000 & 10 & $\mathrm{mg} / \mathrm{kg}$ & Sample \\
\hline & Building 85 & $10 / 16 / 01$ & 37000 & 10 & $\mathrm{mg} / \mathrm{kg}$ & Sample \\
\hline & ENV-B13C & $10 / 16 / 01$ & 18000 & 10 & $\mathrm{mg} / \mathrm{kg}$ & Sample \\
\hline \multirow[t]{4}{*}{ Antimony } & Building 50 & $\begin{array}{l}10 / 16 / 01 \\
10 / 16 / 01\end{array}$ & $\begin{array}{l}N D \\
N D\end{array}$ & $\begin{array}{l}1 \\
1\end{array}$ & $\begin{array}{l}\mathrm{mg} / \mathrm{kg} \\
\mathrm{mg} / \mathrm{kg}\end{array}$ & $\begin{array}{l}\text { Sample } \\
\text { Duplicate }\end{array}$ \\
\hline & Building 69 & $10 / 16 / 01$ & ND & 1 & $\mathrm{mg} / \mathrm{kg}$ & Sample \\
\hline & Building 85 & $10 / 16 / 01$ & ND & 1 & $\mathrm{mg} / \mathrm{kg}$ & Sample \\
\hline & ENV-B13C & $10 / 16 / 01$ & ND & 1 & $\mathrm{mg} / \mathrm{kg}$ & Sample \\
\hline \multirow[t]{4}{*}{ Arsenic } & Building 50 & $\begin{array}{l}10 / 16 / 01 \\
10 / 16 / 01\end{array}$ & $\begin{array}{l}8 \\
9\end{array}$ & $\begin{array}{l}1 \\
1\end{array}$ & $\begin{array}{l}\mathrm{mg} / \mathrm{kg} \\
\mathrm{mg} / \mathrm{kg}\end{array}$ & $\begin{array}{r}\text { Sample } \\
\text { Duplicate }\end{array}$ \\
\hline & Building 69 & 10/16/01 & 3 & 1 & $\mathrm{mg} / \mathrm{kg}$ & Sample \\
\hline & Building 85 & $10 / 16 / 01$ & 3 & 1 & $\mathrm{mg} / \mathrm{kg}$ & Sample \\
\hline & ENV-B13C & $10 / 16 / 01$ & 8 & 1 & $\mathrm{mg} / \mathrm{kg}$ & Sample \\
\hline
\end{tabular}




\section{Analyte Location}

Barium

Building 50

$$
10 / 16 / 01
$$

210

10/16/01

240

5

5

MDA

Result or PQL Units QC Type

\begin{tabular}{|c|c|c|c|c|c|c|}
\hline & Building 69 & $10 / 16 / 01$ & 120 & 5 & $\mathrm{mg} / \mathrm{kg}$ & Sample \\
\hline & Building 85 & 10/16/01 & 140 & 5 & $\mathrm{mg} / \mathrm{kg}$ & Sample \\
\hline & ENV-B13C & $10 / 16 / 01$ & 150 & 5 & $\mathrm{mg} / \mathrm{kg}$ & Sample \\
\hline Beryllium & Building 50 & $\begin{array}{l}10 / 16 / 01 \\
10 / 16 / 01\end{array}$ & $\begin{array}{l}\text { ND } \\
\text { ND }\end{array}$ & $\begin{array}{l}1 \\
1\end{array}$ & $\begin{array}{l}\mathrm{mg} / \mathrm{kg} \\
\mathrm{mg} / \mathrm{kg}\end{array}$ & $\begin{array}{l}\text { Sample } \\
\text { Duplicate }\end{array}$ \\
\hline & Building 69 & 10/16/01 & ND & 1 & $\mathrm{mg} / \mathrm{kg}$ & Sample \\
\hline & Building 85 & 10/16/01 & ND & 1 & $\mathrm{mg} / \mathrm{kg}$ & Sample \\
\hline & ENV-B13C & 10/16/01 & ND & 1 & $\mathrm{mg} / \mathrm{kg}$ & Sample \\
\hline Boron & Building 50 & $\begin{array}{l}10 / 16 / 01 \\
10 / 16 / 01\end{array}$ & $\begin{array}{l}18 \\
20\end{array}$ & $\begin{array}{l}10 \\
10\end{array}$ & $\begin{array}{l}\mathrm{mg} / \mathrm{kg} \\
\mathrm{mg} / \mathrm{kg}\end{array}$ & $\begin{array}{r}\text { Sample } \\
\text { Duplicate }\end{array}$ \\
\hline & Building 69 & 10/16/01 & ND & 10 & $\mathrm{mg} / \mathrm{kg}$ & Sample \\
\hline & Building 85 & $10 / 16 / 01$ & ND & 10 & $\mathrm{mg} / \mathrm{kg}$ & Sample \\
\hline & ENV-B13C & 10/16/01 & 10 & 10 & $\mathrm{mg} / \mathrm{kg}$ & Sample \\
\hline Cadmium & Building 50 & $\begin{array}{l}10 / 16 / 01 \\
10 / 16 / 01\end{array}$ & $\begin{array}{l}2.6 \\
2.9\end{array}$ & $\begin{array}{l}1 \\
1\end{array}$ & $\begin{array}{l}\mathrm{mg} / \mathrm{kg} \\
\mathrm{mg} / \mathrm{kg}\end{array}$ & $\begin{array}{r}\text { Sample } \\
\text { Duplicate }\end{array}$ \\
\hline & Building 69 & 10/16/01 & 1.2 & 1 & $\mathrm{mg} / \mathrm{kg}$ & Sample \\
\hline & Building 85 & $10 / 16 / 01$ & 1.1 & 1 & $\mathrm{mg} / \mathrm{kg}$ & Sample \\
\hline & ENV-B13C & 10/16/01 & ND & 1 & $\mathrm{mg} / \mathrm{kg}$ & Sample \\
\hline Chromium & Building 50 & $\begin{array}{l}10 / 16 / 01 \\
10 / 16 / 01\end{array}$ & $\begin{array}{l}41 \\
48\end{array}$ & $\begin{array}{l}5 \\
5\end{array}$ & $\begin{array}{l}\mathrm{mg} / \mathrm{kg} \\
\mathrm{mg} / \mathrm{kg}\end{array}$ & $\begin{array}{r}\text { Sample } \\
\text { Duplicate }\end{array}$ \\
\hline & Building 69 & 10/16/01 & 110 & 5 & $\mathrm{mg} / \mathrm{kg}$ & Sample \\
\hline & Building 85 & 10/16/01 & 100 & 5 & $\mathrm{mg} / \mathrm{kg}$ & Sample \\
\hline & ENV-B13C & $10 / 16 / 01$ & 36 & 5 & $\mathrm{mg} / \mathrm{kg}$ & Sample \\
\hline
\end{tabular}




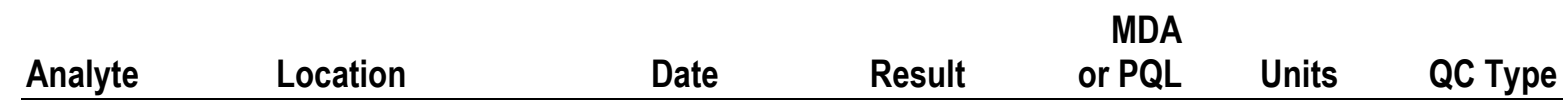

\section{Metals and/or Minerals}

\begin{tabular}{|c|c|c|c|c|c|c|}
\hline \multirow[t]{4}{*}{ Cobalt } & Building 50 & $\begin{array}{l}10 / 16 / 01 \\
10 / 16 / 01\end{array}$ & $\begin{array}{l}10 \\
11\end{array}$ & $\begin{array}{l}5 \\
5\end{array}$ & $\begin{array}{l}\mathrm{mg} / \mathrm{kg} \\
\mathrm{mg} / \mathrm{kg}\end{array}$ & $\begin{array}{r}\text { Sample } \\
\text { Duplicate }\end{array}$ \\
\hline & Building 69 & $10 / 16 / 01$ & 23 & 5 & $\mathrm{mg} / \mathrm{kg}$ & Sample \\
\hline & Building 85 & $10 / 16 / 01$ & 21 & 5 & $\mathrm{mg} / \mathrm{kg}$ & Sample \\
\hline & ENV-B13C & $10 / 16 / 01$ & 8.8 & 5 & $\mathrm{mg} / \mathrm{kg}$ & Sample \\
\hline \multirow[t]{4}{*}{ Copper } & Building 50 & $\begin{array}{l}10 / 16 / 01 \\
10 / 16 / 01\end{array}$ & $\begin{array}{l}140 \\
150\end{array}$ & $\begin{array}{l}5 \\
5\end{array}$ & $\begin{array}{l}\mathrm{mg} / \mathrm{kg} \\
\mathrm{mg} / \mathrm{kg}\end{array}$ & $\begin{array}{c}\text { Sample } \\
\text { Duplicate }\end{array}$ \\
\hline & Building 69 & $10 / 16 / 01$ & 24 & 5 & $\mathrm{mg} / \mathrm{kg}$ & Sample \\
\hline & Building 85 & $10 / 16 / 01$ & 35 & 5 & $\mathrm{mg} / \mathrm{kg}$ & Samp \\
\hline & ENV-B13C & 10/16/01 & 25 & 5 & $\mathrm{mg} / \mathrm{kg}$ & Sample \\
\hline \multirow[t]{4}{*}{ Iron } & Building 50 & $\begin{array}{l}10 / 16 / 01 \\
10 / 16 / 01\end{array}$ & $\begin{array}{l}25000 \\
21000\end{array}$ & $\begin{array}{l}5 \\
5\end{array}$ & $\begin{array}{l}\mathrm{mg} / \mathrm{kg} \\
\mathrm{mg} / \mathrm{kg}\end{array}$ & $\begin{array}{r}\text { Duplicate } \\
\text { Sample }\end{array}$ \\
\hline & Building 69 & $10 / 16 / 01$ & 40000 & 5 & $\mathrm{mg} / \mathrm{kg}$ & Sample \\
\hline & Building 85 & $10 / 16 / 01$ & 36000 & 5 & $\mathrm{mg} / \mathrm{kg}$ & Sample \\
\hline & ENV-B13C & $10 / 16 / 01$ & 20000 & 5 & $\mathrm{mg} / \mathrm{kg}$ & Sample \\
\hline \multirow[t]{4}{*}{ Lead } & Building 50 & $\begin{array}{l}10 / 16 / 01 \\
10 / 16 / 01\end{array}$ & $\begin{array}{l}130 \\
140\end{array}$ & $\begin{array}{l}10 \\
10\end{array}$ & $\begin{array}{l}\mathrm{mg} / \mathrm{kg} \\
\mathrm{mg} / \mathrm{kg}\end{array}$ & $\begin{array}{r}\text { Sample } \\
\text { Duplicate }\end{array}$ \\
\hline & Building 69 & 10/16/01 & ND & 10 & $\mathrm{mg} / \mathrm{kg}$ & Sample \\
\hline & Building 85 & $10 / 16 / 01$ & ND & 10 & $\mathrm{mg} / \mathrm{kg}$ & Sample \\
\hline & ENV-B13C & $10 / 16 / 01$ & 93 & 10 & $\mathrm{mg} / \mathrm{kg}$ & Sample \\
\hline \multirow[t]{4}{*}{ Manganese } & Building 50 & $\begin{array}{l}10 / 16 / 01 \\
10 / 16 / 01\end{array}$ & $\begin{array}{l}1600 \\
1700\end{array}$ & $\begin{array}{l}1 \\
1\end{array}$ & $\begin{array}{l}\mathrm{mg} / \mathrm{kg} \\
\mathrm{mg} / \mathrm{kg}\end{array}$ & $\begin{array}{r}\text { Sample } \\
\text { Duplicate }\end{array}$ \\
\hline & Building 69 & 10/16/01 & 720 & 1 & $\mathrm{mg} / \mathrm{kg}$ & Sample \\
\hline & Building 85 & $10 / 16 / 01$ & 810 & 1 & $\mathrm{mg} / \mathrm{kg}$ & Samr \\
\hline & ENV-B13C & $10 / 16 / 01$ & 390 & 1 & $\mathrm{mg} / \mathrm{kg}$ & Samp \\
\hline
\end{tabular}




\section{Analyte Location}

\begin{tabular}{|c|c|c|c|c|c|c|}
\hline \multirow[t]{4}{*}{ Mercury } & Building 50 & $\begin{array}{l}10 / 16 / 01 \\
10 / 16 / 01\end{array}$ & $\begin{array}{l}0.27 \\
0.23\end{array}$ & $\begin{array}{l}0.05 \\
0.05\end{array}$ & $\begin{array}{l}\mathrm{mg} / \mathrm{kg} \\
\mathrm{mg} / \mathrm{kg}\end{array}$ & $\begin{array}{r}\text { Sample } \\
\text { Duplicate }\end{array}$ \\
\hline & Building 69 & 10/16/01 & ND & 0.05 & $\mathrm{mg} / \mathrm{kg}$ & Sample \\
\hline & Building 85 & 10/16/01 & 0.058 & 0.05 & $\mathrm{mg} / \mathrm{kg}$ & Sample \\
\hline & ENV-B13C & 10/16/01 & 0.12 & 0.05 & $\mathrm{mg} / \mathrm{kg}$ & Sample \\
\hline \multirow[t]{4}{*}{ Molybdenum } & Building 50 & $\begin{array}{l}10 / 16 / 01 \\
10 / 16 / 01\end{array}$ & $\begin{array}{l}\text { ND } \\
\text { ND }\end{array}$ & $\begin{array}{l}5 \\
5\end{array}$ & $\begin{array}{l}\mathrm{mg} / \mathrm{kg} \\
\mathrm{mg} / \mathrm{kg}\end{array}$ & $\begin{array}{r}\text { Sample } \\
\text { Duplicate }\end{array}$ \\
\hline & Building 69 & 10/16/01 & ND & 5 & $\mathrm{mg} / \mathrm{kg}$ & Sample \\
\hline & Building 85 & 10/16/01 & ND & 5 & $\mathrm{mg} / \mathrm{kg}$ & Sample \\
\hline & ENV-B13C & $10 / 16 / 01$ & ND & 5 & $\mathrm{mg} / \mathrm{kg}$ & Sample \\
\hline \multirow[t]{4}{*}{ Nickel } & Building 50 & $\begin{array}{l}10 / 16 / 01 \\
10 / 16 / 01\end{array}$ & $\begin{array}{l}45 \\
49\end{array}$ & $\begin{array}{l}10 \\
10\end{array}$ & $\begin{array}{l}\mathrm{mg} / \mathrm{kg} \\
\mathrm{mg} / \mathrm{kg}\end{array}$ & $\begin{array}{r}\text { Sample } \\
\text { Duplicate }\end{array}$ \\
\hline & Building 69 & 10/16/01 & 65 & 10 & $\mathrm{mg} / \mathrm{kg}$ & Sample \\
\hline & Building 85 & $10 / 16 / 01$ & 60 & 10 & $\mathrm{mg} / \mathrm{kg}$ & Sample \\
\hline & ENV-B13C & 10/16/01 & 30 & 10 & $\mathrm{mg} / \mathrm{kg}$ & Sample \\
\hline \multirow[t]{4}{*}{ Selenium } & Building 50 & $\begin{array}{l}10 / 16 / 01 \\
10 / 16 / 01\end{array}$ & $\begin{array}{l}\text { ND } \\
\text { ND }\end{array}$ & $\begin{array}{l}2 \\
2\end{array}$ & $\begin{array}{l}\mathrm{mg} / \mathrm{kg} \\
\mathrm{mg} / \mathrm{kg}\end{array}$ & $\begin{array}{r}\text { Sample } \\
\text { Duplicate }\end{array}$ \\
\hline & Building 69 & 10/16/01 & ND & 2 & $\mathrm{mg} / \mathrm{kg}$ & Sample \\
\hline & Building 85 & $10 / 16 / 01$ & ND & 2 & $\mathrm{mg} / \mathrm{kg}$ & Sample \\
\hline & ENV-B13C & 10/16/01 & ND & 2 & $\mathrm{mg} / \mathrm{kg}$ & Sample \\
\hline \multirow[t]{4}{*}{ Silver } & Building 50 & $\begin{array}{l}10 / 16 / 01 \\
10 / 16 / 01\end{array}$ & $\begin{array}{l}\text { ND } \\
\text { ND }\end{array}$ & $\begin{array}{l}2 \\
2\end{array}$ & $\begin{array}{l}\mathrm{mg} / \mathrm{kg} \\
\mathrm{mg} / \mathrm{kg}\end{array}$ & $\begin{array}{r}\text { Sample } \\
\text { Duplicate }\end{array}$ \\
\hline & Building 69 & 10/16/01 & ND & 2 & $\mathrm{mg} / \mathrm{kg}$ & Sample \\
\hline & Building 85 & 10/16/01 & ND & 2 & $\mathrm{mg} / \mathrm{kg}$ & Sample \\
\hline & ENV-B13C & $10 / 16 / 01$ & ND & 2 & $\mathrm{mg} / \mathrm{kg}$ & Sample \\
\hline
\end{tabular}




\begin{tabular}{|c|c|c|c|c|c|c|}
\hline Analyte & Location & Date & Result & $\begin{array}{r}\text { MDA } \\
\text { or PQL } \\
\end{array}$ & Units & QC Type \\
\hline \multicolumn{7}{|c|}{ Metals and/or Minerals } \\
\hline \multirow[t]{4}{*}{ Thallium } & Building 50 & $\begin{array}{l}10 / 16 / 01 \\
10 / 16 / 01\end{array}$ & $\begin{array}{l}\text { ND } \\
\text { ND }\end{array}$ & $\begin{array}{l}5 \\
5\end{array}$ & $\begin{array}{l}\mathrm{mg} / \mathrm{kg} \\
\mathrm{mg} / \mathrm{kg}\end{array}$ & $\begin{array}{r}\text { Sample } \\
\text { Duplicate }\end{array}$ \\
\hline & Building 69 & $10 / 16 / 01$ & ND & 5 & $\mathrm{mg} / \mathrm{kg}$ & Sample \\
\hline & Building 85 & $10 / 16 / 01$ & ND & 5 & $\mathrm{mg} / \mathrm{kg}$ & Sample \\
\hline & ENV-B13C & $10 / 16 / 01$ & ND & 5 & $\mathrm{mg} / \mathrm{kg}$ & Sample \\
\hline \multirow[t]{4}{*}{ Vanadium } & Building 50 & $\begin{array}{l}10 / 16 / 01 \\
10 / 16 / 01\end{array}$ & $\begin{array}{l}52 \\
62\end{array}$ & $\begin{array}{l}5 \\
5\end{array}$ & $\begin{array}{l}\mathrm{mg} / \mathrm{kg} \\
\mathrm{mg} / \mathrm{kg}\end{array}$ & $\begin{array}{r}\text { Sample } \\
\text { Duplicate }\end{array}$ \\
\hline & Building 69 & $10 / 16 / 01$ & 100 & 5 & $\mathrm{mg} / \mathrm{kg}$ & Sample \\
\hline & Building 85 & $10 / 16 / 01$ & 110 & 5 & $\mathrm{mg} / \mathrm{kg}$ & Sample \\
\hline & ENV-B13C & 10/16/01 & 44 & 5 & $\mathrm{mg} / \mathrm{kg}$ & Sample \\
\hline \multirow[t]{4}{*}{ Zinc } & Building 50 & $\begin{array}{l}10 / 16 / 01 \\
10 / 16 / 01\end{array}$ & $\begin{array}{l}170 \\
180\end{array}$ & $\begin{array}{l}5 \\
5\end{array}$ & $\begin{array}{l}\mathrm{mg} / \mathrm{kg} \\
\mathrm{mg} / \mathrm{kg}\end{array}$ & $\begin{array}{r}\text { Sample } \\
\text { Duplicate }\end{array}$ \\
\hline & Building 69 & $10 / 16 / 01$ & 66 & 5 & $\mathrm{mg} / \mathrm{kg}$ & Sample \\
\hline & Building 85 & $10 / 16 / 01$ & 57 & 5 & $\mathrm{mg} / \mathrm{kg}$ & Sample \\
\hline & ENV-B13C & $10 / 16 / 01$ & 220 & 5 & $\mathrm{mg} / \mathrm{kg}$ & Sample \\
\hline
\end{tabular}


The following routine sediment data are summarized and discussed in Chapter 7 (Soil and Sediment) of the Site Environmental Report for 2001 (see Volume I). Supplemental sampling data are included in the Supplemental Monitoring section of this volume and discussed in Chapter 10 (Supplemental Monitoring) of Volume I:

\begin{tabular}{|c|c|c|c|c|c|c|}
\hline Analyte & Location & Date & Result & $\begin{array}{r}\text { MDA } \\
\text { or PQL } \\
\end{array}$ & Units & QC Type \\
\hline \multicolumn{7}{|c|}{ Radiological Activity } \\
\hline \multirow[t]{4}{*}{ Actinium 228} & Chicken Creek-Main & $\begin{array}{l}10 / 15 / 01 \\
10 / 15 / 01\end{array}$ & $\begin{array}{l}0.015 \\
\text { ND }\end{array}$ & $\begin{array}{l}0.004 \\
0.003\end{array}$ & $\begin{array}{l}\mathrm{Bq} / \mathrm{g} \\
\mathrm{Bq} / \mathrm{g}\end{array}$ & $\begin{array}{r}\text { Sample } \\
\text { Duplicate }\end{array}$ \\
\hline & Chicken Creek-Trib & $10 / 15 / 01$ & 0.015 & 0.004 & $\mathrm{~Bq} / \mathrm{g}$ & Sample \\
\hline & N. Fork Strawberry-Main & $10 / 15 / 01$ & 0.019 & 0.004 & $\mathrm{~Bq} / \mathrm{g}$ & Sample \\
\hline & N. Fork Strawberry-Trib & $10 / 15 / 01$ & 0.022 & 0.004 & $\mathrm{~Bq} / \mathrm{g}$ & Sample \\
\hline Cesium 137 & Chicken Creek-Trib & $10 / 15 / 01$ & 0.0011 & 0.001 & $\mathrm{~Bq} / \mathrm{g}$ & Sample \\
\hline \multirow[t]{4}{*}{ Gross alpha } & Chicken Creek-Main & $\begin{array}{l}10 / 15 / 01 \\
10 / 15 / 01\end{array}$ & $\begin{array}{l}0.3 \\
0.25\end{array}$ & $\begin{array}{l}0.19 \\
0.19\end{array}$ & $\begin{array}{l}\mathrm{Bq} / \mathrm{g} \\
\mathrm{Bq} / \mathrm{g}\end{array}$ & $\begin{array}{r}\text { Sample } \\
\text { Duplicate }\end{array}$ \\
\hline & Chicken Creek-Trib & $10 / 15 / 01$ & 0.23 & 0.19 & $\mathrm{~Bq} / \mathrm{g}$ & Sample \\
\hline & N. Fork Strawberry-Main & $10 / 15 / 01$ & ND & 0.19 & $\mathrm{~Bq} / \mathrm{g}$ & Sample \\
\hline & N. Fork Strawberry-Trib & 10/15/01 & 0.23 & 0.19 & $\mathrm{~Bq} / \mathrm{g}$ & Sample \\
\hline \multirow[t]{4}{*}{ Gross beta } & Chicken Creek-Main & $\begin{array}{l}10 / 15 / 01 \\
10 / 15 / 01\end{array}$ & $\begin{array}{l}0.63 \\
0.87\end{array}$ & $\begin{array}{l}0.19 \\
0.19\end{array}$ & $\begin{array}{l}\mathrm{Bq} / \mathrm{g} \\
\mathrm{Bq} / \mathrm{g}\end{array}$ & $\begin{array}{r}\text { Sample } \\
\text { Duplicate }\end{array}$ \\
\hline & Chicken Creek-Trib & $10 / 15 / 01$ & 0.867 & 0.19 & $\mathrm{~Bq} / \mathrm{g}$ & Sample \\
\hline & N. Fork Strawberry-Main & $10 / 15 / 01$ & 0.67 & 0.19 & $\mathrm{~Bq} / \mathrm{g}$ & Sample \\
\hline & N. Fork Strawberry-Trib & $10 / 15 / 01$ & 0.67 & 0.19 & $\mathrm{~Bq} / \mathrm{g}$ & Sample \\
\hline
\end{tabular}




\begin{tabular}{|c|c|c|c|c|c|c|}
\hline Analyte & Location & Date & Result & $\begin{array}{r}\text { MDA } \\
\text { or PQL }\end{array}$ & Units & QC Type \\
\hline \multicolumn{7}{|c|}{ Radiological Activity } \\
\hline \multirow[t]{4}{*}{ Lead 214} & Chicken Creek-Main & $\begin{array}{l}10 / 15 / 01 \\
10 / 15 / 01\end{array}$ & $\begin{array}{l}0.003 \\
0.0026\end{array}$ & $\begin{array}{l}0.0004 \\
0.0004\end{array}$ & $\begin{array}{l}\mathrm{Bq} / \mathrm{g} \\
\mathrm{Bq} / \mathrm{g}\end{array}$ & $\begin{array}{r}\text { Sample } \\
\text { Duplicate }\end{array}$ \\
\hline & Chicken Creek-Trib & $10 / 15 / 01$ & 0.0026 & 0.0004 & $\mathrm{~Bq} / \mathrm{g}$ & Sample \\
\hline & N. Fork Strawberry-Main & $10 / 15 / 01$ & 0.0033 & 0.0004 & $\mathrm{~Bq} / \mathrm{g}$ & Sample \\
\hline & N. Fork Strawberry-Trib & $10 / 15 / 01$ & 0.0033 & 0.0004 & $\mathrm{~Bq} / \mathrm{g}$ & Sample \\
\hline \multirow[t]{4}{*}{ Potassium 40} & Chicken Creek-Main & $\begin{array}{l}10 / 15 / 01 \\
10 / 15 / 01\end{array}$ & $\begin{array}{l}0.41 \\
0.37\end{array}$ & $\begin{array}{l}0.011 \\
0.011\end{array}$ & $\begin{array}{l}\mathrm{Bq} / \mathrm{g} \\
\mathrm{Bq} / \mathrm{g}\end{array}$ & $\begin{array}{r}\text { Sample } \\
\text { Duplicate }\end{array}$ \\
\hline & Chicken Creek-Trib & $10 / 15 / 01$ & 0.37 & 0.007 & $\mathrm{~Bq} / \mathrm{g}$ & Sample \\
\hline & N. Fork Strawberry-Main & $10 / 15 / 01$ & 0.44 & 0.011 & $\mathrm{~Bq} / \mathrm{g}$ & Sample \\
\hline & N. Fork Strawberry-Trib & $10 / 15 / 01$ & 0.41 & 0.015 & $\mathrm{~Bq} / \mathrm{g}$ & Sample \\
\hline \multirow[t]{4}{*}{ Radium 226} & Chicken Creek-Main & $\begin{array}{l}10 / 15 / 01 \\
10 / 15 / 01\end{array}$ & $\begin{array}{l}0.0011 \\
0.0011\end{array}$ & $\begin{array}{l}0.00015 \\
0.0004\end{array}$ & $\begin{array}{l}\mathrm{Bq} / \mathrm{g} \\
\mathrm{Bq} / \mathrm{g}\end{array}$ & $\begin{array}{c}\text { Sample } \\
\text { Duplicate }\end{array}$ \\
\hline & Chicken Creek-Trib & 10/15/01 & 0.0011 & 0.00011 & $\mathrm{~Bq} / \mathrm{g}$ & Sample \\
\hline & N. Fork Strawberry-Main & $10 / 15 / 01$ & 0.0011 & 0.00015 & $\mathrm{~Bq} / \mathrm{g}$ & Sample \\
\hline & N. Fork Strawberry-Trib & $10 / 15 / 01$ & 0.0015 & 0.00015 & $\mathrm{~Bq} / \mathrm{g}$ & Sample \\
\hline \multirow[t]{4}{*}{ Tritium } & Chicken Creek-Main & $\begin{array}{l}10 / 15 / 01 \\
10 / 15 / 01\end{array}$ & $\begin{array}{l}\text { ND } \\
\text { ND }\end{array}$ & $\begin{array}{l}0.007 \\
0.007\end{array}$ & $\begin{array}{l}\mathrm{Bq} / \mathrm{g} \\
\mathrm{Bq} / \mathrm{g}\end{array}$ & $\begin{array}{r}\text { Sample } \\
\text { Duplicate }\end{array}$ \\
\hline & Chicken Creek-Trib & $10 / 15 / 01$ & ND & 0.007 & $\mathrm{~Bq} / \mathrm{g}$ & Sample \\
\hline & N. Fork Strawberry-Main & $10 / 15 / 01$ & ND & 0.007 & $\mathrm{~Bq} / \mathrm{g}$ & Sample \\
\hline & N. Fork Strawberry-Trib & $10 / 15 / 01$ & ND & 0.007 & $\mathrm{~Bq} / \mathrm{g}$ & Sample \\
\hline
\end{tabular}

\section{General Indicator Parameters}

Moisture by weight

$\begin{array}{lccccr}\text { Chicken Creek-Main } & 10 / 15 / 01 & 18.24 & 0.05 & \% & \text { Sample } \\ & 10 / 15 / 01 & 13.36 & 0.05 & \% & \text { Duplicate } \\ \text { Chicken Creek-Trib } & 10 / 15 / 01 & 20.44 & 0.05 & \% & \text { Sample } \\ \text { N. Fork Strawberry-Main } & 10 / 15 / 01 & 20.44 & 0.05 & \% & \text { Sample }\end{array}$




\begin{tabular}{|c|c|c|c|c|c|c|}
\hline Analyte & Location & Date & Result & $\begin{array}{r}\text { MDA } \\
\text { or PQL } \\
\end{array}$ & Units & QC Type \\
\hline \multicolumn{7}{|c|}{ General Indicator Parameters } \\
\hline \multicolumn{7}{|c|}{ Moisture by weight } \\
\hline cont. & N. Fork Strawberry-Trib & 10/15/01 & 51.89 & 0.05 & $\%$ & Sample \\
\hline \multirow[t]{4}{*}{$\mathrm{pH}$} & Chicken Creek-Main & $\begin{array}{l}10 / 15 / 01 \\
10 / 15 / 01\end{array}$ & $\begin{array}{l}7.68 \\
7.78\end{array}$ & - & $\begin{array}{l}\text { S.U. } \\
\text { S.U. }\end{array}$ & $\begin{array}{r}\text { Sample } \\
\text { Duplicate }\end{array}$ \\
\hline & Chicken Creek-Trib & 10/15/01 & 7.55 & - & S.U. & Sample \\
\hline & N. Fork Strawberry-Main & $10 / 15 / 01$ & 7.25 & - & S.U. & Sample \\
\hline & N. Fork Strawberry-Trib & $10 / 15 / 01$ & 7.6 & - & S.U. & Sample \\
\hline
\end{tabular}

\section{Metals and/or Minerals}

\begin{tabular}{|c|c|c|c|c|c|c|}
\hline \multirow[t]{4}{*}{ Aluminum } & Chicken Creek-Main & $\begin{array}{l}10 / 15 / 01 \\
10 / 15 / 01\end{array}$ & $\begin{array}{r}8800 \\
14000\end{array}$ & $\begin{array}{l}5 \\
5\end{array}$ & $\begin{array}{l}\mathrm{mg} / \mathrm{kg} \\
\mathrm{mg} / \mathrm{kg}\end{array}$ & $\begin{array}{r}\text { Sample } \\
\text { Duplicate }\end{array}$ \\
\hline & Chicken Creek-Trib & $10 / 15 / 01$ & 11000 & 5 & $\mathrm{mg} / \mathrm{kg}$ & Sample \\
\hline & N. Fork Strawberry-Main & $10 / 15 / 01$ & 8500 & 5 & $\mathrm{mg} / \mathrm{kg}$ & Sample \\
\hline & N. Fork Strawberry-Trib & $10 / 15 / 01$ & 9400 & 5 & $\mathrm{mg} / \mathrm{kg}$ & Sample \\
\hline \multirow[t]{4}{*}{ Antimony } & Chicken Creek-Main & $\begin{array}{l}10 / 15 / 01 \\
10 / 15 / 01\end{array}$ & $\begin{array}{l}\text { ND } \\
\text { ND }\end{array}$ & $\begin{array}{l}1 \\
0.2\end{array}$ & $\begin{array}{l}\mathrm{mg} / \mathrm{kg} \\
\mathrm{mg} / \mathrm{kg}\end{array}$ & $\begin{array}{c}\text { Sample } \\
\text { Duplicate }\end{array}$ \\
\hline & Chicken Creek-Trib & $10 / 15 / 01$ & ND & 1 & $\mathrm{mg} / \mathrm{kg}$ & Sample \\
\hline & N. Fork Strawberry-Main & $10 / 15 / 01$ & ND & 1 & $\mathrm{mg} / \mathrm{kg}$ & Sample \\
\hline & N. Fork Strawberry-Trib & $10 / 15 / 01$ & ND & 1 & $\mathrm{mg} / \mathrm{kg}$ & Samr \\
\hline \multirow[t]{4}{*}{ Arsenic } & Chicken Creek-Main & $\begin{array}{l}10 / 15 / 01 \\
10 / 15 / 01\end{array}$ & $\begin{array}{l}2 \\
3.4\end{array}$ & $\begin{array}{l}0.5 \\
0.5\end{array}$ & $\begin{array}{l}\mathrm{mg} / \mathrm{kg} \\
\mathrm{mg} / \mathrm{kg}\end{array}$ & $\begin{array}{c}\text { Sample } \\
\text { Duplicate }\end{array}$ \\
\hline & Chicken Creek-Trib & $10 / 15 / 01$ & 2 & 0.5 & $\mathrm{mg} / \mathrm{kg}$ & Sampl \\
\hline & N. Fork Strawberry-Main & $10 / 15 / 01$ & 3 & 0.5 & $\mathrm{mg} / \mathrm{kg}$ & Samp \\
\hline & N. Fork Strawberry-Trib & $10 / 15 / 01$ & 3.6 & 0.5 & $\mathrm{mg} / \mathrm{kg}$ & Samp \\
\hline \multirow[t]{2}{*}{ Barium } & Chicken Creek-Main & $\begin{array}{l}10 / 15 / 01 \\
10 / 15 / 01\end{array}$ & $\begin{array}{l}100 \\
130\end{array}$ & $\begin{array}{l}5 \\
5\end{array}$ & $\begin{array}{l}\mathrm{mg} / \mathrm{kg} \\
\mathrm{mg} / \mathrm{kg}\end{array}$ & $\begin{array}{l}\text { Sample } \\
\text { Duplicate }\end{array}$ \\
\hline & Chicken Creek-Trib & $10 / 15 / 01$ & 310 & 5 & $\mathrm{mg} / \mathrm{kg}$ & Sa \\
\hline
\end{tabular}




\begin{tabular}{|c|c|c|c|c|c|c|}
\hline Analyte & Location & Date & Result & $\begin{array}{r}\text { MDA } \\
\text { or } P Q L \\
\end{array}$ & Units & QC Type \\
\hline \multicolumn{7}{|c|}{ Metals and/or Minerals } \\
\hline \multirow{2}{*}{$\begin{array}{l}\text { Barium } \\
\text { cont. }\end{array}$} & N. Fork Strawberry-Main & 10/15/01 & 76 & 5 & $\mathrm{mg} / \mathrm{kg}$ & Sample \\
\hline & N. Fork Strawberry-Trib & $10 / 15 / 01$ & 77 & 5 & $\mathrm{mg} / \mathrm{kg}$ & Sample \\
\hline \multirow[t]{5}{*}{ Beryllium } & Chicken Creek-Main & $10 / 15 / 01$ & ND & 0.5 & $\mathrm{mg} / \mathrm{kg}$ & Sample \\
\hline & & $10 / 15 / 01$ & ND & 0.5 & $\mathrm{mg} / \mathrm{kg}$ & Duplicate \\
\hline & Chicken Creek-Trib & 10/15/01 & ND & 0.5 & $\mathrm{mg} / \mathrm{kg}$ & Sample \\
\hline & N. Fork Strawberry-Main & $10 / 15 / 01$ & ND & 0.5 & $\mathrm{mg} / \mathrm{kg}$ & Sample \\
\hline & N. Fork Strawberry-Trib & $10 / 15 / 01$ & ND & 0.5 & $\mathrm{mg} / \mathrm{kg}$ & Sample \\
\hline \multirow[t]{5}{*}{ Boron } & Chicken Creek-Main & 10/15/01 & ND & 5 & $\mathrm{mg} / \mathrm{kg}$ & Sample \\
\hline & & 10/15/01 & 15 & 5 & $\mathrm{mg} / \mathrm{kg}$ & Duplicate \\
\hline & Chicken Creek-Trib & 10/15/01 & 7.1 & 5 & $\mathrm{mg} / \mathrm{kg}$ & Sample \\
\hline & N. Fork Strawberry-Main & $10 / 15 / 01$ & ND & 5 & $\mathrm{mg} / \mathrm{kg}$ & Sample \\
\hline & N. Fork Strawberry-Trib & $10 / 15 / 01$ & ND & 5 & $\mathrm{mg} / \mathrm{kg}$ & Sample \\
\hline \multirow[t]{5}{*}{ Cadmium } & Chicken Creek-Main & $10 / 15 / 01$ & ND & 1 & $\mathrm{mg} / \mathrm{kg}$ & Sample \\
\hline & & 10/15/01 & ND & 1 & $\mathrm{mg} / \mathrm{kg}$ & Duplicate \\
\hline & Chicken Creek-Trib & $10 / 15 / 01$ & ND & 1 & $\mathrm{mg} / \mathrm{kg}$ & Sample \\
\hline & N. Fork Strawberry-Main & 10/15/01 & ND & 1 & $\mathrm{mg} / \mathrm{kg}$ & Sample \\
\hline & N. Fork Strawberry-Trib & 10/15/01 & ND & 1 & $\mathrm{mg} / \mathrm{kg}$ & Sample \\
\hline \multirow[t]{5}{*}{ Chromium } & Chicken Creek-Main & $10 / 15 / 01$ & 30 & 5 & $\mathrm{mg} / \mathrm{kg}$ & Sample \\
\hline & & 10/15/01 & 48 & 5 & $\mathrm{mg} / \mathrm{kg}$ & Duplicate \\
\hline & Chicken Creek-Trib & $10 / 15 / 01$ & 39 & 5 & $\mathrm{mg} / \mathrm{kg}$ & Sample \\
\hline & N. Fork Strawberry-Main & 10/15/01 & 20 & 5 & $\mathrm{mg} / \mathrm{kg}$ & Sample \\
\hline & N. Fork Strawberry-Trib & $10 / 15 / 01$ & 22 & 5 & $\mathrm{mg} / \mathrm{kg}$ & Sample \\
\hline \multirow[t]{3}{*}{ Cobalt } & Chicken Creek-Main & 10/15/01 & 9.7 & 5 & $\mathrm{mg} / \mathrm{kg}$ & Sample \\
\hline & & 10/15/01 & 8.8 & 5 & $\mathrm{mg} / \mathrm{kg}$ & Duplicate \\
\hline & Chicken Creek-Trib & $10 / 15 / 01$ & 7.9 & 5 & $\mathrm{mg} / \mathrm{kg}$ & Sample \\
\hline
\end{tabular}




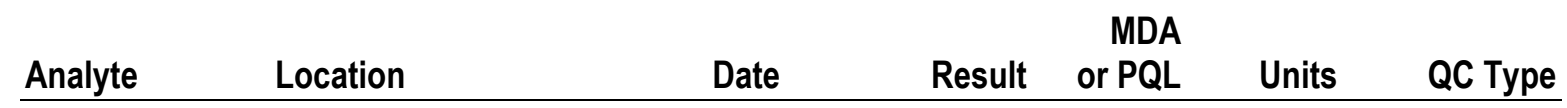

\section{Metals and/or Minerals}

Cobalt

N. Fork Strawberry-Main $\quad$ 10/15/01

$5.1 \quad 5$

$\mathrm{mg} / \mathrm{kg}$

Sample cont.

\begin{tabular}{|c|c|c|c|c|c|c|}
\hline & N. Fork Strawberry-Trib & $10 / 15 / 01$ & 6.1 & 5 & $\mathrm{mg} / \mathrm{kg}$ & Sample \\
\hline \multirow[t]{5}{*}{ Copper } & Chicken Creek-Main & 10/15/01 & 20 & 5 & $\mathrm{mg} / \mathrm{kg}$ & Sample \\
\hline & & 10/15/01 & 21 & 5 & $\mathrm{mg} / \mathrm{kg}$ & Duplicate \\
\hline & Chicken Creek-Trib & 10/15/01 & 19 & 5 & $\mathrm{mg} / \mathrm{kg}$ & Sample \\
\hline & N. Fork Strawberry-Main & 10/15/01 & 13 & 5 & $\mathrm{mg} / \mathrm{kg}$ & Sample \\
\hline & N. Fork Strawberry-Trib & 10/15/01 & 96 & 5 & $\mathrm{mg} / \mathrm{kg}$ & Sample \\
\hline \multirow[t]{5}{*}{ Iron } & Chicken Creek-Main & 10/15/01 & 14000 & 2 & $\mathrm{mg} / \mathrm{kg}$ & Sample \\
\hline & & $10 / 15 / 01$ & 20000 & 2 & $\mathrm{mg} / \mathrm{kg}$ & Duplicate \\
\hline & Chicken Creek-Trib & 10/15/01 & 14000 & 2 & $\mathrm{mg} / \mathrm{kg}$ & Sample \\
\hline & N. Fork Strawberry-Main & 10/15/01 & 17000 & 2 & $\mathrm{mg} / \mathrm{kg}$ & Sample \\
\hline & N. Fork Strawberry-Trib & $10 / 15 / 01$ & 16000 & 2 & $\mathrm{mg} / \mathrm{kg}$ & Sample \\
\hline \multirow[t]{5}{*}{ Lead } & Chicken Creek-Main & $10 / 15 / 01$ & ND & 10 & $\mathrm{mg} / \mathrm{kg}$ & Sample \\
\hline & & 10/15/01 & 41 & 10 & $\mathrm{mg} / \mathrm{kg}$ & Duplicate \\
\hline & Chicken Creek-Trib & 10/15/01 & 15 & 10 & $\mathrm{mg} / \mathrm{kg}$ & Sample \\
\hline & N. Fork Strawberry-Main & 10/15/01 & 20 & 10 & $\mathrm{mg} / \mathrm{kg}$ & Sample \\
\hline & N. Fork Strawberry-Trib & $10 / 15 / 01$ & ND & 10 & $\mathrm{mg} / \mathrm{kg}$ & Sample \\
\hline \multirow[t]{5}{*}{ Manganese } & Chicken Creek-Main & 10/15/01 & 390 & 0.5 & $\mathrm{mg} / \mathrm{kg}$ & Sample \\
\hline & & 10/15/01 & 320 & 0.5 & $\mathrm{mg} / \mathrm{kg}$ & Duplicate \\
\hline & Chicken Creek-Trib & $10 / 15 / 01$ & 490 & 0.5 & $\mathrm{mg} / \mathrm{kg}$ & Sample \\
\hline & N. Fork Strawberry-Main & $10 / 15 / 01$ & 340 & 0.5 & $\mathrm{mg} / \mathrm{kg}$ & Sample \\
\hline & N. Fork Strawberry-Trib & $10 / 15 / 01$ & 480 & 0.5 & $\mathrm{mg} / \mathrm{kg}$ & Sample \\
\hline \multirow[t]{3}{*}{ Mercury } & Chicken Creek-Main & 10/15/01 & ND & 0.05 & $\mathrm{mg} / \mathrm{kg}$ & Sample \\
\hline & & 10/15/01 & ND & 0.05 & $\mathrm{mg} / \mathrm{kg}$ & Duplicate \\
\hline & Chicken Creek-Trib & $10 / 15 / 01$ & ND & 0.05 & $\mathrm{mg} / \mathrm{kg}$ & Sample \\
\hline
\end{tabular}




\begin{tabular}{|c|c|c|c|c|c|c|}
\hline Analyte & Location & Date & Result & $\begin{array}{r}\text { MDA } \\
\text { or } P Q L \\
\end{array}$ & Units & QC Type \\
\hline \multicolumn{7}{|c|}{ Metals and/or Minerals } \\
\hline \multirow{2}{*}{$\begin{array}{l}\text { Mercury } \\
\text { cont. }\end{array}$} & N. Fork Strawberry-Main & 10/15/01 & 0.19 & 0.05 & $\mathrm{mg} / \mathrm{kg}$ & Sample \\
\hline & N. Fork Strawberry-Trib & $10 / 15 / 01$ & 0.13 & 0.05 & $\mathrm{mg} / \mathrm{kg}$ & Sample \\
\hline \multirow[t]{5}{*}{ Molybdenum } & Chicken Creek-Main & $10 / 15 / 01$ & ND & 5 & $\mathrm{mg} / \mathrm{kg}$ & Sample \\
\hline & & 10/15/01 & ND & 5 & $\mathrm{mg} / \mathrm{kg}$ & Duplicate \\
\hline & Chicken Creek-Trib & $10 / 15 / 01$ & ND & 5 & $\mathrm{mg} / \mathrm{kg}$ & Sample \\
\hline & N. Fork Strawberry-Main & $10 / 15 / 01$ & ND & 5 & $\mathrm{mg} / \mathrm{kg}$ & Sample \\
\hline & N. Fork Strawberry-Trib & $10 / 15 / 01$ & ND & 5 & $\mathrm{mg} / \mathrm{kg}$ & Sample \\
\hline \multirow[t]{5}{*}{ Nickel } & Chicken Creek-Main & $10 / 15 / 01$ & 41 & 10 & $\mathrm{mg} / \mathrm{kg}$ & Sample \\
\hline & & 10/15/01 & 43 & 10 & $\mathrm{mg} / \mathrm{kg}$ & Duplicate \\
\hline & Chicken Creek-Trib & $10 / 15 / 01$ & 42 & 10 & $\mathrm{mg} / \mathrm{kg}$ & Sample \\
\hline & N. Fork Strawberry-Main & $10 / 15 / 01$ & 16 & 10 & $\mathrm{mg} / \mathrm{kg}$ & Sample \\
\hline & N. Fork Strawberry-Trib & $10 / 15 / 01$ & 18 & 10 & $\mathrm{mg} / \mathrm{kg}$ & Sample \\
\hline \multirow[t]{5}{*}{ Selenium } & Chicken Creek-Main & $10 / 15 / 01$ & ND & 2 & $\mathrm{mg} / \mathrm{kg}$ & Sample \\
\hline & & $10 / 15 / 01$ & ND & 2 & $\mathrm{mg} / \mathrm{kg}$ & Duplicate \\
\hline & Chicken Creek-Trib & $10 / 15 / 01$ & ND & 2 & $\mathrm{mg} / \mathrm{kg}$ & Sample \\
\hline & N. Fork Strawberry-Main & $10 / 15 / 01$ & ND & 2 & $\mathrm{mg} / \mathrm{kg}$ & Sample \\
\hline & N. Fork Strawberry-Trib & $10 / 15 / 01$ & ND & 2 & $\mathrm{mg} / \mathrm{kg}$ & Sample \\
\hline \multirow[t]{5}{*}{ Silver } & Chicken Creek-Main & $10 / 15 / 01$ & ND & 2 & $\mathrm{mg} / \mathrm{kg}$ & Sample \\
\hline & & 10/15/01 & ND & 2 & $\mathrm{mg} / \mathrm{kg}$ & Duplicate \\
\hline & Chicken Creek-Trib & $10 / 15 / 01$ & ND & 2 & $\mathrm{mg} / \mathrm{kg}$ & Sample \\
\hline & N. Fork Strawberry-Main & $10 / 15 / 01$ & ND & 2 & $\mathrm{mg} / \mathrm{kg}$ & Sample \\
\hline & N. Fork Strawberry-Trib & $10 / 15 / 01$ & ND & 2 & $\mathrm{mg} / \mathrm{kg}$ & Sample \\
\hline \multirow[t]{3}{*}{ Thallium } & Chicken Creek-Main & $10 / 15 / 01$ & ND & 2 & $\mathrm{mg} / \mathrm{kg}$ & Sample \\
\hline & & 10/15/01 & ND & 2 & $\mathrm{mg} / \mathrm{kg}$ & Duplicate \\
\hline & Chicken Creek-Trib & $10 / 15 / 01$ & ND & 2 & $\mathrm{mg} / \mathrm{kg}$ & Sample \\
\hline
\end{tabular}




\begin{tabular}{|c|c|c|c|c|c|c|}
\hline Analyte & Location & Date & Result & $\begin{array}{r}\text { MDA } \\
\text { or } P Q L \\
\end{array}$ & Units & QC Type \\
\hline \multicolumn{7}{|c|}{ Metals and/or Minerals } \\
\hline \multirow{2}{*}{$\begin{array}{l}\text { Thallium } \\
\text { cont. }\end{array}$} & N. Fork Strawberry-Main & $10 / 15 / 01$ & ND & 2 & $\mathrm{mg} / \mathrm{kg}$ & Sample \\
\hline & N. Fork Strawberry-Trib & $10 / 15 / 01$ & ND & 2 & $\mathrm{mg} / \mathrm{kg}$ & Sample \\
\hline \multirow[t]{4}{*}{ Vanadium } & Chicken Creek-Main & $\begin{array}{l}10 / 15 / 01 \\
10 / 15 / 01\end{array}$ & $\begin{array}{l}25 \\
40\end{array}$ & $\begin{array}{l}5 \\
5\end{array}$ & $\begin{array}{l}\mathrm{mg} / \mathrm{kg} \\
\mathrm{mg} / \mathrm{kg}\end{array}$ & $\begin{array}{l}\text { Sample } \\
\text { Duplicate }\end{array}$ \\
\hline & Chicken Creek-Trib & $10 / 15 / 01$ & 31 & 5 & $\mathrm{mg} / \mathrm{kg}$ & Sample \\
\hline & N. Fork Strawberry-Main & $10 / 15 / 01$ & 36 & 5 & $\mathrm{mg} / \mathrm{kg}$ & Sample \\
\hline & N. Fork Strawberry-Trib & $10 / 15 / 01$ & 35 & 5 & $\mathrm{mg} / \mathrm{kg}$ & Sample \\
\hline \multirow[t]{4}{*}{ Zinc } & Chicken Creek-Main & $\begin{array}{l}10 / 15 / 01 \\
10 / 15 / 01\end{array}$ & $\begin{array}{r}99 \\
120\end{array}$ & $\begin{array}{l}5 \\
5\end{array}$ & $\begin{array}{l}\mathrm{mg} / \mathrm{kg} \\
\mathrm{mg} / \mathrm{kg}\end{array}$ & $\begin{array}{r}\text { Sample } \\
\text { Duplicate }\end{array}$ \\
\hline & Chicken Creek-Trib & $10 / 15 / 01$ & 86 & 5 & $\mathrm{mg} / \mathrm{kg}$ & Sample \\
\hline & N. Fork Strawberry-Main & $10 / 15 / 01$ & 110 & 5 & $\mathrm{mg} / \mathrm{kg}$ & Sample \\
\hline & N. Fork Strawberry-Trib & $10 / 15 / 01$ & 140 & 5 & $\mathrm{mg} / \mathrm{kg}$ & Sample \\
\hline
\end{tabular}

\section{Petroleum Hydrocarbons}

Diesel Fuel Chicken Creek-Main

58

10/15/01

40

4

4

$\mathrm{mg} / \mathrm{kg}$

Sample $\mathrm{mg} / \mathrm{kg} \quad$ Duplicate

Chicken Creek-Trib

10/15/01

38

4

$\mathrm{mg} / \mathrm{kg}$

Sample

N. Fork Strawberry-Main

$10 / 15 / 01$

30

4

$\mathrm{mg} / \mathrm{kg}$

Sample

N. Fork Strawberry-Trib

10/15/01

23

4

$\mathrm{mg} / \mathrm{kg}$

Sample

Oil and Grease

Chicken Creek-Main

Chicken Creek-Trib

N. Fork Strawberry-Main

N. Fork Strawberry-Trib
$10 / 15 / 01$

10/15/01

10/15/01

10/15/01

10/15/01
600

59

290

370

570
20

20

$\mathrm{mg} / \mathrm{kg}$

$\mathrm{mg} / \mathrm{kg}$

$\mathrm{mg} / \mathrm{kg}$

$\mathrm{mg} / \mathrm{kg}$

$\mathrm{mg} / \mathrm{kg}$ 20

20

20
Sample Duplicate
Sample

Sample

Sample 


\section{Analyte \\ Location \\ Polychlorinated Biphenyls}

Date

Result or PQL

Units QC Type

\begin{tabular}{|c|c|c|c|c|c|c|}
\hline \multirow[t]{4}{*}{ PCB 1016} & Chicken Creek-Main & $\begin{array}{l}10 / 15 / 01 \\
10 / 15 / 01\end{array}$ & $\begin{array}{l}\text { ND } \\
\text { ND }\end{array}$ & $\begin{array}{l}0.01 \\
0.01\end{array}$ & $\begin{array}{l}\mathrm{mg} / \mathrm{kg} \\
\mathrm{mg} / \mathrm{kg}\end{array}$ & $\begin{array}{l}\text { Sample } \\
\text { Duplicate }\end{array}$ \\
\hline & Chicken Creek-Trib & $10 / 15 / 01$ & ND & 0.01 & $\mathrm{mg} / \mathrm{kg}$ & Sample \\
\hline & N. Fork Strawberry-Main & $10 / 15 / 01$ & ND & 0.01 & $\mathrm{mg} / \mathrm{kg}$ & Sample \\
\hline & N. Fork Strawberry-Trib & $10 / 15 / 01$ & ND & 0.01 & $\mathrm{mg} / \mathrm{kg}$ & Sample \\
\hline \multirow[t]{4}{*}{ PCB 1221} & Chicken Creek-Main & $\begin{array}{l}10 / 15 / 01 \\
10 / 15 / 01\end{array}$ & $\begin{array}{l}\text { ND } \\
\text { ND }\end{array}$ & $\begin{array}{l}0.01 \\
0.01\end{array}$ & $\begin{array}{l}\mathrm{mg} / \mathrm{kg} \\
\mathrm{mg} / \mathrm{kg}\end{array}$ & $\begin{array}{l}\text { Sample } \\
\text { Duplicate }\end{array}$ \\
\hline & Chicken Creek-Trib & $10 / 15 / 01$ & ND & 0.01 & $\mathrm{mg} / \mathrm{kg}$ & Sample \\
\hline & N. Fork Strawberry-Main & 10/15/01 & ND & 0.01 & $\mathrm{mg} / \mathrm{kg}$ & Sample \\
\hline & N. Fork Strawberry-Trib & $10 / 15 / 01$ & ND & 0.01 & $\mathrm{mg} / \mathrm{kg}$ & Sample \\
\hline \multirow[t]{4}{*}{ PCB 1232} & Chicken Creek-Main & $\begin{array}{l}10 / 15 / 01 \\
10 / 15 / 01\end{array}$ & $\begin{array}{l}\text { ND } \\
\text { ND }\end{array}$ & $\begin{array}{l}0.01 \\
0.01\end{array}$ & $\begin{array}{l}\mathrm{mg} / \mathrm{kg} \\
\mathrm{mg} / \mathrm{kg}\end{array}$ & $\begin{array}{r}\text { Sample } \\
\text { Duplicate }\end{array}$ \\
\hline & Chicken Creek-Trib & 10/15/01 & ND & 0.01 & $\mathrm{mg} / \mathrm{kg}$ & Sample \\
\hline & N. Fork Strawberry-Main & $10 / 15 / 01$ & ND & 0.01 & $\mathrm{mg} / \mathrm{kg}$ & Sample \\
\hline & N. Fork Strawberry-Trib & $10 / 15 / 01$ & ND & 0.01 & $\mathrm{mg} / \mathrm{kg}$ & Sample \\
\hline \multirow[t]{4}{*}{ PCB 1242} & Chicken Creek-Main & $\begin{array}{l}10 / 15 / 01 \\
10 / 15 / 01\end{array}$ & $\begin{array}{l}\text { ND } \\
\text { ND }\end{array}$ & $\begin{array}{l}0.01 \\
0.01\end{array}$ & $\begin{array}{l}\mathrm{mg} / \mathrm{kg} \\
\mathrm{mg} / \mathrm{kg}\end{array}$ & $\begin{array}{r}\text { Sample } \\
\text { Duplicate }\end{array}$ \\
\hline & Chicken Creek-Trib & $10 / 15 / 01$ & ND & 0.01 & $\mathrm{mg} / \mathrm{kg}$ & Sample \\
\hline & N. Fork Strawberry-Main & 10/15/01 & ND & 0.01 & $\mathrm{mg} / \mathrm{kg}$ & Sample \\
\hline & N. Fork Strawberry-Trib & $10 / 15 / 01$ & ND & 0.01 & $\mathrm{mg} / \mathrm{kg}$ & Sample \\
\hline \multirow[t]{4}{*}{ PCB 1248} & Chicken Creek-Main & $\begin{array}{l}10 / 15 / 01 \\
10 / 15 / 01\end{array}$ & $\begin{array}{l}\text { ND } \\
\text { ND }\end{array}$ & $\begin{array}{l}0.01 \\
0.01\end{array}$ & $\begin{array}{l}\mathrm{mg} / \mathrm{kg} \\
\mathrm{mg} / \mathrm{kg}\end{array}$ & $\begin{array}{r}\text { Sample } \\
\text { Duplicate }\end{array}$ \\
\hline & Chicken Creek-Trib & $10 / 15 / 01$ & ND & 0.01 & $\mathrm{mg} / \mathrm{kg}$ & Sample \\
\hline & N. Fork Strawberry-Main & $10 / 15 / 01$ & ND & 0.01 & $\mathrm{mg} / \mathrm{kg}$ & Sample \\
\hline & N. Fork Strawberry-Trib & 10/15/01 & ND & 0.01 & $\mathrm{mg} / \mathrm{kg}$ & Sample \\
\hline
\end{tabular}




\begin{tabular}{|c|c|c|c|c|c|c|}
\hline Analyte & Location & Date & Result & $\begin{array}{r}\text { MDA } \\
\text { or PQL } \\
\end{array}$ & Units & QC Type \\
\hline \multicolumn{7}{|c|}{ Polychlorinated Biphenyls } \\
\hline \multirow[t]{4}{*}{ PCB 1254} & Chicken Creek-Main & $\begin{array}{l}10 / 15 / 01 \\
10 / 15 / 01\end{array}$ & $\begin{array}{l}\text { ND } \\
\text { ND }\end{array}$ & $\begin{array}{l}0.01 \\
0.01\end{array}$ & $\begin{array}{l}\mathrm{mg} / \mathrm{kg} \\
\mathrm{mg} / \mathrm{kg}\end{array}$ & $\begin{array}{c}\text { Sample } \\
\text { Duplicate }\end{array}$ \\
\hline & Chicken Creek-Trib & 10/15/01 & ND & 0.01 & $\mathrm{mg} / \mathrm{kg}$ & Sample \\
\hline & N. Fork Strawberry-Main & 10/15/01 & ND & 0.01 & $\mathrm{mg} / \mathrm{kg}$ & Sample \\
\hline & N. Fork Strawberry-Trib & 10/15/01 & ND & 0.01 & $\mathrm{mg} / \mathrm{kg}$ & Sample \\
\hline \multirow[t]{4}{*}{ PCB 1260} & Chicken Creek-Main & $\begin{array}{l}10 / 15 / 01 \\
10 / 15 / 01\end{array}$ & $\begin{array}{l}0.012 \\
N D\end{array}$ & $\begin{array}{l}0.01 \\
0.01\end{array}$ & $\begin{array}{l}\mathrm{mg} / \mathrm{kg} \\
\mathrm{mg} / \mathrm{kg}\end{array}$ & $\begin{array}{r}\text { Sample } \\
\text { Duplicate }\end{array}$ \\
\hline & Chicken Creek-Trib & $10 / 15 / 01$ & ND & 0.01 & $\mathrm{mg} / \mathrm{kg}$ & Sample \\
\hline & N. Fork Strawberry-Main & $10 / 15 / 01$ & ND & 0.01 & $\mathrm{mg} / \mathrm{kg}$ & Sample \\
\hline & N. Fork Strawberry-Trib & $10 / 15 / 01$ & 0.011 & 0.01 & $\mathrm{mg} / \mathrm{kg}$ & Sample \\
\hline \multirow[t]{4}{*}{ Total PCBs } & Chicken Creek-Main & $\begin{array}{l}10 / 15 / 01 \\
10 / 15 / 01\end{array}$ & $\begin{array}{l}0.012 \\
N D\end{array}$ & $\begin{array}{l}0.01 \\
0.01\end{array}$ & $\begin{array}{l}\mathrm{mg} / \mathrm{kg} \\
\mathrm{mg} / \mathrm{kg}\end{array}$ & $\begin{array}{l}\text { Sample } \\
\text { Duplicate }\end{array}$ \\
\hline & Chicken Creek-Trib & $10 / 15 / 01$ & ND & 0.01 & $\mathrm{mg} / \mathrm{kg}$ & Sample \\
\hline & N. Fork Strawberry-Main & $10 / 15 / 01$ & ND & 0.01 & $\mathrm{mg} / \mathrm{kg}$ & Sample \\
\hline & N. Fork Strawberry-Trib & $10 / 15 / 01$ & 0.011 & 0.01 & $\mathrm{mg} / \mathrm{kg}$ & Sample \\
\hline
\end{tabular}




\section{Vegetation}

The following routine vegetation data are summarized and discussed in Chapter 8 (Vegetation and Foodstuffs) of the Site Environmental Report for 2001 (see Volume I). Supplemental sampling data are included in the Supplemental Monitoring section of this volume and discussed in Chapter 10 (Supplemental Monitoring) of Volume I:

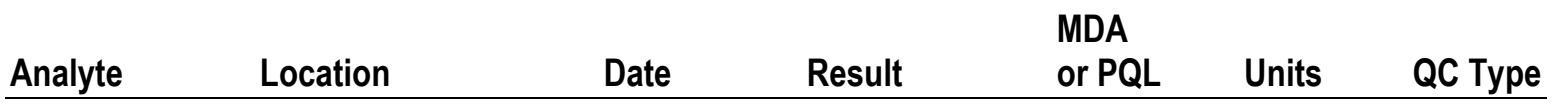

\section{Radiological Activity}

Tritium, Free Water

$\begin{array}{lccccc}\text { B75 Tree A-Core } & 5 / 10 / 01 & 0.0396 & 0.019 & \mathrm{~Bq} / \mathrm{g} & \text { Sample } \\ \text { B75 Tree B-Core } & 5 / 10 / 01 & 0.0415 & 0.019 & \mathrm{~Bq} / \mathrm{g} & \text { Sample } \\ \text { B75 Tree C-Core } & 5 / 10 / 01 & 0.0456 & 0.019 & \mathrm{~Bq} / \mathrm{g} & \text { Sample } \\ \text { B75 Tree D-Core } & 5 / 10 / 01 & 0.0474 & 0.019 & \mathrm{~Bq} / \mathrm{g} & \text { Sample } \\ \text { B75 Tree E-Core } & 5 / 10 / 01 & 0.0389 & 0.019 & \mathrm{~Bq} / \mathrm{g} & \text { Sample } \\ \text { B75 Tree F-Core } & 5 / 10 / 01 & 0.0526 & 0.019 & \mathrm{~Bq} / \mathrm{g} & \text { Sample } \\ & & & & & \\ \text { B75 Tree G-Core } & 5 / 10 / 01 & \mathrm{ND} & 0.019 & \mathrm{~Bq} / \mathrm{g} & \text { Sample } \\ & & & & & \\ \text { B75 Tree H-Core } & 5 / 10 / 01 & 0.0355 & 0.019 & \mathrm{~Bq} / \mathrm{g} & \text { Sample } \\ \text { B75-Tree \#1-Core } & 3 / 1 / 01 & \mathrm{ND} & 0.019 & \mathrm{~Bq} / \mathrm{g} & \text { Sample } \\ \text { B75-Tree \#1-Duff } & 3 / 1 / 01 & 0.0422 & 0.019 & \mathrm{~Bq} / \mathrm{g} & \text { Sample } \\ & & & & & \\ \text { B75-Tree \#2-Core } & 3 / 1 / 01 & \mathrm{ND} & 0.019 & \mathrm{~Bq} / \mathrm{g} & \text { Sample } \\ \text { B75-Tree \#2-Duff } & 3 / 1 / 01 & 0.019 & 0.019 & \mathrm{~Bq} / \mathrm{g} & \text { Sample } \\ \text { B75-Tree \#3-Core } & 3 / 1 / 01 & \mathrm{ND} & 0.019 & \mathrm{~Bq} / \mathrm{g} & \text { Sample } \\ \text { B75-Tree \#3-Duff } & 3 / 1 / 01 & 0.0474 & 0.019 & \mathrm{~Bq} / \mathrm{g} & \text { Sample } \\ & & & & & \\ \text { B75-Tree \#4-Core } & 3 / 1 / 01 & 0.026 & 0.019 & \mathrm{~Bq} / \mathrm{g} & \text { Sample } \\ \text { B75-Tree \#4-Duff } & 3 / 1 / 01 & 0.0336 & 0.019 & \mathrm{~Bq} / \mathrm{g} & \text { Sample }\end{array}$




\section{Analyte \\ Location \\ Radiological Activity}

Date

MDA

Tritium, Free Water cont.

$\begin{array}{llllll}\text { B76-Tree \#5-Core } & 3 / 1 / 01 & \text { ND } & 0.019 & \mathrm{~Bq} / \mathrm{g} & \text { Sample } \\ \text { B76-Tree \#5-Duff } & 3 / 1 / 01 & 0.0504 & 0.019 & \mathrm{~Bq} / \mathrm{g} & \text { Sample } \\ & & & & & \\ \text { B76-Tree \#6-Core } & 3 / 1 / 01 & 0.028 & 0.019 & \mathrm{~Bq} / \mathrm{g} & \text { Sample } \\ \text { B76-Tree \#6-Duff } & 3 / 1 / 01 & 0.0944 & 0.019 & \mathrm{~Bq} / \mathrm{g} & \text { Sample } \\ & & & & & \\ \text { B76-Tree \#7-Core } & 3 / 1 / 01 & 0.021 & 0.019 & \mathrm{~Bq} / \mathrm{g} & \text { Sample } \\ \text { B76-Tree \#7-Duff } & 3 / 1 / 01 & 0.0489 & 0.019 & \mathrm{~Bq} / \mathrm{g} & \text { Sample } \\ & & & & & \\ \text { B76-Tree \#8-Core } & 3 / 1 / 01 & 0.0415 & 0.019 & \mathrm{~Bq} / \mathrm{g} & \text { Sample } \\ \text { B76-Tree \#8-Duff } & 3 / 1 / 01 & 0.0448 & 0.019 & \mathrm{~Bq} / \mathrm{g} & \text { Sample }\end{array}$

Tritium, Organically Bound

$\begin{array}{lcclll}\text { B75-Tree \#1-Core } & 3 / 1 / 01 & \text { ND } & 0.19 & \mathrm{~Bq} / \mathrm{g} & \text { Sample } \\ \text { B75-Tree \#1-Duff } & 3 / 1 / 01 & 1.96 & 0.19 & \mathrm{~Bq} / \mathrm{g} & \text { Sample } \\ \text { B75-Tree \#2-Core } & 3 / 1 / 01 & \mathrm{ND} & 0.2 & \mathrm{~Bq} / \mathrm{g} & \text { Sample } \\ \text { B75-Tree \#2-Duff } & 3 / 1 / 01 & 0.46 & 0.19 & \mathrm{~Bq} / \mathrm{g} & \text { Sample } \\ & & & & & \\ \text { B75-Tree \#3-Core } & 3 / 1 / 01 & \mathrm{ND} & 0.2 & \mathrm{~Bq} / \mathrm{g} & \text { Sample } \\ \text { B75-Tree \#3-Duff } & 3 / 1 / 01 & 0.948 & 0.19 & \mathrm{~Bq} / \mathrm{g} & \text { Sample } \\ \text { B75-Tree \#4-Core } & 3 / 1 / 01 & \mathrm{ND} & 0.2 & \mathrm{~Bq} / \mathrm{g} & \text { Sample } \\ \text { B75-Tree \#4-Duff } & 3 / 1 / 01 & 0.948 & 0.19 & \mathrm{~Bq} / \mathrm{g} & \text { Sample } \\ & & & & & \\ \text { B76-Tree \#5-Core } & 3 / 1 / 01 & \mathrm{ND} & 0.19 & \mathrm{~Bq} / \mathrm{g} & \text { Sample } \\ \text { B76-Tree \#5-Duff } & 3 / 1 / 01 & 0.911 & 0.2 & \mathrm{~Bq} / \mathrm{g} & \text { Sample } \\ \text { B76-Tree \#6-Core } & 3 / 1 / 01 & \mathrm{ND} & 0.2 & \mathrm{~Bq} / \mathrm{g} & \text { Sample } \\ \text { B76-Tree \#6-Duff } & 3 / 1 / 01 & 1.41 & 0.19 & \mathrm{~Bq} / \mathrm{g} & \text { Sample } \\ & & & & & \\ \text { B76-Tree \#7-Core } & 3 / 1 / 01 & \mathrm{ND} & 0.2 & \mathrm{~Bq} / \mathrm{g} & \text { Sample } \\ \text { B76-Tree \#7-Duff } & 3 / 1 / 01 & 1.4 & 0.19 & \mathrm{~Bq} / \mathrm{g} & \text { Sample } \\ \text { B76-Tree \#8-Core } & 3 / 1 / 01 & \mathrm{ND} & 0.2 & \mathrm{~Bq} / \mathrm{g} & \text { Sample } \\ \text { B76-Tree \#8-Duff } & 3 / 1 / 01 & 1.07 & 0.2 & \mathrm{~Bq} / \mathrm{g} & \text { Sample } \\ \text { PMB1a1 } & 3 / 22 / 01 & \mathrm{ND} & 0.19 & \mathrm{~Bq} / \mathrm{g} & \text { Sample } \\ \text { PMB1a2 } & 3 / 22 / 01 & \mathrm{ND} & 0.19 & \mathrm{~Bq} / \mathrm{g} & \text { Sample } \\ \text { PMB1a3 } & 3 / 22 / 01 & \mathrm{ND} & 0.19 & \mathrm{~Bq} / \mathrm{g} & \text { Sample }\end{array}$




\section{Supplemental Monitoring}

The following supplemental monitoring data are summarized and discussed in Chapter 10 (Supplemental Monitoring) of the Site Environmental Report for 2001 (see Volume I):

\begin{tabular}{|c|c|c|c|c|c|c|}
\hline Analyte & Location & Date & Result & MDA or PQL & Units & QC Type \\
\hline \multicolumn{7}{|c|}{ Ambient Air Monitoring } \\
\hline \multirow[t]{30}{*}{ Tritium } & ENV-31 & $6 / 5 / 01$ & ND & 0.15 & $\mathrm{~Bq} / \mathrm{m}^{3}$ & Sample \\
\hline & & $6 / 5 / 01$ & ND & 0.15 & $\mathrm{Bg} / \mathrm{m}^{3}$ & Split \\
\hline & & $7 / 3 / 01$ & ND & 0.18 & $\mathrm{~Bq} / \mathrm{m}^{3}$ & Sample \\
\hline & & $7 / 3 / 01$ & 0.166 & 0.013 & $\mathrm{Bg} / \mathrm{m}^{3}$ & Split \\
\hline & & $8 / 7 / 01$ & ND & 0.15 & $\mathrm{~Bq} / \mathrm{m}^{3}$ & Sample \\
\hline & & $9 / 4 / 01$ & 0.32 & 0.18 & $\mathrm{~Bq} / \mathrm{m}^{3}$ & Sample \\
\hline & & $10 / 2 / 01$ & ND & 0.18 & $\mathrm{~Bq} / \mathrm{m}^{3}$ & Sample \\
\hline & & $10 / 2 / 01$ & ND & 0.18 & $\mathrm{~Bq} / \mathrm{m}^{3}$ & Split \\
\hline & & $11 / 6 / 01$ & ND & 0.15 & $\mathrm{Bg} / \mathrm{m}^{3}$ & Sample \\
\hline & & $12 / 4 / 01$ & ND & 0.19 & $\mathrm{~Bq} / \mathrm{m}^{3}$ & Sample \\
\hline & & $12 / 4 / 01$ & 0.0871 & 0.013 & $\mathrm{~Bq} / \mathrm{m}^{3}$ & Split \\
\hline & & $1 / 8 / 02$ & ND & 0.15 & $\mathrm{~Bq} / \mathrm{m}^{3}$ & Sample \\
\hline & & $1 / 8 / 02$ & 0.115 & 0.011 & $\mathrm{~Bq} / \mathrm{m}^{3}$ & Split \\
\hline & \multirow[t]{11}{*}{ ENV-44 } & $6 / 5 / 01$ & 0.16 & 0.15 & $\mathrm{~Bq} / \mathrm{m}^{3}$ & Sample \\
\hline & & $6 / 5 / 01$ & 0.19 & 0.15 & $\mathrm{~Bq} / \mathrm{m}^{3}$ & Split \\
\hline & & $7 / 3 / 01$ & 0.509 & 0.18 & $\mathrm{~Bq} / \mathrm{m}^{3}$ & Sample \\
\hline & & $8 / 7 / 01$ & ND & 0.15 & $\mathrm{~Bq} / \mathrm{m}^{3}$ & Sample \\
\hline & & $9 / 4 / 01$ & 0.21 & 0.18 & $\mathrm{~Bq} / \mathrm{m}^{3}$ & Sample \\
\hline & & $10 / 2 / 01$ & 0.32 & 0.18 & $\mathrm{~Bq} / \mathrm{m}^{3}$ & Sample \\
\hline & & $10 / 2 / 01$ & 0.6 & 0.09 & $\mathrm{~Bq} / \mathrm{m}^{3}$ & Split \\
\hline & & $11 / 6 / 01$ & 0.29 & 0.15 & $\mathrm{Bg} / \mathrm{m}^{3}$ & Sample \\
\hline & & $11 / 6 / 01$ & 0.29 & 0.15 & $\mathrm{~Bq} / \mathrm{m}^{3}$ & Split \\
\hline & & $12 / 4 / 01$ & ND & 0.2 & $\mathrm{Bg} / \mathrm{m}^{3}$ & Sample \\
\hline & & $1 / 8 / 02$ & 0.283 & 0.15 & $\mathrm{~Bq} / \mathrm{m}^{3}$ & Sample \\
\hline & \multirow[t]{6}{*}{ ENV-69 } & $6 / 5 / 01$ & 0.395 & 0.15 & $\mathrm{~Bq} / \mathrm{m}^{3}$ & Sample \\
\hline & & $6 / 5 / 01$ & 0.506 & 0.08 & $\mathrm{~Bq} / \mathrm{m}^{3}$ & Split \\
\hline & & $7 / 3 / 01$ & 0.614 & 0.18 & $\mathrm{Bg} / \mathrm{m}^{3}$ & Sample \\
\hline & & $7 / 3 / 01$ & 0.588 & 0.18 & $\mathrm{~Bq} / \mathrm{m}^{3}$ & Split \\
\hline & & $8 / 7 / 01$ & 0.682 & 0.15 & $\mathrm{~Bq} / \mathrm{m}^{3}$ & Sample \\
\hline & & $9 / 4 / 01$ & 0.51 & 0.18 & $\mathrm{~Bq} / \mathrm{m}^{3}$ & Sample \\
\hline
\end{tabular}


Analyte

\section{Ambient Air Monitoring}

Tritium

cont.

ENV-69
ENV-75EG

ENV-77

ENV-78

Date Result

MDA or PQL

Units QC Type

$\begin{array}{llllr}10 / 2 / 01 & 0.883 & 0.18 & \mathrm{~Bq} / \mathrm{m}^{3} & \text { Sample } \\ 11 / 6 / 01 & 0.29 & 0.15 & \mathrm{~Bq} / \mathrm{m}^{3} & \text { Sample } \\ 11 / 6 / 01 & 0.25 & 0.15 & \mathrm{~Bq} / \mathrm{m}^{3} & \text { Split } \\ 12 / 4 / 01 & 0.29 & 0.18 & \mathrm{~Bq} / \mathrm{m}^{3} & \text { Sample } \\ \text { Sample } \\ 1 / 8 / 02 & 0.456 & 0.15 & \mathrm{~Bq} / \mathrm{m}^{3} & \\ & & & & \\ 6 / 5 / 01 & 1.29 & 0.15 & \mathrm{~Bq} / \mathrm{m}^{3} & \text { Sample } \\ 6 / 5 / 01 & 1.51 & 0.08 & \mathrm{~Bq} / \mathrm{m}^{3} & \text { Split } \\ 7 / 3 / 01 & 3.34 & 0.18 & \mathrm{~Bq} / \mathrm{m}^{3} & \text { Sample } \\ 7 / 3 / 01 & 4.41 & 0.09 & \mathrm{~Bq} / \mathrm{m}^{3} & \text { Split } \\ 8 / 7 / 01 & 1.53 & 0.15 & \mathrm{~Bq} / \mathrm{m}^{3} & \text { Sample } \\ 8 / 7 / 01 & 2.12 & 0.09 & \mathrm{~Bq} / \mathrm{m}^{3} & \text { Split } \\ 9 / 4 / 01 & 2.3 & 0.18 & \mathrm{~Bq} / \mathrm{m}^{3} & \text { Sample } \\ 9 / 4 / 01 & 2.19 & 0.1 & \mathrm{~Bq} / \mathrm{m}^{3} & \text { Split } \\ 10 / 2 / 01 & 2.85 & 0.18 & \mathrm{~Bq} / \mathrm{m}^{3} & \text { Sample } \\ 10 / 2 / 01 & 5.03 & 0.09 & \mathrm{~Bq} / \mathrm{m}^{3} & \text { Split } \\ 11 / 6 / 01 & 1.25 & 0.15 & \mathrm{~Bq} / \mathrm{m}^{3} & \text { Sample } \\ 11 / 6 / 01 & 1.62 & 0.08 & \mathrm{~Bq} / \mathrm{m}^{3} & \text { Split } \\ 12 / 4 / 01 & 1.17 & 0.18 & \mathrm{~Bq} / \mathrm{m}^{3} & \text { Sample } \\ 12 / 4 / 01 & 1.36 & 0.08 & \mathrm{~Bq} / \mathrm{m}^{3} & \text { Split } \\ 1 / 8 / 02 & 2.88 & 0.15 & \mathrm{~Bq} / \mathrm{m}^{3} & \text { Sample } \\ 1 / 8 / 02 & 2.73 & 0.06 & \mathrm{~Bq} / \mathrm{m}^{3} & \text { Split }\end{array}$

$\begin{array}{llllr}6 / 5 / 01 & 0.679 & 0.15 & \mathrm{~Bq} / \mathrm{m}^{3} & \text { Sample } \\ 7 / 3 / 01 & 1.95 & 0.18 & \mathrm{~Bq} / \mathrm{m}^{3} & \text { Sample } \\ 7 / 3 / 01 & 1.67 & 0.18 & \mathrm{~Bq} / \mathrm{m}^{3} & \text { Split } \\ 8 / 7 / 01 & 0.22 & 0.15 & \mathrm{~Bq} / \mathrm{m}^{3} & \text { Sample } \\ 9 / 4 / 01 & 0.31 & 0.18 & \mathrm{~Bq} / \mathrm{m}^{3} & \text { Sample } \\ 10 / 2 / 01 & 0.893 & 0.18 & \mathrm{~Bq} / \mathrm{m}^{3} & \text { Sample } \\ 11 / 6 / 01 & 0.557 & 0.15 & \mathrm{~Bq} / \mathrm{m}^{3} & \text { Sample } \\ 11 / 6 / 01 & 1.92 & 0.08 & \mathrm{~Bq} / \mathrm{m}^{3} & \text { Split } \\ 12 / 4 / 01 & 0.354 & 0.18 & \mathrm{~Bq} / \mathrm{m}^{3} & \text { Sample } \\ 12 / 4 / 01 & 0.345 & 0.18 & \mathrm{~Bq} / \mathrm{m}^{3} & \text { Split } \\ 1 / 8 / 02 & 0.462 & 0.15 & \mathrm{~Bq} / \mathrm{m}^{3} & \text { Sample } \\ 1 / 8 / 02 & 0.603 & 0.15 & \mathrm{~Bq} / \mathrm{m}^{3} & \text { Split } \\ & & & & \\ 6 / 5 / 01 & 0.8 & 0.15 & \mathrm{~Bq} / \mathrm{m}^{3} & \text { Sample } \\ 7 / 3 / 01 & 2.2 & 0.18 & \mathrm{~Bq} / \mathrm{m}^{3} & \text { Sample } \\ 7 / 3 / 01 & 2.84 & 0.09 & \mathrm{~Bq} / \mathrm{m}^{3} & \text { Split } \\ 8 / 7 / 01 & 0.42 & 0.17 & \mathrm{~Bq} / \mathrm{m}^{3} & \text { Sample } \\ 8 / 7 / 01 & 0.499 & 0.17 & \mathrm{~Bq} / \mathrm{m}^{3} & \text { Split } \\ 9 / 4 / 01 & 1.05 & 0.18 & \mathrm{~Bq} / \mathrm{m}^{3} & \text { Sample } \\ 10 / 2 / 01 & 1.33 & 0.18 & \mathrm{~Bq} / \mathrm{m}^{3} & \text { Sample } \\ 11 / 6 / 01 & 0.755 & 0.15 & \mathrm{~Bq} / \mathrm{m}^{3} & \text { Sample } \\ 12 / 4 / 01 & 0.732 & 0.2 & \mathrm{~Bq} / \mathrm{m}^{3} & \text { Sample } \\ 12 / 4 / 01 & 0.716 & 0.2 & \mathrm{~Bq} / \mathrm{m}^{3} & \text { Split }\end{array}$




\section{Analyte Location}

Tritium

ENV-78

cont.

ENV-85

ENV-AR

ENV-B13A

ENV-B13C

$\begin{array}{llllr}1 / 8 / 02 & 0.809 & 0.15 & \mathrm{~Bq} / \mathrm{m}^{3} & \text { Sample } \\ 1 / 8 / 02 & 0.872 & 0.15 & \mathrm{~Bq} / \mathrm{m}^{3} & \text { Split }\end{array}$

\section{$6 / 5 / 01$}

$7 / 3 / 01$

0.27

0.15

$8 / 7 / 01$

$8 / 7 / 01$

ND

ND

$9 / 4 / 01$

0.0921

0.18

$\mathrm{Bq} / \mathrm{m}^{3}$

Sample

$10 / 2 / 01$

0.31

0.15

$\mathrm{Bq} / \mathrm{m}^{3}$

0.013

$\mathrm{Bq} / \mathrm{m}^{3}$

0.18

$\mathrm{Bq} / \mathrm{m}^{3}$

ND

0.19

$\mathrm{Bq} / \mathrm{m}^{3}$

$11 / 6 / 01$

ND

$12 / 4 / 01$

ND

$12 / 4 / 01$

0.13

0.15

$\mathrm{Bq} / \mathrm{m}^{3}$

0.19

$\mathrm{Bq} / \mathrm{m}^{3}$

$1 / 8 / 02$

ND

0.07

$1 / 8 / 02$

ND

\subsection{5}

0.06

$\mathrm{Bq} / \mathrm{m}^{3}$

$\mathrm{Bq} / \mathrm{m}^{3}$

$\mathrm{Bq} / \mathrm{m}^{3}$

$\mathrm{Bq} / \mathrm{m}^{3}$

Sample

Sample

Split

Sample

Sample

$6 / 5 / 01$

$6 / 5 / 01$

$7 / 3 / 01$

$7 / 3 / 01$

$8 / 7 / 01$

8/7/01

9/5/01

9/5/01

10/2/01

10/2/01

$11 / 6 / 01$

$11 / 6 / 01$

$12 / 4 / 01$

$12 / 4 / 01$

$1 / 8 / 02$

$1 / 8 / 02$

0.2

0.237

0.14

ND

0.202

ND

0.0874

0.25

0.0902

ND

0.0945

ND

0.0474

ND

0.0548

0.88

0.817

0.01

0.18

$\mathrm{Bq} / \mathrm{m}^{3}$

$\mathrm{Bq} / \mathrm{m}^{3}$

$\mathrm{Bq} / \mathrm{m}^{3}$

0.013

0.15

0.013

0.18

0.014

0.19

0.015

0.15

0.01

0.18

0.013

0.15

0.011

$\mathrm{Bq} / \mathrm{m}^{3}$

$\mathrm{Bq} / \mathrm{m}^{3}$

$\mathrm{Bq} / \mathrm{m}^{3}$

$\mathrm{Bq} / \mathrm{m}^{3}$

$\mathrm{Bq} / \mathrm{m}^{3}$

$\mathrm{Bq} / \mathrm{m}^{3}$

$\mathrm{Bq} / \mathrm{m}^{3}$

$\mathrm{Bq} / \mathrm{m}^{3}$

$\mathrm{Bq} / \mathrm{m}^{3}$

$\mathrm{Bq} / \mathrm{m}^{3}$

$\mathrm{Bq} / \mathrm{m}^{3}$

$\mathrm{Bq} / \mathrm{m}^{3}$

$\mathrm{Bq} / \mathrm{m}^{3}$

Sample

Sample

$6 / 5 / 01 \quad 0.2$

$7 / 3 / 01$

$8 / 7 / 01$

9/4/01

9/4/01

10/2/01

10/2/01

$11 / 6 / 01$

$12 / 4 / 01$

$1 / 8 / 02$

0.24
ND

0.15

0.18

$\mathrm{Bq} / \mathrm{m}^{3}$

$\mathrm{Bq} / \mathrm{m}^{3}$

ND

ND

0.15

0.18

ND

ND

0.18

0.18

0.045

0.014

ND

0.15

ND

ND

0.18

0.15

$\mathrm{Bq} / \mathrm{m}^{3}$

$\mathrm{Bq} / \mathrm{m}^{3}$

$\mathrm{Bq} / \mathrm{m}^{3}$

$\mathrm{Bq} / \mathrm{m}^{3}$

$\mathrm{Bq} / \mathrm{m}^{3}$

$\mathrm{Bq} / \mathrm{m}^{3}$

$\mathrm{Bq} / \mathrm{m}^{3}$

$\mathrm{Bq} / \mathrm{m}^{3}$

Split

Sample

Split

$6 / 5 / 01 \quad 0.32$

$6 / 5 / 01$

7/3/01

0.32
0.311
ND

0.15

0.011

0.18

$\mathrm{Bq} / \mathrm{m}^{3}$

$\mathrm{Bq} / \mathrm{m}^{3}$

$\mathrm{Bq} / \mathrm{m}^{3}$

Sample

Split

Sample

Split

Sample

Split

Sample

Split

Sample

Split

Sample

Split

Split

Sample

Split

Sample

Sample

Sample

Sample

Split

Sample

Split

Sample

Sample

Sample

Sample
Split
Sample 
Units QC Type

\section{Ambient Air Monitoring}

Tritium cont.
ENV-B13C

ENV-B13D

ENV-LHS

ENV-MSRI

$\begin{array}{ll}8 / 7 / 01 & \text { ND } \\ 9 / 4 / 01 & \text { ND } \\ 9 / 4 / 01 & \text { ND } \\ 10 / 2 / 01 & \text { ND } \\ 11 / 6 / 01 & \text { ND } \\ 11 / 6 / 01 & 0.037 \\ 12 / 4 / 01 & \text { ND } \\ 1 / 8 / 02 & \text { ND }\end{array}$

6/5/01 ND

$7 / 3 / 01$

$8 / 7 / 01$

$9 / 4 / 01$

$9 / 4 / 01$

$10 / 2 / 01$

$10 / 2 / 01$

$11 / 6 / 01$

$12 / 4 / 01$

$1 / 8 / 02$

$6 / 5 / 01$

$6 / 5 / 01$

$7 / 3 / 01$

$8 / 7 / 01$

$8 / 7 / 01$

$9 / 4 / 01$

9/4/01

10/2/01

10/2/01

$11 / 6 / 01$

$11 / 6 / 01$

$12 / 4 / 01$

$12 / 4 / 01$

$1 / 8 / 02$

$1 / 8 / 02$

$6 / 5 / 01$

$7 / 3 / 01$

$8 / 7 / 01$

$8 / 7 / 01$

$9 / 4 / 01$

10/2/01

$11 / 6 / 01$

$12 / 4 / 01$

$1 / 8 / 02$
ND

0.16

0.22

0.484

0.22

0.23

ND

0.421

ND

0.449
0.572

0.95

1.29

1.29

1.53

0.939

0.988

1.26

5.24

0.52

0.516

0.526

0.808

1.3

1.24

\subsection{4}

0.43

0.54

0.49

0.43

0.623

0.24

ND

ND
0.15

0.18

0.18

0.18

0.15

0.011

0.18

0.15

$0.18 \quad \mathrm{~Bq} / \mathrm{m}^{3}$

$0.18 \mathrm{~Bq} / \mathrm{m}^{3}$

$0.15 \mathrm{~Bq} / \mathrm{m}^{3}$

$0.18 \mathrm{~Bq} / \mathrm{m}^{3}$

$0.09 \quad \mathrm{~Bq} / \mathrm{m}^{3}$

$0.18 \mathrm{~Bq} / \mathrm{m}^{3}$

$0.18 \mathrm{~Bq} / \mathrm{m}^{3}$

$0.15 \quad \mathrm{~Bq} / \mathrm{m}^{3}$

$0.19 \mathrm{~Bq} / \mathrm{m}^{3}$

$0.15 \mathrm{~Bq} / \mathrm{m}^{3}$

$\begin{array}{llr}0.15 & \mathrm{~Bq} / \mathrm{m}^{3} & \text { Sample } \\ 0.08 & \mathrm{~Bq} / \mathrm{m}^{3} & \text { Split } \\ 0.18 & \mathrm{~Bq} / \mathrm{m}^{3} & \text { Sample } \\ 0.09 & \mathrm{~Bq} / \mathrm{m}^{3} & \text { Split } \\ 0.15 & \mathrm{~Bq} / \mathrm{m}^{3} & \text { Sample } \\ 0.09 & \mathrm{~Bq} / \mathrm{m}^{3} & \text { Split } \\ 0.18 & \mathrm{~Bq} / \mathrm{m}^{3} & \text { Sample } \\ 0.1 & \mathrm{~Bq} / \mathrm{m}^{3} & \text { Split } \\ 0.18 & \mathrm{~Bq} / \mathrm{m}^{3} & \text { Sample } \\ 0.09 & \mathrm{~Bq} / \mathrm{m}^{3} & \text { Split } \\ 0.15 & \mathrm{~Bq} / \mathrm{m}^{3} & \text { Sample } \\ 0.07 & \mathrm{~Bq} / \mathrm{m}^{3} & \text { Split } \\ 0.18 & \mathrm{~Bq} / \mathrm{m}^{3} & \text { Sample } \\ 0.08 & \mathrm{~Bq} / \mathrm{m}^{3} & \text { Split } \\ 0.15 & \mathrm{~Bq} / \mathrm{m}^{3} & \text { Sample } \\ 0.06 & \mathrm{~Bq} / \mathrm{m}^{3} & \text { Split }\end{array}$

0.15

0.18

0.15

0.15

0.18

0.2

0.15

0.18

0.15

\section{$\mathrm{Bq} / \mathrm{m}^{3}$}

$\mathrm{Bg} / \mathrm{m}^{3}$

$\mathrm{Bq} / \mathrm{m}^{3}$

$\mathrm{Bq} / \mathrm{m}^{3}$

$\mathrm{Bq} / \mathrm{m}^{3}$

$\mathrm{Bq} / \mathrm{m}^{3}$

$\mathrm{Bq} / \mathrm{m}^{3}$

$\mathrm{Bq} / \mathrm{m}^{3}$

$\mathrm{Bq} / \mathrm{m}^{3}$
Sample

Sample

Split

Sample

Sample

Split

Sample

Sample

Sample

Sample

Sample

Sample

Split

Sample

Split

Sample

Sample

Sample

Sample ample Split mple Split Split ample Split Split mple Split

Split

Sample

Sample

Sample Split Sample Sample Sample Sample Sample 


$\begin{array}{llllll}\text { Analyte } & \text { Location } & \text { Date } & \text { Result } & \text { MDA or PQL Units } & \text { QC Type }\end{array}$

\title{
Ambient Air Monitoring
}

Tritium

cont.
ENV-SSL

ENV-UCBG

TRAVEL BLANK

$\begin{array}{ll}6 / 5 / 01 & 0.687 \\ 7 / 3 / 01 & 0.3 \\ 8 / 7 / 01 & 0.36 \\ 8 / 7 / 01 & 0.506 \\ 9 / 4 / 01 & 0.33 \\ 9 / 4 / 01 & 0.34 \\ 10 / 2 / 01 & 0.34 \\ 11 / 6 / 01 & 0.16 \\ 12 / 4 / 01 & N D \\ 1 / 8 / 02 & N D\end{array}$

$6 / 5 / 01$

$6 / 5 / 01$

$7 / 3 / 01$

$7 / 3 / 01$

$8 / 7 / 01$

$8 / 7 / 01$

$9 / 4 / 01$

$9 / 4 / 01$

$10 / 2 / 01$

$10 / 2 / 01$

$11 / 7 / 01$

$11 / 7 / 01$

$1 / 8 / 02$

$1 / 8 / 02$

$6 / 5 / 01$

$7 / 3 / 01$

$7 / 3 / 01$

$8 / 7 / 01$

$8 / 7 / 01$

$9 / 4 / 01$

$9 / 4 / 01$

10/2/01

$11 / 7 / 01$

$12 / 4 / 01$

$1 / 8 / 02$

$1 / 8 / 02$

$1 / 8 / 02$

ND

ND

0.164

ND

ND

ND

0.136

ND

ND

ND

0.12

ND

0.0744

0.23
0.286
$N D$
0.162
$N D$
0.155
$N D$
0.0976
$N D$
0.193
$N D$
0.0795
$N D$
0.0905

0.15

0.18

0.15

0.09

0.18

0.015

0.18

0.15

0.18

0.15

0.15

0.01

0.18

0.013

0.15

0.011

0.18

0.014

0.18

0.013

0.3

0.02

0.2

0.014

\section{4}

0.4

0.03

0.4

0.04

0.4

0.03

0.4

0.4

0.4

0.08

0.4

0.03
$\mathrm{Bq} / \mathrm{m}^{3}$

$\mathrm{Bq} / \mathrm{m}^{3}$

$\mathrm{Bq} / \mathrm{m}^{3}$

$\mathrm{Bq} / \mathrm{m}^{3}$

$\mathrm{Bq} / \mathrm{m}^{3}$

$\mathrm{Bq} / \mathrm{m}^{3}$

$\mathrm{Bq} / \mathrm{m}^{3}$

$\mathrm{Bq} / \mathrm{m}^{3}$

$\mathrm{Bq} / \mathrm{m}^{3}$

$\mathrm{Bq} / \mathrm{m}^{3}$

$\mathrm{Bq} / \mathrm{m}^{3}$

$\mathrm{Bq} / \mathrm{m}^{3}$

$\mathrm{Bq} / \mathrm{m}^{3}$

$\mathrm{Bq} / \mathrm{m}^{3}$

$\mathrm{Bq} / \mathrm{m}^{3}$

$\mathrm{Bq} / \mathrm{m}^{3}$

$\mathrm{Bq} / \mathrm{m}^{3}$

$\mathrm{Bq} / \mathrm{m}^{3}$

$\mathrm{Bq} / \mathrm{m}^{3}$

$\mathrm{Bq} / \mathrm{m}^{3}$

$\mathrm{Bq} / \mathrm{m}^{3}$

$\mathrm{Bq} / \mathrm{m}^{3}$

$\mathrm{Bq} / \mathrm{m}^{3}$

$\mathrm{Bq} / \mathrm{m}^{3}$

$\mathrm{Bq} / \mathrm{S}$

$\mathrm{Bq} / \mathrm{S}$

$\mathrm{Bq} / \mathrm{S}$

$\mathrm{Bq} / \mathrm{S}$

$\mathrm{Bq} / \mathrm{S}$

$\mathrm{Bq} / \mathrm{S}$

$\mathrm{Bq} / \mathrm{S}$

$\mathrm{Bq} / \mathrm{S}$

$\mathrm{Bq} / \mathrm{S}$

$\mathrm{Bq} / \mathrm{S}$

$\mathrm{Bq} / \mathrm{S}$

$\mathrm{Bq} / \mathrm{S}$

$\mathrm{Bq} / \mathrm{S}$
Sample

Sample

Sample

Split

Sample

Split

Sample

Sample

Sample

Sample

Sample

Split

Sample

Split

Sample

Split

Sample

Split

Sample

Split

Sample

Split

Sample

Split

Blank

Blank

Blank

Blank

Blank

Blank

Blank

Blank

Blank

Blank

Blank

Blank

Blank

\section{Sediment Monitoring}

Tritium, Free Water

\author{
Banana Creek
}

$\begin{array}{ll}4 / 10 / 01 & 0.007 \\ 4 / 10 / 01 & \text { ND } \\ 8 / 31 / 01 & \text { ND } \\ 8 / 31 / 01 & \text { ND }\end{array}$

0.007

0.007

0.007

0.007
$\mathrm{Bq} / \mathrm{g}$
$\mathrm{Bq} / \mathrm{g}$
$\mathrm{Bq} / \mathrm{g}$
$\mathrm{Bq} / \mathrm{g}$

Sample

Duplicate

Sample

Duplicate 
Analyte Location Date Result MDA or PQL Units QC Type

\section{Sediment Monitoring}

Tritium, Free Water

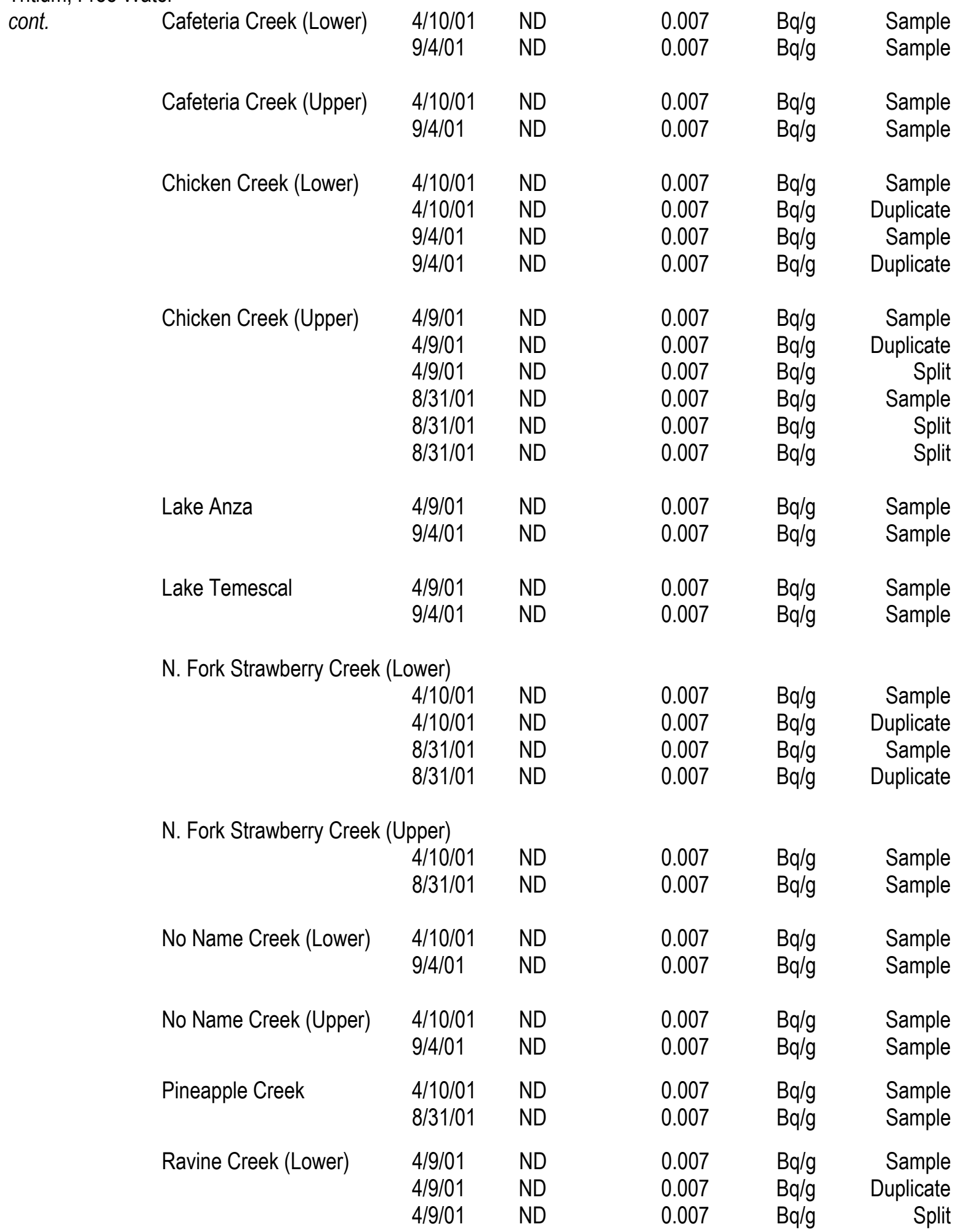




$\begin{array}{llllll}\text { Analyte } & \text { Location } & \text { Date } & \text { Result } & \text { MDA or PQL Units } & \text { QC Type }\end{array}$

\section{Sediment Monitoring}

Tritium, Free Water

\begin{tabular}{|c|c|c|c|c|c|c|}
\hline \multirow[t]{6}{*}{ cont. } & Ravine Creek (Lower) & $\begin{array}{l}8 / 31 / 01 \\
8 / 31 / 01 \\
8 / 31 / 01\end{array}$ & $\begin{array}{l}\text { ND } \\
\text { ND } \\
\text { ND }\end{array}$ & $\begin{array}{l}0.007 \\
0.007 \\
0.007\end{array}$ & $\begin{array}{l}\mathrm{Bq} / \mathrm{g} \\
\mathrm{Bq} / \mathrm{g} \\
\mathrm{Bq} / \mathrm{g}\end{array}$ & $\begin{array}{r}\text { Sample } \\
\text { Split } \\
\text { Split }\end{array}$ \\
\hline & Ravine Creek (Upper) & $\begin{array}{l}4 / 9 / 01 \\
8 / 31 / 01\end{array}$ & $\begin{array}{l}\text { ND } \\
\text { ND }\end{array}$ & $\begin{array}{l}0.007 \\
0.007\end{array}$ & $\begin{array}{l}\mathrm{Bq} / \mathrm{g} \\
\mathrm{Bq} / \mathrm{g}\end{array}$ & $\begin{array}{l}\text { Sample } \\
\text { Sample }\end{array}$ \\
\hline & Strawberry Creek Outfall & $\begin{array}{l}4 / 10 / 01 \\
9 / 4 / 01\end{array}$ & $\begin{array}{l}\text { ND } \\
\text { ND }\end{array}$ & $\begin{array}{l}0.007 \\
0.007\end{array}$ & $\begin{array}{l}\mathrm{Bq} / \mathrm{g} \\
\mathrm{Bq} / \mathrm{g}\end{array}$ & $\begin{array}{l}\text { Sample } \\
\text { Sample }\end{array}$ \\
\hline & Strawberry Creek UC & $\begin{array}{l}4 / 10 / 01 \\
9 / 4 / 01\end{array}$ & $\begin{array}{l}\text { ND } \\
\text { ND }\end{array}$ & $\begin{array}{l}0.007 \\
0.007\end{array}$ & $\begin{array}{l}\mathrm{Bq} / \mathrm{g} \\
\mathrm{Bq} / \mathrm{g}\end{array}$ & $\begin{array}{l}\text { Sample } \\
\text { Sample }\end{array}$ \\
\hline & Ten Inch Creek (Lower) & $\begin{array}{l}4 / 11 / 01 \\
9 / 4 / 01\end{array}$ & $\begin{array}{l}\text { ND } \\
\text { ND }\end{array}$ & $\begin{array}{l}0.007 \\
0.007\end{array}$ & $\begin{array}{l}\mathrm{Bq} / \mathrm{g} \\
\mathrm{Bq} / \mathrm{g}\end{array}$ & $\begin{array}{l}\text { Sample } \\
\text { Sample }\end{array}$ \\
\hline & Ten Inch Creek (Upper) & $\begin{array}{l}4 / 11 / 01 \\
9 / 4 / 01\end{array}$ & $\begin{array}{l}\text { ND } \\
\text { ND }\end{array}$ & $\begin{array}{l}0.007 \\
0.007\end{array}$ & $\begin{array}{l}\mathrm{Bq} / \mathrm{g} \\
\mathrm{Bq} / \mathrm{g}\end{array}$ & $\begin{array}{l}\text { Sample } \\
\text { Sample }\end{array}$ \\
\hline \multirow[t]{3}{*}{ Tritium, Total } & Chicken Creek (Lower) & $\begin{array}{l}4 / 10 / 01 \\
4 / 10 / 01 \\
9 / 4 / 01 \\
9 / 4 / 01\end{array}$ & $\begin{array}{l}\text { ND } \\
\text { ND } \\
\text { ND } \\
\text { ND }\end{array}$ & $\begin{array}{l}0.2 \\
0.2 \\
0.2 \\
0.2\end{array}$ & $\begin{array}{l}\mathrm{Bq} / \mathrm{g} \\
\mathrm{Bq} / \mathrm{g} \\
\mathrm{Bq} / \mathrm{g} \\
\mathrm{Bq} / \mathrm{g}\end{array}$ & $\begin{array}{r}\text { Sample } \\
\text { Duplicate } \\
\text { Sample } \\
\text { Duplicate }\end{array}$ \\
\hline & Chicken Creek (Upper) & $\begin{array}{l}4 / 9 / 01 \\
4 / 9 / 01 \\
4 / 9 / 01 \\
8 / 31 / 01 \\
8 / 31 / 01\end{array}$ & $\begin{array}{l}\text { ND } \\
\text { ND } \\
\text { ND } \\
\text { ND } \\
\text { ND }\end{array}$ & $\begin{array}{l}0.2 \\
0.2 \\
0.2 \\
0.2 \\
0.2\end{array}$ & $\begin{array}{l}\mathrm{Bq} / \mathrm{g} \\
\mathrm{Bq} / \mathrm{g} \\
\mathrm{Bq} / \mathrm{g} \\
\mathrm{Bq} / \mathrm{g} \\
\mathrm{Bq} / \mathrm{g}\end{array}$ & $\begin{array}{r}\text { Sample } \\
\text { Duplicate } \\
\text { Split } \\
\text { Sample } \\
\text { Split }\end{array}$ \\
\hline & N. Fork Strawberry Creek & $\begin{array}{l}\text { lpper) } \\
4 / 10 / 01 \\
8 / 31 / 01\end{array}$ & $\begin{array}{l}\text { ND } \\
\text { ND }\end{array}$ & $\begin{array}{l}0.2 \\
0.2\end{array}$ & $\begin{array}{l}\mathrm{Bq} / \mathrm{g} \\
\mathrm{Bq} / \mathrm{g}\end{array}$ & $\begin{array}{l}\text { Sample } \\
\text { Sample }\end{array}$ \\
\hline
\end{tabular}

\section{Soil Monitoring}

Tritium, Free Water

$\begin{array}{llllll}\text { SSNTLF-01-1-0.5 } & 04 / 4 / 01 & 0.0107 & 0.007 & \mathrm{~Bq} / \mathrm{g} & \text { Sample } \\ \text { SSNTLF-01-1-1.5 } & 04 / 4 / 01 & 0.0132 & 0.007 & \mathrm{~Bq} / \mathrm{g} & \text { Sample } \\ \text { SSNTLF-01-1Comp-0.5 } & & & & & \\ & 04 / 4 / 01 & 0.0201 & 0.007 & \mathrm{~Bq} / \mathrm{g} & \text { Sample } \\ \text { SSNTLF-01-1Comp-1.5 } & & & & & \\ & 04 / 4 / 01 & 0.0155 & 0.007 & \mathrm{~Bq} / \mathrm{g} & \text { Sample }\end{array}$


Date Result MDA or PQL Units QC Type

\section{Soil Monitoring}

Tritium, Free Water cont.

\begin{tabular}{|c|c|c|c|c|c|}
\hline SSNTLF-01-2-0.5 & 04/18/01 & 0.007 & 0.007 & $\mathrm{~Bq} / \mathrm{g}$ & Sample \\
\hline SSNTLF-01-2-1.5 & 04/18/01 & ND & 0.007 & $\mathrm{~Bq} / \mathrm{g}$ & Sample \\
\hline SSNTLF-01-3-0.5 & 04/16/01 & ND & 0.007 & $\mathrm{~Bq} / \mathrm{g}$ & Sample \\
\hline SSNTLF-01-3-1.5 & 04/16/01 & ND & 0.007 & $\mathrm{~Bq} / \mathrm{g}$ & Sample \\
\hline SSNTLF-01-4-0.5 & $\begin{array}{l}04 / 13 / 01 \\
04 / 13 / 01 \\
04 / 13 / 01\end{array}$ & $\begin{array}{l}0.01 \\
0.0081 \\
\text { ND }\end{array}$ & $\begin{array}{l}0.007 \\
0.007 \\
0.007\end{array}$ & $\begin{array}{l}\mathrm{Bq} / \mathrm{g} \\
\mathrm{Bq} / \mathrm{g} \\
\mathrm{Bq} / \mathrm{g}\end{array}$ & $\begin{array}{r}\text { Sample } \\
\text { Split } \\
\text { Split }\end{array}$ \\
\hline SSNTLF-01-4-1.5 & $\begin{array}{l}04 / 13 / 01 \\
04 / 13 / 01 \\
04 / 13 / 01\end{array}$ & $\begin{array}{l}\text { ND } \\
\text { ND } \\
\text { ND }\end{array}$ & $\begin{array}{l}0.007 \\
0.007 \\
0.007\end{array}$ & $\begin{array}{l}\mathrm{Bq} / \mathrm{g} \\
\mathrm{Bq} / \mathrm{g} \\
\mathrm{Bq} / \mathrm{g}\end{array}$ & $\begin{array}{r}\text { Sample } \\
\text { Split } \\
\text { Split }\end{array}$ \\
\hline SSNTLF-01-5-0.5 & $04 / 12 / 01$ & ND & 0.007 & $\mathrm{~Bq} / \mathrm{g}$ & Sample \\
\hline SSNTLF-01-5-1.5 & $04 / 12 / 01$ & ND & 0.007 & $\mathrm{~Bq} / \mathrm{g}$ & Sample \\
\hline SSNTLF-01-6-0.5 & $04 / 18 / 01$ & ND & 0.007 & $\mathrm{~Bq} / \mathrm{g}$ & Sample \\
\hline SSNTLF-01-6-1.5 & $04 / 18 / 01$ & ND & 0.007 & $\mathrm{~Bq} / \mathrm{g}$ & Sample \\
\hline SSNTLF-01-7-0.5 & $\begin{array}{l}04 / 13 / 01 \\
04 / 13 / 01 \\
04 / 13 / 01\end{array}$ & $\begin{array}{l}\text { ND } \\
0.01 \\
\text { ND }\end{array}$ & $\begin{array}{l}0.007 \\
0.007 \\
0.007\end{array}$ & $\begin{array}{l}\mathrm{Bq} / \mathrm{g} \\
\mathrm{Bq} / \mathrm{g} \\
\mathrm{Bq} / \mathrm{g}\end{array}$ & $\begin{array}{r}\text { Sample } \\
\text { Split } \\
\text { Split }\end{array}$ \\
\hline SSNTLF-01-7-1.5 & $\begin{array}{l}04 / 13 / 01 \\
04 / 13 / 01 \\
04 / 13 / 01\end{array}$ & $\begin{array}{l}\text { ND } \\
\text { ND } \\
\text { ND }\end{array}$ & $\begin{array}{l}0.007 \\
0.007 \\
0.007\end{array}$ & $\begin{array}{l}\mathrm{Bq} / \mathrm{g} \\
\mathrm{Bq} / \mathrm{g} \\
\mathrm{Bq} / \mathrm{g}\end{array}$ & $\begin{array}{r}\text { Sample } \\
\text { Split } \\
\text { Split }\end{array}$ \\
\hline SSNTLF-01-8-0.5 & 04/18/01 & ND & 0.007 & $\mathrm{~Bq} / \mathrm{g}$ & Sample \\
\hline SSNTLF-01-8-1.5 & 04/18/01 & ND & 0.007 & $\mathrm{~Bq} / \mathrm{g}$ & Sample \\
\hline SSNTLF-01-9-0.5 & $04 / 12 / 01$ & ND & 0.007 & $\mathrm{~Bq} / \mathrm{g}$ & Sample \\
\hline SSNTLF-01-9-1.5 & $04 / 12 / 01$ & ND & 0.007 & $\mathrm{~Bq} / \mathrm{g}$ & Sample \\
\hline SSNTLF-01-10-0.5 & 04/12/01 & 0.00878 & 0.007 & $\mathrm{~Bq} / \mathrm{g}$ & Sample \\
\hline SSNTLF-01-10-1.5 & $04 / 12 / 01$ & ND & 0.007 & $\mathrm{~Bq} / \mathrm{g}$ & Sample \\
\hline SSNTLF-01-11-0.5 & $04 / 4 / 01$ & 0.0144 & 0.007 & $\mathrm{~Bq} / \mathrm{g}$ & Sample \\
\hline
\end{tabular}




$\begin{array}{llllll}\text { Analyte } & \text { Location } & \text { Date } & \text { Result } & \text { MDA or PQL Units } & \text { QC Type }\end{array}$

\section{Soil Monitoring}

Tritium, Free Water cont.

\begin{tabular}{|c|c|c|c|c|c|}
\hline SSNTLF-01-11-1.5 & $04 / 4 / 01$ & 0.011 & 0.007 & $\mathrm{~Bq} / \mathrm{g}$ & Sample \\
\hline SSNTLF-01-11Comp & & & & & \\
\hline & $04 / 4 / 01$ & 0.0201 & 0.007 & $\mathrm{~Bq} / \mathrm{g}$ & Sample \\
\hline SSNTLF-01-11Comp & & & & & \\
\hline & $04 / 4 / 01$ & 0.014 & 0.007 & $\mathrm{~Bq} / \mathrm{g}$ & Sample \\
\hline SSNTLF-01-12-0.5 & $04 / 13 / 01$ & 0.0326 & 0.007 & $\mathrm{~Bq} / \mathrm{g}$ & Sample \\
\hline & $04 / 13 / 01$ & 0.025 & 0.007 & $\mathrm{~Bq} / \mathrm{g}$ & Split \\
\hline SSNTLF-01-12Comp & & & & & \\
\hline & 04/13/01 & 0.0354 & 0.007 & $\mathrm{~Bq} / \mathrm{g}$ & Sample \\
\hline & $04 / 13 / 01$ & 0.027 & 0.007 & $\mathrm{~Bq} / \mathrm{g}$ & Split \\
\hline & 04/13/01 & 0.016 & 0.007 & $\mathrm{~Bq} / \mathrm{g}$ & Split \\
\hline SSNTLF-01-12-1.5 & $04 / 13 / 01$ & 0.0276 & 0.007 & $\mathrm{~Bq} / \mathrm{g}$ & Sample \\
\hline & $04 / 13 / 01$ & 0.014 & 0.007 & $\mathrm{~Bq} / \mathrm{g}$ & Split \\
\hline SSNTLF-01-12Comp & & & & & \\
\hline & 04/13/01 & 0.031 & 0.007 & $\mathrm{~Bq} / \mathrm{g}$ & Sample \\
\hline & 04/13/01 & 0.025 & 0.007 & $\mathrm{~Bq} / \mathrm{g}$ & Split \\
\hline & $04 / 13 / 01$ & 0.023 & 0.007 & $\mathrm{~Bq} / \mathrm{g}$ & Split \\
\hline SSNTLF-01-13-0.5 & 04/18/01 & ND & 0.007 & $\mathrm{~Bq} / \mathrm{g}$ & Sample \\
\hline SSNTLF-01-13-1.5 & 04/18/01 & ND & 0.007 & $\mathrm{~Bq} / \mathrm{g}$ & Sample \\
\hline SSNTLF-01-14-0.5 & $04 / 24 / 01$ & ND & 0.007 & $\mathrm{~Bq} / \mathrm{g}$ & Sample \\
\hline & $04 / 24 / 01$ & ND & 0.007 & $\mathrm{~Bq} / \mathrm{g}$ & Duplicate \\
\hline SSNTLF-01-14-1.5 & $04 / 24 / 01$ & ND & 0.007 & $\mathrm{~Bq} / \mathrm{g}$ & Sample \\
\hline & $04 / 24 / 01$ & ND & 0.007 & $\mathrm{~Bq} / \mathrm{g}$ & Duplicate \\
\hline SSNTLF-01-15-0.5 & $05 / 3 / 01$ & ND & 0.007 & $\mathrm{~Bq} / \mathrm{g}$ & Sample \\
\hline SSNTLF-01-15-1.5 & $05 / 3 / 01$ & ND & 0.007 & $\mathrm{~Bq} / \mathrm{g}$ & Sample \\
\hline SSNTLF-01-16-0.5 & 05/1/01 & ND & 0.007 & $\mathrm{~Bq} / \mathrm{g}$ & Sample \\
\hline & $05 / 1 / 01$ & ND & 0.007 & $\mathrm{~Bq} / \mathrm{g}$ & Duplicate \\
\hline SSNTLF-01-16-1.5 & 05/1/01 & ND & 0.007 & $\mathrm{~Bq} / \mathrm{g}$ & Sample \\
\hline & $05 / 1 / 01$ & ND & 0.007 & $\mathrm{~Bq} / \mathrm{g}$ & Duplicate \\
\hline
\end{tabular}


Units QC Type

\section{Soil Monitoring}

Tritium, Free Water cont.

\begin{tabular}{|c|c|c|c|c|c|}
\hline SSNTLF-01-17-0.5 & 04/23/01 & ND & 0.007 & $\mathrm{~Bq} / \mathrm{g}$ & Sample \\
\hline SSNTLF-01-17-1.5 & $04 / 23 / 01$ & ND & 0.007 & $\mathrm{~Bq} / \mathrm{g}$ & Sample \\
\hline SSNTLF-01-18-0.5 & $04 / 23 / 01$ & ND & 0.007 & $\mathrm{~Bq} / \mathrm{g}$ & Sample \\
\hline SSNTLF-01-18-1.5 & $04 / 23 / 01$ & ND & 0.007 & $\mathrm{~Bq} / \mathrm{g}$ & Sample \\
\hline SSNTLF-01-19-0.5 & $04 / 23 / 01$ & ND & 0.007 & $\mathrm{~Bq} / \mathrm{g}$ & Sample \\
\hline SSNTLF-01-19-1.5 & $04 / 23 / 01$ & ND & 0.007 & $\mathrm{~Bq} / \mathrm{g}$ & Sample \\
\hline SSNTLF-01-20-0.5 & $04 / 23 / 01$ & ND & 0.007 & $\mathrm{~Bq} / \mathrm{g}$ & Sample \\
\hline SSNTLF-01-20-1.5 & $04 / 23 / 01$ & ND & 0.007 & $\mathrm{~Bq} / \mathrm{g}$ & Sample \\
\hline SSNTLF-01-21-0.5 & $04 / 23 / 01$ & ND & 0.007 & $\mathrm{~Bq} / \mathrm{g}$ & Sample \\
\hline SSNTLF-01-21-1.5 & $04 / 23 / 01$ & ND & 0.007 & $\mathrm{~Bq} / \mathrm{g}$ & Sample \\
\hline SSNTLF-01-22-0.5 & $\begin{array}{l}04 / 23 / 01 \\
04 / 23 / 01\end{array}$ & $\begin{array}{l}\text { ND } \\
\text { ND }\end{array}$ & $\begin{array}{l}0.007 \\
0.007\end{array}$ & $\begin{array}{l}\mathrm{Bq} / \mathrm{g} \\
\mathrm{Bq} / \mathrm{g}\end{array}$ & $\begin{array}{r}\text { Sample } \\
\text { Duplicate }\end{array}$ \\
\hline SSNTLF-01-22-1.5 & $\begin{array}{l}04 / 23 / 01 \\
04 / 23 / 01\end{array}$ & $\begin{array}{l}\text { ND } \\
\text { ND }\end{array}$ & $\begin{array}{l}0.007 \\
0.007\end{array}$ & $\begin{array}{l}\mathrm{Bq} / \mathrm{g} \\
\mathrm{Bq} / \mathrm{g}\end{array}$ & $\begin{array}{r}\text { Sample } \\
\text { Duplicate }\end{array}$ \\
\hline SSNTLF-01-23-0.5 & $\begin{array}{l}04 / 16 / 01 \\
04 / 16 / 01 \\
04 / 16 / 01\end{array}$ & $\begin{array}{l}\text { ND } \\
\text { ND } \\
\text { ND }\end{array}$ & $\begin{array}{l}0.007 \\
0.007 \\
0.007\end{array}$ & $\begin{array}{l}\mathrm{Bq} / \mathrm{g} \\
\mathrm{Bq} / \mathrm{g} \\
\mathrm{Bq} / \mathrm{g}\end{array}$ & $\begin{array}{r}\text { Sample } \\
\text { Split } \\
\text { Split }\end{array}$ \\
\hline SSNTLF-01-23-1.5 & $\begin{array}{l}04 / 16 / 01 \\
04 / 16 / 01 \\
04 / 16 / 01\end{array}$ & $\begin{array}{l}\text { ND } \\
\text { ND } \\
\text { ND }\end{array}$ & $\begin{array}{l}0.007 \\
0.007 \\
0.007\end{array}$ & $\begin{array}{l}\mathrm{Bq} / \mathrm{g} \\
\mathrm{Bq} / \mathrm{g} \\
\mathrm{Bq} / \mathrm{g}\end{array}$ & $\begin{array}{r}\text { Sample } \\
\text { Split } \\
\text { Split }\end{array}$ \\
\hline SSNTLF-01-24-0.5 & $\begin{array}{l}04 / 16 / 01 \\
04 / 16 / 01 \\
04 / 16 / 01\end{array}$ & $\begin{array}{l}\text { ND } \\
\text { ND } \\
\text { ND }\end{array}$ & $\begin{array}{l}0.007 \\
0.007 \\
0.007\end{array}$ & $\begin{array}{l}\mathrm{Bq} / \mathrm{g} \\
\mathrm{Bq} / \mathrm{g} \\
\mathrm{Bq} / \mathrm{g}\end{array}$ & $\begin{array}{r}\text { Sample } \\
\text { Split } \\
\text { Split }\end{array}$ \\
\hline SSNTLF-01-24-1.5 & $\begin{array}{l}04 / 16 / 01 \\
04 / 16 / 01 \\
04 / 16 / 01\end{array}$ & $\begin{array}{l}\text { ND } \\
\text { ND } \\
\text { ND }\end{array}$ & $\begin{array}{l}0.007 \\
0.007 \\
0.007\end{array}$ & $\begin{array}{l}\mathrm{Bq} / \mathrm{g} \\
\mathrm{Bq} / \mathrm{g} \\
\mathrm{Bq} / \mathrm{g}\end{array}$ & $\begin{array}{r}\text { Sample } \\
\text { Split } \\
\text { Split }\end{array}$ \\
\hline SSNTLF-01-25-0.5 & $\begin{array}{l}05 / 3 / 01 \\
05 / 3 / 01\end{array}$ & $\begin{array}{l}\text { ND } \\
\text { ND }\end{array}$ & $\begin{array}{l}0.007 \\
0.007\end{array}$ & $\begin{array}{l}\mathrm{Bq} / \mathrm{g} \\
\mathrm{Bq} / \mathrm{g}\end{array}$ & $\begin{array}{l}\text { Sample } \\
\text { Duplicate }\end{array}$ \\
\hline
\end{tabular}




\begin{tabular}{llllll} 
Analyte & Location & Date & Result & MDA or PQL Units & QC Type \\
\hline
\end{tabular}

\section{Soil Monitoring}

Tritium, Free Water cont.

\begin{tabular}{|c|c|c|c|c|c|}
\hline SSNTLF-01-25-1.5 & $\begin{array}{l}05 / 3 / 01 \\
05 / 3 / 01\end{array}$ & $\begin{array}{l}\text { ND } \\
\text { ND }\end{array}$ & $\begin{array}{l}0.007 \\
0.007\end{array}$ & $\begin{array}{l}\mathrm{Bq} / \mathrm{g} \\
\mathrm{Bq} / \mathrm{g}\end{array}$ & $\begin{array}{r}\text { Sample } \\
\text { Duplicate }\end{array}$ \\
\hline SSNTLF-01-26-0.5 & $05 / 3 / 01$ & ND & 0.007 & $\mathrm{~Bq} / \mathrm{g}$ & Sample \\
\hline SSNTLF-01-26-1.5 & $05 / 3 / 01$ & ND & 0.007 & $\mathrm{~Bq} / \mathrm{g}$ & Sample \\
\hline SSNTLF-01-27-0.5 & $05 / 3 / 01$ & ND & 0.007 & $\mathrm{~Bq} / \mathrm{g}$ & Sample \\
\hline SSNTLF-01-27-1.5 & $05 / 3 / 01$ & ND & 0.007 & $\mathrm{~Bq} / \mathrm{g}$ & Sample \\
\hline SSNTLF-01-28-0.5 & $04 / 24 / 01$ & ND & 0.007 & $\mathrm{~Bq} / \mathrm{g}$ & Sample \\
\hline SSNTLF-01-28-1.5 & 04/24/01 & ND & 0.007 & $\mathrm{~Bq} / \mathrm{g}$ & Sample \\
\hline SSNTLF-01-29-0.5 & $04 / 24 / 01$ & ND & 0.007 & $\mathrm{~Bq} / \mathrm{g}$ & Sample \\
\hline SSNTLF-01-29-1.5 & 04/24/01 & ND & 0.007 & $\mathrm{~Bq} / \mathrm{g}$ & Sample \\
\hline SSNTLF-01-30-0.5 & $04 / 23 / 01$ & ND & 0.007 & $\mathrm{~Bq} / \mathrm{g}$ & Sample \\
\hline SSNTLF-01-30-1.5 & 04/23/01 & ND & 0.007 & $\mathrm{~Bq} / \mathrm{g}$ & Sample \\
\hline SSNTLF-01-31-0.5 & 04/24/01 & ND & 0.007 & $\mathrm{~Bq} / \mathrm{g}$ & Sample \\
\hline SSNTLF-01-31-1.5 & $04 / 24 / 01$ & ND & 0.007 & $\mathrm{~Bq} / \mathrm{g}$ & Sample \\
\hline SSNTLF-01-32-0.5 & $04 / 27 / 01$ & ND & 0.007 & $\mathrm{~Bq} / \mathrm{g}$ & Sample \\
\hline SSNTLF-01-32-1.5 & $04 / 27 / 01$ & ND & 0.007 & $\mathrm{~Bq} / \mathrm{g}$ & Sample \\
\hline SSNTLF-01-33-0.5 & $04 / 23 / 01$ & ND & 0.007 & $\mathrm{~Bq} / \mathrm{g}$ & Sample \\
\hline SSNTLF-01-33-1.5 & 04/23/01 & ND & 0.007 & $\mathrm{~Bq} / \mathrm{g}$ & Sample \\
\hline SSNTLF-01-34-0.5 & $04 / 23 / 01$ & ND & 0.007 & $\mathrm{~Bq} / \mathrm{g}$ & Sample \\
\hline SSNTLF-01-34-1.5 & 04/23/01 & ND & 0.007 & $\mathrm{~Bq} / \mathrm{g}$ & Sample \\
\hline SSNTLF-01-35-0.5 & 04/23/01 & ND & 0.007 & $\mathrm{~Bq} / \mathrm{g}$ & Sample \\
\hline SSNTLF-01-35-1.5 & 04/23/01 & ND & 0.007 & $\mathrm{~Bq} / \mathrm{g}$ & Sample \\
\hline SSNTLF-01-36-0.5 & $\begin{array}{l}05 / 1 / 01 \\
05 / 1 / 01\end{array}$ & $\begin{array}{l}\text { ND } \\
\text { ND }\end{array}$ & $\begin{array}{l}0.007 \\
0.007\end{array}$ & $\begin{array}{l}\mathrm{Bq} / \mathrm{g} \\
\mathrm{Bq} / \mathrm{g}\end{array}$ & $\begin{array}{l}\text { Sample } \\
\text { Duplicate }\end{array}$ \\
\hline
\end{tabular}


Units QC Type

\section{Soil Monitoring}

Tritium, Free Water cont.

\begin{tabular}{|c|c|c|c|c|c|}
\hline SSNTLF-01-36-1.5 & $\begin{array}{l}05 / 1 / 01 \\
05 / 1 / 01\end{array}$ & $\begin{array}{l}\text { ND } \\
\text { ND }\end{array}$ & $\begin{array}{l}0.007 \\
0.007\end{array}$ & $\begin{array}{l}\mathrm{Bq} / \mathrm{g} \\
\mathrm{Bq} / \mathrm{g}\end{array}$ & $\begin{array}{r}\text { Sample } \\
\text { Duplicate }\end{array}$ \\
\hline SSNTLF-01-37-0.5 & $05 / 1 / 01$ & ND & 0.007 & $\mathrm{~Bq} / \mathrm{g}$ & Sample \\
\hline SSNTLF-01-37-1.5 & $05 / 1 / 01$ & ND & 0.007 & $\mathrm{~Bq} / \mathrm{g}$ & Sample \\
\hline SSNTLF-01-38-0.5 & $04 / 27 / 01$ & ND & 0.007 & $\mathrm{~Bq} / \mathrm{g}$ & Sample \\
\hline SSNTLF-01-38-1.5 & $04 / 27 / 01$ & ND & 0.007 & $\mathrm{~Bq} / \mathrm{g}$ & Sample \\
\hline SSNTLF-01-39-0.5 & $04 / 23 / 01$ & ND & 0.007 & $\mathrm{~Bq} / \mathrm{g}$ & Sample \\
\hline SSNTLF-01-39-1.5 & $04 / 23 / 01$ & ND & 0.007 & $\mathrm{~Bq} / \mathrm{g}$ & Sample \\
\hline SSNTLF-01-40-0.5 & $\begin{array}{l}04 / 24 / 01 \\
04 / 24 / 01\end{array}$ & $\begin{array}{l}\text { ND } \\
\text { ND }\end{array}$ & $\begin{array}{l}0.007 \\
0.007\end{array}$ & $\begin{array}{l}\mathrm{Bq} / \mathrm{g} \\
\mathrm{Bq} / \mathrm{g}\end{array}$ & $\begin{array}{r}\text { Sample } \\
\text { Duplicate }\end{array}$ \\
\hline SSNTLF-01-40-1.5 & $\begin{array}{l}04 / 24 / 01 \\
04 / 24 / 01\end{array}$ & $\begin{array}{l}\text { ND } \\
\text { ND }\end{array}$ & $\begin{array}{l}0.007 \\
0.007\end{array}$ & $\begin{array}{l}\mathrm{Bq} / \mathrm{g} \\
\mathrm{Bq} / \mathrm{g}\end{array}$ & $\begin{array}{c}\text { Sample } \\
\text { Duplicate }\end{array}$ \\
\hline SSNTLF-01-41-0.5 & $04 / 23 / 01$ & ND & 0.007 & $\mathrm{~Bq} / \mathrm{g}$ & Sample \\
\hline SSNTLF-01-41-1.5 & $04 / 23 / 01$ & ND & 0.007 & $\mathrm{~Bq} / \mathrm{g}$ & Sample \\
\hline SSNTLF-01-42-0.5 & $\begin{array}{l}05 / 4 / 01 \\
05 / 4 / 01\end{array}$ & $\begin{array}{l}\text { ND } \\
\text { ND }\end{array}$ & $\begin{array}{l}0.007 \\
0.007\end{array}$ & $\begin{array}{l}\mathrm{Bq} / \mathrm{g} \\
\mathrm{Bq} / \mathrm{g}\end{array}$ & $\begin{array}{c}\text { Sample } \\
\text { Duplicate }\end{array}$ \\
\hline SSNTLF-01-42-1.5 & $\begin{array}{l}05 / 4 / 01 \\
05 / 4 / 01\end{array}$ & $\begin{array}{l}\text { ND } \\
\text { ND }\end{array}$ & $\begin{array}{l}0.007 \\
0.007\end{array}$ & $\begin{array}{l}\mathrm{Bq} / \mathrm{g} \\
\mathrm{Bq} / \mathrm{g}\end{array}$ & $\begin{array}{r}\text { Sample } \\
\text { Duplicate }\end{array}$ \\
\hline SSNTLF-01-43-0.5 & $04 / 18 / 01$ & 0.291 & 0.007 & $\mathrm{~Bq} / \mathrm{g}$ & Sample \\
\hline SSNTLF-01-43-1.5 & $04 / 18 / 01$ & 0.37 & 0.007 & $\mathrm{~Bq} / \mathrm{g}$ & Sample \\
\hline SSNTLF-01-44-0.5 & $\begin{array}{l}04 / 13 / 01 \\
04 / 13 / 01\end{array}$ & $\begin{array}{l}0.00967 \\
0.0146\end{array}$ & $\begin{array}{l}0.007 \\
0.007\end{array}$ & $\begin{array}{l}\mathrm{Bq} / \mathrm{g} \\
\mathrm{Bq} / \mathrm{g}\end{array}$ & $\begin{array}{c}\text { Sample } \\
\text { Duplicate }\end{array}$ \\
\hline SSNTLF-01-44-1.5 & $\begin{array}{l}04 / 13 / 01 \\
04 / 13 / 01\end{array}$ & $\begin{array}{l}0.015 \\
0.0113\end{array}$ & $\begin{array}{l}0.007 \\
0.007\end{array}$ & $\begin{array}{l}\mathrm{Bq} / \mathrm{g} \\
\mathrm{Bq} / \mathrm{g}\end{array}$ & $\begin{array}{c}\text { Sample } \\
\text { Duplicate }\end{array}$ \\
\hline SSNTLF-01-45-0.5 & $\begin{array}{l}04 / 13 / 01 \\
04 / 13 / 01 \\
04 / 13 / 01\end{array}$ & $\begin{array}{l}0.00889 \\
\text { ND } \\
0.0093\end{array}$ & $\begin{array}{l}0.007 \\
0.007 \\
0.007\end{array}$ & $\begin{array}{l}\mathrm{Bq} / \mathrm{g} \\
\mathrm{Bq} / \mathrm{g} \\
\mathrm{Bq} / \mathrm{g}\end{array}$ & $\begin{array}{r}\text { Sample } \\
\text { Split } \\
\text { Split }\end{array}$ \\
\hline
\end{tabular}




$\begin{array}{llllll}\text { Analyte } & \text { Location } & \text { Date } & \text { Result } & \text { MDA or PQL Units } & \text { QC Type }\end{array}$

\section{Soil Monitoring}

Tritium, Free Water cont.

\begin{tabular}{|c|c|c|c|c|c|}
\hline SSNTLF-01-45-1.5 & $\begin{array}{l}04 / 13 / 01 \\
04 / 13 / 01 \\
04 / 13 / 01\end{array}$ & $\begin{array}{l}0.0225 \\
0.022 \\
0.022\end{array}$ & $\begin{array}{l}0.007 \\
0.007 \\
0.007\end{array}$ & $\begin{array}{l}\mathrm{Bq} / \mathrm{g} \\
\mathrm{Bq} / \mathrm{g} \\
\mathrm{Bq} / \mathrm{g}\end{array}$ & $\begin{array}{r}\text { Sample } \\
\text { Split } \\
\text { Split }\end{array}$ \\
\hline SSNTLF-01-46-0.5 & $\begin{array}{l}04 / 2 / 01 \\
04 / 2 / 01 \\
04 / 2 / 01\end{array}$ & $\begin{array}{l}0.150 \\
0.131 \\
0.17\end{array}$ & $\begin{array}{l}0.007 \\
0.007 \\
0.007\end{array}$ & $\begin{array}{l}\mathrm{Bq} / \mathrm{g} \\
\mathrm{Bq} / \mathrm{g} \\
\mathrm{Bq} / \mathrm{g}\end{array}$ & $\begin{array}{r}\text { Sample } \\
\text { Split } \\
\text { Split }\end{array}$ \\
\hline SSNTLF-01-46-1.5 & $\begin{array}{l}04 / 2 / 01 \\
04 / 2 / 01 \\
04 / 2 / 01\end{array}$ & $\begin{array}{l}0.164 \\
0.15 \\
0.162\end{array}$ & $\begin{array}{l}0.007 \\
0.007 \\
0.007\end{array}$ & $\begin{array}{l}\mathrm{Bq} / \mathrm{g} \\
\mathrm{Bq} / \mathrm{g} \\
\mathrm{Bq} / \mathrm{g}\end{array}$ & $\begin{array}{r}\text { Sample } \\
\text { Split } \\
\text { Split }\end{array}$ \\
\hline SSNTLF-01-47-0.5 & $\begin{array}{l}04 / 2 / 01 \\
04 / 2 / 01\end{array}$ & $\begin{array}{l}0.0296 \\
0.0104\end{array}$ & $\begin{array}{l}0.007 \\
0.007\end{array}$ & $\begin{array}{l}\mathrm{Bq} / \mathrm{g} \\
\mathrm{Bq} / \mathrm{g}\end{array}$ & $\begin{array}{r}\text { Sample } \\
\text { Split }\end{array}$ \\
\hline SSNTLF-01-47-1.5 & $\begin{array}{l}04 / 2 / 01 \\
04 / 2 / 01\end{array}$ & $\begin{array}{l}0.0283 \\
0.0159\end{array}$ & $\begin{array}{l}0.007 \\
0.007\end{array}$ & $\begin{array}{l}\mathrm{Bq} / \mathrm{g} \\
\mathrm{Bq} / \mathrm{g}\end{array}$ & $\begin{array}{r}\text { Sample } \\
\text { Split }\end{array}$ \\
\hline SSNTLF-01-47Comp-0.5 & $\begin{array}{l}04 / 2 / 01 \\
04 / 2 / 01 \\
04 / 2 / 01\end{array}$ & $\begin{array}{l}0.0319 \\
0.0213 \\
0.0167\end{array}$ & $\begin{array}{l}0.007 \\
0.007 \\
0.007\end{array}$ & $\begin{array}{l}\mathrm{Bq} / \mathrm{g} \\
\mathrm{Bq} / \mathrm{g} \\
\mathrm{Bq} / \mathrm{g}\end{array}$ & $\begin{array}{r}\text { Sample } \\
\text { Split } \\
\text { Split }\end{array}$ \\
\hline SSNTLF-01-47Comp-1.5 & $\begin{array}{l}04 / 2 / 01 \\
04 / 2 / 01 \\
04 / 2 / 01\end{array}$ & $\begin{array}{l}0.0346 \\
0.0257 \\
0.010\end{array}$ & $\begin{array}{l}0.007 \\
0.007 \\
0.007\end{array}$ & $\begin{array}{l}\mathrm{Bq} / \mathrm{g} \\
\mathrm{Bq} / \mathrm{g} \\
\mathrm{Bq} / \mathrm{g}\end{array}$ & $\begin{array}{r}\text { Sample } \\
\text { Split } \\
\text { Split }\end{array}$ \\
\hline SSNTLF-01-48-0.5 & $\begin{array}{l}04 / 2 / 01 \\
04 / 2 / 01\end{array}$ & $\begin{array}{l}0.00818 \\
N D\end{array}$ & $\begin{array}{l}0.007 \\
0.007\end{array}$ & $\begin{array}{l}\mathrm{Bq} / \mathrm{g} \\
\mathrm{Bq} / \mathrm{g}\end{array}$ & $\begin{array}{r}\text { Sample } \\
\text { Split }\end{array}$ \\
\hline SSNTLF-01-48-1.5 & $\begin{array}{l}04 / 2 / 01 \\
04 / 2 / 01\end{array}$ & $\begin{array}{l}\text { ND } \\
\text { ND }\end{array}$ & $\begin{array}{l}0.007 \\
0.007\end{array}$ & $\begin{array}{l}\mathrm{Bq} / \mathrm{g} \\
\mathrm{Bq} / \mathrm{g}\end{array}$ & $\begin{array}{r}\text { Sample } \\
\text { Split }\end{array}$ \\
\hline SSNTLF-01-48Comp-0.5 & $\begin{array}{l}04 / 2 / 01 \\
04 / 2 / 01 \\
04 / 2 / 01\end{array}$ & $\begin{array}{l}0.0105 \\
\text { ND } \\
0.0081\end{array}$ & $\begin{array}{l}0.007 \\
0.007 \\
0.007\end{array}$ & $\begin{array}{l}\mathrm{Bq} / \mathrm{g} \\
\mathrm{Bq} / \mathrm{g} \\
\mathrm{Bq} / \mathrm{g}\end{array}$ & $\begin{array}{r}\text { Sample } \\
\text { Split } \\
\text { Split }\end{array}$ \\
\hline SSNTLF-01-48Comp-1.5 & $\begin{array}{l}04 / 2 / 01 \\
04 / 2 / 01 \\
04 / 2 / 01\end{array}$ & $\begin{array}{l}\text { ND } \\
0.010 \\
\text { ND }\end{array}$ & $\begin{array}{l}0.007 \\
0.007 \\
0.007\end{array}$ & $\begin{array}{l}\mathrm{Bq} / \mathrm{g} \\
\mathrm{Bq} / \mathrm{g} \\
\mathrm{Bq} / \mathrm{g}\end{array}$ & $\begin{array}{r}\text { Sample } \\
\text { Split } \\
\text { Split }\end{array}$ \\
\hline
\end{tabular}


Units QC Type

\section{Soil Monitoring}

Tritium, Free Water cont.

\begin{tabular}{|c|c|c|c|c|c|}
\hline SSNTLF-01-49-0.5 & $\begin{array}{l}05 / 3 / 01 \\
05 / 3 / 01\end{array}$ & $\begin{array}{l}\text { ND } \\
\text { ND }\end{array}$ & $\begin{array}{l}0.007 \\
0.007\end{array}$ & $\begin{array}{l}\mathrm{Bq} / \mathrm{g} \\
\mathrm{Bq} / \mathrm{g}\end{array}$ & $\begin{array}{r}\text { Sample } \\
\text { Duplicate }\end{array}$ \\
\hline \multirow[t]{2}{*}{ SSNTLF-01-49-1.5 } & $05 / 3 / 01$ & ND & 0.007 & $\mathrm{~Bq} / \mathrm{g}$ & Sample \\
\hline & $05 / 3 / 01$ & ND & 0.007 & $\mathrm{~Bq} / \mathrm{g}$ & Duplicate \\
\hline \multirow[t]{2}{*}{ SSNTLF-01-50-0.5 } & $05 / 4 / 01$ & ND & 0.007 & $\mathrm{~Bq} / \mathrm{g}$ & Sample \\
\hline & $05 / 4 / 01$ & ND & 0.007 & $\mathrm{~Bq} / \mathrm{g}$ & Duplicate \\
\hline \multirow[t]{2}{*}{ SSNTLF-01-50-1.5 } & $05 / 4 / 01$ & ND & 0.007 & $\mathrm{~Bq} / \mathrm{g}$ & Sample \\
\hline & $05 / 4 / 01$ & ND & 0.007 & $\mathrm{~Bq} / \mathrm{g}$ & Duplicate \\
\hline SSNTLF-01-51-0.5 & $05 / 4 / 01$ & ND & 0.007 & $\mathrm{~Bq} / \mathrm{g}$ & Sample \\
\hline SSNTLF-01-51-1.5 & $05 / 4 / 01$ & ND & 0.007 & $\mathrm{~Bq} / \mathrm{g}$ & Sample \\
\hline \multirow[t]{2}{*}{ SSNTLF-01-52-0.5 } & $05 / 3 / 01$ & ND & 0.007 & $\mathrm{~Bq} / \mathrm{g}$ & Sample \\
\hline & $05 / 3 / 01$ & ND & 0.007 & $\mathrm{~Bq} / \mathrm{g}$ & Duplicate \\
\hline \multirow[t]{2}{*}{ SSNTLF-01-52-1.5 } & $05 / 3 / 01$ & ND & 0.007 & $\mathrm{~Bq} / \mathrm{g}$ & Sample \\
\hline & $05 / 3 / 01$ & ND & 0.007 & $\mathrm{~Bq} / \mathrm{g}$ & Duplicate \\
\hline SSNTLF-01-53-0.5 & $05 / 4 / 01$ & ND & 0.007 & $\mathrm{~Bq} / \mathrm{g}$ & Sample \\
\hline SSNTLF-01-53-1.5 & $05 / 4 / 01$ & ND & 0.007 & $\mathrm{~Bq} / \mathrm{g}$ & Sample \\
\hline \multirow[t]{3}{*}{ SSNTLF-01-54-0.5 } & $04 / 13 / 01$ & ND & 0.007 & $\mathrm{~Bq} / \mathrm{g}$ & Sample \\
\hline & 04/13/01 & ND & 0.007 & $\mathrm{~Bq} / \mathrm{g}$ & Split \\
\hline & $04 / 13 / 01$ & ND & 0.007 & $\mathrm{~Bq} / \mathrm{g}$ & Split \\
\hline \multirow[t]{3}{*}{ SSNTLF-01-54-1.5 } & $04 / 13 / 01$ & ND & 0.007 & $\mathrm{~Bq} / \mathrm{g}$ & Sample \\
\hline & $04 / 13 / 01$ & ND & 0.007 & $\mathrm{~Bq} / \mathrm{g}$ & Split \\
\hline & $04 / 13 / 01$ & ND & 0.007 & $\mathrm{~Bq} / \mathrm{g}$ & Split \\
\hline SSNTLF-01-55-0.5 & $05 / 4 / 01$ & ND & 0.007 & $\mathrm{~Bq} / \mathrm{g}$ & Sample \\
\hline SSNTLF-01-55-1.5 & $05 / 4 / 01$ & ND & 0.007 & $\mathrm{~Bq} / \mathrm{g}$ & Sample \\
\hline \multirow[t]{2}{*}{ SSNTLF-01-56-0.5 } & $05 / 4 / 01$ & ND & 0.007 & $\mathrm{~Bq} / \mathrm{g}$ & Sample \\
\hline & $05 / 4 / 01$ & ND & 0.007 & $\mathrm{~Bq} / \mathrm{g}$ & Duplicate \\
\hline \multirow[t]{2}{*}{ SSNTLF-01-56-1.5 } & $05 / 4 / 01$ & ND & 0.007 & $\mathrm{~Bq} / \mathrm{g}$ & Sample \\
\hline & $05 / 4 / 01$ & ND & 0.007 & $\mathrm{~Bq} / \mathrm{g}$ & Duplicate \\
\hline SSNTLF-01-57-0.5 & $04 / 13 / 01$ & 0.0128 & 0.007 & $\mathrm{~Bq} / \mathrm{g}$ & Sample \\
\hline
\end{tabular}




$\begin{array}{llllll}\text { Analyte } & \text { Location } & \text { Date } & \text { Result } & \text { MDA or PQL Units } & \text { QC Type }\end{array}$

\section{Soil Monitoring}

Tritium, Free Water cont.

$\begin{array}{llllll}\text { SSNTLF-01-57-1.5 } & 04 / 13 / 01 & 0.0126 & 0.007 & \mathrm{~Bq} / \mathrm{g} & \text { Sample } \\ \text { SSNTLF-01-58-0.5 } & 04 / 13 / 01 & \mathrm{ND} & 0.007 & \mathrm{~Bq} / \mathrm{g} & \text { Sample } \\ \text { SSNTLF-01-58-1.5 } & 04 / 13 / 01 & 0.0102 & 0.007 & \mathrm{~Bq} / \mathrm{g} & \text { Sample } \\ \text { SSNTLF-01-59-0.5 } & 04 / 13 / 01 & 0.0393 & 0.007 & \mathrm{~Bq} / \mathrm{g} & \text { Sample } \\ \text { SSNTLF-01-59-1.5 } & 04 / 13 / 01 & 0.048 & 0.007 & \mathrm{~Bq} / \mathrm{g} & \text { Sample } \\ \text { SSNTLF-01-60-0.5 } & 04 / 18 / 01 & 0.0276 & 0.007 & \mathrm{~Bq} / \mathrm{g} & \text { Sample } \\ \text { SSNTLF-01-60-1.5 } & 04 / 18 / 01 & 0.0362 & 0.007 & \mathrm{~Bq} / \mathrm{g} & \text { Sample } \\ \text { SSNTLF-01-61-0.5 } & 04 / 18 / 01 & 0.105 & 0.007 & \mathrm{~Bq} / \mathrm{g} & \text { Sample } \\ \text { SSNTLF-01-61-1.5 } & 04 / 18 / 01 & 0.0689 & 0.007 & \mathrm{~Bq} / \mathrm{g} & \text { Sample } \\ \text { SSNTLF-01-62-0.5 } & 04 / 4 / 01 & 0.0618 & 0.007 & \mathrm{~Bq} / \mathrm{g} & \text { Sample } \\ \text { SSNTLF-01-62-1.5 } & 04 / 4 / 01 & 0.0618 & 0.007 & \mathrm{~Bq} / \mathrm{g} & \text { Sample } \\ \text { SSNTLF-01-62Comp-0.5 } & 04 / 4 / 01 & 0.0807 & 0.007 & \mathrm{~Bq} / \mathrm{g} & \text { Sample } \\ \text { SSNTLF-01-62Comp-1.5 } & 04 / 4 / 01 & 0.0796 & 0.007 & \mathrm{~Bq} / \mathrm{g} & \text { Sample } \\ \text { SSNTLF-01-63-0.5 } & 04 / 6 / 01 & 0.0238 & 0.007 & \mathrm{~Bq} / \mathrm{g} & \text { Sample } \\ \text { SSNTLF-01-63-1.5 } & 04 / 6 / 01 & 0.0258 & 0.007 & \mathrm{~Bq} / \mathrm{g} & \text { Sample } \\ \text { SSNTLF-01-63Comp-0.5 } & 04 / 6 / 01 & 0.02 & 0.007 & \mathrm{~Bq} / \mathrm{g} & \text { Sample } \\ \text { SSNTLF-01-63Comp-1.5 } & 04 / 6 / 01 & 0.0235 & 0.007 & \mathrm{~Bq} / \mathrm{g} & \text { Sample } \\ \text { SSNTLF-01-64-0.5 } & 04 / 6 / 01 & 0.011 & 0.007 & \mathrm{~Bq} / \mathrm{g} & \text { Sample } \\ \text { SSNTLF-01-64-1.5 } & 04 / 19 / 01 & 0.011 & 0.007 & \mathrm{~Bq} / \mathrm{g} & \text { Sample } \\ \text { SSNTLF-01-65-0.5 } & 04 / 17 / 01 & \mathrm{ND} & 0.007 & \mathrm{~Bq} / \mathrm{g} & \text { Sample } \\ \text { SSNTLF-01-65-1.5 } & 04 / 17 / 01 & \mathrm{ND} & 0.007 & \mathrm{~Bq} / \mathrm{g} & \text { Sample } \\ \text { SSNTLF-01-66-0.5 } & 04 / 17 / 01 & \mathrm{ND} & 0.007 & \mathrm{~Bq} / \mathrm{g} & \text { Sample } \\ \text { SSNTF-01-66-1.5 } & 04 / 17 / 01 & \mathrm{ND} & 0.007 & \mathrm{~Bq} / \mathrm{g} & \text { Sample }\end{array}$


Date Result MDA or PQL Units QC Type

\section{Soil Monitoring}

Tritium, Free Water cont.

Blank

Tritium, Total

$\begin{array}{lllllr}\text { SSNTLF-01-11-0.5 } & 04 / 4 / 01 & \text { ND } & 0.2 & \mathrm{~Bq} / \mathrm{g} & \text { Sample } \\ \text { SSNTLF-01-11-1.5 } & 04 / 4 / 01 & \mathrm{ND} & 0.2 & \mathrm{~Bq} / \mathrm{g} & \text { Sample } \\ \text { SSNTLF-01-11Comp-0.5 } & 04 / 4 / 01 & \mathrm{ND} & 0.2 & \mathrm{~Bq} / \mathrm{g} & \text { Sample } \\ \text { SSNTLF-01-11Comp-1.5 } & 04 / 4 / 01 & \mathrm{ND} & 0.2 & \mathrm{~Bq} / \mathrm{g} & \text { Sample } \\ \text { SSNTLF-01-14-0.5 } & 04 / 24 / 01 & \mathrm{ND} & 0.2 & \mathrm{~Bq} / \mathrm{g} & \text { Sample } \\ & 04 / 24 / 01 & \mathrm{ND} & 0.2 & \mathrm{~Bq} / \mathrm{g} & \text { Duplicate } \\ \text { SSNTLF-01-14-1.5 } & 04 / 24 / 01 & \mathrm{ND} & 0.2 & \mathrm{~Bq} / \mathrm{g} & \text { Sample } \\ & 04 / 24 / 01 & \mathrm{ND} & 0.2 & \mathrm{~Bq} / \mathrm{g} & \text { Duplicate } \\ \text { SSNTLF-01-43-0.5 } & 04 / 18 / 01 & 1.53 & 0.2 & \mathrm{~Bq} / \mathrm{g} & \text { Sample } \\ \text { SSNTLF-01-43-1.5 } & 04 / 18 / 01 & 1.53 & 0.2 & \mathrm{~Bq} / \mathrm{g} & \text { Sample } \\ \text { SSNTLF-01-47-0.5 } & 04 / 2 / 01 & \mathrm{ND} & 0.2 & \mathrm{~Bq} / \mathrm{g} & \text { Sample } \\ & 04 / 2 / 01 & \mathrm{ND} & 0.2 & \mathrm{~Bq} / \mathrm{g} & \text { Split } \\ \text { SSNTLF-01-47-1.5 } & 04 / 2 / 01 & \mathrm{ND} & 0.2 & \mathrm{~Bq} / \mathrm{g} & \text { Sample } \\ & 04 / 2 / 01 & \mathrm{ND} & 0.2 & \mathrm{~Bq} / \mathrm{g} & \text { Split } \\ \text { SSNTLF-01-47Comp-0.5 } & 04 / 2 / 01 & \mathrm{ND} & 0.2 & \mathrm{~Bq} / \mathrm{g} & \text { Sample } \\ & 04 / 2 / 01 & \mathrm{ND} & 0.2 & \mathrm{~Bq} / \mathrm{g} & \text { Split } \\ \text { SSNTLF-01-47Comp-1.5 } & 04 / 2 / 01 & \mathrm{ND} & 0.2 & \mathrm{~Bq} / \mathrm{g} & \text { Sample } \\ & 04 / 2 / 01 & \mathrm{ND} & 0.2 & \mathrm{~Bq} / \mathrm{g} & \text { Split } \\ & 04 / 2 / 01 & \mathrm{ND} & 0.2 & \mathrm{~Bq} / \mathrm{g} & \text { Split }\end{array}$




$\begin{array}{llllll}\text { Analyte } & \text { Location } & \text { Date } & \text { Result } & \text { MDA or PQL Units } & \text { QC Type }\end{array}$

\section{Soil Monitoring}

Tritium, Total cont.

$\begin{array}{llllll}\text { SSNTLF-01-64-0.5 } & 04 / 6 / 01 & \text { ND } & 0.2 & \mathrm{~Bq} / \mathrm{g} & \text { Sample } \\ \text { SSNTLF-01-64-1.5 } & 04 / 19 / 01 & \text { ND } & 0.2 & \mathrm{~Bq} / \mathrm{g} & \text { Sample }\end{array}$

\section{Surface Water Monitoring}

Tritium

Lake Anza

Banana Creek

Cafeteria Creek (Lower)

Cafeteria Creek (Upper)

Chicken Creek (Lower)

$4 / 10 / 0$

$4 / 10 / 01$

$9 / 4 / 01$

$9 / 4 / 01$

$10 / 29 / 01$

$10 / 29 / 01$

$11 / 28 / 01$

$11 / 28 / 01$

$12 / 18 / 01$

ND

ND

ND

ND

ND

ND

ND

ND

14.5

10.7

11

10

10

12

20

14

16

Chicken Creek (Upper)

$$
\text { 4/9/01 }
$$

$4 / 9 / 01$

$4 / 9 / 01$

$8 / 31 / 01$

$8 / 31 / 01$

$8 / 31 / 01$

$10 / 29 / 01$

$10 / 29 / 01$

$10 / 29 / 01$

$11 / 28 / 01$

8.63

14.0

11.7

ND

ND

13.5

ND

10.0

ND

9.6

Chicken Creek (Upper)

$11 / 28 / 01$
$11 / 28 / 01$
$12 / 18 / 01$

12.4

15.5

12

N. Fork Strawberry Creek (Lower)

2.4

ND

ND

ND

\section{7}

7

$\mathrm{Bq} / \mathrm{L}$

$\mathrm{Bq} / \mathrm{L}$

Sample

Sample

$\mathrm{Bq} / \mathrm{L} \quad$ Sample

$\mathrm{Bq} / \mathrm{L} \quad$ Duplicate

$\mathrm{Bq} / \mathrm{L} \quad$ Sample

$\mathrm{Bq} / \mathrm{L} \quad$ Duplicate

7

\section{7}

\section{7}

$\mathrm{Bq} / \mathrm{L}$

$\mathrm{Bq} / \mathrm{L}$

$\mathrm{Bq} / \mathrm{L}$

$\mathrm{Bq} / \mathrm{L}$

$\mathrm{Bq} / \mathrm{L}$

$\mathrm{Bq} / \mathrm{L}$

$\mathrm{Bq} / \mathrm{L}$

$\mathrm{Bq} / \mathrm{L}$

$\mathrm{Bq} / \mathrm{L}$

$\mathrm{Bq} / \mathrm{L}$

$\mathrm{Bq} / \mathrm{L}$

$\mathrm{Bq} / \mathrm{L}$

$\mathrm{Bq} / \mathrm{L}$

$\mathrm{Bq} / \mathrm{L}$

$\mathrm{Bq} / \mathrm{L}$

$\mathrm{Bq} / \mathrm{L}$

$\mathrm{Bq} / \mathrm{L}$

$\mathrm{Bq} / \mathrm{L}$

$\mathrm{Bq} / \mathrm{L}$

$\mathrm{Bq} / \mathrm{L}$

$\mathrm{Bq} / \mathrm{L}$

$\mathrm{Bq} / \mathrm{L}$

$\mathrm{Bq} / \mathrm{L}$

$\mathrm{Bq} / \mathrm{L}$

Sample

Sample

Sample

Duplicate

Sample

Duplicate

Sample

Duplicate

Sample

Duplicate

Sample

Sample Split Split

Sample Split Split

Sample Split Split Sample

7

Split
Split
Sample

$\mathrm{Bq} / \mathrm{L} \quad$ Sample $\mathrm{Bq} / \mathrm{L} \quad$ Duplicate 


\section{Analyte}

Location

Date Result MDA or PQL

Units

QC Type

\section{Surface Water Monitoring}

Tritium cont.

N. Fork Strawberry Creek (Lower)

$\begin{array}{lcllr}8 / 31 / 01 & \text { ND } & 7 & \mathrm{~Bq} / \mathrm{L} & \text { Sample } \\ 8 / 31 / 01 & \text { ND } & 7 & \mathrm{~Bq} / \mathrm{L} & \text { Duplicate } \\ 10 / 29 / 01 & \text { ND } & 7 & \mathrm{~Bq} / \mathrm{L} & \text { Sample } \\ 10 / 29 / 01 & 7.7 & 7 & \mathrm{~Bq} / \mathrm{L} & \text { Duplicate } \\ 11 / 28 / 01 & 10 & 7 & \mathrm{~Bq} / \mathrm{L} & \text { Sample } \\ 11 / 28 / 01 & \text { ND } & 7 & \mathrm{~Bq} / \mathrm{g} & \text { Duplicate } \\ 12 / 18 / 01 & \text { ND } & 7 & \mathrm{~Bq} / \mathrm{L} & \text { Sample }\end{array}$

N. Fork Strawberry Creek (Upper)

$4 / 9 / 01$

$8 / 31 / 01$

$10 / 29 / 01$

$11 / 28 / 01$

$12 / 18 / 01$

ND
ND
ND
7
ND

No Name Creek (Lower)

$4 / 10 / 0$

$9 / 4 / 01$

ND

ND

$4 / 10 / 01$

9/4/01

ND

ND

$4 / 10 / 01$

$8 / 31 / 01$

ND

ND

$4 / 9 / 01$

$4 / 9 / 01$

ND

ND

$4 / 9 / 01$

ND

Ravine Creek (Upper)

Strawberry Creek Outfall

$4 / 9 / 01$

9/4/01

$10 / 29 / 01$

$11 / 28 / 01$

$12 / 18 / 01$

$12 / 18 / 01$

ND

ND

ND

ND

ND

ND

ND

Strawberry Creek UC

$4 / 9 / 01$

$4 / 9 / 01$

ND

ND

\section{$9 / 4 / 01$}

$10 / 29 / 01$

$11 / 28 / 01$

$12 / 18 / 01$

$12 / 18 / 01$
ND

ND

ND

ND

ND
7

7

7

7

7

7

7

7

7

7

7

7

7

7

7

7

7

7

7

7

7

7

7

7

7

7

7

7

7
7
$\mathrm{Bq} / \mathrm{L} \quad$ Sample

$\mathrm{Bq} / \mathrm{L} \quad$ Sample

$\mathrm{Bq} / \mathrm{L} \quad$ Sample

Bq/L Sample

$\mathrm{Bq} / \mathrm{L} \quad$ Sample

$\mathrm{Bq} / \mathrm{L} \quad$ Sample

$\mathrm{Bq} / \mathrm{L} \quad$ Sample

$\mathrm{Bq} / \mathrm{L} \quad$ Sample

$\mathrm{Bq} / \mathrm{L} \quad$ Sample

$\mathrm{Bq} / \mathrm{L} \quad$ Sample

$\mathrm{Bq} / \mathrm{L} \quad$ Sample

$\mathrm{Bq} / \mathrm{L} \quad$ Sample

$\mathrm{Bq} / \mathrm{L} \quad$ Duplicate

$\mathrm{Bq} / \mathrm{L} \quad$ Sample

$\mathrm{Bq} / \mathrm{L} \quad$ Sample

$\mathrm{Bq} / \mathrm{L} \quad$ Duplicate

$\mathrm{Bq} / \mathrm{L} \quad$ Sample

$\mathrm{Bq} / \mathrm{L} \quad$ Sample

$\mathrm{Bq} / \mathrm{L} \quad$ Sample

$\mathrm{Bq} / \mathrm{L} \quad$ Sample

$\mathrm{Bq} / \mathrm{L} \quad$ Duplicate

$\mathrm{Bq} / \mathrm{L} \quad$ Sample

$\mathrm{Bq} / \mathrm{L} \quad$ Duplicate

$\mathrm{Bq} / \mathrm{L} \quad$ Sample

$\mathrm{Bq} / \mathrm{L} \quad$ Sample

$\mathrm{Bq} / \mathrm{L} \quad$ Sample

$\mathrm{Bq} / \mathrm{L} \quad$ Sample

$\mathrm{Bq} / \mathrm{L} \quad$ Duplicate 


$\begin{array}{llllll}\text { Analyte } & \text { Location } & \text { Date } & \text { Result } & \text { MDA or PQL Units } & \text { QC Type }\end{array}$

\section{Surface Water Monitoring}

Tritium cont.

$\begin{array}{llllll}\text { Lake Anza } & 4 / 9 / 01 & \text { ND } & 7 & \mathrm{~Bq} / \mathrm{L} & \text { Sample } \\ & 9 / 4 / 01 & \text { ND } & 7 & \mathrm{~Bq} / \mathrm{L} & \text { Sample } \\ \text { Lake Temescal } & 4 / 9 / 01 & \text { ND } & 7 & \mathrm{~Bq} / \mathrm{L} & \text { Sample } \\ & 9 / 5 / 01 & \mathrm{ND} & 7 & \mathrm{~Bq} / \mathrm{L} & \text { Sample } \\ \text { Ten Inch Creek (Lower) } & 4 / 11 / 01 & \mathrm{ND} & 7 & \mathrm{~Bq} / \mathrm{L} & \\ & 4 / 11 / 01 & \mathrm{ND} & 7 & \mathrm{~Bq} / \mathrm{L} & \text { Sample } \\ & & & & & \\ \text { Blank } & 9 / 4 / 01 & \mathrm{ND} & 7 & \mathrm{~Bq} / \mathrm{L} & \text { Blank } \\ & 9 / 4 / 01 & \mathrm{ND} & 7 & \mathrm{~Bq} / \mathrm{L} & \text { Blank } \\ & 9 / 5 / 01 & \mathrm{ND} & 7 & \mathrm{~Bq} / \mathrm{L} & \text { Blank } \\ & 10 / 29 / 01 & \mathrm{ND} & 7 & \mathrm{~Bq} / \mathrm{L} & \text { Blank } \\ & 11 / 28 / 01 & \mathrm{ND} & 7 & \mathrm{~Bq} / \mathrm{L} & \text { Blank } \\ & 12 / 18 / 01 & \mathrm{ND} & 7 & \mathrm{~Bq} / \mathrm{L} & \text { Blank }\end{array}$

\section{Vegetation Monitoring}

Tritium, Free Water

$\begin{array}{lllllr}\text { EEE6-Chip } & 9 / 12 / 01 & \text { ND } & 0.019 & \mathrm{~Bq} / \mathrm{g} & \text { Sample } \\ & 11 / 28 / 01 & \mathrm{ND} & 0.019 & \mathrm{~Bq} / \mathrm{g} & \text { Sample } \\ \text { EEE6-Duff } & 9 / 12 / 01 & \mathrm{ND} & 0.019 & \mathrm{~Bq} / \mathrm{g} & \text { Sample } \\ & 11 / 28 / 01 & 0.022 & 0.019 & \mathrm{~Bq} / \mathrm{g} & \text { Sample } \\ & 9 / 12 / 01 & 0.0269 & 0.019 & \mathrm{~Bq} / \mathrm{g} & \text { Sample } \\ \text { EEE6-Leaf } & 11 / 28 / 01 & \mathrm{ND} & 0.019 & \mathrm{~Bq} / \mathrm{g} & \text { Sample } \\ & & & & & \\ \text { NEE10-Chip } & 9 / 12 / 01 & \mathrm{ND} & 0.019 & \mathrm{~Bq} / \mathrm{g} & \text { Sample } \\ & 11 / 27 / 01 & \mathrm{ND} & 0.019 & \mathrm{~Bq} / \mathrm{g} & \text { Sample } \\ & & & & & \\ \text { NEE10-Duff } & 9 / 12 / 01 & \mathrm{ND} & 0.019 & \mathrm{~Bq} / \mathrm{g} & \text { Sample } \\ & 11 / 27 / 01 & \mathrm{ND} & 0.019 & \mathrm{~Bq} / \mathrm{g} & \text { Sample } \\ \text { NEE10-Leaf } & 9 / 12 / 01 & \mathrm{ND} & 0.019 & \mathrm{~Bq} / \mathrm{g} & \text { Sample } \\ & 11 / 27 / 01 & \mathrm{ND} & 0.019 & \mathrm{~Bq} / \mathrm{g} & \text { Sample } \\ & & & & & \\ \text { NNN5-Chip } & 9 / 13 / 01 & 0.154 & 0.019 & \mathrm{~Bq} / \mathrm{g} & \text { Sample } \\ & 11 / 29 / 01 & 0.166 & 0.019 & \mathrm{~Bq} / \mathrm{g} & \text { Sample } \\ & 11 / 29 / 01 & 0.167 & 0.002 & \mathrm{~Bq} / \mathrm{g} & \text { Split } \\ & 9 / 13 / 01 & 0.0522 & 0.019 & \mathrm{~Bq} / \mathrm{g} & \text { Sample } \\ \text { NNN5-Duff } & 11 / 29 / 01 & 0.169 & 0.019 & \mathrm{~Bq} / \mathrm{g} & \text { Sample } \\ & 11 / 29 / 01 & 0.136 & 0.003 & \mathrm{~Bq} / \mathrm{g} & \text { Split } \\ & 9 / 13 / 01 & 0.19 & 0.019 & \mathrm{~Bq} / \mathrm{g} & \text { Sample } \\ & 11 / 29 / 01 & 0.186 & 0.019 & \mathrm{~Bq} / \mathrm{g} & \text { Sample } \\ \text { NNN5-Leaf } & 11 / 29 / 01 & 0.694 & 0.003 & \mathrm{~Bq} / \mathrm{g} & \text { Split } \\ & & & & & \end{array}$


Units QC Type

\section{Vegetation Monitoring}

Tritium, Free Water cont.

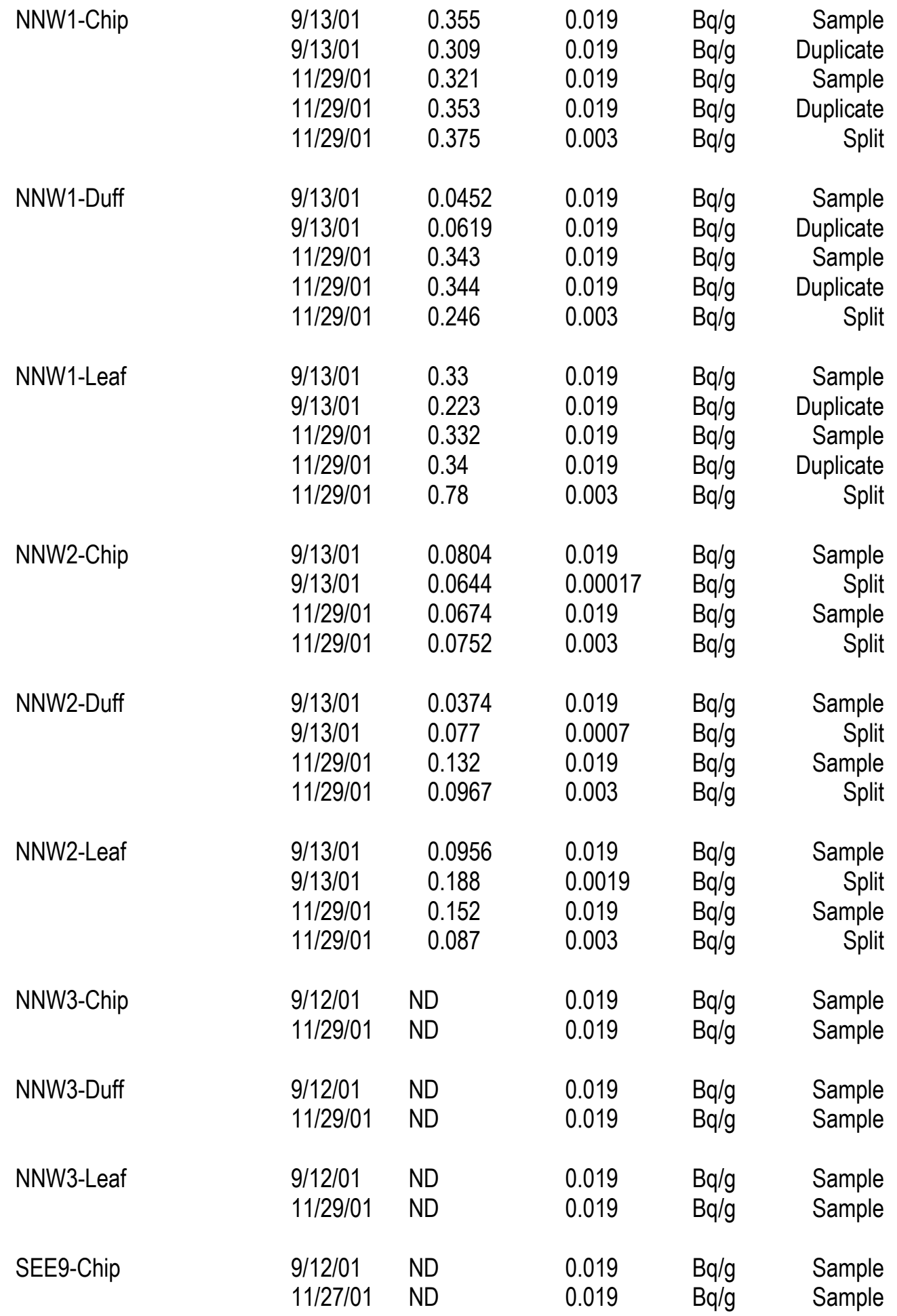




$\begin{array}{llllll}\text { Analyte } & \text { Location } & \text { Date } & \text { Result } & \text { MDA or PQL Units } & \text { QC Type }\end{array}$

\section{Vegetation Monitoring}

Tritium, Free Water cont.

\begin{tabular}{|c|c|c|c|c|c|}
\hline SEE9-Duff & $\begin{array}{l}9 / 12 / 01 \\
11 / 27 / 01\end{array}$ & $\begin{array}{l}\text { ND } \\
\text { ND }\end{array}$ & $\begin{array}{l}0.019 \\
0.019\end{array}$ & $\begin{array}{l}\mathrm{Bq} / \mathrm{g} \\
\mathrm{Bq} / \mathrm{g}\end{array}$ & $\begin{array}{l}\text { Sample } \\
\text { Sample }\end{array}$ \\
\hline SEE9-Leaf & $\begin{array}{l}9 / 12 / 01 \\
11 / 27 / 01\end{array}$ & $\begin{array}{l}\text { ND } \\
\text { ND }\end{array}$ & $\begin{array}{l}0.019 \\
0.019\end{array}$ & $\begin{array}{l}\mathrm{Bq} / \mathrm{g} \\
\mathrm{Bq} / \mathrm{g}\end{array}$ & $\begin{array}{l}\text { Sample } \\
\text { Sample }\end{array}$ \\
\hline SSE7-Chip & $\begin{array}{l}9 / 12 / 01 \\
11 / 28 / 01\end{array}$ & $\begin{array}{l}\text { ND } \\
\text { ND }\end{array}$ & $\begin{array}{l}0.019 \\
0.019\end{array}$ & $\begin{array}{l}\mathrm{Bq} / \mathrm{g} \\
\mathrm{Bq} / \mathrm{g}\end{array}$ & $\begin{array}{l}\text { Sample } \\
\text { Sample }\end{array}$ \\
\hline SSE7-Duff & $\begin{array}{l}9 / 12 / 01 \\
11 / 28 / 01\end{array}$ & $\begin{array}{l}\text { ND } \\
\text { ND }\end{array}$ & $\begin{array}{l}0.019 \\
0.019\end{array}$ & $\begin{array}{l}\mathrm{Bq} / \mathrm{g} \\
\mathrm{Bq} / \mathrm{g}\end{array}$ & $\begin{array}{l}\text { Sample } \\
\text { Sample }\end{array}$ \\
\hline SSE7-Leaf & $\begin{array}{l}9 / 12 / 01 \\
11 / 28 / 01\end{array}$ & $\begin{array}{l}\text { ND } \\
\text { ND }\end{array}$ & $\begin{array}{l}0.019 \\
0.019\end{array}$ & $\begin{array}{l}\mathrm{Bq} / \mathrm{g} \\
\mathrm{Bq} / \mathrm{g}\end{array}$ & $\begin{array}{l}\text { Sample } \\
\text { Sample }\end{array}$ \\
\hline WNW4-Chip & $\begin{array}{l}9 / 13 / 01 \\
11 / 28 / 01\end{array}$ & $\begin{array}{l}0.0297 \\
0.0231\end{array}$ & $\begin{array}{l}0.019 \\
0.019\end{array}$ & $\begin{array}{l}\mathrm{Bq} / \mathrm{g} \\
\mathrm{Bq} / \mathrm{g}\end{array}$ & $\begin{array}{l}\text { Sample } \\
\text { Sample }\end{array}$ \\
\hline WNW4-Duff & $\begin{array}{l}9 / 13 / 01 \\
11 / 28 / 01\end{array}$ & $\begin{array}{l}0.0552 \\
0.05\end{array}$ & $\begin{array}{l}0.019 \\
0.019\end{array}$ & $\begin{array}{l}\mathrm{Bq} / \mathrm{g} \\
\mathrm{Bq} / \mathrm{g}\end{array}$ & $\begin{array}{l}\text { Sample } \\
\text { Sample }\end{array}$ \\
\hline WNW4-Leaf & $\begin{array}{l}9 / 13 / 01 \\
11 / 28 / 01\end{array}$ & $\begin{array}{l}0.0911 \\
0.0481\end{array}$ & $\begin{array}{l}0.019 \\
0.019\end{array}$ & $\begin{array}{l}\mathrm{Bq} / \mathrm{g} \\
\mathrm{Bq} / \mathrm{g}\end{array}$ & $\begin{array}{l}\text { Sample } \\
\text { Sample }\end{array}$ \\
\hline WWW8-Chip & $\begin{array}{l}9 / 12 / 01 \\
11 / 28 / 01\end{array}$ & $\begin{array}{l}\text { ND } \\
\text { ND }\end{array}$ & $\begin{array}{l}0.019 \\
0.019\end{array}$ & $\begin{array}{l}\mathrm{Bq} / \mathrm{g} \\
\mathrm{Bq} / \mathrm{g}\end{array}$ & $\begin{array}{l}\text { Sample } \\
\text { Sample }\end{array}$ \\
\hline WWW8-Duff & $\begin{array}{l}9 / 12 / 01 \\
11 / 28 / 01\end{array}$ & $\begin{array}{l}\text { ND } \\
\text { ND }\end{array}$ & $\begin{array}{l}0.019 \\
0.019\end{array}$ & $\begin{array}{l}\mathrm{Bq} / \mathrm{g} \\
\mathrm{Bq} / \mathrm{g}\end{array}$ & $\begin{array}{l}\text { Sample } \\
\text { Sample }\end{array}$ \\
\hline WWW8-Leaf & $\begin{array}{l}9 / 12 / 01 \\
11 / 28 / 01\end{array}$ & $\begin{array}{l}\text { ND } \\
\text { ND }\end{array}$ & $\begin{array}{l}0.019 \\
0.019\end{array}$ & $\begin{array}{l}\mathrm{Bq} / \mathrm{g} \\
\mathrm{Bq} / \mathrm{g}\end{array}$ & $\begin{array}{l}\text { Sample } \\
\text { Sample }\end{array}$ \\
\hline EEE6-Chip & $\begin{array}{l}9 / 12 / 01 \\
11 / 28 / 01\end{array}$ & $\begin{array}{l}\text { ND } \\
\text { ND }\end{array}$ & $\begin{array}{l}0.19 \\
0.19\end{array}$ & $\begin{array}{l}\mathrm{Bq} / \mathrm{g} \\
\mathrm{Bq} / \mathrm{g}\end{array}$ & $\begin{array}{l}\text { Sample } \\
\text { Sample }\end{array}$ \\
\hline EEE6-Duff & $\begin{array}{l}9 / 12 / 01 \\
11 / 28 / 01\end{array}$ & $\begin{array}{l}0.55 \\
0.35\end{array}$ & $\begin{array}{l}0.2 \\
0.19\end{array}$ & $\begin{array}{l}\mathrm{Bq} / \mathrm{g} \\
\mathrm{Bq} / \mathrm{g}\end{array}$ & $\begin{array}{l}\text { Sample } \\
\text { Sample }\end{array}$ \\
\hline EEE6-Leaf & $\begin{array}{l}9 / 12 / 01 \\
11 / 28 / 01\end{array}$ & $\begin{array}{l}0.26 \\
0.39\end{array}$ & $\begin{array}{l}0.19 \\
0.19\end{array}$ & $\begin{array}{l}\mathrm{Bq} / \mathrm{g} \\
\mathrm{Bq} / \mathrm{g}\end{array}$ & $\begin{array}{l}\text { Sample } \\
\text { Sample }\end{array}$ \\
\hline NEE10-Chip & $\begin{array}{l}9 / 12 / 01 \\
11 / 27 / 01\end{array}$ & $\begin{array}{l}\text { ND } \\
\text { ND }\end{array}$ & $\begin{array}{l}0.19 \\
0.19\end{array}$ & $\begin{array}{l}\mathrm{Bq} / \mathrm{g} \\
\mathrm{Bq} / \mathrm{g}\end{array}$ & $\begin{array}{l}\text { Sample } \\
\text { Sample }\end{array}$ \\
\hline
\end{tabular}


Units QC Type

\section{Vegetation Monitoring}

Tritium, Organically Bound cont.

\begin{tabular}{|c|c|c|c|c|c|}
\hline NEE10-Duff & $\begin{array}{l}9 / 12 / 01 \\
11 / 27 / 01\end{array}$ & $\begin{array}{l}\text { ND } \\
\text { ND }\end{array}$ & $\begin{array}{l}0.19 \\
0.19\end{array}$ & $\begin{array}{l}\mathrm{Bq} / \mathrm{g} \\
\mathrm{Bq} / \mathrm{g}\end{array}$ & $\begin{array}{l}\text { Sample } \\
\text { Sample }\end{array}$ \\
\hline NEE10-Leaf & $\begin{array}{l}9 / 12 / 01 \\
11 / 27 / 01\end{array}$ & $\begin{array}{l}\text { ND } \\
\text { ND }\end{array}$ & $\begin{array}{l}0.19 \\
0.19\end{array}$ & $\begin{array}{l}\mathrm{Bq} / \mathrm{g} \\
\mathrm{Bq} / \mathrm{g}\end{array}$ & $\begin{array}{l}\text { Sample } \\
\text { Sample }\end{array}$ \\
\hline NNN5-Chip & $\begin{array}{l}9 / 13 / 01 \\
11 / 29 / 01 \\
11 / 29 / 01\end{array}$ & $\begin{array}{l}\text { ND } \\
\text { ND } \\
0.0415\end{array}$ & $\begin{array}{l}0.19 \\
0.19 \\
0.0015\end{array}$ & $\begin{array}{l}\mathrm{Bq} / \mathrm{g} \\
\mathrm{Bq} / \mathrm{g} \\
\mathrm{Bq} / \mathrm{g}\end{array}$ & $\begin{array}{r}\text { Sample } \\
\text { Sample } \\
\text { Split }\end{array}$ \\
\hline NNN5-Duff & $\begin{array}{l}9 / 13 / 01 \\
11 / 29 / 01 \\
11 / 29 / 01\end{array}$ & $\begin{array}{l}3.11 \\
2.88 \\
2.30\end{array}$ & $\begin{array}{l}0.19 \\
0.19 \\
0.0011\end{array}$ & $\begin{array}{l}\mathrm{Bq} / \mathrm{g} \\
\mathrm{Bq} / \mathrm{g} \\
\mathrm{Bq} / \mathrm{g}\end{array}$ & $\begin{array}{r}\text { Sample } \\
\text { Sample } \\
\text { Split }\end{array}$ \\
\hline NNN5-Leaf & $\begin{array}{l}9 / 13 / 01 \\
11 / 29 / 01 \\
11 / 29 / 01\end{array}$ & $\begin{array}{l}1.13 \\
1.48 \\
0.593\end{array}$ & $\begin{array}{l}0.19 \\
0.19 \\
0.0011\end{array}$ & $\begin{array}{l}\mathrm{Bq} / \mathrm{g} \\
\mathrm{Bq} / \mathrm{g} \\
\mathrm{Bq} / \mathrm{g}\end{array}$ & $\begin{array}{r}\text { Sample } \\
\text { Sample } \\
\text { Split }\end{array}$ \\
\hline NNW1-Chip & $\begin{array}{l}9 / 13 / 01 \\
9 / 13 / 01 \\
11 / 29 / 01 \\
11 / 29 / 01 \\
11 / 29 / 01\end{array}$ & $\begin{array}{l}\text { ND } \\
\text { ND } \\
0.2 \\
\text { ND } \\
0.143\end{array}$ & $\begin{array}{l}0.19 \\
0.19 \\
0.19 \\
0.19 \\
0.0019\end{array}$ & $\begin{array}{l}\mathrm{Bq} / \mathrm{g} \\
\mathrm{Bq} / \mathrm{g} \\
\mathrm{Bq} / \mathrm{g} \\
\mathrm{Bq} / \mathrm{g} \\
\mathrm{Bq} / \mathrm{g}\end{array}$ & $\begin{array}{r}\text { Sample } \\
\text { Duplicate } \\
\text { Sample } \\
\text { Duplicate } \\
\text { Split }\end{array}$ \\
\hline NNW1-Duff & $\begin{array}{l}9 / 13 / 01 \\
9 / 13 / 01 \\
11 / 29 / 01 \\
11 / 29 / 01 \\
11 / 29 / 01\end{array}$ & $\begin{array}{c}8.81 \\
10.8 \\
4.41 \\
5.26 \\
4.89\end{array}$ & $\begin{array}{l}0.19 \\
0.19 \\
0.19 \\
0.19 \\
0.0011\end{array}$ & $\begin{array}{l}\mathrm{Bq} / \mathrm{g} \\
\mathrm{Bq} / \mathrm{g} \\
\mathrm{Bq} / \mathrm{g} \\
\mathrm{Bq} / \mathrm{g} \\
\mathrm{Bq} / \mathrm{g}\end{array}$ & $\begin{array}{r}\text { Sample } \\
\text { Duplicate } \\
\text { Sample } \\
\text { Duplicate } \\
\text { Split }\end{array}$ \\
\hline NNW1-Leaf & $\begin{array}{l}9 / 13 / 01 \\
9 / 13 / 01 \\
11 / 29 / 01 \\
11 / 29 / 01 \\
11 / 29 / 01\end{array}$ & $\begin{array}{l}1.51 \\
1.81 \\
1.06 \\
1.66 \\
1.44\end{array}$ & $\begin{array}{l}0.19 \\
0.19 \\
0.19 \\
0.19 \\
0.0011\end{array}$ & $\begin{array}{l}\mathrm{Bq} / \mathrm{g} \\
\mathrm{Bq} / \mathrm{g} \\
\mathrm{Bq} / \mathrm{g} \\
\mathrm{Bq} / \mathrm{g} \\
\mathrm{Bq} / \mathrm{g}\end{array}$ & $\begin{array}{r}\text { Sample } \\
\text { Duplicate } \\
\text { Sample } \\
\text { Duplicate } \\
\text { Split }\end{array}$ \\
\hline NNW2-Chip & $\begin{array}{l}9 / 13 / 01 \\
9 / 13 / 01\end{array}$ & $\begin{array}{l}\text { ND } \\
0.03\end{array}$ & $\begin{array}{l}0.19 \\
0.0013\end{array}$ & $\begin{array}{l}\mathrm{Bq} / \mathrm{g} \\
\mathrm{Bq} / \mathrm{g}\end{array}$ & $\begin{array}{r}\text { Sample } \\
\text { Split }\end{array}$ \\
\hline NNW2-Chip & $\begin{array}{l}11 / 29 / 01 \\
11 / 29 / 01\end{array}$ & $\begin{array}{l}\text { ND } \\
0.0374\end{array}$ & $\begin{array}{l}0.19 \\
0.0011\end{array}$ & $\begin{array}{l}\mathrm{Bq} / \mathrm{g} \\
\mathrm{Bq} / \mathrm{g}\end{array}$ & $\begin{array}{r}\text { Sample } \\
\text { Split }\end{array}$ \\
\hline NNW2-Duff & $\begin{array}{l}9 / 13 / 01 \\
9 / 13 / 01 \\
11 / 29 / 01 \\
11 / 29 / 01\end{array}$ & $\begin{array}{l}0.896 \\
1.41 \\
0.893 \\
2.12\end{array}$ & $\begin{array}{l}0.2 \\
0.0016 \\
0.19 \\
0.003\end{array}$ & $\begin{array}{l}\mathrm{Bq} / \mathrm{g} \\
\mathrm{Bq} / \mathrm{g} \\
\mathrm{Bq} / \mathrm{g} \\
\mathrm{Bq} / \mathrm{g}\end{array}$ & $\begin{array}{r}\text { Sample } \\
\text { Split } \\
\text { Sample } \\
\text { Split }\end{array}$ \\
\hline
\end{tabular}




$\begin{array}{llllll}\text { Analyte } & \text { Location } & \text { Date } & \text { Result } & \text { MDA or PQL Units } & \text { QC Type }\end{array}$

\section{Vegetation Monitoring}

Tritium, Organically Bound cont. NNW2-Leaf

$\begin{array}{llllr}\text { 9/13/01 } & 0.874 & 0.19 & \mathrm{~Bq} / \mathrm{g} & \text { Sample } \\ 9 / 13 / 01 & 0.61 & 0.0012 & \mathrm{~Bq} / \mathrm{g} & \text { Split } \\ 11 / 29 / 01 & 0.863 & 0.19 & \mathrm{~Bq} / \mathrm{g} & \text { Sample } \\ 11 / 29 / 01 & 0.706 & 0.0011 & \mathrm{~Bq} / \mathrm{g} & \text { Split }\end{array}$

NNW3-Chip

9/12/01 ND

0.19

$\mathrm{Bq} / \mathrm{g}$

Sample

$11 / 29 / 01 \quad N D$

0.19

$\mathrm{Bq} / \mathrm{g}$

Sample

NNW3-Duff

9/12/01 ND

0.19

$\mathrm{Bq} / \mathrm{g}$

Sample

$11 / 29 / 01 \quad N D$

0.19

$\mathrm{Bq} / \mathrm{g}$

Sample

NNW3-Leaf

9/12/01 ND

0.19

$\mathrm{Bq} / \mathrm{g}$

Sample

$11 / 29 / 01 \quad N D$

0.19

$\mathrm{Bq} / \mathrm{g}$

Sample

SEE9-Chip

9/12/01 ND

0.19

$\mathrm{Bq} / \mathrm{g}$

Sample

11/27/01 ND

0.19

$\mathrm{Bq} / \mathrm{g}$

Sample

SEE9-Duff

ND

0.19

$\mathrm{Bq} / \mathrm{g}$

Sample

11/27/01 ND

0.19

$\mathrm{Bq} / \mathrm{g}$

Sample

SEE9-Leaf

9/12/01 ND

0.19

0.19

$\mathrm{Bq} / \mathrm{g}$

Sample

11/27/01 ND

$\mathrm{Bq} / \mathrm{g}$

Sample

SSE7-Chip

9/12/01 ND

0.19

$\mathrm{Bq} / \mathrm{g}$

Sample

$11 / 28 / 01 \quad N D$

0.19

$\mathrm{Bq} / \mathrm{g}$

Sample

SSE7-Duff

9/12/01 ND

0.19

$\mathrm{Bq} / \mathrm{g}$

Sample

$11 / 28 / 01 \quad N D$

0.19

$\mathrm{Bq} / \mathrm{g}$

Sample

SSE7-Leaf

9/12/01 ND

0.19

$\mathrm{Bq} / \mathrm{g}$

Sample

$11 / 28 / 01$

ND

0.19

$\mathrm{Bq} / \mathrm{g}$

Sample

WNW4-Chip

9/13/01 ND

0.19

$\mathrm{Bq} / \mathrm{g}$

11/28/01 ND

0.19

$\mathrm{Bq} / \mathrm{g}$

Sample

WNW4-Duff

$9 / 13 / 01 \quad 0.49$

0.19

$11 / 28 / 01$

0.46

0.2

$\mathrm{Bq} / \mathrm{g}$

$\mathrm{Bq} / \mathrm{g}$

Sample

WNW4-Leaf

$9 / 13 / 01 \quad 0.656$

$11 / 28 / 01$

0.933

0.19

0.19

$\mathrm{Bq} / \mathrm{g}$

$\mathrm{Bq} / \mathrm{g}$

Sample

WWW8-Chip

9/12/01 ND

0.19

0.19

$\mathrm{Bq} / \mathrm{g}$

$\mathrm{Bq} / \mathrm{g}$

Sample

11/28/01 ND

Sample 
Units QC Type

\section{Vegetation Monitoring}

Tritium, Organically Bound cont WWW8-Duff

$\begin{array}{llllr}9 / 12 / 01 & \text { ND } & 0.19 & \begin{array}{l}\mathrm{Bq} / \mathrm{g} \\ \mathrm{Bq} / \mathrm{g}\end{array} & \begin{array}{r}\text { Sample } \\ \text { Sample }\end{array} \\ \text { 11/28/01 } & \text { ND } & 0.19 & & \\ \text { 9/12/01 } & \text { ND } & 0.19 & \mathrm{~Bq} / \mathrm{g} & \text { Sample } \\ 11 / 28 / 01 & \mathrm{ND} & 0.19 & \mathrm{~Bq} / \mathrm{g} & \text { Sample } \\ & & & & \\ 9 / 19 / 01 & \mathrm{ND} & 7 & \mathrm{~Bq} / \mathrm{L} & \text { Sample } \\ 1 / 17 / 02 & \mathrm{ND} & 7 & \mathrm{~Bq} / \mathrm{L} & \text { Sample } \\ & & & & \\ 9 / 24 / 01 & 504 & 7 & \mathrm{~Bq} / \mathrm{L} & \text { Sample } \\ 9 / 24 / 01 & 481 & 7 & \mathrm{~Bq} / \mathrm{L} & \text { Duplicate } \\ 1 / 3 / 02 & 324 & 7 & \mathrm{~Bq} / \mathrm{L} & \text { Sample } \\ 1 / 3 / 02 & 422 & 7 & \mathrm{~Bq} / \mathrm{L} & \text { Duplicate } \\ 1 / 3 / 02 & 356 & 6 & \mathrm{~Bq} / \mathrm{L} & \text { Split }\end{array}$

NNW2-TW

$9 / 24 / 01 \quad 137$

$9 / 24 / 01 \quad 137$

$1 / 3 / 02 \quad 145$

$1 / 3 / 02 \quad 139$

$\begin{array}{lr}\mathrm{Bq} / \mathrm{L} & \text { Sample } \\ \mathrm{Bq} / \mathrm{L} & \text { Split } \\ \mathrm{Bq} / \mathrm{L} & \text { Sample } \\ \mathrm{Bq} / \mathrm{L} & \text { Split }\end{array}$

NNW3-TW

$9 / 19 / 01 \quad 16$

$1 / 3 / 02 \quad 14$

\section{$\mathrm{Bq} / \mathrm{L} \quad$ Sample} $\mathrm{Bq} / \mathrm{L} \quad$ Sample

SEE9-TW

9/19/01 ND

$1 / 3 / 02$ ND

$\begin{array}{ll}\mathrm{Bq} / \mathrm{L} & \text { Sample } \\ \mathrm{Bq} / \mathrm{L} & \text { Sample }\end{array}$

